\title{
Conjugated Macrocycles in Organic Electronics
}

\author{
Melissa L. Ball
}

Submitted in partial fulfillment of the

requirements for the degree of

Doctor of Philosophy

in the Graduate School of Arts and Sciences

\section{COLUMBIA UNIVERSITY}


(C) 2019

Melissa L. Ball

All Rights Reserved 


\section{ABSTRACT \\ Conjugated Macrocycles in Organic Electronics}

\section{Melissa L. Ball}

The discipline of organic electronics encompasses the design and synthesis of molecules for use in organic field effect transistors, organic photovoltaics, organic photodetectors, single molecule electronics, sensors, and many more. The rationale for studying organic electronic materials is compelling: organics have the potential to be low cost, processable, and flexible complements to silicon technologies to combat some of the most pressing environmental issues.

Organic molecules that transport carriers are used as the active layer in many device applications. Molecules that possess energy levels that allow for electron or hole transport are typically $\pi$-conjugated materials. There has been swift progress on the design and synthesis of $\pi$ conjugated materials that possess a large density of high energy electrons such as acenes. Yet there has been less growth on materials with low energy vacant orbitals to accept an electron. Fullerenes are the ubiquitous acceptor materials used in organic electronics. Over the past few years, there have been several groups, including our own, that have synthesized non-fullerene materials for use in organic field effect transistors and solar cells. In particular, the Nuckolls laboratory has pioneered the design and synthesis of a class of molecules called contorted aromatics and studied these molecules in range of organic electronic applications. Conjugated macrocycles are one subclass of the contorted aromatic family.

This Thesis describes a body of research on the design, synthesis, and application of a new class of electronic materials made from conjugated macrocycles. Each of the macrocycles comprises perylenediimide cores wound together with various electronic linkers. The perylenediimide building block endows each macrocycle with the ability to transport electrons, 
while the synthetic flexibility to install different linkers allows us to create macrocycles with different electronic and physical properties.

We use these materials in organic photovoltaics, field effect transistors, sensors, and photodetectors. The macrocycles possess vivid colors, absorb in the visible range of the solar spectrum, and are an exemplary class of materials to study how rigidity and strain affect device performance. We find that the strained and rigid macrocyclic framework affords each macrocycle with the ability to absorb lower energy visible light with respect to acyclic counterparts and the macrocycles outperform in photovoltaic applications. Rigidity was an important concept in our organic photodetector study: we found rigidity was one of the reasons our macrocycles outperformed both fullerenes and acyclic controls. The macrocycles all possess intramolecular cavities, and our recent studies focused on using this nanospace for sensing applications. Each of the studies described in this Thesis will demonstrate how macrocyclization is a design technique to enhance organic electronic performance. 


\section{Table of Contents}

List of Tables and Figures................................................................................................ ix

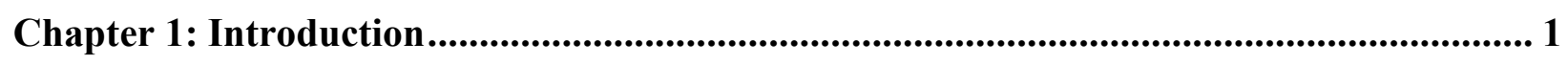

Chapter 1 Section 1A. Contorted Aromatics as Electronic Materials ..................... 1

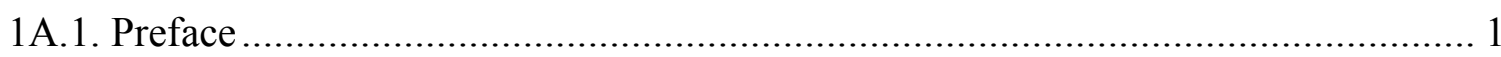

1A.2. Introduction....................................................................................... 1

1A.3. Output and Transport Curves: What Do They Tell Us?................................... 2

1A.4. A Chemist's Approach to Understanding Electronic Materials .......................... 4

1A.6. Contorted Aromatics as Electronic Materials................................................... 7

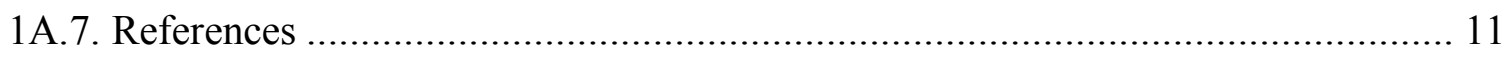

Chapter 1 Section 1B. Conjugated Macrocycles in Organic Electronics................... 15

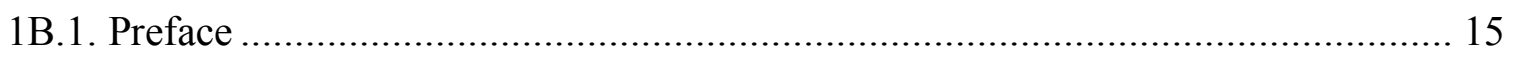

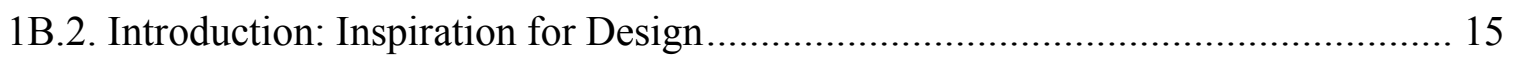

1B.3. Synthetic Approaches to Build in Strain .................................................... 17

1B.4. Bay Substitution of PDI Induces Chirality ................................................. 19

1B.5. Rigidity in the Macrocycles and its Effects on Isomerization .......................... 21

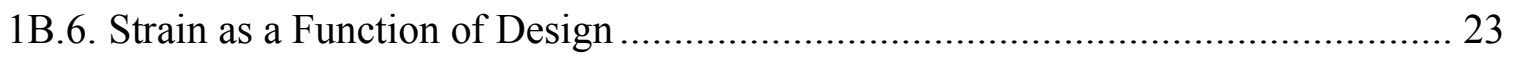

1B.7. Macrocyclic Design Promotes Absorption of Visible Light ........................... 25

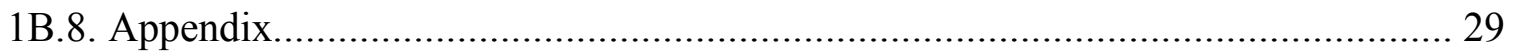




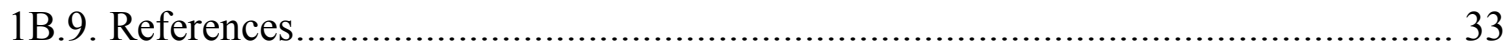

Chapter 2. Chiral Conjugated Corrals.............................................................................................. 37

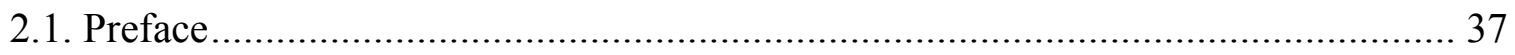

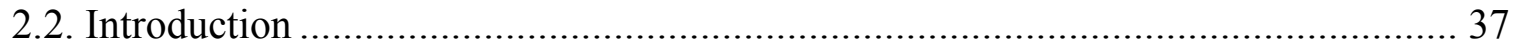

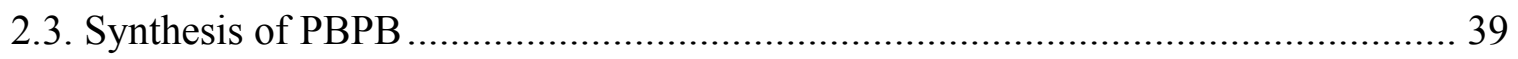

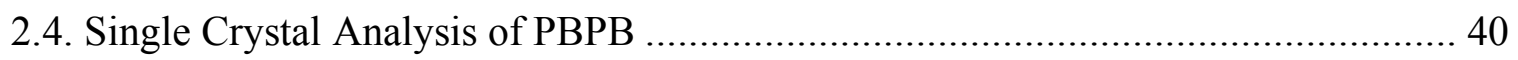

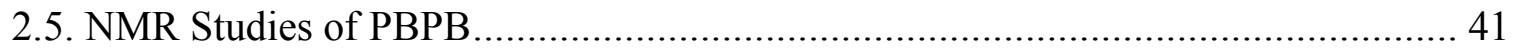

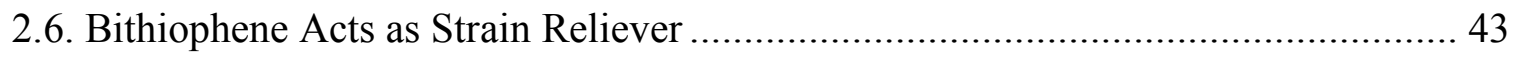

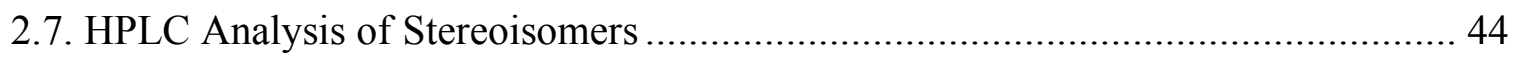

2.8. Intramolecular Somersault Mode of Isomerization .............................................. 46

2.9. Charge Transfer Characteristics in PBPB ……….............................................. 47

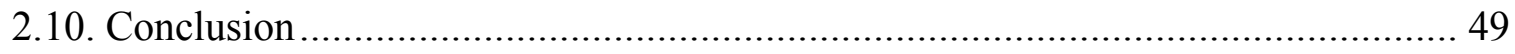

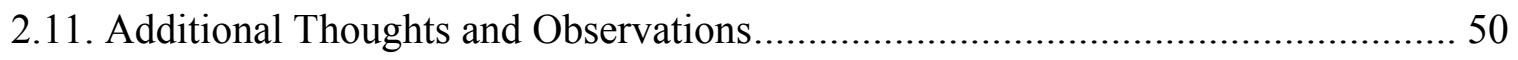

2.12. Appendix - Supplementary Figures.................................................................. 51

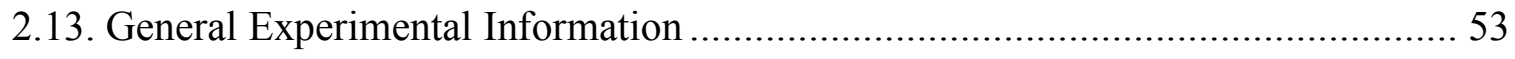

2.14. Synthetic Procedures and Characterization .................................................... 58

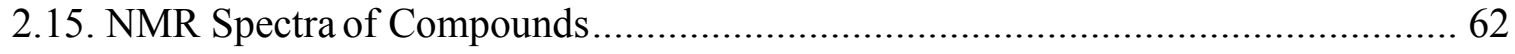

2.16. DFT Output

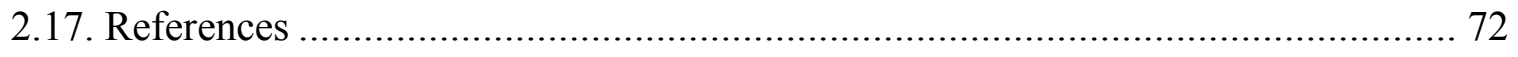


Chapter 3. Macrocyclization in the Design of Organic n-Type Electronic Materials......... 76

3.1. Preface

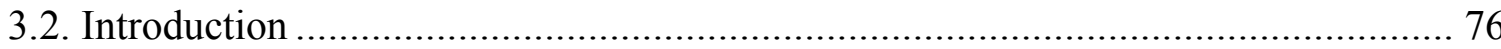

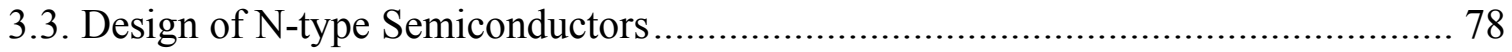

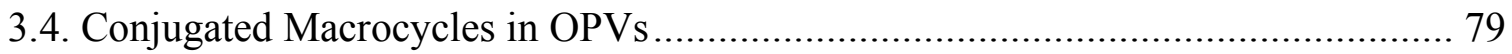

3.5. Electrochemistry of N-type Semiconductors.......................................... 83

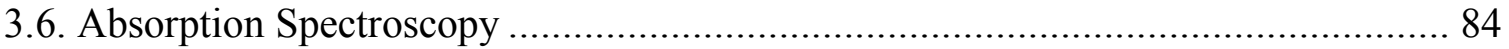

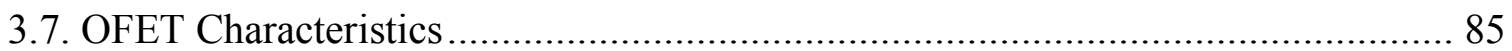

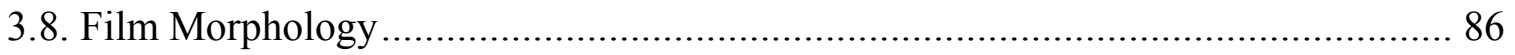

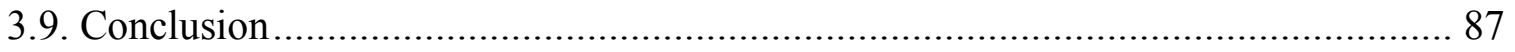

3.10. Appendix - Supplementary Figures.................................................. 88

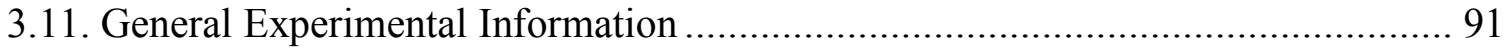

3.12. Synthetic Procedures and Characterization ................................................... 94

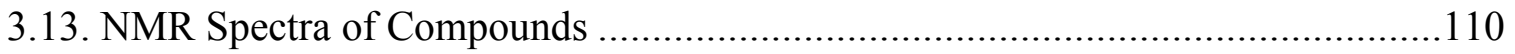

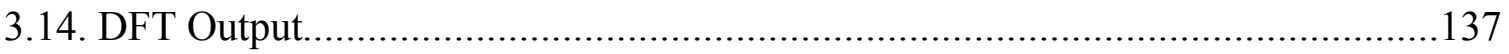

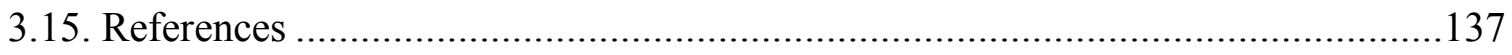

Chapter 4. The Importance of Intramolecular Conductivity in Three Dimensional Molecular

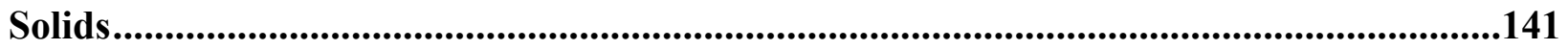

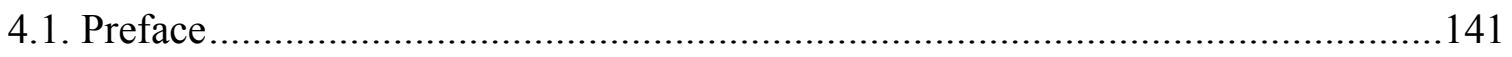

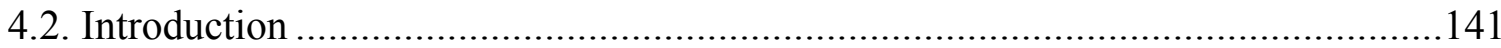


4.3. OFETs - Trans Linkage Produces Higher Current

.143

4.4. Film Morphology

4.5. Molecular Structures of Isomeric Macrocycles.

4.6. Cyclic Voltammetry Shows Similar Reduction Potentials

4.7. STM-BJ Studies Show Trans Conducts Better Than Cis Isomer.

4.8. Acyclic Controls Possess Analogous Electron Mobility in OFETs 150

4.9. Appendix - Supplementary Figures

4.10. General Experimental Information .....

4.11. Synthetic Procedures \& Characterization. 156

4.12. NMR Spectra of Compounds 163

4.13. DFT Output...... 170

4.14. References .196

Chapter 5. Rigid, Conjugated Macrocycles for High Performance Organic Photodetectors

5.1. Preface. .201

5.2. Introduction .201

5.3. OPD Results for $\left(\mathrm{PPh}_{2}\right)_{4}$ .203

5.4. Transient Absorption Shows Fast Charge Separation ..... 206

5.5 $\mathrm{PC}_{71} \mathrm{BM}$ Shows High Dark Current Density .208

5.6. Rigid Macrocyclic Design Impedes Charge Defects .209 
5.7. Conclusion

5.8. Appendix - Supplementary Figures

5.9. General Experimental Information.

5.10. DFT Output

5.11. References

6.1. Preface.

6.2. Introduction

6.3. Capsule Construction .....

6.4. Capsule Structure.

6.5. Cellular Solids from Capsular Nanostructures.

6.6. Electron Transport through Cellular Films.

6.7. Conclusion.

6.8. Appendix - Supplementary Figures

6.9. General Experimental Information

6.10. Synthetic Procedures and Characterizations

6.13. References 
Chapter 7. Chiral PDI as Electron Deficient Moiety in Locked Cycloparaphenylene .......258

7.1. Preface .258

7.2. Introduction .258

7.3. Design of Conformationally Restricted Macrocycle .259

7.4. Synthesis of $\mathrm{PPh}_{6}-\mathrm{PhHex}$ .260

7.5. Strain Originates from Aryl-PDI Dihedral .261

7.6. Size-Dependent Isomerization .262

7.7. CD Measurements Show $\mathrm{PPh}_{6}-\mathrm{PhHex}$ is Conformationally Locked .263

7.8. Incorporation of PDI Alters Electronic Structure of $\mathrm{PPh}_{6}-\mathrm{PhHex}$ .264

7.9. Conclusion. 266

7.10. Appendix - Supplementary Figures....... .266

7.11. General Experimental Information .268

7.12. Synthetic Procedures and Characterization. .269

7.13. NMR Spectra of Compounds .272

7.14. DFT Output. .274

7.15. References .309

Chapter 8. The Influence of Molecular Conformation on Electron Transport in Giant, Conjugated Macrocycles.

8.1. Preface. .312

8.2. Introduction .312 
8.10. Conclusion

8.11. Appendix - Supplementary Figures

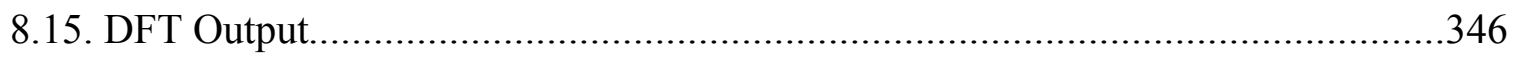

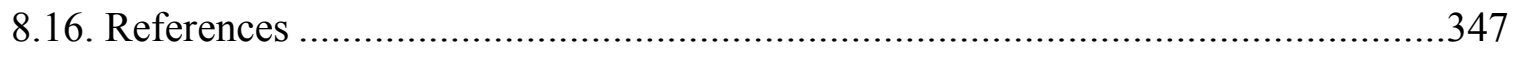

Chapter 9. Conjugated PDI Macrocycles as Electronic Hosts ...................................................350

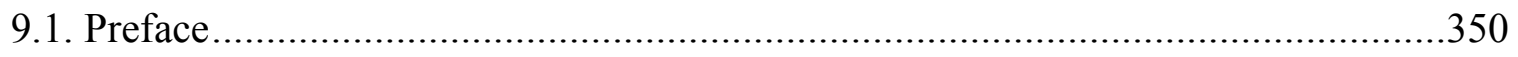

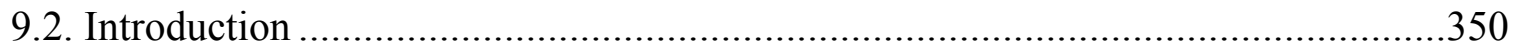

9.3. NMR Spectroscopy Shows Formation of Supramolecular Complex ........................351

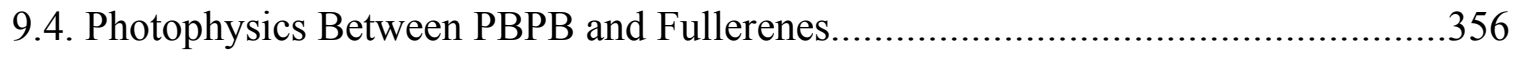


9.5. Potential Applications for PBPB-Fullerene Complex

9.6. Future Directions for Conjugated Macrocycles.

9.7. Appendix - Supplementary Figures...

9.8. General Experimental Information. .372

9.9. NMR Spectra of aryl-PBPB . .374

9.10. DFT Output 374

9.11. References ...... .395 


\section{List of Tables and Figures}

Figure 1A.1. (a) A schematic of an OFET device. The semiconducting layer comprises a conjugated molecule with p-type characteristics, n-type characteristics, or both; (b) a representative output curve showing the current between the source and drain electrodes as a function of a gate bias; we show the device is saturated at a gate bias of $80 \mathrm{~V}$ and transports electrons; and (c) a representative transfer curve that shows the current generated between the source and drain electrodes as a function of sweeping the gate.

Figure 1A.2. (a) Common donor molecules and (b) common acceptor molecules used to construct small molecules and polymers for organic electronics; (c) design strategies to create electronic materials; and (d) $\mathrm{C}_{60}$.

Figure 1A.3. Representative molecule of two contorted aromatics (a) HBC and (b) DBTCC. Both HBC and DBTCC are composed of [4] helicene structures outlined in red; (c) hPDI tetramer. One of the helical PDI series that showed exceptional solar cell performance; and (d) a donor-acceptor $\mathrm{hPDI}_{3}$-based ribbon, where the HOMO resides on a pyrene-centered orbital and the LUMO resides on a $\mathrm{hPDI}_{3}$-centered orbital.

Figure 1A.4. (a) Helical PDI oligomer family. The imide position creates an area of low-energy unoccupied space on the molecule, the bay position can be readily substituted. (b) The most common substitution patterns are 1,7 and 1,6 which will be discussed in the following chapters. The R groups can be altered to affect processing of material and photophysical properties; (c) the helical conformation of the hPDI oligomers using DFT performed by Dr. Michael L. Steigerwald.

Figure 1B.1. The five conjugated macrocycles: (a) PPh 6 -PhHex; (b) PBPB; (c) (PBBr 4$)_{3}$; (d) $\left(\mathbf{P P h}_{2}\right)_{4}$; and (e) DBDB. 16

Figure 1B.2. (a) Synthetic approach utilizing an unstrained precursor to perform the macrocyclization before the final aromatization step; (b) a Pt-based strategy that relies on transmetalation from an aryl-tin bond to an aryl- Pt bond. $\mathrm{R}=$ alkyl, alkoxy groups. 18

Figure 1B.3. (a) $P$-diphenyl PDI $(P$-1.1) and (b) $M$-diphenyl PDI $(M-1.1)$ from DFT calculations carried out at 6-31G/B3LYP level of theory; (c) and (d) show how the $P$ and $M$ designations are assigned. One looks down the stereogenic axis (indicated with a yellow dot) and assigns the groups off the top naphthyl (solid line) a 1 or 2 priority before assigning 3 and 4 to the bottom naphthyl (dashed line) ring substituents. 20

Figure 1B.4. Monomeric PDI-based macrocycles. The smallest macrocycles are (a) $\left(\mathbf{P B B r}_{4}\right)_{3}$ and (b) $\mathbf{P P h} 6-\mathbf{P h H e x}$; (c) PBPB; and the largest cavity is for (d) (PPh $\mathbf{P h}_{4}$. 22

Figure 1B.5. (a) An example of an homodesmotic reaction to calculate the strain in cyclobutane. Two carbon-carbon bonds must break and then form in order to yield the hexane product. The two products possess a $\Delta \mathrm{H}_{\mathrm{f}}$ of -237.061 hartrees $(1$ hartree $=627.51 \mathrm{kcal} / \mathrm{mol})$, and $\Delta \mathrm{H}_{\mathrm{f}}$ is -237.104 for hexane. The difference of $43 \mathrm{mH} / 27.2 \mathrm{kcal} / \mathrm{mol}$ is close to the experimentally determined value of $26.5 \mathrm{kcal} / \mathrm{mol}$. (b) The aryl-aryl distance in an unsubstituted diphenyl PDI (Compound 
1.1) and $\mathbf{P P h}$-PhHex. Our findings suggest a negative correlation between aryl-aryl distance and strain: as this distance increases, the strain energy decreases................................................... 23

Table 1B.1. The transannular aryl-aryl distance from the phenyl rings adjacent to the PDI ...... 24

Figure 1B.6. (a) Vials containing the macrocycles in micromolar solutions. (b) The UV-Vis absorption spectrum of the four PDI-based macrocycles are at $10^{-5} \mathrm{M}$. ................................... 25

Table 1B.2: Electrochemistry, UV/vis and DFT Data for Conjugated Macrocycles.................. 26

Figure 2.1. (a) Schematic structure of the -a-b-a-b pattern for PBPB. (b) The stereoisomerism of PBPB results from the chirality of the $(P)$ - and $(M)$-1,7-diphenyl-PDI (1.1) subunits. (c) PBPB exists in chiral $[(P, P)-\mathbf{P B P B}$ and $(M, M)$-PBPB $]$ and meso $[(M, P)$-PBPB $]$ forms.................... 38

Figure 2.2. Scheme for synthesis of PBPB. (a) $\mathrm{Pt}(\mathrm{COD}) \mathrm{Cl}_{2}$, toluene, $100{ }^{\circ} \mathrm{C}, 24.5 \mathrm{~h} 63 \%$ yield. (b) 5,5' bis(trimethylstannyl)-2,2'-bithiophene, THF, $55{ }^{\circ} \mathrm{C}, 48 \mathrm{~h}$. (c) $\mathrm{PPh}_{3}$, toluene, $100{ }^{\circ} \mathrm{C}, 12 \mathrm{~h}$,

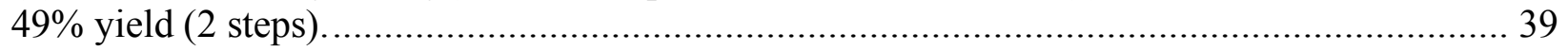

Figure 2.3. (a) Two adjacent diphenyl PDIs. The shortest PDI-PDI distance is $\sim 3.7 \AA$. Only one PDI unit of $(P, P)$-PBPB (red) and $(M, M)$-PBPB (blue) is shown; (b) a one-dimensional molecular wire formed from $\pi-\pi$ interactions between neighboring macrocycles viewed down the a axis. Side chains and hydrogen atoms have been removed for clarity; and (c) a view down the $b$ axis showing adjacent PBPB wires. Carbon $=$ gray, nitrogen $=$ blue, oxygen $=$ red, sulfur $=$ yellow. Hydrogen atoms have been removed to clarify the view. 41

Figure 2.4. (a) Downfield region of the ${ }^{1} \mathrm{HNMR}$ spectrum of macrocycle PBPB recorded at 88 ${ }^{\circ} \mathrm{C}$. The red squares (chiral) and blue circles (achiral) identify resonances from the two stereoisomers. (b) DFT minimized model (side-on and face-on views) of chiral stereoisomer $(M, M)$-PBPB. (c) DFT minimized model (side-on and face-on views) of meso stereoisomer $(P, M)$ PBPB (undecyl sidechains have been truncated to a methyl group to simplify the calculations). Carbon $=$ grey, nitrogen $=$ blue, oxygen $=$ red, sulfur $=$ yellow. Hydrogens have been removed to clarify the view..... 43

Figure 2.5. (a) HPLC chromatogram of PBPB using a CHIRALPAK ${ }^{\circledR}$ IA-3 column. (b) HPLCCD chromatogram of PBPB showing peaks A and C exhibit opposite optical activity (270-410 $\mathrm{nm}$ ). (c) CD spectra for both enantiomers of PBPB. (D) Interconversion of the stereoisomers of PBPB by sequential rotation of one PDI subunit through the macrocycle and then rotation of the other PDI to convert between enantiomers. The hydrocarbon sidechains have been replaced with a methyl group in the DFT structures. Carbon $=$ grey, nitrogen $=$ blue, oxygen $=$ red, sulfur $=$ yellow. Hydrogen atoms have been removed to clarify the view. 45

Figure 2.6. (a) The interconversion of $\mathbf{C}$ to $\mathbf{A}$ through the intermediacy of $\mathbf{B}$. (b) Plot of relative intensities (via integration of the area under each peak) as a function of time.... 47

Figure 2.7. (a) UV-vis absorption spectrum of PBPB (black line), 5,5'-bis(tributylstannyl)-2,2'bithiophene (yellow) and 1.1 (diphenyl PDI), red) in $\mathrm{CH}_{2} \mathrm{Cl}_{2}$ (concentration $=1.6 \times 10^{-5} \mathrm{M}, 1.3 \times$ $10^{-4}$ and $1.5 \times 10^{-5}$, respectively, path length $=1.0 \mathrm{~cm}$ ). The yellow trace is plotted with respect to the right ordinate, and the black and red traces are plotted with respect to the left ordinate; (b) CV 
of PBPB showing two reversible reduction and one reversible oxidation peak; (c) and (d) the HOMO and LUMO orbitals of PBPB from TDDFT, respectively........................................ 48

Figure 2.8. (a) A proposed mechanism for the reversibility of the transmetalation step when $\mathrm{R}_{1}$ and $\mathrm{R}_{2}$ are different substituents, but have similar electronics; and (b) reversibility is not observed in our system as there is a large difference in the aryl groups electronics e.g. one if electron rich and the other is electron deficient. 50

Figure 2.9. (a) ${ }^{1} \mathrm{H} N M R$ of $\mathbf{P B P B}$ at $300 \mathrm{~K}$ in $\mathrm{C}_{2} \mathrm{D}_{2} \mathrm{Cl}_{4}$. (b) ${ }^{1} \mathrm{HNMR}$ of $\mathbf{P B P B}$ at $360 \mathrm{~K}$ in $\mathrm{C}_{2} \mathrm{D}_{2} \mathrm{Cl}_{4}$. 51

Figure 2.10. Single crystal X-ray structure of $N, N^{\prime}$-di(6-undecyl)-1,7-di(4-bromophenyl)perylene-3,4:9,10-tetracarboxylic diimide. (a) Face-on view. (b) Side-on view 52

Figure 2.11. (a) Conversion of B into $A$ and C. (b) Plot and linear fit of $\ln \left(\mathrm{de}_{\mathrm{o}} / \mathrm{de}_{\mathrm{t}}\right)$ vs. time. ... 53

Table 2.1. Homodesmotic Calculations for Strain ................................................................. 71

Table 2.2. Thermochemical Calculations for Strain............................................................... 71

Figure 3.1. Structures of compounds designed and synthesized to compare acyclic and cyclic, $\pi$ conjugated molecules for n-type electronic materials. (a) PBPB; (b) a monomer version (3.1) of the macrocycle; (c) acyclic PBPB where the bond between one phenyl and a bithiophene is cut (3.2); and (d) a polymeric version (3.3). $\mathrm{R}=\mathrm{C}_{11} \mathrm{H}_{23}$ side chains.

Figure 3.2. Structures of compounds designed and synthesized to compare acyclic and cyclic, $\pi$ conjugated molecules for n-type electronic materials. (a) $\left(\mathbf{P P h}_{2}\right)_{4}$; (b) a monomer version (1.1) of the macrocycle introduced in Chapter 1B; $(\mathrm{c})$ acyclic $\left(\mathbf{P} \mathbf{P h}_{2}\right)_{4}$ where the bond between two phenyl groups is cut (3.5); and (d) a polymeric version (3.6). 78

Figure 3.3. (a) Energy minimized structures from DFT for PBPB. The $(M, M)$-stereoisomer is shown. ${ }^{20}$ (b) Cavity view of PBPB. (c) Energy minimized structures from DFT for $\left(\mathbf{P P h} \mathbf{~}_{4}\right.$. The $(M, M, M, M)$-stereoisomer is shown. (d) Cavity view for $\left(\mathbf{P P h}_{2}\right)_{4}$. Carbon $=$ gray, nitrogen $=$ blue, oxygen $=$ red, sulfur $=$ yellow. Hydrogen atoms have been removed to clarify the view. A methyl group substitutes the sidechains in the calculations. The methyl group, too, has been removed to clarify the view in the structures presented here. 79

Figure 3.4. (a) Schematic of the solar cell device fabricated in this study. (b-e) J-V curves for (b) PBPB-series and (c) $\left(\mathbf{P P h}_{2}\right)_{4}$-series solar cells under simulated AM 1.5G irradiation (100 $\left.\mathrm{mWcm}^{-2}\right)$. EQE spectra for (d) PBPB -series and (e) $\left(\mathbf{P P h}_{2}\right)_{4}$-series solar cells ........................ 81

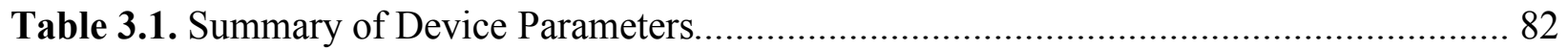

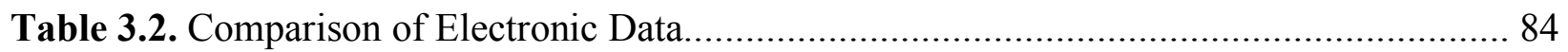

Figure 3.5. UV-vis absorption spectra measured in solution: (a) for PBPB, 3.1, 3.2, and 3.3; (b) for $\left(\mathbf{P P h}_{2}\right)_{4}, \mathbf{1 . 1}, 3.5$, and 3.6 normalized to each absorption maxima, where absorption $\max =1$. 
Figure 3.6. OFET transfer characteristics plotted in $\left(\mathrm{I}_{\mathrm{DS}}\right)^{1 / 2}-\mathrm{V}_{\mathrm{G}}$ axes for (a) PBPB, 3.1 and $\mathbf{3 . 3}$ and (b) for $\left(\mathbf{P P h}_{2}\right)_{4}, \mathbf{1 . 1}$, and 3.6. The $\mathrm{V}_{\mathrm{DS}}$ is $80 \mathrm{~V}$.

Figure 3.7. AFM phase images of bulkjunction films for (a) PBPB, (b) $\mathbf{3 . 1}$, (c) 3.3, (d) $\left(\mathbf{P P h}_{2}\right)_{4}$, (e) 1.1, and (f) 3.6. The mass ratio of donor-to-acceptor is fixed at 1:2.1\% CN additive was used. The scale bar is $200 \mathrm{~nm}$.

Figure 3.8. UV-vis absorption spectra measured in films: for (a) PBPB, 3.1, 3.2, and 3.3; and (b) $\left(\mathbf{P P h}_{2}\right)_{4}, \mathbf{1 . 1}, \mathbf{3 . 5}$, and $\mathbf{3 . 6}$ normalized to each absorption maxima, where absorption $\max =1 \ldots 88$

Figure 3.9. Device performance of PTB7-Th:PBPB solar cells. (a) Current density versus voltage $(J-V)$ characteristics of PTB7-Th:PBPB solar cells with different blend ratios (PTB7-Th to PBPB) and $1 \% \mathrm{v} / \mathrm{v}$ CN. (b) EQE spectra of corresponding PTB7-Th:PBPB solar cells. 88

Table 3.3. Summary of Device Parameters for PTB7-Th:PBPB solar cell 89

Figure 3.10. Device performance of PTB7-Th:(PPh $)_{4}$ solar cells. (a) Current density versus voltage $(J-V)$ characteristics of PTB7-Th:(PPh $)_{4}$ solar cells with different blend ratios (PTB7-Th to $\left.\left(\mathbf{P P h}_{2}\right)_{4}\right)$ and $1 \%$ v/v CN. (b) EQE spectra of corresponding PTB7-Th:(PPh $)_{4}$ solar cells... 89

Table 3.4. Summary of Device Parameters for PTB7-Th:(PPh $)_{4}$ solar cell.

Figure 3.11. AFM height images of PTB7-Th:PBPB films at a donor:acceptor ratio of $1: 2$ (a) without $\mathrm{CN}$ and (C) with $1 \% \mathrm{CN}$. AFM phase images of PTB7-Th:PBPB films at a donor:acceptor ratio of $1: 2(\mathrm{~B})$ without $\mathrm{CN}$ and (d) with $1 \% \mathrm{CN}$. The scale bar is $200 \mathrm{~nm}$. Both of the active layers have very smooth surface, with root-mean-square roughness (RMS) of $0.5 \mathrm{~nm}$ and $2.6 \mathrm{~nm}$ for films both with/without $1 \% \mathrm{CN}$, respectively. When turning to the phase images, we observed fibrous domains in the active layer without $\mathrm{CN}$. However, this feature is too fine to be quantified under the instrument limit $(\sim 8 \mathrm{~nm})$. In contrast, the active layer with the $1 \% \mathrm{CN}$ additive possesses clear phase separation as shown in $\mathrm{d}$. The average domain size is estimated to be $20-40 \mathrm{~nm}$. Here $\mathrm{CN}$ plays a critical role in phase aggregation, which is essential to the device performance. 90

Figure 3.12. CV (a) for PBPB, 3.1, 3.2, and 3.3; (b) for $\left(\mathbf{P P h}_{2}\right)_{4}, \mathbf{1 . 1}, \mathbf{3 . 5}$, and 3.6. ............... 90

Figure 3.13. AFM height images of bulkjunction films for (a) PBPB, (b) 3.1, (c) 3.3, (d) $\left(\mathbf{P P h}_{2}\right)_{4}$, (e) 1.1, and (f) 3.6. The mass ratio of donor-to-acceptor is fixed at 1:2.1\% CN additive was used. The scale bar is $200 \mathrm{~nm}$. 91

Figure 4.1. Structures of (a) 1,6 and 1,7-dibromo PDI, with the cis/trans orientation indicated in red; (b) cis-PBPB and trans-PBPB; (c) molecules used for single molecule conductance measurements cis-4.1 and trans-4.1; and (d) structures of acyclic PDI semiconductors cis-4.2 and trans-4.2. Cis- and trans-based molecules are derived from 1,6-dibromo PDI and 1,7-dibromo PDI, respectively. $\mathrm{R}=$ branched $\mathrm{C}_{11} \mathrm{H}_{23}$ side chains. 142

Figure 4.2. Output curves for (a) cis-PBPB and (b) trans-PBPB films from OFETs. In both devices, the source-drain voltage saturates at $80 \mathrm{~V}$. 144 
Figure 4.3. (a) Transfer curve for cis-PBPB; (b) transfer curve for trans-PBPB. Device current (left axis, black) and square root of current (right axis, red or blue) measured as a function of gate voltage at a constant source-drain voltage of $80 \mathrm{~V}$. (c) Height image for cis-PBPB and (d) transPBPB. Both films are continuous and smooth and have a root mean square roughness of 0.35 and $0.37 \mathrm{~nm}$ for the cis and trans-based devices, respectively. The scale bar is $1.0 \mu \mathrm{m}$. (e) PXRD of both films showing no obvious signs of crystallinity. Films drop-cast from $\mathrm{CDCl}_{3}$. 145

Figure 4.4. Molecular structures obtained with DFT using 6-31G/B3LYP basis set. (a) cis-PBPB and (b) trans-PBPB. (c) SCXRD solid-state packing of trans-PBPB as viewed down the $a$ axis. Blue and red are the two enantiomers of the diphenyl PDI packing down the axis. Red = oxygen, blue $=$ nitrogen, black $=$ carbon, and yellow $=$ sulfur. Hydrogens and side chains have been removed for clarity. 147

Figure 4.5. CV of (a) trans-PBPB; (b) trans-4.2; (c) cis-PBPB; and (d) cis-4.2. CVs taken in $\mathrm{CH}_{2} \mathrm{Cl}_{2}$ containing $0.1 \mathrm{M} \mathrm{NBu}_{4} \mathrm{PF}_{6}$ as the electrolyte. .148

Figure 4.6. UV of (a) trans-PBPB and cis-PBPB; (b) trans-4.2 and cis-4.2. 148

Figure 4.7. (a) Schematic of a single-molecule junction showing trans-4.1 in the break junction. The diphenyl PDI contains two aurophilic amino groups on the aryl rings to bind the gold electrodes in the junction; (b) Logarithm conductance histograms for cis-4.1 (yellow) and trans4.1 (purple) measured with an applied bias of $450 \mathrm{mV}$ in a 1,2,4-trichlorobenzene solution.....149

Table 4.1. Comparison of trans-PBPB and cis-PBPB Electronic Data................................150

Table 4.2. OFET Characteristics for Semiconductors .................................................... 151

Figure 4.8. Molecular structure of trans-PBPB. One of the two independent molecules is shown. Thermal ellipsoids are rendered at the $20 \%$ probability level. Black, carbon; red, oxygen; blue, nitrogen; yellow, sulfur. Hydrogen atoms and the minor positions of disordered atoms are omitted. Crystallographic data is provided in Chapter 2. .152

Figure 4.9. The two-dimensional conductance-displacement histograms of (a) cis-4.1 and (b) trans-4.1.

Figure 4.10. Transfer characteristics for (a) cis-4.2 and (b) trans-4.2. The mobilities are similar: $2.1 \times 10^{-4} \mathrm{~cm}^{2} \mathrm{~V}^{-1} \mathrm{~s}^{-1}$ and $1.8 \times 10^{-4} \mathrm{~cm}^{2} \mathrm{~V}^{-1} \mathrm{~s}^{-1}$ for cis-4.2 and trans-4.2, respectively. .153

Figure 4.11. AFM micrograph height image for cis-4.2 and (b) trans-4.2. Both films are continuous and smooth and have a root mean square roughness of 0.43 and $0.45 \mathrm{~nm}$ for the $c i s$ and trans-based films, respectively. The scale bar is $1.0 \mu \mathrm{m}$. 153

Table 4.3. Homodesmotic Calculations for Strain .196

Figure 5.1. (a) Molecular structure of $\left(\mathbf{P P h}_{2}\right)_{4}$. (b) Energy minimized structures from DFT for $\left(\mathbf{P P h}_{2}\right)_{4}$. Carbon $=$ gray, nitrogen $=$ blue, oxygen $=$ red. Hydrogen atoms have been removed to clarify the view. A methyl group substitutes the sidechains in the calculations. The methyl group, too, has been removed to clarify the view in the structures presented here. .202 
Figure 5.2. (a) Normalized film absorption spectra of PTB7-Th and (PPh $)_{4}$. (b) Device structure for the inverted organic photodetector. (c) Schematic of the energy levels of ITO, ZnO, PTB7-Th, $\left(\mathbf{P P h}_{2}\right)_{4}, \mathrm{MoO}_{3}$ and Al. Energy levels of PTB7-Th and $\left(\mathbf{P P h}_{2}\right)_{4}$ were estimated from cyclic voltammetry measurements in solution and adopted from ref. 16 and ref. 15, respectively. (d) Current density-voltage curves under dark condition and simulated AM $1.5 \mathrm{G}$ irradiation $\left(100 \mathrm{mWcm}^{-2}\right)$. (e) External quantum efficiency and specific detectivity spectra calculated at -0.1 $\mathrm{V}$ bias voltage. (f) Comparison of detectivity and working voltage in reported organic photodetectors and this work.

.204

Table 5.1. A Summary of Responsivity, Detectivity, and EQE. .205

Figure 5.3. Transient absorption spectra (b) and dynamics (b) for the neat film of PTB7-Th and blended film of $\left(\mathbf{P P h}_{2}\right)_{4}$ and PTB7-Th pumped at $710 \mathrm{~nm}$. The dashed-black curves are from PTB7-Th which were scaled for comparison. 206

Figure 5.4. (a) Dark current density-voltage curves for $\mathrm{PC}_{71} \mathrm{BM}, \mathbf{3 . 6}$, and $\left(\mathbf{P P h} \mathbf{2}_{4}\right.$ based photodetectors with the same device structure as shown in Figure 5.2b. (b) Specific detectivity spectra for $\left(\mathbf{P P h}_{2}\right)_{4}, \mathbf{3 . 6}$ and $\mathrm{PC}_{71} \mathrm{BM}$ based OPDs calculated at $-0.1 \mathrm{~V}$ bias voltage. .209

Figure 5.5. Linear dynamic range of the $\left(\mathbf{P P h}_{2}\right)_{4}$-based OPD. A red light emitting diode $(\lambda=625 \mathrm{~nm})$ was used as the light source. 211

Figure 5.6. Frequency response of the $\left(\mathbf{P P h}_{2}\right)_{4}$-based OPD. The device area is $0.09 \mathrm{~cm}^{2}$. .211

Figure 5.7. Transient absorption spectra and dynamics for the neat $\left(\mathbf{P P h}_{2}\right)_{4}(\mathrm{a}, \mathrm{b})$ and the blended film of (PPh $)_{4}$ and PTB7-Th (c,d) upon $560 \mathrm{~nm}$ excitation. The dashed-black curve in (c) is the spectrum from PTB7-Th upon the excitation of $710 \mathrm{~nm}$. The signal from PTB7-th was scaled for comparison.

Figure 5.8. The device structure is ITO/ZnO/PBDTT-TT:(PPh $)_{4} / \mathrm{MoO}_{3} / \mathrm{Al}$. The original $J-V$ curve is shown in Figure 5.2c Plot of $\mathrm{d} V / \mathrm{d} J$ vs $J^{-1}$ and linear fitting. (b) $\ln (J)$ vs $\left(V-J R_{\mathrm{S}}\right)$ and linear fitting. The $J_{0}$ is calculated to be $7.68 \times 10^{-13} \mathrm{Acm}^{-2}$. .214

Figure 5.9. The dependence of the dark current density of the $\left(\mathbf{P P h} \mathbf{P}_{\mathbf{4}}\right.$-based OPD on temperature measured at $-0.5 \mathrm{~V}$ and $-2.0 \mathrm{~V}$. .214

Figure 5.10. External quantum efficiency at different wavelength with inverted OPD structure at $-0.1 \mathrm{~V}$. .215

Figure 5.11. Calculated responsivity $\left(R\right.$ in $\left.\mathrm{AW}^{-1}\right)$ spectra for different OPDs. .215

Figure 6.1. (a) Structure of $\left(\mathbf{P B B r}_{4}\right)_{3}$; (b) - (g) van der Waals Surface of $\left(\mathbf{P B B r}_{4}\right)_{3}$ seen from the side and top, trimmed to reveal the cavity $(\mathrm{g}) . \mathrm{R}=\mathrm{C}_{11} \mathrm{H}_{23}$ side chains. .222

Figure 6.2. (a) $\mathrm{Pd}\left(\mathrm{PPh}_{3}\right)_{4}$, Toluene, $110{ }^{\circ} \mathrm{C}, 13 \mathrm{~h}, 69 \%$ yield; (b) $\mathrm{PtCODCl}_{2}$, 1,2-dichloroethane, $85^{\circ} \mathrm{C}, 72 \mathrm{~h}$; (c) 1,1'-ferrocenediyl-bis(diphenylphophine), $\mathrm{CH}_{2} \mathrm{Cl}_{2}, \mathrm{rt}, 24 \mathrm{~h}$; (d) $\mathrm{PPh}_{3}$, Toluene, $110{ }^{\circ} \mathrm{C}, 48 \mathrm{~h}, 15 \%$ yield for $\left(\mathrm{PBBr}_{4}\right)_{3}$; (e) $\mathrm{Br}_{2}, \mathrm{I}_{2}, \mathrm{CH}_{2} \mathrm{Cl}_{2}, \mathrm{rt}, 80 \%$ yield. .223 
Figure 6.3. (a) CD of 6.2; (b) $\mathrm{CD}$ of $\left(\mathrm{PBBr}_{4}\right)_{3}$. Both spectra show each enantiomer preferentially absorbs left or right-handed circularly polarized light. .223

Figure 6.4. Molecular structure from SCXRD of $\left(\mathbf{P B B r}_{4}\right)_{3}$. (a) Side and $\mathrm{b}$, top view of $(S S S)$ $\left(\mathbf{P B B r}_{4}\right)_{3} . \mathrm{C}, \mathrm{N}, \mathrm{O}, \mathrm{S}$, and $\mathrm{Br}$ atoms are colored in grey, blue, red, yellow, and brown, respectively. Hydrogen atoms have been removed to clarify the view. The alkyl chains on the imide are refined to only nine of the eleven carbon atoms due to disorder. .224

Figure 6.5. Structural packing of $\left(\mathrm{PBBr}_{4}\right)_{3}$. (a) view of the honeycomb structure in the ab plane for $\left(\mathbf{P B B r}_{4}\right)_{3}$. The capsule and $i$ corresponds to the internal cavity of $\left(\mathbf{P B B r}_{4}\right)_{3}$ and the cavity formed by the packing of $\left(\mathbf{P B B r}_{4}\right)_{3}$, respectively. The remaining sulfur atoms are colored in yellow to provide a marker to identify the macrocycle cavities. See bottom left cartoon. Highlighted in green are the imide side chains (some of the sidechains have been removed to clarify the view of the cavity). In red are the thiophene rings. (b) Surface map of the void space in the ab plane of $\left(\mathbf{P B B r}_{4}\right)_{3}$. (c) Two molecules of $\left(\mathbf{P B B r}_{4}\right)_{3}$ showing the $\mathrm{C}=\mathrm{O} \ldots \mathrm{Br}-\mathrm{C}$ halogen interaction responsible for the $2 \mathrm{D}$ assembly. (d) View of the packing of $\left(\mathbf{P B B r}_{4}\right)_{3}$. As shown, the vertical stacking follows the $\mathrm{c}$ axis. The alkyl sidechains of the imide are shown in green. Hydrogen atoms have been removed from all structures to clarify the view.....

.225

Figure 6.6. $P X R D$ of $\left(\mathrm{PBBr}_{4}\right)_{3}$ and 6.1. (a) Films drop cast from a chloroform solution; (b) data obtained from powder samples. The black line represents the predicted pattern for $\left(\mathbf{P B B r}_{\mathbf{4}} \mathbf{)}_{3}\right.$. .226

Figure 6.7. Charge transport for $\left(\mathbf{P B B r}_{4}\right)_{3}$. (a) Transfer characteristics of OFET device for (PBBr 4$)_{3}$; (b) device cycling response under vacuum (red circles) and $\mathrm{N}_{2}$ atmosphere (blue triangles); and (c) normalized behavior of the device response under vacuum (step 1), $\mathrm{N}_{2}$ (step 2), and different analytes atmosphere (step 3: n-hexane, 3-hexyne and 1-hexyne). Error bars represent the standard error obtained in three measurements. 227

Figure 6.8. (a) $\mathrm{I} / \mathrm{I}_{0}$ for $\left(\mathrm{PBBr}_{4}\right)_{3}$ under $n$-hexane, 3-hexyne and 1-hexyne. (b) Device cycling response for $\left(\mathbf{P B B r}_{4}\right)_{3}$ under vacuum, $\mathrm{N}_{2}$ and hexane atmosphere. Error bars represent the standard error obtained in three measurements. (c) Recovery response for $\left(\mathbf{P B B r}_{4}\right)_{3}$ under vacuum after exposure to 1-hexyne. The films do not recover their original current levels. .229

Figure 6.9. Coherence length for $\left(\mathbf{P B B r}_{4}\right)_{3}$. We can approximate the coherence length lower bound at $335 \AA$ (black dotted line) and upper bound at $385 \AA$ (green dotted line). ${ }^{42}$ The online Supporting Information contains the additional PDF analysis details and spectra for $\mathbf{6 . 2}$ and for $\left(\mathrm{PBBr}_{4}\right)_{3}{ }^{1}$. .231

Figure 6.10. Transfer characteristics for 6.2. .231

Figure 6.11. (a) AFM height images of spin-cast films for (a) $\left(\mathbf{P B B r}_{4}\right)_{3}$ and (b) 6.2. The root mean square roughness are $3.2 \mathrm{~nm}$ for $\left(\mathbf{P B B r}_{4}\right)_{3}$ and $0.347 \mathrm{~nm}$ for 6.2, respectively. .231

Figure 6.12. (a) Device cycling response for 6.2 under vacuum and $\mathrm{N}_{2}$ atmosphere. (b) The average current of response for $\left(\mathbf{P B B r}_{4}\right)_{3}$ OFET to series of analytes at a concentration of 3000 PPM. Error bars represent the standard error obtained in three measurements. .232

Figure 6.13. Sensitivity for $\left(\mathrm{PBBr}_{4}\right)_{3}$ OFET under different concentrations of ethanol. .232 
Table 6.1. Crystallographic data for $\left(\mathrm{PBBr}_{4}\right)_{3}$.

Figure 7.1. (a) The two pieces that were joined to make PPh6-PhHex: a diphenyl PDI (Compound 1.1) and five-aryl precursor that possess analogous distance between substituents; (b) a PDIsubstituted CPP called $\mathbf{P P h} \mathbf{p h}_{\mathbf{6}} \mathbf{P h H e x}$, where the PDI is the electron poor and the belt is the electron rich portion of the molecule. Red = relative electron poor subunit = lower energy available space to accept an electron and blue = relative electron rich subunit e.g. larger density of high energy electrons. $\mathrm{R}=\mathrm{C}_{11} \mathrm{H}_{23}$ for $\mathrm{PDI}$ and $\mathrm{R}=\mathrm{OC}_{6} \mathrm{H}_{13}$ for aryl ring. .259

Figure 7.3. DFT calculations showing (a) the twisting of $(P)-\mathbf{P P h}_{\mathbf{6}}-\mathbf{P h H e x}$. The torsional angle is reduced in $\mathbf{P P h} \mathbf{h}_{\mathbf{6}} \mathbf{P h H e x}$. (b) The cavity measures $12.3 \AA$ along the long axis from PDI to alkoxysubstituted aryl ring. Orange circles indicate the distance between the PDI phenyl appendages. Red $=$ oxygen, blue $=$ nitrogen, black $=$ carbon, and yellow $=$ sulfur. Hydrogens and side chains have been removed for clarity. Computations at the using 6-31G/B3LYP level of theory......

.262

Figure 7.4. (a) HPLC chromatogram showing the retention times of both $(P)-\mathbf{P P h} \mathbf{P}_{\mathbf{6}} \mathbf{- P h H e x}$ and $(M)$ - PPh $_{6}$-PhHex with a mobile phase of $18 \%$ DCM/82\% Hexanes using an IA-3 Chiralpak column. We call them Enantiomer 1 and 2, arbitrarily assigned; (b) CD chromatogram $(P)-\mathbf{P P h}_{6^{-}}$ PhHex and $(M)$ - $\mathbf{P P h}_{6}-\mathbf{P h H e x}$ in toluene $\left(1 \mathrm{~cm}\right.$ path length, $10^{-6} \mathrm{M}$ for Enantiomer 1 and $10^{-5} \mathrm{M}$ for Enantiomer 2); and (c) UV-Vis absorption spectrum of $\mathbf{P P h} \mathbf{h}_{\mathbf{6}} \mathbf{P h H e x}$ (dichloromethane, $1.0 \mathrm{~cm}$ path length, $1.37 \times 10^{-5} \mathrm{M}$ ). .264

Figure 7.5. (a) HOMO energy level showing a high density of high energy electrons on the hexoxy-substituted aryl ring and (b) LUMO energy level showing unoccupied low energy space on the PDI. Red = oxygen, blue $=$ nitrogen, black $=$ carbon, and yellow $=$ sulfur. Hydrogens and side chains have been removed for clarity. .265

Figure 7.6. DFT minimized geometry for SI-7.1. Red = oxygen, blue = nitrogen, black = carbon, and yellow $=$ sulfur. Hydrogens and side chains have been removed for clarity. .266

Figure 7.7. Proton NMR and chemical structure of the major byproduct from the reaction SI-7.1. This byproduct suggests there is some degree of reversibility in the reaction. It contains two bisplatinum, diphenyl-PDIs and one belt linker. ${ }^{1} \mathrm{H}$ NMR taken in $\mathrm{C}_{2} \mathrm{D}_{2} \mathrm{Cl}_{4}$ at $410 \mathrm{~K}$. HRMS (MALDI ) calculated $\mathrm{m} / \mathrm{z}$ for $\left[\mathrm{C}_{158} \mathrm{H}_{164} \mathrm{~N}_{4} \mathrm{O}_{10}\right]^{-}$is 2276.873 , found 2277.274.

Figure 7.8. (a) The calculated UV-Vis for $\mathbf{1 . 1}$ (b) and $\mathbf{P P h} \mathbf{h}_{\mathbf{6}}-\mathbf{P h H e x}$. The PDI $\rightarrow$ PDI transition is shifted to lower energy in the macrocycle as a result of a higher energy HOMO. 267

Figure 7.9. (a) UV-vis absorption spectrum of $\mathbf{P} \mathbf{P h}_{6}-\mathbf{P h H e x}\left(\mathrm{DCM}, 1.0 \mathrm{~cm}\right.$ path length, $1.37 \times 10^{-}$ ${ }^{5} \mathrm{M}$ ), diphenyl-PDI (1.1), and [10]CPP; (b) HOMO-LUMO energy gaps for the three compounds showing the electronic effect of incorporating a PDI moiety into a CPP nanobelt. .268

Table 7.1. Homodesmotic Calculations for Strain .309

Figure 8.1. (a) Macrocycle building blocks comprising a phenyl-bithiophene-phenyl linker (B) and an hPDI dimer (D). The macrocycles are formed from these two molecular components wrapped into a -D-B-D-B- arrangement; (b) chemical structure of cis-DBDB; and (c) trans-DBDB. 
Figure 8.2. Synthesis of DBDB. key: a) 1,4-bis(tributylstannyl)benzene, P-(2-furyl) $3, \mathrm{Pd}_{2} \mathrm{dba}_{3}$, THF, $55^{\circ} \mathrm{C}, 12 \mathrm{~h}$; b) $\mathrm{Pt}(\mathrm{COD}) \mathrm{Cl}_{2}$, toluene, $10{ }^{\circ} \mathrm{C}, 12 \mathrm{~h}$; c) 5,5'-bis(tributylstannyl)-2,2'bithiophene, THF, $55^{\circ} \mathrm{C}, 40 \mathrm{~h}$. (d) $\mathrm{PPh}_{3}$, toluene, $100{ }^{\circ} \mathrm{C}, 12 \mathrm{~h}$.

Figure 8.3. (a) Schematic of the OFET device and (b) transfer characteristics for trans-DBDB and cis-DBDB, showing a four-fold increase in electron mobility for the latter. 316

Figure 8.4. (a) DFT calculated lowest energy geometry for trans-DBDB; (b) and cis-DBDB. Red $=$ oxygen, blue $=$ nitrogen, black $=$ carbon, and yellow $=$ sulfur. Hydrogens and side chains have been removed for clarity; (c) Schematics of both trans-DBDB and cis-DBDB, respectively; (d) an overlay of the tetracyclic linker of both trans-DBDB and cis-DBDB. trans-DBDB shows a pronounced bowing relative to the cis isomer. Dashed black line indicates no bowing...... .318

Figure 8.5. (a) Molecular building blocks for DBDB macrocycles; (b) Schematics the two conformers of cis-DBDB; (c) and trans-DBDB that interconvert through a somersault mechanism; and (d) VT-NMR of both macrocycles at $380 \mathrm{~K}$. trans-DBDB shows the presence of both conformers at $380 \mathrm{~K}$ at 9.1 and $8.7 \mathrm{ppm}$, denoted with green circles. .319

Figure 8.6. The dependence of source-drain current of the cis-DBDB and trans-DBDB-based OFET on temperature (a) from $290 \mathrm{~K}$ to $250 \mathrm{~K}$ and (b) from $250 \mathrm{~K}$ to $290 \mathrm{~K}$, measured at gate voltage of $40 \mathrm{~V}$ and bias voltage of $80 \mathrm{~V}$. The slope of the best fit line for a) is $6.6 \times 10^{-2}$ and $7.6 \mathrm{x}$ $10^{-3}$ for cis-DBDB and trans-DBDB, suggesting the cis corral is more temperature sensitive than the trans corral. The dependence of mobility of the cis-DBDB and trans-DBDB-based OFET on temperature (c) from $290 \mathrm{~K}$ to $250 \mathrm{~K}$ and (d) from $250 \mathrm{~K}$ to $290 \mathrm{~K}$, measured at bias voltage of 80 V.

Figure 8.7: Synthetic details for the two dibromo $\mathrm{hPDI}_{2}$ isomers (8.1a and 8.2a) showing an enriched mixture of $8.2 \mathrm{a}(2: 1$ trans:cis)..... .323

Figure 8.8. ${ }^{1} \mathrm{H}$ NMR spectrum of the enriched mixture of 8.2a:8.1a's precursors, showing an approximate $2: 1$ ratio of the downfield protons at $9.65-9.70 \mathrm{ppm}$. These peaks correspond to the red starred protons that are deshielded due to the bromine atoms. .324

Figure 8.9: The two dibromo $\mathrm{hPDI}_{2}$ isomers are separated using a CHIRALPAK ${ }^{\circledR}$ IA-3 column (4.6 mm I.D. x $250 \mathrm{~mm}, 3 \mu \mathrm{m}$ ), using an isocratic method of $22 \%$ methylene chloride: $78 \%$ hexanes. Using an enriched mixture (2:1 trans isomer:cis isomer), we were able to confirm the first peak from HPLC is 8.2a. .324

Figure 8.10. Thermal ellipsoid plot of hPDI-Br 2 . The two independent sites are disordered over 4 and 3 positions. All atoms were refined with isotropic ADPs due to the extensive disorder. Thermal ellipsoids are depicted at the $40 \%$ level. The $\mathrm{C}_{11} \mathrm{H}_{23}$ side chains were omitted from the refinement. Hydrogen atoms are omitted for clarity...... .325

Table 8.1. Table of crystallographic data for Compound 8.2a .........................................325

Figure 8.11. CV for cis-DBDB and trans-DBDB relative to a hPDI monomer.....................326 
Figure 8.12. Output curves for (a) cis-DBDB and (b) trans-DBDB. Both devices show current saturation at $80 \mathrm{~V}$.

Table 8.2. Transfer characteristics for (a) cis-DBDB and (b) trans-DBDB. .327

Figure 8.13. Transfer curves for (a) 8.1a and (b) 8.2a. The mobilities are essentially identical at $2.0 \times 10^{-3}$ and $1.6 \times 10^{-3}$ for 8.1a and 8.2a, respectively.

Figure 8.14. (a) DSC showing broad features at temperatures below $160{ }^{\circ} \mathrm{C}$; (b) PXRD of cisDBDB and trans-DBDB at three temperatures, showing the materials are amorphous at higher temperatures $\sim 160{ }^{\circ} \mathrm{C}$; (c) AFM of height images of cis-DBDB and (d) trans-DBDB. .328

Figure 8.15. VT-NMR for cis-DBDB and trans-DBDB. Both room temperature spectra show the presence of multiple conformers. Yet, at 400K, the spectrum for cis-DBDB shows coalescence, while trans-DBDB shows multiple peaks at 8.7 and $9.1 \mathrm{ppm}$, denoted with pink stars. 329

Figure 8.16. UV-Vis absorption spectra for cis-DBDB, trans-DBDB, and a $\mathrm{hPDI}_{2}$. .329

Table 8.3. Homodesmotic Calculations for Strain .346

Figure 9.1. The recognition of fullerenes $\mathbf{C}_{70}, \mathbf{C}_{60}$ or PCBM by bis-PDI macrocycle PBPB to form a supramolecular complex $\left(\mathrm{R}=\mathrm{C}_{11} \mathrm{H}_{23}\right)$..... .350

Figure 9.2. (a) DFT structure of the supramolecular complex showing non-covalent interactions present. (b) $\mathrm{CH}-\boldsymbol{\pi}$ interactions from aryl rings to $\mathbf{C}_{\mathbf{7 0}}$; (c) sulfur lone pair- $\pi$ interactions; (d) $\mathrm{CH}-\boldsymbol{\pi}$ interaction between methylene and fullerene; and (e) Truncated ${ }^{1} \mathrm{H}$ NMR spectra from the titration of 1 eq. of $\mathbf{C}_{70}$ fullerene guest into a $0.5 \mathrm{mM}$ host solution of $\mathbf{P B P B}$ (start point $=$ red, end point $=$ blue, $\mathrm{d}_{8}$-toluene, $298 \mathrm{~K}, 500 \mathrm{MHz}$. .353

Table 9.1. Fullerene Association Constants .355

Figure 9.3. (a) Electronic absorption spectra of PBPB upon the addition of $\mathbf{C}_{70}$ (toluene, corrected for $\mathbf{C}_{\mathbf{7 0}}$ absorptions); (b) Fluorescence emission spectra of $\mathbf{P B P B}$ upon the titration with $\mathbf{C}_{\mathbf{7 0}}$ (toluene, $4 \mu \mathrm{M}, \lambda \mathrm{ex}=600 \mathrm{~nm}$ ); and (c) HOMO energy level showing electron delocalization.357

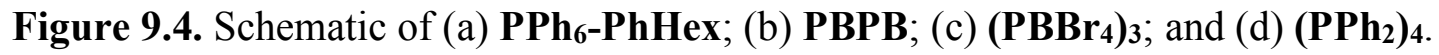
.359

Figure 9.5. (a) Literature synthesis to make 9.1; (b) First synthesis to make macrocycle 9.2. ..360

Figure 9.6. The DFT energy minimized structures for (a) 9.2; (b) 9.3; and (c) 9.4. One hypothesis for not forming $\mathbf{9 . 2}$ could be due to strain. The aryl-aryl distance serves as a quick tool to assess strain. The smallest PDI-based macrocycle synthesized has an aryl-aryl distance at $0.97 \mathrm{~nm} . .361$

Figure 9.7. (a) A proposed synthesis to form 9.2 using a nickel-based strategy; (b) one could also employ oxidative coupling conditions to yield a catenane structure. .362

Figure 9.8. The ${ }^{1} \mathrm{H}$ NMR of PBPB and $\mathbf{C}_{70}$ from 0.0 to 3.2 equivalents. The aryl protons shift dramatically as the concentration of $\mathbf{C}_{\mathbf{7 0}}$ increases. .363 
Figure 9.9. The ${ }^{1} \mathrm{H}$ NMR of PBPB and $\mathrm{C}_{70}$ from 0.0 to 3.2 equivalents. The alkyl protons split into two as the concentration of $\mathrm{C}_{70}$ increases, indicative of outside and inside environments for methylenes. .363

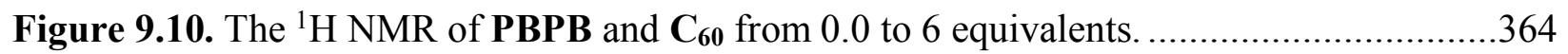

Figure 9.11. The ${ }^{1} \mathrm{H}$ NMR of PBPB and PCBM from 0.0 to 6 equivalents. .........................364

Figure 9.12. The ${ }^{1} \mathrm{H}$ NMR of PBPB alkyl region showing the methylene protons ortho to the methine $\left(\mathrm{H}_{\mathrm{f}}\right.$ and $\left.\mathrm{H}_{\mathrm{g}}\right)$ as two broad peaks as they are diastereotopic. Each integrates to eight protons.

Figure 9.13. The ${ }^{1} \mathrm{H}$ NMR of PBPB and 1.0 equivalent of $\mathbf{C}_{70}$. The methylene protons $\left(\mathrm{H}_{\mathrm{f}}\right.$ and $\mathrm{H}_{\mathrm{g}}$ ) ortho to the methine split into two broad peaks and are now inequivalent.........................365

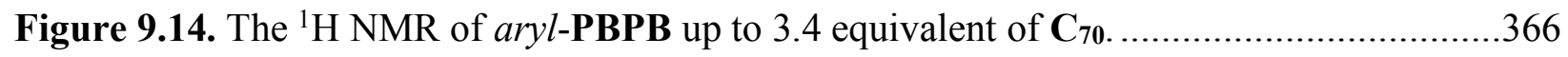

Figure 9.15. Truncated ${ }^{1} \mathrm{H}$ NMR spectra of Compound $\mathbf{3 . 4}$ (bottom) and after the addition of four equivalents of $\mathbf{C}_{70}$ fullerene (top) (d8-toluene, $\left.298 \mathrm{~K}, 500 \mathrm{MHz}, \mathrm{R}=\mathrm{C}_{11} \mathrm{H}_{23}\right) \ldots \ldots \ldots \ldots \ldots \ldots \ldots . . . . . . . . .66$

Figure 9.16. Truncated ${ }^{1} \mathrm{HNMR}$ spectra of trans-DBDB (Chapter 8) and after the addition of four equivalents of $\mathbf{C}_{70}$ fullerene ( $\mathrm{d}_{8}$-toluene, $298 \mathrm{~K}, 500 \mathrm{MHz}$ ). $\Delta \delta \mathrm{H}_{\mathrm{a}}=0.01 \mathrm{ppm}$ and so too small to be accurately fitted. .367

Figure 9.17. ${ }^{1} \mathrm{H}$ NMR spectroscopic titration curves and residuals for PBPB and aryl-PBPB upon titration with fullerene guests. Solid points represent experimental data, continuous line represents theoretical binding isotherm. Errors for association constants are given in brackets. Job's plots for complexes indicate a 1:1 binding stoichiometry; the maximum change in chemical shift occurs when the mole fraction of fullerene guest $\chi(\mathrm{F})=0.5$. .369

Figure 9.18. Top: The theoretical ESI mass spectrum of the $1: 1$ stoichiometric host guest complex [PBPB] $\supset\left[\mathbf{C}_{70}\right]$. Bottom: Experimental ESI mass spectrum of the 1:1 stoichiometric host guest complex $[\mathbf{P B P B}] \supset\left[\mathbf{C}_{70}\right]$. .369

Figure 9.19. The ESI MS/MS spectrum of $[\mathbf{P B P B}] \supset\left[\mathbf{C}_{70}\right]$ as the complex is broken into its constituent parts (collision energy $=80-100 \mathrm{eV})$. .370

Figure 9.20. (a) Truncated ${ }^{13} \mathrm{C}$ NMR spectra showing $\mathbf{C}_{70}$ fullerene and a 1:1.5 PBPB:C $\mathbf{C}_{70}$ stoichiometric mixture (d8-toluene, $298 \mathrm{~K}, 125 \mathrm{MHz}$ ). Carbon labels for $\mathbf{C}_{\mathbf{7 0}}$ are also given. (b) Truncated ${ }^{13} \mathrm{C}$ NMR spectra showing $\mathbf{C}_{60}$ fullerene, $\mathbf{C}_{70}$ fullerene and a 1:1.5:1.5 PBPB:C $\mathbf{C}_{60}: \mathbf{C}_{70}$ stoichiometric mixture ( $\mathrm{d}_{8}$-toluene, $298 \mathrm{~K}, 125 \mathrm{MHz}$ ). 370

Figure 9.21. (top) Electronic absorption spectrum for PBPB upon addition of $\mathbf{C}_{60}$ correct for $\mathbf{C}_{60}$ absorptions; (bottom) Florescence emission spectrum of PBPB with titration of $\mathbf{C}_{60}$ (toluene, 4 $\left.\mu \mathrm{M}, \lambda_{\mathrm{ex}}=600 \mathrm{~nm}\right)$.

Figure 9.22. Florescence emission spectrum of $\mathbf{3 . 4}$ with titration of $\mathbf{C}_{60}$ (toluene, $4 \mu \mathrm{M}, \boldsymbol{\lambda}_{\mathrm{ex}}=600$ $\mathrm{nm})$. .372 
Figure 9.23. MALDI from first attempt to synthesize PDI-phenanthroline macrocycle showing many products formed due to the reversibility of the reaction..........................................372 


\section{ACKNOWLEDGEMENTS}

I am indebted to all mentors, mentees, teachers, friends, and family who have supported, encouraged and inspired me throughout my life. In particular, I am beholden to those who provided me courage to pursue a doctorate despite possessing a non-traditional background. I have always been motivated by those around me, and academia has provided endless reasons to be motivated over the past five years.

I remain in disbelief that my advisor Colin Nuckolls took me - a pre-medical, economics students - into his group five years ago. That one decision has steered my life into a different direction, and I am not sure how to convey in words what this has meant to me. His unconditional support of me as a scientist has made me who I am today; he was never too busy to discuss science and macrocycles together. I am proud of what we accomplished, and its fulfilling to see other students admitted from non-traditional backgrounds over the past five years. I had the unique experience of being an undergraduate in CHEM 3444 with Colin, and this is where my passion for organic chemistry was developed, in a large part thanks to him.

Mike Steigerwald served as a friend, colleague, and, especially, mentor over the past five years. I am appreciative for all the hours together discussing science; his office served as one of the best classrooms I have experienced. Yet I am most grateful for his support and friendship. He supported me through some difficult times in graduate school, not least, with the death of my father, and provided a space where I could just be.

I am indebted to my Committee members. I know I am a better scientist for their mentorship and encouragement. Tristan Lambert was one of the first people to give me courage to change my career and pursue a doctorate. I am indebted to him for his continued support. Tomislav Rovis is an academic mentor. I am grateful for all of our conversations, his support, and candor. 
Both their examples excite me for a career in academia. I am thankful to Xavier Roy for his support and his guidance on my future over the past five years.

Latha Venkataraman has served as a role model throughout graduate school. I am grateful for our frank and open conversations; her encouragement helped me decide to pursue a postdoctoral position. Fay Ng was my first mentor in lab. She taught me how to be a rigorous scientist in the laboratory and shaped how I approach lab work. We started the conjugated macrocycles project, and I have her to thank for an amazing five years of learning. I am thankful to David Reichman and Laura Kaufman for their openness to listen to the students and their desire to improve our community as the Chair and Director of Graduate Studies. I am grateful to Ruben Gonzalez for his support of my candidacy as DGS. Willem Buiter was my first academic mentor at the London School of Economics. His support and friendship have taught me how special this mentor-mentee bond can be.

I am also indebted to David Reichman for his support of Women in Chemistry. Cofounding this group with Anastasia Voevodin is one of the things I am most proud of during my time at Columbia University. The Chemistry Department's support was invaluable. I am grateful to all the female students, post-docs, and professors who attended our events: this group would be nothing without each of you.

I have been fortunate to collaborate with wonderful scientist and friends. Brandon Fowler and $\mathrm{Yu}$ Zhong served as mentors and friends both in my early days and now. Boyuan Zhang has been my better half in lab for five years. I am fortunate to have been in the same year as her, as her support and friendship have been invaluable. Brandon, Yu, Boyuan and I were the first students and post-docs to develop and work on the macrocycle project. I am proud to be part of this team. 
I am indebted to Cary Ritter, Ayden Schattman, and Pan Pan Li. Mentoring these three women was one of most joyful experiences of graduate school. I am thankful to all my students as a teaching assistant. I loved being in the classroom because of them.

Thank you to my graduating class and accepting me as one of you despite having pursued a different path to get here. In particular, I am thankful to Anastasia Voevodin and Evan Doud for their friendship. Some of my best memories in graduate school are with you both. I am thankful for both Makeda Tekle-Smith and Prakriti Joshi for their support and excitement for academia; they both are inspiring peers and I am proud to be their friend. I can't imagine graduate school without the friendship of Anouck Champsaur. Tim Su, Nate Schuster, Dan Paley, Raul Hernandez Sanchez, and Michael Inkpen served as mentors and friends; I am grateful for their support. I am thankful for Ilana Berenson Stone ("Mini"). She was another student that followed the path from post-bac to Ph.D. Watching her grow as a scientist has been fulfilling. Natalia Gadjieva has been by my side for five years. I am indebted to her for her friendship. I am thankful to all past and present lab members; it has been a wonderful environment to pursue science. I am thankful for Ryan Hastie's advice and friendship through the years, but mostly for all the laughs together. Thank you to all those that keep the department running, especially Alison Doyle for keeping me organized. I am thankful for the Guthikonda family for their financial support in my fifth year. Thank you to Brandon Fowler and Nate Schuster too for their helpful edits in Chapter 1.

I am thankful for all my friendships. My group of friends have been my biggest supporters. There are too many to name, but I could not have done this without their love. I am particularly thankful for the love and support over the past decade from both Paul and Jo Parsonson.

I owe everything to my mother and father, Alicia and Mitchell. Neither of them completed high school, yet instilled a work ethic and love of education into me and my twin sister. My mother 
sacrificed her entire life so I would have one. When I was seven, she told me I could be whatever I put my mind to, and I believed her. My father was in disbelief when I told him I was pursuing a Ph.D. at Columbia University, and I am grateful I was able to share my experiences with him before he passed away in my third year. I know he is proud today. I am who I am today because of these two individuals. I am not sure either parent knew they would raise two doctors.

My twin sister Melinda is my best friend and biggest supporter. She has been my partner throughout my life. We have conquered many obstacles and accomplished many feats together, and without one another's support, I have no doubt our lives would be fundamentally different. Nothing in life seems too difficult when you have Melinda next to you.

Last, none of this would be possible without my loving husband Mark Parsonson. His unconditional love and support fuel me daily. This year we celebrated my ten year remission anniversary from cancer. Experiencing an illness at a young age impacted us; we wanted our lives to mean more. I will never forget where we were in Notting Hill when we decided to quit our finance jobs, and apply to Columbia University together. I am more fulfilled now than ever, and am indebted to him for pushing me forward, then and now, and picking me up when needed. Life is the best adventure with him. I love you. 
For my mother and father:

Future generations and I are forever indebted to you. 


\section{Chapter 1: Introduction}

\section{Chapter 1 Section 1A. Contorted Aromatics as Electronic Materials}

\section{A.1. Preface}

Chapter 1A is inspired by conversations with both Prof. Colin Nuckolls and Dr. Michael L. Steigerwald and a recent published Accounts article titled "Conjugated Macrocycles in Organic Electronics” by Melissa L. Ball, Boyuan Zhang, Yu Zhong, Brandon Fowler, Shengxiong Xiao, Fay Ng, Michael L. Steigerwald, and Colin Nuckolls, published in the Accounts of Chemical Research (2019). ${ }^{1}$

\section{A.2. Introduction}

The discipline of organic electronics encompasses the design and synthesis of molecules for use in organic field effect transistors (OFETs), organic photovoltaics (OPVs), organic photodetectors (OPDs), single molecule electronics (SME), sensors, and many more. The rationale for synthesizing organic electronic materials is compelling: organics have the potential to be low cost, processable, and flexible complements to silicon technologies to combat some of the most pressing environmental issues and be used in logic-based systems and memory storage. A bottomup approach to synthesis is beneficial as it allows one to understand the electronic and physical structure of a molecule and provides a better chance of rationalizing device properties. One can also tune both the energetics and structural properties of the molecule and this can result in different physical properties. A bottom-up approach also provides an opportunity to study device physics on a fundamental level, as chemists can make nuanced changes to a molecular backbone and see how the device properties change.

This Thesis describes our efforts to design and study a new class of conjugated macrocycles materials that incorporate perylenediimide (PDI). ${ }^{1-6}$ Our conjugated macrocycles are enveloped within a broader class of contorted aromatics that include discs and ribbons from the Nuckolls 
labortory. ${ }^{7,8}$ The macrocycles absorb low energy visible light, and function as electron-accepting materials for device applications. Before I provide a general overview of the conjugated macrocycles in Chapter 1B, I provide a brief background on OFETs and the importance of $\pi$ conjugated materials for electronic applications.

\section{A.3. Output and Transport Curves: What Do They Tell Us?}

A necessary condition for a molecule to be considered for use in (opto)electronic applications is whether the molecule can carry current. ${ }^{9-14}$ One device to test carrier mobility is an OFET. An idealized transistor can function as a binary logic gate, operating in either an "on" state, where there is current, or in an "off" state in the absence of current. Figure 1A.1 shows a schematic of a bottom-contact OFET. For the data presented in this Thesis, we used a three-probe setup to bias the device: a source electrode $\left(\mathrm{V}_{\mathrm{s}}\right)$, a drain electrode $\left(\mathrm{V}_{\mathrm{d}}\right)$, and a gate electrode $\left(\mathrm{V}_{\mathrm{g}}\right)$. Current flows through a conduction channel comprised of the semiconducting material.

The semiconducting material in the channel ("Semiconducting layer" in Figure 1A.1) can be either a p-type molecule (a molecule that transports holes, or the absence of an electron) or ntype molecule (a molecule that transports electrons) or both (ambipolar). The semiconducting material lies within a channel between a source and drain electrode (gold in Figure 1A.1). In the case of electron transport, we empirically know that a positive electrical potential is applied to the gate electrode (silicon), and electrons, which are attracted to the positive gate bias, collect to form a conduction pathway between the source and drain electrodes to form a current. A negative gate potential is required to transport holes for p-type materials for the analogous electrostatic reasons.

Two types of curves are often presented for OFETs: the output curve and the transfer curve.

Figure 1A.1 shows representative data for both an output and transfer curve. The output curve 
shows the amount of current produced by sweeping the source-drain bias at a constant gate potential and is used to assess where the saturation point is in the device.

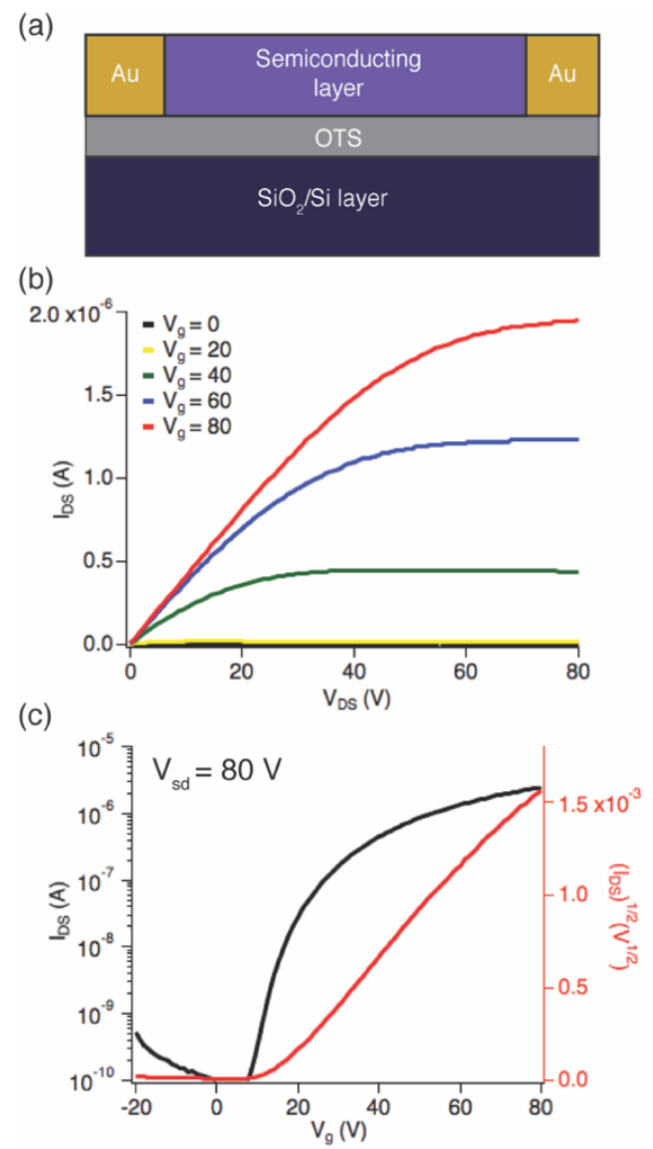

Figure 1A.1. (a) A schematic of an OFET device. The semiconducting layer comprises a conjugated molecule with p-type characteristics, n-type characteristics, or both; (b) a representative output curve showing the current between the source and drain electrodes as a function of a gate bias; we show the device is saturated at a gate bias of $80 \mathrm{~V}$ and transports electrons; and (c) a representative transfer curve that shows the current generated between the source and drain electrodes as a function of sweeping the gate.

In the example presented in Figure 1A.1b, a gate potential ranging from $0 \mathrm{~V}-80 \mathrm{~V}$ is applied, and one can observe the device transports electrons and saturates (or plateaus) at $80 \mathrm{~V}$. We use the saturation current to produce a transfer curve by sweeping the gate potential. Figure 1A.1c shows a device with a constant source-drain bias of $80 \mathrm{~V}$. One sweeps the gate potential from $-20 \mathrm{~V}-80 \mathrm{~V}$ to produce transfer characteristics (black line in Figure 1A.1c). In order to calculate the mobility of the device, one takes the square root of the IDs curve to produce the red 
line (transfer curve) in Figure 1A.1c. The mobility is calculated from the transfer curve in the saturation regime using $I_{\mathrm{DS}}=(W / 2 L) C_{\mathrm{i}} \mu\left(V_{\mathrm{G}}-V_{\mathrm{T}}\right)^{2}$, where $W$ and $L$ are the width and length of the channel, $C_{\mathrm{i}}\left(11.5 \mathrm{nFcm}^{-2}\right), \mu$, and $V_{\mathrm{T}}$ correspond to the capacitance per unit area of the gate insulator, the field effect mobility, and the threshold voltage, respectively. ${ }^{11,15}$

Each application discussed in this Thesis (OFETs, OPVs, OPDs, and sensors) requires the efficient transport of a carrier. Transport requires an otherwise localized carrier to be "freed" so it can move through a conduction channel and collected at an electrode, and this is where $\pi$ conjugation and energy levels matter. There are additional requirements (and often overlapping) for each application: the absorption of visible light, too, is important for OPVs (Chapter 3) and a rigid structure is important for OPDs (Chapter 5).

\section{A.4. A Chemist's Approach to Understanding Electronic Materials}

Section 1A.3 defined n-type materials as electron-transporting and p-type materials as hole transporting. A molecule can be "electron transporting" if it possesses low energy unoccupied space to accept an electron that has been "freed" from its local environment; this energy level is often referred to as the lowest unoccupied molecular orbital (LUMO). Likewise, a molecule is "hole transporting" if it possesses a large density of high energy electrons or a high energy highest occupied molecular orbital (HOMO) that provides an electron (often after prompted by a stimulus e.g. light or a potential).

As the basic principles of molecular electronics require transport of a charge carrier, the first question then is what molecular design causes a molecule to possess a high energy HOMO and low energy LUMO? A second related question is why do these two energy levels matter for organic electronics? Pi-conjugated molecules remain the ubiquitous materials for organic electronics. ${ }^{11,16-19} \mathrm{Pi}$-conjugation comprises alternating single and double bonds within the same 
plane (or nearly so), which facilitates carrier transport through the molecular backbone. All else being equal, for a homologous series of conjugated molecules, a longer conjugation length gives a higher energy HOMO and a lower energy LUMO. ${ }^{20}$

A higher energy HOMO is advantageous in organic electronics as it suggests the molecule is easier to oxidize, and a lower energy LUMO suggests the molecule is easier to reduce (the HOMO and LUMO are often referred to as the frontier orbitals). Both properties are helpful if the goal is to create a conduction channel in an OFET as we need molecules that can rapidly accept and give up electrons (or holes). Thus, we have answered the first two questions: $\pi$-conjugated materials endow a molecule with hole or electron transport properties because conjugation can raise the energy of the HOMO and lower the energy of the LUMO, all else being equal. ${ }^{19,21,22}$

The third question to address is what molecular designs can be utilized to tune the frontier orbitals energy levels and decrease the HOMO/LUMO gap? Through the past several decades, a donor/acceptor strategy remains the primary method to tune the energy levels of molecules. I define donor as a molecule with a relatively high energy HOMO and an acceptor as a molecule (or part of a molecule) with a low energy LUMO. Given their large density of high energy electrons, donor molecules are often referred to as p-type materials. Analogously, acceptor molecules and ntype molecules are used interchangeably. ${ }^{17}$ Arrow-pushing may help classify some molecules (or part of a molecule) as donors or acceptors (donor and acceptor classifications can be misleading as it often can depend on the state of reference e.g. ground or excited state). For example, a molecule with a lone pair could be a potent donor; such as a thiophene, as the lone pair can be pushed or "donated" into an acceptor. Molecules or a part of a molecule that can accept or "pull" electron density are often classified as acceptors; for example, atoms with high electron affinities like the oxygen of a carbonyl group. Often cyclic voltammetry (CV) is used to approximate HOMO 
and LUMO levels of a molecule. ${ }^{23}$ Thus, chemists design acceptor molecules because they can possess low energy LUMOs, which would make them potential candidates as n-type materials, and design donor molecules because they have high energy HOMOs to function as p-type materials in organic electronics.

\section{A.5. Evolution of Molecules for Organic Electronics}

(a)

Electron-rich Molecules
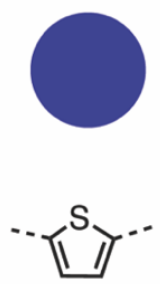

Thiophenes<smiles>Cc1cc2sc(C)cc2s1</smiles>

Thieno(3,2-b)thiophene

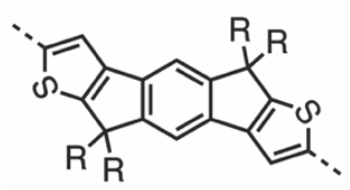

Indocenodithiophene (b)

Electron-poor Molecules
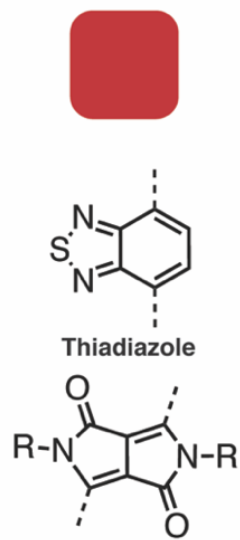

Diketopyrrolopyrrole

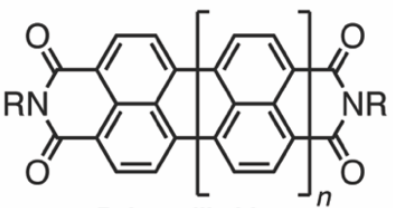

Rylene diimides (c) Design Strategies to Create Electronic Materials
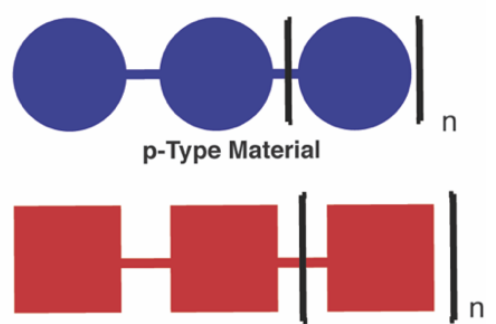

$\mathrm{n}$-Type Material

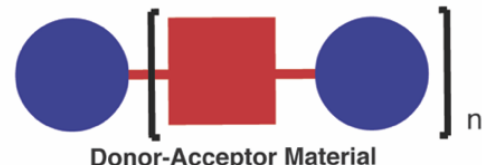

(d)

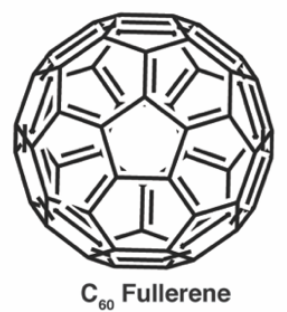

Figure 1A.2. (a) Common donor molecules and (b) common acceptor molecules used to construct small molecules and polymers for organic electronics; (c) design strategies to create electronic materials; and (d) $\mathrm{C}_{60}$.

Typical donor molecules for small molecules and polymers include thiophenes, pentacene, indaceno-dithiophene (IDT), hexabenzocoronene, and thieno(3,2-b)thiophene (TT). Each of these molecules possess either a lone pair that can add electron density, or donate to an adjacent atom or molecule, or possess extended conjugation. ${ }^{15,24,25}$

N-type molecules are often composed of thiadiazole, diketopyrrolopyrrole, rylene diimides-based structures, and $\mathrm{C}_{60} / 70$ and their derivatives. ${ }^{26-31}$ Figure $1 \mathrm{~A} .2$ provides examples of common donor and acceptor molecules used to build small molecules and polymers used in 
OFETs. This is by no means an exhaustive list, but illustrative of the types of molecules chemists consider in their design of materials. The evolution of bottom-up synthesis to synthesize materials that exhibit p-type characteristics has been rapid over the past few decades, with n-type materials recently garnering greater attention.

\section{A.6. Contorted Aromatics as Electronic Materials}

Fullerene derivatives, such as [6,6]-phenyl- $\mathrm{C}_{61} /{ }_{71}$-butyric acid methyl ester $\left(\mathrm{PC}_{61} \mathrm{BM}\right.$ and $\mathrm{PC}_{71} \mathrm{BM}$ ), have been used historically as the electron acceptor in bulk heterojunction due to efficient charge separation in fullerenes. ${ }^{27,32-37}$ Fullerenes present four useful structural and electronic properties: (1) a three-dimensional shape with a $\pi$-surface that can participate in $\pi$-to- $\pi$ interactions; (2) a conjugated and fully delocalized $\pi$-space; (3) the presence of an interior and exterior to the $\pi$-surface; and (4) a low-energy unoccupied molecular orbital(s) allowing them to accept electrons. These electronic and structural elements have inspired chemists to design nonfullerene molecules that exhibit "fullerene-like" properties.

Over the past few years, there have been several groups, including our own, that have synthesized non-fullerene materials for use in OFETs and OPVs. ${ }^{8,8,28,30,31,38-50}$ In particular, the Nuckolls laboratory has pioneered the design and synthesis of new contorted aromatics for materials applications. We named these compounds 'contorted aromatics' because steric congestion in their periphery results in non-planar structures. We have synthesized both discs and ribbon versions of contorted aromatics (Figure 1A.3.). ${ }^{8}$ These molecules have been an exciting complement to planar polyaromatic hydrocarbons. Contorted structures change both the physical and electronic structure of the molecules. This allows one to build different supramolecular assemblies with different binding motifs to probe on a fundamental level the relationship between structure and function in device applications. 


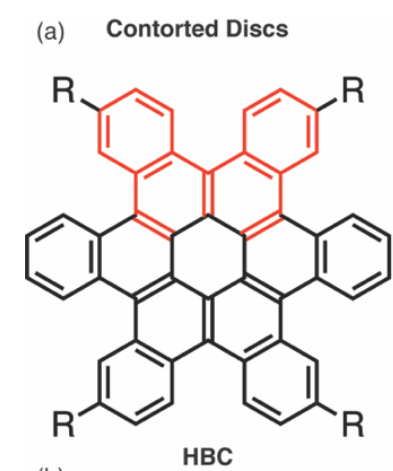

(c)

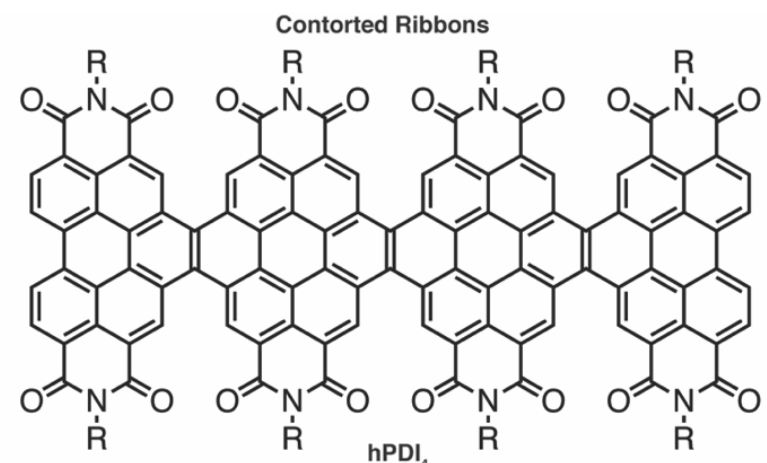

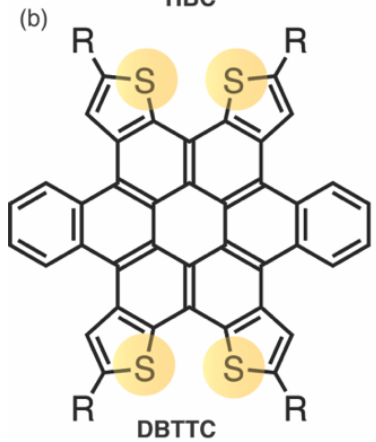

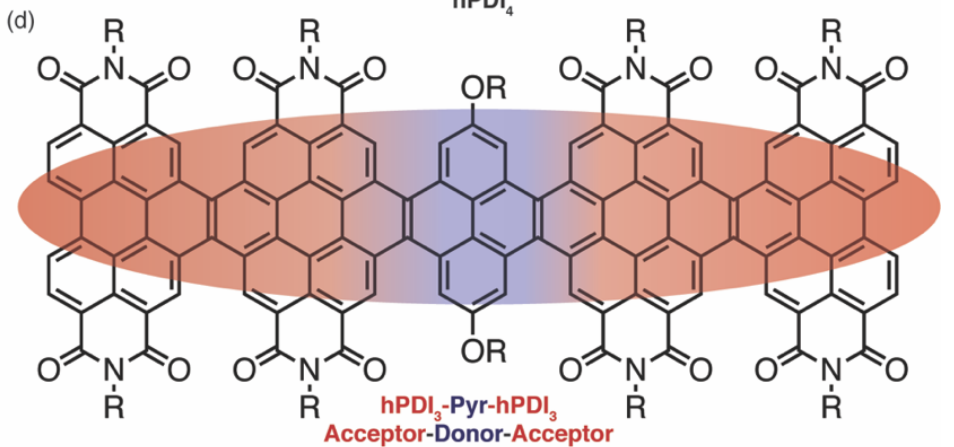

Figure 1A.3. Representative molecule of two contorted aromatics (a) HBC and (b) DBTCC. Both HBC and DBTCC are composed of [4] helicene structures outlined in red; (c) hPDI tetramer. One of the helical PDI series that showed exceptional solar cell performance; and (d) a donor-acceptor $\mathrm{hPDI}_{3}$-based ribbon, where the HOMO resides on a pyrene-centered orbital and the LUMO resides on a $\mathrm{hPDI}_{3}$-centered orbital.

The disc-shaped contorted molecules have well-defined conformations that create concave $\pi$-surfaces. The molecular feature incorporated into the design of these contorted structures is benzophenanthrene, also known as [4]helicene, the smallest carbohelicene (indicated in red in Figure 1A.3a). ${ }^{51}$ Steric congestion in the bay positions causes the molecule to bend out of planarity. Hexabenzocoronene (HBC) and dibenzotetrathienocoronene (DBTTC) are two examples of discshaped molecules in Figure 1A.3a,b. ${ }^{7,52,53}$ The concave surfaces of these disc-shaped contorted molecules form ideal receptors for the molecular recognition and assembly with shape-matched molecules. $^{54}$

The ribbon-shaped, contorted molecules can be conceptualized as ultra-narrow pieces of graphene. ${ }^{55}$ PDI is the building block for the ribbons. PDI has many advantages: (1) it is an efficient material in n-type organic field effect transistors, ${ }^{33,42,44,56,57}$ (2) it has high molar 
absorptivities; ${ }^{8,55,58}(3)$ it is an efficient electron acceptor in organic photovoltaics; ${ }^{41,44}$ and (4) it is easily functionalized from inexpensive starting materials and photostable. ${ }^{59-61}$ Upon substitution in the bay region of the molecule, PDI exists as two isomeric forms: 1,6 and 1,7-substituted PDI that possess different electronic properties (Figure 1A.4). ${ }^{62-65}$ 1,6-PDI and 1,7-PDI are also referred to as cis and trans-PDI, respectively. Chapter 4 and Chapter 8 both present interesting studies where we found substitution patterns impacted device performance.

The Nuckolls lab fuses PDI moieties to make atomically-defined graphene ribbons, the helical-PDI family $\left(\mathrm{hPDI}_{n}\right)^{8,55}$ The bottom-up approach allows for exquisite structural control and versatility to synthesize oligomeric derivatives. Figure 1A.4 shows the three oligomers synthesized: the dimer $\left(\mathrm{hPDI}_{2}\right)$, trimer $\left(\mathrm{hPDI}_{3}\right)$, and tetramer $(\mathrm{hPDI})$. The contortion in these ribbons causes them to wind into helical ribbons and exists in multiple conformations. Density Functional Theory (DFT) calculations performed by Dr. Nathanial Schuster in the Nuckolls laboratory suggest the barrier to inversion is approximately $11 \mathrm{kcal} / \mathrm{mol}$ between conformers. The PDI ribbons have been used in OFETs ${ }^{28} \mathrm{OPVs}^{28,41,66}$ and $\mathrm{OPDs}^{67,68}$. 

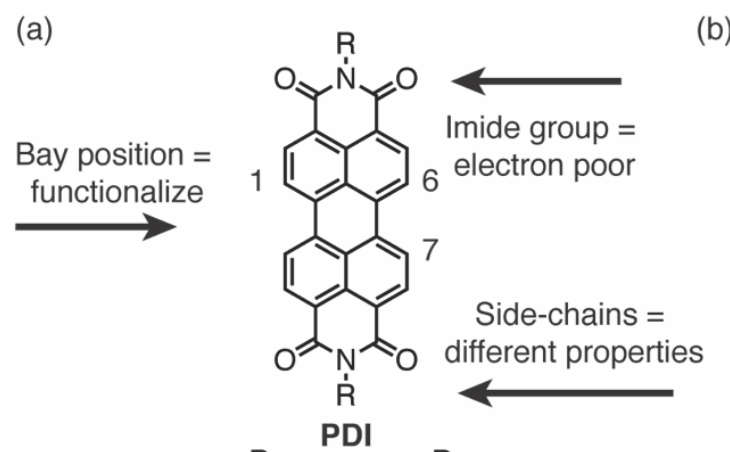

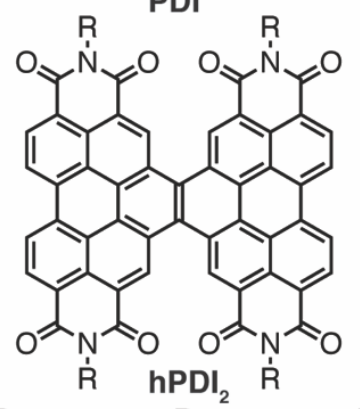

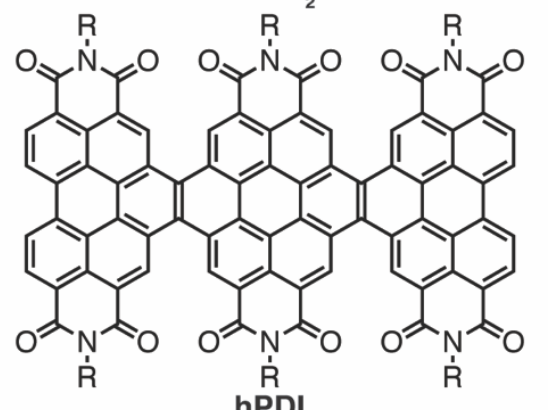

(c)

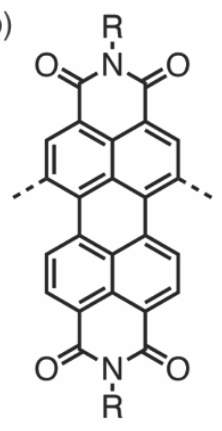<smiles>[R]N1C(=O)c2ccc3c4c(C)cc5c6c(ccc(c7c(C)cc(c2c37)C1=O)c64)C(=O)N(P)C5=O</smiles><smiles>[Te][Pb]</smiles>
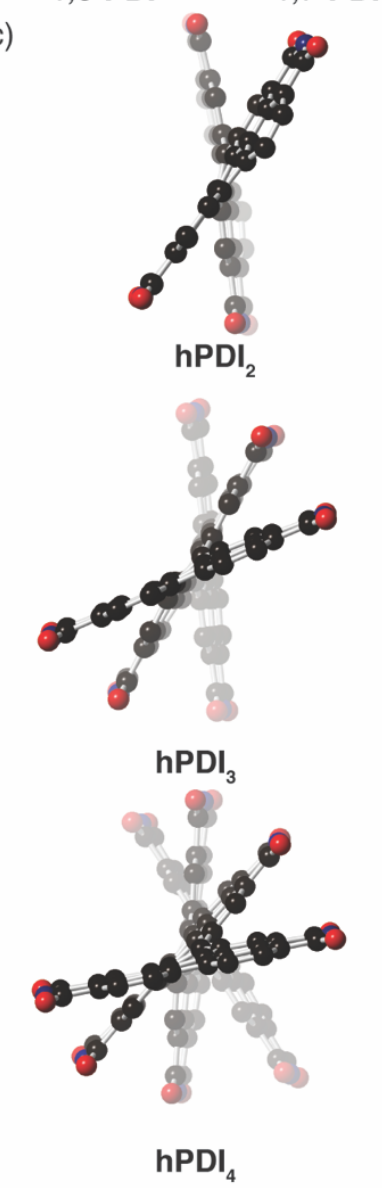

Figure 1A.4. (a) Helical PDI oligomer family. The imide position creates an area of low-energy unoccupied space on the molecule, the bay position can be readily substituted. (b) The most common substitution patterns are 1,7 and 1,6 which will be discussed in the following chapters. The $\mathrm{R}$ groups can be altered to affect processing of material and photophysical properties; (c) the helical conformation of the hPDI oligomers using DFT performed by Dr. Michael L. Steigerwald.

My research into the design and synthesis of the PDI-based conjugated macrocycles was inspired by much of the work produced in the Nuckolls laboratory over the past two decades. Ultimately, I was interested in how physical organic principles such as strain, rigidity, and chirality 
impact materials applications in three-dimensional conjugated macrocycles. Conjugated macrocycles had not been used in (opto)electronic devices until our studies. Chapter 1B provides an overview of the conjugated macrocycles; including, the design and inspiration for the materials. I also provide an overview of key concepts that feature in this thesis such as strain and rigidity.

\section{A.7. References}

1. Ball, M. et al. Conjugated Macrocycles in Organic Electronics. Acc. Chem. Res. (2019).

2. $\quad$ Ball, M. et al. Chiral Conjugated Corrals. J. Am. Chem. Soc. 137, 9982-9987 (2015).

3. Ball, M. et al. Macrocyclization in the Design of Organic n-Type Electronic Materials. $J$. Am. Chem. Soc. 138, 12861-12867 (2016).

4. Zhang, B. et al. Rigid, Conjugated Macrocycles for High Performance Organic Photodetectors. J. Am. Chem. Soc. 138, 16426-16431 (2016).

5. Zhang, B. et al. Hollow organic capsules assemble into cellular semiconductors. Nat. Commun. 9, 1957 (2018).

6. Ball, M. L. et al. Influence of Molecular Conformation on Electron Transport in Giant, Conjugated Macrocycles. J. Am. Chem. Soc. 140, 10135-10139 (2018).

7. Kang, S. J. et al. A Supramolecular Complex in Small-Molecule Solar Cells based on Contorted Aromatic Molecules. Angew. Chem. Int. Ed. 51, 8594-8597 (2012).

8. Ball, M. et al. Contorted Polycyclic Aromatics. Acc. Chem. Res. 48, 267-276 (2015).

9. Brédas, J. L. et al. Organic semiconductors: A theoretical characterization of the basic parameters governing charge transport. Proc. Natl. Acad. Sci. 99, 5804 LP - 5809 (2002).

10. Bendikov, M. et al. Tetrathiafulvalenes, Oligoacenenes, and Their Buckminsterfullerene Derivatives: The Brick and Mortar of Organic Electronics. Chem. Rev. 104, 4891-4946 (2004).

11. Newman, C. R. et al. Introduction to Organic Thin Film Transistors and Design of nChannel Organic Semiconductors. Chem. Mater. 16, 4436-4451 (2004).

12. Anthony, J. E. Functionalized Acenes and Heteroacenes for Organic Electronics. Chem. Rev. 106, 5028-5048 (2006).

13. Gundlach, D. J. et al. Thin-film transistors based on well-ordered thermally evaporated naphthacene films. Appl. Phys. Lett. 80, 2925-2927 (2002).

14. Lin, Y. et al. Stacked pentacene layer organic thin-film transistors with improved characteristics. IEEE Electron Device Lett. 18, 606-608 (1997).

15. Zaumseil, J. et al. Electron and Ambipolar Transport in Organic Field-Effect Transistors. Chem. Rev. 107, 1296-1323 (2007).

16. Brédas, J.-L. et al. Charge-Transfer and Energy-Transfer Processes in $\pi$-Conjugated Oligomers and Polymers: A Molecular Picture. Chem. Rev. 104, 4971-5004 (2004). 
17. Coropceanu, V. et al. Charge Transport in Organic Semiconductors. Chem. Rev. 107, 926952 (2007).

18. Roncali, J. Molecular engineering of the band gap of pi-conjugated systems: Facing technological applications. Macromol. Rapid Commun. 28, 1761-1775 (2007).

19. Havinga, E. E. et al. A new class of small band gap organic polymer conductors. Polym. Bull. 29, 119-126 (1992).

20. Anslyn, E. V. et al. Modern Physical Organic Chemistry. (University Science Books, 2006).

21. Guo, X. et al. Designing $\pi$-conjugated polymers for organic electronics. Prog. Polym. Sci. 38, 1832-1908 (2013).

22. Havinga, E. E. et al. Alternate donor-acceptor small-band-gap semiconducting polymers; Polysquaraines and polycroconaines. Synth. Met. 55, 299-306 (1993).

23. You, J. B. et al. A polymer tandem solar cell with $10.6 \%$ power conversion efficiency. Nat. Commun. 4, 10 (2013).

24. Facchetti, A. $\pi$-Conjugated Polymers for Organic Electronics and Photovoltaic Cell Applications. Chem. Mater. 23, 733-758 (2011).

25. Dou, L. et al. 25th Anniversary Article: A Decade of Organic/Polymeric Photovoltaic Research. Adv. Mater. 25, 6642-6671 (2013).

26. Kroto, H. W. et al. C60: Buckminsterfullerene. Nature 318, 162-163 (1985).

27. Haddon, R. C. et al. C60 thin film transistors. Appl. Phys. Lett. 67, 121-123 (1995).

28. Zhong, Y. et al. Molecular helices as electron acceptors in high-performance bulk heterojunction solar cells. Nat Commun. 6, 8242 (2015).

29. Lin, Y. Z. et al. High-performance fullerene-free polymer solar cells with $6.31 \%$ efficiency. Energy Environ. Sci. 8, 610-616 (2015).

30. Nielsen, C. B. et al. Non-Fullerene Electron Acceptors for Use in Organic Solar Cells. Acc. Chem. Res. 48, 2803-2812 (2015).

31. Yan, C. et al. Non-fullerene acceptors for organic solar cells. Nat. Rev. Mater. 3, 18003 (2018).

32. Savoie, B. M. et al. Unequal Partnership: Asymmetric Roles of Polymeric Donor and Fullerene Acceptor in Generating Free Charge. J. Am. Chem. Soc. 136, 2876-2884 (2014).

33. Anthony, J. E. et al. n-Type Organic Semiconductors in Organic Electronics. Adv. Mater. 22, 3876-3892 (2010).

34. Huang, Q. et al. Photoconductive Curved-Nanographene/Fullerene Supramolecular Heterojunctions. Angew. Chem. Int. Ed. 58, 6244 (2019).

35. Li, H. et al. High-Mobility Field-Effect Transistors from Large-Area Solution-Grown Aligned C60 Single Crystals. J. Am. Chem. Soc. 134, 2760-2765 (2012).

36. Zhang, Y. et al. Development of fullerenes and their derivatives as semiconductors in fieldeffect transistors: exploring the molecular design. J. Mater. Chem. C 6, 3514-3537 (2018). 
37. Zhao, Y. et al. 25th Anniversary Article: Recent Advances in n-Type and Ambipolar Organic Field-Effect Transistors. Adv. Mater. 25, 5372-5391 (2013).

38. Hou, J. et al. Organic solar cells based on non-fullerene acceptors. Nat. Mater. 17, 119 (2018).

39. Matsuno, T. et al. Self-Sorting of Two Hydrocarbon Receptors with One Carbonaceous Ligand. Angew. Chem. Int. Ed. 55, 15339-15343 (2016).

40. Zhong, Y. et al. Efficient Organic Solar Cells with Helical Perylene Diimide Electron Acceptors. J. Am. Chem. Soc. 136, 15215-15221 (2014).

41. Zhong, Y. et al. Efficient Organic Solar Cells with Helical Perylene Diimide Electron Acceptors. J. Am. Chem. Soc. 136, 15215 (2014).

42. Sharenko, A. et al. A High-Performing Solution-Processed Small Molecule: Perylene Diimide Bulk Heterojunction Solar Cell. Adv. Mater. 25, 4403-4406 (2013).

43. Lin, Y. et al. A Star-Shaped Perylene Diimide Electron Acceptor for High-Performance Organic Solar Cells. Adv. Mater. 26, 5137-5142 (2014).

44. Cai, Y. et al. High Performance Organic Solar Cells Based on a Twisted Bay-Substituted Tetraphenyl Functionalized Perylenediimide Electron Acceptor. Adv. Energy Mater. 5, 1500032 (2015).

45. Segawa, Y. et al. Efficient Organic Solar Cells with Helical Perylene Diimide Electron Acceptors. J. Am. Chem. Soc. 137, 3122-3125 (2015).

46. Lin, H. et al. Reduced Intramolecular Twisting Improves the Performance of 3D Molecular Acceptors in Non-Fullerene Organic Solar Cells. Adv. Mater. 28, 8546-8551 (2016).

47. Meng, D. et al. High-Performance Solution-Processed Non-Fullerene Organic Solar Cells Based on Selenophene-Containing Perylene Bisimide Acceptor. J. Am. Chem. Soc. 138, 375-380 (2016).

48. Lin, Y. et al. Non-fullerene acceptors for organic photovoltaics: an emerging horizon. Mater. Horizons 1, 470-488 (2014).

49. Chen, W. et al. A perylene diimide (PDI)-based small molecule with tetrahedral configuration as a non-fullerene acceptor for organic solar cells. J. Mater. Chem. C 3, 4698-4705 (2015).

50. Zhang, J. et al. Material insights and challenges for non-fullerene organic solar cells based on small molecular acceptors. Nat. Energy 3, 720-731 (2018).

51. Gingras, M. One hundred years of helicene chemistry. Part 1: non-stereoselective syntheses of carbohelicenes. Chem. Soc. Rev. 42, 968-1006 (2013).

52. Chiu, C.-Y. et al. Shape-shifting in contorted dibenzotetrathienocoronenes. Chem. Sci. 2, 1480-1486 (2011).

53. Roncali, J. Synthetic Principles for Bandgap Control in Linear $\pi$-Conjugated Systems. Chem. Rev. 97, 173-206 (1997).

54. Tremblay, N. J. et al. Photovoltaic Universal Joints: Ball-and-Socket Interfaces in Molecular Photovoltaic Cells. ChemPhysChem 11, 799-803 (2010). 
55. Zhong, Y. et al. Helical Ribbons for Molecular Electronics. J. Am. Chem. Soc. 136, 8122 8130 (2014).

56. Zhou, E. J. et al. All-Polymer Solar Cells from Perylene Diimide Based Copolymers: Material Design and Phase Separation Control. Angew. Chem. Int. Ed. 50, 2799-2803 (2011).

57. Li, C. \& Wonneberger, H. Perylene Imides for Organic Photovoltaics: Yesterday, Today, and Tomorrow. Adv. Mater. 24, 613-636 (2012).

58. Nolde, F. et al. Synthesis and self-organization of core-extended perylene tetracarboxdiimides with branched alkyl substituents. Chem. Mater. 18, 3715-3725 (2006).

59. Yan, Q. F. et al. Conjugated Dimeric and Trimeric Perylenediimide Oligomers. Org. Lett. 11, 3426-3429 (2009).

60. Huo, L. J. et al. Synthesis and absorption spectra of n-type conjugated polymers based on perylene diimide. Macromol. Rapid Commun. 29, 1444-1448 (2008).

61. Würthner, F. et al. Perylene Bisimide Dye Assemblies as Archetype Functional Supramolecular Materials. Chem. Rev. 116, 962-1052 (2016).

62. Ahrens, M. J. et al. R. Bis(n-octylamino)perylene-3,4:9,10-bis(dicarboximide)s and Their Radical Cations: Synthesis, Electrochemistry, and ENDOR Spectroscopy. J. Org. Chem. 71, 2107-2114 (2006).

63. Osswald, P. et al. Effects of Bay Substituents on the Racemization Barriers of Perylene Bisimides: Resolution of Atropo-Enantiomers. J. Am. Chem. Soc. 129, 14319-14326 (2007).

64. Schmidt, R. et al. Synthetic Routes to Core-fluorinated Perylene Bisimide Dyes and their Properties. Z. Naturforsch. B 64, 735-746 (2009).

65. Chen, Z. et al. Effect of Core Twisting on Self-Assembly and Optical Properties of Perylene Bisimide Dyes in Solution and Columnar Liquid Crystalline Phases. Chem. - A Eur. J. 13, 450-465 (2007).

66. Edison, C. et al. Cove-Edge Nanoribbon Materials for Efficient Inverted Halide Perovskite Solar Cells. Angew. Chem. Int. Ed. 56, 14648-14652 (2017).

67. Zhong, Y. et al. Helical Nanoribbons for Ultra-Narrowband Photodetectors. J. Am. Chem. Soc. 139, 5644-5647 (2017). 


\section{Chapter 1 Section 1B. Conjugated Macrocycles in Organic Electronics}

\section{B.1. Preface}

Chapter 1B contains excerpts from a published accounts article entitled "Conjugated Macrocycles in Organic Electronics” by Melissa L. Ball, Boyuan Zhang, Yu Zhong, Brandon Fowler, Shengxiong Xiao, Fay Ng, Michael Steigerwald, and Colin Nuckolls, published in the Accounts of Chemical Research. ${ }^{1}$ Copyright 2019 American Chemical Society.

\section{B.2. Introduction: Inspiration for Design}

Here I describe our efforts to design and synthesize a new class of n-type materials: conjugated macrocycles. Conjugated macrocycles ${ }^{2-28}$ have several potential advantages as organic electronic materials. ${ }^{11,28-33}$ Our macrocyclic design takes into consideration the useful attributes of fullerenes and what properties make fullerenes efficient n-type materials. In order to create electronic materials that complement fullerenes, we asked ourselves what makes fullerenes effective as n-type materials, and then used the power of synthetic chemistry to try to install these features into new materials. Fullerenes present four useful structural and electronic properties: 1) a three-dimensional shape; 2) a conjugated and fully delocalized pi-space; 3) the presence of an interior and exterior to the pi-surface; and 4) a low-energy unoccupied molecular orbital(s) allowing them to accept electrons. ${ }^{34,35}$

While designing the macrocycles, we wondered how "fullerene-like" the macrocycles needed to be in order to remain "fullerene-like" electronic materials. We synthesized the macrocycles to mimic the three dimensional shape of fullerenes, yet our design offers several advantages: synthetic flexibility (allowing us to control the electronic properties of the macrocycles), extensive pi-conjugation, and intramolecular cavities that facilitate guest incorporation and sensing. My research goal was to design and synthesize three-dimensional 
conjugated macrocycles that could absorb visible light and be used as the n-type material in electronic applications.

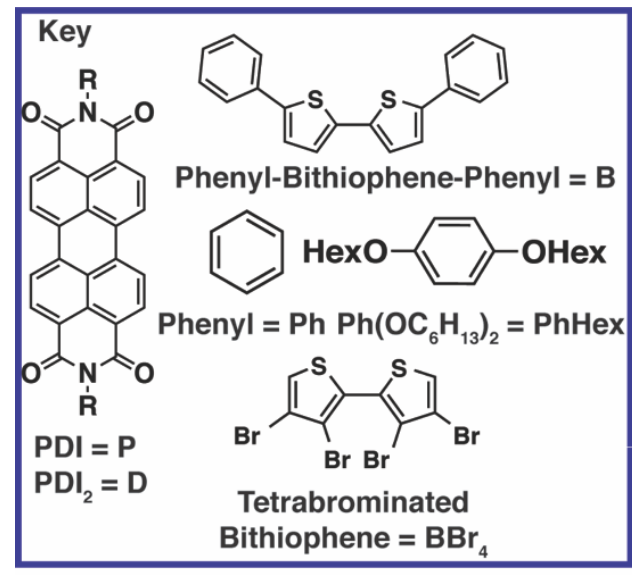

(c)

(d) (a)

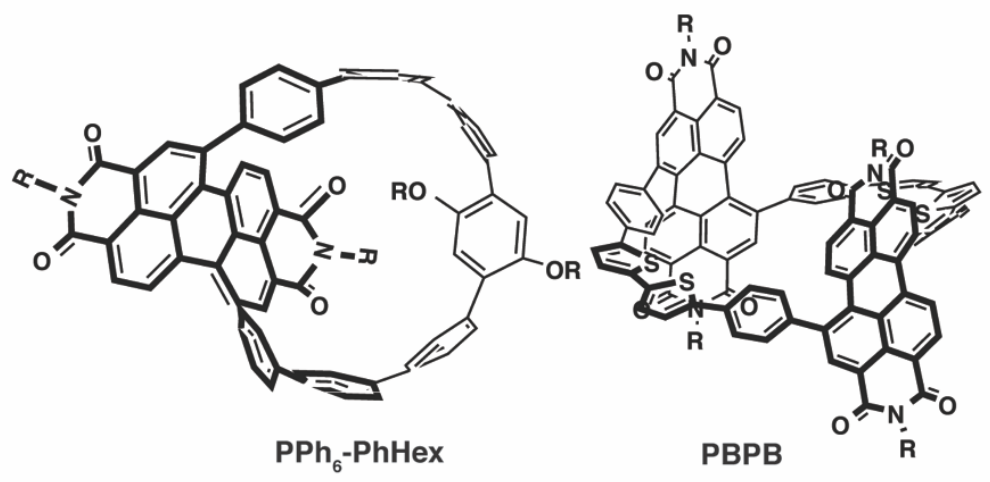

(e)
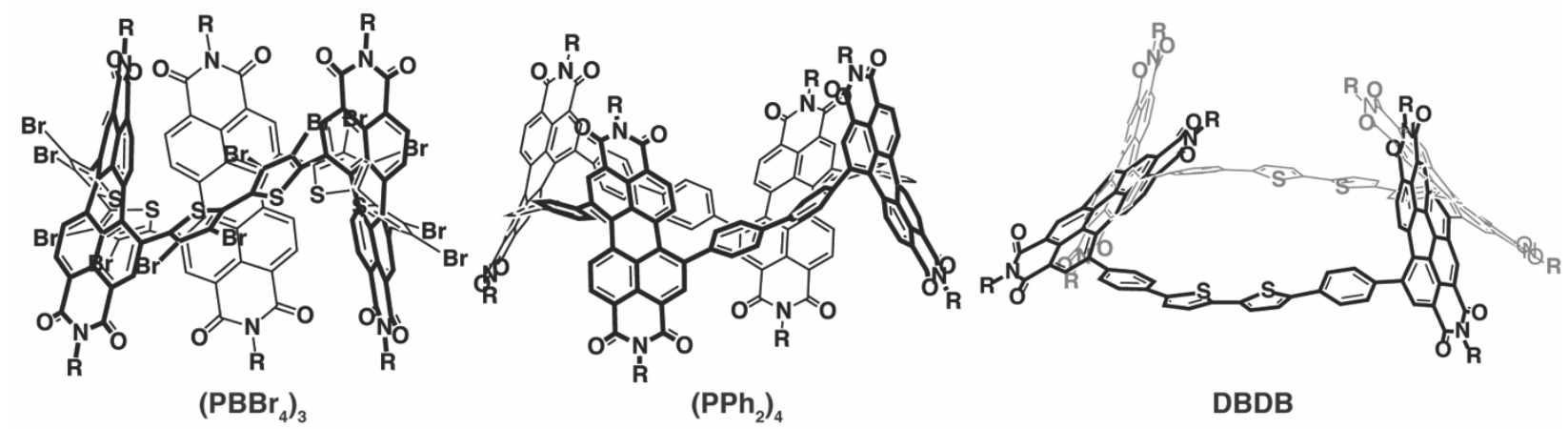

Figure 1B.1. The five conjugated macrocycles: (a) PPh6-PhHex; (b) PBPB; (c) (PBBr 4$)_{3}$; (d) $\left(\mathbf{P P h}_{2}\right)_{4}$; and (e) DBDB.

Figures 1B.1a-d show the four macrocycles studied that comprise monomeric PDI. The four molecules differ in the number of PDI (PDI $=$ "P" in the names of each macrocycle) and linking groups that wind them into a macrocyclic structure. I studied three main linkers: a PhenylBithiophene-Phenyl belt (B), a phenyl group (Ph and PhHex), and a tetrabrominated bithiophene $\left(\mathbf{B B r}_{4}\right)$ (Figure 1B.1a-d). We also synthesized and studied a $\mathrm{hPDI}_{2}$-based $\left(\mathrm{hPDI}_{2}=\right.$ "D") macrocycle that exchanged monomeric PDI for $\mathbf{h} \mathbf{P D I}_{2}$ (Figure 1A.4), and called this macrocycle DBDB (Figure 1B.1e).

There was both a structural and electronic consideration in the design of the conjugated macrocycles. First, the choice of subunits was paramount. We considered subunits that absorbed 
visible light as this would allow provide utility in optoelectronic application. Each of the macrocycles contain at least one PDI (or hPDI 2 ) due to its many advantages: it is an efficient material in n-type organic field effect transistors; ${ }^{36-40}$ (2) it has high molar absorptivities; ${ }^{41-43}$ (3) it is an efficient electron acceptor in organic photovoltaics; ${ }^{10,44}$ (4) it forms small band gap polymers with electron-donating moieties; and (5) it is easily functionalized from inexpensive starting materials and photostable. ${ }^{42,45-47}$ The choice of linker between the PDI moieties was both a structural and electronic decision. The geometry of the linking group can facilitate macrocyclization by reducing strain. The linker groups, too, had a profound effect on the electronic structure of each macrocycle by modulating the HOMO-LUMO gap (Section 1B.7).

The macrocycles exist as chiral materials and exhibit size-dependent isomerization between stereoisomers (Section 1B.4.). Two of the monomer PDI macrocycles $\mathbf{P P h}_{6}-\mathbf{P h H e x}$ and $\left(\mathbf{P B B r}_{4}\right)_{3}$ are conformationally rigid, and unable to isomerize, while both $\mathbf{P B P B}$ and $\left(\mathbf{P P h}_{2}\right)_{4}$ show dynamic stereochemistry (Sections 1B.4 and 1B.5).

The conjugated macrocycles, too, are electronic and optoelectronic materials, and these "pseudo-fullerenes" are useful in an array of devices. This Thesis will describe how the macrocycles are effective n-type semiconductors in OFETs, ${ }^{11,33,48}$ and can act as the electronaccepting materials in a bulk heterojunction. ${ }^{11}$ Moreover, we find the internal cavities can be used as a locus of guest binding to create sensors and nanoscale reaction chambers (Chapter 6 and 9). ${ }^{33}$

\section{B.3. Synthetic Approaches to Build in Strain}

We based our synthetic strategy on the pioneering studies by the Yamago ${ }^{21,49}$ and the Jasti laboratories $^{50,51}$ in their syntheses of cycloparaphenylenes (CPPs). CPPs possess interesting topologies, but do not absorb in the visible portion of the solar spectrum and thus have not been explored in optoelectronic devices. 
Figure 1B.2 shows two synthetic approaches to synthesize strained CPPs. The first approach builds in strain by performing the macrocyclization and reductive elimination steps before a final reductive aromatization to yield their desired product. One of the interesting design features is the two-fold addition of 4-bromo-4'-lithiobiphenyl to 1,4-benzoquinone: the two biphenyl appendages are on the same face of the aryl ring aiding the macrocyclization step. The hypothesis is that after the first addition, the oxygen anion is on one face of the aryl ring, and as the second mole of the lithio-biphenyl approaches, it preferentially attacks the carbonyl from the side anti to the oxygen anion as it is electronically favored due to Coulombic repulsion.

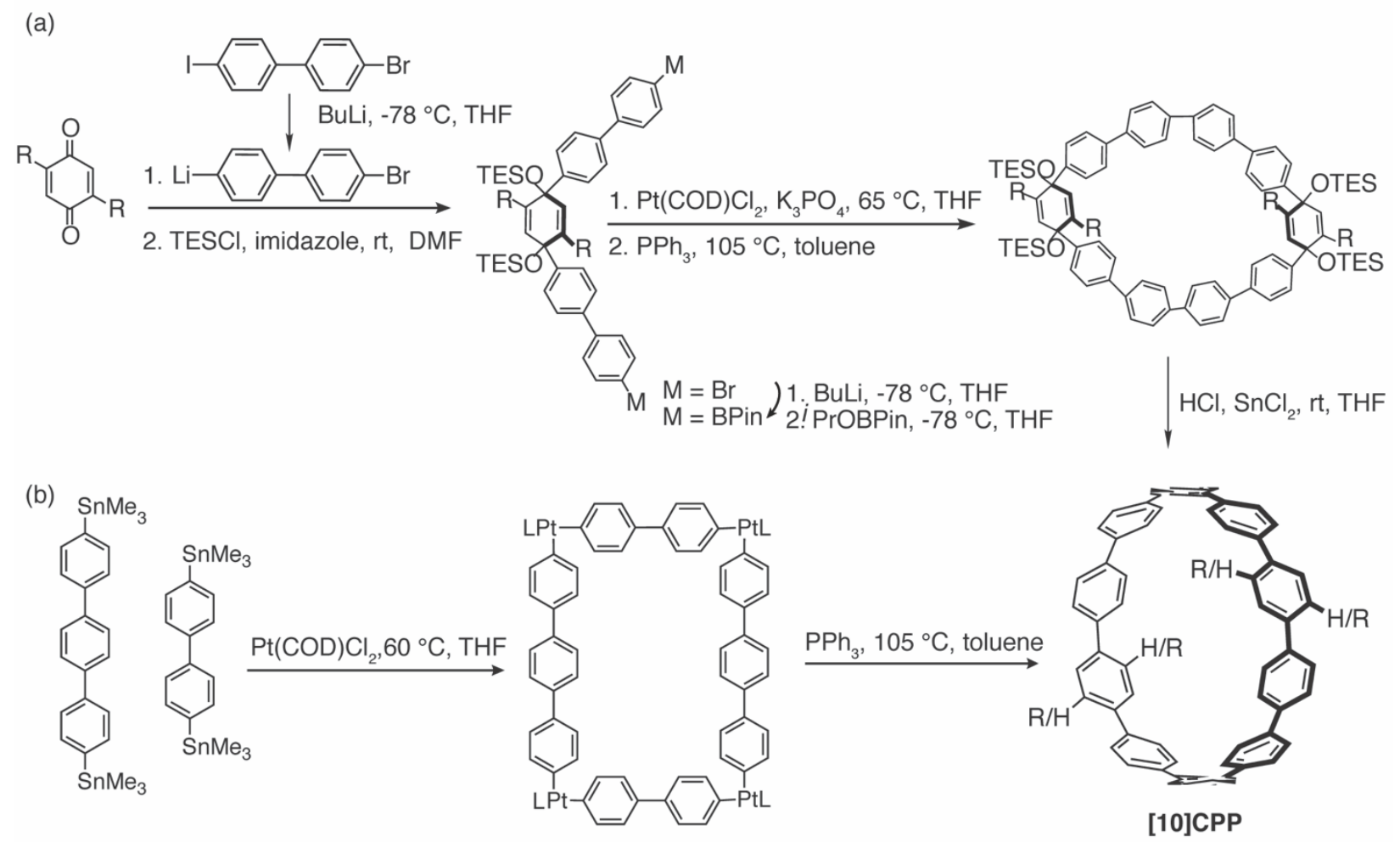

Figure 1B.2. (a) Synthetic approach utilizing an unstrained precursor to perform the macrocyclization before the final aromatization step; (b) a Pt-based strategy that relies on transmetalation from an aryl-tin bond to an aryl- Pt bond. $\mathrm{R}=$ alkyl, alkoxy groups.

The second strategy involves the transmetalation of a tin group onto a platinum $(\mathrm{Pt})$. While both approaches take advantage of the square planar geometry of $\mathrm{Pt}$ in the macrocyclization step, the latter strategy does not possess a final reductive aromatization step. One of the theoretical 
downsides to both strategies is the reversibility of the transmetalation step, which can lead to multiple unwanted byproducts, yet it only seemed to impact the latter approach. ${ }^{21,52}$ We employed both approaches in the syntheses of the conjugated macrocycles, and the following chapters provide the details of each macrocycle's synthesis.

Several important physical organic concepts arise in each chapter of this Thesis including strain, rigidity, conjugation, and chirality, and each have interesting effects on the macrocycles structural and electronic properties. Thus, Sections 1B.4 - IB.7 provide a brief background on these topics insomuch as how they relate to the conjugated macrocycles. This knowledge will support and aid one's understanding of the remaining chapters.

\section{B.4. Bay Substitution of PDI Induces Chirality}

The PDI macrocycles possess chiral axes about each PDI as described below. For macrocycles that are sufficiently rigid, these chiral axes are locked; however, for more flexible macrocycles, mechanisms exist for stereoisomerization of these chiral axes. The stereoisomers of macrocyclic PDIs arise from di-substitution of the PDI bay positions, which results in two observed isomeric forms, a 1,6- and a 1,7-substituted PDI or cis- and trans-substituted PDI (Figure 1A.4). The 1,7-substitution exhibits axial chirality (or helicity), with a stereogenic axis down the

long axis of the PDI moeity. ${ }^{53-58}$ Axial chirality arises when a molecules possesses a stereogenic axis, rather than a stereogenic center, and the substituents about this axis are spatially arranged so they are not superimposable. ${ }^{59}$ When 1,7-substituted PDI (1,7-PDI, for short) possesses a bulky group in the bay position (phenyl groups in Figure 1B.3), the two naphthyl groups that comprise the perylene backbone twist out of planarity to avoid steric congestion between the substituent and the hydrogen in the adjacent bay position (top naphthyl is in a solid rectangle and the bottom naphthyl is indicated with a dashed rectangle in Figure 1B.3a,b). The twist angle is approximately 
$22^{\circ}$ for a 1,7-phenyl PDI (Compound 1.1). The twisting of the naphthyl groups places the bay substituents on the same face of the PDI molecule. Figure 1B.3c and Figure 1B.3d provide a view of the stereogenic axis and a schematic detailing the designation of substituents. The top naphthyl contains the two "near" groups and the bottom naphthyl contains the two "far" groups.

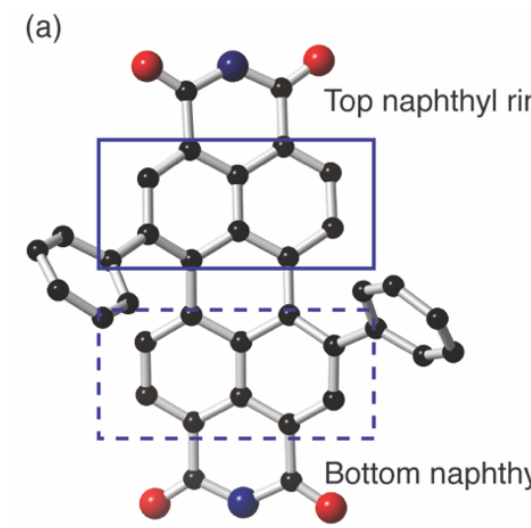

(c)
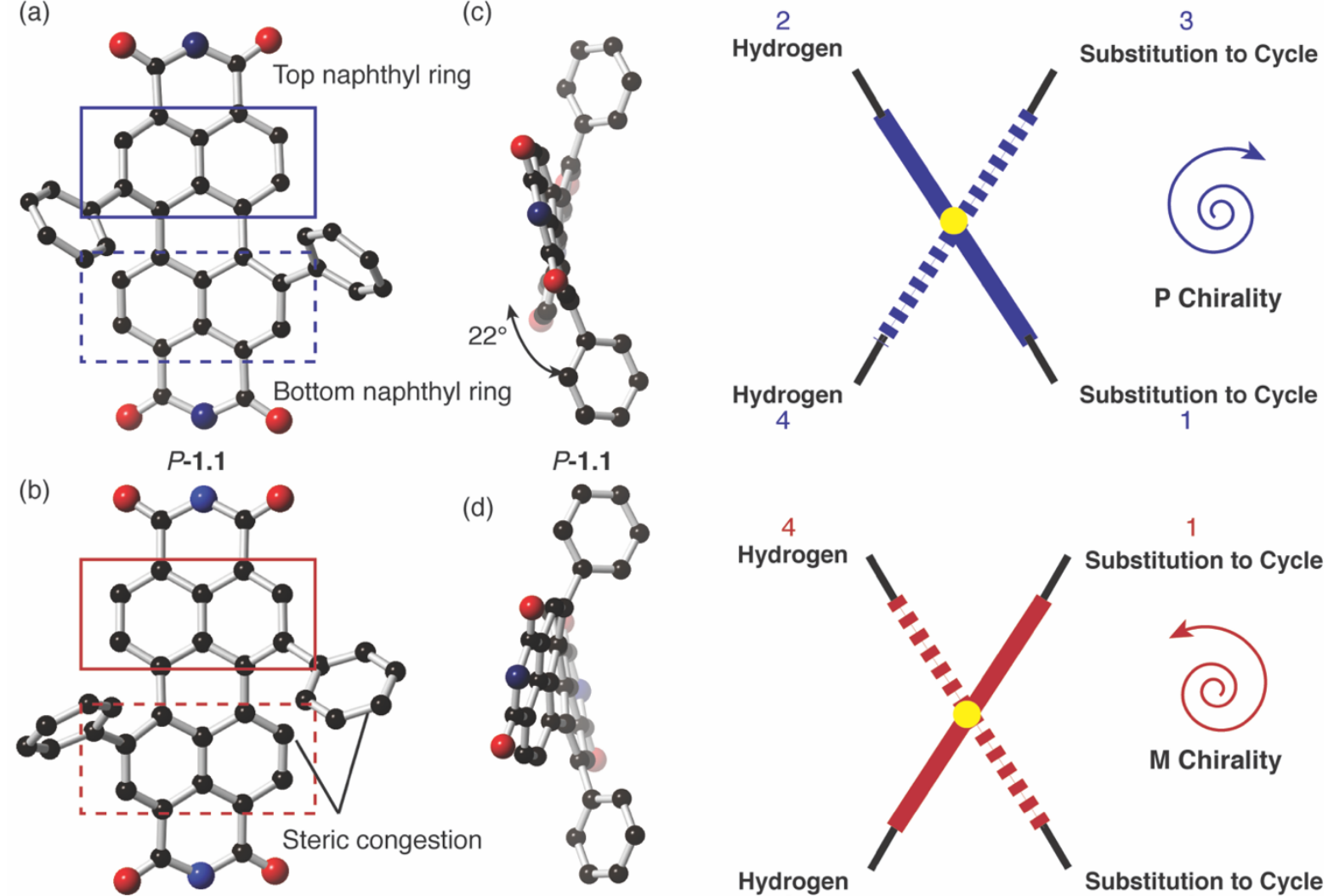

M-1.1

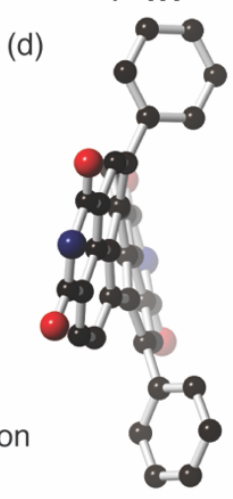

$M-1.1$

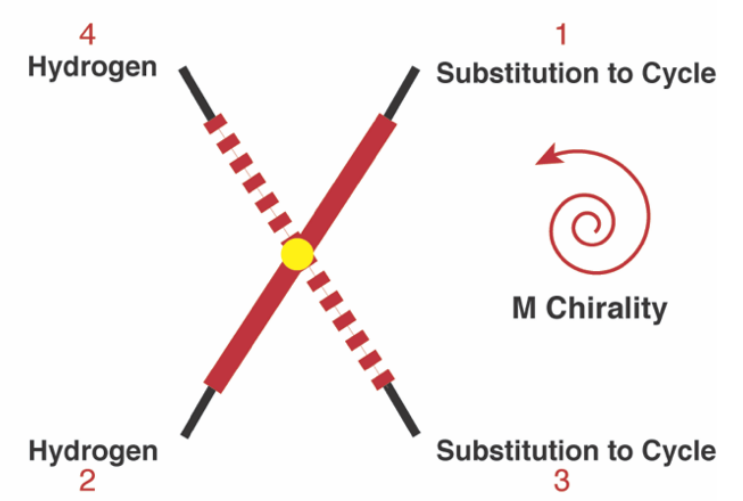

Figure 1B.3. (a) $P$-diphenyl PDI $(P$-1.1) and (b) $M$-diphenyl PDI $(M-1.1)$ from DFT calculations carried out at 6-31G/B3LYP level of theory; (c) and (d) show how the $P$ and $M$ designations are assigned. One looks down the stereogenic axis (indicated with a yellow dot) and assigns the groups off the top naphthyl (solid line) a 1 or 2 priority before assigning 3 and 4 to the bottom naphthyl (dashed line) ring substituents.

One assigns the substituents from $1-4$ with the near groups taking priority. If the assignment produces a clockwise rotation, this produces a right-handed, $P$ (or Plus) helix, and a counterclockwise rotation yields a left-handed helix $M$ (or Minus). An analogous procedure can be used to assign the helicity of DBDB. When conformationally restricted in a macrocycle, 
isomerization between a $P$ or $M$ enantiomer depends on the size of the cavity. Section 1B.5 introduces how rigidity affects the ability to isomerize between stereoisomers.

\section{B.5. Rigidity in the Macrocycles and its Effects on Isomerization}

The number of stereoisomers is directly related to the number of PDI subunits constrained within the cycle. For example, PBPB contains two PDI subunits and each can take a $P$ or $M$ designation. Thus, theoretically in solution, there are the enantio-pair $(P, P)-,(M, M)$-, and meso diastereomer $(P, M)$. Likewise $\left(\mathbf{P P h}_{2}\right)_{4}$ possesses four 1,7-PDI units, and exists as six stereoisomers in solution (two pairs of enantiomers, $P, P, P, P, / M, M, M, M$ and $P, P, P, M / M, M, M, P$ and two meso compounds, $P, M, P, M$ and $P, P, M, M)$.

One of the most obvious differences among the macrocycles is the size of their intramolecular cavities. According to structures determined DFT, the cavity is the smallest in

$\left(\mathbf{P B B r}_{4}\right)_{3}\left(1.1 \mathrm{~nm}\right.$ from PDI to thiophene), largest in $\left(\mathbf{P P h}_{2}\right)_{4}(2.0 \mathrm{~nm}$ across its transannular axis from PDI to PDI), and intermediate (1.6 nm) in PBPB and (1.3 nm) in $\mathbf{P P h} 6-\mathbf{P h H e x}$. The size of the cavity directly affects the intramolecular fluctional behavior and the macrocycles ability to isomerize between stereoisomers. 


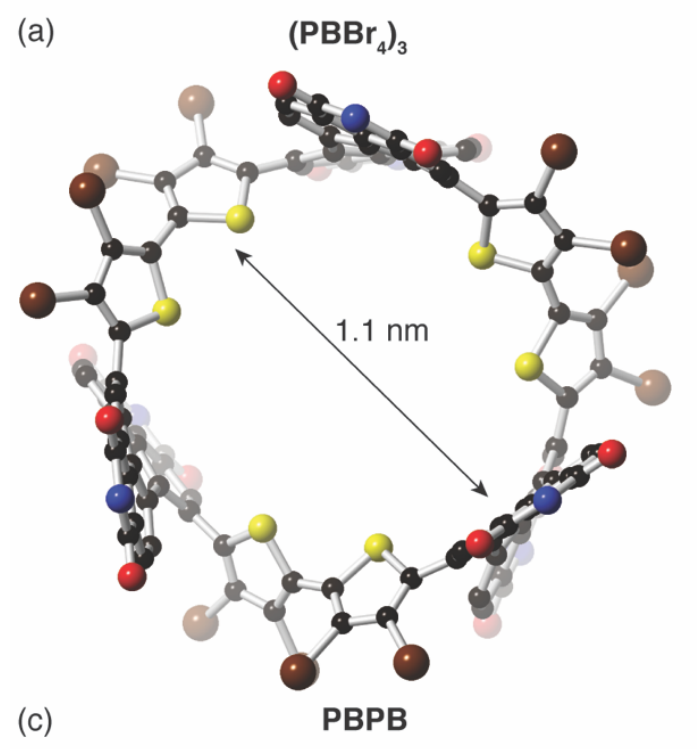

(b) $\quad \mathrm{PPh}_{6}-\mathrm{PhHex}$
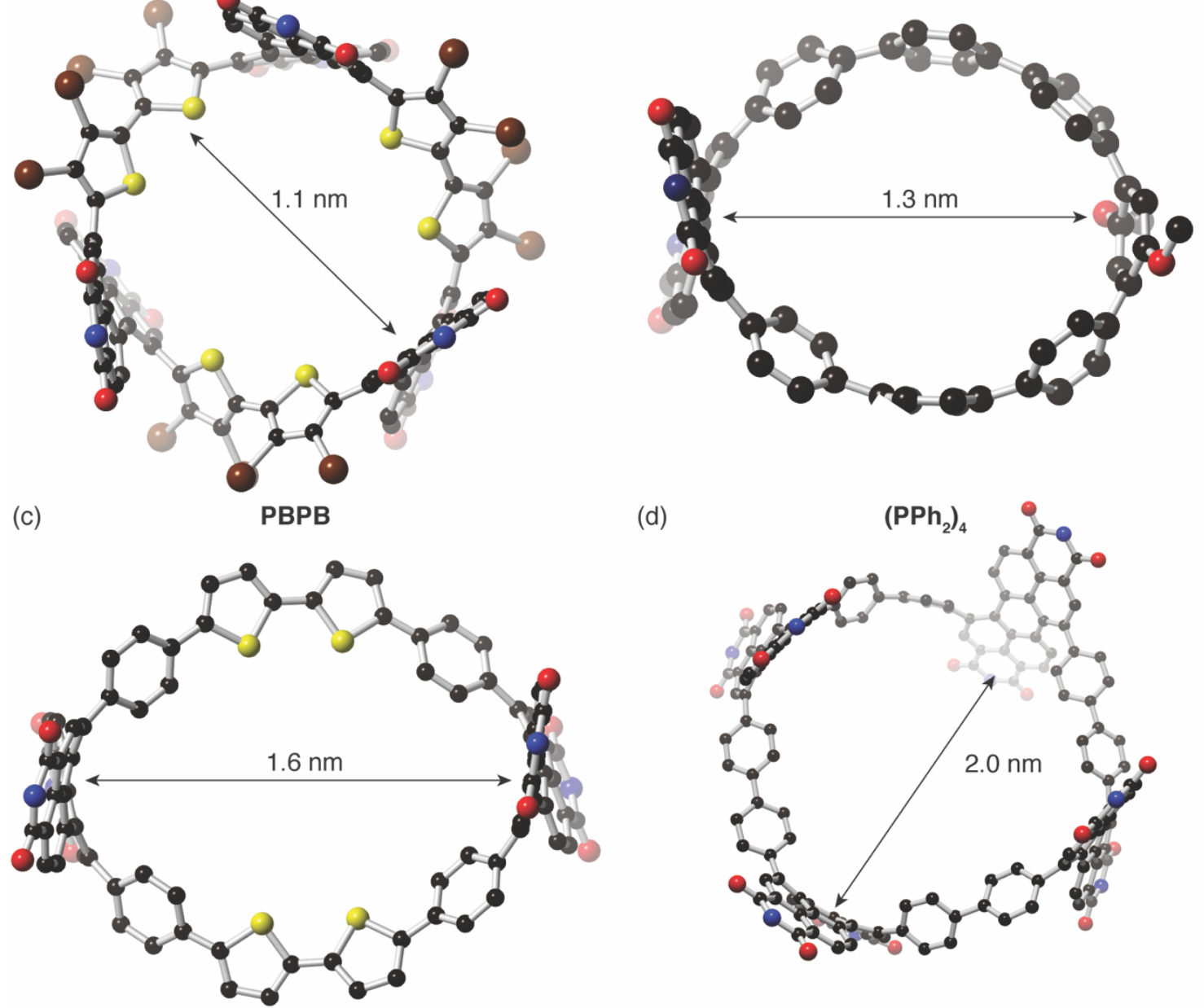

(d)

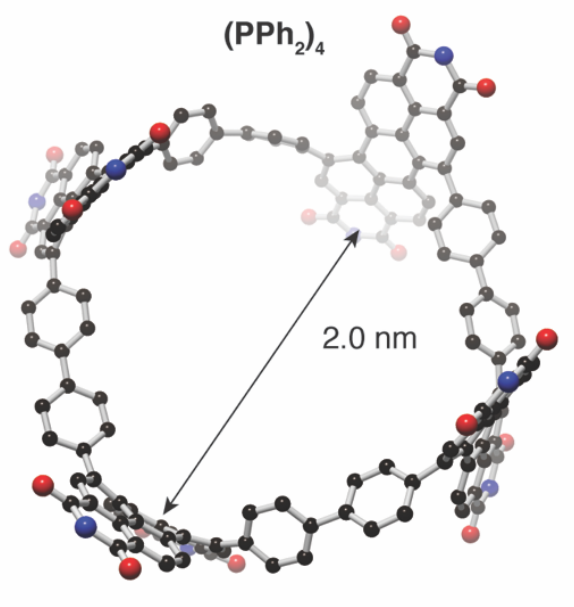

Figure 1B.4. Monomeric PDI-based macrocycles. The smallest macrocycles are (a) $\left(\mathbf{P B B r}_{4}\right)_{3}$ and (b) $\mathbf{P P h} \mathbf{h}_{6}-\mathbf{P h H e x}$; (c) PBPB; and the largest cavity is for (d) $(\mathbf{P P h})_{4}$.

Both $\left(\mathbf{P B B r}_{4}\right)_{3}$ and $\mathbf{P P h}_{6}-\mathbf{P h H e x}$ possess fewer rotational degrees of freedom, resulting in conformationally locked macrocycles that are unable to isomerize between stereoisomers. We are able to isolate the two enantiomers of $\mathbf{P P h}_{6}-\mathbf{P h H e x}$ from chiral high performance liquid chromatography (HPLC), and find the two enantiomers do not interconvert at temperature up to $100{ }^{\circ} \mathrm{C}$. We also are able to isolate one pair of the enantiomers for $\left(\mathbf{P B B r}_{4}\right)_{3}$, and find no interconversion up to $160^{\circ} \mathrm{C}$. With larger cavities, $\mathbf{P B P B},(\mathbf{P P h})_{4}$, and DBDB exhibit dynamic stereo-isomerization. These macrocycles isomerize rapidly at room temperature, and are we are unable to isolate individual stereoisomers. 


\section{B.6. Strain as a Function of Design}

The macrocycles are an exemplary model system to test the interplay between rigidity and strain. As the macrocycles' topologies differ, so does the amount of strain within each system. We calculate the amount of strain within the macrocycles using a group equivalent homodesmotic calculation. ${ }^{49,60-62}$ Before discussing the macrocycles, Figure 1B.5 shows an example of an homodesmotic calculations using a cyclobutane molecule.
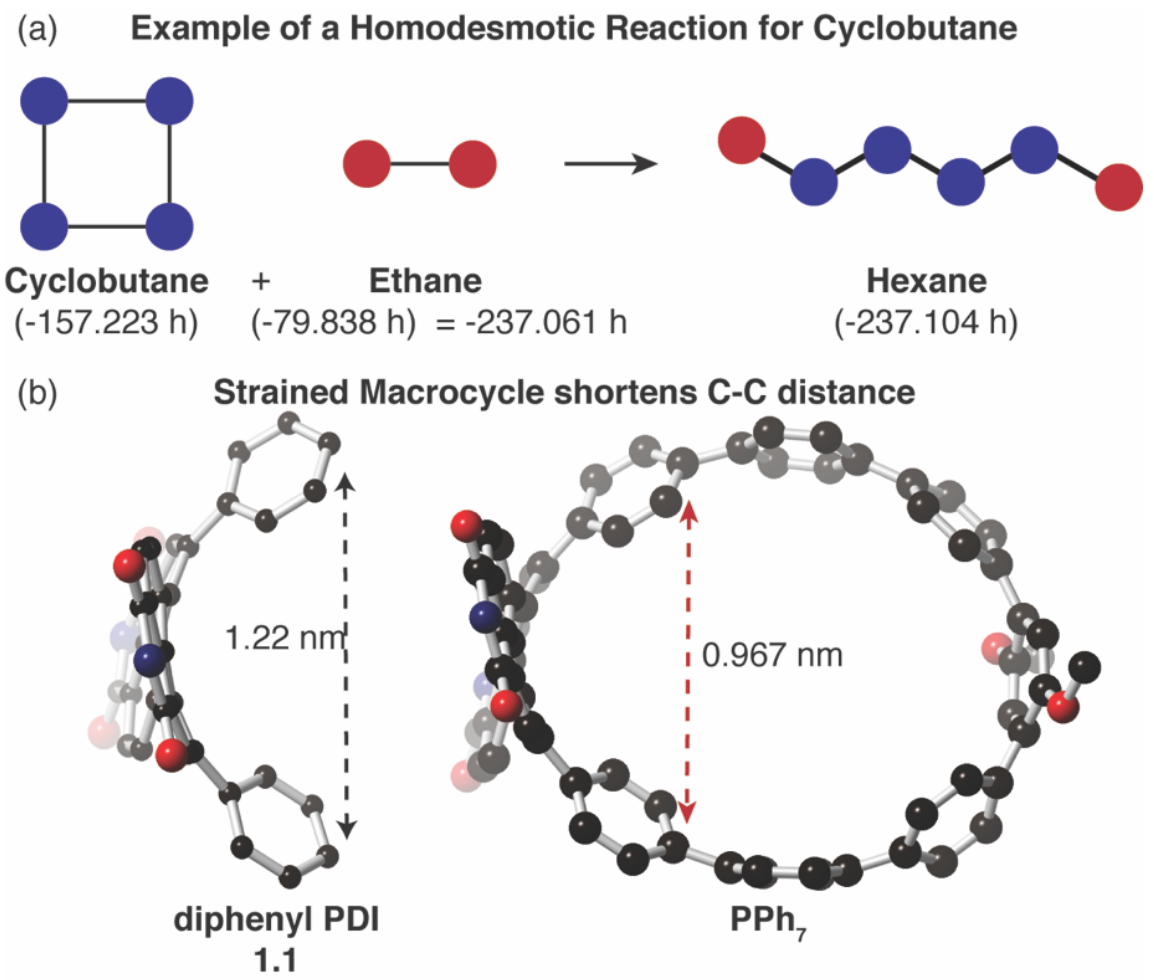

Figure 1B.5. (a) An example of an homodesmotic reaction to calculate the strain in cyclobutane. Two carbon-carbon bonds must break and then form in order to yield the hexane product. The two products possess a $\Delta \mathrm{H}_{\mathrm{f}}$ of -237.061 hartrees $(1$ hartree $=627.51 \mathrm{kcal} / \mathrm{mol})$, and $\Delta \mathrm{H}_{\mathrm{f}}$ is -237.104 for hexane. The difference of $43 \mathrm{mH} / 27.2 \mathrm{kcal} / \mathrm{mol}$ is close to the experimentally determined value of $26.5 \mathrm{kcal} / \mathrm{mol}$. (b) The aryl-aryl distance in an unsubstituted diphenyl PDI (Compound 1.1) and $\mathbf{P P h} \mathbf{h}_{6}-\mathbf{P h H e x}$. Our findings suggest a negative correlation between aryl-aryl distance and strain: as this distance increases, the strain energy decreases.

Both the reactants and products contain six sp $\rightarrow \mathrm{sp}^{3}$ with the same number of carbons and hydrogens. One can envision breaking and forming two $\mathrm{C}-\mathrm{C}$ bonds on the reactants side to form hexane (Figure 1B.5). According to DFT, the difference in energy between an unstrained hexane 
molecule and its group equivalent cyclobutane and ethane is $27.2 \mathrm{kcal} / \mathrm{mol}$, consistent with -26.4 $\mathrm{kcal} / \mathrm{mol}$ experimentally determined, and considered to be the amount of strain in the molecule. The strain energy encompasses deviations from ideal bond lengths and angles by restricting the four carbon atoms into a cyclic geometry. ${ }^{59}$

Both $\left(\mathbf{P B B r}_{4}\right)_{3}(2 \mathrm{kcal} / \mathrm{mol})$ and $\left(\mathbf{P P h}_{2}\right)_{4}(\sim 0 \mathrm{kcal} / \mathrm{mol})$ possess a small amount of strain by DFT, while PBPB (21 kcal/mol) and $\mathbf{P P h} \mathbf{h}_{6}-\mathbf{P h H e x}(63 \mathrm{kcal} / \mathrm{mol})$ contain a relatively significant amount of strain. It is interesting to note that although $\left(\mathbf{P B B r}_{4}\right)_{3}$ possesses the smallest cavity, the syn geometry of the three bithiophene subunits alleviates strain in the macrocycle as the syn geometry provides a natural curvature. The role of the bithiophene in macrocyclization is discussed in Chapter 2.

Table 1B.1. The transannular aryl-aryl distance from the phenyl rings adjacent to the PDI

\begin{tabular}{|c|c|c|c|}
\hline & \multicolumn{2}{|c|}{ Aryl-Aryl Distances } & Strain \\
\hline & $\begin{array}{c}\text { Distance } \\
(\mathrm{nm})\end{array}$ & $\begin{array}{c}\text { Deviation } \\
(\mathrm{nm})\end{array}$ & $\begin{array}{c}\text { Energy } \\
(\mathrm{kcal} / \mathrm{mol})\end{array}$ \\
\hline $\mathbf{1 . 1}$ & 1.22 & - & - \\
\hline $\mathbf{P P h}_{\mathbf{6}}-\mathbf{P h H e x}$ & 0.97 & 0.26 & 63 \\
\hline $\mathbf{P B P B}$ & 0.99 & 0.24 & 23 \\
\hline $\left.\mathbf{( P B B r}_{4}\right)_{3}$ & 1.0 & 0.18 & 2 \\
\hline $\left.\mathbf{( P P h}_{\mathbf{2}}\right)_{4}$ & 1.1 & 0.10 & 0 \\
\hline
\end{tabular}

Table 1B.1. The transannular aryl-aryl distance from the phenyl rings adjacent to the PDI. There is a negative correlation with distance and strain: as the distance increases, the strain decreases in the conjugated macrocycles.

One possibility to assess strain visually in these systems is to consider the para carboncarbon distance from the adjacent phenyl rings to the PDI. In an unsubstituted diphenyl PDI (1.1), this distance is approximately $1.22 \mathrm{~nm}$ (Figure 1B.5b); however, this distance changes when restricted into a macrocyclic framework: for the least strained macrocycles $\left(\mathbf{P P h}_{2}\right)_{4}$ and $\left(\mathbf{P B B r}_{4}\right)_{3}$, the carbon-carbon distance is the least perturbed, and measures 1.13 and $1.05 \mathrm{~nm}$, respectively. 
There is a greater perturbation in $\mathbf{P P h}$ - $\mathbf{P h H e x}(0.26 \mathrm{~nm})$ followed by PBPB $(0.24 \mathrm{~nm})$, analogous to the same trend in strain energy.

\section{B.7. Macrocyclic Design Promotes Absorption of Visible Light}

The first indication that the macrocycles could be efficient optoelectronic materials was their color. Figure 1B.6 shows the colors of the conjugated macrocycles: by visual inspection, PBPB is black, $\mathbf{P P h} 6-\mathbf{P h H e x}$ is teal, $\left(\mathbf{P P h}_{2}\right)_{4}$ and $\mathbf{D B D B}$ are purple, and $(\mathbf{P B B r})_{3}$ is red. The UVVisible spectra confirm light absorption across much of the visible range (Figure 1B.6b).

(a)

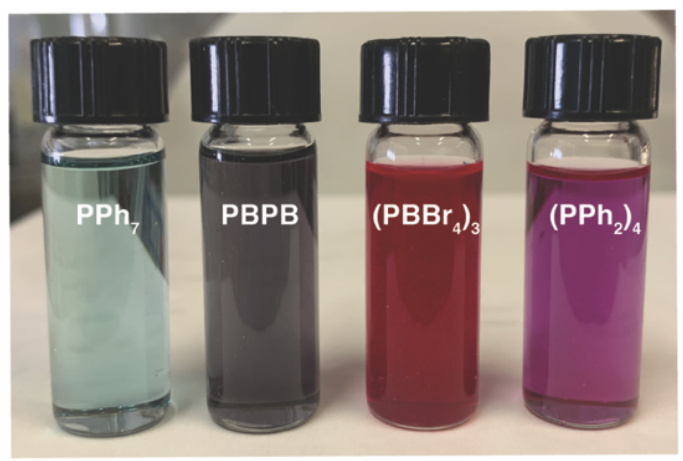

(b)

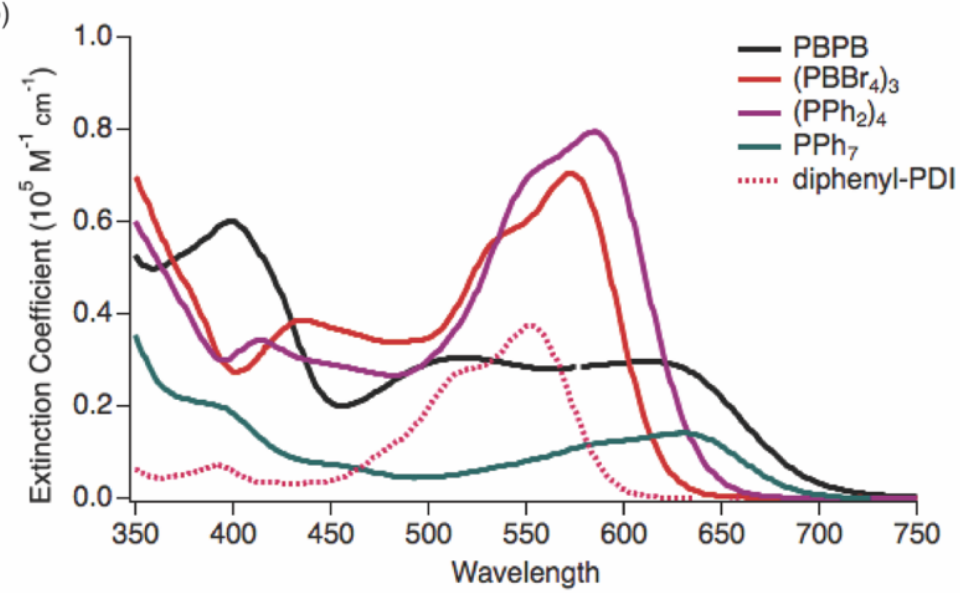

Figure 1B.6. (a) Vials containing the macrocycles in micromolar solutions. (b) The UV-Vis absorption spectrum of the four PDI-based macrocycles are at $10^{-5} \mathrm{M}$.

Each macrocycle functions as a n-type material in devices. DFT calculations and estimates from electrochemistry reveal that each molecule possesses a LUMO energy level that can accept at least two electrons per PDI (or four electrons for each $\mathrm{hPDI}_{2}$ molecule) due to PDI's electron accepting ability (Table 1B.2). From the potential of the first reduction peak in the CV, we estimate 
the LUMO energy levels to be approximately $-3.9 \mathrm{eV}$ for $\mathbf{P B P B},(\mathbf{P P h})_{4}$, and $(\mathbf{P B B r})_{3}$. For DBDB, the LUMO value is $-3.90 \mathrm{eV}$. These values are similar to the common n-type semiconductors like $[6,6]$-phenyl $\mathrm{C}_{61}$-butyric acid methyl ester (PCBM), whose LUMO is also $3.9 \mathrm{eV}$, and an unsubstituted PDI at $-3.89 .^{63,64}$

Table 1B.2: Electrochemistry, UV/vis and DFT Data for Conjugated Macrocycles

\begin{tabular}{|c|c|c|c|c|c|c|c|}
\hline & \multicolumn{3}{|c|}{ Electrochemical $^{\mathrm{a}}$} & \multicolumn{3}{|c|}{$\mathrm{DFT}^{\mathrm{b}}$} & \multirow{2}{*}{$\begin{array}{c}\text { Optical }^{\mathrm{c}} \\
\mathrm{E}_{\text {gap }} / \mathrm{eV}\end{array}$} \\
\hline & $\mathrm{E}_{\text {LUMO }} / \mathrm{eV}$ & $\mathrm{E}_{\text {номо }} / \mathrm{eV}$ & $\mathrm{E}_{\mathrm{gap}} / \mathrm{eV}$ & $\mathrm{E}_{\text {LUMO}} / \mathrm{eV}$ & $\mathrm{E}_{\mathrm{HOMO}} / \mathrm{eV}$ & $\mathrm{E}_{\text {gap }} / \mathrm{eV}$ & \\
\hline PBPB & -3.87 & -5.39 & 1.52 & -3.46 & -5.31 & 1.85 & 1.79 \\
\hline $\begin{array}{c}\text { PPh6- }^{-} \\
\text {PhHex }\end{array}$ & - & - & - & -3.46 & -5.28 & 1.81 & 1.82 \\
\hline$\left(\mathrm{PPh}_{2}\right)_{4}$ & -3.90 & -5.69 & 1.79 & -3.54 & -5.74 & 2.20 & 1.94 \\
\hline$\left(\mathrm{PBBr}_{4}\right)_{3}$ & -3.90 & -6.06 & 2.16 & -3.78 & -6.12 & 2.34 & 2.00 \\
\hline
\end{tabular}

Table 1B.2. ${ }^{a}$ DFT calculations carried out at 6-31G/B3LYP level of theory. ${ }^{b}$ HOMO and LUMO levels were estimated from onset of the first oxidation and reduction peaks and are relative to $\mathrm{Fc} / \mathrm{Fc}+{ }^{\mathrm{c}}$ Optical band gaps were estimated from the onset of absorption.

We care about strain because strain affects the electronic structure and properties of the macrocycles. For example, we find the electronic structures of the macrocycles differ from their acyclic counterparts, with the macrocycles absorbing more visible light (Chapter 3). Chapter 1A discussed the effects of conjugation on the HOMO/LUMO levels and the importance of a narrow energy gap for electronics. These same principles apply to the conjugated macrocycles and are discussed throughout this Thesis. I spent five years trying to understand the overlap between strain, rigidity, and the effects of conjugation. These concepts matter because we observe an appreciable effect on device performance that we can attribute to these physical organic concepts. Yet I would 
not say that these concepts alone are sufficient to design materials that perform well in devices: devices and how they work are complicated. But these molecules allowed us to probe on a fundamental level, from a physical organic chemistry point of view, what design features may be important for organic materials applications, and for this I am grateful.

Chapters 1A provided background on non-fullerene, contorted aromatics. It also described the role of conjugation and its effects on molecules. Chapter 1B describes an overview of the conjugated macrocycles. Here, I discussed the role of strain and rigidity on the macrocycles' electronic and physical structures. This background will be advantageous for Chapters 2-8, as I show how macrocyclization impacts device performance. The most interesting results described in this Thesis involve the interplay between strain and rigidity and device performance.

Chapters 2 and 3 focus on PBPB as an electronic material. Chapter 2 will describe the molecular structure of PBPB, and the exciting stereochemistry observed for this molecule. This Chapter will also show how the electronic structure of PBPB is not just a sum of the two linker components. Chapter 3 describes PBPB and $\left(\mathbf{P P h}_{2}\right)_{4}$ in both OFETs and OPVs. Here we study how the macrocyclic framework affects device performance by comparing $\left(\mathbf{P P h}_{2}\right)_{4}$ and $\mathbf{P B P B}$ to several acyclic control molecules. The macrocycles outperform the acyclic controls in each of the parameters tested.

The focus in Chapter 4 is how a 1,7 or 1,6 substitution affects OFET mobility. We design a cis analog to PBPB, and using a combination of single molecule junction conductance measurements of the components of the macrocycles, control experiments with acyclic counterparts to the macrocycles, and analyses of each of the materials using spectroscopy, electrochemistry, and DFT, we show the difference in electron mobility is due to a difference in intramolecular conductivity between the two isomers. The ability to synthesize and probe the 
effects from nuanced differences in the macrocyclic framework is an advantage to this bottom-up approach.

Chapter 5 describes a direct relationship between structure and function for $\left(\mathbf{P P h}_{2}\right)_{4}$. We compare the macrocycle relative to an acyclic control and show that the rigidity of the macrocycle aides its ability to function as a photodetector. This is a beautiful demonstration of structure and function. The macrocyclic structure minimizes the number of charged defects from deformed $\mathrm{sp}^{2}$ bonds, resulting in a low dark current and a detectivity comparable to the best fullerene-based photodetectors.

Chapter 6 focuses on $\left(\mathbf{P B B r}_{4}\right)_{3}$ and its role as a sensor of small molecule guests. The macrocycle detects subtle differences in a series of alkanes (n-hexanes, 1-hexyne, and 3-hexyne), and is the first demonstration of the utility of designing a macrocycle with open pores. One of the key features of $\left(\mathbf{P B B r}_{4}\right)_{3}$ is that it is conformationally restricted, which could help aide its ability to function as a sensor. Chapter 7 describes the synthesis and characterization of the second

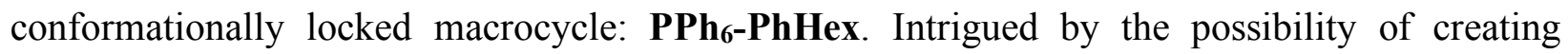
enantio-pure sensors, we sought to make this synthetically-challenging macrocycle. The cavity of $\mathbf{P P h}_{6}-\mathbf{P h H e x}$ is larger than $\left(\mathbf{P B B r}_{4}\right)_{3}$, and future work will include using $\mathbf{P P h} \mathbf{h}_{6}-\mathbf{P h H e x}$ in sensing applications with larger guests.

Chapter 8 includes our work on incorporating hPDI 2 within a macrocyclic framework. The motivation for this design was based off the success of the hPDI oligomers in electronic applications, as discussed in Chapter 1A. The final chapter (Chapter 9) discusses recent work using PBPB as a host system with fullerenes, and shows the utility of the conjugated macrocycles in sensing applications. Future work will explore how fullerene encapsulation affects electron mobility in OFET devices. 
1B.8. Appendix

\begin{tabular}{|c|c|c|}
\hline \multicolumn{3}{|c|}{$\begin{array}{c}\text { Diphenyl PDI - M enantiomer } \\
M-1.1\end{array}$} \\
\hline \multicolumn{3}{|c|}{ Heat of Formation $=-1871.869720$} \\
\hline 2.421580 & -5.686988 & -0.551058 \\
\hline & -8.435283 & \\
\hline 046 & $-7.75^{\prime}$ & \\
\hline & -5.00 & \\
\hline-5.235350 & -4.313 & \\
\hline$-3 .($ & -5.4 & \\
\hline 0.0 & -8.65 & \\
\hline 1.3 & -9.09 & \\
\hline & -7.29 & \\
\hline 3.2 & -6.14 & \\
\hline-2.4 & -7.9 & \\
\hline-1.6 & -7.47 & \\
\hline-2. & -7.7 & \\
\hline-0.3 & -7.31 & \\
\hline & -8.3 & \\
\hline-3.1 & -8.2 & \\
\hline 2. & -8.82 & \\
\hline-1. & -7.75 & \\
\hline-0.6 & -8.8 & \\
\hline & -9.2 & \\
\hline-3.4 & -8.1 & \\
\hline & -7.3 & \\
\hline-1.8 & -8.0 & \\
\hline & -7.4 & \\
\hline-0.0 & -7.46 & \\
\hline-3.8 & -7.93 & \\
\hline-1.5 & -8.76 & \\
\hline-3.9 & -5.91 & \\
\hline & -6.3 & \\
\hline & -9.45 & \\
\hline-6.2 & -7.0 & \\
\hline & -9.30 & \\
\hline & -7.7 & \\
\hline & -7.04 & 9222 \\
\hline 2.97 & -7.12 & -2.0 \\
\hline-6.3 & -5.86 & 829 \\
\hline & -9.63 & \\
\hline 010 & -9.864893 & 4.311023 \\
\hline-3.85 & -7.148013 & \\
\hline-5.182302 & -5.273796 & 0.429999 \\
\hline 260 & -5.762086 & -0.810 \\
\hline
\end{tabular}




$\begin{array}{rrrr}\mathrm{C} & 4.052702 & -7.684487 & -2.762991 \\ \mathrm{C} & -0.228581 & -8.336572 & 0.824523 \\ \mathrm{C} & -2.527987 & -7.780686 & 1.454983 \\ \mathrm{C} & -1.572964 & -8.020981 & 0.443818 \\ \mathrm{C} & -2.195747 & -8.065949 & 2.797319 \\ \mathrm{C} & -0.792754 & -7.885935 & -1.931405 \\ \mathrm{C} & -0.951585 & -8.538327 & 3.160011 \\ \mathrm{C} & 0.573956 & -7.865349 & -1.500910 \\ \mathrm{H} & -2.930259 & -7.902932 & 3.578354 \\ \mathrm{H} & 2.889882 & -8.897009 & -0.446732 \\ \mathrm{H} & -4.446242 & -8.338187 & -3.240152 \\ \mathrm{H} & 3.316019 & -9.584932 & 1.884983 \\ \mathrm{H} & 1.984016 & -6.998213 & -4.491384 \\ \mathrm{H} & -3.974710 & -8.422972 & -0.817385 \\ \mathrm{H} & -4.971899 & -8.677025 & 2.266916 \\ \mathrm{H} & -7.157630 & -7.549857 & 2.002095 \\ \mathrm{C} & -1.961508 & -7.315325 & -7.510367 \\ \mathrm{H} & -1.656182 & -6.318177 & -7.834399 \\ \mathrm{H} & 1.904695 & -9.942237 & 6.383852 \\ \mathrm{H} & 0.670165 & -8.757095 & 6.919588 \\ \mathrm{H} & 0.198223 & -10.435131 & 6.617291 \\ \mathrm{H} & -3.027841 & -7.460423 & -7.663121 \\ \mathrm{H} & -1.388093 & -8.049013 & -8.080981 \\ \mathrm{H} & -7.300991 & -5.365623 & 0.824024 \\ \mathrm{H} & 6.639889 & -6.042220 & -1.284028\end{array}$

\section{Diphenyl PDI - P enantiomer $P-1.1$}

Heat of Formation $=-1871.869710$

$\begin{array}{rrrr}\mathrm{H} & 4.322695 & -4.627155 & -2.086531 \\ \mathrm{H} & 6.480589 & -5.862076 & -2.055867 \\ \mathrm{H} & 4.650901 & -8.975413 & 0.274756 \\ \mathrm{H} & -4.891827 & -4.606365 & 1.896083 \\ \mathrm{H} & -3.018507 & -5.461131 & 0.529059 \\ \mathrm{C} & 0.036188 & -8.549338 & 2.206044 \\ \mathrm{C} & 1.360381 & -8.489346 & 2.697902 \\ \mathrm{C} & 1.249969 & -8.317575 & -3.846104 \\ \mathrm{C} & 3.350203 & -7.345556 & -0.272590 \\ \mathrm{C} & -2.353403 & -7.449330 & -3.769315 \\ \mathrm{~N} & -1.621648 & -7.593664 & -6.106551 \\ \mathrm{C} & -2.674522 & -7.340354 & -5.214359 \\ \mathrm{C} & -0.311331 & -7.924194 & -5.733733 \\ \mathrm{C} & 0.879325 & -8.039485 & -0.063985 \\ \mathrm{C} & -3.111843 & -7.313839 & -1.451999 \\ \mathrm{C} & 2.171546 & -7.866160 & 0.479106 \\ \mathrm{C} & -1.047709 & -7.773041 & -3.332459\end{array}$




$\begin{array}{lrrr}\mathrm{C} & -0.756740 & -9.211659 & 4.501099 \\ \mathrm{C} & 2.384079 & -8.113769 & 1.852792 \\ \mathrm{C} & -3.337629 & -7.184319 & -2.839593 \\ \mathrm{C} & 5.608811 & -6.275320 & -1.557396 \\ \mathrm{C} & -1.853697 & -7.763373 & -0.995845 \\ \mathrm{C} & 1.544893 & -8.339257 & -2.478450 \\ \mathrm{C} & -0.024960 & -8.001632 & -4.281475 \\ \mathrm{O} & -3.793546 & -7.047292 & -5.614377 \\ \mathrm{O} & -1.637249 & -9.539410 & 5.285896 \\ \mathrm{C} & -4.016481 & -5.866593 & 0.393221 \\ \mathrm{C} & 5.696657 & -7.494934 & -0.887807 \\ \mathrm{C} & 1.663560 & -8.773137 & 4.122977 \\ \mathrm{C} & -6.585148 & -6.883830 & 0.027340 \\ \mathrm{~N} & 0.572123 & -9.113920 & 4.936071 \\ \mathrm{C} & -5.528854 & -7.361337 & -0.749333 \\ \mathrm{O} & 0.536459 & -8.132376 & -6.591711 \\ \mathrm{C} & 3.276631 & -6.113740 & -0.942459 \\ \mathrm{C} & -6.360320 & -5.896162 & 0.985156 \\ \mathrm{C} & 0.815217 & -9.409690 & 6.351669 \\ \mathrm{O} & 2.799872 & -8.713242 & 4.573569 \\ \mathrm{C} & -4.228087 & -6.868107 & -0.568471 \\ \mathrm{C} & -5.072527 & -5.385789 & 1.161639 \\ \mathrm{C} & 4.577663 & -8.023362 & -0.243505 \\ \mathrm{C} & 4.395533 & -5.584000 & -1.577943 \\ \mathrm{C} & -0.217747 & -8.261087 & 0.831885 \\ \mathrm{H} & -1.628667 & -8.460776 & -8.022649 \\ \mathrm{H} & -2.586272 & -8.600041 & 1.263823 \\ \mathrm{C} & -1.576556 & -8.199940 & 0.382779 \\ \mathrm{C} & -2.316233 & -8.958394 & 2.588984 \\ \mathrm{C} & -0.776681 & -7.869792 & -1.935325 \\ \mathrm{C} & -1.022638 & -8.897038 & 3.077478 \\ \mathrm{C} & 0.575327 & -8.073823 & -1.506675 \\ \mathrm{H} & -3.113243 & -9.264222 & 3.257586 \\ \mathrm{H} & -4.308019 & -6.858907 & -3.197689 \\ \mathrm{H} & 3.377808 & -7.998465 & 2.270927 \\ \mathrm{H} & 2.016802 & -8.525811 & -4.583933 \\ \mathrm{H} & -5.709908 & -8.132118 & -1.493412 \\ \mathrm{H} & -7.583986 & -7.284223 & -0.119677 \\ \mathrm{H} & -1.2687900 & -7.512086 & -7.546949 \\ \mathrm{H} & -6.728588 & -7.994165 \\ \mathrm{H} & -8.038191 & -0.864073\end{array}$




$$
\begin{array}{rrrr}
H & -3.610913 & -8.631871 & 0.923819 \\
H & 2.556709 & -8.565503 & -2.176293 \\
H & 2.336950 & -5.569898 & -0.955767
\end{array}
$$

\section{Cyclobutane}

Heat of Formation $=-157.2230$

$\begin{array}{lrrr}\mathrm{C} & -0.58524 & 0.51356 & 0.77860 \\ \mathrm{C} & -0.58085 & 0.51852 & -0.77860 \\ \mathrm{C} & 0.58085 & -0.51852 & -0.77860 \\ \mathrm{C} & 0.58524 & -0.51356 & 0.77860 \\ \mathrm{H} & -1.51202 & 0.14734 & 1.22988 \\ \mathrm{H} & -0.34799 & 1.47692 & 1.23946 \\ \mathrm{H} & -0.32995 & 1.48292 & -1.22992 \\ \mathrm{H} & -1.50826 & 0.16598 & -1.23943 \\ \mathrm{H} & 1.50826 & -0.16598 & -1.23943 \\ \mathrm{H} & 0.32995 & -1.48292 & -1.22992 \\ \mathrm{H} & 1.51202 & -0.14734 & 1.22988 \\ \mathrm{H} & 0.34799 & -1.47692 & 1.23946\end{array}$

\section{Ethane}

Heat of Formation $=-79.83871$

$\begin{array}{lrrr}\mathrm{C} & -0.14076 & -0.65454 & 0.37030 \\ \mathrm{C} & 0.14076 & 0.65454 & -0.37030 \\ \mathrm{H} & 0.20291 & -1.52032 & -0.20548 \\ \mathrm{H} & 0.36684 & -0.68106 & 1.34028 \\ \mathrm{H} & 1.21215 & 0.78569 & -0.55513 \\ \mathrm{H} & -0.36684 & 0.68106 & -1.34028 \\ \mathrm{H} & -0.20291 & 1.52032 & 0.20548 \\ \mathrm{H} & -1.21215 & -0.78569 & 0.55513\end{array}$

Hexanes

Heat of Formation $=-237.10497$

$\begin{array}{lrrr}\mathrm{C} & -0.76632 & 0.02456 & 0.00000 \\ \mathrm{C} & -1.33586 & 1.44885 & 0.00000 \\ \mathrm{C} & 0.76632 & -0.02456 & 0.00000 \\ \mathrm{C} & 1.33586 & -1.44885 & 0.00000 \\ \mathrm{C} & 2.86683 & -1.48805 & 0.00000 \\ \mathrm{C} & -2.86683 & 1.48805 & 0.00000 \\ \mathrm{H} & -1.14729 & -0.51733 & 0.87764 \\ \mathrm{H} & -1.14729 & -0.51733 & -0.87764 \\ \mathrm{H} & -0.95626 & 1.99032 & -0.87716 \\ \mathrm{H} & -0.95626 & 1.99032 & 0.87716 \\ \mathrm{H} & 1.14729 & 0.51733 & 0.87764 \\ \mathrm{H} & 1.14729 & 0.51733 & -0.87764 \\ \mathrm{H} & 0.95626 & -1.99032 & -0.87716 \\ \mathrm{H} & 0.95626 & -1.99032 & 0.87716\end{array}$




\section{B.9. References}

$\begin{array}{rrrr}\mathrm{H} & 3.24204 & -2.51681 & 0.00000 \\ \mathrm{H} & 3.27553 & -0.98490 & -0.88375 \\ \mathrm{H} & 3.27553 & -0.98490 & 0.88375 \\ \mathrm{H} & -3.24204 & 2.51681 & 0.00000 \\ \mathrm{H} & -3.27553 & 0.98490 & -0.88375 \\ \mathrm{H} & -3.27553 & 0.98490 & 0.88375\end{array}$

1. Ball, M. et al. Conjugated Macrocycles in Organic Electronics. Acc. Chem. Res. (2019). doi:10.1021/acs.accounts.9b00017

2. Krömer, J. et al. Synthesis of the First Fully $\alpha$-Conjugated Macrocyclic Oligothiophenes: Cyclo[n]thiophenes with Tunable Cavities in the Nanometer Regime. Angew. Chem. Int. Ed. 39, 3481-3486 (2000).

3. Nakao, K. et al. Giant Macrocycles Composed of Thiophene, Acetylene, and Ethylene Building Blocks. J. Am. Chem. Soc. 128, 16740-16747 (2006).

4. Darzi, E. R. et al. Synthesis, Properties, and Design Principles of Donor-Acceptor Nanohoops. ACS Cent. Sci. 1, 335-342 (2015).

5. Ball, M. et al. Chiral Conjugated Corrals. J. Am. Chem. Soc. 137, $9982-9987$ (2015).

6. Ito, H. et al. Thiophene-Based, Radial pi-Conjugation: Synthesis, Structure, and Photophysical Properties of Cyclo-1,4-phenylene-2 ',5'-thienylenes. Angew. Chem. Int. Ed. 54, 159-163 (2015).

7. Xue, J. Y. et al. Aromatic hydrocarbon macrocycles for highly efficient organic lightemitting devices with single-layer architectures. Chem. Sci. 7, 896-904 (2016).

8. Kayahara, E. et al. Synthesis and Characterization of $\{[\} \mathrm{n}] \mathrm{CPP}(\mathrm{n}=5,6,8,10$, and 12) Radical Cation and Dications: Size-Dependent Absorption, Spin, and Charge Delocalization. J. Am. Chem. Soc. 138, 338-344 (2016).

9. Kayahara, E. et al. Size Dependence of $\{[\} n]$ Cycloparaphenylenes $(n=5-12)$ in Electrochemical Oxidation. Chem. Asian J. 11, 1793-1797 (2016).

10. Zhang, B. et al. Rigid, Conjugated Macrocycles for High Performance Organic Photodetectors. J. Am. Chem. Soc. 138, 16426-16431 (2016).

11. Ball, M. et al. Macrocyclization in the Design of Organic n-Type Electronic Materials. $J$. Am. Chem. Soc. 138, 12861-12867 (2016).

12. Van Raden, J. et al. Synthesis and characterization of a highly strained donor-acceptor nanohoop. Org. Biomol. Chem. 14, 5721 (2016).

13. Peeks, M. D. et al. Aromatic and antiaromatic ring currents in a molecular nanoring. Nature 541, 200 (2017).

14. Zang, L. et al. One-Dimensional Self-Assembly of Planar $\pi$-Conjugated Molecules: Adaptable Building Blocks for Organic Nanodevices. Acc. Chem. Res. 41, 1596-1608 (2008).

15. Izumi, T. et al. Efficient Blue Electroluminescence from a Single-layer Organic Device 
Composed Solely of Hydrocarbons. Chem. Asian J. 12, 730-733 (2017).

16. Nishigaki, S. et al. Synthesis, Structures, and Photophysical Properties of Alternating Donor-Acceptor Cycloparaphenylenes. Chem. Eur. J. 23, 7227-7231 (2017).

17. Thakellapalli, H. et al. Synthesis and Properties of Conjugated Macrocycles Containing 2,7Bis(2-thienyl)-9H-fluoren-9-one Units. Org. Lett. 19, 2674-2677 (2017).

18. Li, T. et al. Synthesis, solvent-dependent emission and two- photon absorption of a triangular - \{[\}D-pi-A](3)- macrocycle. Org. Chem. Front. 4, 737-742 (2017).

19. Hahn, S. et al. Synthesis and Characterization of Heterobenzenacyclooctaphanes Derived from Cyclotetrabenzoin. Chem. Eur. J. 23, 10543-10550 (2017).

20. Van Raden, J. et al. 2,2'-Bipyridyl-Embedded Cycloparaphenylenes as a General Strategy To Investigate Nanohoop-Based Coordination Complexes. J. Am. Chem. Soc. 139, 29362939 (2017).

21. Kayahara, E. et al. Gram-Scale Syntheses and Conductivities of [10]Cycloparaphenylene and Its Tetraalkoxy Derivatives. J. Am. Chem. Soc. 139, 18480-18483 (2017).

22. Zhang, F. et al. Giant Cyclo $\mathrm{n}$ thiophenes with Extended pi Conjugation. Angew. Chem. Int. Ed. 48, 6632-6635 (2009).

23. Fenwick, O. et al. Linear and Cyclic Porphyrin Hexamers as Near-Infrared Emitters in Organic Light-Emitting Diodes. Nano Lett. 11, 2451-2456 (2011).

24. Zhang, F. et al. Molecular and electronic structure of cyclo[10]thiophene in various oxidation states: polaron pair vs. bipolaron. Chem. Sci. 2, 781-784 (2011).

25. Iyoda, M. et al. Conjugated Macrocycles: Concepts and Applications. Angew. Chem. Int. Ed. 50, 10522-10553 (2011).

26. Jiang, H.-W. et al. Cyclic 2,12-Porphyrinylene Nanorings as a Porphyrin Analogue of Cycloparaphenylenes. J. Am. Chem. Soc. 137, 2219-2222 (2015).

27. Kuwabara, T. et al. Curved Oligophenylenes as Donors in Shape-Persistent DonorAcceptor Macrocycles with Solvatofluorochromic Properties. Angew. Chem. Int. Ed. 54, 9646-9649 (2015).

28. Chen, Q. et al. Strain-Induced Stereoselective Formation of Blue-Emitting Cyclostilbenes. J. Am. Chem. Soc. 137, 12282-12288 (2015).

29. Iwamoto, T. et al. Size-Selective Encapsulation of C60 by [10]Cycloparaphenylene: Formation of the Shortest Fullerene-Peapod. Angew. Chem. Int. Ed. 50, 8342-8344 (2011).

30. Kylberg, W. et al. Oligothiophene dendron-decorated squaraine dyes: Synthesis, thin film formation, and performance in organic solar cells. Org. Electron. 13, 1204-1212 (2012).

31. Iwamoto, T. et al. Size- and Orientation-Selective Encapsulation of C70 by Cycloparaphenylenes. Chem. Eur. J. 19, 14061-14068 (2013).

32. Nakanishi, Y. et al. Size-Selective Complexation and Extraction of Endohedral Metallofullerenes with Cycloparaphenylene. Angew. Chem. Int. Ed. 53, 3102-3106 (2014).

33. Zhang, B. et al. Hollow organic capsules assemble into cellular semiconductors. Nat. Commun. 9, 1957 (2018). 
34. Dou, L. et al. 25th Anniversary Article: A Decade of Organic/Polymeric Photovoltaic Research. Adv. Mater. 25, 6642-6671 (2013).

35. Savoie, B. M. et al. Unequal Partnership: Asymmetric Roles of Polymeric Donor and Fullerene Acceptor in Generating Free Charge. J. Am. Chem. Soc. 136, 2876-2884 (2014).

36. Anthony, J. E. et al. n-Type Organic Semiconductors in Organic Electronics. Adv. Mater. 22, 3876-3892 (2010).

37. Sharenko, A. et al. A High-Performing Solution-Processed Small Molecule: Perylene Diimide Bulk Heterojunction Solar Cell. Adv. Mater. 25, 4403-4406 (2013).

38. Cai, Y. et al. High Performance Organic Solar Cells Based on a Twisted Bay-Substituted Tetraphenyl Functionalized Perylenediimide Electron Acceptor. Adv. Energy Mater. 5, 1500032 (2015).

39. Zhou, E. J. et al. All-Polymer Solar Cells from Perylene Diimide Based Copolymers: Material Design and Phase Separation Control. Angew. Chem. Int. Ed. 50, 2799-2803 (2011).

40. Li, C. et al. Perylene Imides for Organic Photovoltaics: Yesterday, Today, and Tomorrow. Adv. Mater. 24, 613-636 (2012).

41. Ball, M. et al. Contorted Polycyclic Aromatics. Acc. Chem. Res. 48, 267-276 (2015).

42. Zhong, Y. et al. Helical Ribbons for Molecular Electronics. J. Am. Chem. Soc. 136, 81228130 (2014).

43. Nolde, F. et al. Synthesis and self-organization of core-extended perylene tetracarboxdiimides with branched alkyl substituents. Chem. Mater. 18, 3715-3725 (2006).

44. Meng, D. et al. High-Performance Solution-Processed Non-Fullerene Organic Solar Cells Based on Selenophene-Containing Perylene Bisimide Acceptor. J. Am. Chem. Soc. 138, 375-380 (2016).

45. Yan, Q. F. et al. Conjugated Dimeric and Trimeric Perylenediimide Oligomers. Org. Lett. 11, 3426-3429 (2009).

46. Huo, L. J. et al. Synthesis and absorption spectra of n-type conjugated polymers based on perylene diimide. Macromol. Rapid Commun. 29, 1444-1448 (2008).

47. Wuerthner, F. et al. Perylene Bisimide Dye Assemblies as Archetype Functional Supramolecular Materials. Chem. Rev. 116, 962-1052 (2016).

48. Ball, M. L. et al. Influence of Molecular Conformation on Electron Transport in Giant, Conjugated Macrocycles. J. Am. Chem. Soc. 140, 10135-10139 (2018).

49. Fujitsuka, M. et al. Size-dependent fluorescence properties of n cycloparaphenylenes $(\mathrm{n}=8$ 13), hoop-shaped pi-conjugated molecules. Phys. Chem. Chem. Phys. 14, 14585-14588 (2012).

50. Darzi, E. R. et al. An Operationally Simple and Mild Oxidative Homocoupling of Aryl Boronic Esters To Access Conformationally Constrained Macrocycles. J. Am. Chem. Soc. 139, 3106-3114 (2017).

51. Evans, P. J. et al. Efficient room-temperature synthesis of a highly strained carbon 
nanohoop fragment of buckminsterfullerene. Nat. Chem. 6, 404-408 (2014).

52. Iwamoto, T. et al. Selective and Random Syntheses of n Cycloparaphenylenes $(\mathrm{n}=8-13)$ and Size Dependence of Their Electronic Properties. J. Am. Chem. Soc. 133, 8354-8361 (2011).

53. Ahrens, M. J. et al. Bis(n-octylamino)perylene-3,4:9,10-bis(dicarboximide)s and Their Radical Cations: Synthesis, Electrochemistry, and ENDOR Spectroscopy. J. Org. Chem. 71, 2107-2114 (2006).

54. Osswald, P. et al. Effects of Bay Substituents on the Racemization Barriers of Perylene Bisimides: Resolution of Atropo-Enantiomers. J. Am. Chem. Soc. 129, 14319-14326 (2007).

55. Chen, Z. et al. Effect of Core Twisting on Self-Assembly and Optical Properties of Perylene Bisimide Dyes in Solution and Columnar Liquid Crystalline Phases. Chem. Eur. J. 13, 450465 (2007).

56. Goretzki, G. et al. Bis-morpholine-Substituted Perylene Bisimides: Impact of Isomeric Arrangement on Electrochemical and Spectroelectrochemical Properties. J. Org. Chem. 73, 8808-8814 (2008).

57. Dubey, R. K. et al. 1,7- And 1,6-Regioisomers of Diphenoxy and Dipyrrolidinyl Substituted Perylene Diimides: Synthesis, Separation, Characterization, and Comparison of Electrochemical and Optical Properties. Chem. Mater. 23, 778-788 (2011).

58. Würthner, F. et al. Perylene Bisimide Dye Assemblies as Archetype Functional Supramolecular Materials. Chem. Rev. 116, 962-1052 (2016).

59. Anslyn, E. V. \& Dougherty, D. A. Modern Physical Organic Chemistry. (University Science Books, 2006).

60. George, P. et al. An alternative approach to the problem of assessing stabilization energies in cyclic conjugated hydrocarbons. Theor. Chim. Acta 38, 121-129 (1975).

61. Bachrach, S. M. et al. DFT Study of Cycloparaphenylenes and Heteroatom-Substituted Nanohoops. J. Org. Chem. 75, 6595-6604 (2010).

62. Segawa, Y. et al. Theoretical Studies on the Structures and Strain Energies of Cycloparaphenylenes. Org. Lett. 12, 2262-2265 (2010).

63. Li, Y. et al. Synthesis and Properties of Ethylene-Annulated Di(perylene diimides). Org. Lett. 14, 5278-5281 (2012).

64. White, B. M. et al. Expanding the Chemical Space of Biocompatible Fluorophores: Nanohoops in Cells. ACS Cent. Sci. 4, 1173-1178 (2018). 


\section{Chapter 2. Chiral Conjugated Corrals}

\subsection{Preface}

Chapter 2 is reproduced with permission from the authors: Melissa Ball, Brandon Fowler, Panpan Li, Leo A. Joyce, Fang Li, Taifeng Liu, Daniel W. Paley, Yu Zhong, Hexing Li, Shengxiong Xiao, Fay Ng, Michael L. Steigerwald, and Colin Nuckolls published in the Journal of the American Chemical Society. ${ }^{1}$ Copyright 2015 American Chemical Society. I synthesized all compounds, with valuable input from Shengxiong Xiao and coworkers. I performed calculations with essential input from Michael L. Steigerwald. Brandon Fowler and I performed HPLC experiments. Daniel W. Paley performed XRD analysis. Leo A. Joyce performed circular dichroism experiments. Yu Zhong performed CV.

\subsection{Introduction}

This manuscript describes the design, synthesis and study of a new type of conjugated aromatic macrocycle formed from the linkage of donor and acceptor subunits into a strained cycle (Figure 2.1.). These macrocycles are members of an ever-growing class of cyclic, conjugated belts, such as, CPPs ${ }^{2-24}$ and cycloporphyrins (CPs). ${ }^{25-34}$ CPPs consist of para-connected phenylene rings and possess size-dependent optical and electronic properties. ${ }^{21}$ However, CPPs do not absorb at a sufficiently broad part of the visible spectrum for them to be useful in optoelectronic devices. The optical gaps for CPs are smaller, but they have not been used in devices. ${ }^{17}$ Alternation of electron donor and acceptor subunits is a proven strategy to engineer the energetics of linear, conjugated oligomers and polymers, but it has not been explored in the context of conjugated, cyclic oligomers. ${ }^{35-37}$ 

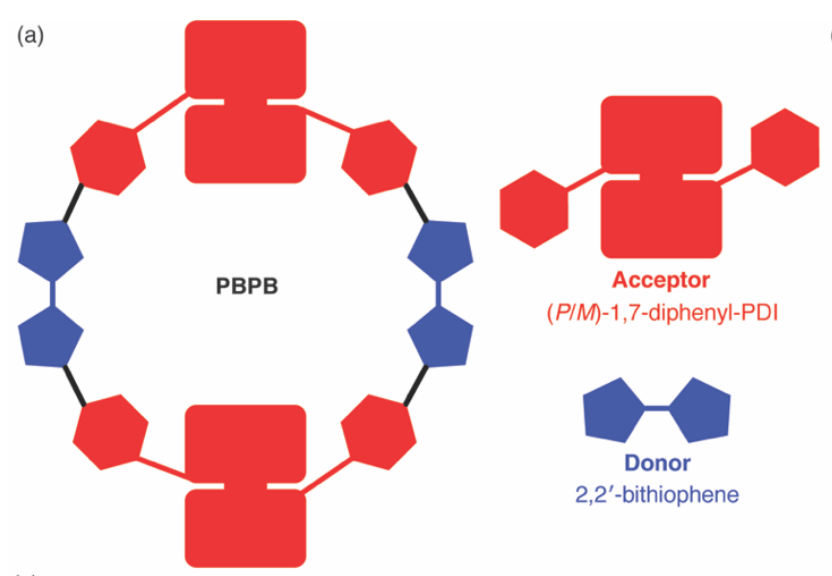

(b)
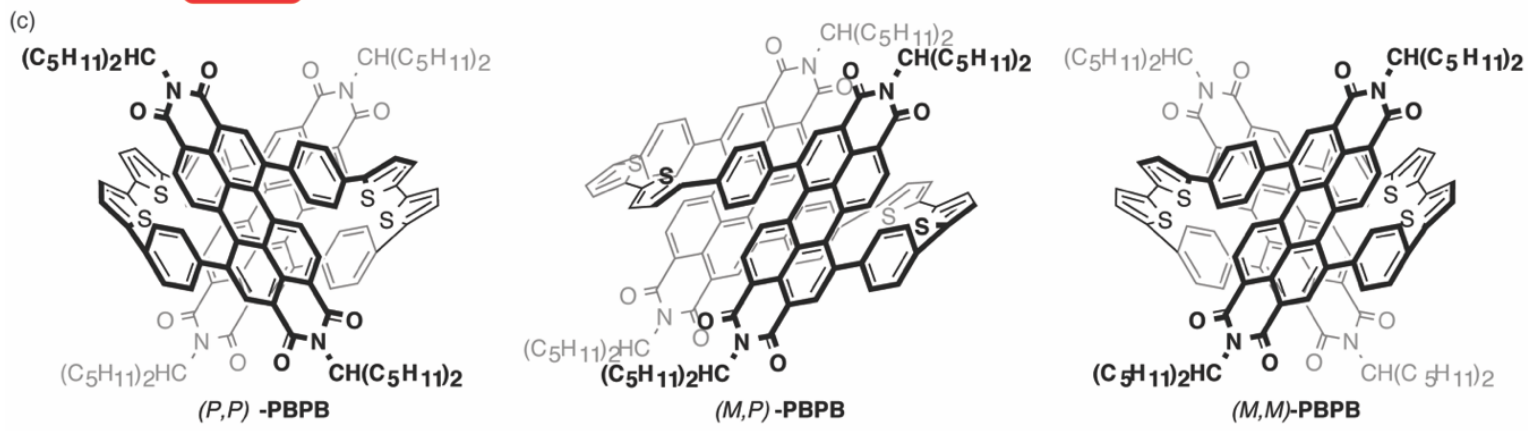

Figure 2.1. (a) Schematic structure of the -a-b-a-b pattern for PBPB. (b) The stereoisomerism of PBPB results from the chirality of the $(P)$ - and $(M)$-1,7-diphenyl-PDI (1.1) subunits. (c) PBPB exists in chiral $[(P, P)-\mathbf{P B P B}$ and $(M, M)$-PBPB $]$ and meso $[(M, P)-\mathbf{P B P B}]$ forms.

For this study, we designed and synthesized the molecules shown in Figure 2.1, comprising a PDI and a phenyl-bithiophene-phenyl linker. We incorporated PDI as the electron acceptor in PBPB's design because PDI derivatives have shown a number of desirable behaviors for organic electronics, as discussed in Chapter 1. We employed oligothiophenes as the electron-rich counterpart because they are ubiquitous as electron-donor molecules in electronic materials. Each of these macrocycles has a persistent, elliptiform cavity that is lined with electron deficient $\pi$-faces of the PDI and the lone pairs of the sulfur atoms from the thiophene subunits. We named these molecules PBPB reflecting their ability to perform intramolecular charge transfer upon excitation. The macrocycle is black in color and has a broad absorption spectrum that spans the entire visible range. The onset of strong absorbance occurs at approximately $700 \mathrm{~nm}$ (HOMO-to-LUMO excitation at $1.8 \mathrm{eV}$ ), hinting at PBPB's favorable prospects as optical materials (vida infra 
Chapter 3). Furthermore, we find PBPB exists in a dynamical equilibrium between both chiral$[(P, P)$-PBPB and $(M, M)$-PBPB $]$ and meso- $[(M, P)$-PBPB $]$ forms due to the handedness of the helical PDI subunits (Chapter 1B.4, Figure 2.1). The essential point of this study is that this new a-b-a-b- motif for cyclic conjugated molecules (Figure 2.1a) provides interesting chiral information and an unusual electronic structure.

\subsection{Synthesis of PBPB}

Our synthesis is based on the strategies developed by Bäuerle ${ }^{2,18}$ and $\mathrm{Yamago}^{21}$ in the syntheses of conjugated macrocycles via square planar platinum intermediates. Figure 2.2 displays the synthesis of PBPB.

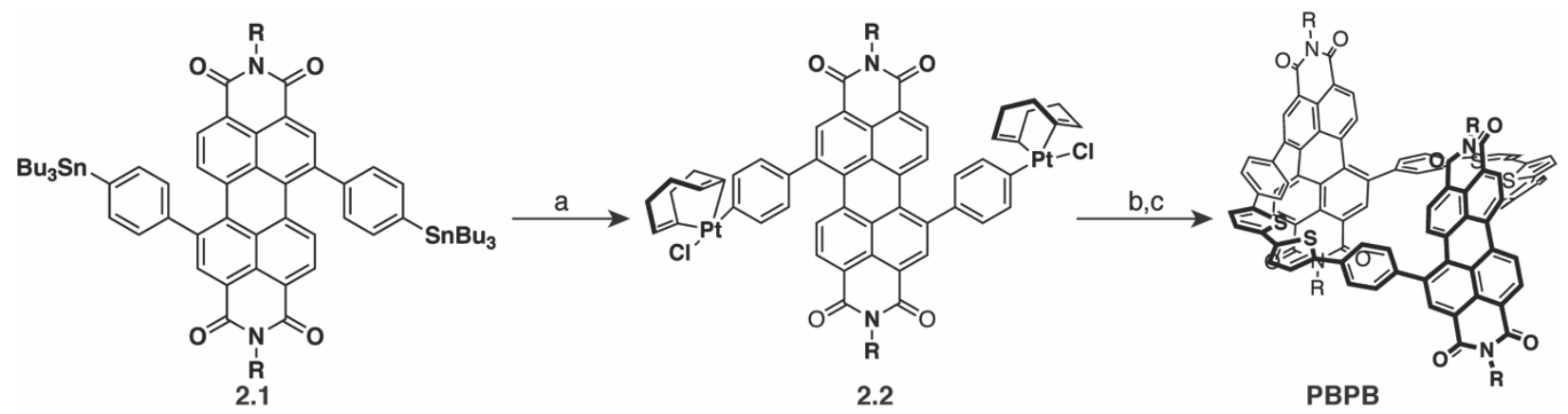

Figure 2.2. Scheme for synthesis of PBPB. (a) $\mathrm{Pt}(\mathrm{COD}) \mathrm{Cl}_{2}$, toluene, $100{ }^{\circ} \mathrm{C}, 24.5 \mathrm{~h} 63 \%$ yield. (b) 5,5' bis(trimethylstannyl)-2,2'-bithiophene, THF, $55^{\circ} \mathrm{C}, 48 \mathrm{~h}$. (c) $\mathrm{PPh}_{3}$, toluene, $100{ }^{\circ} \mathrm{C}, 12 \mathrm{~h}$, $49 \%$ yield ( 2 steps).

The key intermediate is the bis-platinated diphenyl-PDI, 2.2. We synthesize this important building block by a double Stille coupling of 1,4-bis(tributylstannyl)benzene with the 1,7dibromo-PDI to afford the bis-tributyltin substituted aromatic, 2.1. Transmetalation of 2.1 with dichloro(1,5-cyclooctadiene)platinum(II) provides 2.2. We heat equimolar amounts of $\mathbf{2 . 2}$ and 5,5'-bis(trimethylstannyl)-2,2'-bithiophene for $48 \mathrm{~h}$ to afford a tetra-platinum intermediate. This organometallic intermediate macrocycle proved difficult to isolate, so we carried the crude material on to the ultimate product by heating it with excess triphenylphosphine. This initiates the four-fold reductive elimination that forms PBPB. I found the yield was concentration dependent. 
If we perform the macrocyclization step at 20 mili-molar $(\mathrm{mM})$, then we isolate PBPB in a $16 \%$ yield over the two steps. Yet if we run the macrocyclization step at $3.0 \mathrm{mM}$, PBPB is isolated in a $49 \%$ yield (over the two steps), as lower concentrations promote macrocyclization. This yield is exceptional given that it incorporates two steps (macrocyclization and reductive elimination) without a template to yield a complex, strained macrocycle.

Scrambling of aryl-tin/aryl-platinum bonds has been observed in the formation of platinum macrocycles from unsubstituted aromatics; ${ }^{21,24}$ such scrambling is suppressed here due to the electron deficiency of the PDI subunit. Others have found scrambling less pronounced when aryl groups possessed electron-withdrawing substituents. ${ }^{38,39} \mathrm{We}$ find no evidence of scrambling in the Pt-phenyl bond in 2.2 when heated to $50{ }^{\circ} \mathrm{C}$ with tributyltin chloride for $48 \mathrm{~h}$. The lack of reversibility is a new design feature that allows the synthesis, for the first time, of conjugated macrocycles with an alternating -a-b-a-b- pattern.

\subsection{Single Crystal Analysis of PBPB}

The structure of PBPB was unambiguously confirmed via single crystal XRD (SCXRD). Figure 2.3 shows the relationship between two PBPB adjacent diphenyl subunits with their $\pi$ faces orthogonal to the $\mathrm{b}$ axis, the direction of the $\pi$-stacked wires. PBPB can exist as one of three different stereoisomers (a $P$ - or $M$-enantiomer and a meso form), but exists as the two enantiomers in the solid-state. Neighboring diphenyl PDIs have opposite handedness, such that, a $P$ enantiomer (red molecule in Figure 2.3a) always packs with the $M$ stereoisomer (blue molecule). ${ }^{40}$ The interaction between adjacent PDIs in the stack can be thought of as a left hand clasping a right hand. This, in turn, maximizes the $\pi-\pi$ interaction between adjacent molecules by positioning the $\pi$ faces within $\sim 3.7 \AA$ of each other. Neighboring macrocycles are orthogonal and create a onedimensional wires down the $\mathrm{b}$ axis. Section 2.12 contains crystallographic information. 
(a)

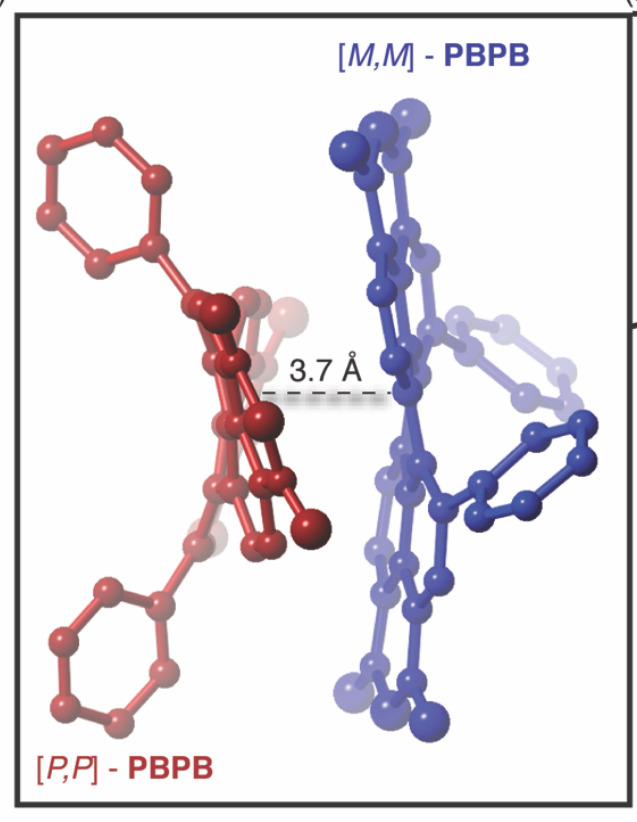

(b)

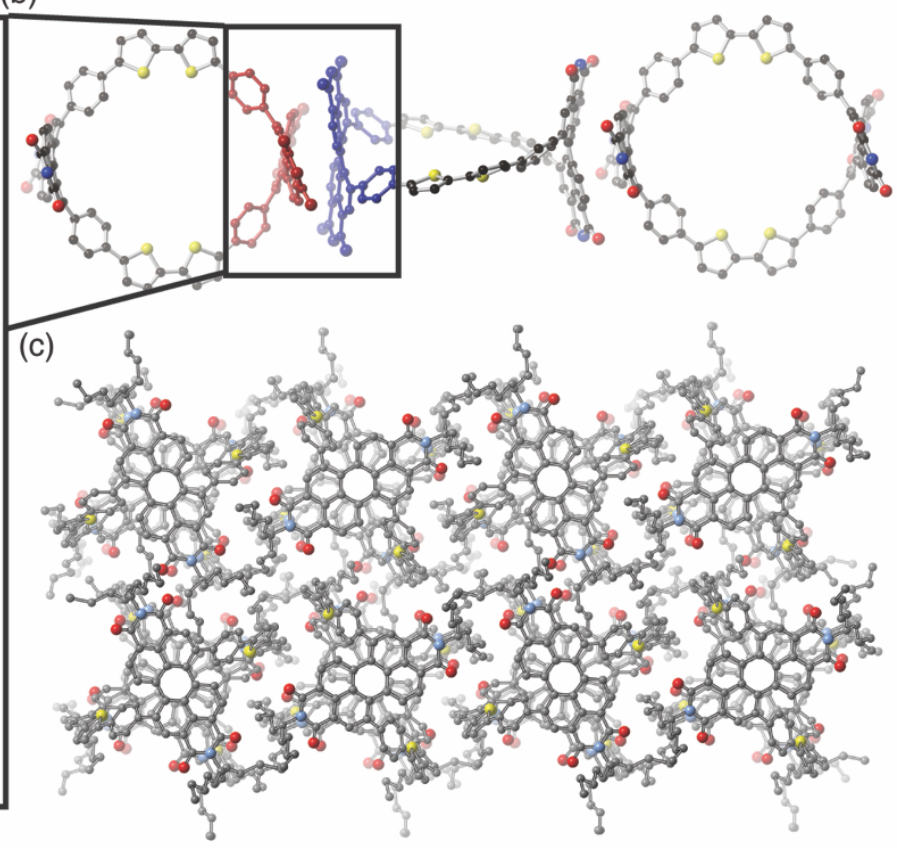

Figure 2.3. (a) Two adjacent diphenyl PDIs. The shortest PDI-PDI distance is $\sim 3.7 \AA$. Only one PDI unit of $(P, P)$-PBPB (red) and $(M, M)$-PBPB (blue) is shown; (b) a one-dimensional molecular wire formed from $\pi-\pi$ interactions between neighboring macrocycles viewed down the a axis. Side chains and hydrogen atoms have been removed for clarity; and (c) a view down the b axis showing adjacent PBPB wires. Carbon $=$ gray, nitrogen $=$ blue, oxygen $=$ red, sulfur $=$ yellow. Hydrogen atoms have been removed to clarify the view.

\subsection{NMR Studies of PBPB}

While the ${ }^{1} \mathrm{H}$ NMR spectrum of PBPB contains several broad peaks in the aromatic region at room temperature, suggesting that dynamical processes (rotation of the phenyl rings and the undecyl sidechains) ${ }^{41}$ occur on the NMR timescale (Figure 2.9), acquiring the NMR data at $88^{\circ} \mathrm{C}$ (in 1,1,2,2-tetrachloroethane- $\mathrm{d}_{2}$ ), however, provides a well-resolved ${ }^{1} \mathrm{H}$ NMR spectrum (Figure 2.4a). In the downfield region of the spectrum (Figure 2.4a), we observe resonances consistent with the macrocycle alongside a smaller set of resonances that have analogous splitting and multiplicity. This pattern suggests that PBPB exists as two closely related isomers, and integration of the resonances reveals that the two isomers occur in a ratio of approximately $6: 1\left(\right.$ at $\left.87^{\circ} \mathrm{C}\right)$. The gas phase calculations provide a ratio of $316: 1$ at $87^{\circ} \mathrm{C}$ (Section 2.16$)$. The effects of the medium, 
solvation, dielectric constant, and guest inclusion must account for the difference between the calculated and the observed ratios. We attempted to find the coalescence point of the two isomers using variable temperature NMR, however, full coalescence was not observed even at temperatures of $147^{\circ} \mathrm{C}$.

The two sets of resonances we observe in the NMR in Figure 2.4a are a result of stereoisomerism. The diphenyl PDI moiety, when linked in the 1,7-position and constrained in a macrocycle, introduces chirality (Chapter 1B, Figure 1B.3). With this information in hand, we used DFT to determine energy-optimized structures for the stereoisomers, and these are represented in Figures $2.4 \mathrm{~b}$ and $2.4 \mathrm{c}$. When linked together by a pair of bithiophenes, two diphenyl-PDI subunits possessing the same chirality produce a pair of enantiomers $[(P, P)-\mathbf{P B P B}$ and $(M, M)-\mathbf{P B P B}]$. Alternatively, linking two PDI subunits with opposite chirality produces an achiral, meso form $[(P, M)$-PBPB, Figure 2.4c.]. The enantiomeric forms of PBPB have an effective $\mathrm{C}_{2}$ axis with respect to two PDIs in the macrocycle. The achiral, meso isomer, contains a mirror plane between the two PDIs. Both the chiral and meso forms of PBPB have PDI core structures that resemble the diphenyl-PDI conformation. The twist of the PDI core in the meso isomer is the same as that of the diphenyl PDI derivative, $22^{\circ}$ (Figure 1B.3 and Figure 2.7); whereas, the chiral isomer has a larger twist in its PDI subunit $\left(25^{\circ}\right.$, shown in Figure $\left.2.4 \mathrm{~b}\right)$. We believe this twist and orientation of the phenyls facilitates formation of PBPB by giving curvature to the otherwise planar PDI. 

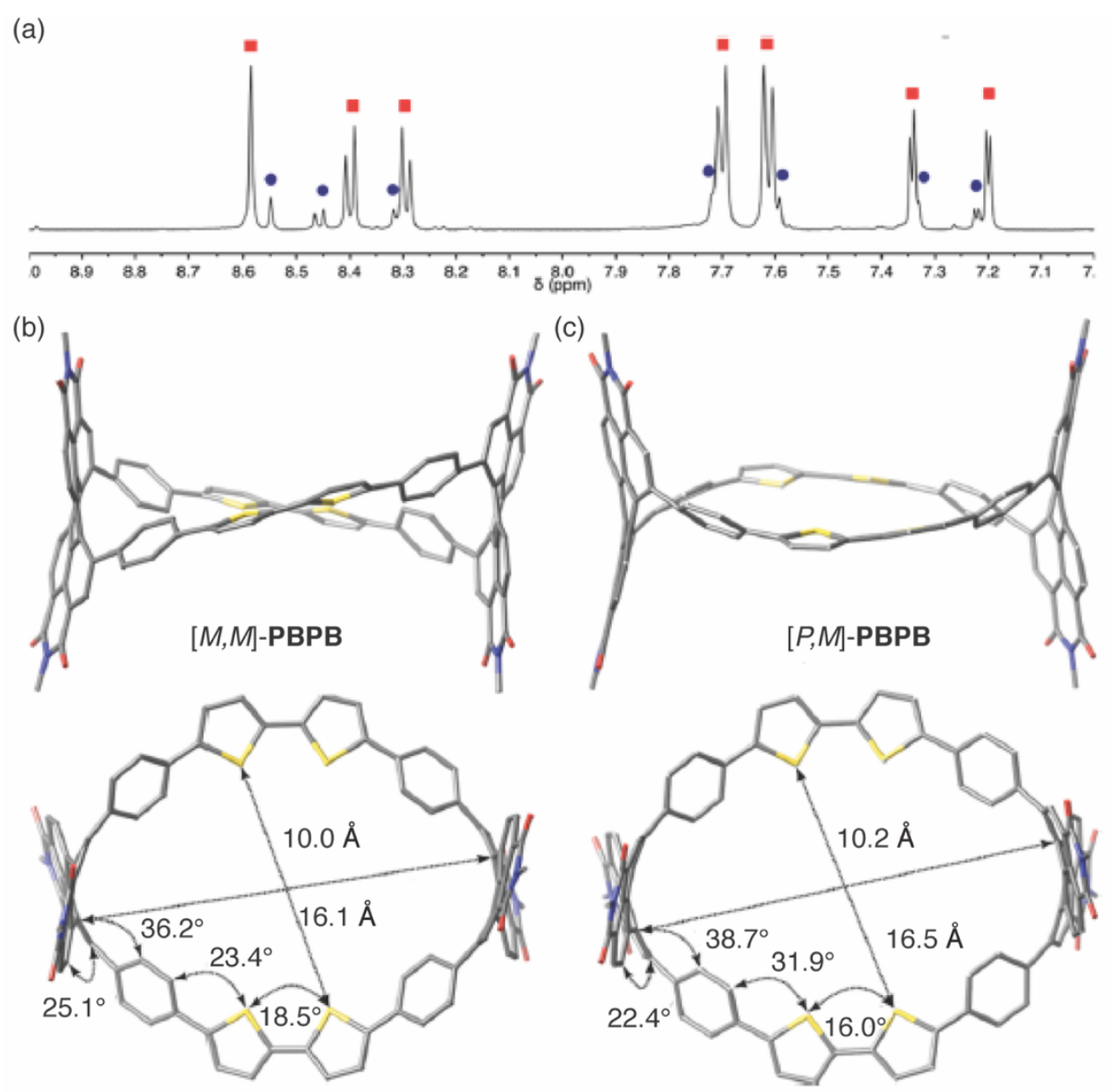

Figure 2.4. (a) Downfield region of the ${ }^{1} \mathrm{HNMR}$ spectrum of macrocycle PBPB recorded at 88 ${ }^{\circ} \mathrm{C}$. The red squares (chiral) and blue circles (achiral) identify resonances from the two stereoisomers. (b) DFT minimized model (side-on and face-on views) of chiral stereoisomer $(M, M)$-PBPB. (c) DFT minimized model (side-on and face-on views) of meso stereoisomer $(P, M)$ PBPB (undecyl sidechains have been truncated to a methyl group to simplify the calculations). Carbon $=$ grey, nitrogen $=$ blue, oxygen $=$ red, sulfur $=$ yellow. Hydrogens have been removed to clarify the view.

\subsection{Bithiophene Acts as Strain Reliever}

In both stereoisomers of PBPB, the macrocycle has a persistent elliptiform cavity, measuring $1.6 \mathrm{~nm}$ from PDI to PDI and $1.2 \mathrm{~nm}$ from bithiophene to bithiophene. One striking difference between these two stereoisomers is the apparent strain in the tetracyclic linkers connecting the PDIs. The meso isomer forces the linkers to bow in order to accommodate the rigidity of the cycle (Figure 2.4c), while in the chiral isomer the tetracyclic linkers are essentially 
flat (Figure 2.4b), which make the chiral isomer appear less strained. In both isomers, the sulfur atoms of the four thiophenes point into the cavity while the diphenyl PDIs cap the ends of the macrocycle. Oligothiophenes typically possess an anti-geometry of their sulfur atoms rather than syn orientation, reflecting decreased steric repulsion in the former. ${ }^{42-44}$ However, this is not the case for PBPB. The bithiophene orientation plays a crucial role in the assembly of PBPB by relieving strain. In order to quantify this effect, we modeled the syn and anti conformers of simple bithiophene, $\mathrm{C}_{8} \mathrm{H}_{6} \mathrm{~S}_{2}$. The angle between the two terminal C-H bond vectors defines the curvature supported by the bithiophene. This angle is $117^{\circ}$ in the syn geometry and $180^{\circ}$ in the anti geometry. Thus, the syn orientation of the bithiophene promotes the formation and stabilization of PBPB. Conversely, the incorporation of the bithiophenes into PBPB promotes the otherwise unfavorable syn orientation in the bithiophenes. Reports from Bäuerle and Itami describe a similar conformation preference in macrocyclic, 2,5-linked oligothiophenes. ${ }^{9,18}$

\subsection{HPLC Analysis of Stereoisomers}

Chiral Higher Performance Liquid Chromatography (HPLC) allowed us to separate and characterize the stereoisomers of PBPB. The chromatogram of PBPB (Figure 2.5a) shows both the pair of enantiomers and the meso isomer. In the chromatogram, peaks $\mathbf{A}$ and $\mathbf{C}$ have equal integrated intensity and therefore represent the enantiomeric pair. A third peak, B, corresponds to the meso stereoisomer. Using HPLC with a circular dichroism (CD) detector, we were able to confirm this assignment (Figure 2.5b). The two peaks in the chromatogram assigned to the two enantiomers gave opposite CD signals $(225-800 \mathrm{~nm}$, Figures $2.5 \mathrm{~b} \& 2.5 \mathrm{c})$. As anticipated, the peak assigned to the meso compound had no detectable CD signal. 


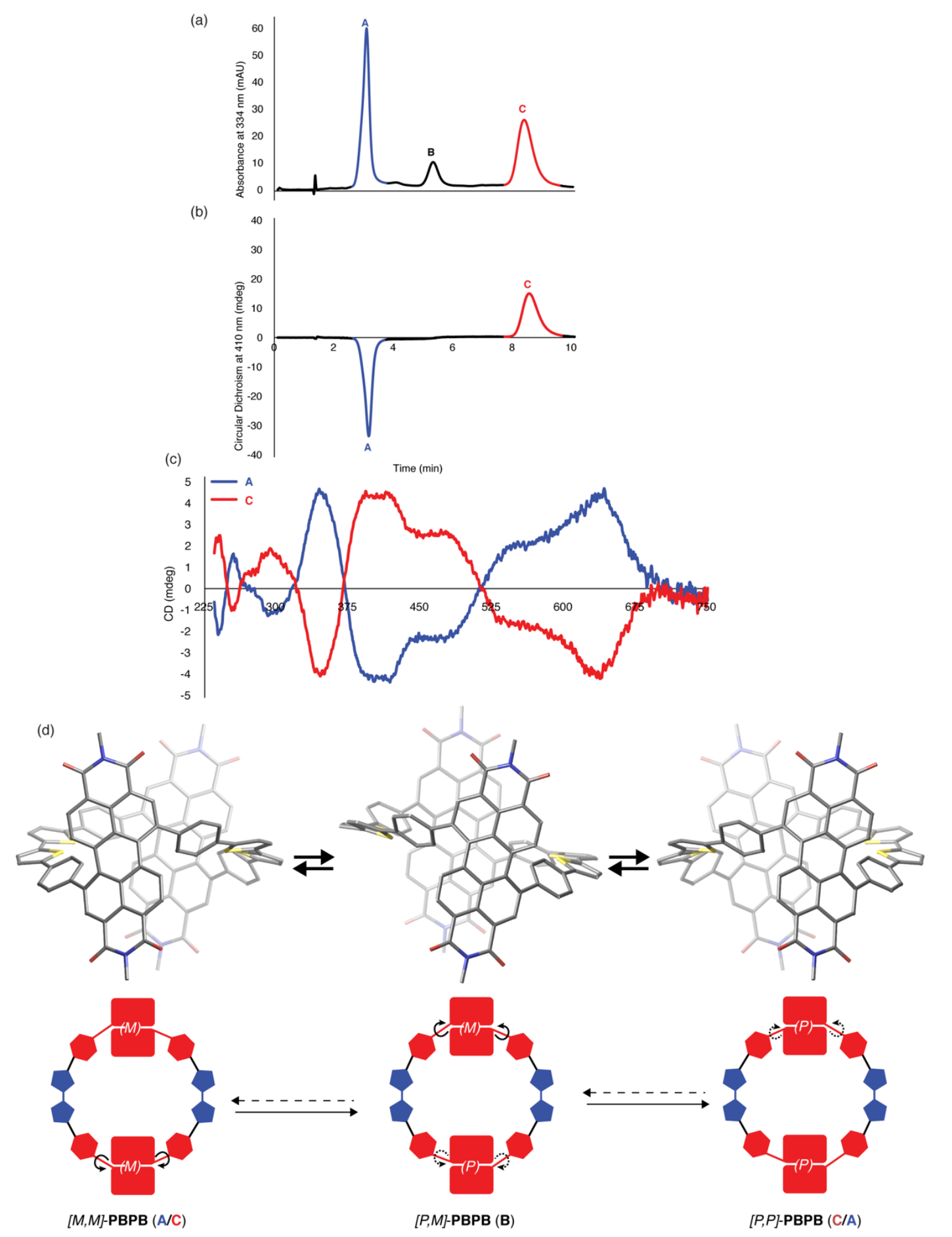

Figure 2.5. (a) HPLC chromatogram of PBPB using a CHIRALPAK ${ }^{\circledR}$ IA-3 column. (b) HPLCCD chromatogram of PBPB showing peaks A and C exhibit opposite optical activity (270-410 $\mathrm{nm}$ ). (c) CD spectra for both enantiomers of PBPB. (D) Interconversion of the stereoisomers of PBPB by sequential rotation of one PDI subunit through the macrocycle and then rotation of the other PDI to convert between enantiomers. The hydrocarbon sidechains have been replaced with a methyl group in the DFT structures. Carbon $=$ grey, nitrogen $=$ blue, oxygen $=$ red, sulfur $=$ yellow. Hydrogen atoms have been removed to clarify the view. 


\subsection{Intramolecular Somersault Mode of Isomerization}

It is interesting that $\mathbf{A}$ and $\mathbf{C}$ interconvert at room temperature, albeit not directly; the interconversion requires the intermediacy of $\mathbf{B}$, the meso compound. To confirm the intermediacy of $\mathbf{B}$, we used preparative HPLC. We obtained a pure fraction of $\mathbf{C}$, and immediately re-injected it into the HPLC and monitored for the appearance of the $\mathbf{A}$ and $\mathbf{B}$ stereoisomers (Figure 2.6). Within 20 min, the concentration of $\mathbf{B}$ had already reached $62 \%$ of its equilibrium value, yet that of $\mathbf{A}$ had reached only $8 \%$ of its equilibrium value. After $2 \mathrm{~h}$ at room temperature, all peaks had reached their equilibrium intensities (Figure 2.6b). This result is remarkable because it suggests the mode of isomerization is a PDI 'intramolecular somersault' in which one of the PDIs rotates around its transverse axis, thereby moving one of its diimide heads through the plane of the cavity. This somersault converts a chiral molecule into an achiral one and vice versa. Figure $2.5 \mathrm{~d}$ shows a schematic of this conversion process between $\mathbf{A}$ and $\mathbf{B}$.

We were also interested in the rate of interconversion, and we used variable temperature HPLC to study this process. Maintaining the temperature of both the HPLC column and the sample at $0{ }^{\circ} \mathrm{C}$, we analyzed the freshly isolated meso isomer, $\mathbf{B}$, monitoring its conversion to both of the enantiomers $(\mathbf{A} / \mathbf{C})$ over time. Figure 2.11 shows the natural log of the diastereomeric excess as a function of time. If we assume this process is a simple first-order reaction, the rate constant was $\sim 10^{-4} \mathrm{~s}^{-1}$ at $0{ }^{\circ} \mathrm{C}$. It is difficult to relate this rate to other macrocycles, given there have been just a few kinetic studies concerning the racemization of conjugated belt-like compounds. Yet, we see experimentally that equilibrium is reached within two hours at room temperature as discussed above. 
(a)

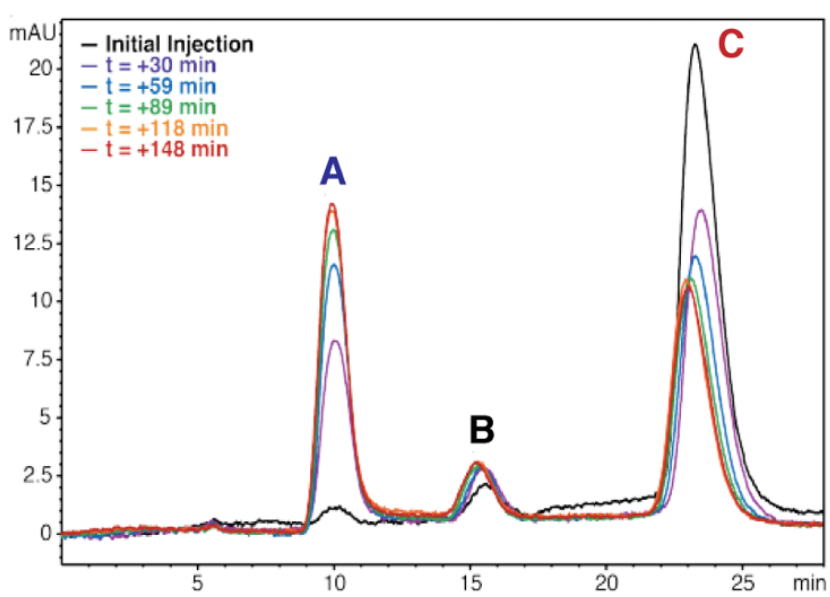

(b)

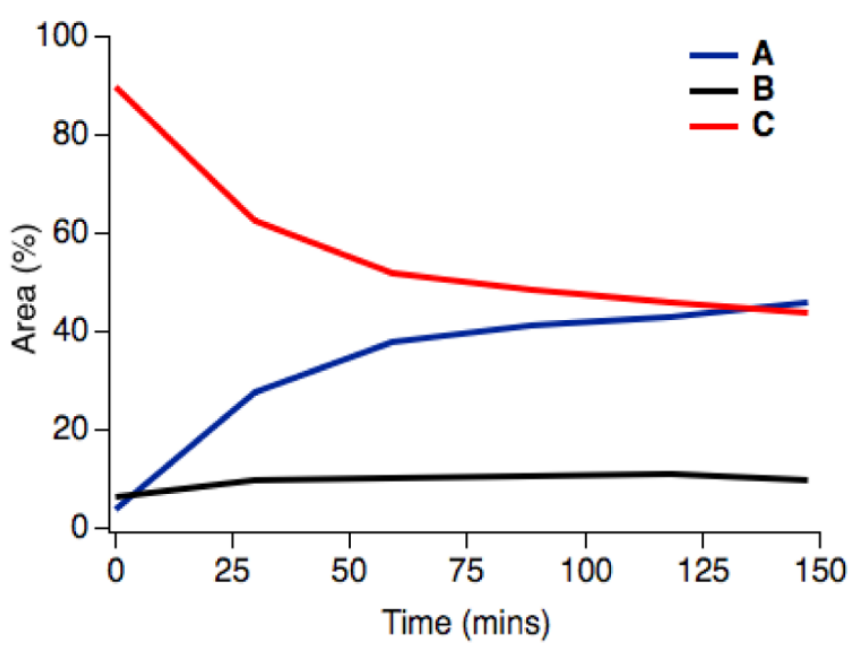

Figure 2.6. (a) The interconversion of $\mathbf{C}$ to $\mathbf{A}$ through the intermediacy of $\mathbf{B}$. (b) Plot of relative intensities (via integration of the area under each peak) as a function of time.

\subsection{Charge Transfer Characteristics in PBPB}

The electronic properties of PBPB can be appreciated by consideration of its UV-Vis absorption spectrum (Figure 2.7), particularly when compared to the spectra of its constituent parts, viz., 1.1, and a bithiophene derivative. PBPB retains the shorter-wavelength absorptions $(\sim 300-400 \mathrm{~nm})$ characteristic of bithiophene and the intermediate-wavelength absorptions $(\sim 450-$ $550 \mathrm{~nm}$ ) characteristic of PDIs, but a new, strong absorption band appears at longer wavelength $(\sim 550-700 \mathrm{~nm})$. The optical gap is estimated to be $1.8 \mathrm{eV}$ from the absorption band edge. It is the addition of this latter band that completes the absorption of the visible and renders PBPB black. 
(18)

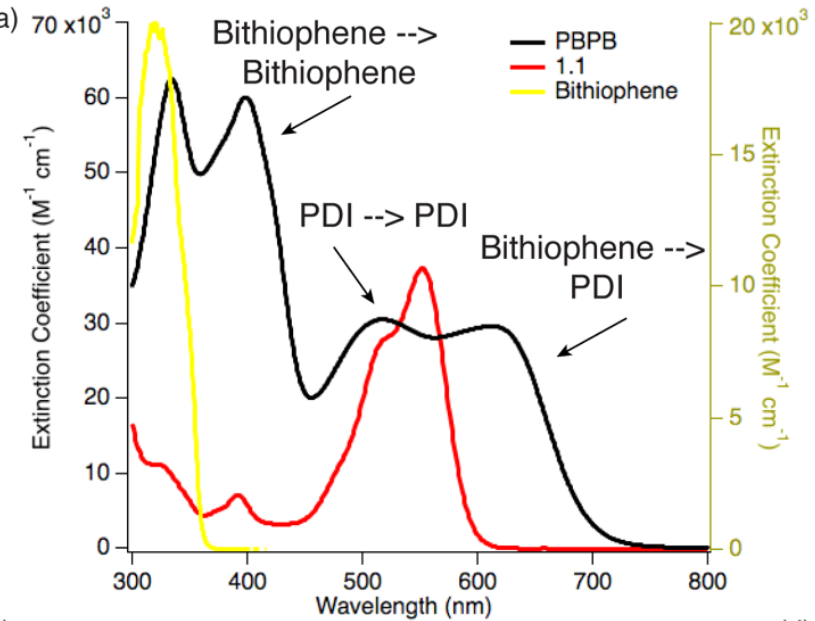

(c)

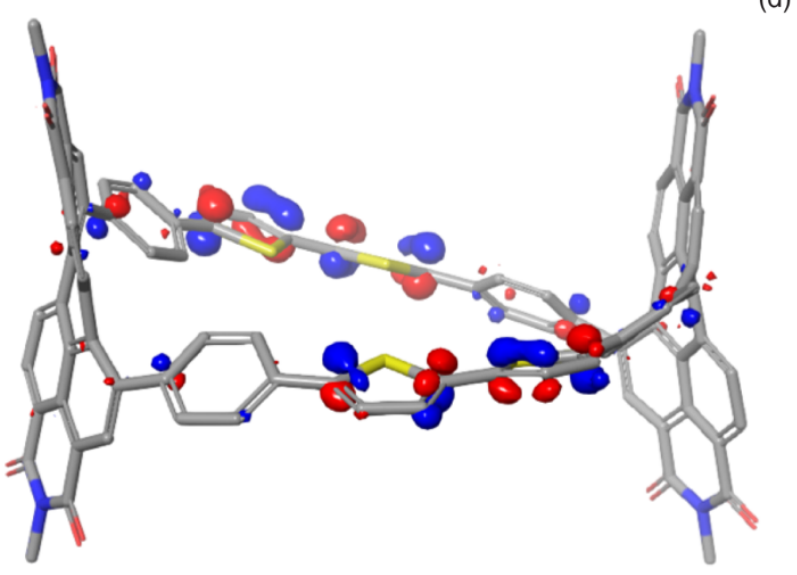

(d) (b)

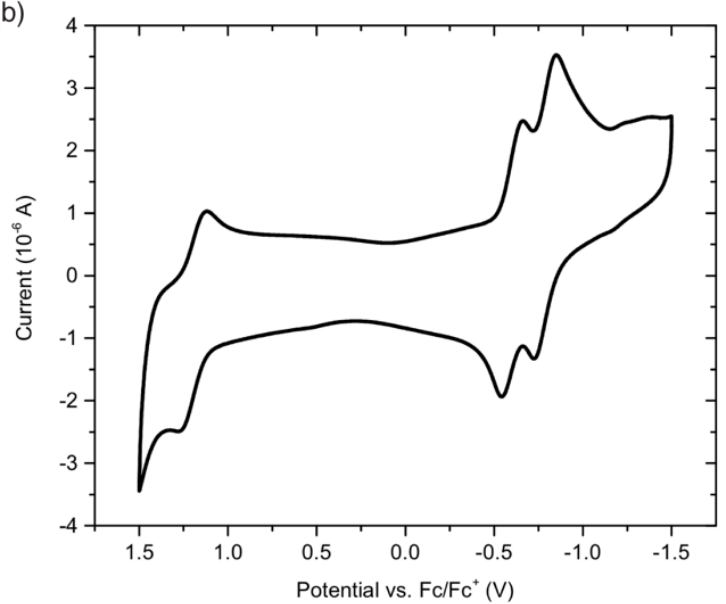

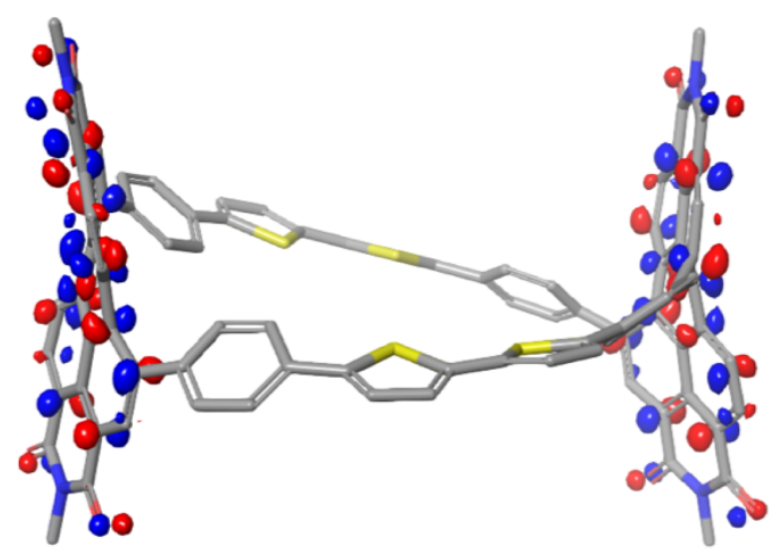

Figure 2.7. (a) UV-vis absorption spectrum of PBPB (black line), 5,5'-bis(tributylstannyl)-2,2'bithiophene (yellow) and 1.1 (diphenyl PDI), red) in $\mathrm{CH}_{2} \mathrm{Cl}_{2}$ (concentration $=1.6 \times 10^{-5} \mathrm{M}, 1.3 \times$ $10^{-4}$ and $1.5 \times 10^{-5}$, respectively, path length $\left.=1.0 \mathrm{~cm}\right)$. The yellow trace is plotted with respect to the right ordinate, and the black and red traces are plotted with respect to the left ordinate; (b) $\mathrm{CV}$ of PBPB showing two reversible reduction and one reversible oxidation peak; (c) and (d) the HOMO and LUMO orbitals of PBPB from TDDFT, respectively.

The electronic properties of PBPB can be appreciated by consideration of its UV-vis absorption spectrum (Figure 2.7a), particularly when compared to the spectra of its constituent parts, viz., a helically-deformed PDI (1.1), and a bithiophene derivative. PBPB retains the shorterwavelength absorptions $(\sim 300-400 \mathrm{~nm})$ characteristic of bithiophene and the intermediatewavelength absorptions ( 450-550 nm) characteristic of PDIs, but a new, strong absorption band appears at longer wavelength $(\sim 550-700 \mathrm{~nm})$. The optical gap is estimated to be $1.8 \mathrm{eV}$ from the absorption band edge. It is the addition of this latter band that completes the absorption of the 
visible and renders PBPB black.

We also studied the energetics of the frontier orbitals of PBPB using CV (Figure 2.7b). The onset of the first oxidation $(0.58 \mathrm{~V})$ and reduction $(-1.07 \mathrm{~V})$ peaks relative to $\mathrm{Fc} / \mathrm{Fc}^{+}$provide an estimate of the HOMO-LUMO gap $(1.6 \mathrm{eV})$. Similar to other aryl-substituted PDI compounds, PBPB has one oxidation and two one-electron reductions, revealing that the PDIs within PBPB retain their ability to accept two electrons. ${ }^{45,46} \mathrm{We}$ also used quantum chemical calculations (TDDFT) to characterize the low-lying excited states of PBPB, and found that while there is little charge-transfer from the electron-rich thiophenes to the electron-accepting PDIs in the ground state of the molecule, there is such charge-transfer in the lowest-energy excited states: the longwavelength absorptions are best viewed as forming bithiophene-to-PDI charge-transfer states (Figure 2.7c-d).

\subsection{Conclusion}

This Chapter describes a new class of fully-conjugated macrocycles that incorporate donor and acceptors subunits to form a chiral conjugated corral. PBPB is the first example of a conjugated macrocycle with alternating aromatic subunits in an -a-b-a-b- pattern. These new macrocycles possess an interesting topology and useful electronic properties that is a result of the pattern written into the cycle. The macrocycle's unusual electronic structure provides a small HOMO/LUMO gap and broad absorbance across the entire visible light spectrum. Excited-state DFT calculations reveal intramolecular charge transfer in the frontier orbitals. Not only does PBPB's conformational dynamics allow for the interconversion of this macrocycle between chiral and achiral forms, but this easy propensity for shape-shifting offers the appealing opportunity for sterically-forgiving host-guest chemistry (Chapter 9). This unusual set of properties makes the macrocycles exciting new candidates for use in energy applications such as in photovoltaics in the 
following chapter.

\subsection{Additional Thoughts and Observations}

It is suggested that the reversibility of the transmetalation step is akin to electrophilic aromatic substitution. ${ }^{38,39}$ In the reaction for PBPB, once the bithiophene has been transferred to the platinum center, the transmetalation process is reversible, yet he lack of scrambled products in suggest that the mechanism for the back reaction is selective. Figure 2.8 shows a proposed mechanism. In Figure 2.8a, $\mathrm{R}_{1}$ and $\mathrm{R}_{2}$ are different structures (e.g. a biphenyl and $p$-terphenyl) with similar electronics. The reversibility step can result in scrambled products. ${ }^{21}$

(a) Transmetalation Step \& Reversibility $R_{1} \neq R_{2}$ but electronics $R_{1}=R_{2}$

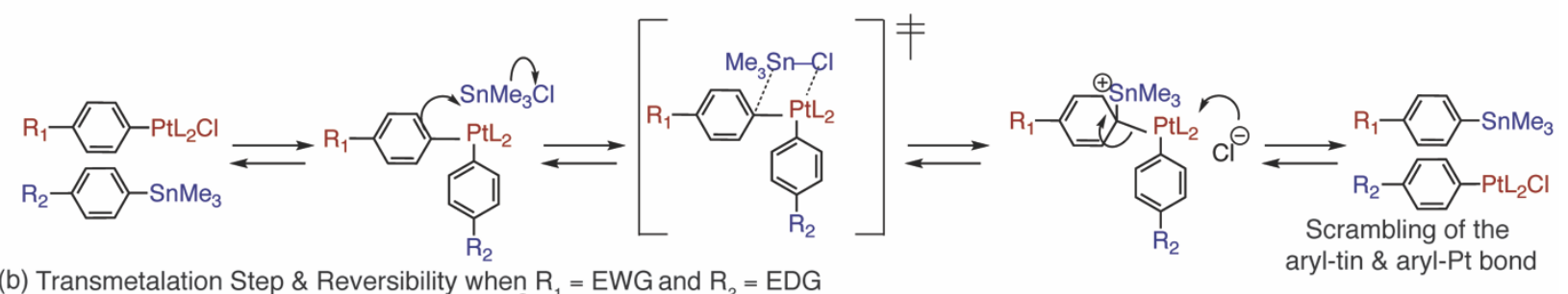

(b) Transmetalation Step \& Reversibility when $R_{1}=E W G$ and $R_{2}=E D G$

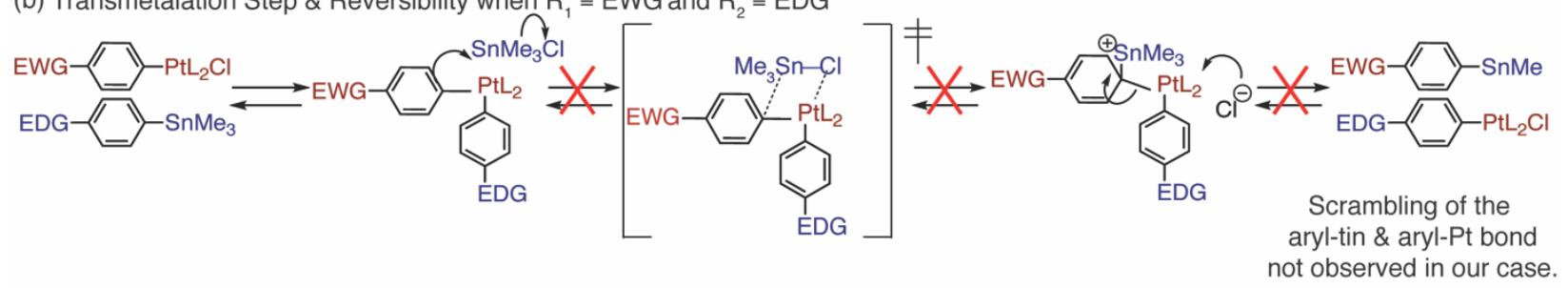

Figure 2.8. (a) A proposed mechanism for the reversibility of the transmetalation step when $\mathrm{R}_{1}$ and $\mathrm{R}_{2}$ are different substituents, but have similar electronics; and (b) reversibility is not observed in our system as there is a large difference in the aryl groups electronics e.g. one if electron rich and the other is electron deficient.

Figure $2.8 \mathrm{~b}$ is an analogous situation to the synthesis of $\mathbf{P B P B}$. If $\mathrm{R}_{1}$ and $\mathrm{R}_{2}$ possess different electronics e.g. one is electron rich and the other is electron poor, then the electrophilic addition will likely occur on the more electron rich ring (larger density of high energy electrons) theoretically and not from the electron deficient ring as shown in Figure $2.8 \mathrm{~b}$. This results in starting material and doesn't provide the opportunity to create scrambled products. This is one possible mechanism that explains the lack of reversibility in the synthesis of PBPB. 


\subsection{Appendix - Supplementary Figures}

(a)

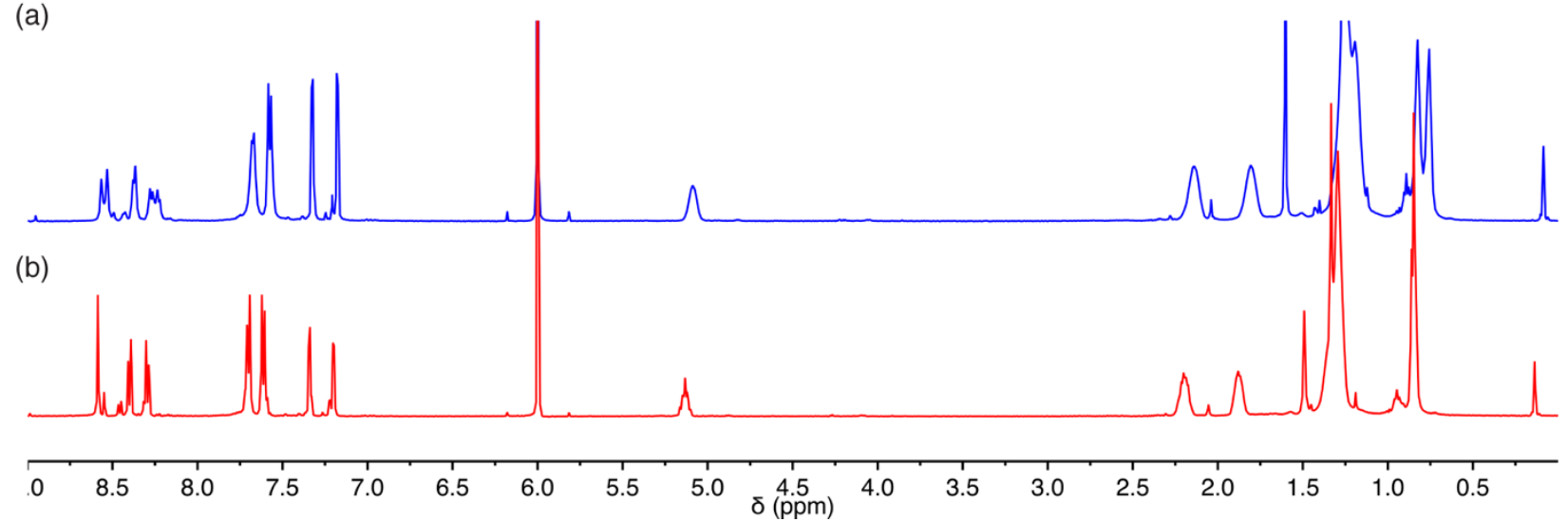

Figure 2.9. (a) ${ }^{1} \mathrm{H} N M R$ of PBPB at $300 \mathrm{~K}$ in $\mathrm{C}_{2} \mathrm{D}_{2} \mathrm{Cl}_{4}$. (b) ${ }^{1} \mathrm{HNMR}$ of $\mathbf{P B P B}$ at $360 \mathrm{~K}$ in $\mathrm{C}_{2} \mathrm{D}_{2} \mathrm{Cl}_{4}$.

Section 2.5 describes the twist angle of the PDI in PBPB relative to diphenyl PDI. Below is also the SCXRD structure of dibromo-diphenyl PDI that shows a $22^{\circ}$ twist angle of the two naphthyl units. Two views of the single crystal structure of N,N'-di(6-undecyl)-1,7-di(4bromophenyl)-perylene-3,4:9,10-tetracarboxylic diimide. Carbon $=$ black, oxygen $=$ red, nitrogen $=$ blue, bromine $=$ brown. Hydrogen atoms are omitted for clarity; in the side-on view the $\mathrm{C}_{11}$ chains are also omitted. The $\mathrm{C}_{11} \mathrm{H}_{23}$ chains were disordered over several positions. In addition, the crystal contained $25 \%$ of $\mathrm{PDI}\left(\mathrm{C}_{6} \mathrm{H}_{4} \mathrm{Br}\right)\left(\mathrm{C}_{6} \mathrm{H}_{5}\right)$. The minor positions of these disordered atoms are omitted. Thermal ellipsoids are rendered at the 50\% level. 


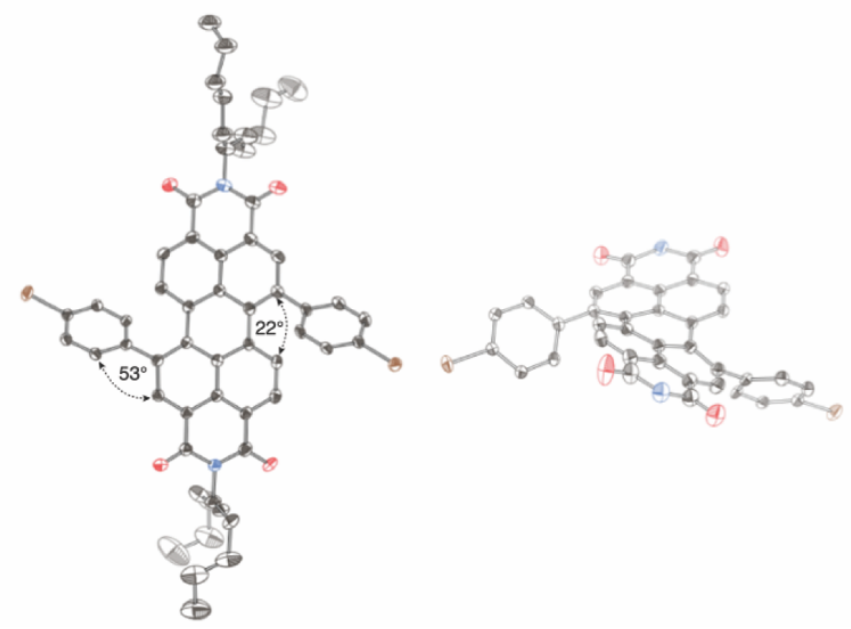

Figure 2.10. Single crystal X-ray structure of $N, N^{\prime}$-di(6-undecyl)-1,7-di(4-bromophenyl)perylene-3,4:9,10-tetracarboxylic diimide. (a) Face-on view. (b) Side-on view.

The rapid equilibration of $(P, M)$-PBPB into $(P, P)$-PBPB and $(M, M)$-PBPB was monitored by analytical $\mathrm{HPLC}$ using $30 \% \mathrm{CH}_{2} \mathrm{Cl}_{2} /$ hexanes at $0 \pm 0.2{ }^{\circ} \mathrm{C}$ at $1.5 \mathrm{~mL} / \mathrm{min}$ on a CHIRALPAK ${ }^{\circledR}$ IA-3 column ( $4.6 \mathrm{~mm}$ I.D. x $250 \mathrm{~mm}, 3 \mu \mathrm{m})$. The meso peak was isolated from a preparative HPLC column and injected onto the analytical HPLC within 1 minute before placing in an ice bath. The sample was kept in an ice bath for the first 185 minutes after the initial injection, then allowed to warm to room temperature $\left(\right.$ solid lines $=$ sample kept at $0{ }^{\circ} \mathrm{C}$; dashed lines $=$ sample kept at $25^{\circ} \mathrm{C}$ ). Diastereomeric excess (de) was calculated for each HPLC injection and plotted as $\ln \left(\mathrm{de}_{0} / \mathrm{de}_{\mathrm{t}}\right)$ vs. time, where $\mathrm{de}_{0}$ is the $\mathrm{d}_{\mathrm{e}}$ value for the initial injection and $\mathrm{de}_{\mathrm{t}}$ is the $\mathrm{d}_{\mathrm{e}}$ at a given time after the initial injection. The rate constant for conversion of the meso diastereomer to the chiral diastereomers is given by the slope.

Equation: $\ln \left(\mathrm{de}_{0} / \mathrm{de}_{\mathrm{t}}\right)=2 \mathrm{kt}$.

$$
\text { Slope }=4.0 \times 10^{-4} ; \mathrm{R}^{2}=0.9911 \mathrm{k}=1.7 \times 10^{-4} \mathrm{~s}^{-1}
$$


(a)

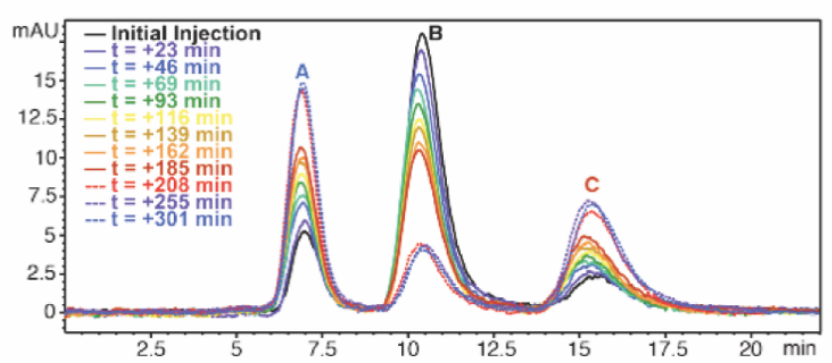

(b)

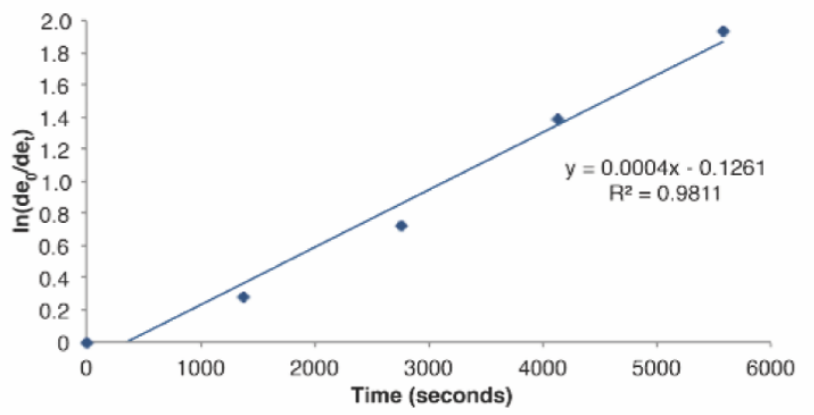

Figure 2.11. (a) Conversion of $B$ into $A$ and C. (b) Plot and linear fit of $\ln \left(\mathrm{de}_{0} / \mathrm{de}_{\mathrm{t}}\right)$ vs. time.

\subsection{General Experimental Information}

Synthesis. All reactions were performed in oven-dried or flame-dried round bottom flasks, unless otherwise noted. The flasks were fitted with rubber septa and reactions were conducted under a positive pressure of nitrogen or argon, unless otherwise noted. Anhydrous and anaerobic solvents were obtained from a Glass Contour solvent system consisting of a Schlenk manifold with purification columns packed with activated alumina and supported copper catalyst. Reaction monitoring by thin layer chromatography (TLC) was performed on J.T. Baker Baker-flex Silica Gel IB2-F (25 mm x $75 \mathrm{~mm})$ TLC plates. TLC visualization was accomplished by visible observation and irradiation with a UV lamp. Commercial reagents were used without further purification. $\mathrm{Pt}(\mathrm{COD}) \mathrm{Cl}_{2}$ was purchased from Strem Chemicals, and all other reagents were purchased from Sigma-Aldrich

Purification. Automated flash chromatography was performed using a Teledyne Isco Combiflash Rf200 and Redisep Rf Silica columns. Preparative high performance liquid chromatography (HPLC) was performed on a Waters Prep150 instrument equipped with a UV-vis detector (335 
$\mathrm{nm}$ ), an automated fraction collector, and a Nacalai Tesque COSMOSIL Buckyprep column (20 mm I.D. x $250 \mathrm{~mm}, 5 \mu \mathrm{m})$.

Spectrometers. ${ }^{1} \mathrm{H}$ NMR spectra were recorded on a Bruker 400 or $500 \mathrm{MHz}$ spectrometer. ${ }^{13} \mathrm{C}$ NMR spectra were recorded on a Bruker 125 or $100 \mathrm{MHz}$ spectrometer with complete proton decoupling. NMR spectra were recorded at $300 \mathrm{~K}$ unless otherwise noted. Chemical shifts are reported in parts per million (ppm) Data are represented as follows: chemical shift, multiplicity (s $=$ singlet, $\mathrm{d}=$ doublet, $\mathrm{dd}=$ doublet of doublets, $\mathrm{t}=$ triplet, $\mathrm{m}=$ multiplet $)$, coupling constants in $\mathrm{Hz}$, and integration. Broadening (br) of peaks in the $1 \mathrm{H}$ NMR spectrum is due to rotational isomers about the imide side chains. **Multiple peaks for the same carbon in the ${ }^{13} \mathrm{C}$ NMR spectrum (indicated by $* *)$ reflect rotational isomers about the imide side chains that have been seen previously. ${ }^{41}$ This was further confirmed by the HSQC spectrum of Compound 2.2 (pages 64). High-resolution mass spectrometry (HRMS) was performed on a Waters XEVO G2XS instrument equipped with a UPC SFC inlet, electrospray (ESI) and atmospheric pressure chemical (APCI) ionization, and a QToF mass spectrometer. UV-Vis absorption spectra were recorded on a Shimadzu UV-1800 spectrophotometer. Infrared (IR) spectra were recorded on a Perkin Elmer Spectrum400 FTIR spectrometer using a PIKE ATR attachment.

Chirality Analyses. Analytical HPLC was performed on an Agilent 1200 Series instrument equipped with a diode array detector $(300 \mathrm{~nm}$ to $900 \mathrm{~nm})$ and a CHIRALPAK ${ }^{\circledR}$ IA-3 column (4.6 mm I.D. x 250 mm, $3 \mu \mathrm{m}$ ) from Chiral Technologies. Analytical HPLC-circular dichroism (HPLCCD) was performed on an Agilent 1100 system comprised of a G1311A quaternary pump, G1367A WPALS auto sampler, G1315B diode array detector, a CD-1595 circular dichroism detector, and a CHIRALPAK ${ }^{\circledR}$ IA-3 column (4.6 mm I.D. x 150 mm, $\left.3 \mu \mathrm{m}\right)$ from Chiral Technologies. Full CD spectra (225 $\mathrm{nm}$ to $750 \mathrm{~nm}$ ) were measured on a Jasco 810 CD spectropolarimeter, equipped 
with a $0.1 \mathrm{~cm}$ microcell and a Julabo thermostat.

HPLC-CD Instrument Method and CD Spectra: PBPB Separation was achieved using 30\% $\mathrm{CH}_{2} \mathrm{Cl}_{2} /$ hexanes at $20{ }^{\circ} \mathrm{C}$ at $1.5 \mathrm{~mL} / \mathrm{min}$ on a CHIRALPAK ${ }^{\circ}$ IA-3 column (4.6 mm I.D. x 150 $\mathrm{mm}, 3 \mu \mathrm{m})$. Analytical experiments were performed by injecting $5 \mu \mathrm{L}$ of a $0.5 \mathrm{mg} / \mathrm{mL}$ solution of the macrocycle in $\mathrm{CH}_{2} \mathrm{Cl}_{2}$. Using this same method of separation, $10 \mu \mathrm{L}$ of the sample at 0.75 $\mathrm{mg} / \mathrm{mL}$ were injected, and the corresponding peak fractions were collected manually. The solvent from each fraction (approximately 1-2 mL) was immediately removed, and the remaining compound dissolved in approximately $200 \mu \mathrm{L} \mathrm{CH}_{2} \mathrm{Cl}_{2}$. The $\mathrm{CD}$ spectrum at $25^{\circ} \mathrm{C}$ was taken as quickly as possible, and baseline corrected against the $\mathrm{CH}_{2} \mathrm{Cl}_{2}$ blank.

Cyclic Voltammetry. CVs were recorded on a $\mathrm{CH} 166$ electrochemical workstation using $\mathrm{Ag} / \mathrm{AgCl}$ electrode as the reference electrode at room temperature. Experiments were performed in $\mathrm{CH}_{2} \mathrm{Cl}_{2}$ with $\mathrm{NBu}_{4} \mathrm{PF}_{6}$ as the supporting electrolyte at a scan rate of $0.1 \mathrm{~V} / \mathrm{s} .{ }^{47}$

\section{Single crystal X-ray diffraction.}

\section{Diphenyl-dibromo PDI}

Data for all compounds was collected on an Agilent SuperNova diffractometer using mirrormonochromated $\mathrm{Cu} \mathrm{K} \alpha$ or $\mathrm{Mo} \mathrm{K} \alpha$ radiation. Data collection, integration, scaling (ABSPACK) and absorption correction (face-indexed Gaussian integration ${ }^{48}$ or numeric analytical methods ${ }^{49}$ ) were performed in CrysAlisPro.${ }^{50}$ Structure solution was performed using ShelXS,${ }^{51}$ ShelXT, ${ }^{52}$ or SuperFlip. ${ }^{53}$ Subsequent refinement was performed by full-matrix least-squares on $\mathrm{F}^{2}$ in ShelXL. Olex $2^{54}$ was used for viewing and to prepare CIF files. PLATON ${ }^{55}$ was used extensively for SQUEEZE,${ }^{56}$ ADDSYM and TwinRotMat. Many disordered solvent molecules were modeled as rigid fragments from the Idealized Molecular Geometry Library. ORTEP graphics were prepared in CrystalMaker. ${ }^{57}$ Thermal ellipsoids are rendered at the $50 \%$ probability level. A toluene solution 
of PDI $\left(\mathrm{C}_{6} \mathrm{H}_{4} \mathrm{Br}\right)_{2}$ was diluted with methanol by vapor diffusion to afford bright red prisms. Part of a crystal $(0.06 \times 0.04 \times 0.02 \mathrm{~mm})$ was separated carefully, mounted with STP oil treatment, and cooled to $100 \mathrm{~K}$ on the diffractometer. Complete data $(99.7 \%)$ were collected to $0.815 \AA .37417$ reflections were collected (9236 unique, 7420 observed) with $\mathrm{R}$ (int) $5.8 \%$ and $\mathrm{R}$ (sigma) 5.2\% after analytical absorption correction (Tmax 0.968 , Tmin 0.914). The space group was assigned as $\mathrm{P} 2{ }_{1} / \mathrm{c}$ based on the systematic absences. The PDI(Ar $)_{2}$ core was located easily and the $\mathrm{C}_{11}$ chains were located in two disordered positions. The geometries of the disordered side chains were stabilized with DFIX restraints on 1,2 and 1,3 distances for the intermediate refinements, which were converted to SAME restraints in the final refinement. The last three atoms of one chain were disordered over 3 positions, which were modeled with the aid of SUMP to restrain their total occupancy. All ADPs in the disordered side chains were stabilized with RIGU and SIMU.

In addition, the bromine atoms in the $\mathrm{C}_{6} \mathrm{H}_{4} \mathrm{Br}$ groups had significant negative Fourier peaks. The model was revised by splitting the entire group into two positions and freely refining the occupancy of the $\mathrm{Br}$ atom in the minor position. This amounted to a three-part disorder with the ratios (major,C-Br):(minor,C-Br):(minor,C-H) 73:13:14 and 76:14:10 for the two independent $\mathrm{C}_{6} \mathrm{H}_{4} \mathrm{Br}$ groups. Therefore the crystal was a 76:24 mixture of 1,7-bis(bromophenyl)-PDI and 1bromophenyl-7-phenyl-PDI. Hydrodehalogenation is a known side reaction in Suzuki couplings. ${ }^{58}$ All $\mathrm{C}-\mathrm{H}$ hydrogens were placed in calculated positions and refined with riding coordinates and isotropic ADPs. The final refinement (9236 data, 2058 restraints, 926 parameters) converged with $\mathrm{R}_{1}\left(\mathrm{~F}_{\mathrm{o}}>4 \sigma\left(\mathrm{F}_{\mathrm{o}}\right)\right)=5.8 \%, \mathrm{wR}_{2}=13.5 \%, \mathrm{~S}=1.11$. The largest Fourier features were 0.59 and -0.45 $\mathrm{e}^{-} \mathrm{A}^{-3}$. 


\section{PBPB}

Data for trans-PBPB was collected on an Agilent SuperNova diffractometer using mirrormonochromated $\mathrm{Cu} \mathrm{K} \alpha$ radiation. Data integration, scaling (ABSPACK) and absorption correction (face-indexed Gaussian integration ${ }^{48}$ or numeric analytical methods ${ }^{49}$ ) were performed in CrysAlisPro. ${ }^{50}$ Structure solution was performed using ShelXT. ${ }^{51}$ Subsequent refinement was performed by full-matrix least-squares on F2 in ShelXL. ${ }^{54}$ Olex $2^{54}$ was used for viewing and to prepare CIF files. PLATON ${ }^{55}$ was used extensively for SQUEEZE. ${ }^{56}$ ORTEP graphics were prepared in CrystalMaker. ${ }^{57}$ Thermal ellipsoids are rendered at the $20 \%$ probability level.

We grew crystals of PBPB for crystallography from a solution of toluene vapor diffused with methanol. A natural crystal $(0.10 \times 0.07 \times 0.05 \mathrm{~mm})$ was mounted with STP oil treatment and cooled to $100 \mathrm{~K}$ on the diffractometer. The diffraction was extremely weak and extended to low resolution, with no detectable intensity beyond $1 \AA$ resolution. Complete data (99.3\%) were collected to $0.985 \AA$ A. 116722 reflections were collected (15869 unique, 8603 observed) with R(int) 10.0\% and R(sigma) 6.3\% after analytical absorption correction (Tmax .979, Tmin .965).

According to the systematic absences, the space group was Cc or C2/c. Using ShelXT, the structure was solved readily in $\mathrm{C} 2 / \mathrm{c}$ with two half-molecules in the asymmetric unit. Each molecule lies on a twofold axis with the PDI fragments normal to the axis. (Most non-H atoms appeared in the initial solution, but all four independent $\mathrm{C}_{11}$ side chains were disordered over two or three positions. These disorders were modeled with the aid of absolute (DFIX) restraints on all 1,2 and 1,3 distances, SAME similarity restraints for the two (three) components of each disordered chain, and SIMU restraints on the ADPs of all disordered atoms. In view of the poor data-to-parameters ratio, a global RIGU restraint was applied. $\mathrm{C}-\mathrm{H}$ hydrogens were placed in calculated positions and refined with riding coordinates and ADPs. 
The structure contained large voids with no Fourier peak larger than $1.1 \mathrm{e}-\AA^{-3}$. Since there were no recognizable solvent molecules in the Fourier maps, the voids were treated with PLATON SQUEEZE. The unit cell contains $8853 \AA 3$ of solvent-accessible volume with 2555 e- (equivalent to 51 toluene molecules) in the void space, giving 1 toluene per $174 \AA 3$ of void space. Since crystalline toluene packs with 1 molecule per $145 \AA 3$, the results of the SQUEEZE analysis are reasonable. When the solvent in void space had been included as a diffuse contribution to the scattering, R1 improved from $15.0 \%$ to $9.6 \%$. The final refinement (15869 data, 3138 restraints, 1789 parameters) converged with $\mathrm{R} 1(\mathrm{Fo}>4 \sigma(\mathrm{Fo}))=9.7 \%$, $\mathrm{wR} 2=33.4 \%, \mathrm{~S}=1.08$. The largest Fourier features were 0.54 and -0.31 e- A-3.

\subsection{Synthetic Procedures and Characterization}

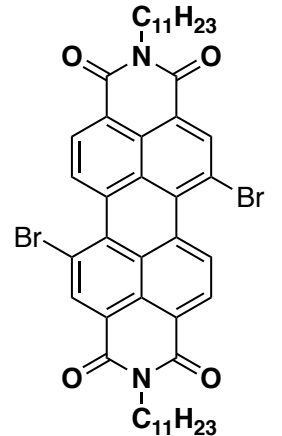

1,7-dibromo PDI

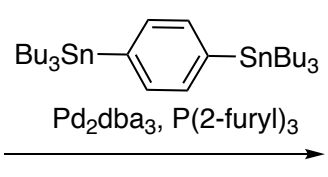

THF, $50^{\circ} \mathrm{C}, 14 \mathrm{~h}$

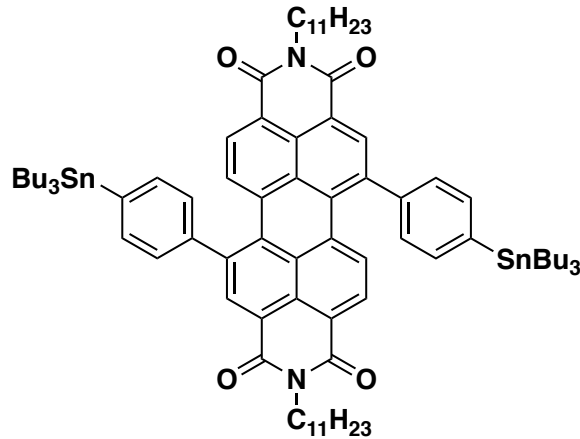

2.1

Synthesis of 1,7-Bis[4-(tributylstannyl)-phenyl]-PDI (2.1): Regio-pure 1,7-Bis[4(tributylstannyl)-phenyl]-PDI was synthesized from regio-pure $N, N$-'di(6-undecyl)-1,7dibromoperylene-3,4:9,10-tetracarboxylic diimide. The 1,6- and 1,7-regioisomers of $N, N^{\prime}$-di(6undecyl)-dibromoperylene-3,4:9,10-tetracarboxylic diimide were separated using prep HPLC on a COSMOSIL Buckyprep $20 \times 250 \mathrm{~mm}, 18.9 \mathrm{~mL} / \mathrm{min}$ and $12: 88 \mathrm{CH}_{2} \mathrm{Cl}_{2}$ :hexanes prior to the Stille coupling. 1,7-dibromo PDI (1.00 equiv, $0.350 \mathrm{mmol}, 0.300 \mathrm{~g}$ ), 1,4-bis(tributylstannyl)benzene (4.00 equiv, $1.40 \mathrm{mmol}, 0.800 \mathrm{~mL}$ ), tri(2-furyl)phosphine (0.400 equiv, $0.140 \mathrm{mmol}, 0.0325 \mathrm{~g}$ ), and THF $(18.0 \mathrm{~mL})$ were added to an oven-dried round bottom flask equipped with a stir bar. The 
mixture was degassed for thirty minutes. While under $\mathrm{N}_{2}$, tris(dibenzylideneacetone)dipalladium, $\mathrm{Pd}_{2} \mathrm{dba}_{3},(0.100$ equiv, $0.0350 \mathrm{mmol}, 0.0321 \mathrm{~g})$ was added. The mixture was further degassed for ten minutes before being placed in a $55^{\circ} \mathrm{C}$ oil bath and allowed to stir for $14 \mathrm{~h}$. The crude mixture was concentrated and purified by column chromatography (Redisep Rf Silica) using a gradient from $0 \%$ to $60 \% \mathrm{CH}_{2} \mathrm{Cl}_{2} /$ hexanes to yield the Compound 2.1 as a magenta solid $(0.276 \mathrm{~g}, 0.193$ mmol, $55.1 \%$ yield). ${ }^{1} \mathbf{H}$ NMR (500 MHz, $\left.\mathrm{CDCl}_{3}\right) \delta 8.64(\mathrm{br}, 2 \mathrm{H}), 8.12(\mathrm{br}, 2 \mathrm{H}), 7.91$ (br s, $\left.2 \mathrm{H}\right)$, 7.61* (d, J = 7.3 Hz, 4H), $7.54(\mathrm{~d}, \mathrm{~J}=7.4 \mathrm{~Hz}, 4 \mathrm{H}), 5.18$ (br m, 2H), 2.25 (br m, 4H), 1.84 (br m, 4H), 1.64* (m, 12H), $1.42(\mathrm{~m}, 12 \mathrm{H}), 1.29$ (br m, 24H), 1.17* (m, 12H), $0.96(\mathrm{~m}, 18 \mathrm{H}), 0.85$ (br m, 12H). ${ }^{13} \mathrm{C}$ NMR (125 MHz, $\left.\mathrm{CDCl}_{3}\right) \delta 164.76$ (br), 163.67 (br), 143.09, 141.73, 141.25, 138.07*, $135.99^{* *}, 135.29^{* *}, 134.96,132.46,130.15,129.86^{* *}, 129.28,129.10^{* *}, 128.31 *, 127.89$, $122.80^{* *}, 122.49^{* *}, 122.08^{* *}, 121.73^{* *}, 54.60,32.34,31.78,29.16^{*}, 27.38^{*}, 26.60,22.57$, 14.06, 13.75, 9.76*. IR $\left(\mathrm{cm}^{-1}\right) 2956,2925,2856,1697,1659,1598,1587,1465,1409,1326$, 1241, 1183, 1070, 863, 813. HRMS (APCI+) calculated $\mathrm{m} / \mathrm{z}$ for $\left[\mathrm{C}_{82} \mathrm{H}_{114} \mathrm{~N}_{2} \mathrm{O}_{4} \mathrm{Sn}_{2}+\mathrm{Na}\right]^{+}$is 1453.6720, found $1453.6720 .{ }^{*}$ Tin satellite peaks visible.

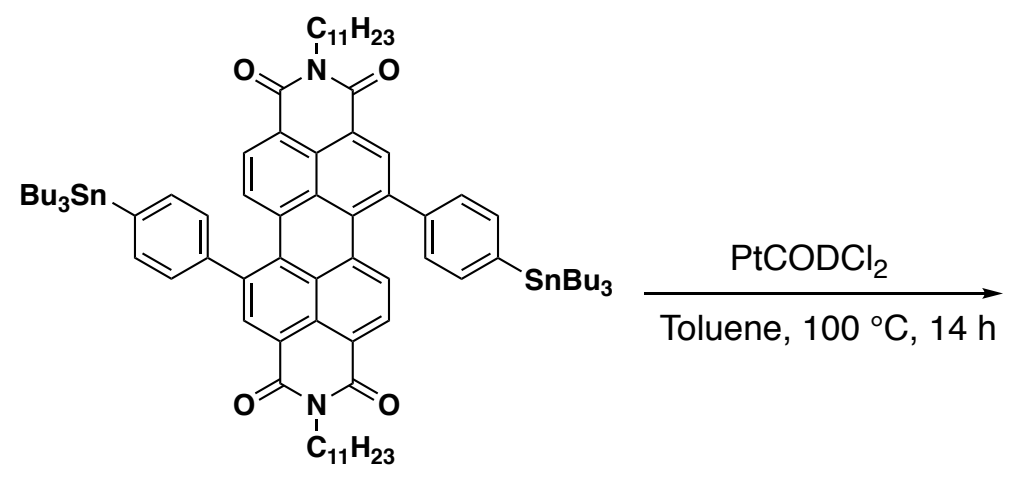

2.1

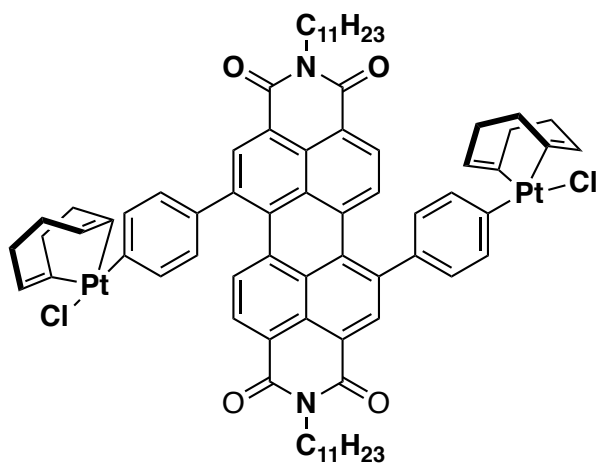

2.2

Synthesis of 1,7-Bis[4-(Pt(COD)Cl)-phenyl]-PDI (2.2). 1,7-Bis[4-(tributylstannyl)-phenyl]-PDI (2.1) (1.00 equiv, $0.193 \mathrm{mmol}, 0.276 \mathrm{~g}$ ) was added to an oven-dried round bottom flask equipped with a stir bar. Dichloro(1,5-cyclooctadiene)platinum(II) (2.20 equiv, $0.424 \mathrm{mmol}, 0.158 \mathrm{~g}$ ) was 
added to the flask with anhydrous toluene $(20 \mathrm{~mL})$. The mixture was degassed for thirty minutes and then placed in a $100{ }^{\circ} \mathrm{C}$ oil bath and allowed to stir for overnight. The crude mixture was allowed to cool to room temperature and concentrated in vacuo. The crude product was purified by column chromatography using a gradient from $0 \%$ to $100 \% \mathrm{CH}_{2} \mathrm{Cl}_{2}$ / hexanes to elute the desired product. The product is re-precipitated from methanol three times to remove excess $\mathrm{PtCODCl}_{2}$. The product is a purple solid (0.192 g, $0.121 \mathrm{mmol}, 62.5 \%$ yield). ${ }^{1} \mathbf{H}$ NMR $\left(500 \mathrm{MHz}, \mathrm{CDCl}_{3}\right) \delta$ $8.58(\mathrm{br}, 2 \mathrm{H}), 8.07$ (br d, J = 8.2 Hz, 2H), $7.88(\mathrm{br}, 2 \mathrm{H}), 7.38(\mathrm{~d}, \mathrm{~J}=7.8 \mathrm{~Hz}, 4 \mathrm{H}), 7.29(\mathrm{~d}, \mathrm{~J}=7.7$ Hz, 4H), 5.87 (s, 4H), 5.14 (br m, 2H), 4.72 (s, 4H), 2.75 (br m, 4H), 2.59 (br m, 4H), 2.45 (br m, 8H), 2.19 (br m, 4H), 1.80 (br m, 4H), 1.26 (br m, 24H), 0.82 (br m, 12H). ${ }^{13} \mathbf{C}$ NMR (125 MHz, $\left.\mathrm{CDCl}_{3}\right) \delta 164.89^{* *}, 164.77^{* *}, 163.81^{* *}, 163.66^{* *}, 145.66,141.21,138.16,136.15^{* *}, 135.64$ $135.40^{* *}, 135.16,132.39,129.84,129.70^{* *}, 129.34,128.93^{* *}, 128.68,127.76,122.65^{* *}$, $122.25^{* *}, 121.89^{* *}, 121.48^{* *}, 115.92,87.94,54.55^{* *}, 54.45^{* *}, 32.37,32.30,31.80,28.04$, 26.62, 22.60, 14.13. IR $\left(\mathrm{cm}^{-1}\right)$ 2953, 2924, 2854, 1694, 1652, 1598, 1584, 1408, 1323, 1236, 1181 , 1015, 812. HRMS $(E S I+)$ calculated $\mathrm{m} / \mathrm{z}$ for $\left[\mathrm{C}_{74} \mathrm{H}_{84} \mathrm{Cl}_{2} \mathrm{~N}_{2} \mathrm{O}_{4} \mathrm{Pt}_{2}+\mathrm{Na}\right]^{+}$is 1547.5001 , found 1547.4994.

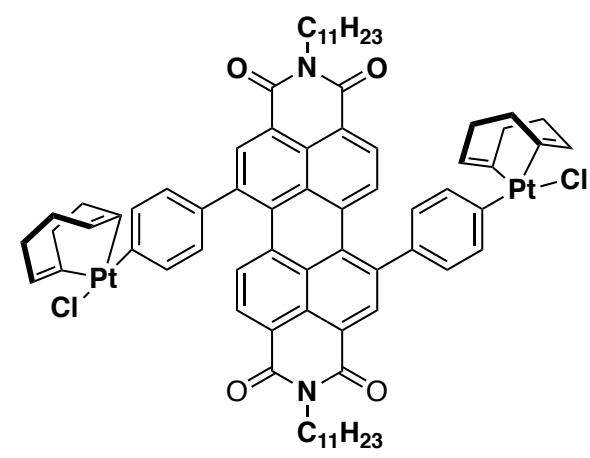

2.2

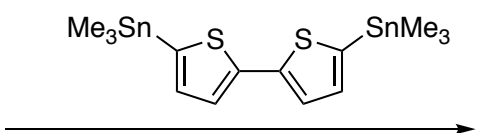

1. THF, $55^{\circ} \mathrm{C}, 48 \mathrm{~h}$

2. $\mathrm{PPh}_{3}$, Toluene, $100^{\circ} \mathrm{C}, 14 \mathrm{~h}$

Synthesis of PBPB. 1,7-Bis[4-(Pt(COD)Cl)-phenyl]-PDI (2.2) (1.00 equiv, $0.331 \mathrm{mmol}, 0.505$ g), commercially available 5,5'-bis(trimethylstannyl)-2,2'-bithiophene (1.00 equivalent, 0.331 
mmol, 0.163 grams) and THF (110. mL) were added to a $250 \mathrm{ml}$ two-neck round bottom flask. The mixture was degassed for thirty minutes and, then, stirred in a $55^{\circ} \mathrm{C}$ oil bath for $48 \mathrm{~h}$. The crude mixture was concentrated, and triphenylphosphine (20.0 equiv, $6.62 \mathrm{mmol}, 1.93 \mathrm{~g})$ and toluene $(110 . \mathrm{mL})$ were added. The mixture was degassed for fifteen minutes, then stirred for overnight in a $100{ }^{\circ} \mathrm{C}$ oil bath. The crude mixture was first purified by column chromatography using a gradient from $0 \%$ to $85 \% \mathrm{CH}_{2} \mathrm{Cl}_{2} /$ hexanes. Fractions that contained PBPB were collected and further purified on by preparative TLC using 70\%:30\% $\mathrm{CH}_{2} \mathrm{Cl}_{2} /$ hexanes. The product is a black solid (0.163 g, $0.0805 \mathrm{mmol}, 48.6 \%$ yield). ${ }^{1} \mathbf{H}$ NMR $\left(500 \mathrm{MHz}, 370 \mathrm{~K}, \mathrm{C}_{2} \mathrm{D}_{2} \mathrm{Cl}_{4}\right) \delta[$ major stereoisomer] 8.59 (br s, 4H), 8.40 (br d, $J=7.9 \mathrm{~Hz}, 4 \mathrm{H}), 8.30$ (br d, $J=7.9 \mathrm{~Hz}, 4 \mathrm{H}), 7.70$ (d, $J=$ $7.8 \mathrm{~Hz}, 8 \mathrm{H}), 7.62(\mathrm{~d}, J=8.0 \mathrm{~Hz}, 8 \mathrm{H}), 7.34(\mathrm{~d}, J=3.5 \mathrm{~Hz}, 4 \mathrm{H}), 7.22(\mathrm{~d}, J=3.7 \mathrm{~Hz}, 4 \mathrm{H}), 5.14$ (br m, 4H), 2.19 (br m, 8H), 1.88 (br m, 8H), 1.30 (br m, 48H), 0.85 (br m, 24H); [distinguishable minor stereoisomer peaks] 8.55, 8.45 (br d), 7.54, 7.22 (d, J=3.7 Hz). ${ }^{13} \mathbf{C}$ NMR (125 MHz, 300 $\left.\mathrm{K}, \mathrm{C}_{2} \mathrm{D}_{2} \mathrm{Cl}_{4}\right)$ [mixture of interconverting meso and racemic stereoisomers] $\delta 164.22,163.93$, $163.10,162.82,141.89,140.32,140.05,137.30,134.92,134.24,134.14,133.18,132.15,130.63$, $129.78,129.75,129.30,128.87,128.45,126.91,126.84,125.89,123.26,122.76,122.25,121.82$, $121.49,121.05,119.77,54.09,33.68,31.69,31.40,31.17,29.60,29.19,29.15,26.06,22.21,22.02$, 20.41, 13.74, 13.61, 13.58. IR $\left(\mathrm{cm}^{-1}\right) 2952,2924,2855,1695,1657,1583,1456,1405,1323$, 1239, 1181, 1126, 1094, 853, 836, 812, 794, 762. HRMS (ESI+) calculated $\mathrm{m} / \mathrm{z}$ for $\left[\mathrm{C}_{132} \mathrm{H}_{128} \mathrm{~N}_{4} \mathrm{O}_{8} \mathrm{~S}_{4}+\mathrm{Na}\right]^{+}$is 2047.8513 , found 2047.8458. The list of ${ }^{13} \mathrm{C}$ NMR peaks above includes all distinguishable peaks for both diastereomers of PBPB. 


\subsection{NMR Spectra of Compounds}
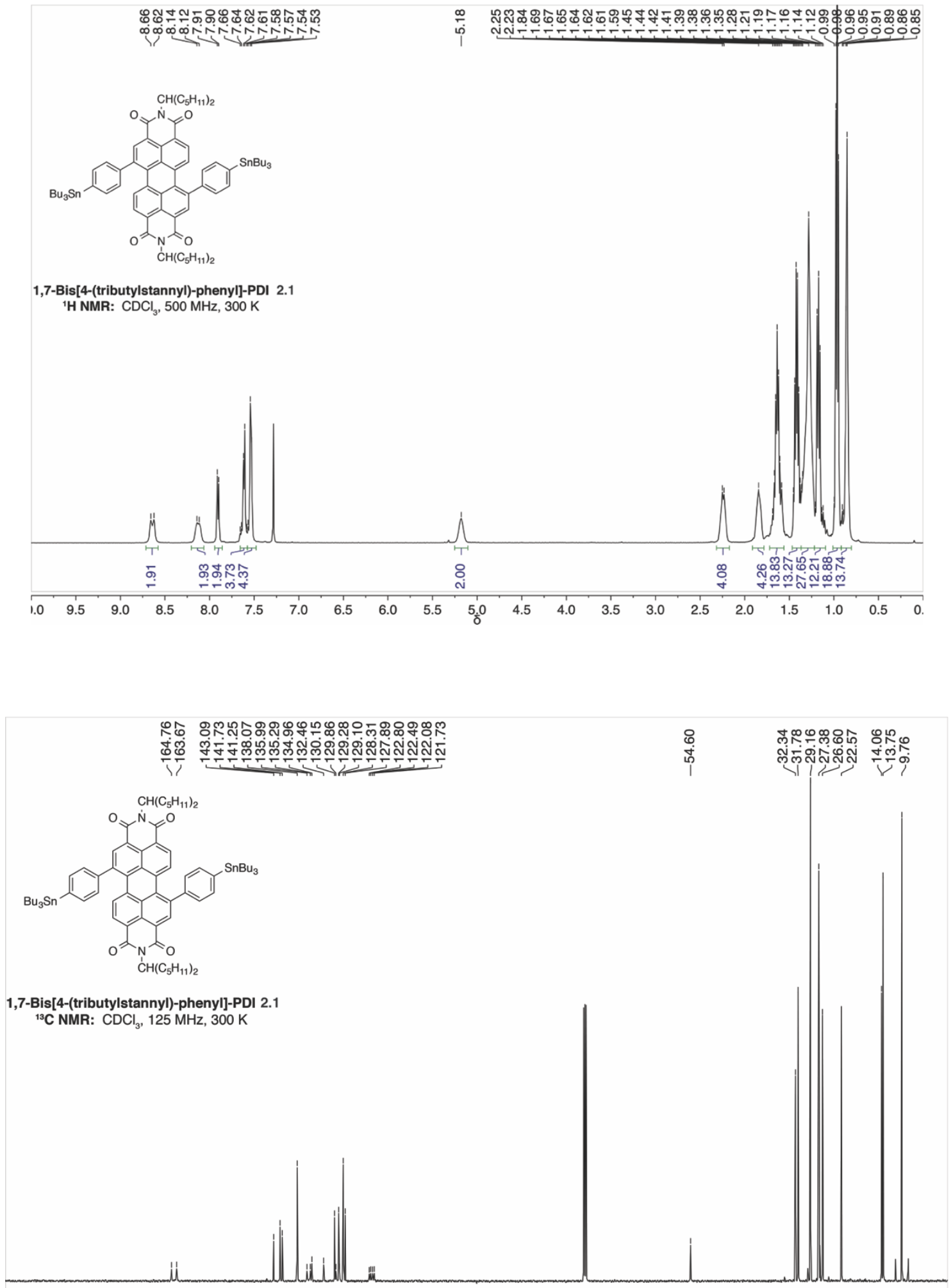

\begin{tabular}{|llllllllllllllllllllll}
\hline 0 & 190 & 180 & 170 & 160 & 150 & 140 & 130 & 120 & 110 & 100 & 90 & 80 & 70 & 60 & 50 & 40 & 30 & 20 & 10 & (
\end{tabular} 

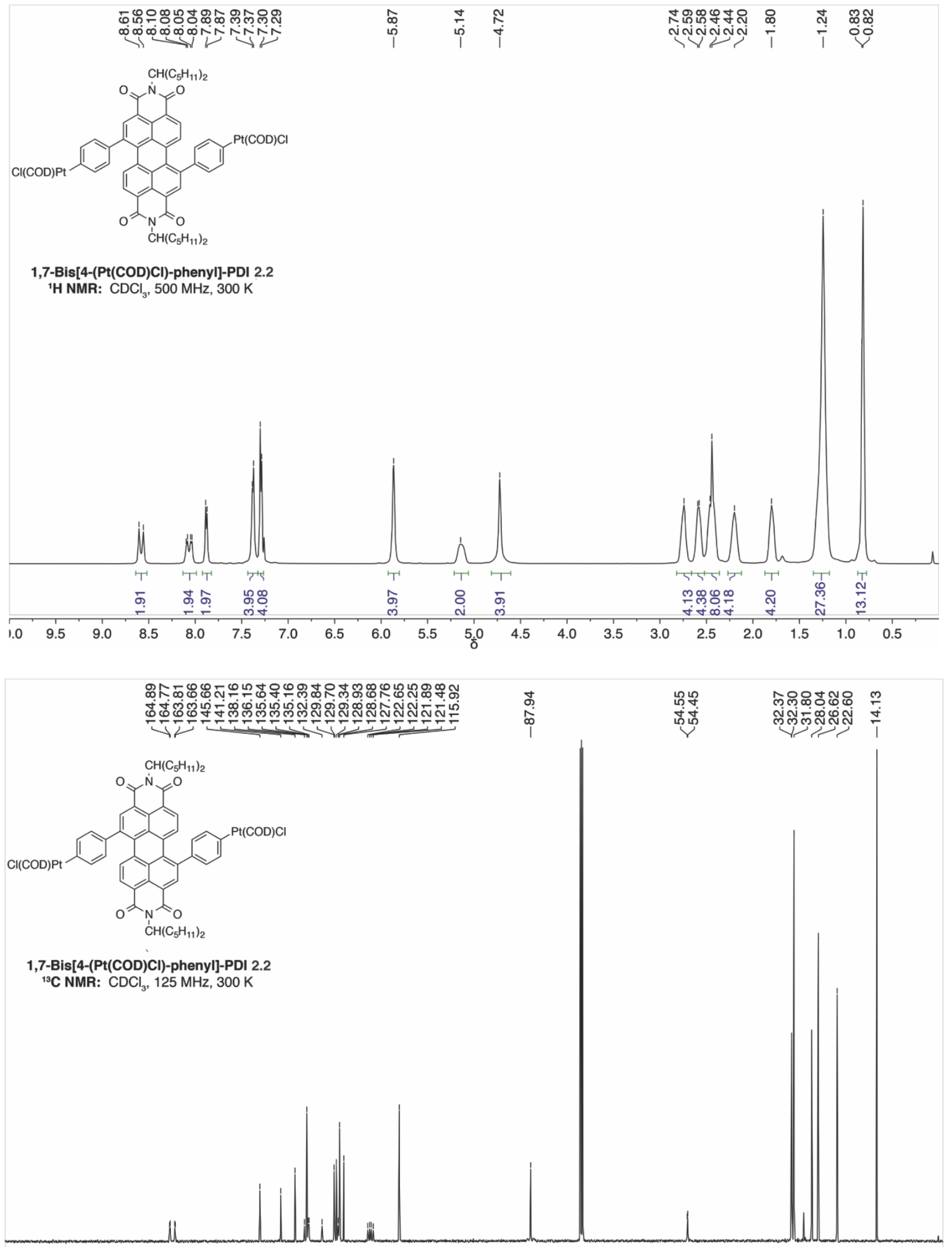

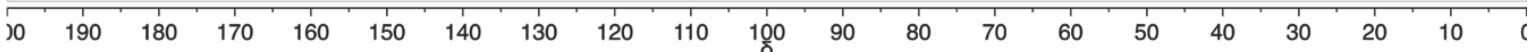




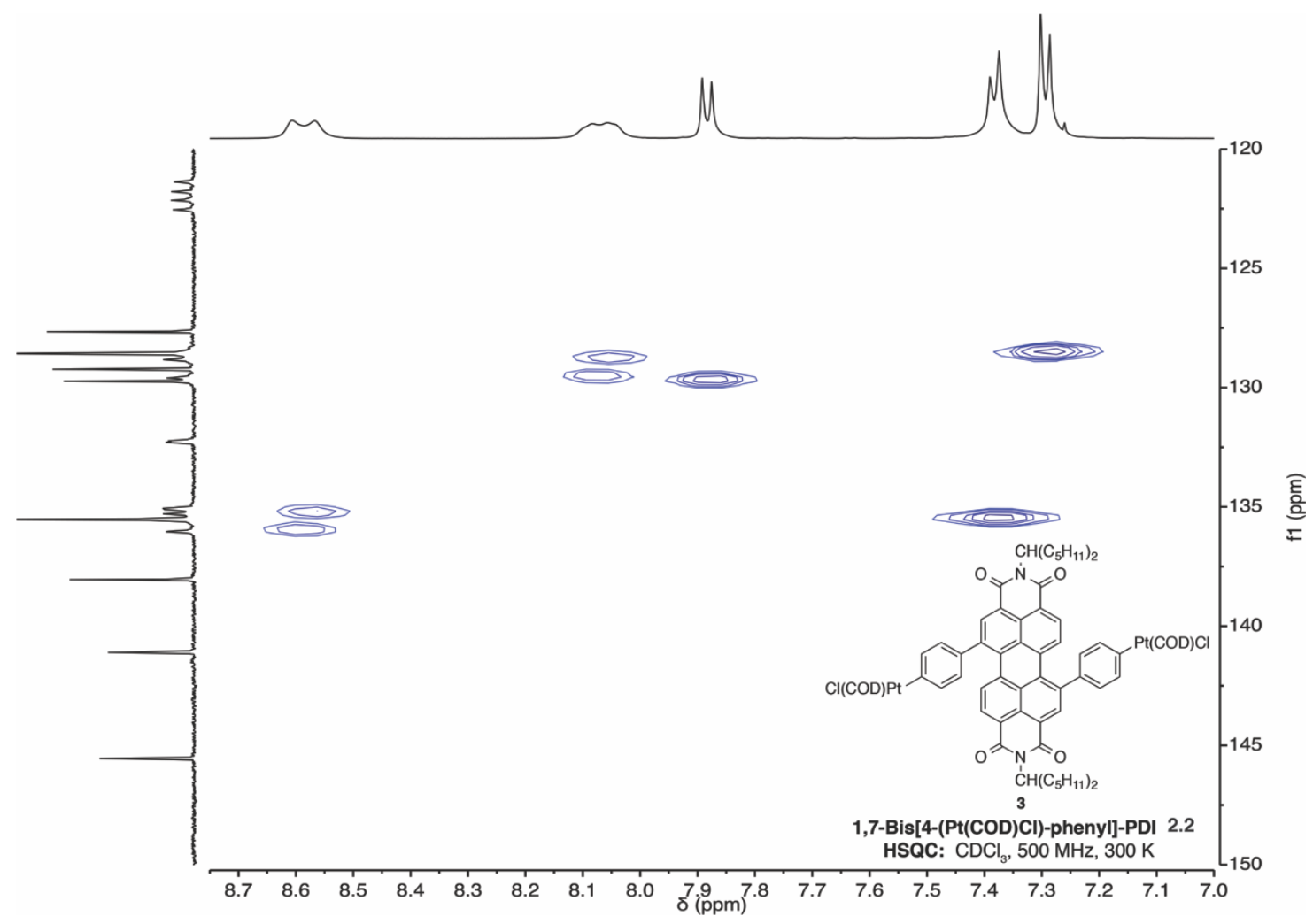




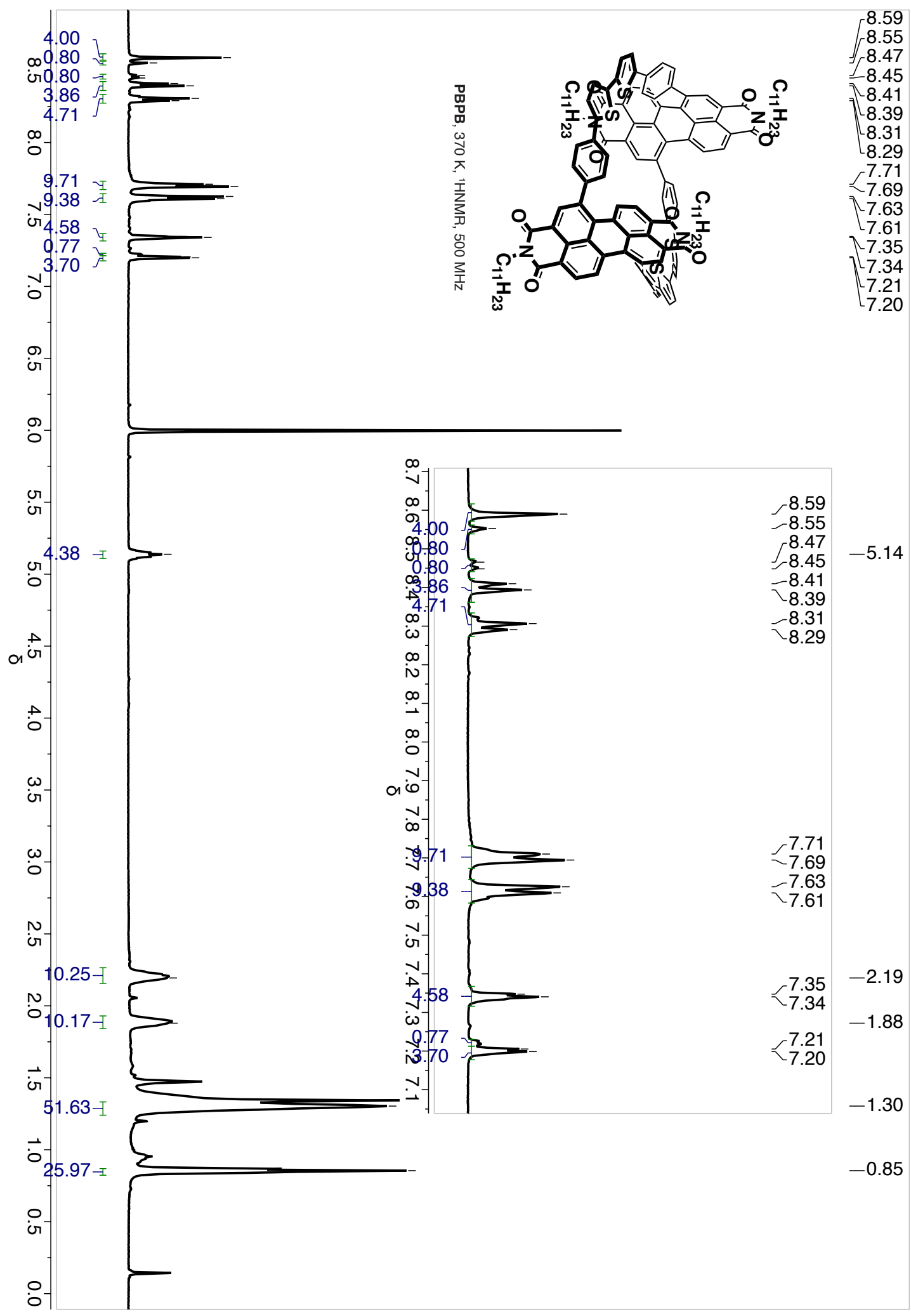




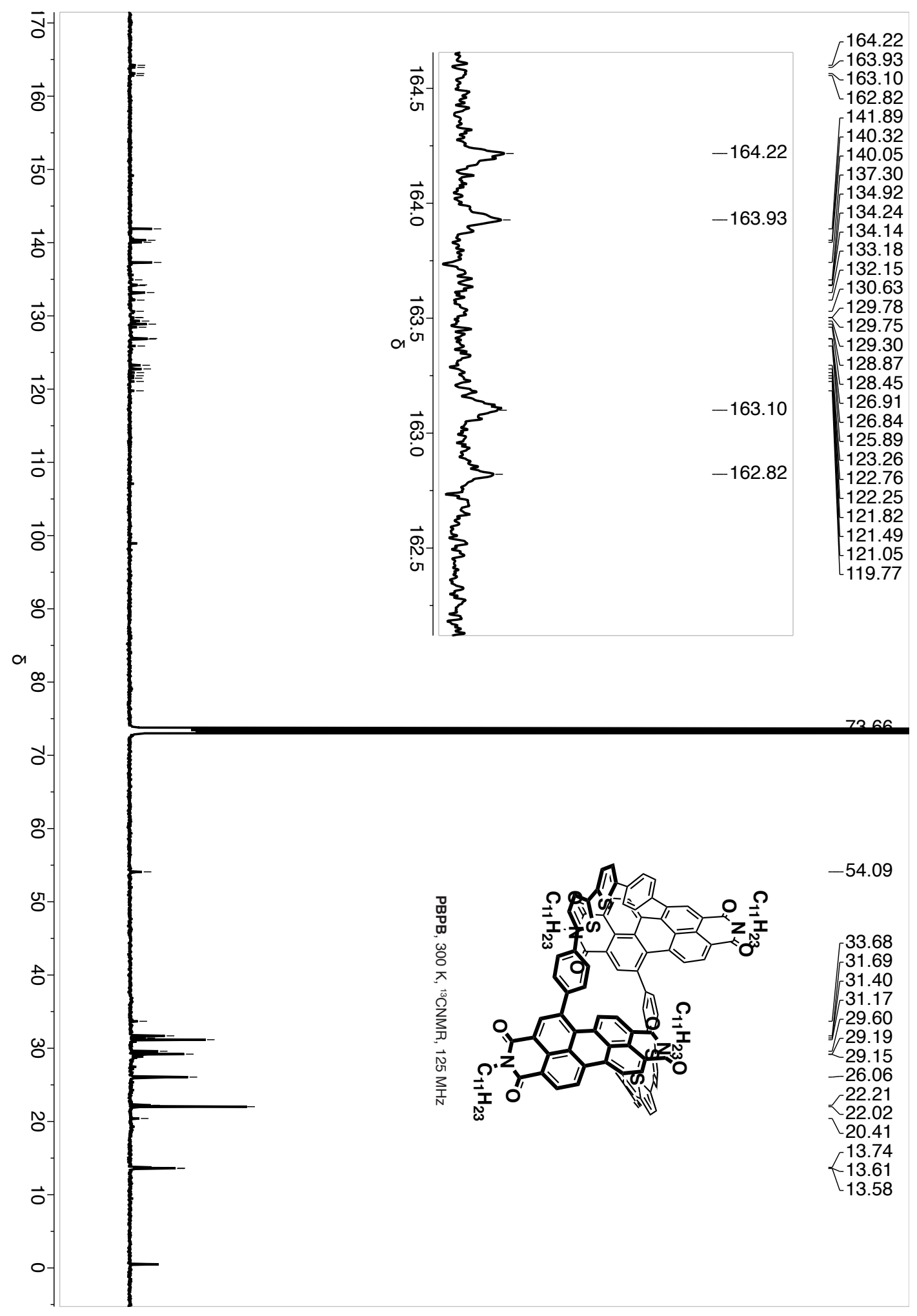




\subsection{DFT Output}

All quantum chemical calculations were performed using Jaguar, version 8.3, Schrodinger, Inc., New York, NY, 2013. (See A. D. Bochevarov, E. Harder, T. F. Hughes, J. R. Greenwood, D. A. Braden, D. M. Philipp, D. Rinaldo, M. D. Halls, J. Zhang, R. A. Friesner, "Jaguar: A High Performance Quantum Chemistry Software Program with Strengths in Life and Materials Sciences", Int. J. Quantum Chem., 2013, 113(18), 2110-2142). All geometries were optimized using the B3LYP functional and the $6-31 \mathrm{G}^{* *}$ basis set. Geometry optimization output information can be found in the online Supporting Information of Ref. $1 .{ }^{1}$ For PBPB, we present the fifteen lowest energy roots determined by the TDDFT calculations. The HOMO is the doubly occupied orbital numbered 378.

\section{TDDFT Output}

Restricted Singlet Excited State 1: $\quad 1.5318 \mathrm{eV} \quad 809.41 \mathrm{~nm}$

excitation X coeff.

$378=>379 \quad-0.98886$

Transition dipole moment (debye):

$\mathrm{X}=-0.0890 \mathrm{Y}=-0.0862 \quad \mathrm{Z}=-3.2537$ Tot $=3.2560$

Oscillator strength, $\mathrm{f}=\quad 0.0616$

Restricted Singlet Excited State 2: $1.6320 \mathrm{eV} \quad 759.68 \mathrm{~nm}$ excitation X coeff.

$378=>380 \quad 0.99758$

Transition dipole moment (debye):

$\mathrm{X}=4.2970 \quad \mathrm{Y}=-0.1207 \quad \mathrm{Z}=-0.1417 \quad \mathrm{Tot}=4.3011$

Oscillator strength, $\mathrm{f}=0.1145$

\begin{tabular}{|c|c|c|}
\hline $\begin{array}{l}\text { Restricted Singlet Excited State } 3 \text { : } \\
\text { excitation X coeff. }\end{array}$ & $1.7453 \mathrm{eV}$ & $710.40 \mathrm{~nm}$ \\
\hline $377=>379 \quad 0.99575$ & & \\
\hline Transition dipole moment (debye & & \\
\hline $\begin{array}{ccc}\mathrm{X}=0.3000 \quad \mathrm{Y}= & 6.6914 & \mathrm{Z}= \\
\text { Oscillator strength, } \mathrm{f}= & 0.2972\end{array}$ & 0.2086 Tot $=$ & 6.7013 \\
\hline
\end{tabular}




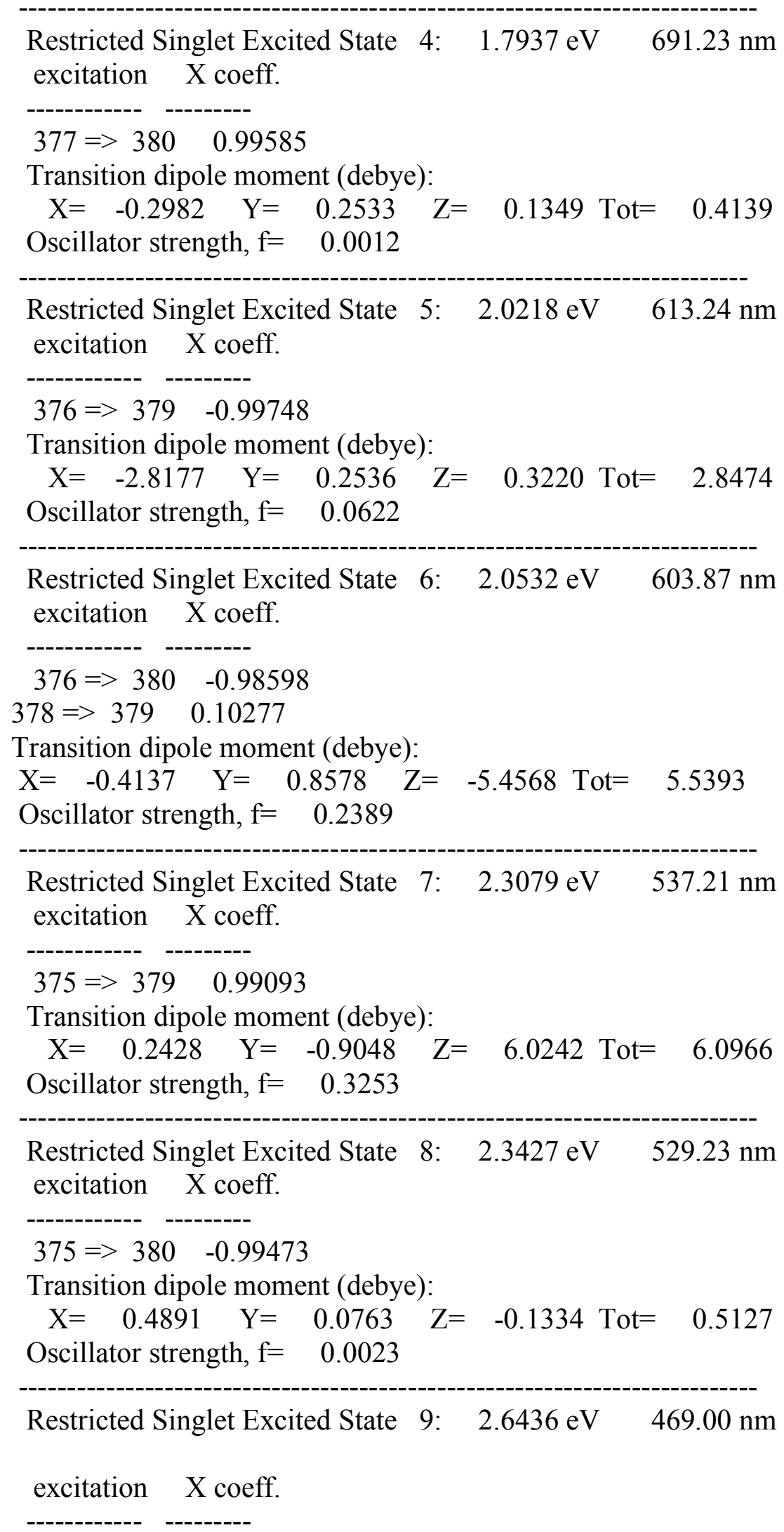


$372=>380 \quad-0.18272$

$374=>379 \quad 0.96735$

Transition dipole moment (debye):

$\mathrm{X}=-0.2332 \mathrm{Y}=-0.1825 \mathrm{Z}=-0.0543$ Tot $=0.3011$

Oscillator strength, $\mathrm{f}=0.0009$

Restricted Singlet Excited State 10: $2.6744 \mathrm{eV} \quad 463.60 \mathrm{~nm}$ excitation X coeff.

$372=>379 \quad 0.19523$

$374=>380 \quad-0.96112$

Transition dipole moment (debye):

$\mathrm{X}=-0.1319 \mathrm{Y}=-3.5869 \mathrm{Z}=-0.3162 \mathrm{Tot}=3.6032$

Oscillator strength, $\mathrm{f}=0.1317$

Restricted Singlet Excited State 11: $2.7637 \mathrm{eV} \quad 448.62 \mathrm{~nm}$ excitation X coeff.

\begin{tabular}{|c|c|}
\hline $377=>381$ & 0.29688 \\
\hline $378 \Rightarrow>381$ & 0.79213 \\
\hline $378=>38$ & 0.4 \\
\hline
\end{tabular}

Transition dipole moment (debye):

$\mathrm{X}=0.0625 \mathrm{Y}=7.5012 \quad \mathrm{Z}=-3.1504 \mathrm{Tot}=8.1362$ Oscillator strength, $\mathrm{f}=0.6938$

Restricted Singlet Excited State 12: $\quad 2.8174 \mathrm{eV} \quad 440.07 \mathrm{~nm}$ excitation X coeff.

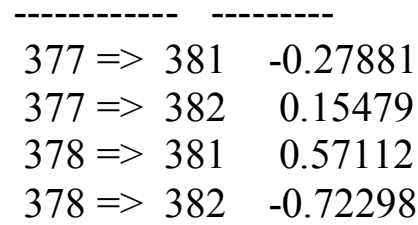

Transition dipole moment (debye):

$\mathrm{X}=0.4220 \mathrm{Y}=8.0581 \quad \mathrm{Z}=2.7629$ Tot $=8.5290$

Oscillator strength, $\mathrm{f}=0.7772$

Restricted Singlet Excited State 13: $\quad 2.9360 \mathrm{eV} \quad 422.29 \mathrm{~nm}$ excitation X coeff.

$\begin{array}{rlr}369 & =>380 & 0.14851 \\ 371 & =>380 & 0.10405 \\ 373 & =>379 & 0.93743 \\ 376 & =>382 & -0.15916 \\ 378 & =>387 & 0.10576\end{array}$

Transition dipole moment (debye): 


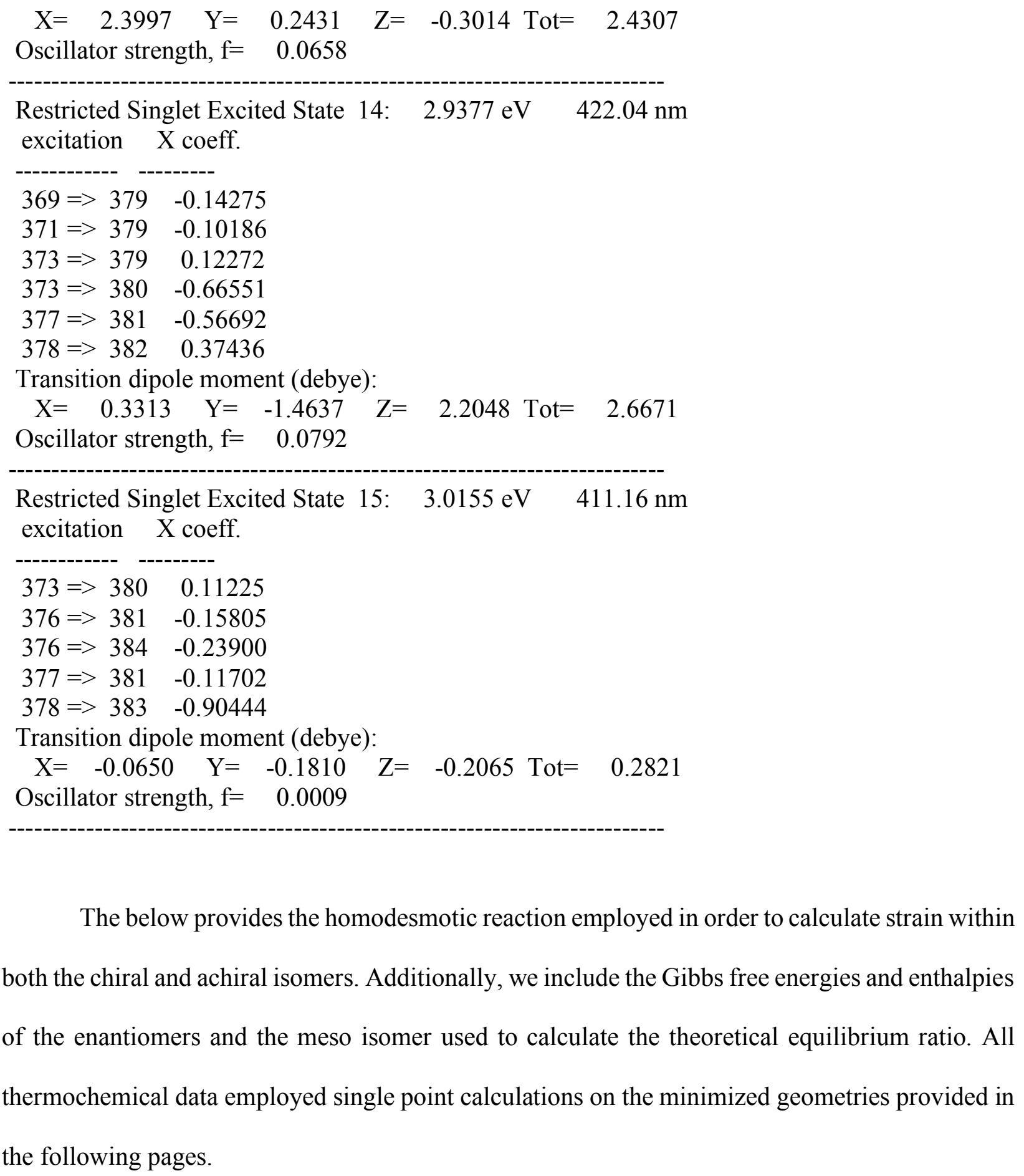




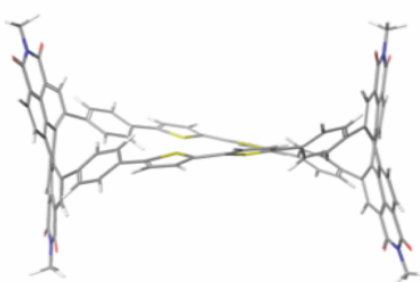

$(P, P)$-PBPB \& $(M, M)$-PBPB

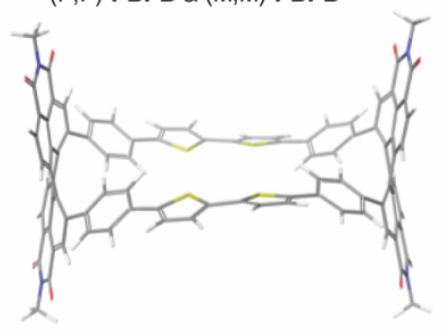

(P,M)-PBPB

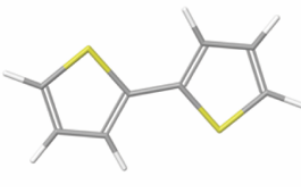

Bithiophene

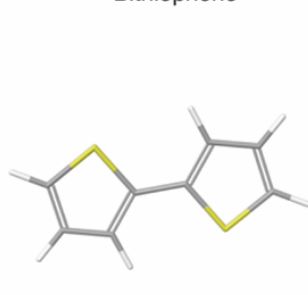

Bithiophene

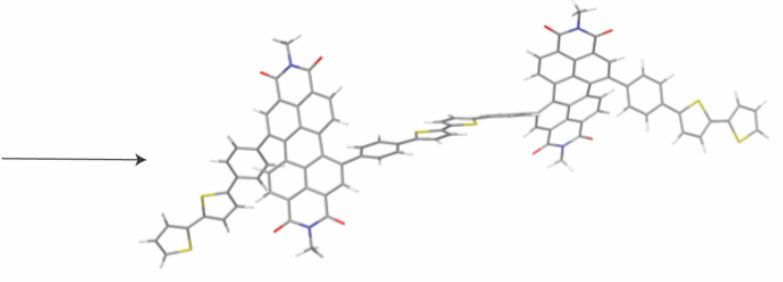

Acyclic

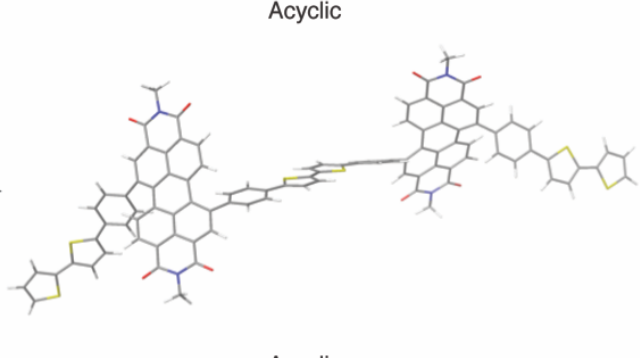

Acyclic

Table 2.1. Homodesmotic Calculations for Strain

\begin{tabular}{|l|c|c|c|}
\hline Compound & $\begin{array}{c}\text { Total energy } \\
\text { (hartrees) }\end{array}$ & $\begin{array}{c}\text { Strain energy } \\
\text { (hartrees) }\end{array}$ & $\begin{array}{c}\text { Strain energy } \\
(\mathrm{kcal} / \mathrm{mol})\end{array}$ \\
\hline $\begin{array}{l}(P, P)-\text { PBPB/}(M, M)- \\
\text { PBPB }\end{array}$ & -5948.582452 & 0.032223 & 20 \\
\hline$(P, M)-$ PBPB & -5948.579539 & 0.035138 & 22 \\
\hline Bithiophene & -1104.826802 & - & - \\
\hline Acyclic & -7053.441477 & - & - \\
\hline
\end{tabular}

Table of energies for the Homodesmotic Reaction (enthalpy)

Table 2.2. Thermochemical Calculations for Strain

\begin{tabular}{|c|c|c|}
\hline Compound & $\begin{array}{c}\text { Gibbs free energy } \\
\text { (hartrees })\end{array}$ & $\begin{array}{c}\text { Enthalpy } \\
\text { (hartrees) }\end{array}$ \\
\hline$(P, P)-\mathbf{P B P B} /(M, M)-\mathbf{P B P B}$ & -5947.593171 & -5947.309814 \\
\hline$(P, M)-\mathbf{P B P B}$ & -5947.587400 & -5947.306842 \\
\hline Difference $(\boldsymbol{M}, \boldsymbol{M}) /(\boldsymbol{P}, \boldsymbol{P})-(\boldsymbol{M}, \boldsymbol{P})$ & -0.005771 & -0.002972 \\
\hline $\mathrm{K}_{[\mathrm{P} / \mathrm{M} \text { enantiomers }]}=\mathrm{e}^{-\Delta \mathrm{G} / \mathrm{RT}}$ & $158: 1$ & $14: 1$ \\
\hline$(\mathrm{P}+\mathrm{M}): \mathrm{M}$ & $316: 1$ & $28: 1$ \\
\hline
\end{tabular}

Temperature $=360 \mathrm{~K}, \mathrm{R}=8.3145 \mathrm{~J} \mathrm{~mol}^{-1} \mathrm{~K}^{-1}$ 


\subsection{References}

1. Ball, M. et al. Chiral Conjugated Corrals. J. Am. Chem. Soc. 137, 9982-9987 (2015).

2. Krömer, J. et al. Synthesis of the First Fully $\alpha$-Conjugated Macrocyclic Oligothiophenes: Cyclo[n]thiophenes with Tunable Cavities in the Nanometer Regime. Angew. Chem. Int. Ed. 39, 3481-3486 (2000).

3. Nakao, K. et al. Giant Macrocycles Composed of Thiophene, Acetylene, and Ethylene Building Blocks. J. Am. Chem. Soc. 128, 16740-16747 (2006).

4. Hitosugi, S. et al. Bottom-up Synthesis and Thread-in-Bead Structures of Finite (n,0)Zigzag Single-Wall Carbon Nanotubes. J. Am. Chem. Soc. 134, 12442-12445 (2012).

5. Omachi, H. et al. Synthesis of Cycloparaphenylenes and Related Carbon Nanorings: A Step toward the Controlled Synthesis of Carbon Nanotubes. Acc. Chem. Res. 45, 1378-1389 (2012).

6. Ishii, Y. et al. Size-selective synthesis of 9 - 11 and 13 cycloparaphenylenes. Chem. Sci. 3, 2340-2345 (2012).

7. Evans, P. J. et al. Efficient room-temperature synthesis of a highly strained carbon nanohoop fragment of buckminsterfullerene. Nat. Chem. 6, 404-408 (2014).

8. Kayahara, E. et al. Synthesis and Characterization of 5 Cycloparaphenylene. J. Am. Chem. Soc. 136, 2284-2287 (2014).

9. Kuwabara, T. et al. Curved Oligophenylenes as Donors in Shape-Persistent DonorAcceptor Macrocycles with Solvatofluorochromic Properties. Angew. Chem. Int. Ed. 54, 9646-9649 (2015).

10. Ito, H. et al. Thiophene-Based, Radial pi-Conjugation: Synthesis, Structure, and Photophysical Properties of Cyclo-1,4-phenylene-2',5'-thienylenes. Angew. Chem. Int. Ed. 54, 159-163 (2015).

11. Darzi, E. R. et al. Synthesis, Properties, and Design Principles of Donor-Acceptor Nanohoops. ACS Cent. Sci. 1, 335-342 (2015).

12. Chen, Q. et al. Strain-Induced Stereoselective Formation of Blue-Emitting Cyclostilbenes. J. Am. Chem. Soc. 137, 12282-12288 (2015).

13. Chang, S.-W. et al. A Donor-Acceptor Conjugated Block Copolymer of Poly(arylenevinylene)s by Ring-opening Metathesis Polymerization. Chem. Commun. 51, 9113-9116 (2015).

14. Jasti, R. et al. Synthesis, Characterization, and Theory of 9 -, 12 -, and 18 Cycloparaphenylene: Carbon Nanohoop Structures. J. Am. Chem. Soc. 130, 17646-17647 (2008).

15. Asai, K. et al. A Cyclic Octithiophene Containing B,B'-linkages. Chem. Commun. 51, 6096 (2015).

16. Nishigaki, S. et al. Synthesis, Structures, and Photophysical Properties of Alternating Donor-Acceptor Cycloparaphenylenes. Chem. Eur. J. 23, 7227-7231 (2017).

17. Kayahara, E. et al. Gram-Scale Syntheses and Conductivities of [10]Cycloparaphenylene 
and Its Tetraalkoxy Derivatives. J. Am. Chem. Soc. 139, 18480-18483 (2017).

18. Zhang, F. et al. Giant Cyclo $\mathrm{n}$ thiophenes with Extended pi Conjugation. Angew. Chem. Int. Ed. 48, 6632-6635 (2009).

19. Segawa, Y. et al. [9]Cycloparaphenylene: Nickel-mediated Synthesis and Crystal Structure. Chem. Lett. 40, 423-425 (2011).

20. Iwamoto, T. et al. Size-Selective Encapsulation of C60 by [10]Cycloparaphenylene: Formation of the Shortest Fullerene-Peapod. Angew. Chem. Int. Ed. 50, 8342-8344 (2011).

21. Iwamoto, T. et al. Selective and Random Syntheses of n Cycloparaphenylenes ( $\mathrm{n}=8-13)$ and Size Dependence of Their Electronic Properties. J. Am. Chem. Soc. 133, 8354-8361 (2011).

22. Segawa, Y. et al. Concise Synthesis and Crystal Structure of 12 Cycloparaphenylene. Angew. Chem. Int. Ed. 50, 3244-3248 (2011).

23. Hitosugi, S. et al Atropisomerism in a Belt-Persistent Nanohoop Molecule: Rotational Restriction Forced by Macrocyclic Ring Strain. Chem. Asian J. 7, 1550-1552 (2012).

24. Kayahara, E. et al. Selective Synthesis and Crystal Structure of [10]Cycloparaphenylene. Org. Lett. 14, 3284-3287 (2012).

25. van der Boom, T. et al. Charge Transport in Photofunctional Nanoparticles Self-Assembled from Zinc 5,10,15,20-Tetrakis(perylenediimide)porphyrin Building Blocks. J. Am. Chem. Soc. 124, 9582-9590 (2002).

26. Campbell, W. M. et al. Highly efficient porphyrin sensitizers for dye-sensitized solar cells. J. Phys. Chem. C 111, 11760-11762 (2007).

27. Hoffmann, M. et al. Template-Directed Synthesis of a $\pi$-Conjugated Porphyrin Nanoring. Angew. Chem. Int. Ed. 46, 3122-3125 (2007).

28. Bessho, T. et al. Highly Efficient Mesoscopic Dye-Sensitized Solar Cells Based on DonorAcceptor-Substituted Porphyrins. Angew. Chem. Int. Ed. 49, 6646-6649 (2010).

29. Song, J. et al. A Porphyrin Nanobarrel That Encapsulates C60. J. Am. Chem. Soc. 132, 16356-16357 (2010).

30. Yella, A. et al. Porphyrin-Sensitized Solar Cells with Cobalt (II/III)-Based Redox Electrolyte Exceed 12 Percent Efficiency. Science 334, 629-634 (2011).

31. Saito, S. et al. Expanded Porphyrins: Intriguing Structures, Electronic Properties, and Reactivities. Angew. Chem. Int. Ed. 50, 4342-4373 (2011).

32. Fenwick, O. et al. Linear and Cyclic Porphyrin Hexamers as Near-Infrared Emitters in Organic Light-Emitting Diodes. Nano Lett. 11, 2451-2456 (2011).

33. Sprafke, J. K. et al. Belt-Shaped $\pi$-Systems: Relating Geometry to Electronic Structure in a Six-Porphyrin Nanoring. J. Am. Chem. Soc. 133, 17262-17273 (2011).

34. Jiang, H.-W. et al. Cyclic 2,12-Porphyrinylene Nanorings as a Porphyrin Analogue of Cycloparaphenylenes. J. Am. Chem. Soc. 137, 2219-2222 (2015).

35. Havinga, E. E. et al. A New Class of Small Band Gap Organic Polymer Conductors. Polym. Bull. 29, 119-126 (1992). 
36. Havinga, E. E. et al. Alternate Donor-Acceptor Small Band-gap Semiconducting Polymers; Polysquaraines and Polycroconaines. Synth. Met. 55, 299-306 (1993).

37. Roncali, J. Synthetic Principles for Bandgap Control in Linear $\pi$-Conjugated Systems. Chem. Rev. 97, 173-206 (1997).

38. Eaborn, C. et al. Preparation of Aryl-Platinum(II) Complexes - Iinteraction of dichloro-(etacyclco-octa-1,5-diene)platinum(II) and Aryltrimethylstannanes. J. Chem. Soc. Trans. 357368 (1978).

39. Shekhar, S. et al. Distinct electronic effects on reductive eliminations of symmetrical and unsymmetrical bis-aryl platinum complexes. J. Am. Chem. Soc. 126, 13016-13027 (2004).

40. Würthner, F. et al. Perylene Bisimide Dye Assemblies as Archetype Functional Supramolecular Materials. Chem. Rev. 116, 962-1052 (2016).

41. Rajasingh, P. et al. Selective Bromination of Perylene Diimides under Mild Conditions. $J$. Org. Chem. 72, 5973-5979 (2007).

42. Samdal, S. et al. Molecular Conformation of 2,2'-Bithiophene Determined by by Gas-Phase Electron Diffraction and ab-initio calculations. Synth. Met. 59, 259-265 (1993).

43. Quattrocchi, C. et al. Theoretical Investigation of the Conformaitonal Behavior of 2,2'Bithiophene. Chem. Phys. Lett. 208, 120-124 (1993).

44. Capozzi, B. et al. Length-Dependent Conductance of Oligothiophenes. J. Am. Chem. Soc. 136, 10486-10492 (2014).

45. Chao, C. C. et al. Photophysical and electrochemical properties of 1,7-diaryl-substituted perylene diimides. J. Org. Chem. 70, 4323-4331 (2005).

46. Goretzki, G. et al. Bis-morpholine-Substituted Perylene Bisimides: Impact of Isomeric Arrangement on Electrochemical and Spectroelectrochemical Properties. J. Org. Chem. 73, 8808-8814 (2008).

47. You, J. B. et al. A polymer tandem solar cell with $10.6 \%$ power conversion efficiency. Nat. Commun. 4, 10 (2013).

48. Blanc, E. et al. The evaluation of transmission factors and their first derivatives with respect to crystal shape parameters. J. Appl. Crystallogr. 24, 1035-1041 (1991).

49. Clark, R. C. et al. The analytical calculation of absorption in multifaceted crystals. Acta Crystallogr. Sect. A 51, 887-897 (1995).

50. Oxford Diffraction/Agilent Technologies UK Ltd. Version 1.171.37.35. (2014).

51. Sheldrick, G. M. Crystal structure refinement with \{lit SHELXL\}. Acta Crystallogr. Sect. C 71, 3-8 (2015).

52. Sheldrick, G. M. \{lit SHELXT $\} \quad\{--\}$ Integrated space-group and crystal-structure determination. Acta Crystallogr. Sect. A 71, 3-8 (2015).

53. Palatinus, L. et al. \{lit SUPERFLIP $\}\{--\}$ a computer program for the solution of crystal structures by charge flipping in arbitrary dimensions. J. Appl. Crystallogr. 40, 786-790 (2007).

54. Dolomanov, O. V. et al. \{\it OLEX2\}: A complete structure solution, refinement and 
analysis program. J. Appl. Crystallogr. 42, 339-341 (2009).

55. Spek, A. L. Structure validation in chemical crystallography. Acta Crystallogr. Sect. D 65, 148-155 (2009).

56. van der Sluis, P. et al. BYPASS: an effective method for the refinement of crystal structures containing disordered solvent regions. Acta Crystallogr. Sect. A 46, 194-201 (1990).

57. Crystalmaker Software Ltd.

58. Ahmadi, Z. et al. A mechanistic investigation of hydrodehalogenation using ESI-MS. Chem. Commun. 49, 11488-11490 (2013). 


\section{Chapter 3. Macrocyclization in the Design of Organic n-Type Electronic Materials}

\subsection{Preface}

Chapter 3 is reproduced with permission from the authors: Melissa Ball, Yu Zhong, Brandon Fowler, Boyuan Zhang, Panpan Li, Grisha Etkin, Daniel W. Paley, John Decatur, Ankur K. Dalsania, Hexing Li, Shengxiong Xiao, Fay Ng, Michael L. Steigerwald and Colin Nuckolls published in the Journal of the American Chemical Society. ${ }^{1}$ Copyright 2016 American Chemical Society. Brandon Fowler and I carried out the synthesis and characterization of all compounds. Yu

Zhong, Boyuan Zhang and Ankur K. Dalsania performed all device fabrication and characterization. Grisha Etkin performed GPC on polymer materials.

\subsection{Introduction}

We compare cyclic and acyclic $\pi$-conjugated molecules as n-type electronic materials and find that the cyclic molecules have numerous benefits in OPVS. Conjugated macrocycles ${ }^{2-28}$ have several potential advantages as organic electronic materials: (1) their contorted structure ${ }^{29}$ should facilitate intermolecular contacts and charge transport; (2) they lack endgroups that are known to create defects in linear polymers and act as trap-sites for charges as they move through materials; ${ }^{30-}$ 34 (3) often they have an altered electronic structure, ${ }^{2,25}$ and (4) they have a defined cavity that can be a host for electronically useful guest molecules. ${ }^{16,24,35-37}$ Figure 3.1 and 3.2 display the cyclic and acyclic molecules designed and synthesized for this study. The two conjugated cycles here are $\left(\mathbf{P P h}_{2}\right)_{4}$ and PBPB. Section 3.12 contains the synthetic details for all compounds. 


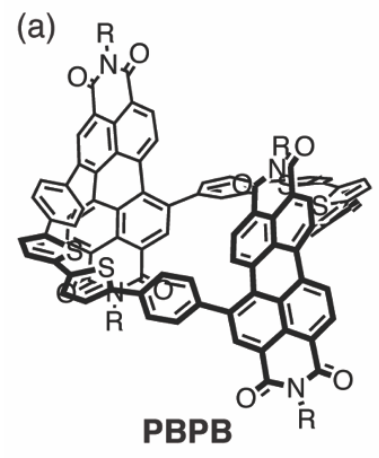

(b)

(d)

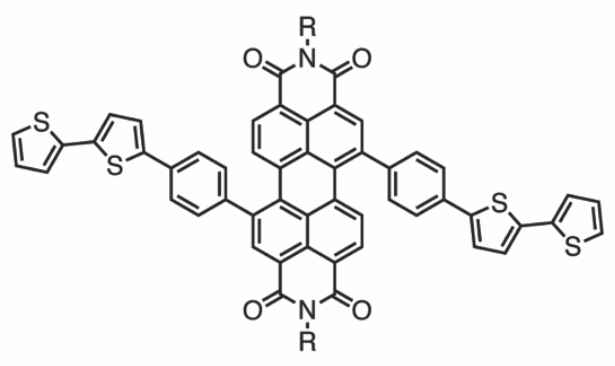

3.1

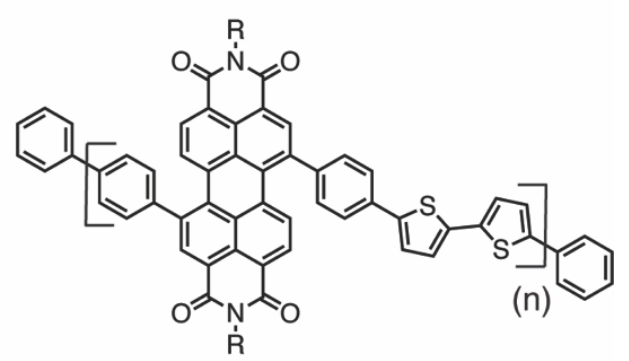

3.3

(c)

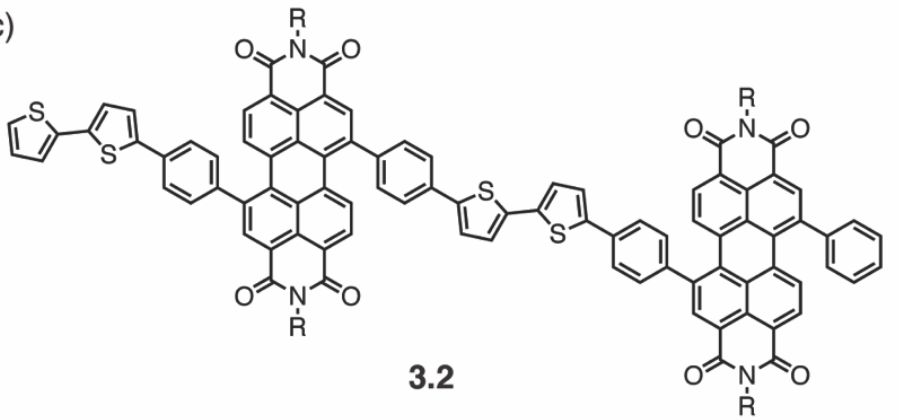

Figure 3.1. Structures of compounds designed and synthesized to compare acyclic and cyclic, $\pi$ conjugated molecules for n-type electronic materials. (a) PBPB; (b) a monomer version (3.1) of the macrocycle; (c) acyclic PBPB where the bond between one phenyl and a bithiophene is cut (3.2); and (d) a polymeric version (3.3). $\mathrm{R}=\mathrm{C}_{11} \mathrm{H}_{23}$ side chains.

We compare these macrocycles to a series of acyclic molecules that link varying numbers of $\mathbf{P}$ subunits and find that the conjugated cycles have bathochromically shifted UV-vis absorbances, are more easily reduced, have higher electron mobility and have better morphology in blended films. All of these factors contribute to the more than doubling of the power conversion efficiency observed in solar cells using these macrocycles as n-type, electron-transporting material. This is first report directly comparing analogous cyclic and acyclic $\pi$-conjugated molecules as ntype materials in OPVs. 
(a)

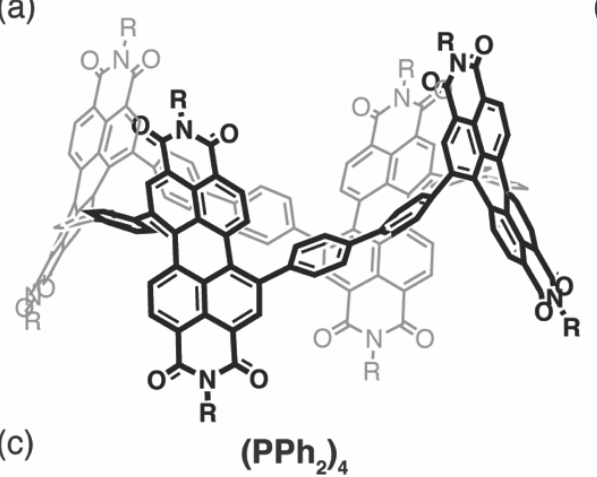

(b)

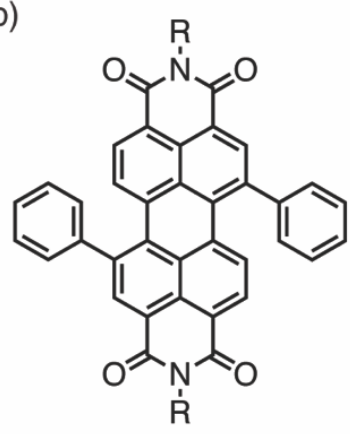

1.1 (d)

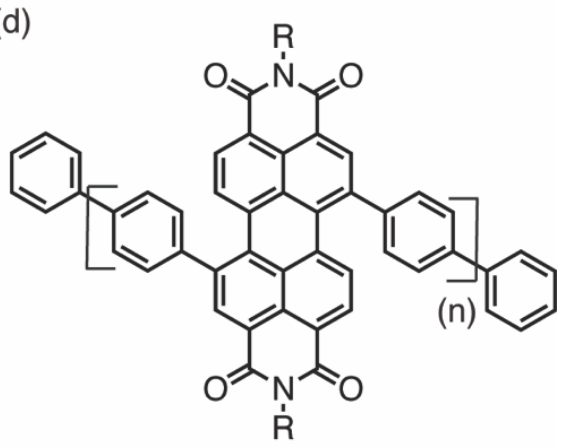

3.6

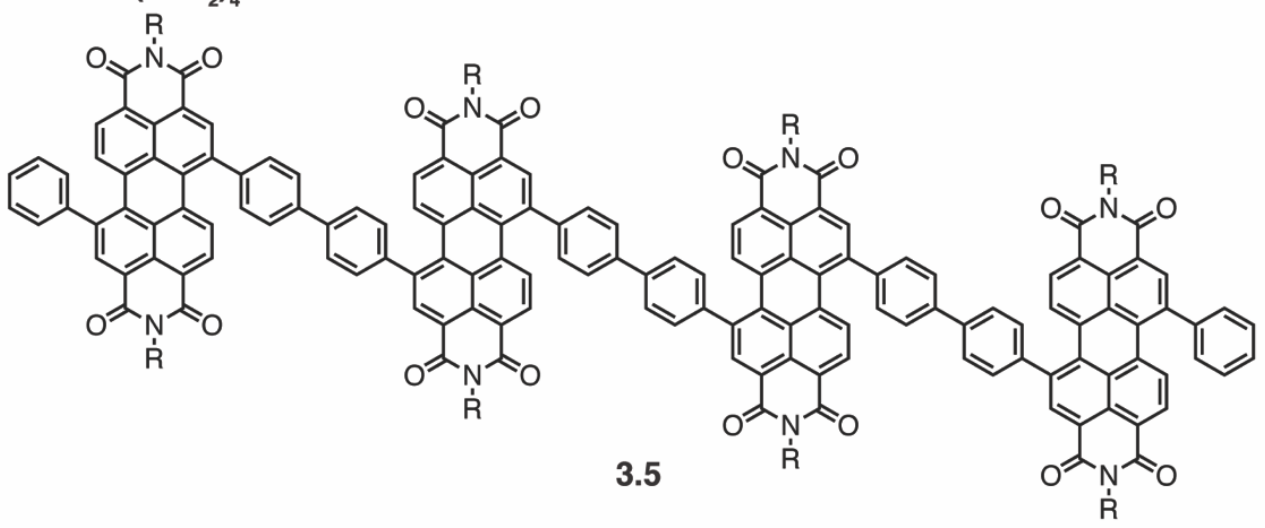

Figure 3.2. Structures of compounds designed and synthesized to compare acyclic and cyclic, $\pi$ conjugated molecules for n-type electronic materials. (a) (PPh $\mathbf{P A}_{4}$; (b) a monomer version (1.1) of the macrocycle introduced in Chapter 1B; (c) acyclic $(\mathbf{P P h})_{4}$ where the bond between two phenyl groups is cut (3.5); and (d) a polymeric version (3.6).

\subsection{Design of N-type Semiconductors}

We designed a series of cyclic and acyclic $\pi$-conjugated molecules (Figures 3.1 and 3.2) that contain the redox active PDI subunit. PDIs possess many desirable properties such as efficient electron transport, ${ }^{38-42}$ high molar absorptivities, ${ }^{29,43,44}$ and ease of functionalization. ${ }^{45-47}$ Derivatives of PDI are efficacious in solar cells when combined with electron rich conjugated polymers. ${ }^{44,48-50}$ From these diaryl substituted PDI subunits, we build the two macrocycles, PBPB and $(\mathbf{P P h})_{4}$, using a tetranucleur platinum macrocyclization followed by reductive eliminations, analogous to the synthetic approach described in Chapter $1 \mathrm{~B}$ and Chapter 2..$^{7,22,25}$ The DFT minimized structures for $\mathbf{P B P B}{ }^{20}$ and $\left(\mathbf{P P h}_{2}\right)_{4}$ are shown in Figure 3.3. 

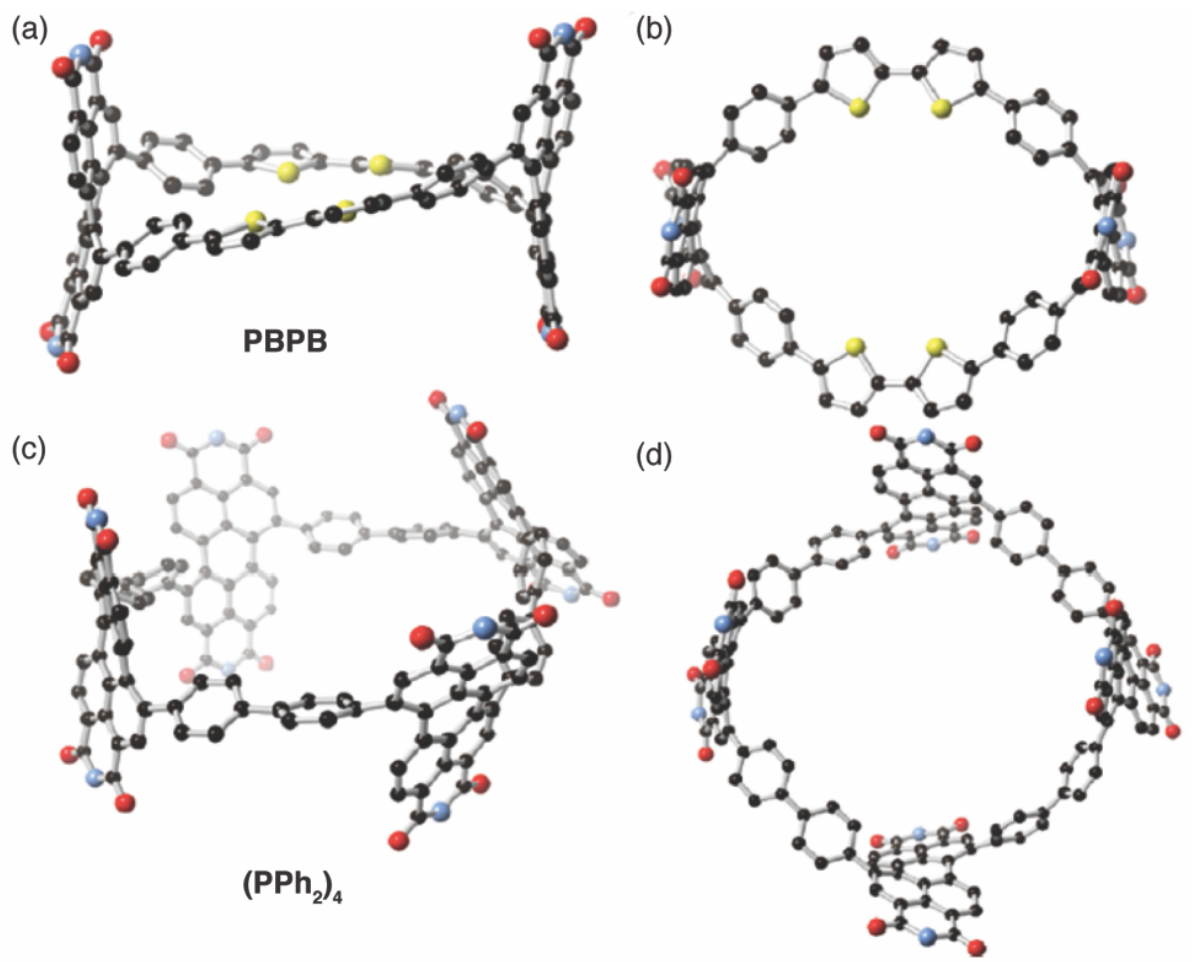

Figure 3.3. (a) Energy minimized structures from DFT for PBPB. The $(M, M)$-stereoisomer is shown. ${ }^{20}$ (b) Cavity view of PBPB. (c) Energy minimized structures from DFT for (PPh $\mathbf{P}_{4}$. The $(M, M, M, M)$-stereoisomer is shown. (d) Cavity view for $\left(\mathbf{P P h}_{2}\right)_{4}$. Carbon $=$ gray, nitrogen $=$ blue, oxygen $=$ red, sulfur $=$ yellow. Hydrogen atoms have been removed to clarify the view. A methyl group substitutes the sidechains in the calculations. The methyl group, too, has been removed to clarify the view in the structures presented here.

For comparison to PBPB and $(\mathbf{P P h})_{4}$, we synthesized a series of acyclic molecules. The simplest structures are Compounds 3.1 and 1.1; each possessing one PDI subunit (1.1 was introduced in Chapter 1B). We created the polymeric version of the macrocycles [3.3 and 3.6] too, as these molecules would theoretically possess "infinite conjugation". As a final point of comparison, we also synthesized the precise analogs, "unfolded" macrocycles, that have one of their bonds cleaved and terminated with hydrogen atoms (3.2 and 3.5).

\subsection{Conjugated Macrocycles in OPVs}

We fabricated devices from each of these macrocyclic and acyclic molecules. We chose the low-bandgap semiconducting polymer poly[4,8-bis(5-(2-ethylhexyl)thiophen-2-yl)benzo[1,2b;4,5-b']dithiophene-2,6-diyl-alt-(4-(2-ethylhexyl)-3-fluorothieno[3,4-b]thiophene)-2- 
carboxylate-2,6-diyl] (PTB7-Th) ${ }^{51,52}$ as the electron donating component in our devices. PTB7-Th is widely used as a high-performance donor material in both fullerene and non-fullerene based solar cells. ${ }^{53,54}$ PTB7-Th is complementary to all molecules synthesized, as it absorbs more low energy light ( $\sim 550 \mathrm{~nm}$ to $800 \mathrm{~nm})$. Figure 3.8 (in the Appendix) contains the film absorption spectra for all the compounds. We spin cast the mixture of PTB7-Th and the cyclic or acyclic molecules to form a bulk heterojunction (BHJ) solar cell. ${ }^{55} \mathrm{We}$ used an inverted configuration of ITO/ZnO(20 nm)/PTB7-Th:acceptor/ $\mathrm{MoO}_{3}(7 \mathrm{~nm}) / \mathrm{Ag}(100 \mathrm{~nm})$ for all of our solar cell devices. ${ }^{56}$ A schematic of the device is shown in Figure 3.4a.

Figure 3.4b-e displays the OPV properties and the EQE measurements for each of the cyclic and acyclic molecules. Details for the optimization including varying the ratio of donor and acceptor, the additives for annealing and the film thickness are included in the Supporting Information. The optimal active layers were $\sim 100 \mathrm{~nm}$ in thickness. For the cyclic molecules, the optimal mass ratio was 1:2 for donor:acceptor. Furthermore, we optimized the films by using 1chloronaphthalene $(\mathrm{CN})$ as a solvent additive to control film morphology (Figure 3.11 in the Appendix). ${ }^{42}$ PBPB's PCE increases to $3.3 \%$ on average with a maximal value of $3.5 \%$. Using an analogous procedure, we were able to achieve a PCE of 3.6\% for $(\mathbf{P P h})_{4}$ (see Figure 3.9, Figure 3.10, Table 3.3, and Table 3.4). This is the first example of a macrocycle being used as the electron acceptor in an OPV. 
(a)

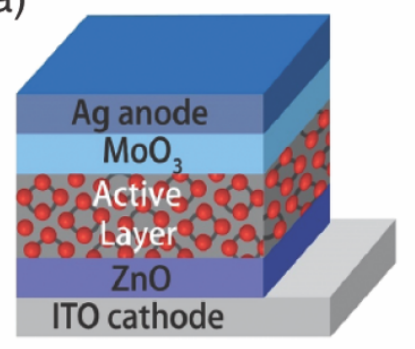

(b)

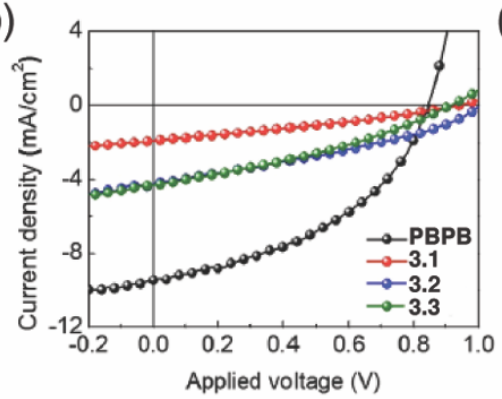

(c)

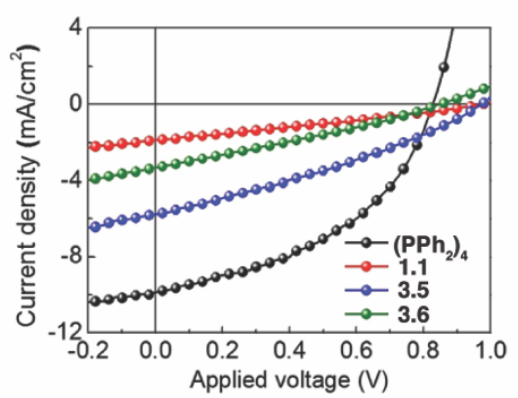

(d)

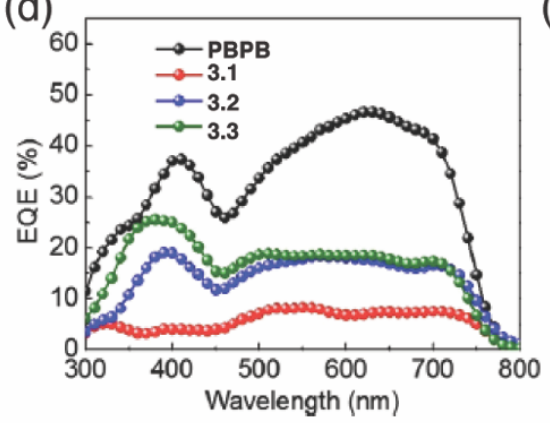

(e)

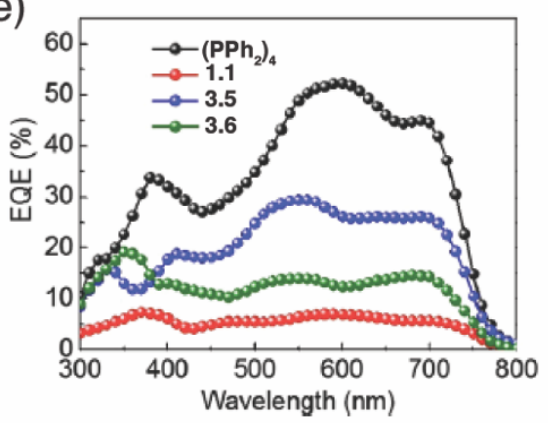

Figure 3.4. (a) Schematic of the solar cell device fabricated in this study. (b-e) J-V curves for (b) PBPB-series and (c) $\left(\mathbf{P P h}_{2}\right)_{4}$-series solar cells under simulated AM 1.5G irradiation (100 $\mathrm{mWcm}^{-2}$ ). EQE spectra for (d) PBPB -series and (e) $\left(\mathbf{P P h}_{2}\right)_{4}$-series solar cells

Figure 3.4d and Figure 3.4e show the external quantum efficiency (EQE) curves for PTB7Th:PBPB and the PTB7-Th:( $\left.\mathbf{P P h}_{2}\right)_{4}$ solar cells. All the devices show broad photo-response from $350 \mathrm{~nm}$ to $800 \mathrm{~nm}$, consistent with the absorption spectra (Figure 3.8). Each EQE spectrum shows two transitions; a narrow band centered at $\sim 400 \mathrm{~nm}$ and a broad band centered at $620 \mathrm{~nm}$ for PBPB and at $600 \mathrm{~nm}$ for $\left(\mathbf{P P h}_{2}\right)_{4}$. The EQE spectrum for $\left(\mathbf{P P h}_{2}\right)_{4}$ shows an increase relative to $\mathbf{P B P B}$ at $\sim 700 \mathrm{~nm}$. We note that both macrocycles show strong absorption from $400 \mathrm{~nm}$ to $650 \mathrm{~nm}$ (see Figure 3.5), indicating that photoexcitation in acceptor domains contributes to photocurrent in this type of solar cell. The integrated $J_{\mathrm{sc}}$ values are 9.2 and $9.8 \mathrm{mAcm}^{-2}$ for PTB7-Th:PBPB and the PTB7-Th:(PPh $)_{4}$ solar cells, respectively. These values agree well with the measured $J_{\mathrm{sc}}$, with mismatch $<3 \%$. Upon addition of the $\mathrm{CN}$ additive, the $\mathrm{EQE}$ enhances over a broad range of wavelengths, particularly from $550 \mathrm{~nm}$ to $750 \mathrm{~nm}$ (Figure 3.9 and Figure 3.10). Atomic force microscopy (AFM) of the films confirms that $\mathrm{CN}$ changes the film morphology, resulting in more efficient charge dissociation and transport (Figure 3.11). Like $\mathrm{PC}_{71} \mathrm{BM}$ and some non-fullerene 
acceptors, ${ }^{48,53}$ complementary absorption between the macrocycles and the donor material is beneficial for harvesting light in the visible light region to maximize photocurrent.

Table 3.1. Summary of Device Parameters

\begin{tabular}{|l|c|c|c|c|}
\hline & $J_{\text {sc }}\left(\mathrm{mA} \mathrm{cm}^{-2}\right)$ & $V_{\text {oc }}(\mathrm{V})$ & FF & PCE (\%) \\
\hline PBPB & $9.2 \pm 0.3$ & $0.84 \pm 0.01$ & $0.44 \pm 0.01$ & $3.3 \pm 0.2(3.5)$ \\
\hline $\mathbf{3 . 1}$ & $1.6 \pm 0.2$ & $0.94 \pm 0.01$ & $0.30 \pm 0.01$ & $0.46 \pm 0.04(0.53)$ \\
\hline $\mathbf{3 . 2}$ & $4.2 \pm 0.1$ & $1.00 \pm 0.01$ & $0.33 \pm 0.01$ & $1.3 \pm 0.1(1.4)$ \\
\hline $\mathbf{3 . 3}$ & $4.2 \pm 0.2$ & $0.90 \pm 0.01$ & $0.33 \pm 0.01$ & $1.1 \pm 0.2(1.3)$ \\
\hline $\mathbf{( P P h})_{4}$ & $9.7 \pm 0.2$ & $0.83 \pm 0.01$ & $0.44 \pm 0.01$ & $3.5 \pm 0.1(3.6)$ \\
\hline $\mathbf{1 . 1}$ & $1.7 \pm 0.1$ & $0.97 \pm 0.02$ & $0.28 \pm 0.01$ & $0.46 \pm 0.03(0.51)$ \\
\hline $\mathbf{3 . 5}$ & $5.8 \pm 0.1$ & $0.97 \pm 0.01$ & $0.32 \pm 0.01$ & $1.6 \pm 0.2(1.8)$ \\
\hline $\mathbf{3 . 6}$ & $3.2 \pm 0.1$ & $0.85 \pm 0.01$ & $0.28 \pm 0.01$ & $0.73 \pm 0.04(0.78)$ \\
\hline
\end{tabular}

Table 3.1: Summary of device parameters of the solar cells based on the cycles and acyclic molecules. Highest PCE values are shown in parentheses.

We next compare the OPV results from the cyclic molecules to the acyclic molecules.

Table 3.1 summarizes the device data. The key finding is that all of the acyclic molecules showed poor device performance on both an absolute and relative basis. Figure $3.4 \mathrm{~b}$ and $3.4 \mathrm{c}$ display the $J-V$ curves for all the devices. We observe a couple of trends from this study: (1) smaller oligomer acyclic molecules (3.1, 3.2, 1.1, and 3.5) and the polymers (3.3 and 3.6) show decreased $J_{\mathrm{sc}}$ relative to the cyclic compounds; (2) the acyclic molecules also show higher $V_{\text {oc }}$ values as compared to the cyclic acceptors; and (3) the poor PCEs in the devices from acyclic molecules are mainly attributed to the reduced $J_{\mathrm{sc}}$ and FFs relative to the cyclic ones. Figure $3.3 \mathrm{~d}$ and $3.3 \mathrm{e}$ display comparison of external quantum spectra of the cyclics versus the acyclics. Overall, the photocurrent generation 
in cyclic-based devices is much larger than the acyclic-based devices. These results indicate that the cyclic acceptors have enhanced photocarrier generation and better charge transport.

To better understand the performance difference between the cyclic and acyclic molecules, we examined the electrochemistry, UV-vis absorption, electron mobility and morphology of the films. These studies are described below.

\subsection{Electrochemistry of N-type Semiconductors}

We probe the variations in the frontier orbital energies for the macrocycles and their acyclic analogues using CV (see Figure 3.12 in the Appendix). The onset of the first oxidation and reduction peaks provide an estimate of the $\mathrm{HOMO}$ and LUMO levels, respectively. ${ }^{57} \mathrm{We}$ find the acyclic molecules possess a more negative first reduction potential than the cyclic molecules. As a result, we observe higher energies for the LUMO for each of the acyclic molecules. The electrochemical data are summarized in Table 3.2.

The energy offset between the donor's HOMO and acceptor's LUMO is one of the factors that determines the $V_{\mathrm{oc}}$ in BHJ solar cells. ${ }^{58}$ The values obtained from $\mathrm{CV}$ results are in good agreement with the $V_{\text {oc }}$ trend from the devices. Previous studies show a direct correlation between relatively large $V_{\text {oc }}$ values coupled with low $J_{\text {sc }}$ when the band offset does not provide sufficient driving force for exciton dissociation at the donor/acceptor interfaces. ${ }^{59,60}$ Here, the trend observed suggests that the high LUMO levels, particularly in the short acyclic compounds, result in a higher occurrence of recombination and lower $J_{\text {sc. }}{ }^{60,61}$ 
Table 3.2. Comparison of Electronic Data

\begin{tabular}{|l|cc|c|c|c|}
\hline \multirow{2}{*}{} & \multicolumn{3}{|c|}{ Electrochemical $^{a}$} & Optical $^{b}$ & FET \\
\cline { 2 - 6 } & $\mathrm{E}_{\mathrm{LUMO}} / \mathrm{eV}$ & $\mathrm{E}_{\mathrm{HOMO}} / \mathrm{eV}$ & $\begin{array}{c}\mathrm{E}_{\text {gap }} \\
/ \mathrm{eV}\end{array}$ & $\begin{array}{c}\mathrm{E}_{\text {gap }} \\
/ \mathrm{eV}\end{array}$ & $\begin{array}{c}\mu \\
/ \mathrm{cm}^{2} \mathrm{~V}^{-1} \mathrm{~s}^{-1}\end{array}$ \\
\hline $\mathbf{P B P B}$ & -3.87 & -5.39 & 1.52 & 2.02 & $(1.5 \pm 0.2) \times 10^{-3}$ \\
\hline $\mathbf{3 . 1}$ & -3.80 & -5.42 & 1.62 & 2.18 & $(4.3 \pm 0.2) \times 10^{-4}$ \\
\hline $\mathbf{3 . 3}$ & -3.80 & -5.40 & 1.60 & 1.79 & - \\
\hline $\mathbf{( P P h})_{4}$ & -3.86 & -5.45 & 1.59 & 2.21 & $(2.3 \pm 0.3) \times 10^{-4}$ \\
\hline $\mathbf{1 . 1}$ & -3.90 & -5.69 & 1.79 & 2.12 & $(1.5 \pm 0.2) \times 10^{-3}$ \\
\hline $\mathbf{3 . 5}$ & -3.75 & - & - & 2.25 & $(2.0 \pm 0.3) \times 10^{-5}$ \\
\hline $\mathbf{3 . 6}$ & -3.82 & -5.77 & 1.95 & 2.23 & - \\
\hline
\end{tabular}

Table 3.2: Comparison of the band-gaps estimated from CV and UV-vis absorption spectroscopy, and OFET performance. $a \mathrm{HOMO}$ and LUMO levels were estimated from onset of the first oxidation and reduction peaks. cOptical band gaps were estimated from the wavelength at the absorption maximum.

\subsection{Absorption Spectroscopy}

Figure 3.5 compares the UV-vis absorption spectra of $\mathbf{P B P B}$ and $\left(\mathbf{P P h} \mathbf{h}_{\mathbf{4}}\right.$ to their acyclic counterparts. It is well documented that contorting linear molecules into cyclic structures significantly alters the electronic properties. ${ }^{2,25}$ Absorptions in the cyclic compounds are shifted to lower energy relative to the linear, unstrained acyclic molecules. The $\mathrm{CV}$ data is also consistent with the UV-vis data. PBPB and (PPh $)_{4}$ have smaller HOMO-LUMO gaps relative to each of the corresponding acyclic molecules studied (Figure 3.12). Greater visible light absorption contributes to the more efficient solar cells for the cyclic molecules, providing the higher $J_{\text {sc }}$ parameter for the cyclic molecules relative to the acyclic molecules. 

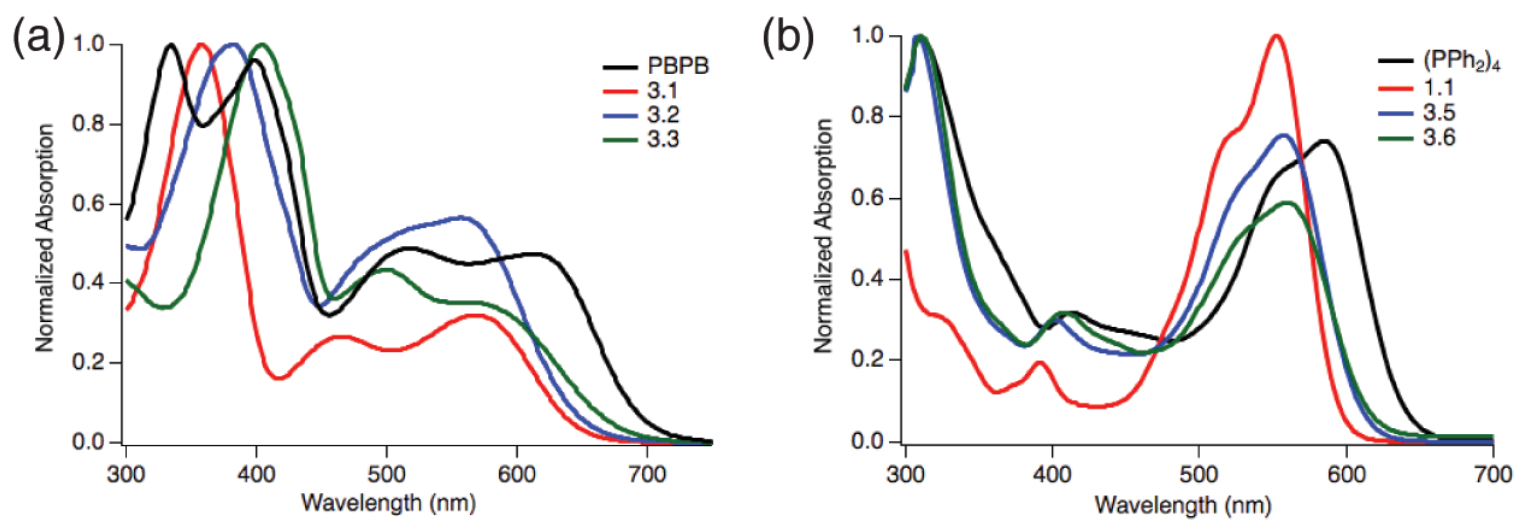

Figure 3.5. UV-vis absorption spectra measured in solution: (a) for PBPB, 3.1, 3.2, and 3.3; (b) for $\left(\mathbf{P P h}_{2}\right)_{4}, \mathbf{1 . 1}, 3.5$, and 3.6 normalized to each absorption maxima, where absorption $\max =1$.

\subsection{OFET Characteristics}

Another factor that is critical for OPV device performance is electron transport through the acceptor phase. Poor carrier mobility impedes the carrier extraction and results in increased carrier recombination inside OPV devices. This negatively impacts the $J_{\mathrm{sc}}$, FF and overall solar cell performance. To investigate the electron mobility of these compounds, we fabricated OFETs. The Supporting Information describes the preparation of the devices and the methodology used to extract the OFET characteristics. All molecules measured form n-type, electron-transporting thin-

film semiconductors. ${ }^{38,62}$ Figure 3.6 displays typical transfer curves in the saturation regime. The mobility was calculated in the saturation regime using $\mathrm{I}_{\mathrm{DS}}=(\mathrm{W} / 2 \mathrm{~L}) \mathrm{C}_{\mathrm{i}} \mu\left(\mathrm{V}_{\mathrm{G}}-\mathrm{V}_{\mathrm{T}}\right)^{2}$, where $\mathrm{W}$ and $\mathrm{L}$ are the width and length of the channel, $\mathrm{C}_{\mathrm{i}}\left(11.5 \mathrm{nFcm}^{-2}\right), \mu$, and $\mathrm{V}_{\mathrm{T}}$ correspond to the capacitance per unit area of the gate insulator, the field effect mobility, and the threshold voltage, respectively. ${ }^{63}$ Both $\mathbf{P B P B}$ and $(\mathbf{P P h})_{4}$ show electron mobility of $(1.5 \pm 0.2) \times 10^{-3} \mathrm{~cm}^{2} \mathrm{~V}^{-1} \mathrm{~s}^{-1}$. One of the key findings is the cyclic molecules have a far greater ability to transport electrons in thin-film devices relative to the acyclic molecules. Table 3.2 shows electron mobility for six of the compounds studied. PBPB's average mobility is five-fold higher than its acyclic counterparts; $\left(\mathbf{P P h}_{2}\right)_{4}$ 's mobility is nearly two orders of magnitude higher than its counterparts. The cyclic 
structures far greater ability to transport electrons contributes to the overall better solar cell performance.
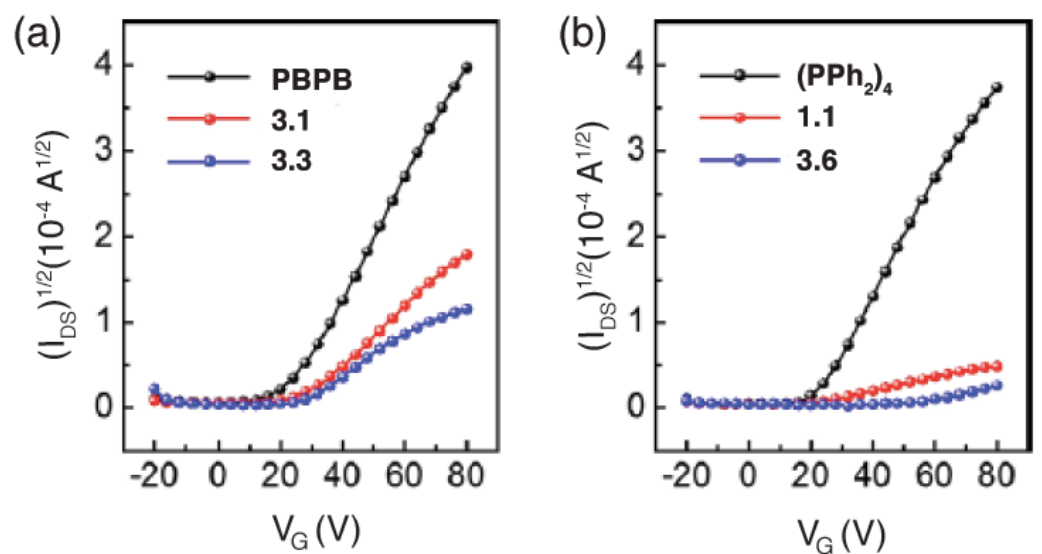

Figure 3.6. OFET transfer characteristics plotted in $\left(\mathrm{I}_{\mathrm{DS}}\right)^{1 / 2}-\mathrm{V}_{\mathrm{G}}$ axes for (a) PBPB, 3.1 and $\mathbf{3 . 3}$ and (b) for $\left(\mathbf{P P h}_{2}\right)_{4}, \mathbf{1 . 1}$, and 3.6. The $\mathrm{V}_{\mathrm{DS}}$ is $80 \mathrm{~V}$.

\subsection{Film Morphology}

At the nanoscale level, phase separation between the donor and the acceptor plays an important role in providing an efficient donor/acceptor interface and a continuous pathway for carrier transport. Appropriate aggregation and phase separation is critical to device performance of BHJs in terms of charge dissociation and carrier transport. We performed AFM to study the surface morphology of the active layers. Figure 3.7 displays phase images of the six active layers studied. The corresponding height images are displayed in Figure 3.13. For both PBPB and $(\mathbf{P P h})_{4}$, the active layers possess clear phase separation as shown in Figure 3.7a and 3.7d. The average domain size is estimated to be $20-40 \mathrm{~nm}$. For the polymeric $\mathbf{3 . 3}$ and $\mathbf{3 . 6}$, the domain sizes are relatively small $(10-30 \mathrm{~nm})$ (Figure 3.7c,f). It is difficult to detect efficient phase segregation in these films. The active layers containing 3.1 and 1.1 have large domain sizes; they are in the range of 50-70 nm, as shown in Figure $3.7 \mathrm{~b}$ and $3.7 \mathrm{e}$. These features exceed twice the typical exciton diffusion length (ca. 10-20 nm) in organic semiconductors. Thus, photogenerated excitons within the domain recombine before they reach the donor/acceptor interface. ${ }^{55,64}$ The over- 
aggregation in the $\mathbf{3 . 1}$ and $\mathbf{1 . 1}$ solar cells likely results in carrier recombination and poor device performance. In the PBPB- and ( $\mathbf{P P h} \mathbf{2}_{\mathbf{4}}$-based BHJ systems, phase aggregation is essential to the device performance as it enables an efficient donor/acceptor interface and a three dimensional continuous pathway for efficient carrier transport.
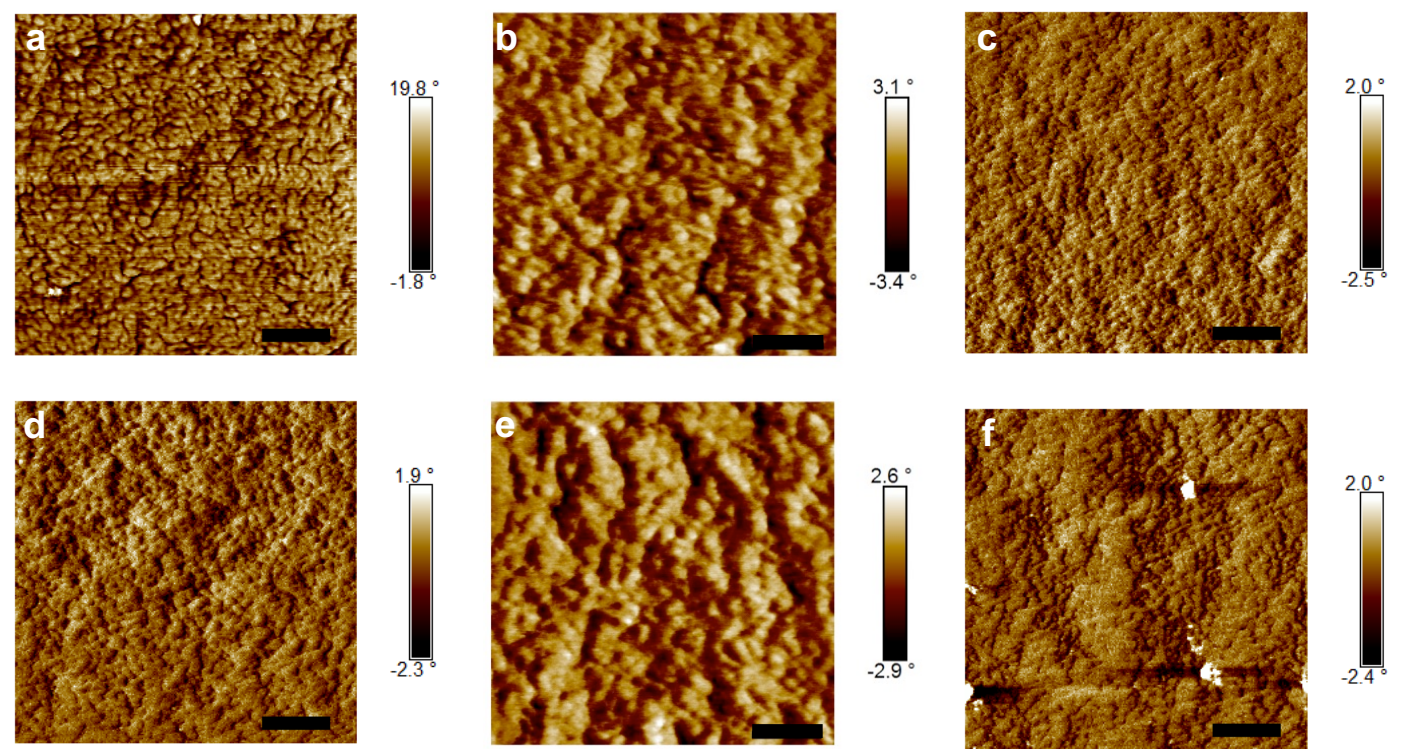

Figure 3.7. AFM phase images of bulkjunction films for (a) PBPB, (b) 3.1, (c) 3.3, (d) $\left(\mathbf{P P h}_{2}\right)_{4}$, (e) 1.1, and (f) 3.6. The mass ratio of donor-to-acceptor is fixed at $1: 2.1 \% \mathrm{CN}$ additive was used. The scale bar is $200 \mathrm{~nm}$.

\subsection{Conclusion}

This is the first study comparing cyclic structures to their acyclic counterparts in OPVs. We found that the cyclic structures far outperform the acyclic controls in organic photovoltaics. We find it interesting that $\mathbf{P B P B}$ and $\left(\mathbf{P P h}_{2}\right)_{4}$ perform similarly as the electron transporting material in OPVs even though PBPB's UV-vis is shifted to lower energy compared to that of $\left(\mathbf{P P h}_{2}\right)_{4}$. The origin of the increase in the efficiency of the devices when cyclic molecules are used in place of acyclic ones is multifaceted. When compared to the acyclic molecules, the macrocycles: (1) have better energy alignment with the donor material; (2) absorb more visible light; (3) are more efficient at transporting electrons; and (4) shows optimal phase separation for BHJ solar cells. The ease with which we can tune the energetics and therefore the properties of these 
macrocycles - through a different linker subunit or incorporating oligomeric PDI subunits - will magnify these initial findings. ${ }^{54}$ This study also offers the intriguing possibility of tuning the geometry of the donor so that it is shape matched to these cyclic electron accepting structures as a means to creating highly efficient devices.

\subsection{Appendix - Supplementary Figures}
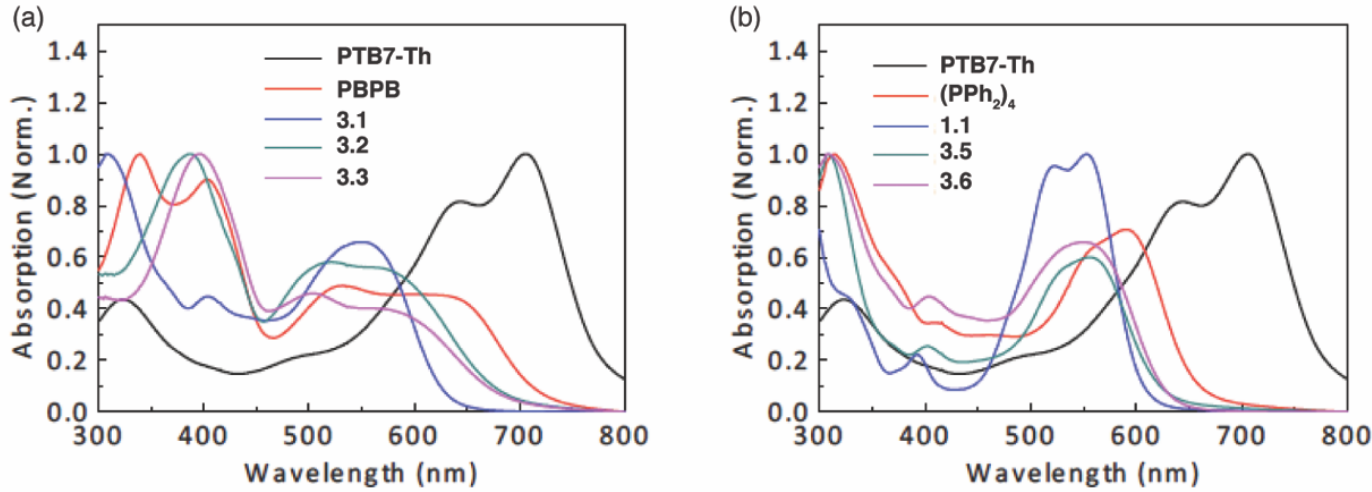

Figure 3.8. UV-vis absorption spectra measured in films: for (a) PBPB, 3.1, 3.2, and 3.3; and (b) $\left(\mathbf{P P h}_{2}\right)_{4}, \mathbf{1 . 1}, 3.5$, and 3.6 normalized to each absorption maxima, where absorption $\max =1$.

(a)

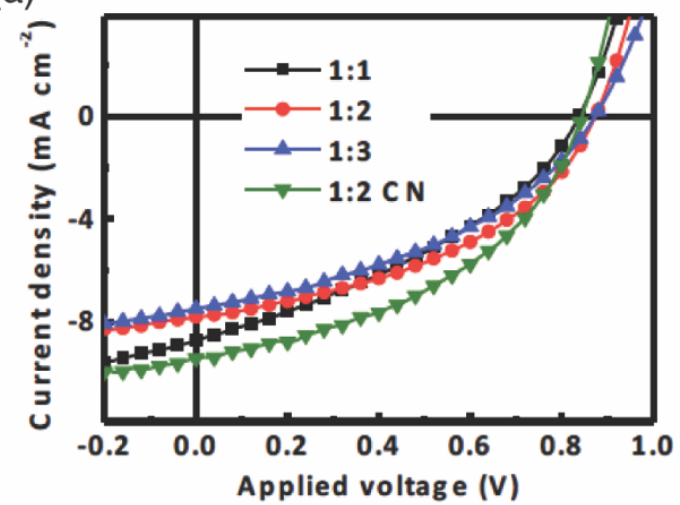

(b)

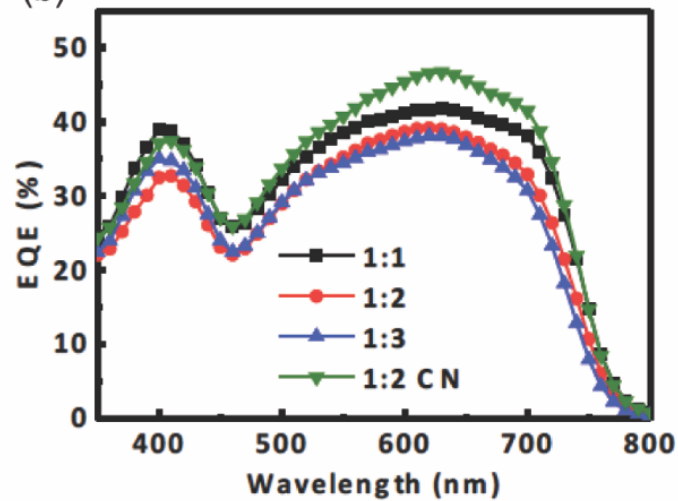

Figure 3.9. Device performance of PTB7-Th:PBPB solar cells. (a) Current density versus voltage $(J-V)$ characteristics of PTB7-Th:PBPB solar cells with different blend ratios (PTB7-Th to PBPB) and $1 \% \mathrm{v} / \mathrm{v}$ CN. (b) EQE spectra of corresponding PTB7-Th:PBPB solar cells. 
Table 3.3. Summary of Device Parameters for PTB7-Th:PBPB solar cell.

\begin{tabular}{|l|c|c|c|c|}
\hline Mass ratio & $J_{s c}\left(\mathrm{mAcm}^{-2}\right)$ & $V_{o c}(\mathrm{~V})$ & FF & PCE (\%) \\
\hline $1: 1$ & $8.5 \pm 0.3$ & $0.83 \pm 0.01$ & $0.36 \pm 0.01$ & $2.5 \pm 0.1(2.6)$ \\
\hline $1: 2$ & $7.6 \pm 0.2$ & $0.87 \pm 0.01$ & $0.43 \pm 0.01$ & $2.8 \pm 0.1(2.9)$ \\
\hline $1: 3$ & $7.4 \pm 0.1$ & $0.87 \pm 0.01$ & $0.40 \pm 0.01$ & $2.5 \pm 0.1(2.6)$ \\
\hline $1: 2$ with CN & $9.2 \pm 0.3$ & $0.84 \pm 0.01$ & $0.44 \pm 0.01$ & $3.3 \pm 0.2(3.5)$ \\
\hline
\end{tabular}

Table 3.3: Summary of device parameters of best PTB7-Th:PBPB solar cells with different conditions. Average PCE values were calculated from six devices for each condition; the highest PCE values are shown in parentheses.

(a)

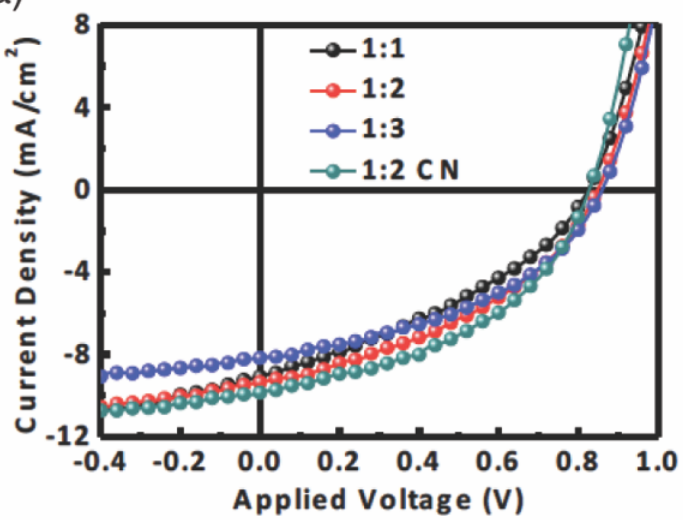

(b)

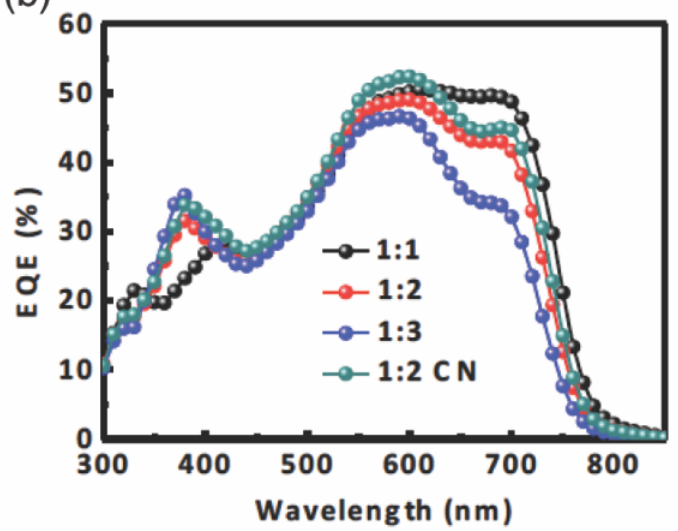

Figure 3.10. Device performance of PTB7-Th:( $\left(\mathbf{P P h}_{2}\right)_{4}$ solar cells. (a) Current density versus voltage $(J-V)$ characteristics of PTB7-Th:(PPh $)_{4}$ solar cells with different blend ratios (PTB7-Th to $\left.\left(\mathbf{P P h}_{2}\right)_{4}\right)$ and $1 \% \mathrm{v} / \mathrm{v}$ CN. (b) EQE spectra of corresponding PTB7-Th:( $\left(\mathbf{P P h}_{2}\right)_{4}$ solar cells.

Table 3.4. Summary of Device Parameters for PTB7-Th:(PPh $)_{4}$ solar cell.

\begin{tabular}{|l|c|c|c|c|}
\hline Mass ratio & $J_{s c}\left(\mathrm{mAcm}^{-2}\right)$ & $V_{o c}(\mathrm{~V})$ & FF & PCE (\%) \\
\hline $1: 1$ & $8.6 \pm 0.3$ & $0.82 \pm 0.01$ & $0.37 \pm 0.01$ & $2.6 \pm 0.2(2.8)$ \\
\hline $1: 2$ & $8.8 \pm 0.2$ & $0.85 \pm 0.01$ & $0.41 \pm 0.01$ & $3.0 \pm 0.1(3.2)$ \\
\hline $1: 3$ & $7.6 \pm 0.2$ & $0.86 \pm 0.01$ & $0.43 \pm 0.01$ & $2.8 \pm 0.2(3.0)$ \\
\hline $1: 2$ with CN & $9.7 \pm 0.2$ & $0.83 \pm 0.01$ & $0.44 \pm 0.01$ & $3.5 \pm 0.1(3.6)$ \\
\hline
\end{tabular}

Table 3.4: Summary of device parameters of best PTB7-Th:( $\left(\mathbf{P P h}_{2}\right)_{4}$ solar cells with different conditions. Average PCE values were calculated from six devices for each condition; the highest PCE values are shown in parentheses. 

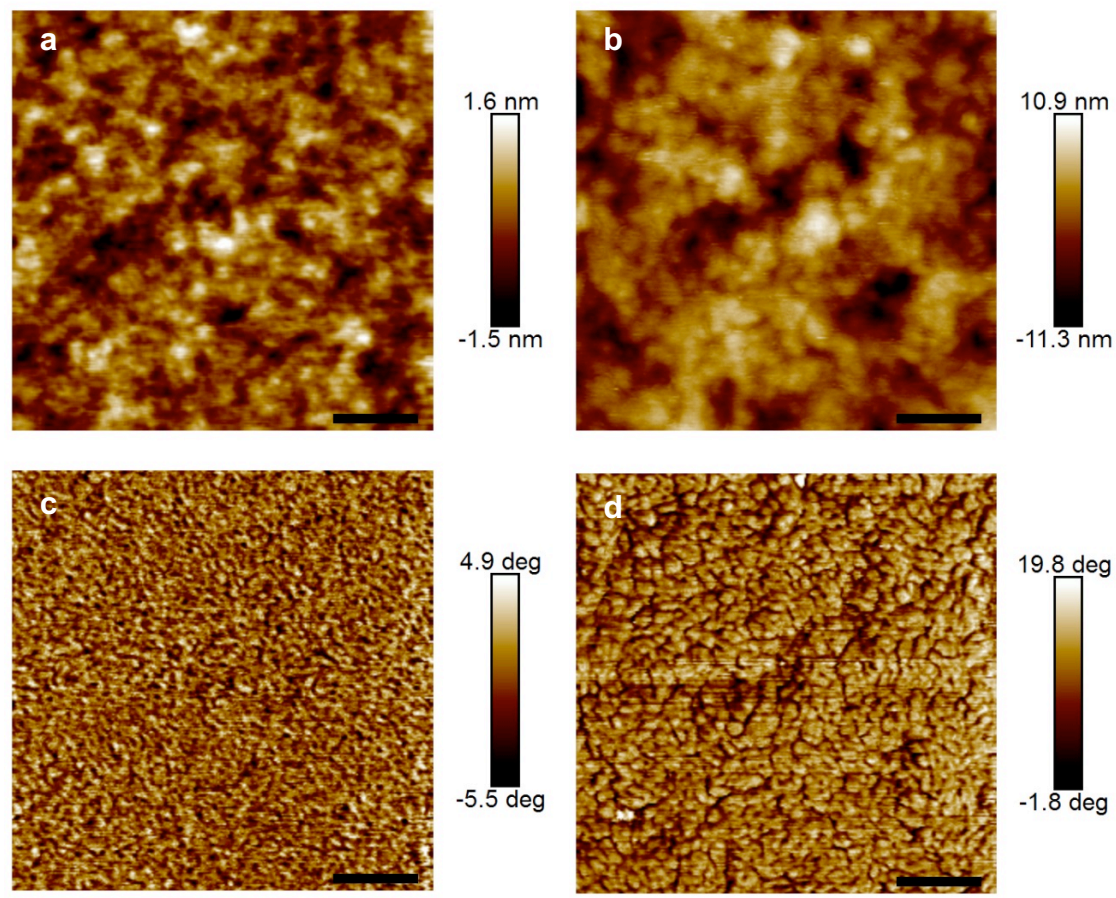

Figure 3.11. AFM height images of PTB7-Th:PBPB films at a donor:acceptor ratio of 1:2 (a) without $\mathrm{CN}$ and (C) with $1 \% \mathrm{CN}$. AFM phase images of PTB7-Th:PBPB films at a donor:acceptor ratio of 1:2 (B) without $\mathrm{CN}$ and (d) with $1 \% \mathrm{CN}$. The scale bar is $200 \mathrm{~nm}$. Both of the active layers have very smooth surface, with root-mean-square roughness (RMS) of $0.5 \mathrm{~nm}$ and $2.6 \mathrm{~nm}$ for films both with/without $1 \% \mathrm{CN}$, respectively. When turning to the phase images, we observed fibrous domains in the active layer without $\mathrm{CN}$. However, this feature is too fine to be quantified under the instrument limit $(\sim 8 \mathrm{~nm})$. In contrast, the active layer with the $1 \% \mathrm{CN}$ additive possesses clear phase separation as shown in $\mathrm{d}$. The average domain size is estimated to be $20-40 \mathrm{~nm}$. Here $\mathrm{CN}$ plays a critical role in phase aggregation, which is essential to the device performance.

(a)
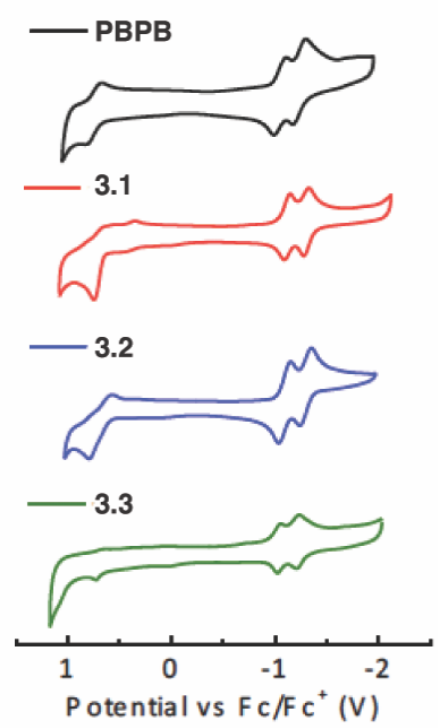

(b)
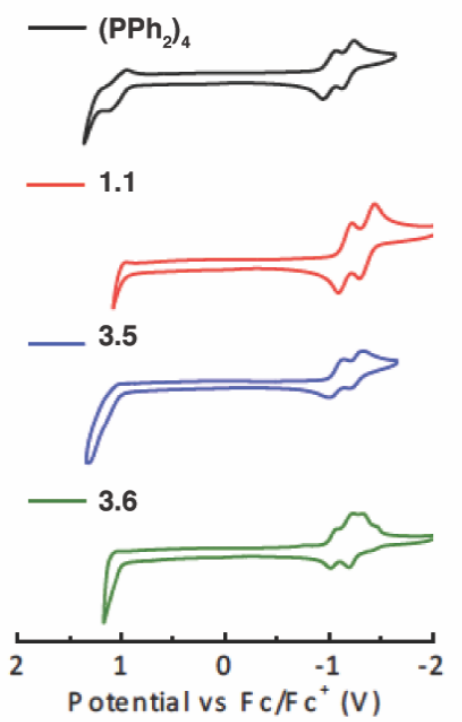

Figure 3.12. CV (a) for PBPB, 3.1, 3.2, and 3.3; (b) for $\left(\mathbf{P P h}_{2}\right)_{4}, 1.1,3.5$, and 3.6. 

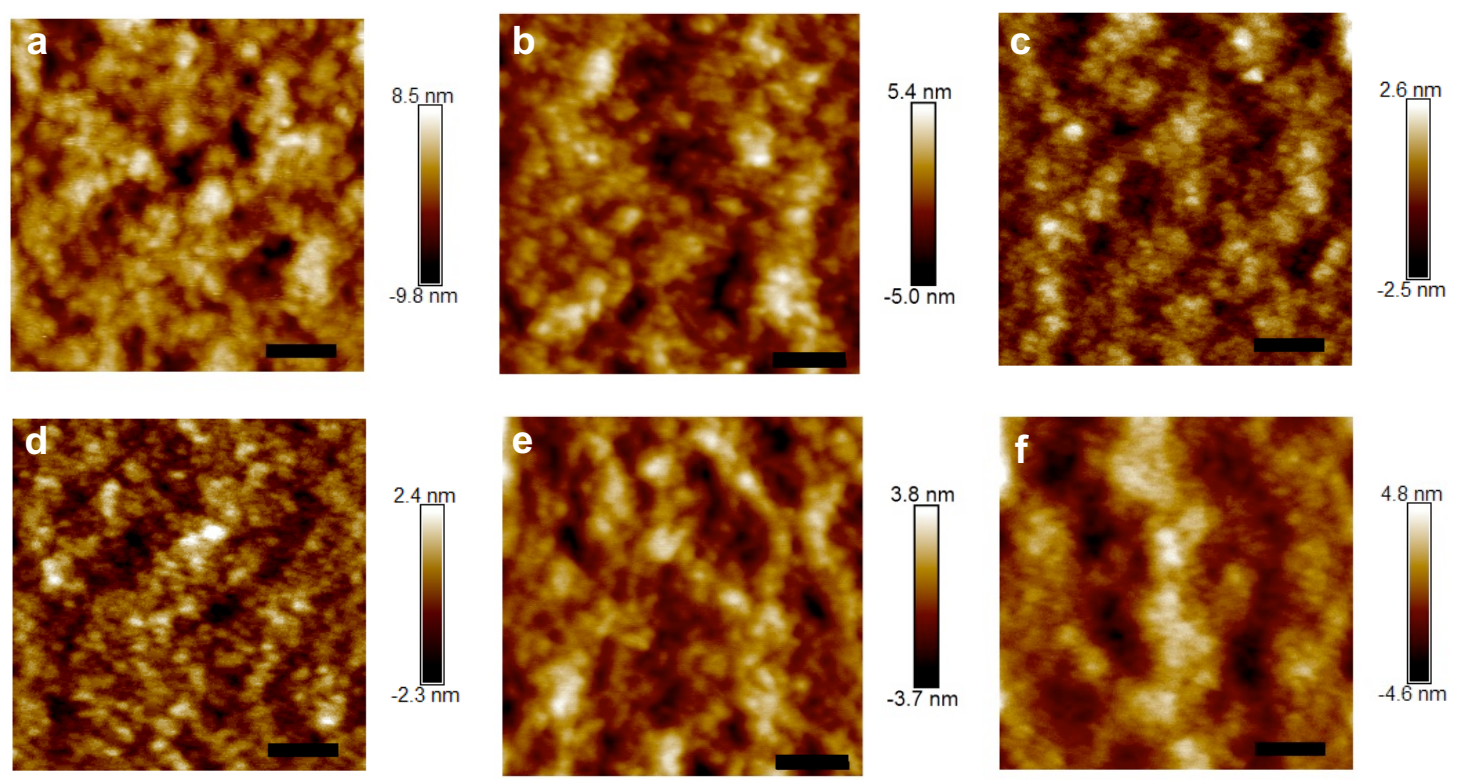

Figure 3.13. AFM height images of bulkjunction films for (a) PBPB, (b) 3.1, (c) 3.3, (d) (PPh2)4, (e) 1.1, and (f) 3.6. The mass ratio of donor-to-acceptor is fixed at 1:2.1\% CN additive was used. The scale bar is $200 \mathrm{~nm}$.

\subsection{General Experimental Information}

Synthesis. All reactions were performed in oven-dried or flame-dried round bottom flasks, unless otherwise noted. The flasks were fitted with rubber septa and reactions were conducted under a positive pressure of nitrogen or argon, unless otherwise noted. Anhydrous and anaerobic solvents were obtained from a Glass Contour solvent system consisting of a Schlenk manifold with purification columns packed with activated alumina and supported copper catalyst. Reaction monitoring by TLC was performed on J.T. Baker Baker-flex Silica Gel IB2-F (25 mm x 75 mm) TLC plates. TLC visualization was accomplished by visible observation and irradiation with a UV lamp. Commercial reagents were used without further purification. $\mathrm{Pt}(\mathrm{COD}) \mathrm{Cl}_{2}$ was purchased from Strem Chemicals, and all other reagents were purchased from Sigma-Aldrich.

Purification. Automated flash chromatography was performed using a Teledyne Isco Combiflash Rf200 and Redisep Rf Silica columns. Preparative high performance liquid chromatography (HPLC) was performed on a Waters Prep150 instrument equipped with a UV-vis 
detector, an automated fraction collector, and a Nacalai Tesque COSMOSIL Buckyprep column (20 mm I.D. x $250 \mathrm{~mm}, 5 \mu \mathrm{m})$.

Spectrometers. ${ }^{1} \mathrm{H}$ NMR spectra were recorded on a Bruker 400 or $500 \mathrm{MHz}$ spectrometer. ${ }^{13} \mathrm{C}$ NMR spectra were recorded on a Bruker 125 or $100 \mathrm{MHz}$ spectrometer with complete proton decoupling. NMR spectra were recorded at $300 \mathrm{~K}$ unless otherwise noted. Chemical shifts are reported in parts per million (ppm) Data are represented as follows: chemical shift, multiplicity (s $=$ singlet, $\mathrm{d}=$ doublet, $\mathrm{dd}=$ doublet of doublets, $\mathrm{t}=$ triplet, $\mathrm{m}=$ multiplet $)$, coupling constants in $\mathrm{Hz}$, and integration. Broadening (br) of peaks in the ${ }^{1} \mathrm{HNMR}$ spectrum is due to rotational isomers about the imide side chains. Multiple peaks for the same carbon in the ${ }^{13} \mathrm{C}$ NMR spectrum arise due to rotational isomers about the imide side chains that have been seen previously. ${ }^{13,65}$ HRMS was performed on a (1) Waters XEVO G2-XS QTOF instrument equipped with a UPC SFC inlet, and ESI and APCI ionization sources; or (2) a Bruker UltrafleXtreme MALDI TOF/TOF instrument using a dithranol matrix. UV-vis absorption spectra were recorded on a Shimadzu UV1800 spectrophotometer using a $1.0 \mathrm{~cm}$ quartz cell. IR spectra were recorded on a Perkin Elmer Spectrum400 FTIR spectrometer using a PIKE ATR attachment. GPC analysis was done on an Agilent Technologies 1200 Infinity Series GPC fitted with a refractive index detector and a UVvisible detector. All samples were eluted at $1.0 \mathrm{~mL} / \mathrm{min}$ through two Agilent Technologies PLgel $5 \mu \mathrm{m}$ MIXED-D 300x7.5mm columns and monitored by their $U V$-vis signal. $M_{n}$ and $M_{w}$ were assigned based off of polystyrene standards.

Atomic force microscopy. AFM measurements were carried out in tapping mode on a Bruker Multi-Mode AFM at ambient conditions. A commercial silicon cantilever (RTESPA, MPP$11120-10$, Bruker) was used in this study with a typical radius of curvature of $\sim 8 \mathrm{~nm}$ and a nominal spring constant of $\sim 40 \mathrm{Nm}^{-1}$. 
Cyclic Voltammetry. CVs were recorded on a CH166 electrochemical workstation using an $\mathrm{Ag} / \mathrm{AgCl}$ electrode as the reference electrode at room temperature. Experiments were performed in $\mathrm{CH}_{2} \mathrm{Cl}_{2}$ with $\mathrm{NBu}_{4} \mathrm{PF}_{6}$ as the supporting electrolyte at a scan rate of $0.1 \mathrm{~V} / \mathrm{s} .{ }^{57}$

Thin film transistors. To create the devices, we first silanize the substrate $\left(300 \mathrm{~nm}\right.$ of $\mathrm{SiO}_{2}$ on a Si wafer) with octadecyltrichlorosilane (OTS). Au is deposited onto the substrate as bottomcontact source and drain electrodes $(40 \mathrm{~nm})$ with a width of $105 \mu \mathrm{m}$ and length of $20 \mu \mathrm{m}$. Next, we spin-cast organic films onto the surface at 1,000 r.p.m. for $1 \mathrm{~min}$, to form transistors using the silicon wafer as the global back gate for the device. Finally, the samples were annealed under inert atmosphere at $160^{\circ} \mathrm{C}$ for 10 minutes to optimize device performance except that the $\mathbf{3 . 5}$ film was annealed at $120^{\circ} \mathrm{C}$. The thin film transistors were tested on the Agilent $4155 \mathrm{C}$ semiconductor parameter analyzer.

Solar cell fabrication. PTB7-Th was purchased from 1-Material Inc. Synthesis of $\mathrm{ZnO}$ sol-gel precursor was described elsewhere. ${ }^{56}$ Zinc acetate dihydrate, ethanolamine, 2methoxyethanol, DIO and all of the solvents were purchased from Sigma Aldrich. Pre-patterned ITO-coated glass with a sheet resistance of $\sim 15 \Omega \mathrm{sq}^{-1}$ was cleaned with detergent and ultrasonicated in deionized water, acetone and isopropanol for $30 \mathrm{~min}$, respectively. Subsequently, we treated the substrates by ultraviolet-ozone for $10 \mathrm{~min}$. The prepared $\mathrm{ZnO}$ precursor was spincast onto the ITO substrate at 3,000 r.p.m. for $1 \mathrm{~min}$, followed by annealing at $200^{\circ} \mathrm{C}$ for $1 \mathrm{~h}$ in air, to form a thin film with approximate thickness of $20 \mathrm{~nm}$. Active layers were prepared by spincoating a mixed solution containing polymer and acceptor in chlorobenzene at a total concentration of $25 \mathrm{mgml}^{-1}$. The thickness of the prepared active layers is about $100 \mathrm{~nm}$. Finally, a $7 \mathrm{~nm} \mathrm{MoO}$ layer was deposited first and then a 100-nm Ag electrode was subsequently deposited through a shadow mask by thermal evaporation under a vacuum about $1 \times 10^{-6}$ torr. The current density- 
voltage $(J-V)$ curves were measured by a Keithley 2635A source measure unit. The photocurrent was measured under AM 1.5G illumination at $100 \mathrm{mWcm}^{-2}$ under a Newport solar Simulator. A KG5-Si reference cell traceable to Newport was used to calibrate light intensity. The effective device area was defined as $6.25 \mathrm{~mm}^{2}$ by an aperture mask. EQE measurements were performed using a QEXL system from PV Measurements Inc.

\subsection{Synthetic Procedures and Characterization}

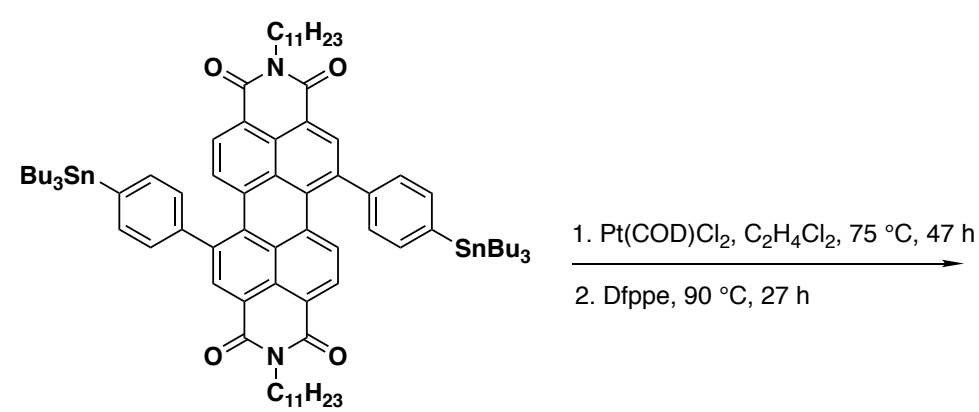

2.1

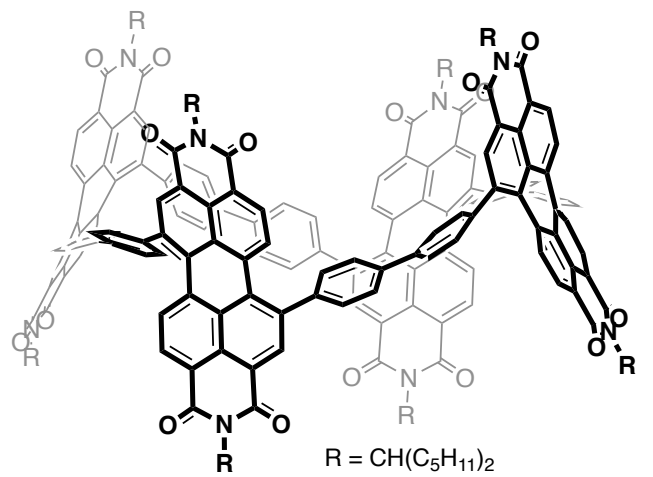

Synthesis of $\left(\mathbf{P P h}_{2}\right)_{4}$ : Compound 2.1 (1.04 g, $0.727 \mathrm{mmol}, 1.00$ equiv) and $\mathrm{Pt}(\mathrm{COD}) \mathrm{Cl}_{2}(0.272$ a stir bar. 1,2-Dichloroethane $(300 \mathrm{~mL})$ was added, and the mixture was sparged with $\mathrm{N}_{2}$ for 30 min at ambient temperature. Under positive pressure of $\mathrm{N}_{2}$, the flask was placed in an oil bath at $75^{\circ} \mathrm{C}$ with stirring for $47 \mathrm{~h}$. The mixture was allowed to cool to ambient temperature before adding 1,2-bis[bis(pentafluorophenyl)phosphino]ethane $(2.76 \mathrm{~g}, 3.63 \mathrm{mmol}, 5.00$ equiv). The mixture was stirred at room temperature for $30 \mathrm{~min}$, fitted with a reflux condenser, and placed in an oil bath at $90{ }^{\circ} \mathrm{C}$ for $27 \mathrm{~h}$. The mixture was allowed to cool to room temperature then concentrated under reduced pressure. The crude mixture was loaded onto a silica column (120 g Redisep Rf Silica) and purified with a gradient from 9:1 $\mathrm{CH}_{2} \mathrm{Cl}_{2} /$ hexanes to $\mathrm{CH}_{2} \mathrm{Cl}_{2}$ to $9: 1 \mathrm{CH}_{2} \mathrm{Cl}_{2} / \mathrm{MeOH}$ at $85 \mathrm{~mL} / \mathrm{min}$. Fractions containing $\left(\mathbf{P} \mathbf{P h}_{2}\right)_{4}$ were identified by MALDI-TOF MS and were collected and concentrated. These fractions were further purified by preparative HPLC on a COSMOSIL 
Buckyprep column ( $20 \times 250 \mathrm{~mm})$ with an eluent of $12 \% \mathrm{CH}_{2} \mathrm{Cl}_{2} /$ hexanes (v/v) at a flow rate of $18.9 \mathrm{~mL} / \mathrm{min}$. The pure fractions were concentrated to give $\left(\mathbf{P P h}_{2}\right)_{4}$ as a dark purple solid $(0.0530$ g, $0.0150 \mathrm{mmol}, 8 \%$ yield). ${ }^{1} \mathrm{H}$ NMR $\left(500 \mathrm{MHz}, \mathrm{C}_{2} \mathrm{D}_{2} \mathrm{Cl}_{4}, 413 \mathrm{~K}\right) \delta 8.62(\mathrm{~s}, 8 \mathrm{H}), 8.39$ (d, J = 8.0 $\mathrm{Hz}, 8 \mathrm{H}), 8.32(\mathrm{~d}, \mathrm{~J}=8.0 \mathrm{~Hz}, 8 \mathrm{H}), 7.75(\mathrm{~d}, \mathrm{~J}=8.4 \mathrm{~Hz}, 16 \mathrm{H}), 7.64(\mathrm{br} \mathrm{d}, \mathrm{J}=7.7 \mathrm{~Hz}, 16 \mathrm{H}), 5.21-$ $5.13(\mathrm{~m}, 8 \mathrm{H}), 2.30-2.18(\mathrm{~m}, 16 \mathrm{H}), 2.00-1.89(\mathrm{~m}, 16 \mathrm{H}), 1.44-1.29(\mathrm{~m}, 96 \mathrm{H}), 0.89(\mathrm{t}, \mathrm{J}=7.0$ $\mathrm{Hz}, 48 \mathrm{H}) .{ }^{13} \mathrm{C}$ NMR $(125 \mathrm{MHz}, \mathrm{C} 2 \mathrm{D} 2 \mathrm{Cl} 4,383 \mathrm{~K}) \delta 163.82,163.57,141.33,140.40,134.93$, $134.52,132.57,130.13,129.89,129.52,129.35,128.46$ (br), 127.45, 122.64, 122.30, 54.74, 32.34, 31.44, 26.38, 22.13, 13.53. IR (cm-1) 2955, 2927, 2860, 1695, 1657, 1595, 1585, 1456, 1407, $1324,1238,1180,1127,1096,1003,812$. HRMS (MALDI-) calculated $m / z$ for $\left[\mathrm{C}_{232} \mathrm{H}_{240} \mathrm{~N}_{8} \mathrm{O}_{16}\right]^{-}$ 3393.821, found 3393.715 .

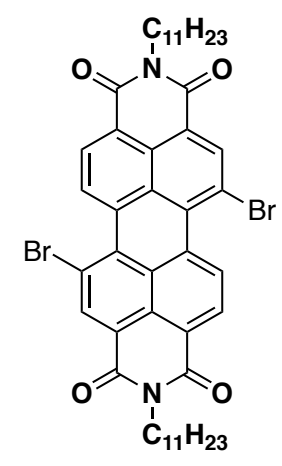

1,7-dibromo PDI

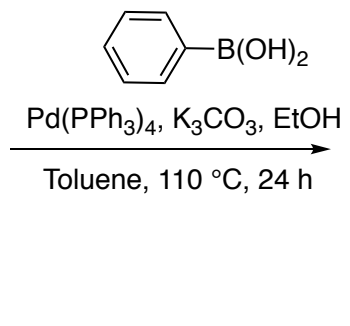
.

Synthesis of SI-3.1 and 1.1: $N, N^{\prime}$-Di(6-undecyl)-1,7-dibromoperylene-3,4:9,10-tetracarboxylic diimide ( $0.600 \mathrm{~g}, 0.703 \mathrm{mmol}, 1.00$ equiv), phenyl boronic acid (0.0430 g, $0.352 \mathrm{mmol}, 0.500$ equiv), aqueous $\mathrm{K}_{2} \mathrm{CO}_{3}(2 \mathrm{M}, 6.00 \mathrm{~mL})$, $\mathrm{EtOH}(1.20 \mathrm{~mL})$ and toluene $(18.0 \mathrm{~mL})$ were added to a two-neck 100-mL round bottom flask equipped with a stir bar. The mixture was sparged with $\mathrm{N}_{2}$ for thirty minutes. While under $\mathrm{N}_{2}$, tetrakis(triphenylphosphine)palladium(0) $(0.0810 \mathrm{~g}, 0.0703$ mmol, 0.100 equiv) was added. The mixture was further sparged for ten minutes before being fitted with a condenser and placed in a $100{ }^{\circ} \mathrm{C}$ oil bath under $\mathrm{N}_{2}$, stirring for twenty-four hours. The 
crude mixture was extracted with ethyl acetate and brine, concentrated and purified by column chromatography (40 g Redisep Rf Silica) using a gradient from $0 \%$ to $100 \% \mathrm{CH}_{2} \mathrm{Cl}_{2} /$ hexanes at $40 \mathrm{~mL} / \mathrm{min}$. Further purification by prep HPLC (COSMOSIL Buckyprep 20 x $250 \mathrm{~mm}, 18.9$ $\mathrm{mL} / \mathrm{min}$ and 3:93 $\mathrm{CH}_{2} \mathrm{Cl}_{2}:$ hexanes $)$ yielded SI-3.1 (0.0930 g, $\left.0.109 \mathrm{mmol}, 16 \%\right)$ and $\mathbf{1 . 1}(0.0290$ g, 0.0341, 5.0\%) SI-3.1: ${ }^{1} \mathbf{H}$ NMR (400 MHz, $\left.\mathrm{CDCl}_{3}\right) \delta 9.54(\mathrm{~d}, \mathrm{~J}=8.2 \mathrm{~Hz}, 1 \mathrm{H}), 8.90$ (br, $\left.1 \mathrm{H}\right)$, $8.66(\mathrm{br}, 2 \mathrm{H}), 8.11(\mathrm{br}, 1 \mathrm{H}), 7.80(\mathrm{~d}, \mathrm{~J}=8.2 \mathrm{~Hz}, 1 \mathrm{H}), 7.49(\mathrm{br}, 5 \mathrm{H}), 5.24-5.16(\mathrm{~m}, 1 \mathrm{H}), 5.12(\mathrm{br}$, 1H), $2.30-2.17(\mathrm{~m}, 4 \mathrm{H}), 1.88-1.79(\mathrm{~m}, 4 \mathrm{H}), 1.26(\mathrm{br}, 24 \mathrm{H}), 0.85(\mathrm{br}, 12 \mathrm{H}) .{ }^{13} \mathbf{C}$ NMR $(100$ $\left.\mathrm{MHz}, \mathrm{CDCl}_{3}\right) \delta 164.61,164.26,163.53,162.54,141.96,141.43,138.48,137.83,135.74,135.07$, $133.91,133.70,133.63,131.62,130.37,130.30,130.18,129.99,129.77,129.58,129.28,128.99$, $128.80,128.67,128.24,127.70,127.41,123.87,123.16,122.45,121.76,120.29,54.87,54.76$, $32.34,32.26,31.75,31.72,26.58,22.57,22.54,14.04$. IR $\left(\mathrm{cm}^{-1}\right) 2955,2925,2857,1698,1657$, 1596, 1587, 1456, 1447, 1403, 1326, 1239, 1184, 914, 812, 734. HRMS (APCI+) calculated $\mathrm{m} / \mathrm{z}$ for $\left[\mathrm{C}_{52} \mathrm{H}_{57} \mathrm{~N}_{2} \mathrm{O}_{4} \mathrm{Br}+\mathrm{H}\right]^{+}$853.3580, found 853.3572 .3 .1 : ${ }^{1} \mathbf{H}$ NMR $\left(400 \mathrm{MHz}, \mathrm{CDCl}_{3}\right) \delta 8.62(\mathrm{br}$, 2H), $8.12(\mathrm{br}, 2 \mathrm{H}), 7.82(\mathrm{~d}, \mathrm{~J}=8.2 \mathrm{~Hz}, 2 \mathrm{H}), 7.59-7.55(\mathrm{~m}, 4 \mathrm{H}), 7.54-7.45(\mathrm{~m}, 6 \mathrm{H}), 5.15(\mathrm{br}$, 2H), $2.29-2.16(\mathrm{~m}, 4 \mathrm{H}), 1.88-1.76(\mathrm{~m}, 4 \mathrm{H}), 1.37-1.18(\mathrm{~m}, 24 \mathrm{H}), 0.83(\mathrm{t}, \mathrm{J}=6.6 \mathrm{~Hz}, 12 \mathrm{H}) .{ }^{13} \mathrm{C}$ NMR $\left(100 \mathrm{MHz}, \mathrm{CDCl}_{3}\right) \delta 164.70,163.63,142.23,141.08,135.78,135.10,134.81,132.49$, $130.22,130.16,129.91,129.23,129.17,129.03,128.59,127.90,122.87,122.53,122.13,121.77$, $54.65,32.32,31.75,26.59,22.55,14.04$. IR $\left(\mathrm{cm}^{-1}\right) 2956,2925,2860,1695,1657,1598,1585$, $1409,1325,1242,910,814$. HRMS (APCI-) calculated $m / z$ for $\left[\mathrm{C}_{58} \mathrm{H}_{62} \mathrm{~N}_{2} \mathrm{O}_{4}\right]^{-} 850.4710$, found 850.4704. 1.1 was synthesized in Chapter 4 in a 97\% yield using different conditions. 


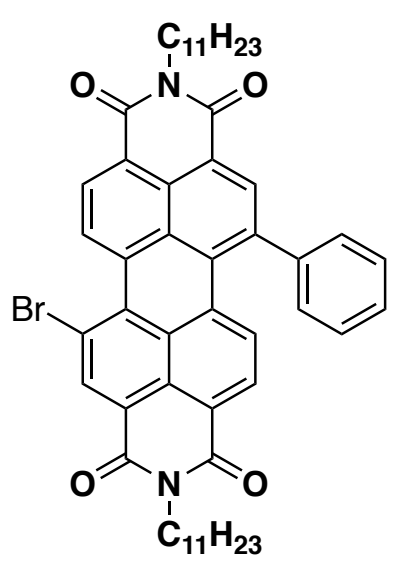

SI-3.1

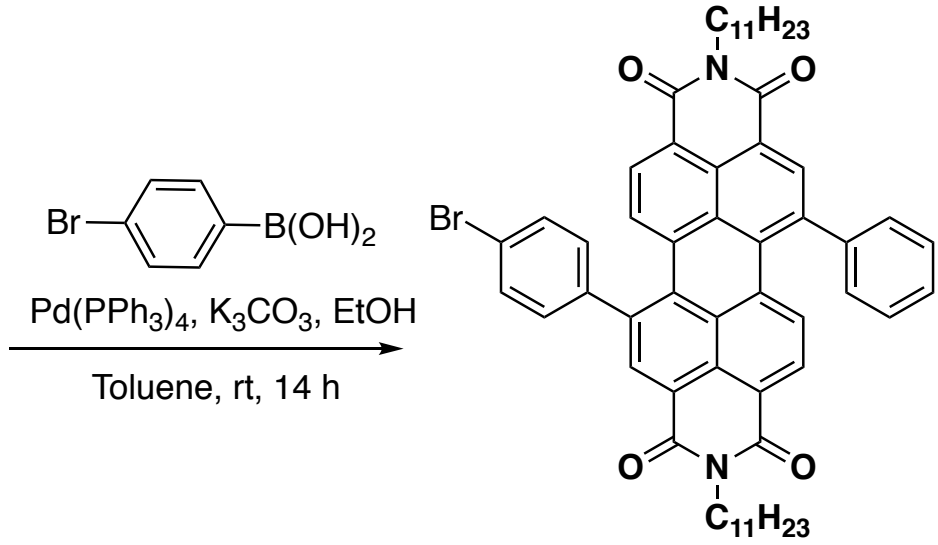

SI-3.2

Synthesis of SI-3.2: SI-3.1 ( $0.120 \mathrm{~g}, 0.141 \mathrm{mmol}, 1.00$ equiv), 4-bromophenylboronic acid (0.0850 g, $0.422 \mathrm{mmol}, 3.00$ equiv), aqueous $\mathrm{K}_{2} \mathrm{CO}_{3}(2 \mathrm{M}, 1.20 \mathrm{~mL}), \mathrm{EtOH}(0.240 \mathrm{~mL})$ and toluene $(3.60 \mathrm{~mL})$ were added to $20 \mathrm{~mL}$ scintillation vial equipped with a stir bar. The mixture was sparged with $\mathrm{N}_{2}$ for thirty minutes. While under $\mathrm{N}_{2}$, tetrakis(triphenylphosphine)palladium(0) $(0.0160 \mathrm{~g}$, $0.0104 \mathrm{mmol}, 0.100$ equiv) was added. The mixture was further sparged for ten minutes and stirred overnight at room temperature. The crude mixture was extracted with ethyl acetate and brine, concentrated and purified by column chromatography (40 g Redisep Rf Silica) using a gradient from $0 \%$ to $100 \% \mathrm{CH}_{2} \mathrm{Cl}_{2} /$ hexanes at $40 \mathrm{~mL} / \mathrm{min}$. Impure fractions were further purified by prep HPLC (COSMOSIL Buckyprep $20 \times 250 \mathrm{~mm}, 18.9 \mathrm{~mL} / \mathrm{min}$ and 7:93 $\mathrm{CH}_{2} \mathrm{Cl}_{2}$ :hexanes) to yield a total $0.0700 \mathrm{~g}(0.0750 \mathrm{mmol}, 54 \%)$ of SI-3.2. ${ }^{1} \mathbf{H}$ NMR $\left(400 \mathrm{MHz}, \mathrm{C}_{2} \mathrm{D}_{2} \mathrm{Cl}_{4}, 390 \mathrm{~K}\right) \delta 8.65(\mathrm{~s}, 1 \mathrm{H})$, $8.60(\mathrm{~s}, 1 \mathrm{H}), 8.24(\mathrm{~d}, \mathrm{~J}=8.2 \mathrm{~Hz}, 1 \mathrm{H}), 8.18(\mathrm{~d}, \mathrm{~J}=8.2 \mathrm{~Hz}, 1 \mathrm{H}), 7.92(\mathrm{~d}, \mathrm{~J}=8.1 \mathrm{~Hz}, 2 \mathrm{H}), 7.74(\mathrm{~d}, \mathrm{~J}$ $=8.4 \mathrm{~Hz}, 2 \mathrm{H}), 7.65-7.51(\mathrm{~m}, 7 \mathrm{H}), 5.23-5.14(\mathrm{~m}, 2 \mathrm{H}), 2.32-2.24(\mathrm{~m}, 4 \mathrm{H}), 1.98-1.93(\mathrm{~m}, 4 \mathrm{H})$, $1.38(\mathrm{br}, 24 \mathrm{H}), 0.93(\mathrm{br}, 12 \mathrm{H}) .{ }^{13} \mathrm{C} \mathbf{N M R}\left(100 \mathrm{MHz}, \mathrm{C}_{2} \mathrm{D}_{2} \mathrm{Cl}_{4}, 390 \mathrm{~K}\right) \delta 163.98,163.92,163.88$, $142.54,141.56,141.36,139.62,135.32,134.90,134.83,134.54,133.46,132.51,132.34,130.79$, $130.22,130.19,130.10,129.63,129.44,129.34,129.04,128.70,128.19,128.15,123.17,123.14$, 123.08, 123.01, 122.75, 55.43, 32.67, 31.74, 26.68, 22.42, 13.80. IR $\left(\mathrm{cm}^{-1}\right) 2955,2926,2860$, 
1697, 1658, 1597, 1587, 1459, 1408, 1325, 1241, 1185, 813. HRMS (APCI+) calculated $\mathrm{m} / z$ for $\left[\mathrm{C}_{58} \mathrm{H}_{61} \mathrm{~N}_{2} \mathrm{O}_{4} \mathrm{Br}+\mathrm{H}\right]^{+}$929.3893, found 929.3885.

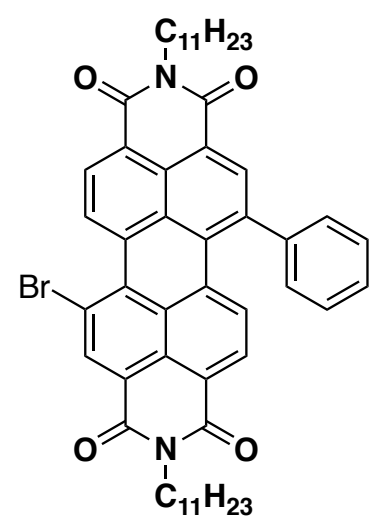

SI-3.1

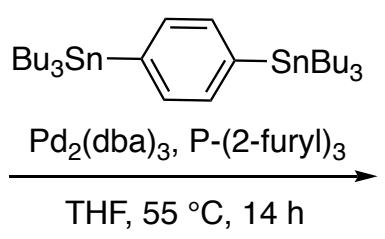

THF, $55^{\circ} \mathrm{C}, 14 \mathrm{~h}$

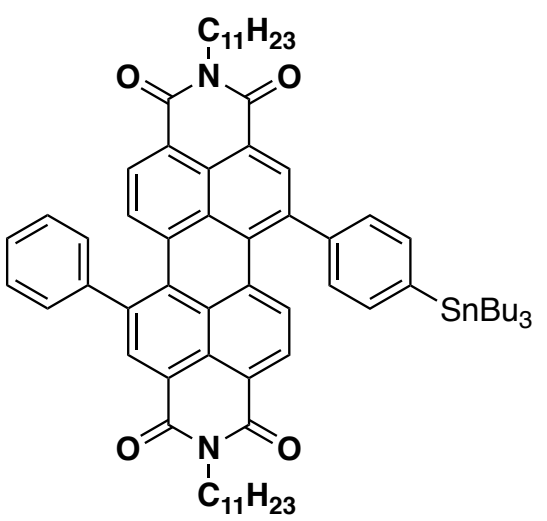

SI-3.3

Synthesis of SI-3.3: SI-3.1 (0.0930 g, $0.109 \mathrm{mmol}, 1.00$ equiv), 1,4-bis(tributylstannyl)benzene (0.0600 mL, $0.0720 \mathrm{mmol}, 2.50$ equiv) and THF $(90.0 \mathrm{~mL})$ were added to a $20 \mathrm{~mL}$ scintillation vial equipped with a stir bar. The mixture was sparged with $\mathrm{N}_{2}$ for thirty minutes. While under $\mathrm{N}_{2}$, tris(dibenzylideneacetone)dipalladium $(0.0100$ g, $0.0109 \mathrm{mmol}, 0.100$ equiv) and tri(2furyl)phosphine $(0.0110 \mathrm{~g}, 0.0436 \mathrm{mmol}, 0.400$ equiv) were added. The mixture was further sparged for ten minutes before being placed in a $55^{\circ} \mathrm{C}$ oil bath and stirred overnight. The crude mixture was condensed and purified by column chromatography (40 g Redisep Rf Silica) using a gradient from $0 \%$ to $100 \% \mathrm{CH}_{2} \mathrm{Cl}_{2} /$ hexanes at $40 \mathrm{~mL} / \mathrm{min}$ to yield $\mathbf{S I - 3 . 3}(0.0770 \mathrm{~g}, 0.0680 \mathrm{mmol}$, 62\% yield). ${ }^{1} \mathrm{H}$ NMR (500 MHz, $\left.\mathrm{CDCl}_{3}\right) \delta 8.62(\mathrm{br}, 2 \mathrm{H}), 8.11(\mathrm{br}, 2 \mathrm{H}), 7.88(\mathrm{~d}, \mathrm{~J}=8.5 \mathrm{~Hz}, 1 \mathrm{H})$, $7.81(\mathrm{~d}, \mathrm{~J}=6.3 \mathrm{~Hz}, 1 \mathrm{H}), 7.63-7.54 *(\mathrm{~m}, \mathrm{~J}=7.1 \mathrm{~Hz}, 4 \mathrm{H}), 7.52-7.48(\mathrm{~m}, 5 \mathrm{H}), 5.15(\mathrm{br}, 2 \mathrm{H}), 2.25$ $-2.21(\mathrm{~m}, 4 \mathrm{H}), 1.82(\mathrm{br}, 4 \mathrm{H}), 1.66-1.54 *(\mathrm{~m}, 6 \mathrm{H}), 1.43-1.35(\mathrm{~m}, 6 \mathrm{H}), 1.26(\mathrm{br}, 24 \mathrm{H}), 1.18-$ 1.09* (m, 6H), $0.94(\mathrm{t}, \mathrm{J}=7.3 \mathrm{~Hz}, 9 \mathrm{H}), 0.83$ (br t, J = 6.6 Hz, 12H). ${ }^{13} \mathbf{C} \mathbf{N M R}\left(125 \mathrm{MHz}, \mathrm{CDCl}_{3}\right)$ $\delta 164.77,163.66,143.14,142.28,141.68,141.33,141.00,138.19,138.08,135.87,135.41,135.16$, $134.91,134.76,132.57,132.40,130.20,130.17,130.15,129.97,129.84,129.26,129.06,128.58$, $128.28,127.92,127.87,122.83,122.50,122.14,121.77,54.63,32.34,31.77,29.14^{*}, 27.37^{*}$, 
26.60, 26.59, 22.56, 14.05, 13.73, 9.75*. IR $\left(\mathrm{cm}^{-1}\right) 2956,2925,2855,1697,1657,1598,1586$, $1459,1447,1408,1325,1264,1239,863,814,739$. HRMS (APCI+) calculated $m / z$ for $\left[\mathrm{C}_{70} \mathrm{H}_{88} \mathrm{~N}_{2} \mathrm{O}_{4} \mathrm{Sn}+\mathrm{H}\right]^{+} \quad 1141.5844$, found 1141.5859. ${ }^{*}$ Tin satellite peaks visible.

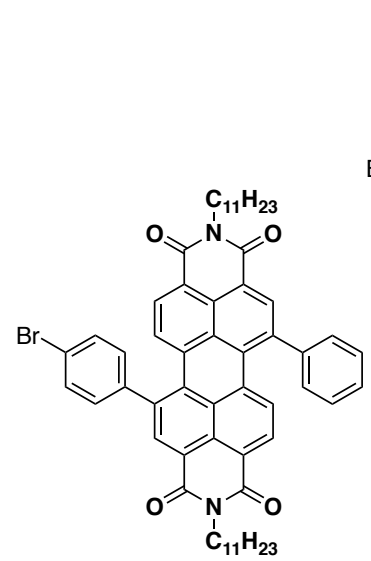

SI-3.2

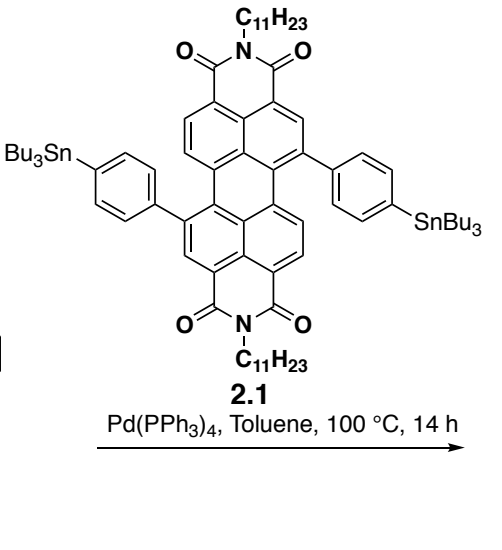

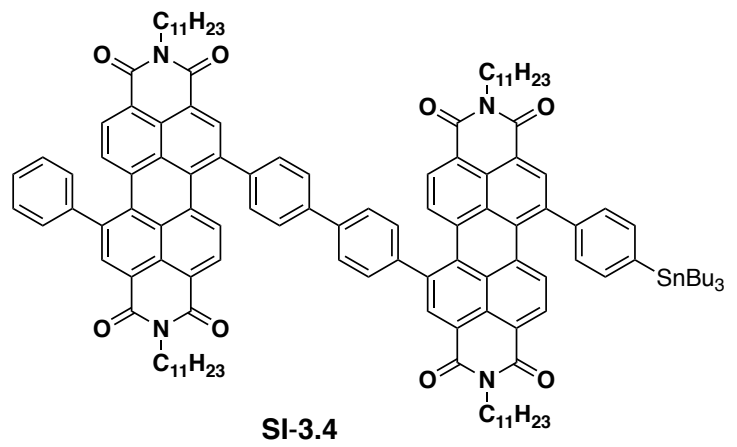

SI-3.4

Synthesis of SI-3.4: SI-3.2 ( $0.0750 \mathrm{~g}, 0.0810 \mathrm{mmol}, 1.00$ equiv) and $\mathbf{2 . 1}(0.463 \mathrm{~g}, 0.324 \mathrm{mmol}$, 4.00 equiv) were dissolved in toluene $(8.00 \mathrm{~mL})$ in a $20 \mathrm{~mL}$ scintillation vial equipped with a stir bar. The mixture was sparged with $\mathrm{N}_{2}$ for thirty minutes. While under $\mathrm{N}_{2}$, tetrakis(triphenylphosphine)palladium( 0$)(0.00900 \mathrm{~g}, 0.00810 \mathrm{mmol}, 0.100$ equiv) was added. The mixture was further sparged for ten minutes before being placed in a $110^{\circ} \mathrm{C}$ oil bath and stirred overnight. The crude mixture was condensed and purified by column chromatography ( $40 \mathrm{~g}$ Redisep Rf Silica) using a gradient from $0 \%$ to $100 \% \mathrm{CH}_{2} \mathrm{Cl}_{2} /$ hexanes at $40 \mathrm{~mL} / \mathrm{min}$. Further purification by preparative HPLC (COSMOSIL Buckyprep 20 x $250 \mathrm{~mm}, 18.9 \mathrm{~mL} / \mathrm{min}, 7: 93$ $\mathrm{CH}_{2} \mathrm{Cl}_{2}$ :hexanes) yielded SI-3.4 (0.0270 g, $0.0140 \mathrm{mmol}, 17 \%$ yield). ${ }^{1} \mathbf{H}$ NMR (500 MHz, $\left.\mathrm{CDCl}_{3}\right)$ $\delta 8.67$ (br, 4H), $8.17(\mathrm{br}, 4 \mathrm{H}), 8.03(\mathrm{~d}, J=3.2 \mathrm{~Hz}, 1 \mathrm{H}), 8.01(\mathrm{~d}, J=3.2 \mathrm{~Hz}, 1 \mathrm{H}), 7.91(\mathrm{~d}, \mathrm{~J}=8.5$ $\mathrm{Hz}, 1 \mathrm{H}), 7.87(\mathrm{~d}, \mathrm{~J}=7.6,4 \mathrm{H}), 7.85(\mathrm{~d}, \mathrm{~J}=9.1 \mathrm{~Hz}, 1 \mathrm{H}), 7.74(\mathrm{~d}, \mathrm{~J}=2.0 \mathrm{~Hz}, 2 \mathrm{H}), 7.73(\mathrm{~d}, \mathrm{~J}=2.3$ $\mathrm{Hz}, 2 \mathrm{H}), 7.61-7.59^{*}(\mathrm{~m}, 4 \mathrm{H}), 7.54-7.50^{*}(\mathrm{~m}, 5 \mathrm{H}), 5.17$ (br, 4H), $2.27-2.19$ (br m, 8H), 1.83 (br, 8H), $1.67-1.60 *(\mathrm{~m}, 6 \mathrm{H}), 1.44-1.35(\mathrm{~m}, 6 \mathrm{H}), 1.35-1.21$ (br m, 48H), $1.19-1.11 *(\mathrm{~m}$, 6H), $0.95(\mathrm{t}, \mathrm{J}=7.3 \mathrm{~Hz}, 9 \mathrm{H}), 0.86-0.81(\mathrm{~m}, 24 \mathrm{H}) .{ }^{13} \mathbf{C} \mathbf{N M R}\left(100 \mathrm{MHz}, \mathrm{CDCl}_{3}\right) \delta$ 164.70, 163.64 
$143.19,142.22,141.78,141.72,141.66,141.40,141.15,140.48,140.39,140.20,140.15,138.10$ $135.82,135.01,134.82,132.57,130.28,130.21,130.03,129.79,129.35,129.34,129.28,129.16$ $129.07,128.75,128.64,128.30,127.97,127.93,122.90,122.62,122.21,121.90,54.68,32.34$, $31.78,29.15^{*}, 27.38^{*}, 26.61,22.58,14.07,13.75,9.76^{*} . \mathbf{I R}\left(\mathrm{cm}^{-1}\right) 2955,2927,2859,1698,1659$, $1599,1587,1457,1409,1326,1240,1183,814$. HRMS (APCI+) calculated $\mathrm{m} / \mathrm{z}$ for $\left[\mathrm{C}_{128} \mathrm{H}_{148} \mathrm{~N}_{4} \mathrm{O}_{8} \mathrm{Sn}+\mathrm{Na}\right]^{+}$2012.0217, found 2012.0212. *Tin satellite peaks visible. that have been seen previously.
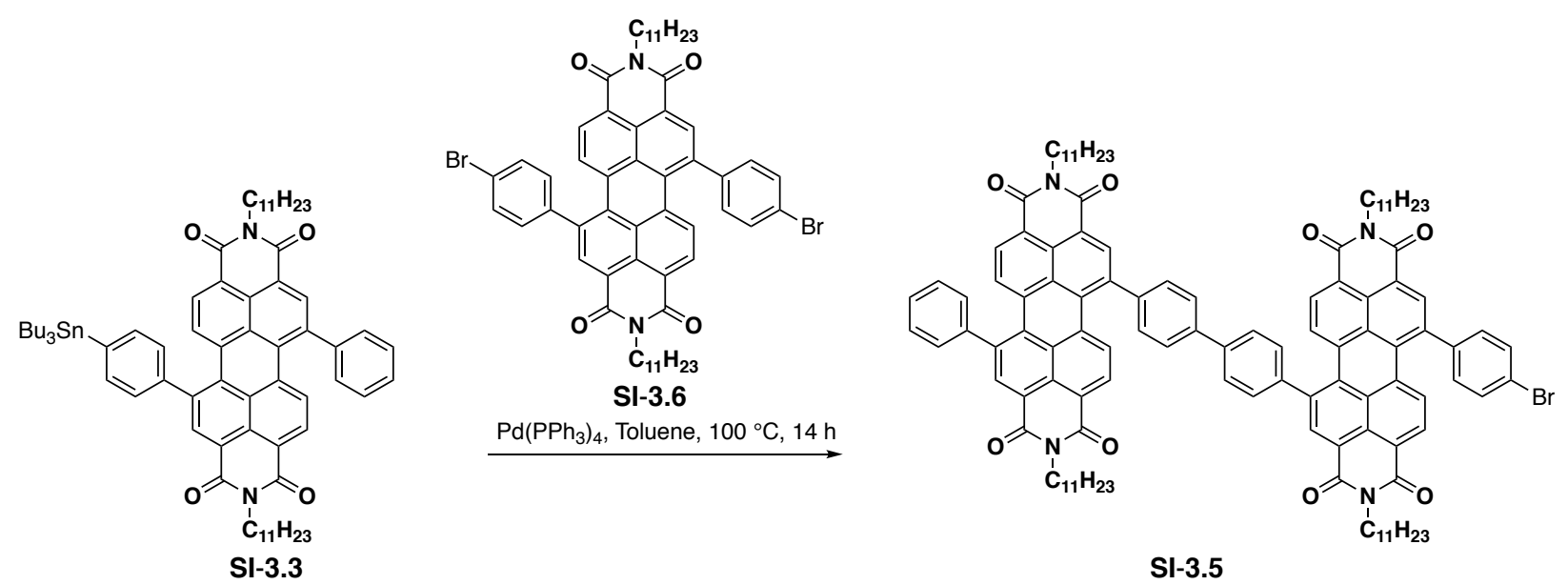

Synthesis of SI-3.5. SI-3.3 (0.0550 g, $0.0480 \mathrm{mmol}, 1.00$ equiv) and $N, N^{\prime}$-di(6-undecyl)-1,7-di(4bromophenyl)- perylene-3,4:9,10-tetracarboxylic diimide (SI-3.6) (0.194 g, $0.193 \mathrm{mmol}, 4.00$ equiv) were dissolved in toluene $(6 \mathrm{~mL})$ in a $20 \mathrm{~mL}$ scintillation vial equipped with a stir bar. The mixture was sparged with $\mathrm{N}_{2}$ for thirty minutes. While under $\mathrm{N}_{2}$, tetrakis(triphenylphosphine)palladium( 0$)(0.00600 \mathrm{~g}, 0.00480 \mathrm{mmol}, 0.100$ equiv) was added. The mixture was further sparged for ten minutes before being placed in a $110{ }^{\circ} \mathrm{C}$ oil bath and stirred overnight. The crude mixture was condensed and purified by column chromatography ( $24 \mathrm{~g}$ Redisep Rf Silica) using a gradient from $0 \%$ to $100 \% \mathrm{CH}_{2} \mathrm{Cl}_{2} /$ hexanes at $40 \mathrm{~mL} / \mathrm{min}$. Further purification using preparative HPLC (Buckyprep, isocratic 15:85 $\mathrm{CH}_{2} \mathrm{Cl}_{2}$ :hexane) yielded SI-3.5 (0.0150 g, 0.00800 mmol, $18 \%$ yield). ${ }^{1} \mathbf{H}$ NMR $\left(500 \mathrm{MHz}, \mathrm{CDCl}_{3}\right) \delta 8.71-8.58$ (br m, 4H), 8.24 
$-8.12($ br m, 4H), $8.02(\mathrm{~d}, J=8.4 \mathrm{~Hz}, 2 \mathrm{H}), 7.87(\mathrm{~d}, J=7.9 \mathrm{~Hz}, 4 \mathrm{H}), 7.85(\mathrm{~d}, J=8.2 \mathrm{~Hz}, 2 \mathrm{H}), 7.74$ $-7.72(\mathrm{~m}, 4 \mathrm{H}), 7.67(\mathrm{~d}, J=8.0 \mathrm{~Hz}, 2 \mathrm{H}), 7.60(\mathrm{~d}, J=7.1 \mathrm{~Hz}, 2 \mathrm{H}), 7.54-7.48(\mathrm{~m}, 5 \mathrm{H}), 5.17(\mathrm{br}$, 4H), 2.23 (br, 8H), 1.83 (br, 8H), 1.26 (br, 48H), $0.86-0.81$ (br m, 24H). ${ }^{13}$ C NMR (100 MHz, $\left.\mathrm{CDCl}_{3}\right) \delta 164.70,163.59,142.20,141.75,141.60,141.16,141.07,140.65,140.45,140.24,140.12$, $139.64,135.89,135.47,135.17,134.81,134.49,133.43,132.53,130.81,130.36,130.29,130.20$ $130.06,130.01,129.80,129.76,129.50,129.33,129.30,129.26,129.07,128.79,128.75,128.65$, $128.04,127.99,127.97,123.08,122.99,122.65,122.32,121.88,54.70,32.33,31.78,26.62,22.59$, 14.09. IR $\left(\mathrm{cm}^{-1}\right)$ 2954, 2924, 2858, 1697, 1657, 1598, 1587, 1457, 1408, 1324, 1239, 1183, 814. HRMS (APCI + ) calculated $\mathrm{m} / z$ for $\left[\mathrm{C}_{116} \mathrm{H}_{121} \mathrm{~N}_{4} \mathrm{O}_{8} \mathrm{Br}+\mathrm{Na}\right]^{+}$1799.8265, found 1799.8259 . Broadening (br) of peaks in the ${ }^{1} \mathrm{H}$ NMR spectrum is due to rotational isomers about the imide side chains.

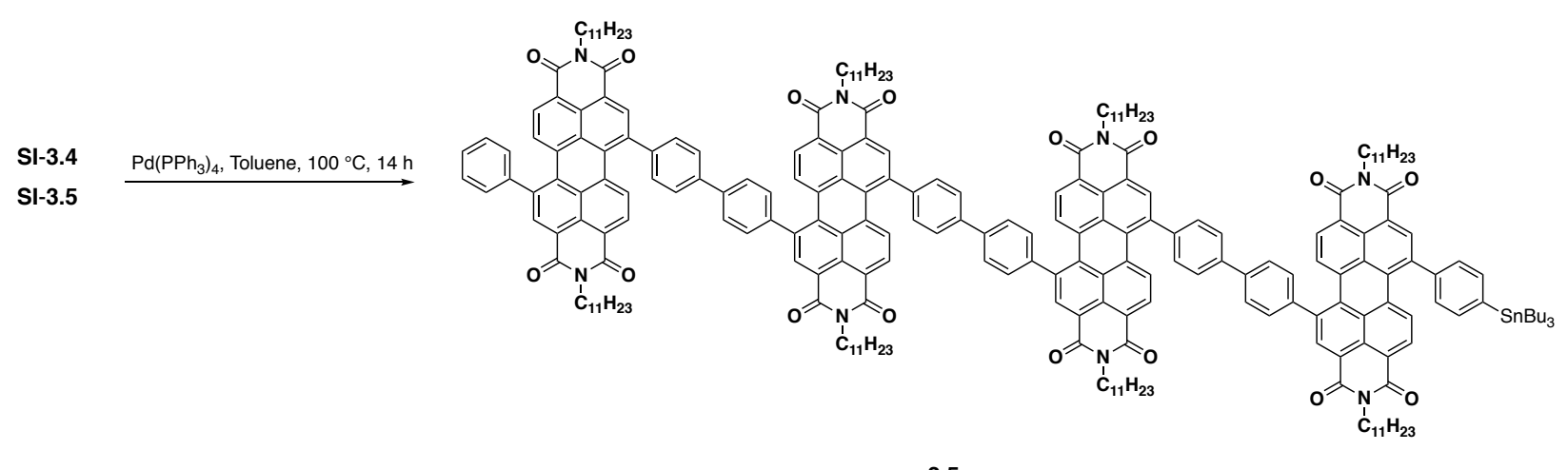

3.5

Synthesis of 3.5: SI-3.4 (0.0170 g, $0.00800 \mathrm{mmol}, 1.00$ equiv) and SI-3.5 (0.0150 g, 0.00800 mmol, 1.00 equiv) were dissolved in toluene $(2.0 \mathrm{~mL})$ in a $20 \mathrm{~mL}$ scintillation vial equipped with a stir bar. The mixture was sparged with $\mathrm{N}_{2}$ for thirty minutes. While under $\mathrm{N}_{2}$, tetrakis(triphenylphosphine)palladium( 0$)(0.00600 \mathrm{~g}, 0.00480 \mathrm{mmol}, 0.100$ equiv) was added. The mixture was further sparged for ten minutes before being placed in a $110{ }^{\circ} \mathrm{C}$ oil bath and stirred for eight hours. The crude mixture was filtered through Celite, concentrated and purified by preparative TLC using a mobile phase of $70: 30 \mathrm{CH}_{2} \mathrm{Cl}_{2}$ :hexanes. The product was washed with 
hexanes and precipitated from methanol to yield $3.5(0.00900 \mathrm{~g}, 0.00300 \mathrm{mmol}, 62 \%$ yield $) .{ }^{1} \mathbf{H}$ NMR $\left(500 \mathrm{MHz}, \mathrm{C}_{2} \mathrm{D}_{2} \mathrm{Cl}_{4}, 370 \mathrm{~K}\right) \delta 8.75(\mathrm{~s}, 4 \mathrm{H}), 8.72(\mathrm{~s}, 2 \mathrm{H}), 8.67(\mathrm{~s}, 2 \mathrm{H}), 8.32-8.26(\mathrm{~m}, 6 \mathrm{H})$, $8.19(\mathrm{~d}, J=8.2 \mathrm{~Hz}, 2 \mathrm{H}), 8.14(\mathrm{~d}, \mathrm{~J}=8.0 \mathrm{~Hz}, 2 \mathrm{H}), 8.14(\mathrm{~d}, \mathrm{~J}=8.2 \mathrm{~Hz}, 2 \mathrm{H}), 8.12(\mathrm{~d}, J=8.3 \mathrm{~Hz}$, 2H), $7.99-7.91(\mathrm{~m}, 14 \mathrm{H}), 7.84-7.78(\mathrm{~m}, 12 \mathrm{H}), 7.66(\mathrm{~d}, J=7.0 \mathrm{~Hz}, 4 \mathrm{H}), 7.62-7.56(\mathrm{~m}, 6 \mathrm{H})$, $5.25-5.15$ (br m, 8H), $2.33-2.21$ (br m, 16H), $2.00-1.90$ (br m, 16H), $1.45-1.27$ (br m, 96H), $0.98-0.88$ (br m, 48H). ${ }^{13} \mathbf{C}$ NMR $\left(125 \mathrm{MHz}, \mathrm{C}_{2} \mathrm{D}_{2} \mathrm{Cl}_{4}, 370 \mathrm{~K}\right) \delta 163.81,142.26,141.79,140.98$, $140.40,140.30,140.27,135.11,134.68,134.64,132.34,132.31,132.26,130.04,129.97,129.52$, $129.23,129.13,128.83,128.63,128.46,127.96,127.88,122.77,122.50,54.70,32.36,31.53$, 26.47, 26.45, 22.24, 22.23, 13.69. IR ( $\left.\mathrm{cm}^{-1}\right)$ 2956, 2924, 2856, 1696, 1657, 1598, 1587, 1457, 1408, 1324, 1262, 1239, 1182, 1097, 1029, 861, 813, 740. HRMS (MALDI-) calculated $m / z$ for $\left[\mathrm{C}_{232} \mathrm{H}_{242} \mathrm{~N}_{8} \mathrm{O}_{16}\right]^{-} 3395.8369$, found 3395.8363 .

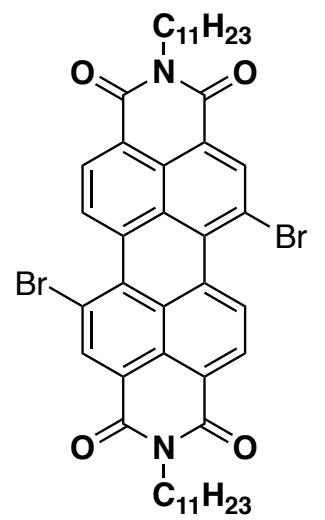

1,7-dibromo PDI

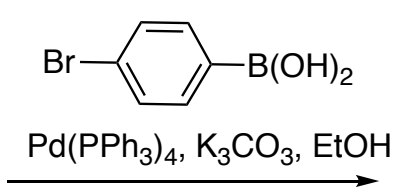

Toluene, rt, $72 \mathrm{~h}$

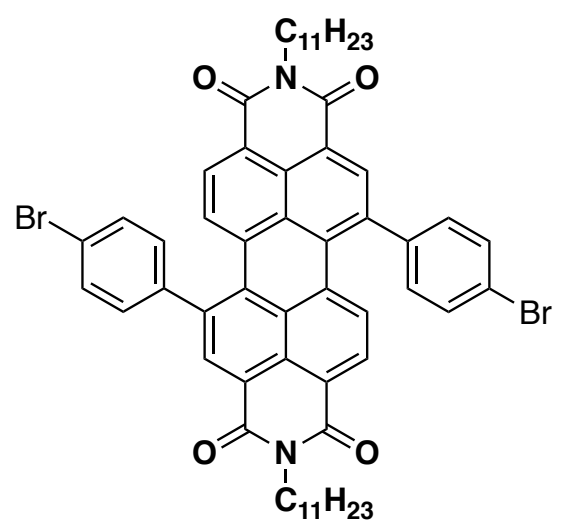

SI-3.6

Synthesis of 1,7-N,N'-di(6-undecyl)-1,7-di(4-bromophenyl)- perylene-3,4:9,10-tetracarboxylic diimide (SI-3.6): $\quad N, N^{\prime}$-Di(6-undecyl)-1,7-dibromoperylene-3,4:9,10-tetracarboxylic diimide ( $0.200 \mathrm{~g}, 0.234 \mathrm{mmol}, 1.00$ equiv), 4-bromophenylboronic acid ( $0.141 \mathrm{~g}, 7.01 \mathrm{mmol}, 3.00$ equiv), aqueous $\mathrm{K}_{2} \mathrm{CO}_{3}(2 \mathrm{M}, 2.00 \mathrm{~mL})$, EtOH $(0.400 \mathrm{~mL})$ and toluene $(6.00 \mathrm{~mL})$ were added to $20 \mathrm{~mL}$ scintillation vial equipped with a stir bar. The mixture was sparged with $\mathrm{N}_{2}$ for thirty minutes. While under $\mathrm{N}_{2}$, tetrakis(triphenylphosphine)palladium(0) (0.0270 g, $0.0234 \mathrm{mmol}, 0.100$ equiv) 
was added. The mixture was further sparged for ten minutes and stirred overnight at room temperature. A small aliquot was taken 24 hours later and showed $\sim 10 \%$ conversion to product by ${ }^{1} \mathrm{H}$ NMR. Additional tetrakis(triphenylphosphine)palladium(0) (0.108 g, $0.0936 \mathrm{mmol}, 0.400$ equiv) was added. The reaction was monitored for forty-eight hours and another 0.400 equiv of catalyst was added. In total, the reaction completed in 72 hours. The crude mixture was extracted with ethyl acetate and brine, concentrated and purified by column chromatography ( $40 \mathrm{~g}$ Redisep Rf Silica) using a gradient from $0 \%$ to $100 \% \mathrm{CH}_{2} \mathrm{Cl}_{2} /$ hexanes at $40 \mathrm{~mL} / \mathrm{min}$. Impure fractions were further purified by preparative TLC to yield a total $0.091 \mathrm{~g}(0.0905 \mathrm{mmol}, 39 \%)$ of SI-3.6. ${ }^{\mathbf{1}} \mathbf{H}$ NMR $\left(500 \mathrm{MHz}, \mathrm{CDCl}_{3}\right) \delta 8.58(\mathrm{br}, 2 \mathrm{H}), 8.18(\mathrm{br}, 2 \mathrm{H}), 7.82(\mathrm{~d}, \mathrm{~J}=8.1 \mathrm{~Hz}, 2 \mathrm{H}), 7.65(\mathrm{~d}, \mathrm{~J}=8.3$ Hz, 4H), 7.45 (d, J = 8.4 Hz, 4H). $5.16(\mathrm{br}, 2 \mathrm{H}), 2.28-2.16(\mathrm{~m}, 4 \mathrm{H}), 1.88-1.77(\mathrm{~m}, 4 \mathrm{H}), 1.38-$ 1.18 (br m, 24H) 0.81 (br t, $\mathrm{J}=6.9 \mathrm{~Hz}, 12 \mathrm{H}) .{ }^{13} \mathbf{C} \mathbf{N M R}\left(125 \mathrm{MHz}, \mathrm{CDCl}_{3}\right) \delta 164.52,163.43$, $140.98,139.73,135.45,134.73,134.42,133.43,132.36,130.74,130.28,130.10,129.34,129.20$, 128.00, 123.11, 122.76, 122.38, 122.01, 54.76, 32.30, 31.74, 26.58, 22.56, 14.04. IR $\left(\mathrm{cm}^{-1}\right) 2955$, 2928, 2858, 1697, 1657, 1598, 1587, 1487, 1456, 1409, 1324, 1239, 1183, 1072, 1012, 814. HRMS (APCI+) calculated $m / z$ for $\left[\mathrm{C}_{58} \mathrm{H}_{60} \mathrm{~N}_{2} \mathrm{O}_{4} \mathrm{Br}_{2}+\mathrm{H}\right]^{+}$1007.2998, found 1007.2994.

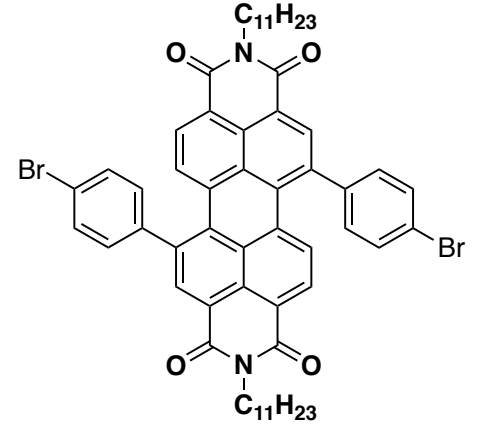

SI-3.6

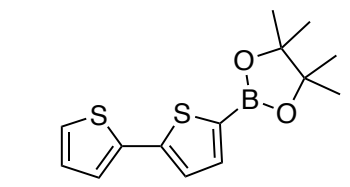

$\underset{\mathrm{THF}, \mathrm{rt}, 14 \mathrm{~h}}{\stackrel{\mathrm{Pd}\left(\mathrm{PPh}_{3}\right)_{2} \mathrm{Cl}_{2}, \mathrm{~K}_{3} \mathrm{CO}_{3}, \mathrm{H}_{2} \mathrm{O}}{\longrightarrow}}$

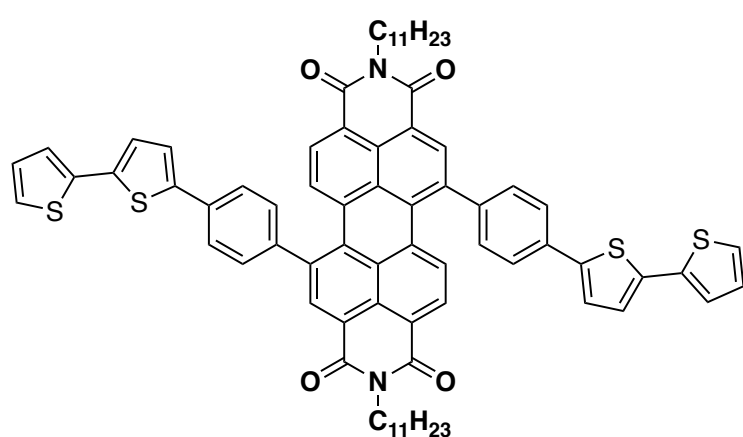

3.1

Synthesis of 3.1: SI-3.6 (0.100 g, $0.0990 \mathrm{mmol}, 1.00$ equiv), 2,2'-bithiophene-5-boronic acid pinacol ester (1.74 g, 0.596 mmol, 6.00 equiv), $\mathrm{K}_{2} \mathrm{CO}_{3}\left(0.218 \mathrm{~g}, 1.58 \mathrm{mmol}, 16.0\right.$ equiv), $\mathrm{H}_{2} \mathrm{O}$ $(1.00 \mathrm{~mL})$ and THF (3.00 mL) were added to $20 \mathrm{~mL}$ scintillation vial equipped with a stir bar. The 
mixture was sparged with $\mathrm{N}_{2}$ for thirty minutes. While under $\mathrm{N}_{2}$, bis(triphenylphosphine)palladium chloride (0.0110 g, $0.0150 \mathrm{mmol}, 0.150$ equiv) was added. The mixture was further sparged for ten minutes and stirred overnight at room temperature. The crude mixture was extracted with ethyl acetate and brine, concentrated and purified by column chromatography using a gradient from $0 \%$ to $100 \% \mathrm{CH}_{2} \mathrm{Cl}_{2} /$ hexanes to yield $3.1(0.112 \mathrm{~g}, 0.0950 \mathrm{mmol}, 94 \%) .{ }^{1} \mathbf{H} \mathbf{~ N M R}\left(500 \mathrm{MHz}, \mathrm{C}_{2} \mathrm{D}_{2} \mathrm{Cl}_{4}\right)$ $\delta 8.64$ (br, 2H), 8.17 (br, 2H), 7.99 (br, 2H), $7.76(\mathrm{~d}, \mathrm{~J}=7.9 \mathrm{~Hz}, 4 \mathrm{H}), 7.65$ (d, J = 8.3 Hz, 4H), 7.40 $(\mathrm{d}, \mathrm{J}=3.8 \mathrm{~Hz}, 2 \mathrm{H}), 7.30(\mathrm{dd}, \mathrm{J}=5.1,1.1 \mathrm{~Hz}, 2 \mathrm{H}), 7.28(\mathrm{dd}, \mathrm{J}=3.6,1.1 \mathrm{~Hz}, 2 \mathrm{H}), 7.24(\mathrm{~d}, \mathrm{~J}=3.7$ Hz, 2H), 7.09 (dd, J = 5.1, 3.6 Hz, 2H), 5.12 (br, 2H), 2.26 - 2.11 (br m, 4H), 1.86 (br, 4H), 1.37 - 1.22 (br m, 24H), $0.88-0.82$ (br m, 12H). $\left.{ }^{13} \mathrm{C} \mathrm{NMR} \mathrm{(125} \mathrm{MHz,} \mathrm{C}_{2} \mathrm{D}_{2} \mathrm{Cl}_{4}\right) \delta 164.47,164.37$, $163.41,163.30,141.70,140.97,140.11,137.25,136.99,135.48,134.77,134.55,134.08,132.26$, $130.00,129.67,129.24,129.09,128.00,127.78,126.91,124.80,124.77,124.63,123.91,122.83$, $122.42,122.10,121.74,54.52,32.16,31.66,26.53,22.49,14.09$. IR $\left(\mathrm{cm}^{-1}\right) 2927,2857,1694$, 1654, 1586, 1497, 1409, 1326, 1240, 1006, 950, 887, 838. HRMS (APCI+) calculated $m / z$ for $\left[\mathrm{C}_{74} \mathrm{H}_{70} \mathrm{~N}_{2} \mathrm{O}_{4} \mathrm{~S}_{4}+\mathrm{H}\right]^{+}$1179.4297, found 1179.4301 .

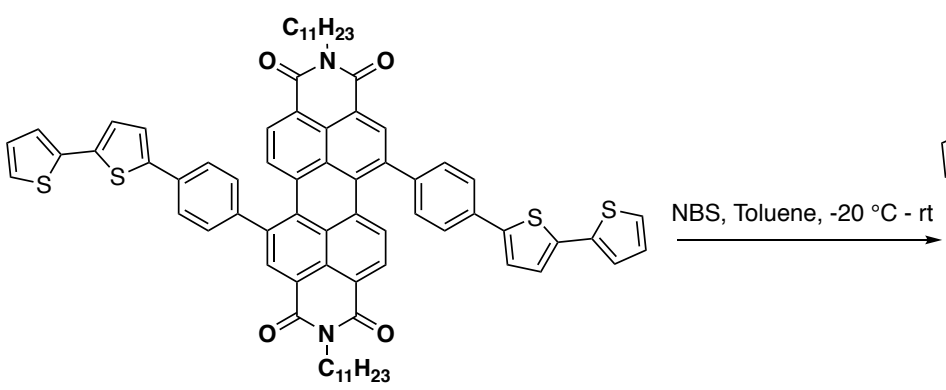

3.1

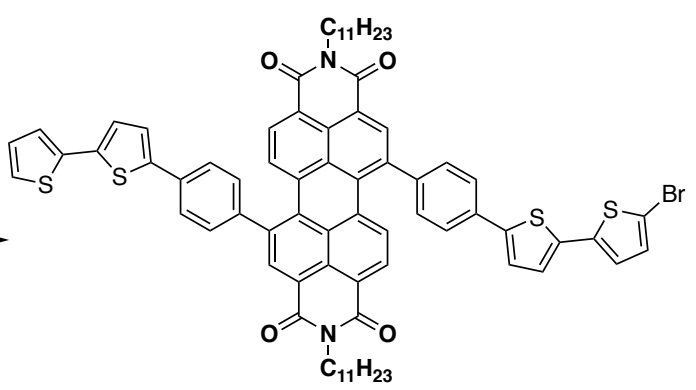

SI-3.7

Synthesis of SI-3.7: 3.1 ( $0.0740 \mathrm{~g}, 0.0640 \mathrm{mmol}, 1.00$ equiv) was dissolved in toluene $(6.00 \mathrm{~mL})$ and sparged with $\mathrm{N}_{2}$ for fifteen minutes while at $-20{ }^{\circ} \mathrm{C} . \mathrm{N}$-Bromosuccinimide $(0.0110 \mathrm{~g}, 0.0640$ mmol, 1.00 equiv) was added and aluminum was placed over the reaction flask to protect from light. The reaction was stirred for twenty-four hours and allowed to warm to room temperature. The reaction mixture was concentrated and purified by column chromatography using a gradient 
from $0 \%$ to $100 \% \mathrm{CH}_{2} \mathrm{Cl}_{2} /$ hexanes to yield SI-3.7 (0.00800 g, $\left.0.00600 \mathrm{mmol}, 10 \%\right)$. ${ }^{1} \mathbf{H}$ NMR $\left(500 \mathrm{MHz}, \mathrm{CD}_{2} \mathrm{Cl}_{2}\right) \delta 8.62(\mathrm{br}, 2 \mathrm{H}), 8.15(\mathrm{br}, 2 \mathrm{H}), 8.00(\mathrm{~d}, \mathrm{~J}=6.9 \mathrm{~Hz}, 1 \mathrm{H}), 7.98(\mathrm{~d}, \mathrm{~J}=6.9 \mathrm{~Hz}$, 1H), $7.80-7.77(\mathrm{~m}, 4 \mathrm{H}), 7.65(\mathrm{~d}, \mathrm{~J}=8.1 \mathrm{~Hz}, 4 \mathrm{H}), 7.41(\mathrm{~d}, \mathrm{~J}=3.8 \mathrm{~Hz}, 1 \mathrm{H}), 7.39$ (d, J = $3.8 \mathrm{~Hz}$, 1H), $7.31(\mathrm{~d}, \mathrm{~J}=4.7 \mathrm{~Hz}, 1 \mathrm{H}), 7.29(\mathrm{~d}, \mathrm{~J}=3.5 \mathrm{~Hz}, 1 \mathrm{H}), 7.25(\mathrm{~d}, \mathrm{~J}=3.7 \mathrm{~Hz}, 1 \mathrm{H}), 7.19(\mathrm{~d}, \mathrm{~J}=3.8$ Hz, 1H), $7.09(\mathrm{dd}, \mathrm{J}=5.1,3.6 \mathrm{~Hz}, 1 \mathrm{H}), 7.06(\mathrm{~d}, \mathrm{~J}=3.8 \mathrm{~Hz}, 1 \mathrm{H}), 7.03(\mathrm{~d}, \mathrm{~J}=3.8 \mathrm{~Hz}, 1 \mathrm{H}), 5.12(\mathrm{br}$, 2H), $2.24-2.12$ (br m, 4H), 1.86 (br, 4H), $1.33-1.24$ (br m, 24H), $0.89-0.83$ (br m, 12H). ${ }^{13} \mathrm{C}$ NMR* $\left(100 \mathrm{MHz}, \mathrm{C}_{2} \mathrm{D}_{2} \mathrm{Cl}_{4}\right) \delta 142.25,141.70,141.17,140.13,138.53,137.27,136.99,136.10$, $134.92,134.58,134.10,133.88,132.30,130.84,130.02,129.69,129.09,128.00,127.79,126.96$, $125.05,124.79,124.66,123.96,123.91,111.12,54.55,32.16,31.65,26.53,22.48,14.08 . \mathbf{I R}\left(\mathrm{cm}^{-}\right.$ 1) $2954,2926,2857,1697,1656,1597,1586,1457,1409,1326,1241,1185,838,813,796$. HRMS (ESI+) calculated $m / z$ for $\left[\mathrm{C}_{74} \mathrm{H}_{69} \mathrm{~N}_{2} \mathrm{O}_{4} \mathrm{~S}_{4} \mathrm{Br}+\mathrm{H}\right]^{+} 1257.3402$, found $1257.3392 . *$ Partial spectrum reported due to limited solubility.
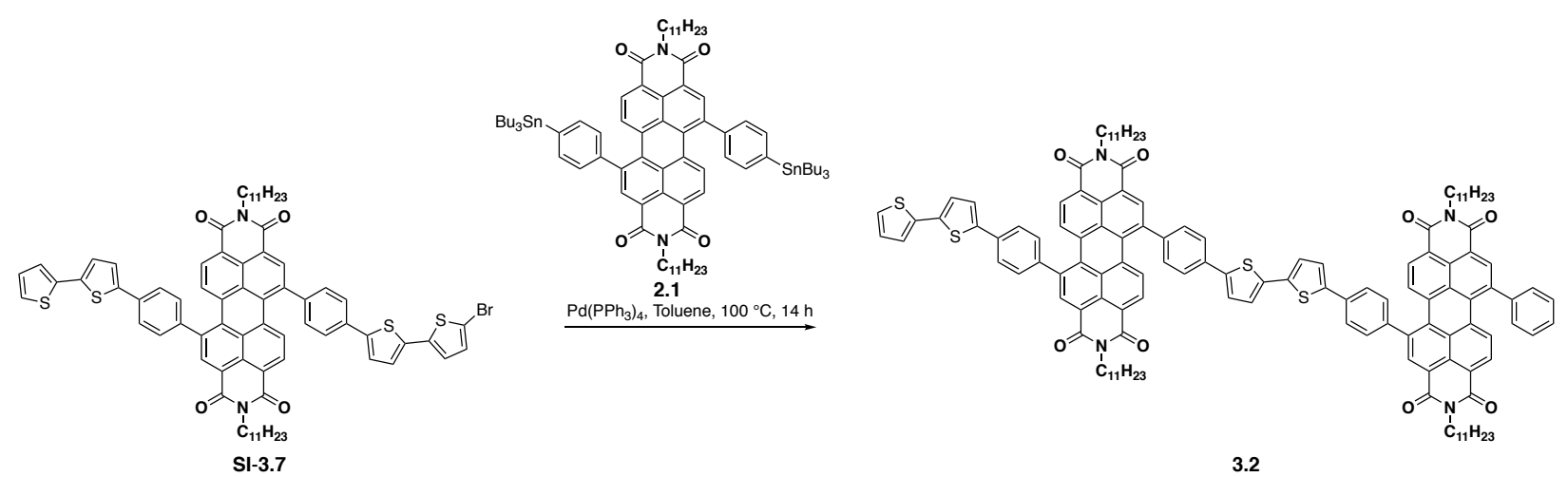

Synthesis of 3.2: SI-3.7 (0.0230 g, $0.0180 \mathrm{mmol}, 1.00$ equiv) and $2.1(0.105 \mathrm{~g}, 0.0780 \mathrm{mmol}, 4.00$ equiv) were dissolved in toluene $(6.00 \mathrm{~mL})$ in a $20 \mathrm{~mL}$ scintillation vial equipped with a stir bar. The mixture was sparged with $\mathrm{N}_{2}$ for thirty minutes. While under $\mathrm{N}_{2}$, tetrakis(triphenylphosphine)palladium(0) $(0.00200 \mathrm{~g}, 0.00200 \mathrm{mmol}, 0.100$ equiv) was added. The mixture was further sparged for ten minutes before being placed in a $107^{\circ} \mathrm{C}$ oil bath and stirred overnight. The crude mixture was concentrated and purified by preparative TLC using a mobile 
phase of 50:50 $\mathrm{CH}_{2} \mathrm{Cl}_{2}$ :hexanes. The product was passed through a small silica plug to remove residual impurities. The product was then dissolved in $\mathrm{CH}_{2} \mathrm{Cl}_{2}(2.0 \mathrm{~mL})$ and $\mathrm{HCl}(2.0 \mathrm{~mL})$ and stirred at room temperature for two days. The crude mixture was extracted with water and $\mathrm{CH}_{2} \mathrm{Cl}_{2}$, concentrated and purified by column chromatography ( $4 \mathrm{~g}$ Redisep Rf Silica) using a gradient from $0 \%$ to $100 \% \mathrm{CH}_{2} \mathrm{Cl}_{2} /$ hexanes. The product was precipitated from $\mathrm{CH}_{2} \mathrm{Cl}_{2}$ and methanol to yield 3.2 as a purple solid $(0.0050 \mathrm{~g}, 0.0020 \mathrm{mmol}, 14 \%) .{ }^{1} \mathbf{H}$ NMR $\left(400 \mathrm{MHz}, \mathrm{C}_{2} \mathrm{D}_{2} \mathrm{Cl}_{4}\right) \delta 8.62(\mathrm{br}$, 4H), $8.17(\mathrm{br}, 4 \mathrm{H}), 8.03-7.98(\mathrm{~m}, 3 \mathrm{H}), 7.84(\mathrm{~d}, \mathrm{~J}=8.4 \mathrm{~Hz}, 1 \mathrm{H}), 7.83-7.77(\mathrm{~m}, 6 \mathrm{H}), 7.70-7.63$ (m, 6H), $7.61($ br d, J = 6.3 Hz, 2H), $7.58-7.51(\mathrm{~m}, 3 \mathrm{H}), 7.45$ (br d, J = 3.1 Hz, 2H), $7.41(\mathrm{~d}, \mathrm{~J}=$ $3.8 \mathrm{~Hz}, 1 \mathrm{H}), 7.32(\mathrm{~d}, \mathrm{~J}=3.9 \mathrm{~Hz}, 2 \mathrm{H}), 7.30-7.27(\mathrm{~m}, 2 \mathrm{H}), 7.25(\mathrm{~d}, \mathrm{~J}=3.7 \mathrm{~Hz}, 1 \mathrm{H}), 7.11-7.06$ (m, 1H), $5.16(\mathrm{br}, 4 \mathrm{H}), 2.31-2.13(\mathrm{br} \mathrm{m}, 8 \mathrm{H}), 1.89-1.76(\mathrm{br} \mathrm{m}, 8 \mathrm{H}), 1.27$ (br, 48H), 0.84 (br t, $\mathrm{J}=6.6 \mathrm{~Hz}, 24 \mathrm{H}) .{ }^{13} \mathbf{C} \mathbf{N M R}\left(100 \mathrm{MHz}, \mathrm{C}_{2} \mathrm{D}_{2} \mathrm{Cl}_{4}\right) \delta 165.13,164.00,142.97,142.87,142.53,142.18$, $142.14,142.03,141.56,140.82,140.79,140.76,137.89,137.71,137.67,137.66,135.90,135.31$, 134.72, 134.65, 134.62, 133.09, 130.79, 130.63, 130.47, 130.43, 129.89, 129.81, 129.66, 129.07, $128.56,128.53,128.49,127.51,127.47,125.52,125.34,125.30,125.23,125.08,124.45,123.60$, $123.26,122.88,122.52,54.99,54.48,54.40,32.84,32.34,27.14,23.16,14.38$. IR $\left(\mathrm{cm}^{-1}\right) 2954$, 2925, 2854, 1697, 1659, 1597, 1587, 1459, 1409, 1326, 1265, 1248, 1240, 814, 740. HRMS (ESI+) calculated $m / z$ for $\left[\mathrm{C}_{132} \mathrm{H}_{130} \mathrm{~N}_{4} \mathrm{O}_{8} \mathrm{~S}_{4}+\mathrm{H}\right]^{+} 2027.8850$, found 2027.8820 .
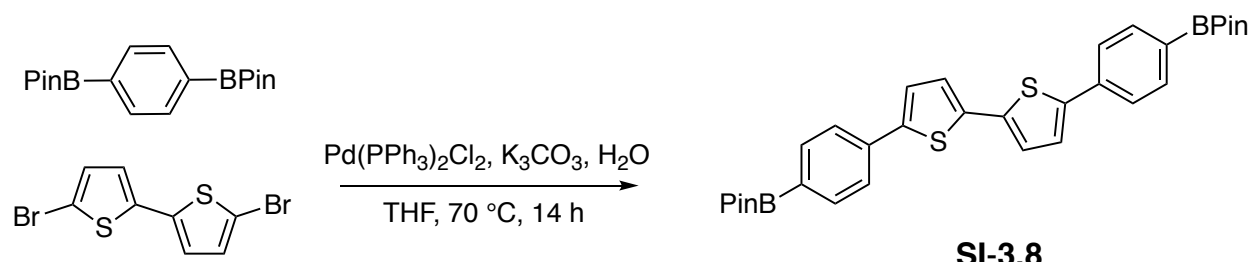

SI-3.8

Synthesis of SI-3.8: 1,4-Benzenediboronic acid bis(pinacol) ester (0.815 g, $2.47 \mathrm{mmol}, 4.00$ equiv), 5,5'-dibromo-2,2'-bithiophene ( $0.200 \mathrm{~g}, 0.617 \mathrm{mmol}, 1.00$ equiv) and $\mathrm{K}_{2} \mathrm{CO}_{3}(2.12 \mathrm{~g}, 15.0$ mmol, 25.00 equiv) were dissolved in THF $(21.0 \mathrm{~mL})$ and $\mathrm{H}_{2} \mathrm{O}(6.00 \mathrm{ml})$ in a $100 \mathrm{~mL}$ round bottom 
flask equipped with a stir bar. The mixture was sparged with $\mathrm{N}_{2}$ for thirty minutes. While under $\mathrm{N}_{2}$, bis(triphenylphosphine)palladium chloride ( $0.0430 \mathrm{~g}, 0.0620 \mathrm{mmol}, 0.100$ equiv) was added. The mixture was further sparged for thirty minutes before being placed in a $70{ }^{\circ} \mathrm{C}$ oil bath and stirred overnight. The crude mixture was extracted with brine and ethyl acetate. The organic layer was collected, concentrated and purified by column chromatography using a gradient of $0-100 \%$ of hexanes/DCM. The final product was a pale yellow solid $(0.030 \mathrm{~g}, 0.0526,9 \%) .{ }^{1} \mathbf{H}$ NMR (400 $\left.\mathrm{MHz}, \mathrm{CD}_{2} \mathrm{Cl}_{2}\right) \delta 7.80(\mathrm{~d}, \mathrm{~J}=8.2 \mathrm{~Hz}, 2 \mathrm{H}), 7.64(\mathrm{~d}, \mathrm{~J}=8.2 \mathrm{~Hz}, 2 \mathrm{H}), 7.36(\mathrm{~d}, \mathrm{~J}=3.8 \mathrm{~Hz}, 1 \mathrm{H}), 7.24$ $(\mathrm{d}, \mathrm{J}=3.8 \mathrm{~Hz}, 1 \mathrm{H}), 1.36(\mathrm{~s}, 24 \mathrm{H}) . \mathbf{I R}\left(\mathrm{cm}^{-1}\right)$ 2973, 2934, 2857, 1605, 1399, 1362, 1145, 1093, 963, 859, 806. HRMS (ESI+) calculated $m / z$ for $\left[\mathrm{C}_{32} \mathrm{H}_{36} \mathrm{~B}_{2} \mathrm{O}_{4} \mathrm{~S}_{2}\right]^{+} 570.2241$, found 570.2252 .

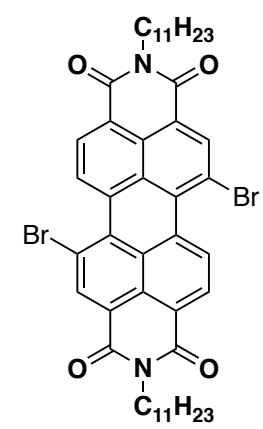

1,7-dibromo PDI

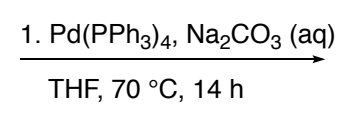

2.

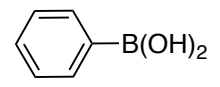

3.

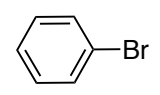

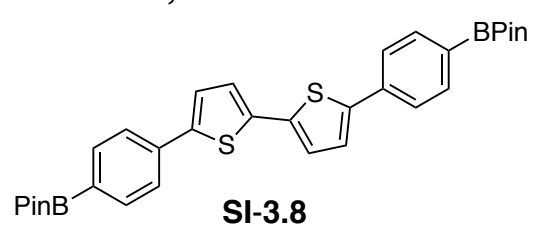

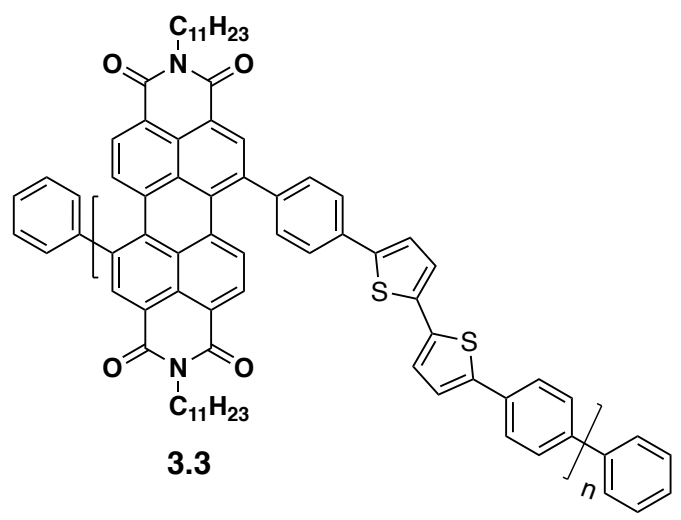

Synthesis of 3.3: $N, N^{\prime}$-Di(6-undecyl)-1,7-dibromoperylene-3,4:9,10-tetracarboxylic diimide ( $0.0420 \mathrm{~g}, 0.0490 \mathrm{mmol}, 1.03$ equiv), SI-3.8 ( $0.027 \mathrm{~g}, 0.0474 \mathrm{mmol}, 1.00$ equiv), aqueous $\mathrm{Na}_{2} \mathrm{CO}_{3}$ $(2 \mathrm{M}, 2.20 \mathrm{~mL})$ and THF $(4.00 \mathrm{~mL})$ were added to a $20 \mathrm{~mL}$ scintillation vial equipped with a stir bar. The mixture was sparged with $\mathrm{N}_{2}$ for thirty minutes. While under $\mathrm{N}_{2}$, tetrakis(triphenylphosphine)palladium(0) $(0.002 \mathrm{~g}, 0.002 \mathrm{mmol}, 0.0400$ equiv) was added. The mixture was further sparged for ten minutes and stirred for 48 hours at $82{ }^{\circ} \mathrm{C}$. Then phenylboronic acid $(0.0170 \mathrm{~g}, 0.142 \mathrm{mmol}, 3.30$ equiv) was added to the reaction and allowed to stir for three 
hours at $82{ }^{\circ} \mathrm{C}$, followed by bromobenzene $(0.200 \mathrm{~mL}, 1.90 \mathrm{mmol}, 40$ equiv). The reaction was left stirring overnight. The crude mixture was extracted with ethyl acetate and brine and concentrated. Solids were crashed out with methanol and further purified using soxhlet extraction using methanol, followed by hexanes. The final product was recovered using chloroform. The reaction yielded $0.040 \mathrm{~g}, 82 \%$ yield*. ${ }^{1} \mathbf{H}$ NMR* $\left(500 \mathrm{MHz}, \mathrm{C}_{2} \mathrm{D}_{2} \mathrm{Cl}_{4}\right) \delta 8.71(\mathrm{~s}, 2 \mathrm{H}), 8.27(\mathrm{~d}, \mathrm{~J}=$ $8.0 \mathrm{~Hz}, 2 \mathrm{H}), 8.10(\mathrm{~d}, \mathrm{~J}=8.0 \mathrm{~Hz}, 2 \mathrm{H}), 7.86(\mathrm{~d}, \mathrm{~J}=7.5 \mathrm{~Hz}, 4 \mathrm{H}), 7.71(\mathrm{~d}, \mathrm{~J}=8.0 \mathrm{~Hz}, 4 \mathrm{H}), 7.47$ (d, J $=3.0 \mathrm{~Hz}, 2 \mathrm{H}), 7.37(\mathrm{~d}, \mathrm{~J}=3.4 \mathrm{~Hz}, 2 \mathrm{H}), 5.26-5.16(\mathrm{~m}, 2 \mathrm{H}), 2.28(\mathrm{br}, 4 \mathrm{H}), 1.99(\mathrm{br}, 4 \mathrm{H}), 1.43(\mathrm{br}$, 24H)**, 0.95 (br, 12H). GPC: $\mathrm{Mn}=6,388 ; \mathrm{Mw}=30,238$ and $\mathrm{PDI}=4.73$; solvent = chloroform; $\lambda=440 \mathrm{~nm}$. HRMS (MALDI) shows oligomers with repeating units of $1012 \mathrm{~m} / \mathrm{z}$ up to the eightmer. * Polymer yields do not incorporate phenyl caps.

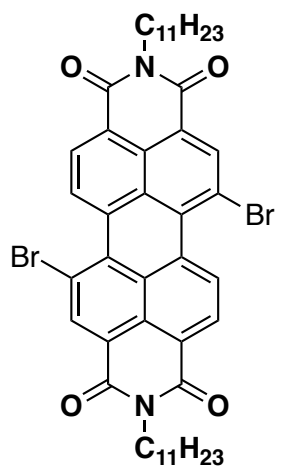

1,7-dibromo PDI

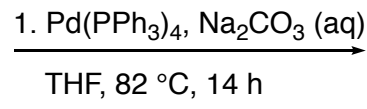

2.<smiles>O[BiH2]c1ccccc1</smiles>

3.

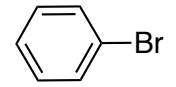

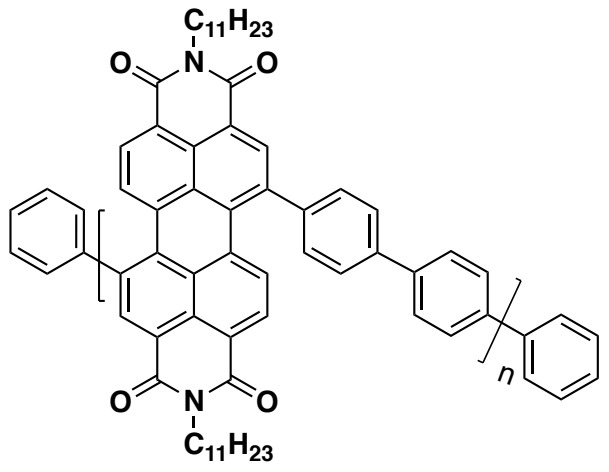

3.6

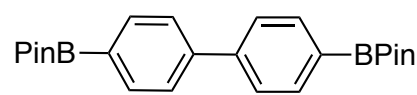

Synthesis of 3.6: $N, N^{\prime}$-Di(6-undecyl)-1,7-dibromoperylene-3,4:9,10-tetracarboxylic diimide (0.200 g, $0.234 \mathrm{mmol}, 1.00$ equiv), 4,4'-biphenyldiboronic acid bis(pinacol) ester (0.0950 g, 0.234 mmol, 1.00 equiv), aqueous $\mathrm{Na}_{2} \mathrm{CO}_{3}(2 \mathrm{M}, 11.0 \mathrm{~mL})$ and $\mathrm{THF}(19.0 \mathrm{~mL})$ were added to $100 \mathrm{~mL}$ two-neck, round bottom flask equipped with a stir bar. The mixture was sparged with $\mathrm{N}_{2}$ for thirty minutes. While under $\mathrm{N}_{2}$, tetrakis(triphenylphosphine)palladium(0) $(0.0110 \mathrm{~g}, 0.009 \mathrm{mmol}$, 0.0400 equiv) was added. The mixture was further sparged for ten minutes and stirred for 48 hours 
at $78{ }^{\circ} \mathrm{C}$. Then, commercially available phenylboronic acid $(0.0940 \mathrm{~g}, 0.767 \mathrm{mmol}, 3.30$ equiv) was added to the reaction and allowed to stir for two hours at $78^{\circ} \mathrm{C}$, followed by bromobenzene (1.0 ml, $9.55 \mathrm{mmol}, 41.0$ equiv). The reaction was left stirring overnight. The crude mixture was extracted with ethyl acetate and brine and concentrated. Solids were crashed out with methanol and further purified using soxhlet extraction using methanol, followed by hexanes. The final product was recovered using chloroform. The reaction yielded $0.181 \mathrm{~g}$ for a $91 \%$ yield*. ${ }^{1} \mathbf{H} \mathbf{N M R}^{*}$ $\left(500 \mathrm{MHz}, \mathrm{C}_{2} \mathrm{D}_{2} \mathrm{Cl}_{4}\right) \delta 8.77(\mathrm{~s}, 2 \mathrm{H}), 8.33(\mathrm{~d}, \mathrm{~J}=8.5 \mathrm{~Hz}, 2 \mathrm{H}), 8.18(\mathrm{~d}, \mathrm{~J}=8.3 \mathrm{~Hz}, 2 \mathrm{H}), 7.99(\mathrm{~d}, \mathrm{~J}=$ 8.1 Hz, 4H), $7.84(\mathrm{~d}, \mathrm{~J}=7.8 \mathrm{~Hz}, 4 \mathrm{H}), 5.27-5.20(\mathrm{~m}, 2 \mathrm{H}), 2.31$ (br, 4H), 1.99 (br, 4H), 1.42 (br, $24 \mathrm{H})^{* *}, 0.95(\mathrm{br}, 12 \mathrm{H})$. GPC: $\mathrm{M}_{\mathrm{n}}=16,950 ; \mathrm{M}_{\mathrm{w}}=61,169$ and PDI $=3.61$; solvent $=\mathrm{THF} ; \lambda=500$ nm. HRMS (MALDI) shows oligomers with repeating units of $849 \mathrm{~m} / \mathrm{z}$ up to the nine-mer. *Polymer yields do not incorporate phenyl caps. **The broad peak at $1.43 \mathrm{ppm}$ indicates water in the sample and prevents integration of the overlapping alkyl protons. 


\subsection{NMR Spectra of Compounds}

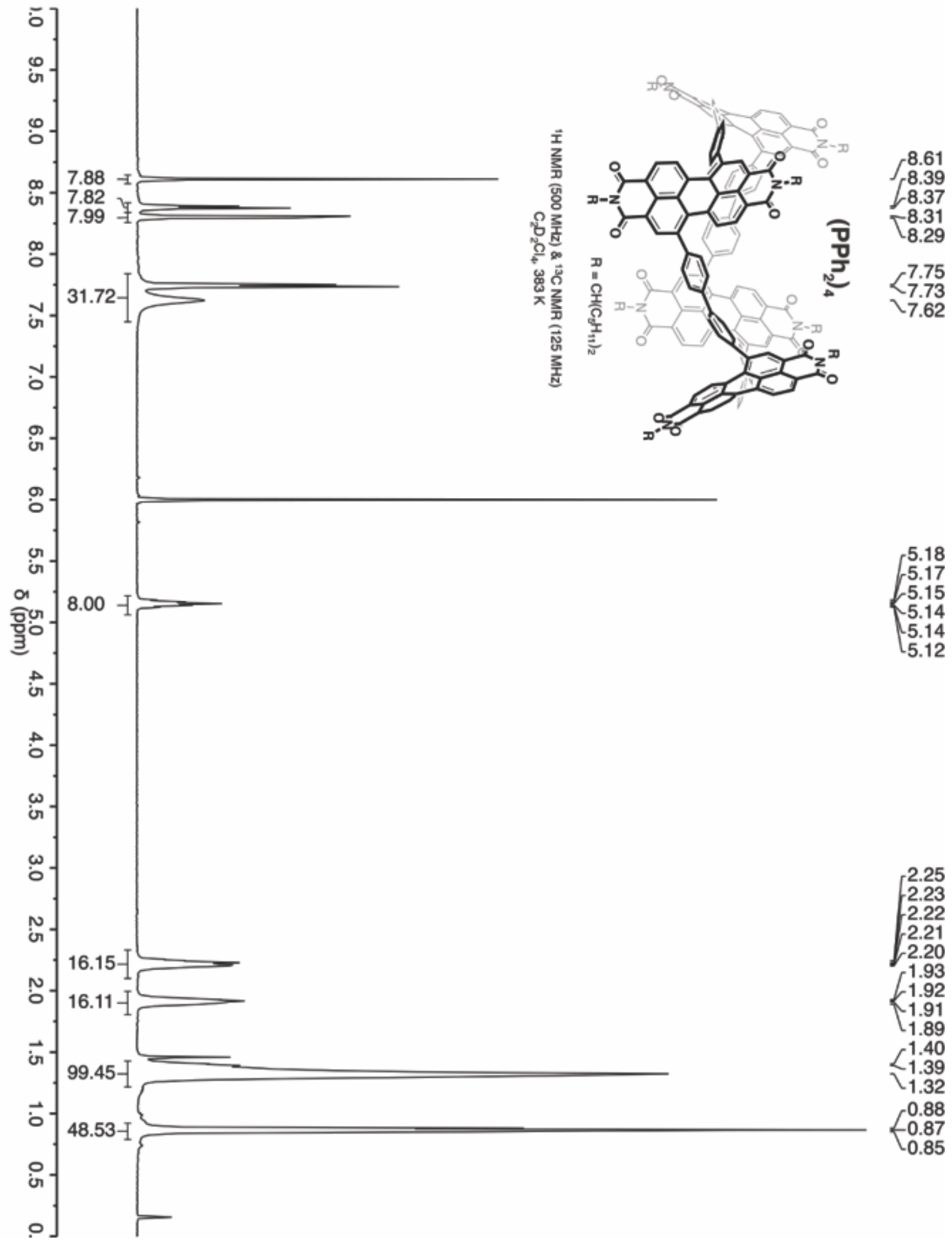




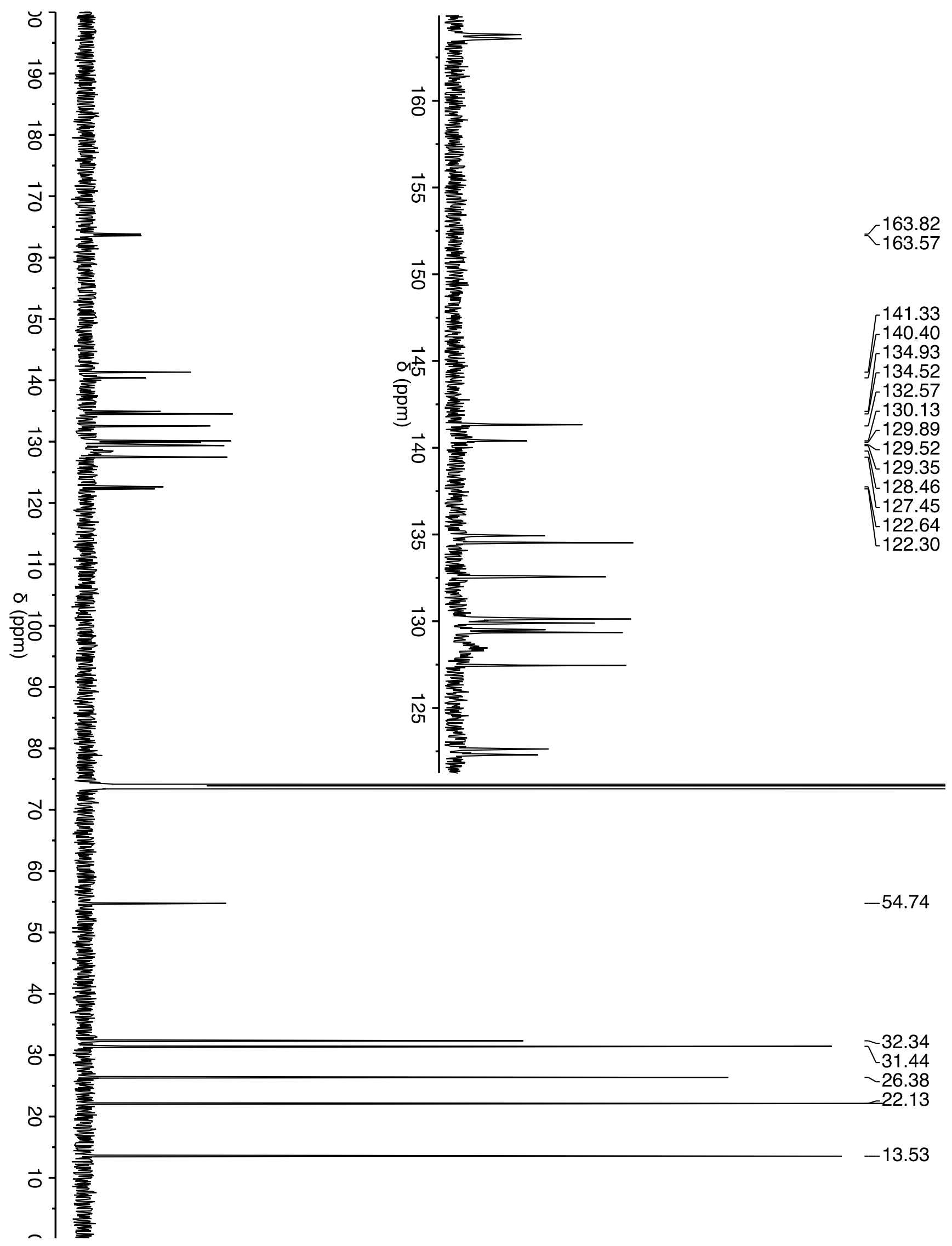




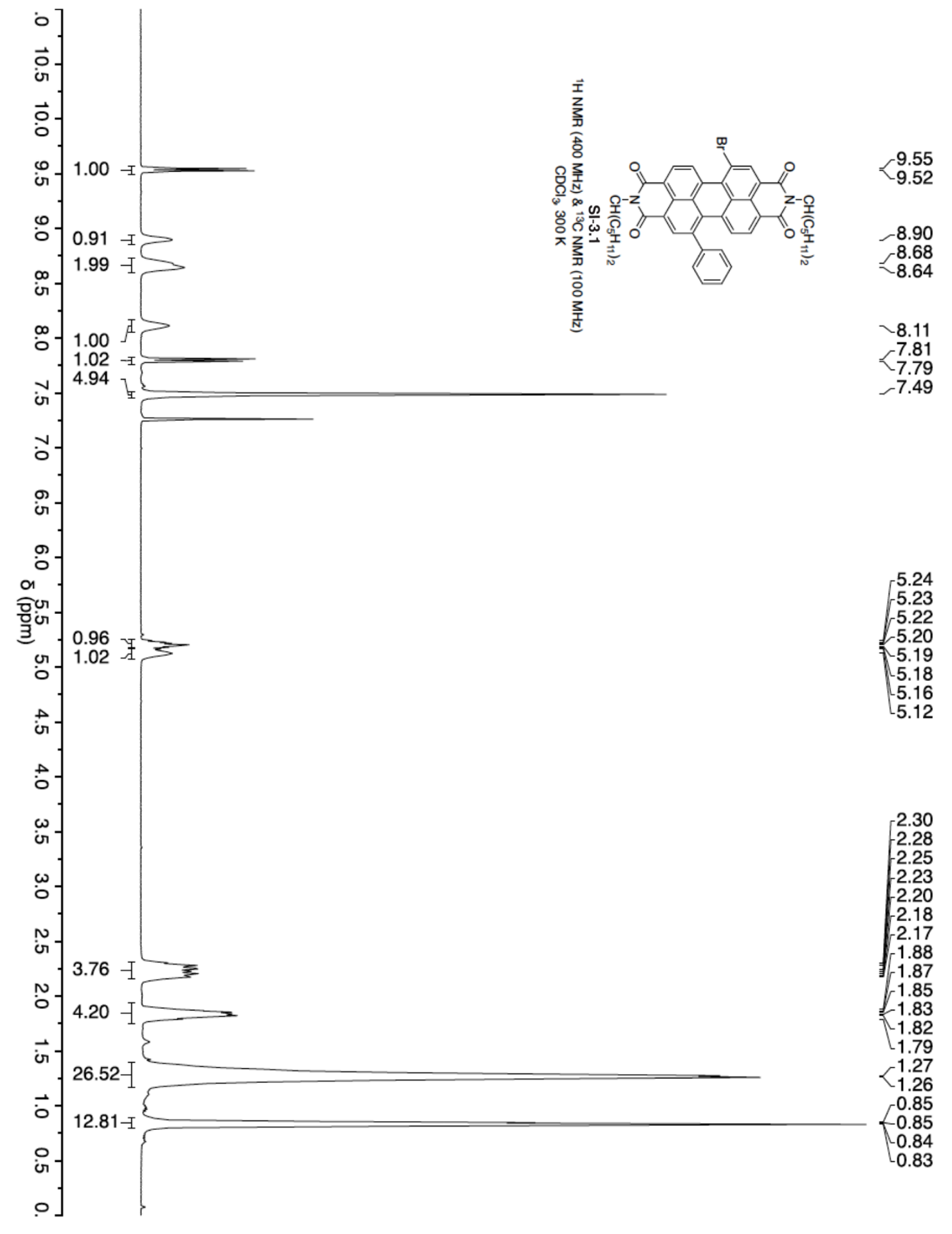




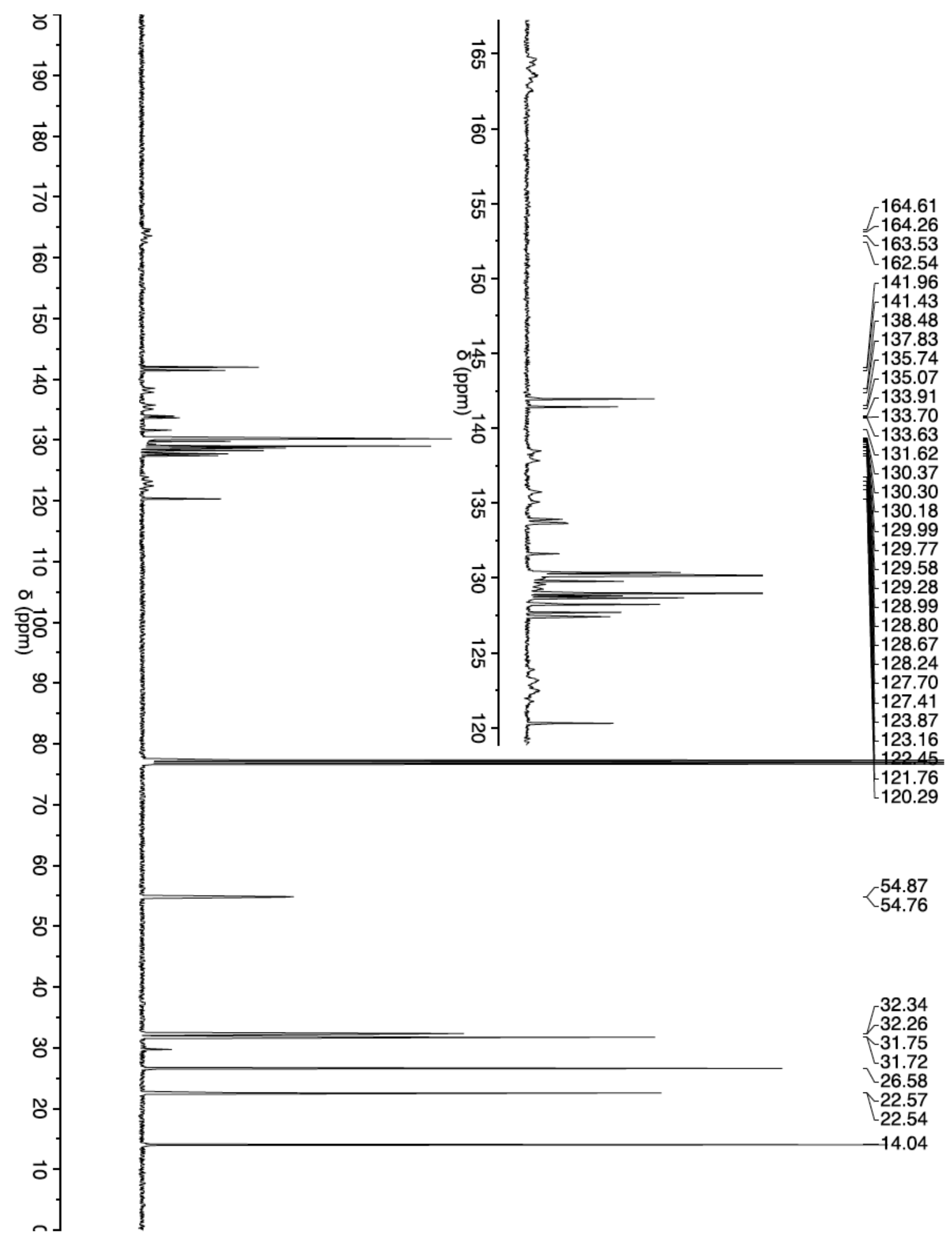




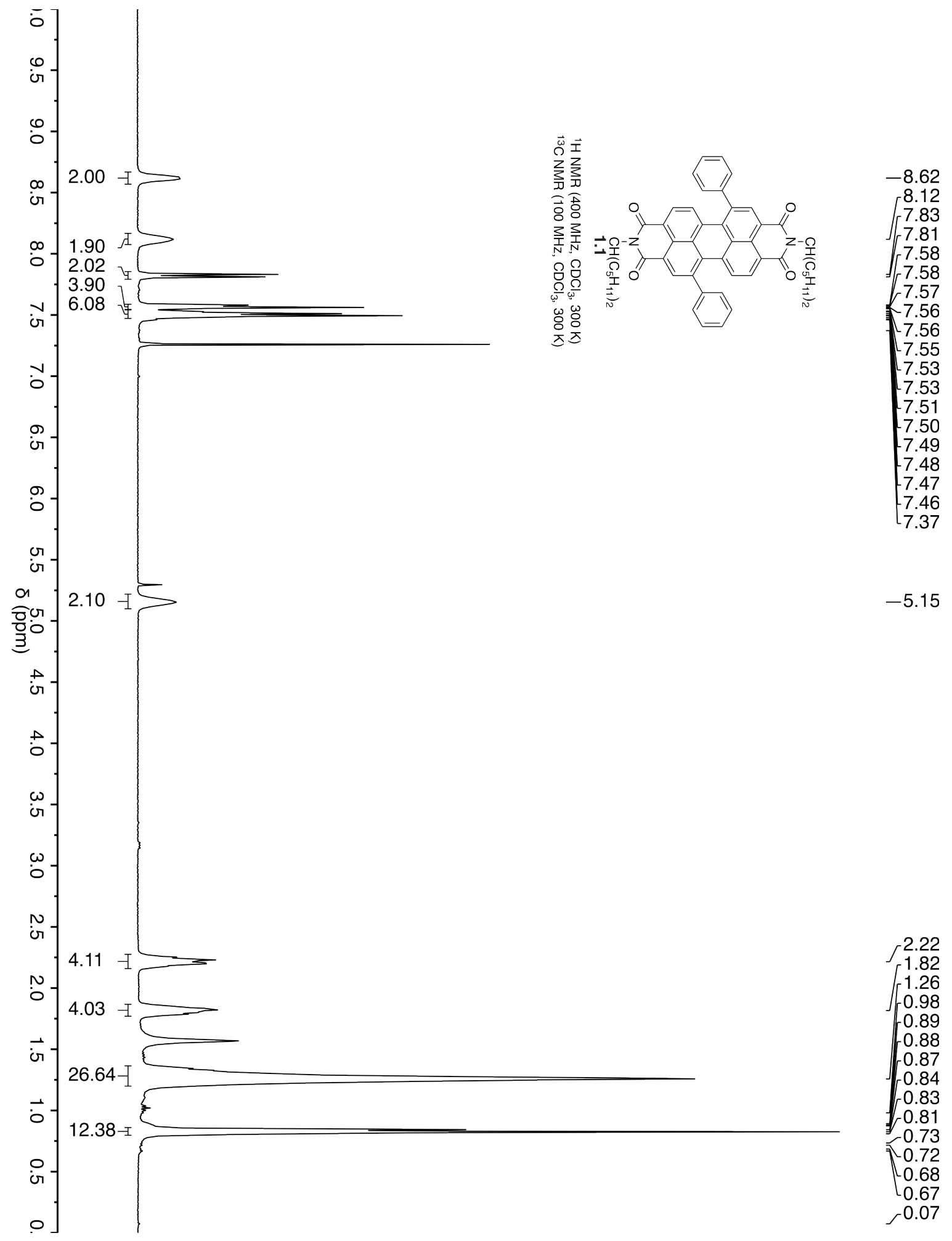




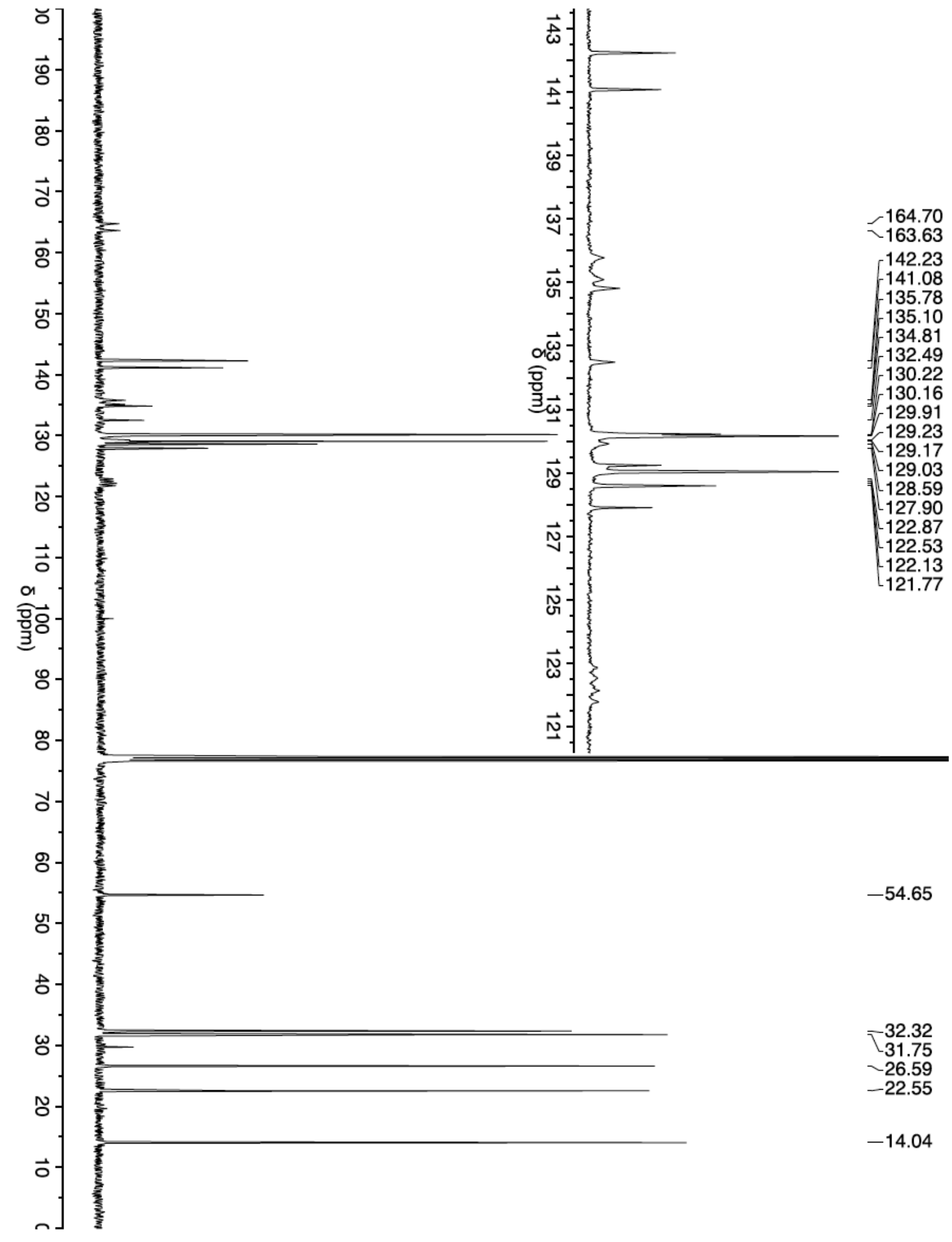




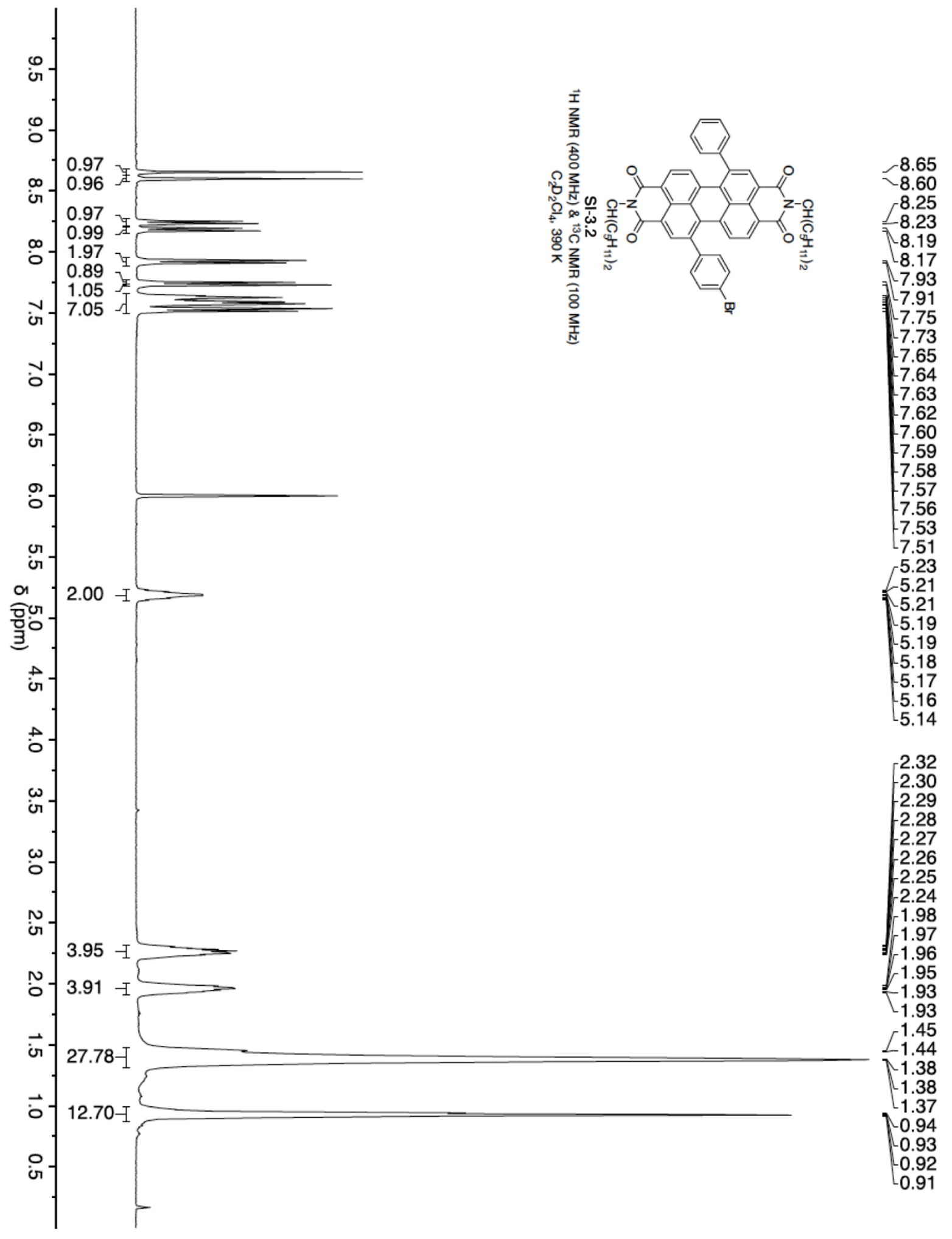




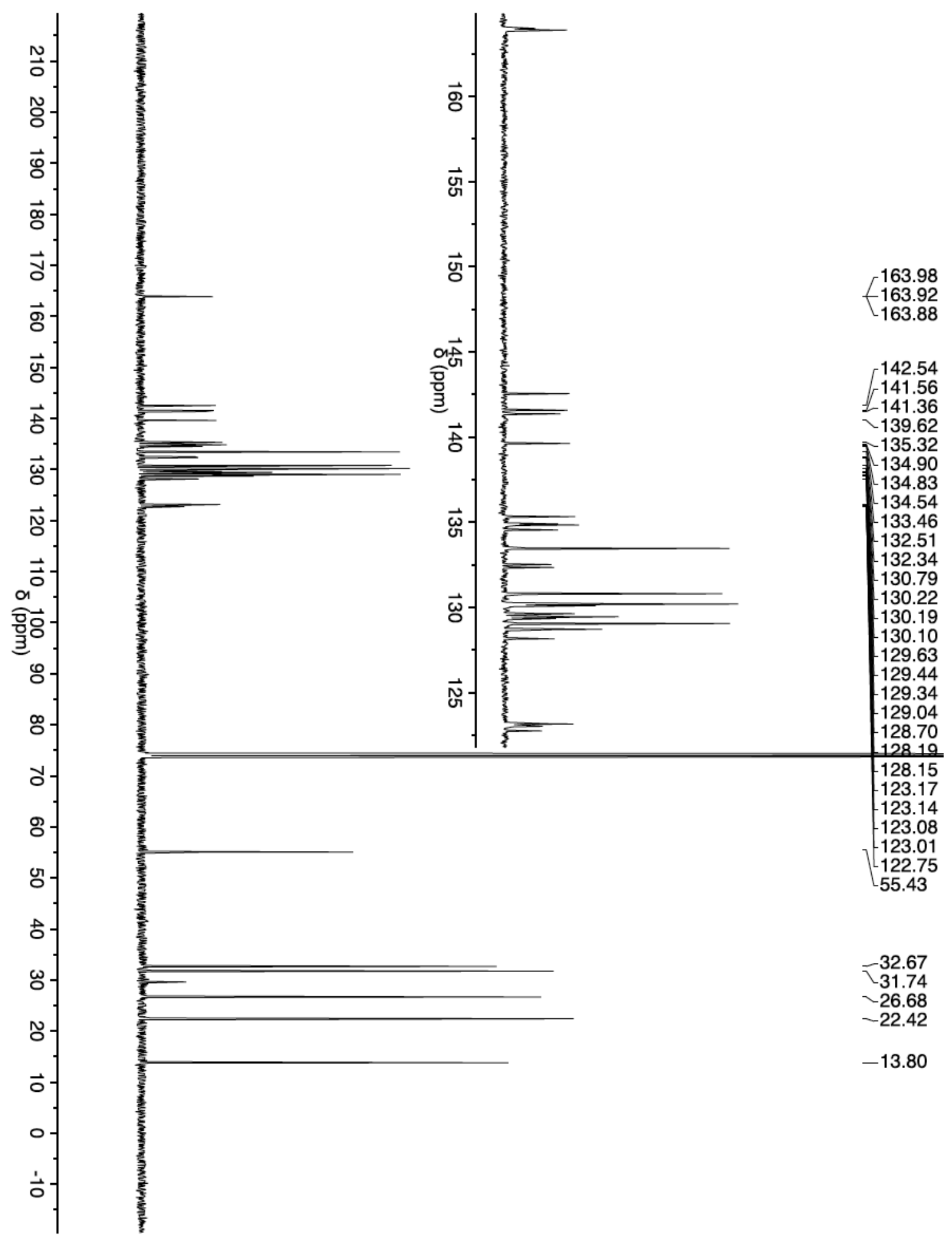




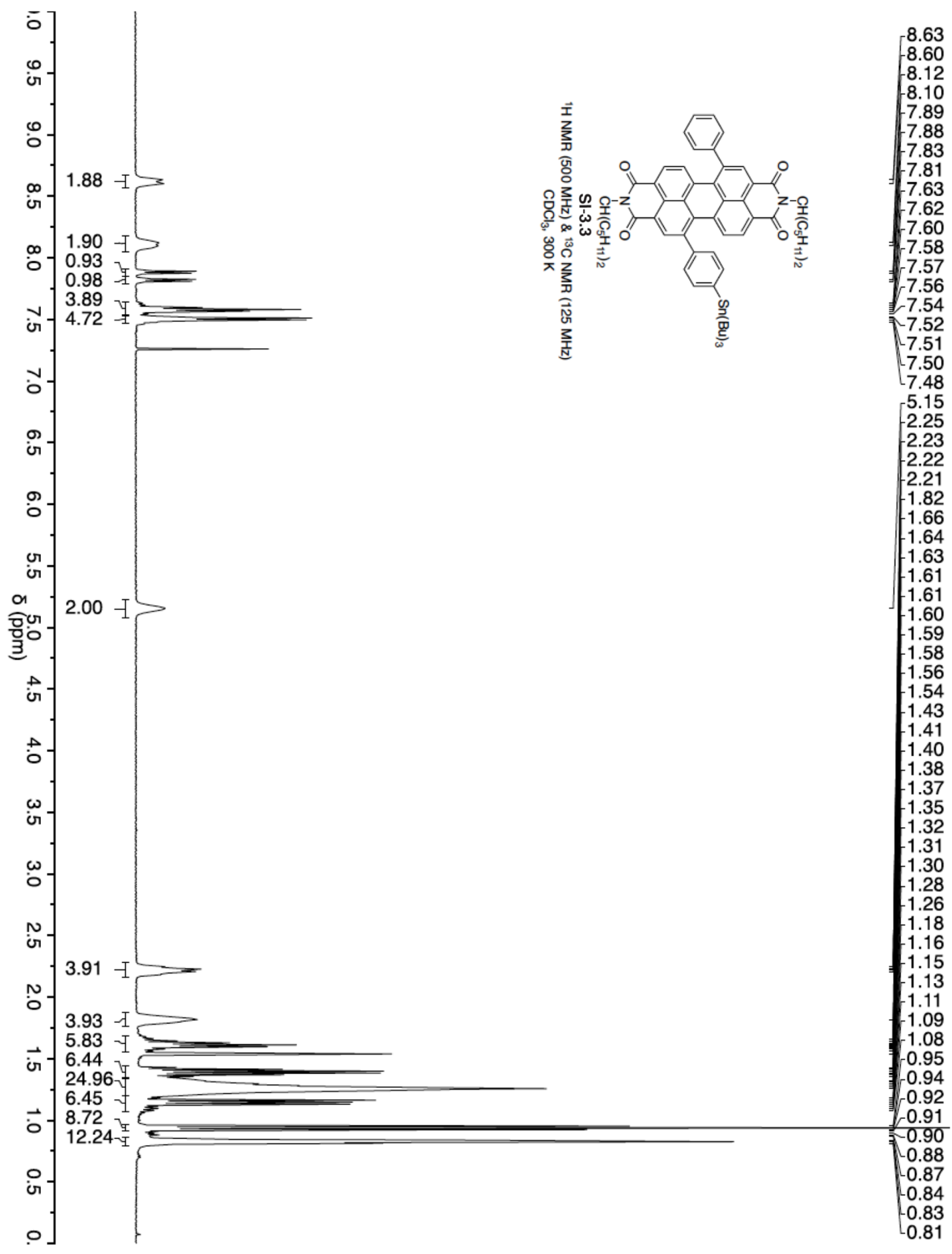




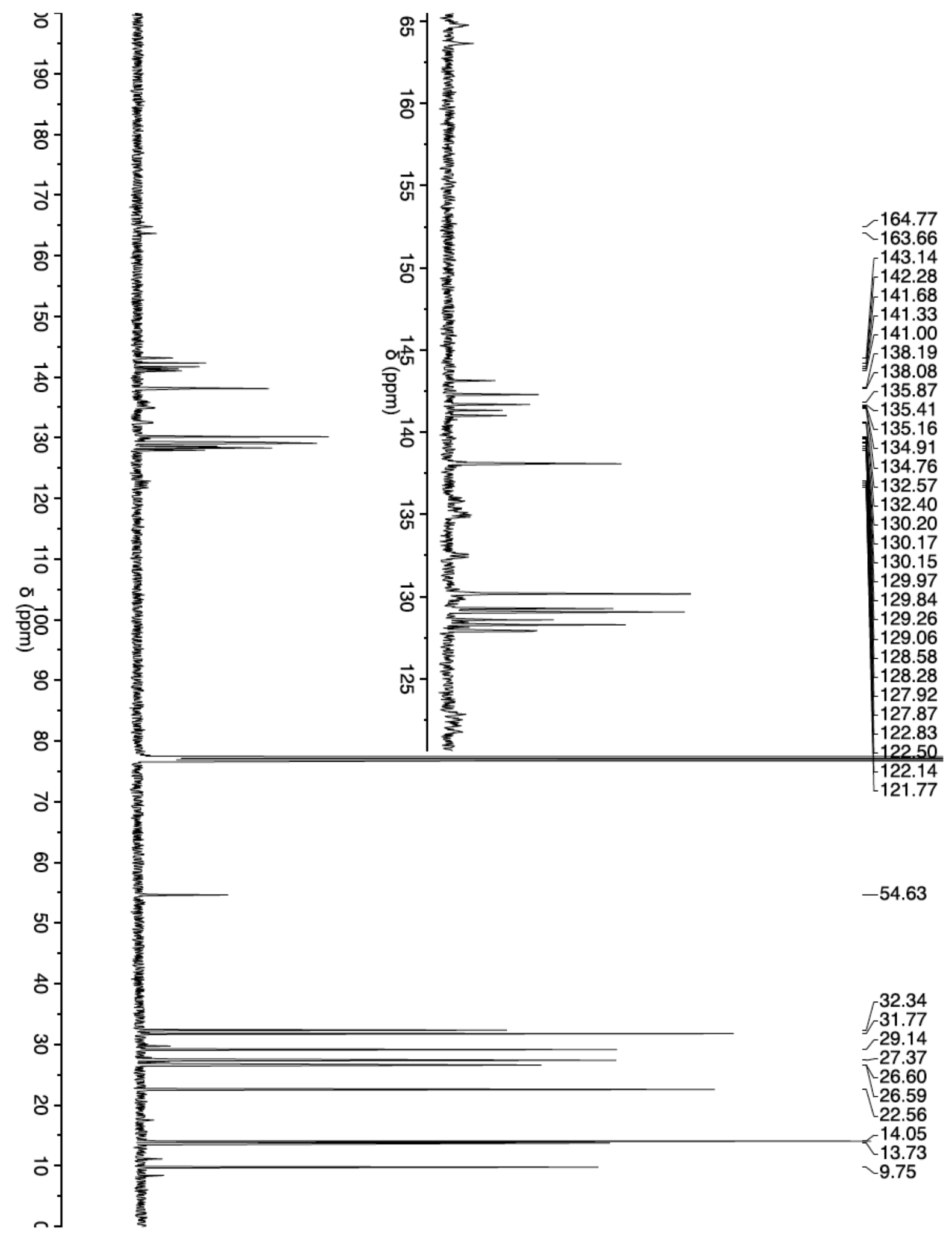




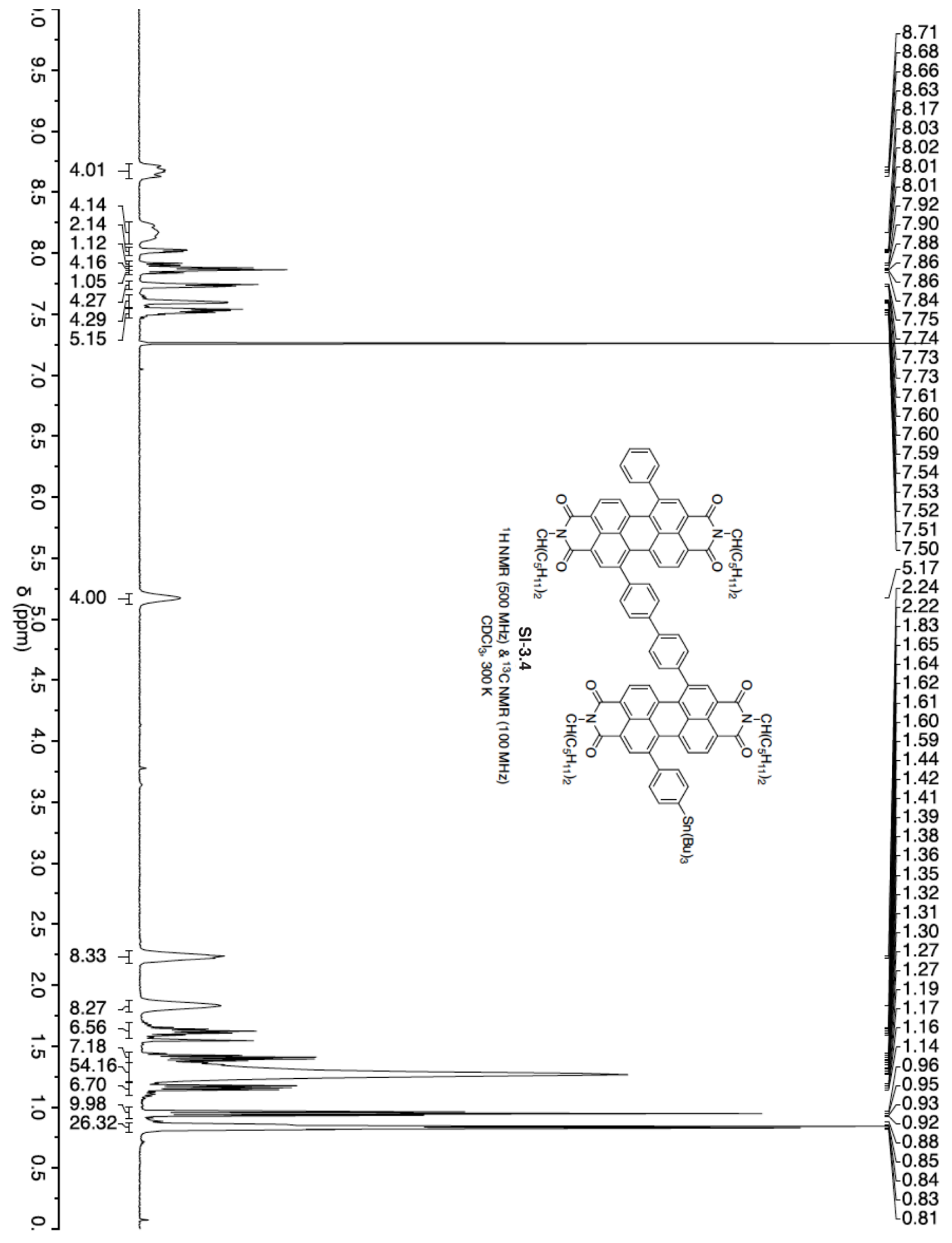




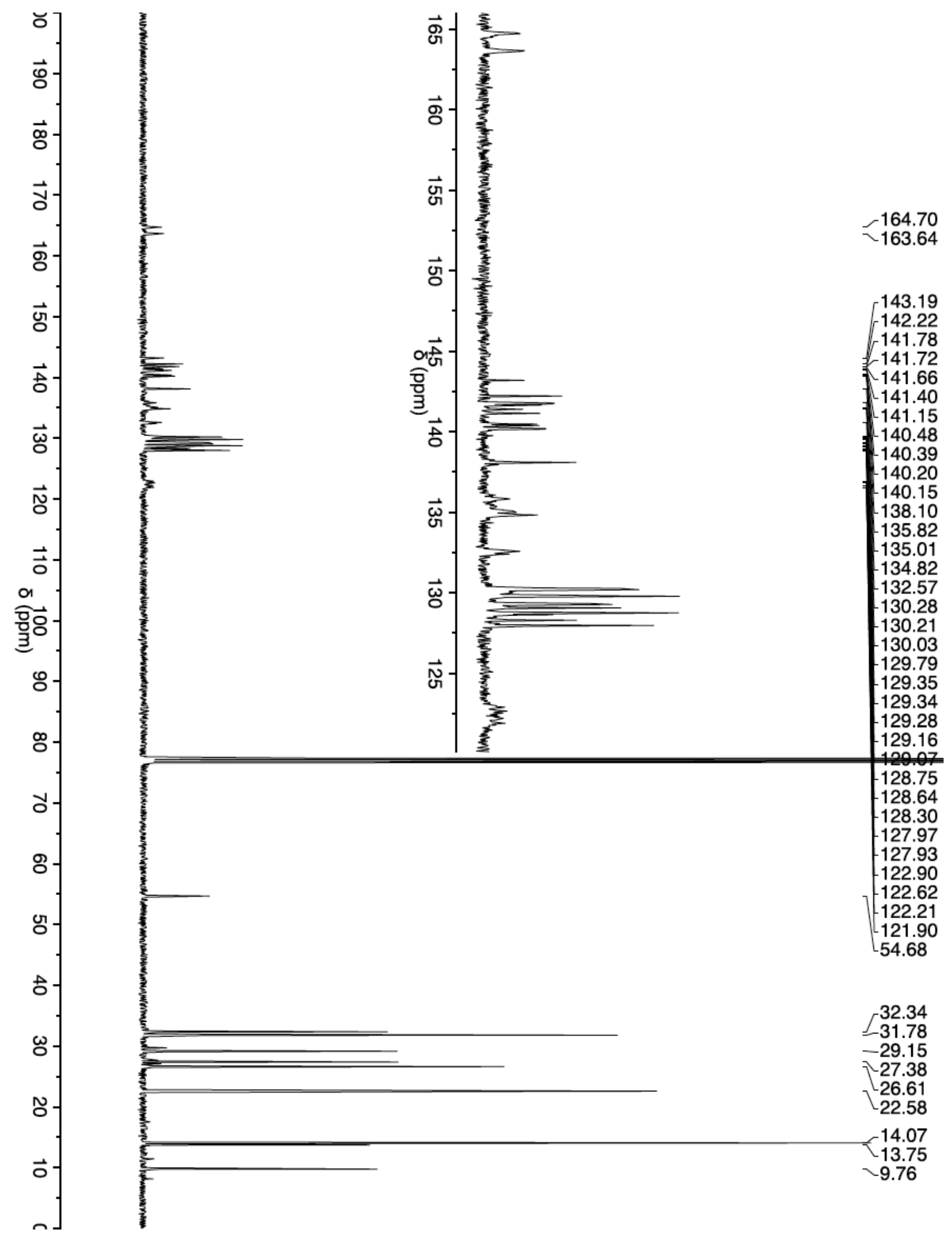




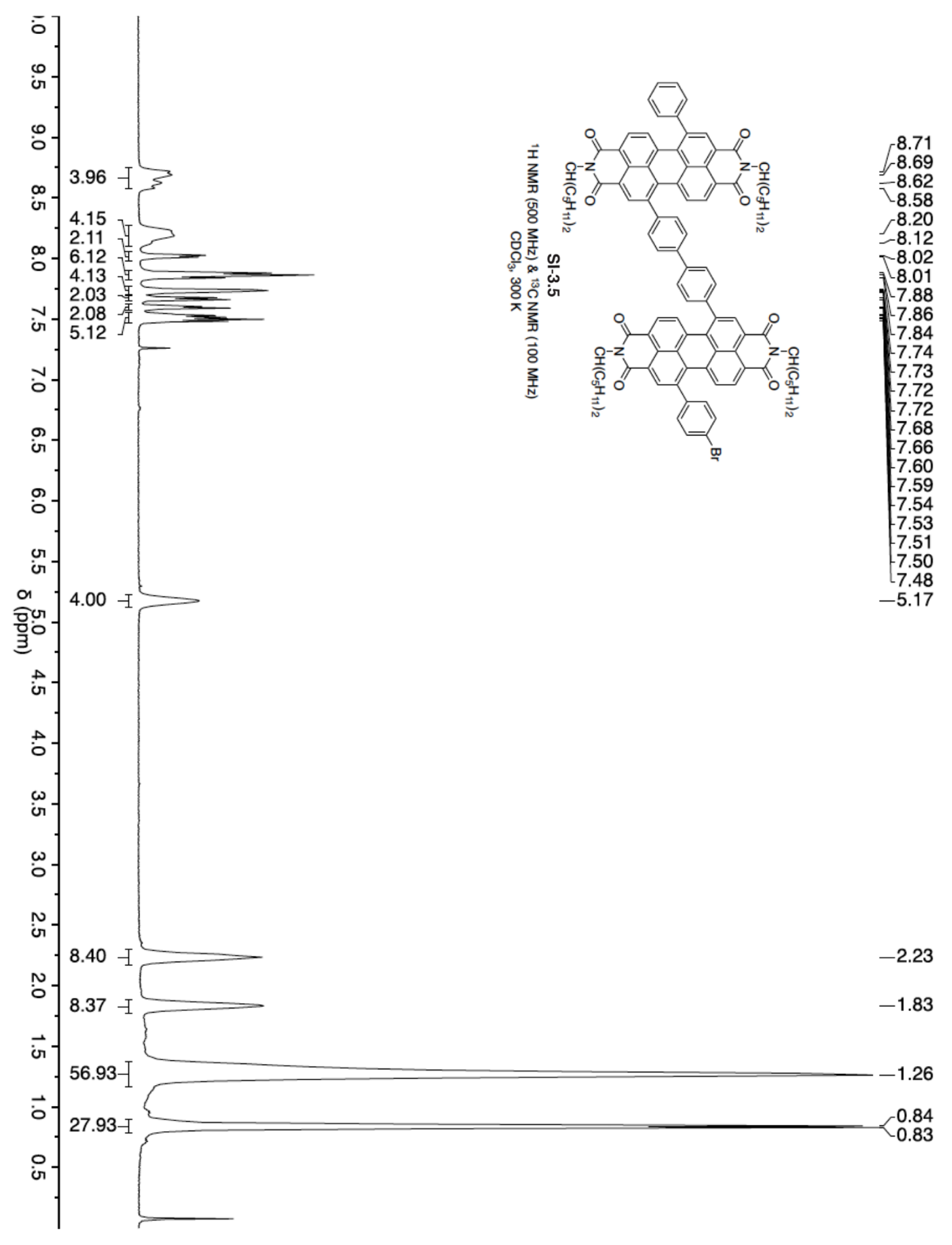




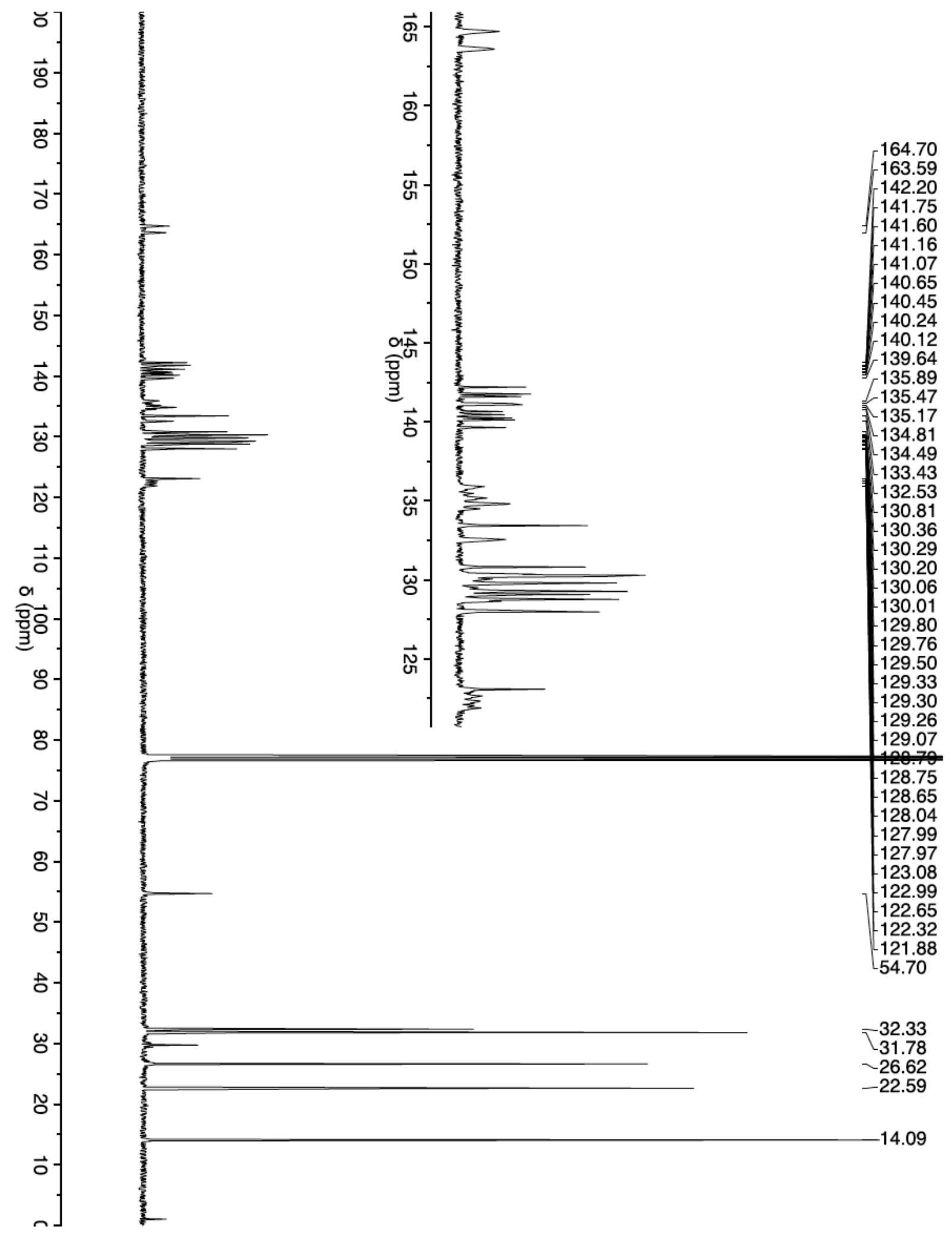




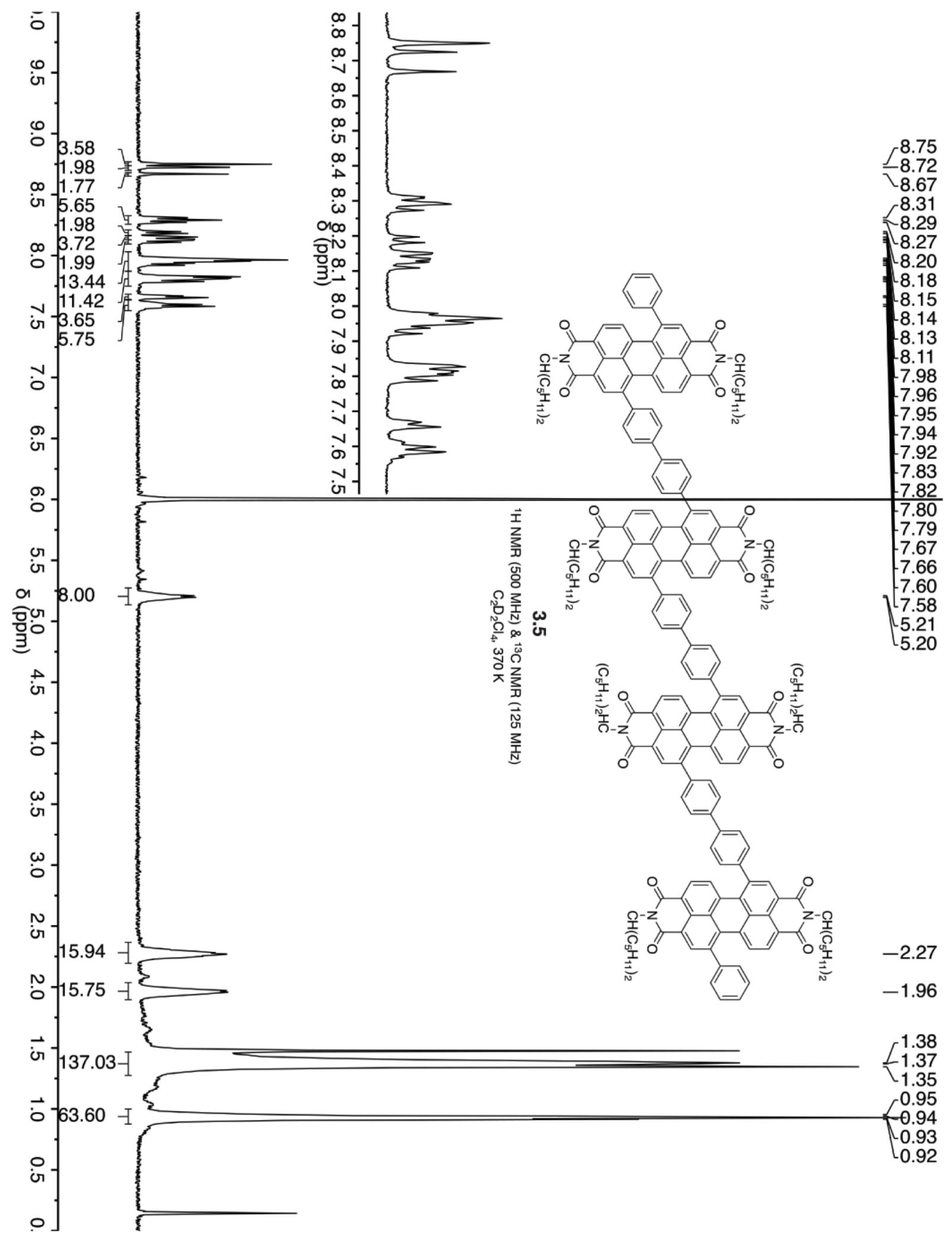




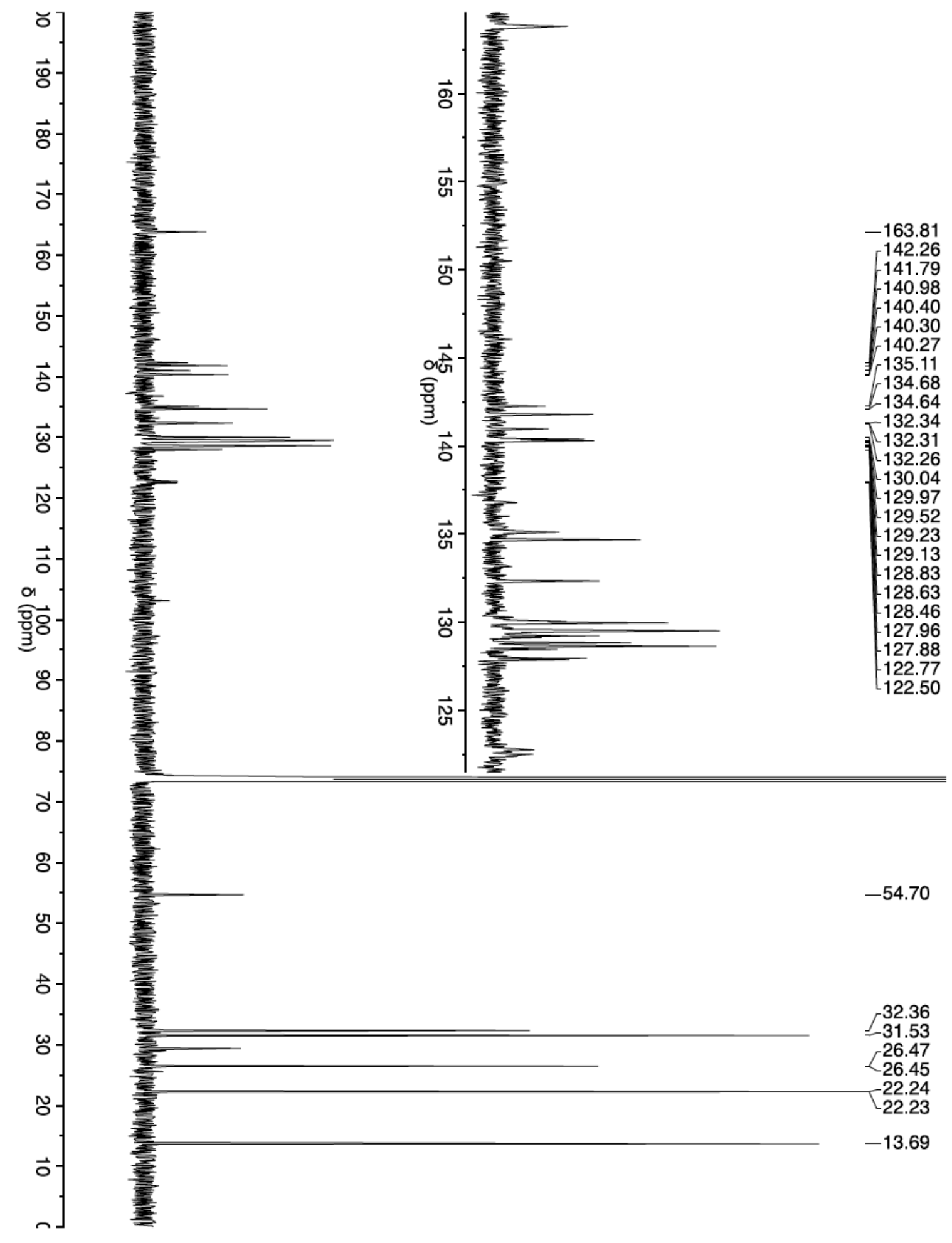




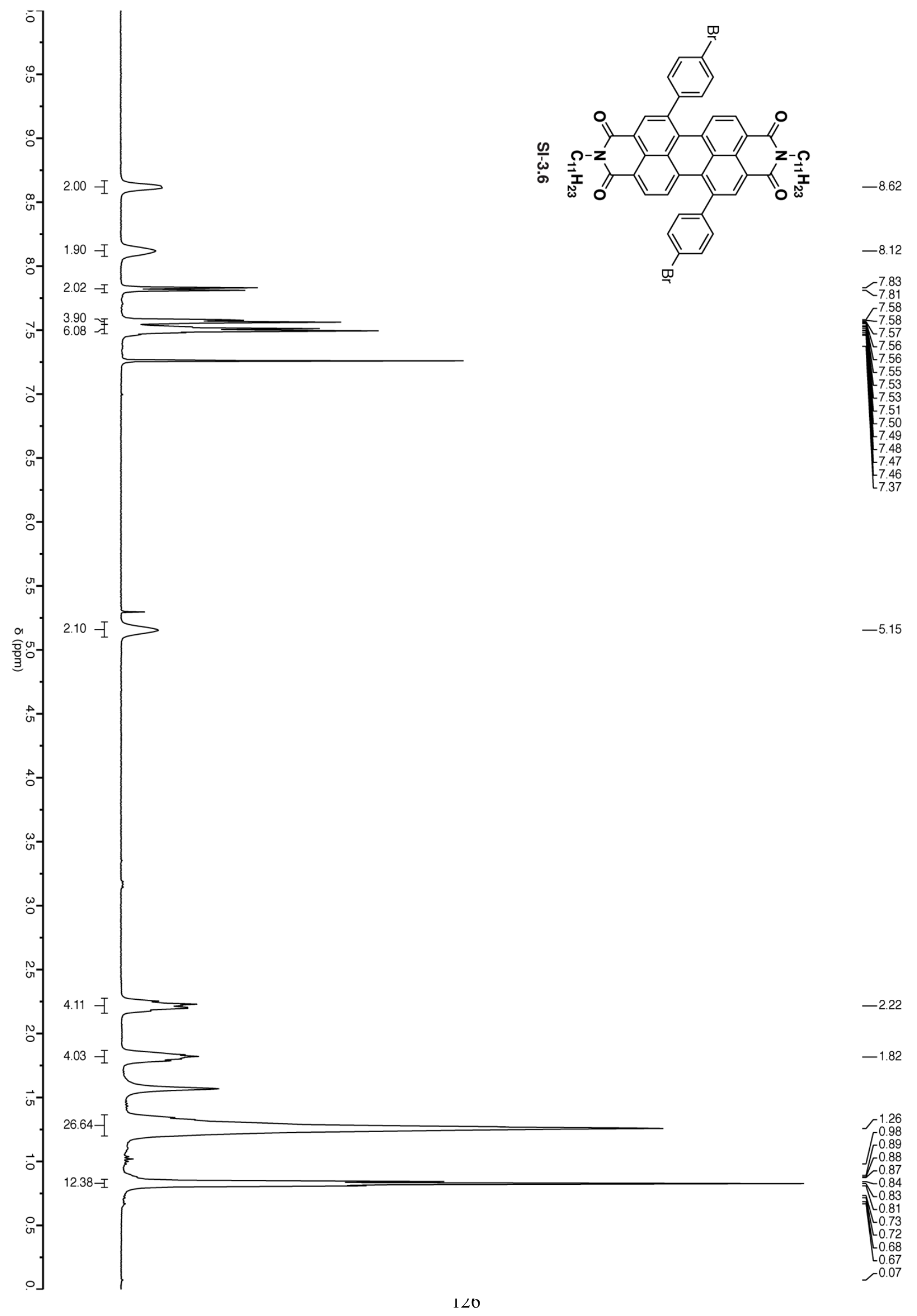




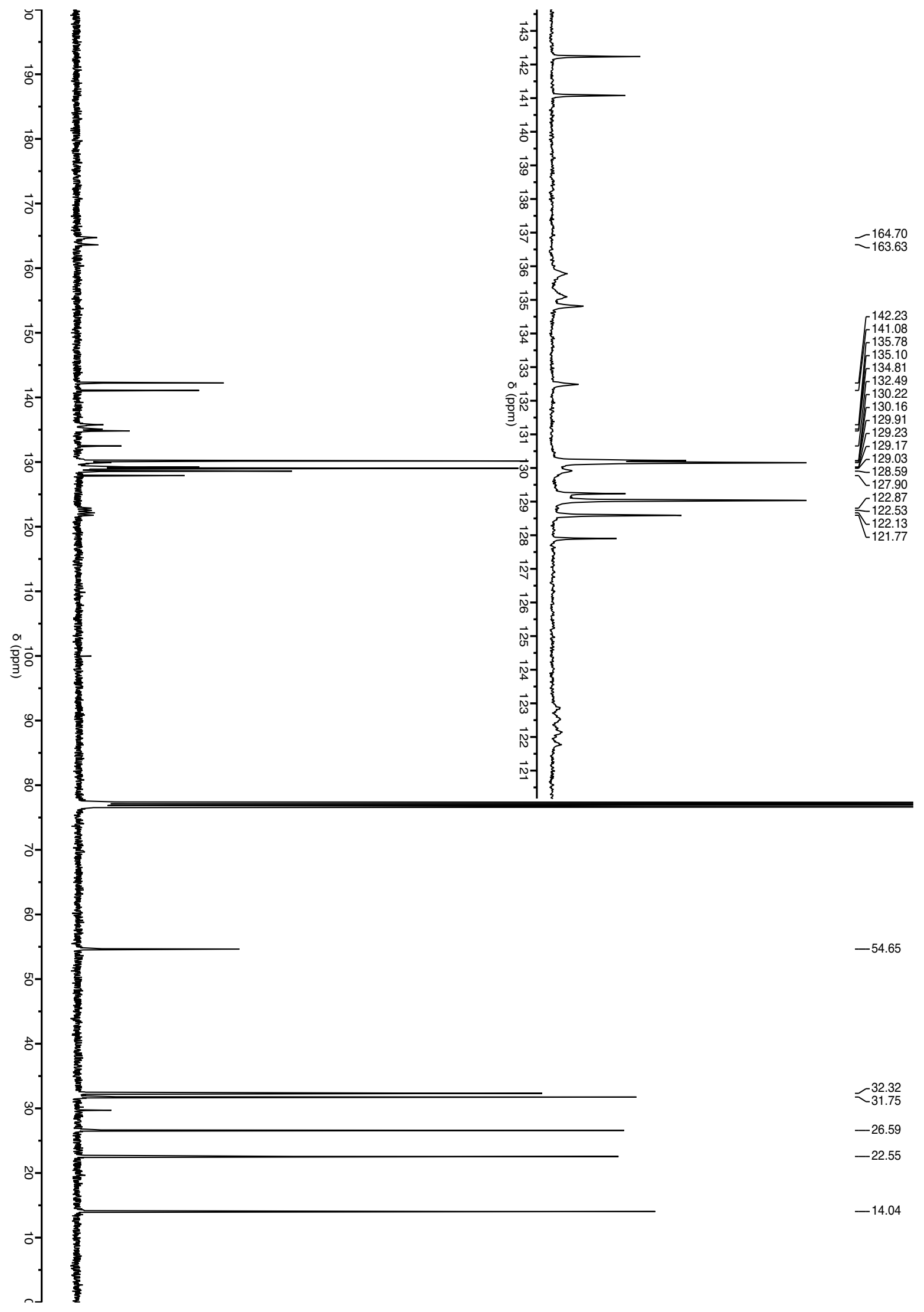




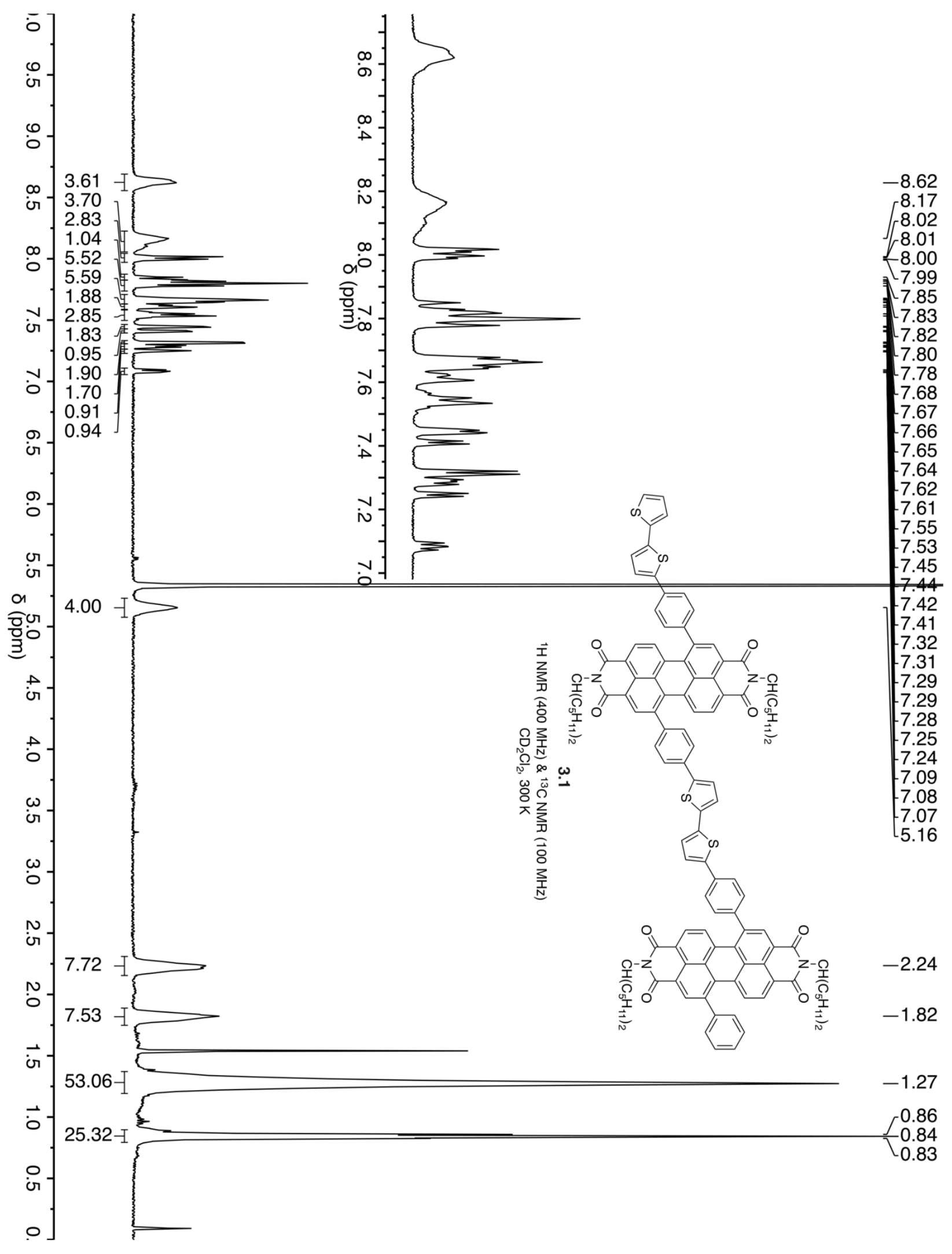




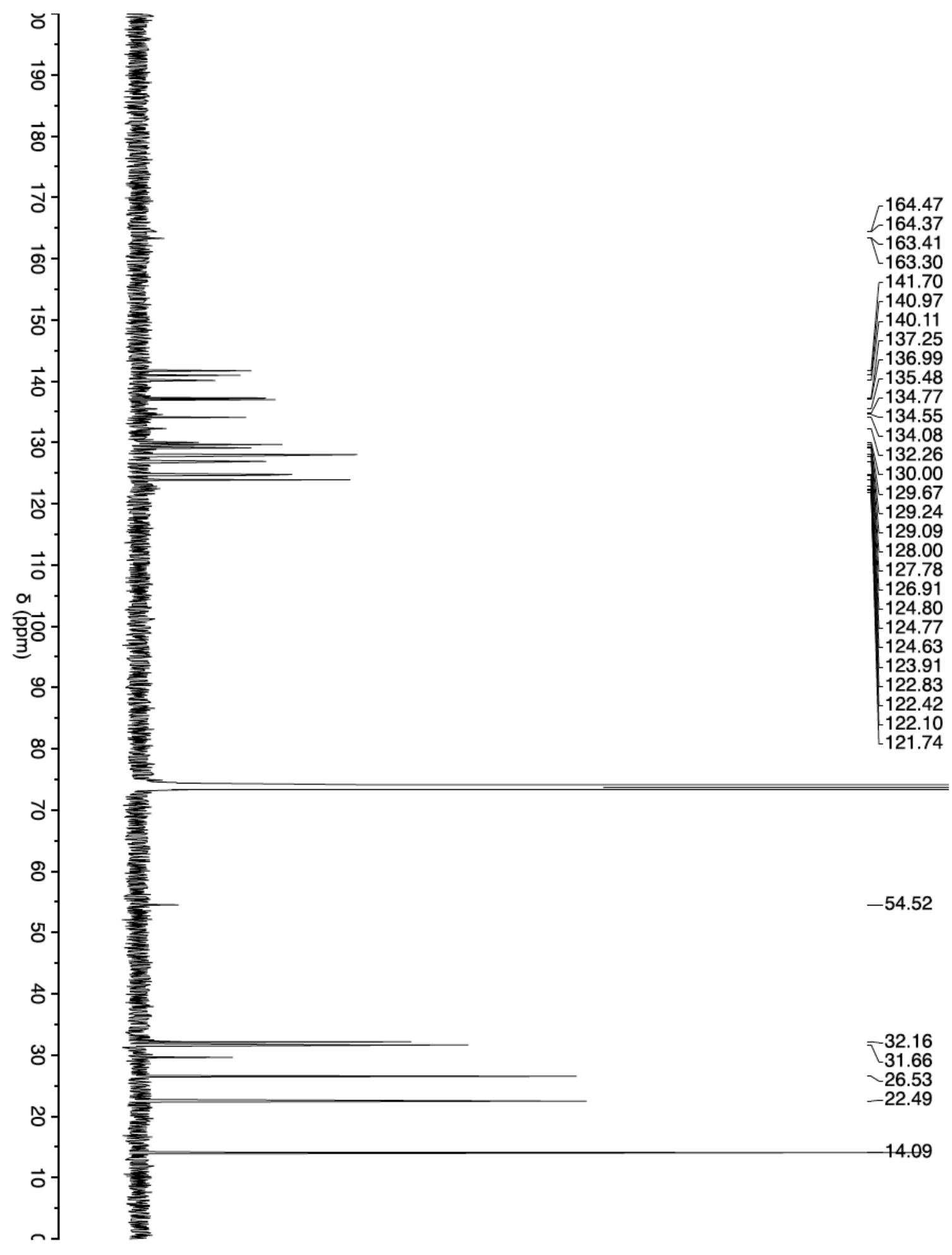




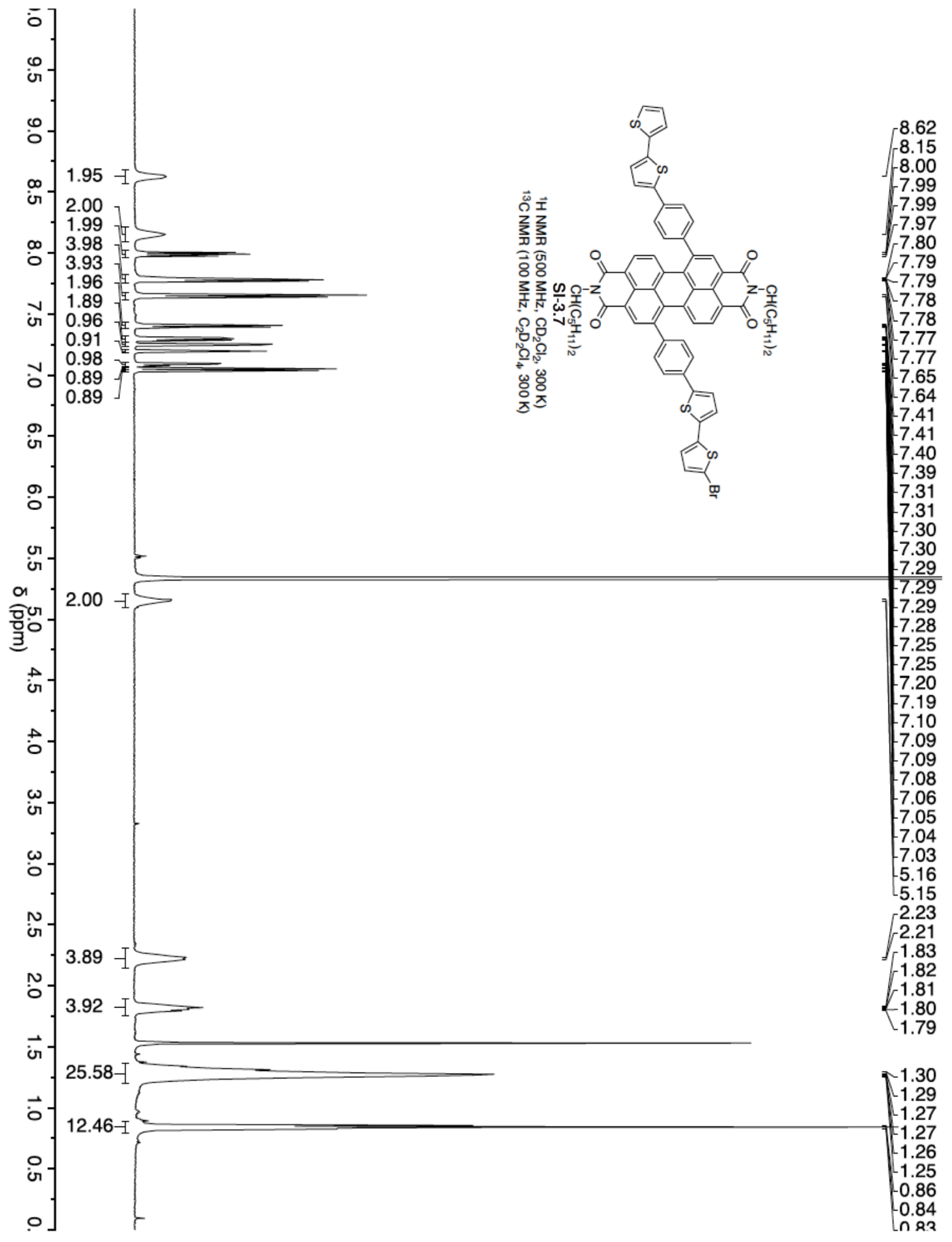




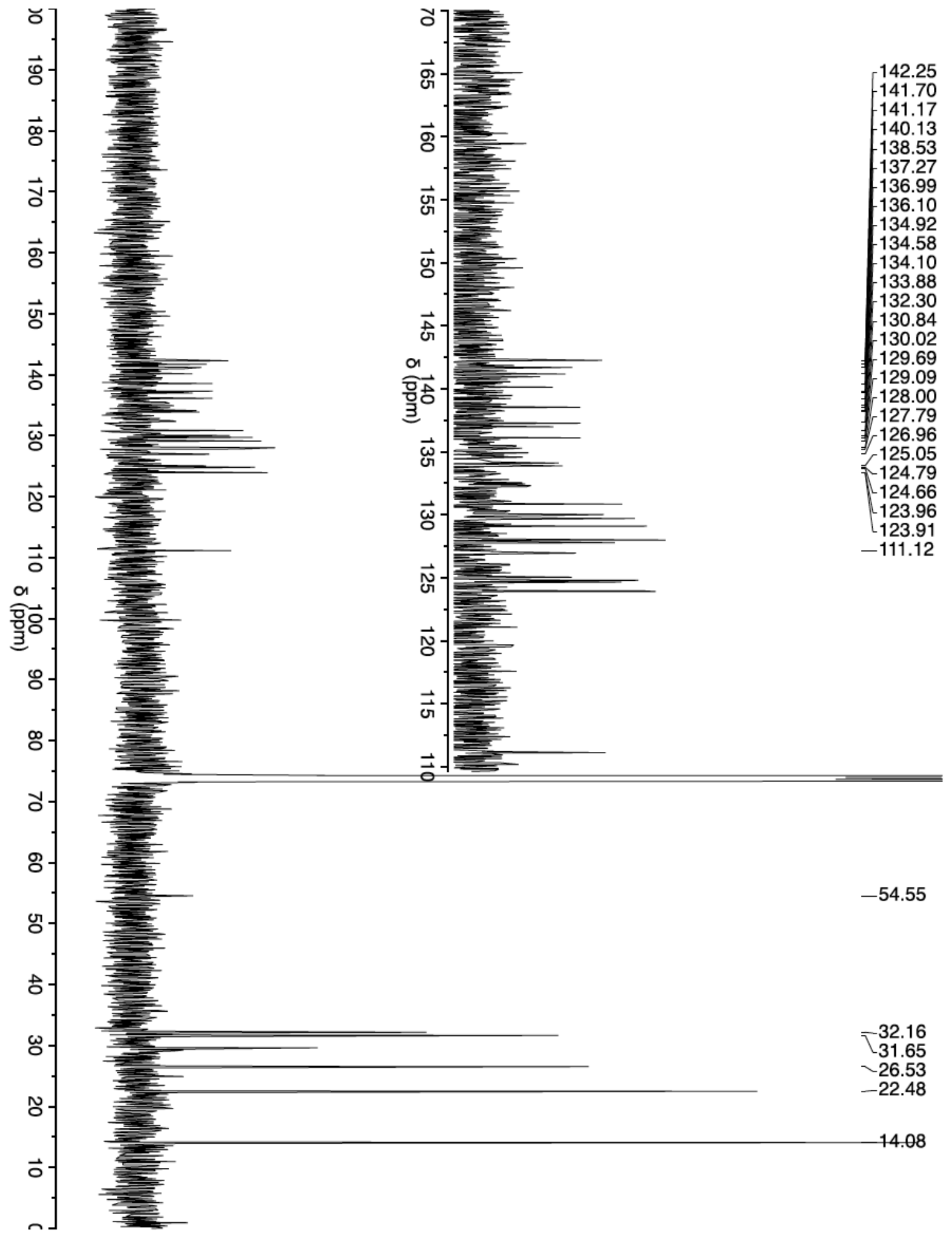




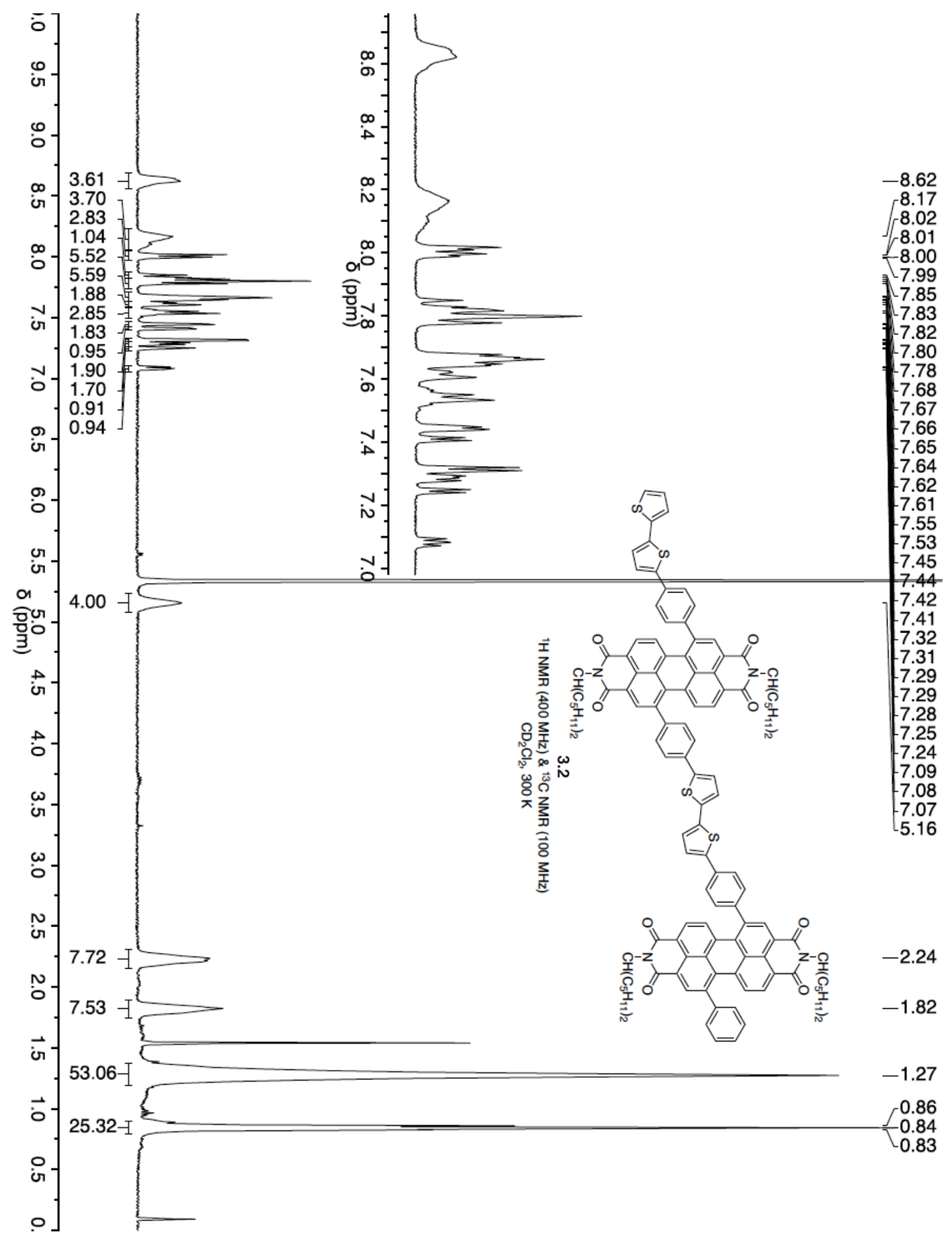




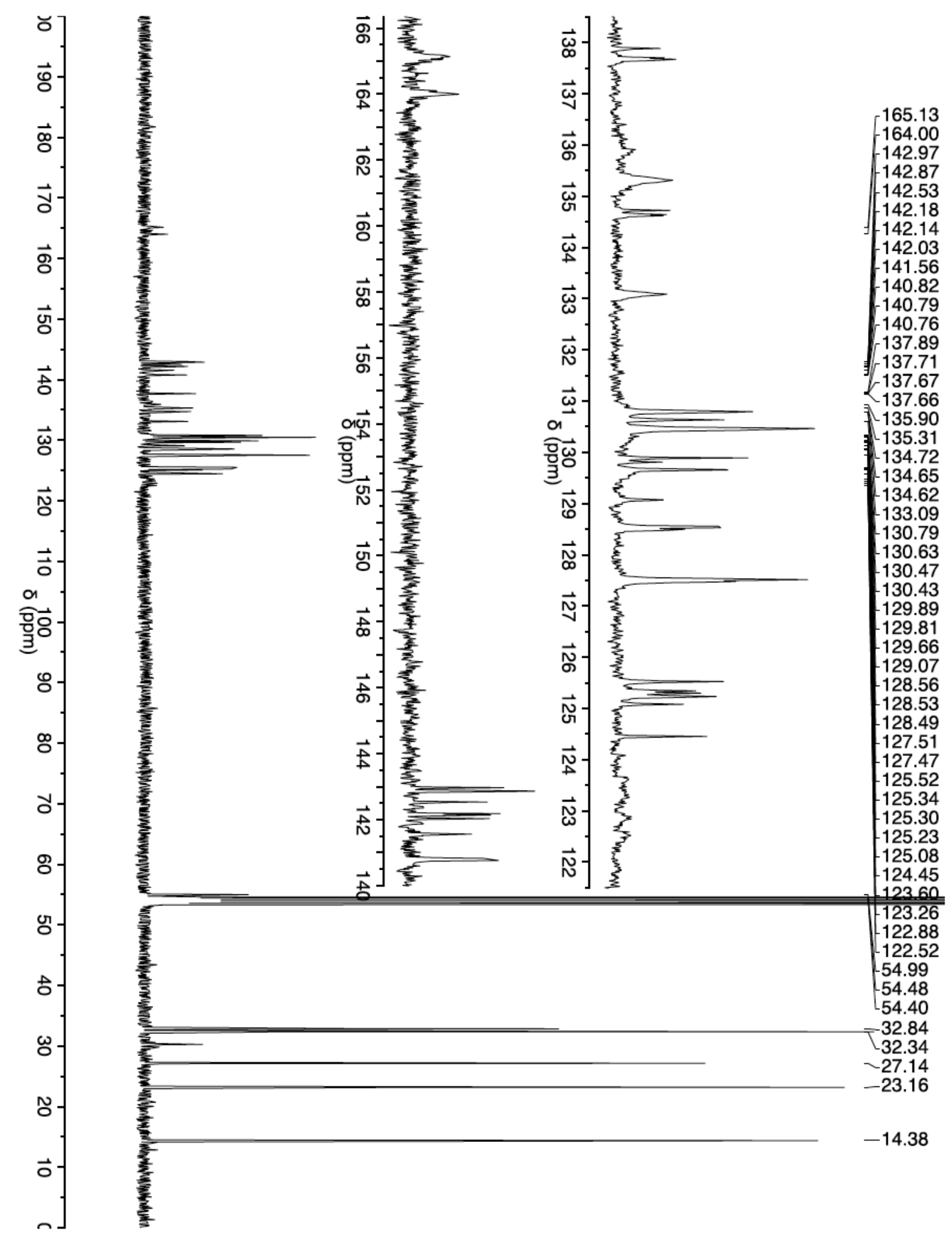




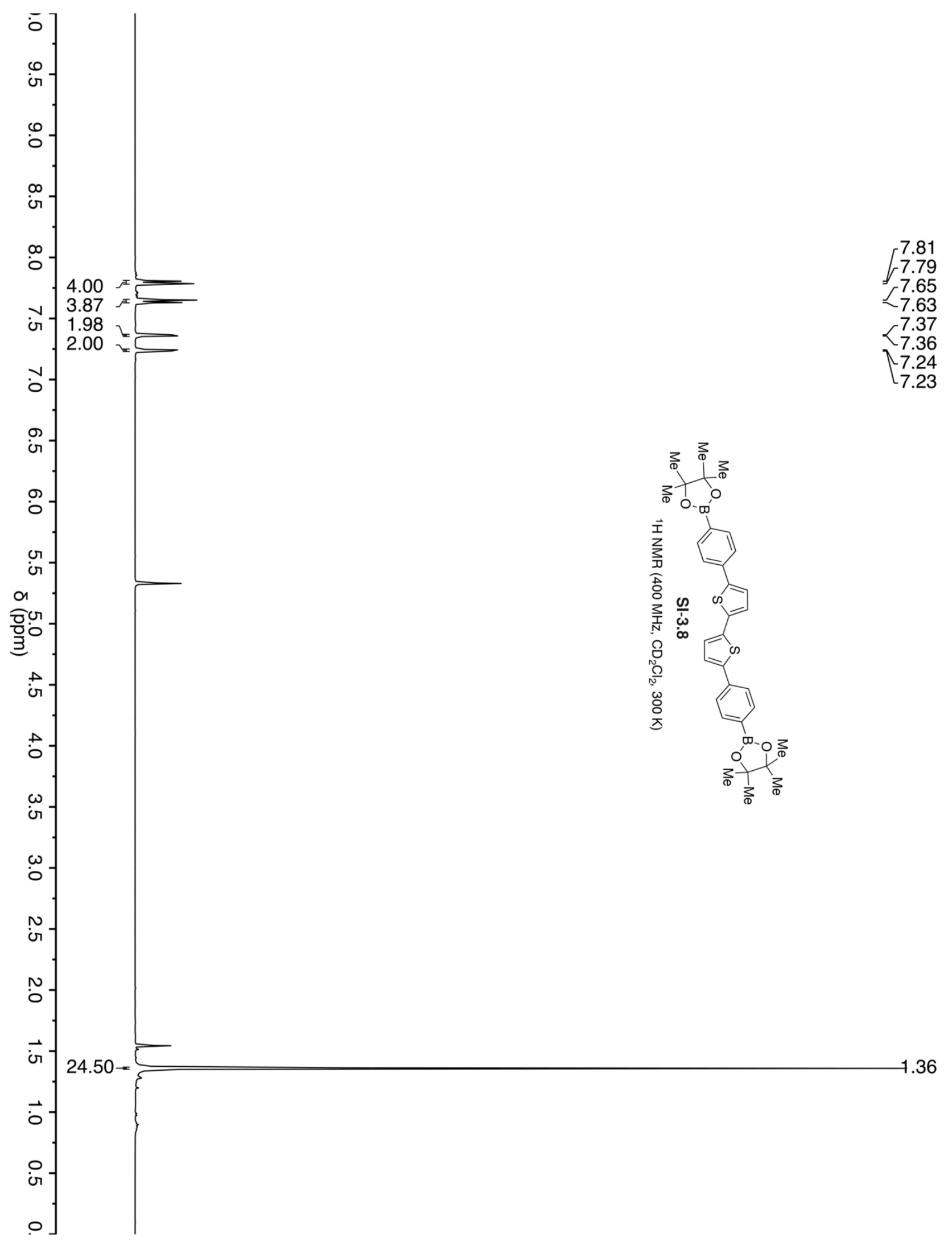




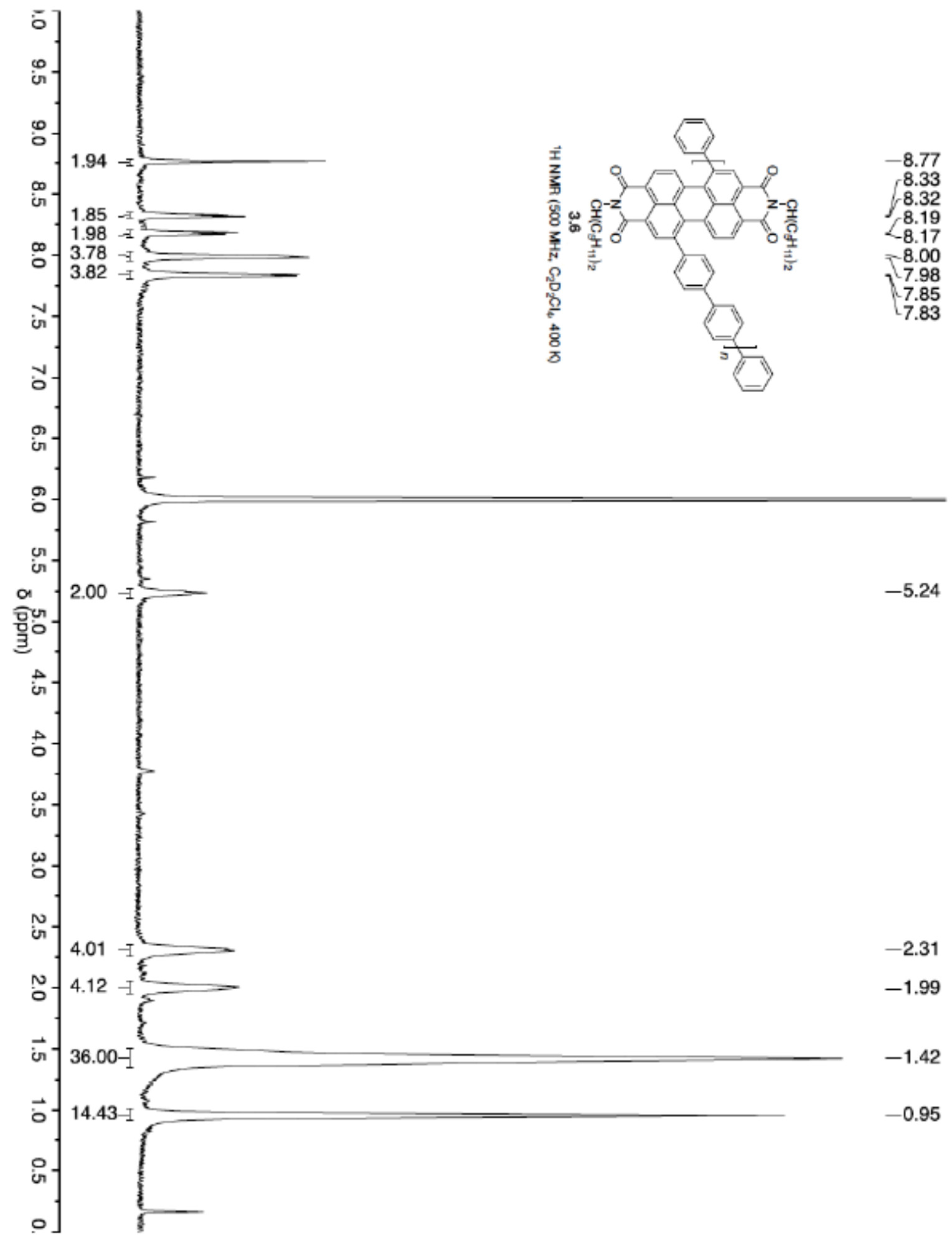




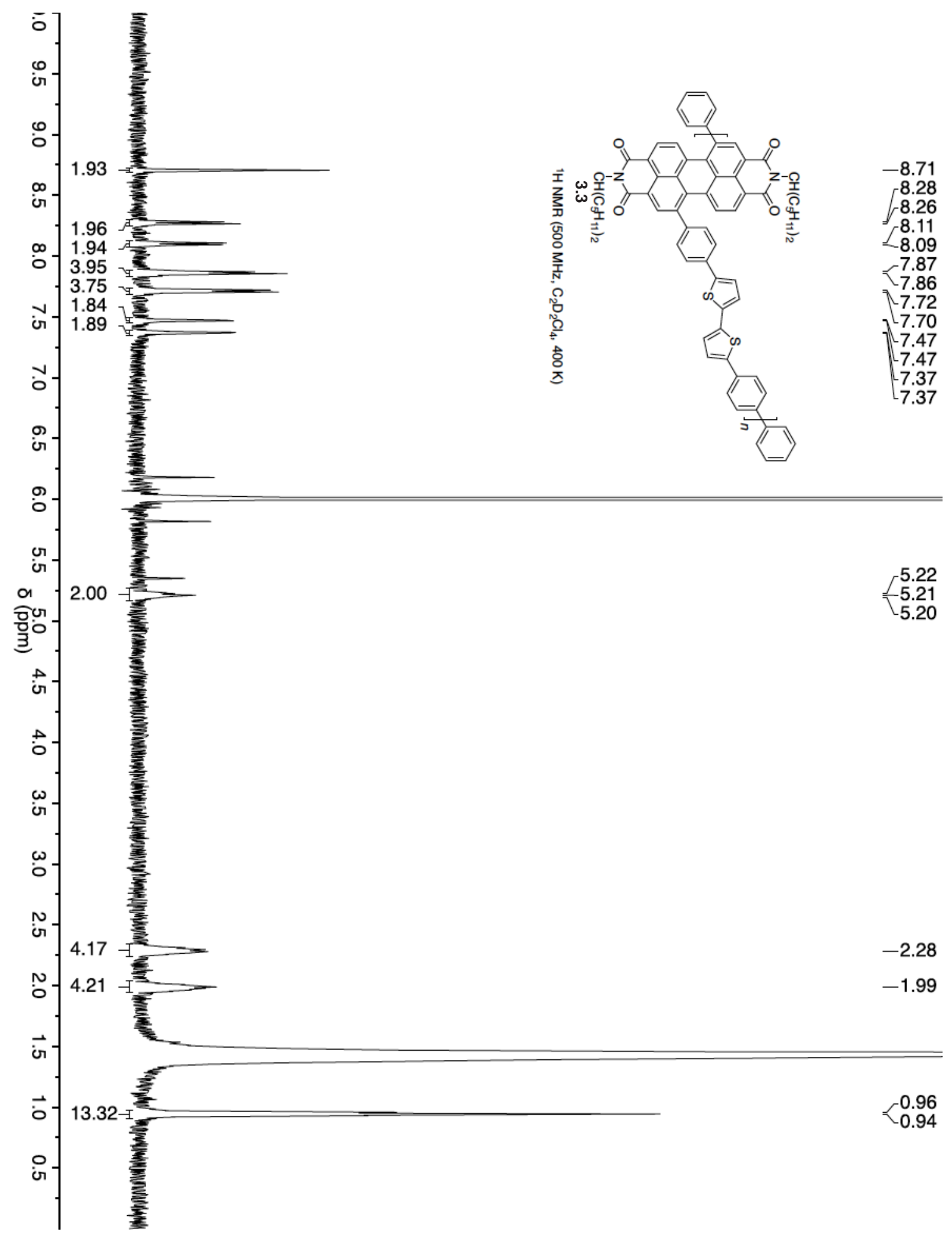




\subsection{DFT Output}

All quantum chemical calculations were performed using Jaguar, version 8.3, Schrodinger, Inc., New York, NY,2013. (See A. D. Bochevarov, E. Harder, T. F. Hughes, J. R. Greenwood, D. A. Braden, D. M. Philipp, D. Rinaldo, M. D. Halls, J. Zhang, R. A. Friesner, "Jaguar: A High Performance Quantum Chemistry Software Program with Strengths in Life and Materials Sciences", Int. J. Quantum Chem., 2013, 113(18), 2110-2142). All geometries were optimized using the B3LYP functional and the 6-31G basis set. The optimized geometry for (PPh $\mathbf{~}_{4}$ 's can be found online at Reference 1 and PBPB can be found at Reference $13 .{ }^{13}$

\subsection{References}

1. Ball, M. et al. Macrocyclization in the Design of Organic n-Type Electronic Materials. $J$. Am. Chem. Soc. 138, 12861-12867 (2016).

2. Krömer, J. et al. Synthesis of the First Fully $\alpha$-Conjugated Macrocyclic Oligothiophenes: Cyclo[n]thiophenes with Tunable Cavities in the Nanometer Regime. Angew. Chem. Int. Ed. 39, 3481-3486 (2000).

3. Nakao, K. et al. Giant Macrocycles Composed of Thiophene, Acetylene, and Ethylene Building Blocks. J. Am. Chem. Soc. 128, 16740-16747 (2006).

4. Hitosugi, S. et al. Atropisomerism in a Belt-Persistent Nanohoop Molecule: Rotational Restriction Forced by Macrocyclic Ring Strain. Chem. Asian J. 7, 1550-1552 (2012).

5. Hitosugi, S. et al. Bottom-up Synthesis and Thread-in-Bead Structures of Finite (n,0)Zigzag Single-Wall Carbon Nanotubes. J. Am. Chem. Soc. 134, 12442-12445 (2012).

6. Ishii, Y. et al. Size-selective synthesis of 9 - 11 and 13 cycloparaphenylenes. Chem. Sci. 3, 2340-2345 (2012).

7. Kayahara, E. et al. Selective Synthesis and Crystal Structure of 10 Cycloparaphenylene. Org. Lett. 14, 3284-3287 (2012).

8. Omachi, H. et al. Synthesis of Cycloparaphenylenes and Related Carbon Nanorings: A Step toward the Controlled Synthesis of Carbon Nanotubes. Acc. Chem. Res. 45, 1378-1389 (2012).

9. Evans, P. J. et al. Efficient room-temperature synthesis of a highly strained carbon nanohoop fragment of buckminsterfullerene. Nat. Chem. 6, 404-408 (2014).

10. Kayahara, E. et al. Synthesis and Characterization of 5 Cycloparaphenylene. J. Am. Chem. Soc. 136, 2284-2287 (2014).

11. Yamago, S. et al. Organoplatinum-Mediated Synthesis of Cyclic pi-Conjugated Molecules: Towards a New Era of Three-Dimensional Aromatic Compounds. Chem. Rec. 14, 84-100 
(2014).

12. Asai, K. et al. A Cyclic Octithiophene Containing B,B'-linkages Chem. Commun. 51, 6096 (2015).

13. Ball, M. et al. Chiral Conjugated Corrals. J. Am. Chem. Soc. 137, 9982-9987 (2015).

14. Jasti, R. et al. Synthesis, Characterization, and Theory of 9 -, 12 -, and 18 Cycloparaphenylene: Carbon Nanohoop Structures. J. Am. Chem. Soc. 130, 17646-17647 (2008).

15. Chang, S.-W. et al. A Donor-Acceptor Conjugated Block Copolymer of Poly(arylenevinylene)s by Ring-opening Metathesis Polymerization. Chem. Commun. 51, 9113-9116 (2015).

16. Chen, Q. et al. Strain-Induced Stereoselective Formation of Blue-Emitting Cyclostilbenes. J. Am. Chem. Soc. 137, 12282-12288 (2015).

17. Darzi, E. R. et al. Synthesis, Properties, and Design Principles of Donor-Acceptor Nanohoops. ACS Cent. Sci. 1, 335-342 (2015).

18. Ito, H. et al. Thiophene-Based, Radial pi-Conjugation: Synthesis, Structure, and Photophysical Properties of Cyclo-1,4-phenylene-2 ',5'-thienylenes. Angew. Chem. Int. Ed. 54, 159-163 (2015).

19. Jiang, H.-W. et al. Cyclic 2,12-Porphyrinylene Nanorings as a Porphyrin Analogue of Cycloparaphenylenes. J. Am. Chem. Soc. 137, 2219-2222 (2015).

20. Kuwabara, T. et al. Curved Oligophenylenes as Donors in Shape-Persistent DonorAcceptor Macrocycles with Solvatofluorochromic Properties. Angew. Chem. Int. Ed. 54, 9646-9649 (2015).

21. Van Raden, J. M. et al. Synthesis and characterization of a highly strained donor-acceptor nanohoop. Org. Biomol. Chem. 14, 5721 (2016).

22. Zhang, F. et al. Giant Cyclo n thiophenes with Extended pi Conjugation. Angew. Chem. Int. Ed. 48, 6632-6635 (2009).

23. Omachi, H. et al. A Modular and Size-Selective Synthesis of [n]Cycloparaphenylenes: A Step toward the Selective Synthesis of n, n Single-Walled Carbon Nanotubes. Angew. Chem. Int. Ed. 49, 10202-10205 (2010).

24. Iwamoto, T. et al. Size-Selective Encapsulation of C60 by [10]Cycloparaphenylene: Formation of the Shortest Fullerene-Peapod. Angew. Chem. Int. Ed. 50, 8342-8344 (2011).

25. Iwamoto, T. et al. Selective and Random Syntheses of $n$ Cycloparaphenylenes $(\mathrm{n}=8-13)$ and Size Dependence of Their Electronic Properties. J. Am. Chem. Soc. 133, 8354-8361 (2011).

26. Segawa, Y. et al. Concise Synthesis and Crystal Structure of [12]Cycloparaphenylene. Angew. Chem. Int. Ed. 50, 3244-3248 (2011).

27. Segawa, Y. et al. [9]Cycloparaphenylene: Nickel-mediated Synthesis and Crystal Structure. Chem. Lett. 40, 423-425 (2011).

28. Sprafke, J. K. et al. Belt-Shaped $\pi$-Systems: Relating Geometry to Electronic Structure in a Six-Porphyrin Nanoring. J. Am. Chem. Soc. 133, 17262-17273 (2011). 
29. Ball, M. et al. Contorted Polycyclic Aromatics. Acc. Chem. Res. 48, 267-276 (2015).

30. Nicolai, H. T. et al. Unification of trap-limited electron transport in semiconducting polymers. Nat. Mater 11, 882-887 (2012).

31. Mandoc, M. M. et al. Trap-limited electron transport in disordered semiconducting polymers. Phys. Rev. B 75, 193202 (2007).

32. Arias, A. C. et al. Materials and Applications for Large Area Electronics: Solution-Based Approaches. Chem. Rev. 110, 3-24 (2010).

33. Kaake, L. G. et al. Intrinsic Charge Trapping in Organic and Polymeric Semiconductors: A Physical Chemistry Perspective. J. Phys. Chem. Lett. 1, 628-635 (2010).

34. Sirringhaus, H. et al. Device Physics of Solution-Processed Organic Field-Effect Transistors. Adv. Mater. 17, 2411-2425 (2005).

35. Iyoda, M. et al. Conjugated Macrocycles: Concepts and Applications. Angew. Chem. Int. Ed. 50, 10522-10553 (2011).

36. Iwamoto, T. et al. Size- and Orientation-Selective Encapsulation of C70 by Cycloparaphenylenes. Chem. Eur. J. 19, 14061-14068 (2013).

37. Nakanishi, Y. et al. Size-Selective Complexation and Extraction of Endohedral Metallofullerenes with Cycloparaphenylene. Angew. Chem. Int. Ed. 53, 3102-3106 (2014).

38. Anthony, J. E. et al. n-Type Organic Semiconductors in Organic Electronics. Adv. Mater. 22, 3876-3892 (2010).

39. Zhou, E. J. et al. All-Polymer Solar Cells from Perylene Diimide Based Copolymers: Material Design and Phase Separation Control. Angew. Chem. Int. Ed. 50, 2799-2803 (2011).

40. Li, C. \& Wonneberger, H. Perylene Imides for Organic Photovoltaics: Yesterday, Today, and Tomorrow. Adv. Mater. 24, 613-636 (2012).

41. Sharenko, A. et al. A High-Performing Solution-Processed Small Molecule: Perylene Diimide Bulk Heterojunction Solar Cell. Adv. Mater. 25, $4403-4406$ (2013).

42. Cai, Y. et al. High Performance Organic Solar Cells Based on a Twisted Bay-Substituted Tetraphenyl Functionalized Perylenediimide Electron Acceptor. Adv. Energy Mater. 5, 1500032 (2015).

43. Nolde, F. et al. Synthesis and self-organization of core-extended perylene tetracarboxdiimides with branched alkyl substituents. Chem. Mater. 18, 3715-3725 (2006).

44. Zhong, Y. et al. Helical Ribbons for Molecular Electronics. J. Am. Chem. Soc. 136, 81228130 (2014).

45. Yan, Q. F. et al. Conjugated Dimeric and Trimeric Perylenediimide Oligomers. Org. Lett. 11, 3426-3429 (2009).

46. Huo, L. J. et al. Synthesis and absorption spectra of n-type conjugated polymers based on perylene diimide. Macromol. Rapid Commun. 29, 1444-1448 (2008).

47. Würthner, F. et al. Perylene Bisimide Dye Assemblies as Archetype Functional Supramolecular Materials. Chem. Rev. 116, 962-1052 (2016). 
48. Zhong, Y. et al. Efficient Organic Solar Cells with Helical Perylene Diimide Electron Acceptors. J. Am. Chem. Soc. 136, 15215 (2014).

49. Meng, D. et al. High-Performance Solution-Processed Non-Fullerene Organic Solar Cells Based on Selenophene-Containing Perylene Bisimide Acceptor. J. Am. Chem. Soc. 138, 375-380 (2016).

50. Wu, Q. et al. Covalently Bound Clusters of Alpha-Substituted PDI-Rival Electron Acceptors to Fullerene for Organic Solar Cells. J. Am. Chem. Soc. 138, 7248-7251 (2016).

51. Liang, Y. et al. For the Bright Future-Bulk Heterojunction Polymer Solar Cells with Power Conversion Efficiency of 7.4\%. Adv. Mater. 22, E135--E138 (2010).

52. Liao, S.-H. et al. Fullerene Derivative-Doped Zinc Oxide Nanofilm as the Cathode of Inverted Polymer Solar Cells with Low-Bandgap Polymer (PTB7-Th) for High Performance. Adv. Mater. 25, 4766-4771 (2013).

53. He, Z. et al. Single-junction polymer solar cells with high efficiency and photovoltage. Nat Phot. 9, 174-179 (2015).

54. Zhong, Y. et al. Molecular helices as electron acceptors in high-performance bulk heterojunction solar cells. Nat Commun. 6, 8242 (2015).

55. Heeger, A. J. 25th Anniversary Article: Bulk Heterojunction Solar Cells: Understanding the Mechanism of Operation. Adv. Mater. 26, 10-28 (2014).

56. Sun, Y. et al. Inverted Polymer Solar Cells Integrated with a Low-Temperature-Annealed Sol-Gel-Derived ZnO Film as an Electron Transport Layer. Adv. Mater. 23, 1679-1683 (2011).

57. You, J. B. et al. A polymer tandem solar cell with $10.6 \%$ power conversion efficiency. Nat. Commun. 4, 10 (2013).

58. Elumalai, N. K. et al. Open circuit voltage of organic solar cells: An in-depth review. Energy Environ. Sci. 9, 291 (2015).

59. Wang, M. et al. High Open Circuit Voltage in Regioregular Narrow Band Gap Polymer Solar Cells. J. Am. Chem. Soc. 136, 12576-12579 (2014).

60. Li, W. et al. High Quantum Efficiencies in Polymer Solar Cells at Energy Losses below 0.6 eV. J. Am. Chem. Soc. 137, 2231 (2015).

61. Hendriks, K. H. et al. Dichotomous Role of Exciting the Donor or the Acceptor on Charge Generation in Organic Solar Cells. J. Am. Chem. Soc. 138, 10026-10031 (2016).

62. Zhao, Y. et al. 25th Anniversary Article: Recent Advances in n-Type and Ambipolar Organic Field-Effect Transistors. Adv. Mater. 25, 5372-5391 (2013).

63. Newman, C. R. et al. Introduction to Organic Thin Film Transistors and Design of nChannel Organic Semiconductors. Chem. Mater. 16, 4436-4451 (2004).

64. Dou, L. et al. 25th Anniversary Article: A Decade of Organic/Polymeric Photovoltaic Research. Adv. Mater. 25, 6642-6671 (2013).

65. Rajasingh, P. et al. Selective Bromination of Perylene Diimides under Mild Conditions. $J$. Org. Chem. 72, 5973-5979 (2007). 


\section{Chapter 4. The Importance of Intramolecular Conductivity in Three Dimensional Molecular Solids}

\subsection{Preface}

Chapter 4 is under review at Chemical Science. The manuscript was prepared by Melissa L. Ball, Boyuan Zhang, Tianren Fu, Ayden M. Schattman, Daniel W. Paley, Fay Ng, Latha Venkataraman, Colin Nuckolls, Michael L. Steigerwald. I synthesized cis-PBPB and its intermediates, with help from Ayden M. Schattman. Fay Ng synthesized the two single molecule control compounds. Boyuan Zhang fabricated the OFETs, and Tianren Fu performed the single molecule conductance measurements. Daniel W. Paley solved the crystal structure for PBPB.

\subsection{Introduction}

Understanding how molecular structure impacts mobility in OFETs has garnered much attention in recent years. ${ }^{1-6}$ Small, flat aromatic molecules, such as linear acenes, have been widely used as the active layer in organic semiconductors due to their relatively high carrier mobilities in both films and single crystal devices. The high carrier mobilities are attributed to strong intermolecular interaction amongst adjacent molecules and low intramolecular reorganization energy. $^{7-9}$ While these two requirements govern charge transport for small, flat aromatic molecules, they are insufficient for complex, three dimensional molecules. In the latter, carriers can become localized, impeding transport. Examples of three dimensional molecular prototype are fullerene and its derivatives, ${ }^{10}$ which are n-type materials used in OFETs, ${ }^{11-13}$ OPVs, ${ }^{14-16}$ and OPDs. ${ }^{16,17}$ However, fullerenes are difficult to synthesize and functionalize, and their optical properties cannot be easily tuned. This prompts the search for alternatives that both absorb visible light and retain structural features, such as a three-dimensional shape. ${ }^{18-22}$ 

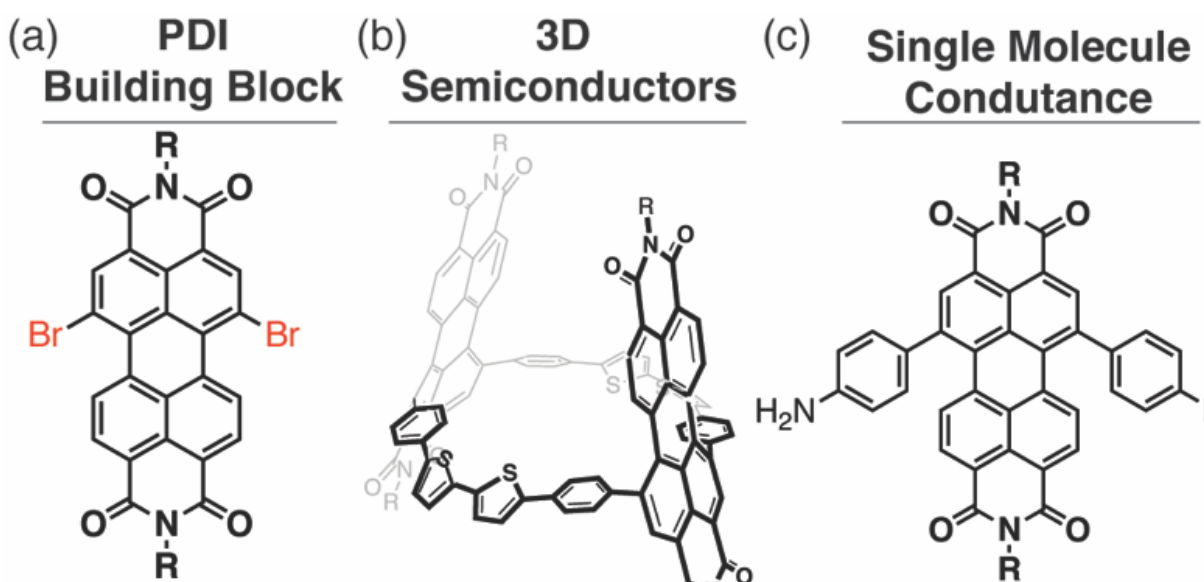

(d)

Acyclic
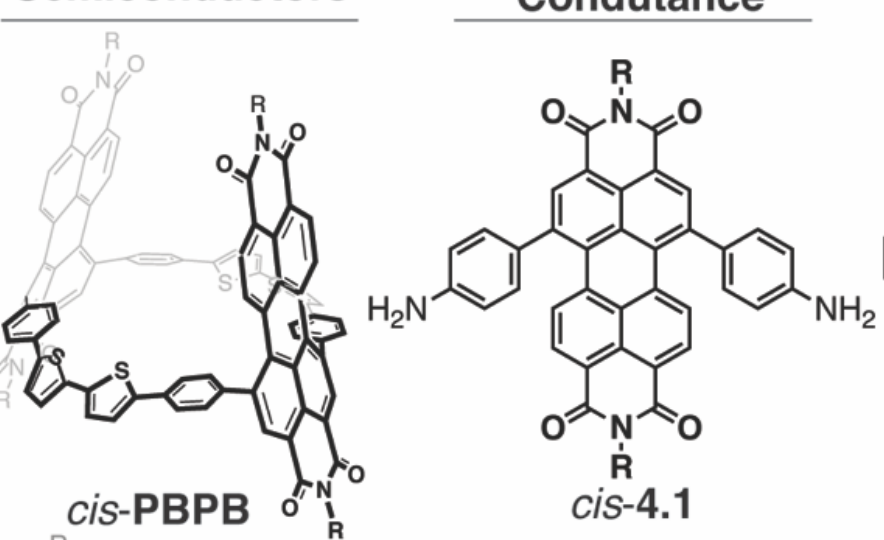

cis-4.1

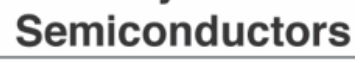

1,6-dibromo PDI<smiles>[R]N1C(=O)c2ccc3c4c(Br)cc5c6c(ccc(c7c(Br)cc(c2c37)C1=O)c64)C(=O)N([R])C5=O</smiles>

1,7-dibromo PDI

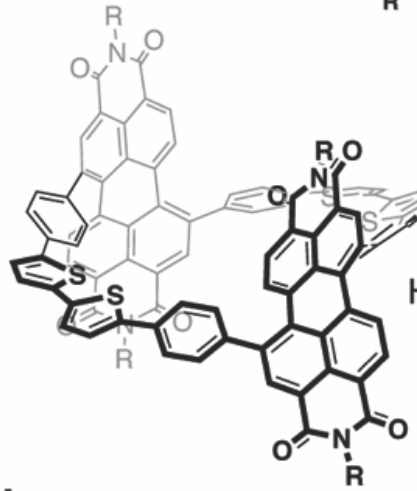

trans-PBPB<smiles>[R]N1C(=O)c2ccc3c4c(-c5ccc(N)cc5)cc5c6c(ccc(c7c(-c8ccc(N)cc8)cc(c2c37)C1=O)c64)C(=O)N(P)C5=O</smiles>

trans-4.1

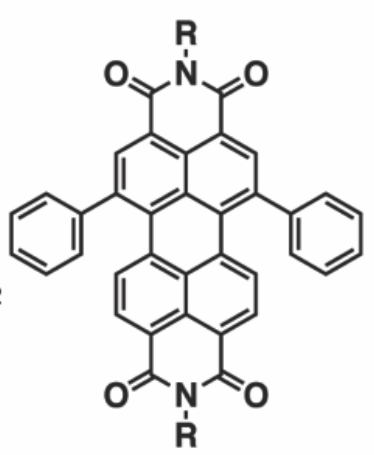

cis-4.2

Figure 4.1. Structures of (a) 1,6 and 1,7-dibromo PDI, with the cis/trans orientation indicated in red; (b) cis-PBPB and trans-PBPB; (c) molecules used for single molecule conductance measurements cis-4.1 and trans-4.1; and (d) structures of acyclic PDI semiconductors cis-4.2 and trans-4.2. Cis- and trans-based molecules are derived from 1,6-dibromo PDI and 1,7-dibromo PDI, respectively. $\mathrm{R}=$ branched $\mathrm{C}_{11} \mathrm{H}_{23}$ side chains.

Here, we study a sub-class of three dimensional, organic materials called conjugated macrocycles. Conjugated macrocycles possess several structural and electronic advantages over acyclic molecules: 1) their contorted structure can facilitate intermolecular contact and charge transport; ${ }^{19,23}$ 2) they contain no end groups that can act as trap sites in linear molecules; ${ }^{24-27} 3$ ) they often absorb more visible light than linear molecules; ${ }^{28,29}$ and 4) their intramolecular cavities can act as a host for electronic guests. ${ }^{30-35}$ We create OFETs with three dimensional molecular solids made from macrocyclic organic semiconductors illustrated in Figure 4.1, and describe the role of intramolecular conductivity on their performance. While electronically active macrocycles 
have been used in organic devices such as transistors, photovoltaics and detectors in recent

years, ${ }^{23,29,33,36-41}$ the impact of molecular structure on device performance is an ongoing field of research. ${ }^{41}$ Our macrocycles were designed to enhance intermolecular interactions through $\pi-\pi$ coupling while allowing for synthetic flexibility to control their electronic properties.

We utilize two types of PDI macrocycles that differ in their connectivity to the phenylbithiophene-phenyl linker: the PDI and linker are in a trans orientation for trans-PBPB and cis orientation for cis-PBPB (Figure 4.1b). Trans-PBPB incorporates a 1,7-substituted PDI isomer into the synthesis while cis-PBPB comprises a 1,6-substituted PDI isomer (Figure 4.1a). We call these macrocycles PBPB, where, $\mathbf{P}$ is PDI, and $\mathbf{B}$ is phenyl-bithiophene-phenyl belt. We previously reported the synthesis of trans-PBPB (Chapter 2). ${ }^{42}$. We measure the device performance in OFETs, and show that electrical mobilities are three times higher in the transbased devices than in the cis-based devices. We study the materials on a single molecule level with macrocyclic components, use control experiments, computations, and spectroscopy to determine that the difference in electron mobility in OFETs made with the two macrocyclic isomers is due to the difference in intramolecular conductivity. This study demonstrates that intramolecular carrier pathways affect electron transport in three-dimensional molecular solids.

\subsection{OFETs - Trans Linkage Produces Higher Current}

We first investigate the impact from the cis- or trans-linkage on the electrical properties of OFETs made using trans- and cis-PBPB (Figure 4.2). Both trans-PBPB and cis-PBPB exhibit ntype characteristics and not p-type characteristics. To validate if the materials show any p-type characteristics, we set the source voltage at $-80 \mathrm{~V}$ and swept the gate voltage to $-80 \mathrm{~V}$. From this measurement, we didn't observe any current in the negative gate region, which confirms the material doesn't show p-type characteristics. The devices show some leakage current due to the 
large difference between the gate voltage when we sweep from $80 \mathrm{~V}$ to $-20 \mathrm{~V}$ and the source-drain current $(80 \mathrm{~V})$. Figure 4.2 shows the output curves for both macrocycles, and shows both devices saturate at a source/drain voltage of $80 \mathrm{~V}$.
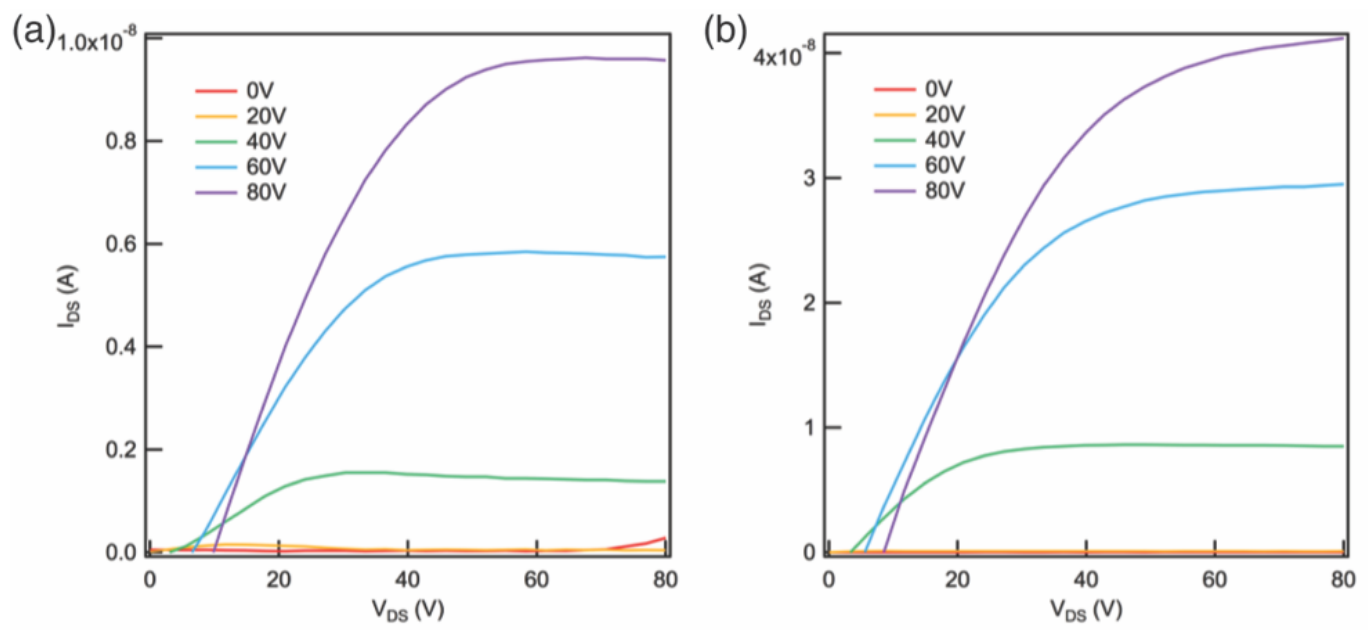

Figure 4.2. Output curves for (a) cis-PBPB and (b) trans-PBPB films from OFETs. In both devices, the source-drain voltage saturates at $80 \mathrm{~V}$.

Figures 4.3a,b display the current versus applied gate voltages (transfer curves) for a trans and cis device. We collected the data for these transfer curves using a source-drain voltage of 80 $\mathrm{V}$ while sweeping the gate voltage from $-20 \mathrm{~V}$ to $80 \mathrm{~V}$. The mobility was calculated in the saturation regime ${ }^{3,43}$ using $I_{D S}=(W / 2 L) C_{i} \mu\left(V_{G^{-}} V_{T}\right)^{2}$, where $W$ and $L$ are the width and length of the channel, $C_{i}\left(11.5 \mathrm{nFcm}^{-2}\right), \mu, V_{G}$, and $V_{T}$ correspond to the capacitance per unit area of the gate insulator, the field effect mobility, the gate voltage, and the threshold voltage, respectively. We find the mobility in trans-PBPB is three times that in cis-PBPB $\left(1.3 \times 10^{-3} \mathrm{~cm}^{2} / \mathrm{V} \bullet \mathrm{s}\right.$ versus $0.4 \times$ $\left.10^{-3} \mathrm{~cm}^{2} / \mathrm{V} \cdot \mathrm{s}\right)$. We reproduce these mobility measurements across many samples. For example, we made ten devices with each isomer and found that the same values for the mobilities. Table 4.1 provides the averaged data for each macrocycle and Table 4.2 provides the OFET characteristics of both devices. 


\subsection{Film Morphology}

(a)

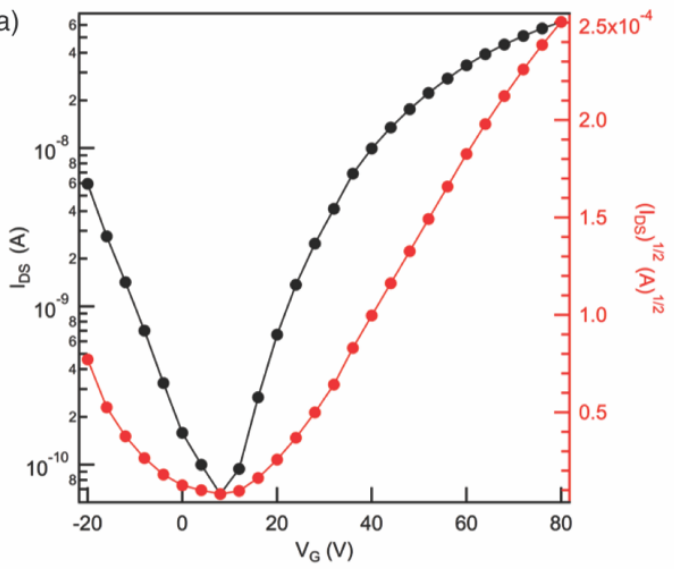

(c)

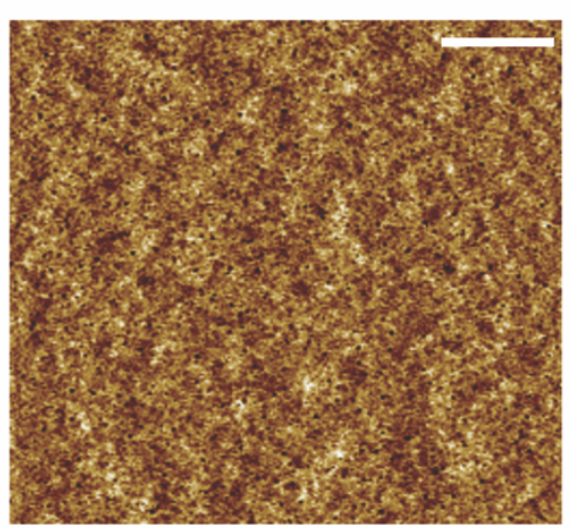

(b)

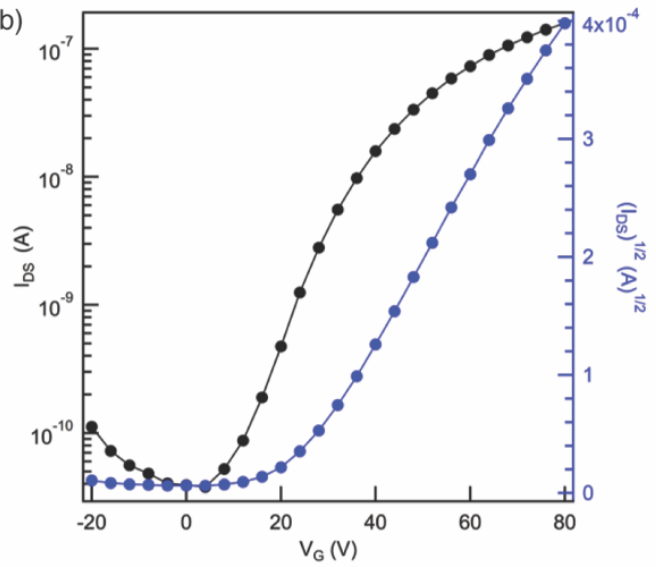

(d)

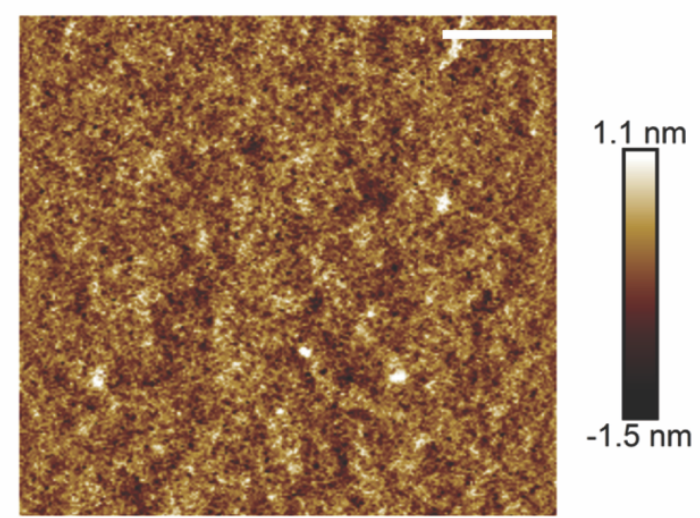

(e)
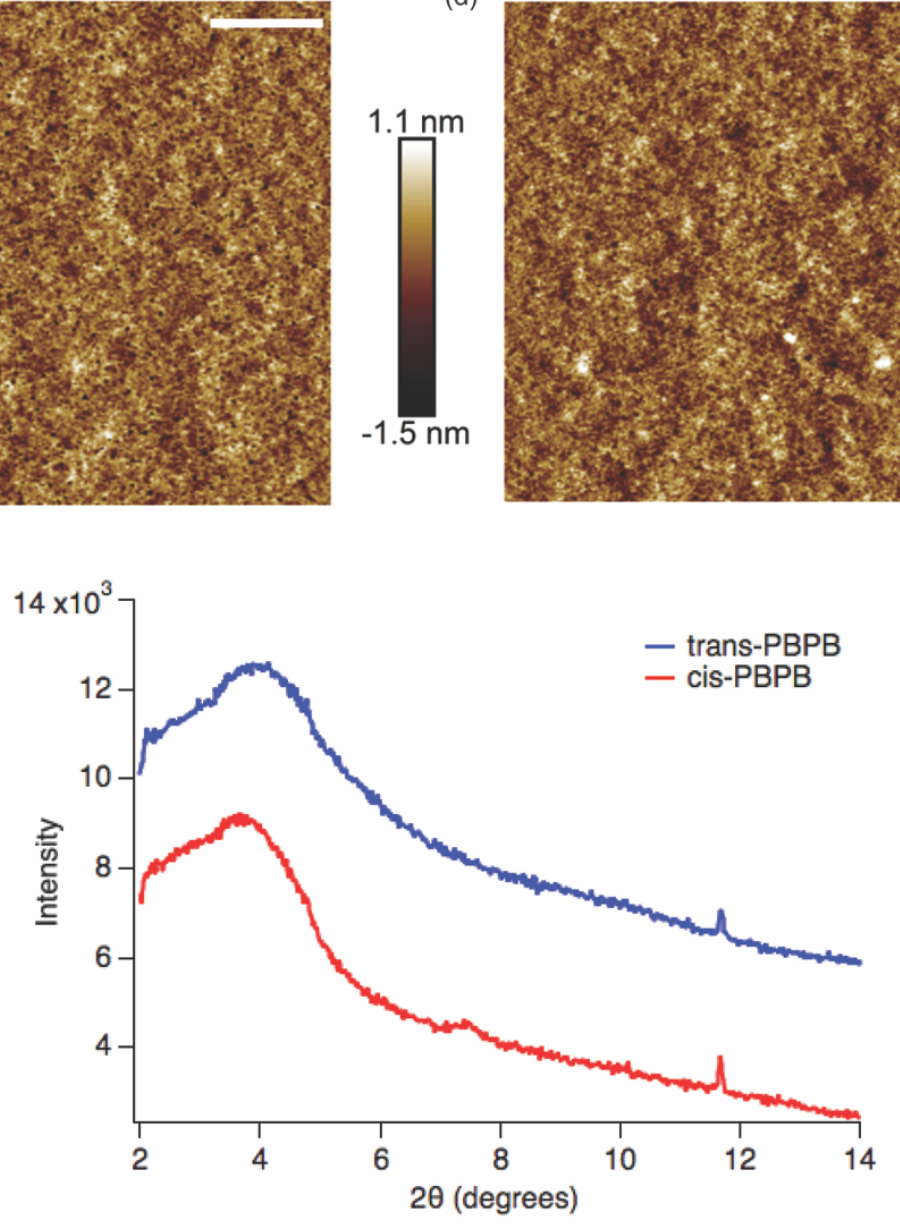

Figure 4.3. (a) Transfer curve for cis-PBPB; (b) transfer curve for trans-PBPB. Device current (left axis, black) and square root of current (right axis, red or blue) measured as a function of gate voltage at a constant source-drain voltage of $80 \mathrm{~V}$. (c) Height image for cis-PBPB and (d) transPBPB. Both films are continuous and smooth and have a root mean square roughness of 0.35 and $0.37 \mathrm{~nm}$ for the cis and trans-based devices, respectively. The scale bar is $1.0 \mu \mathrm{m}$. (e) PXRD of both films showing no obvious signs of crystallinity. Films drop-cast from $\mathrm{CDCl}_{3}$. 
As morphology is known to have a profound effect on mobility, we first examined the film morphology using AFM to see if morphological differences could explain the difference in

mobility. ${ }^{2,5,44,45}$ Both films were continuous and smooth, and had a root mean square roughness of $0.35 \mathrm{~nm}$ and $0.37 \mathrm{~nm}$ for cis-PBPB and trans-PBPB, respectively (Figures $4.3 \mathrm{c}, \mathrm{d}$ ). The Powder/thin-film X-ray Diffraction (PXRD) of both films too show no obvious signs of crystallinity (Figure 4.3e). This suggests that morphology does not explain the different transport characteristics for the three-dimensional semiconductors.

\subsection{Molecular Structures of Isomeric Macrocycles}

The synthesis for cis-PBPB is based off our earlier synthesis of trans-PBPB. ${ }^{42}$ We next considered the molecular structures of trans-PBPB and cis-PBPB using DFT calculations. Figure 4.4 contains the lowest energy structures for cis-PBPB and trans-PBPB determined from DFT using 6-31G/B3LYP level of computation. We see that the PDI units remain upright in transPBPB while they bow inward toward the cavity in cis-PBPB. The PDI-linker connection differs between the two isomers. The torsional angle is greater in the cis molecule relative to trans-PBPB. This causes the PDI and linker to possess a relatively more orthogonal relationship, and decreases the electronic coupling in cis-PBPB (Figures 4.4a,b). The colors of the macrocycles support transPBPB is more conjugated: cis-PBPB is purple by visual inspection, and trans-PBPB is black.

We next consider the packing of these macrocycles with the crystal structure of transPBPB (shown in Figure 4.4c and Figure 4.8 in the Appendix). We see that the macrocycles pack with the PDI face of one adjacent to that of another, though with opposite chirality. ${ }^{46-48}$ We were unable to obtain crystal structures of $c i s$-PBPB but anticipate a similar face-to-face packing, given the DFT-based structure presented here. 

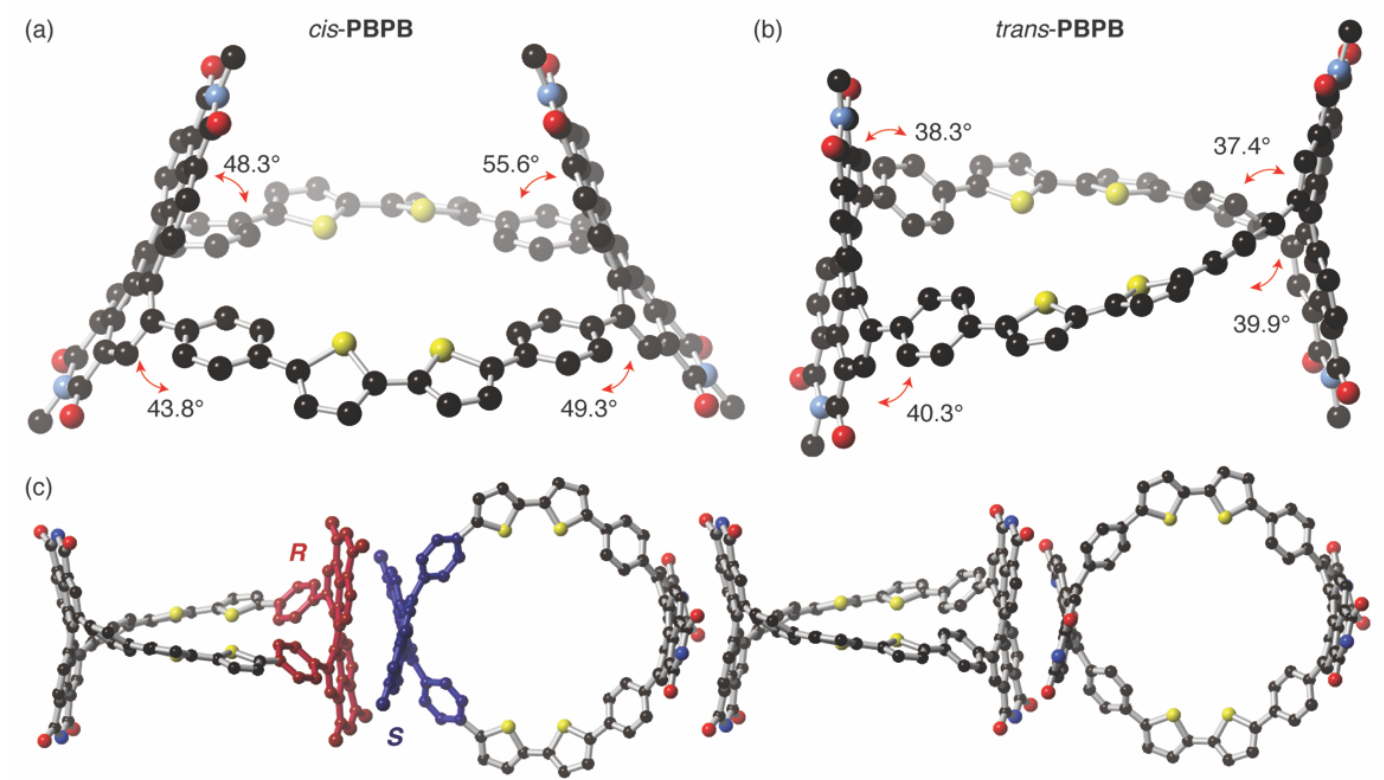

Figure 4.4. Molecular structures obtained with DFT using 6-31G/B3LYP basis set. (a) cis-PBPB and (b) trans-PBPB. (c) SCXRD solid-state packing of trans-PBPB as viewed down the $a$ axis. Blue and red are the two enantiomers of the diphenyl PDI packing down the axis. Red = oxygen, blue $=$ nitrogen, black $=$ carbon, and yellow $=$ sulfur. Hydrogens and side chains have been removed for clarity.

In addition, its known that molecular strain and rigidity can influence charge transport in macrocyclic semiconductor with the more strained systems having lower intermolecular coupling and hence lower intermolecular coupling and lower mobility. ${ }^{41}$ We calculate the enthalpy difference between the macrocycle and an acyclic analog (i.e., a homodesmotic calculation ${ }^{28,49-51}$ ) to assess the strain energy in trans-PBPB and cis-PBPB. We found only a small $(2 \mathrm{kcal} / \mathrm{mol})$ difference in strain energy and therefore conclude that this does not explain the difference in mobility. Section 4.13 contains the details of the calculations used to assess the strain energy.

\subsection{Cyclic Voltammetry Shows Similar Reduction Potentials}

As these macrocyclic materials are n-type semiconductors, we wondered if a difference in reduction potentials would explain the difference in mobility. We used CV to estimate the LUMO energies for both trans- and cis-PBPB (Table 4.1, Figure 4.5). Trans-PBPB and cis-PBPB have similar reduction potentials, as estimated from the onset of the first reduction peak. ${ }^{52}$ We also 
studied the electronic structure using UV-Vis. The UV-vis spectrum suggests trans-PBPB is more conjugated: the lowest energy transition is at lower energy in trans-PBPB than in cis-PBPB. Moreover, trans-PBPB has a smaller optical gap than the cis-PBPB macrocycle (Table 4.1, Figure 4.6). This likely reflects greater orbital overlap between the linker and PDI, given the smaller torsional angle between the linker and the PDI (Figures 4.4a,b).
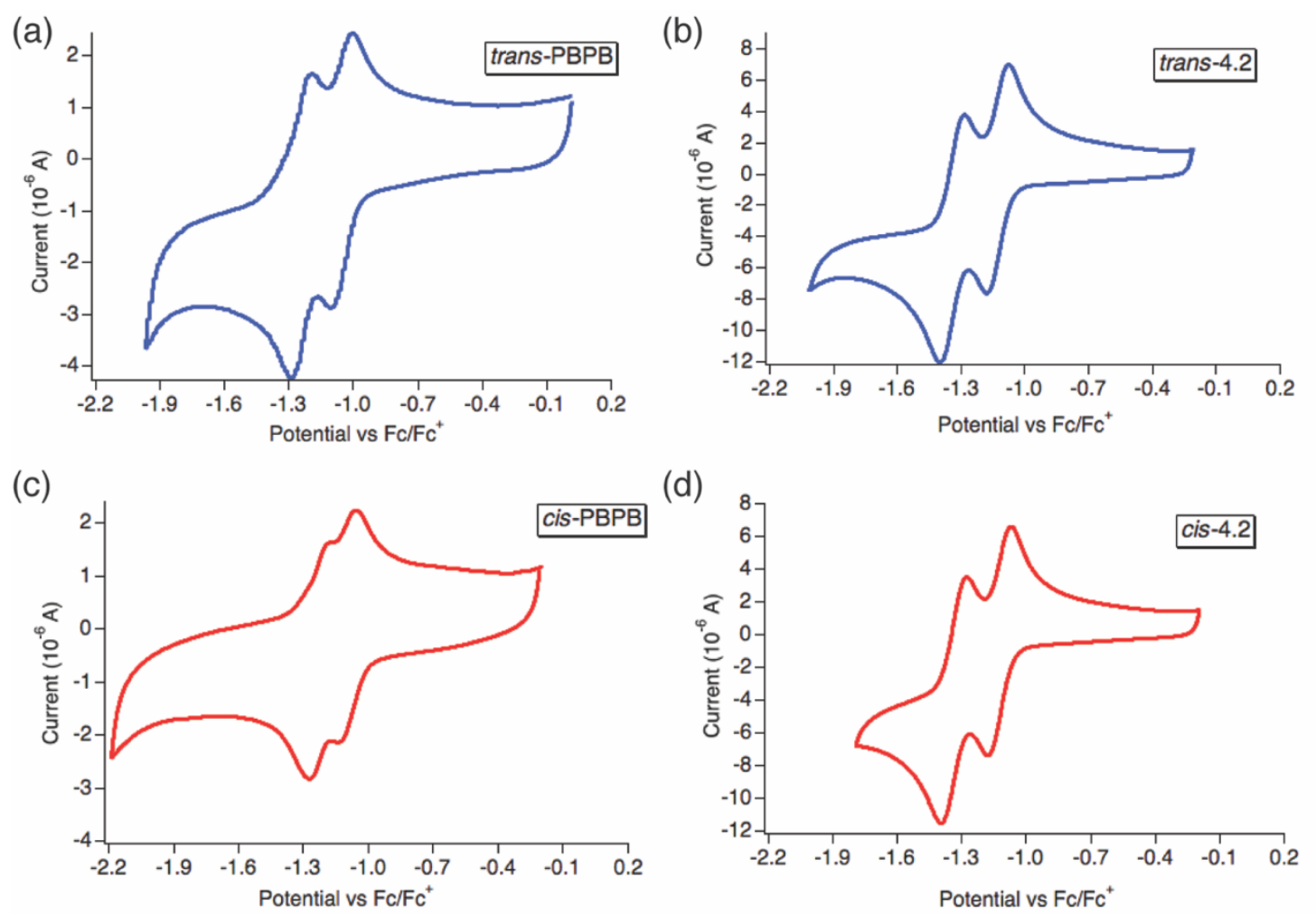

Figure 4.5. CV of (a) trans-PBPB; (b) trans-4.2; (c) cis-PBPB; and (d) cis-4.2. CVs taken in $\mathrm{CH}_{2} \mathrm{Cl}_{2}$ containing $0.1 \mathrm{M} \mathrm{NBu}_{4} \mathrm{PF}_{6}$ as the electrolyte.

(a)

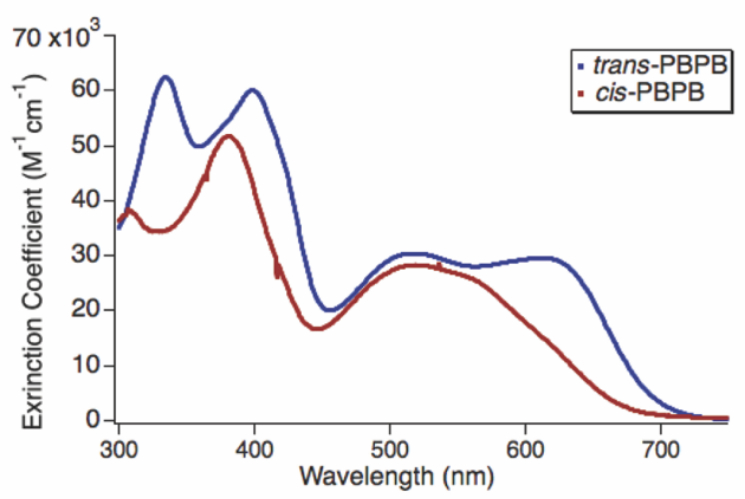

(b)

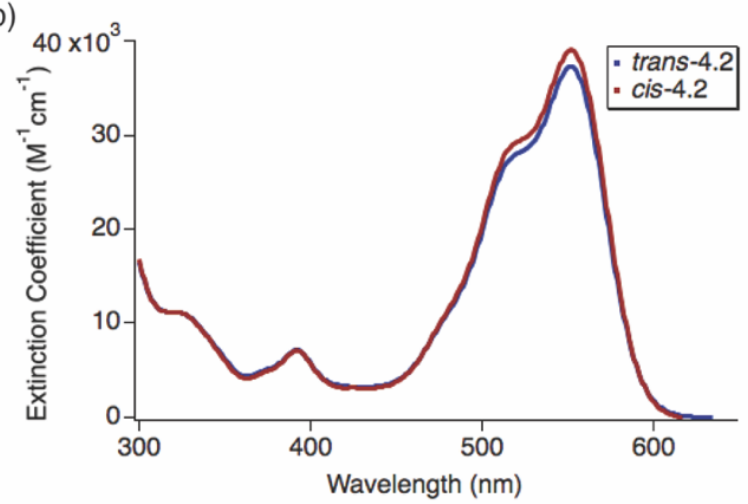

Figure 4.6. UV of (a) trans-PBPB and cis-PBPB; (b) trans-4.2 and cis-4.2. 


\subsection{STM-BJ Studies Show Trans Conducts Better Than Cis Isomer}

We next evaluate the intramolecular conductivity by deconstructing the macrocycles into 1,6- and 1,7-diphenyl PDI monomers that possess two aurophilic amino groups on the aryl rings. We refer to these molecules as trans-4.1 and cis-4.1 (Figure 4.7a). While the cis and trans PDI isomers are well known, ${ }^{53-56}$ the difference in intramolecular conduction between the cis and trans isomers has not been reported until now. The two aurophilic amino groups on the aryl rings bind the Au electrodes in the STM-BJ setup ${ }^{57-60}$ to form Au-4.1-Au- junctions (Fig. 4.7a). We found that trans-4.1 has a conductance nearly one order of magnitude higher than cis-4.1 at $\sim 8.6 \times 10^{-5} \mathrm{G}_{0}$ compared with $\sim 1.0 \times 10^{-5} \mathrm{G}_{0}$ (Fig. $4.7 \mathrm{~b}$ ), where $\mathrm{G}_{0}=e^{2} / h$ is the conductance quantum. Figure 4.8 contains the two-dimensional histograms for cis- and trans-4.1.
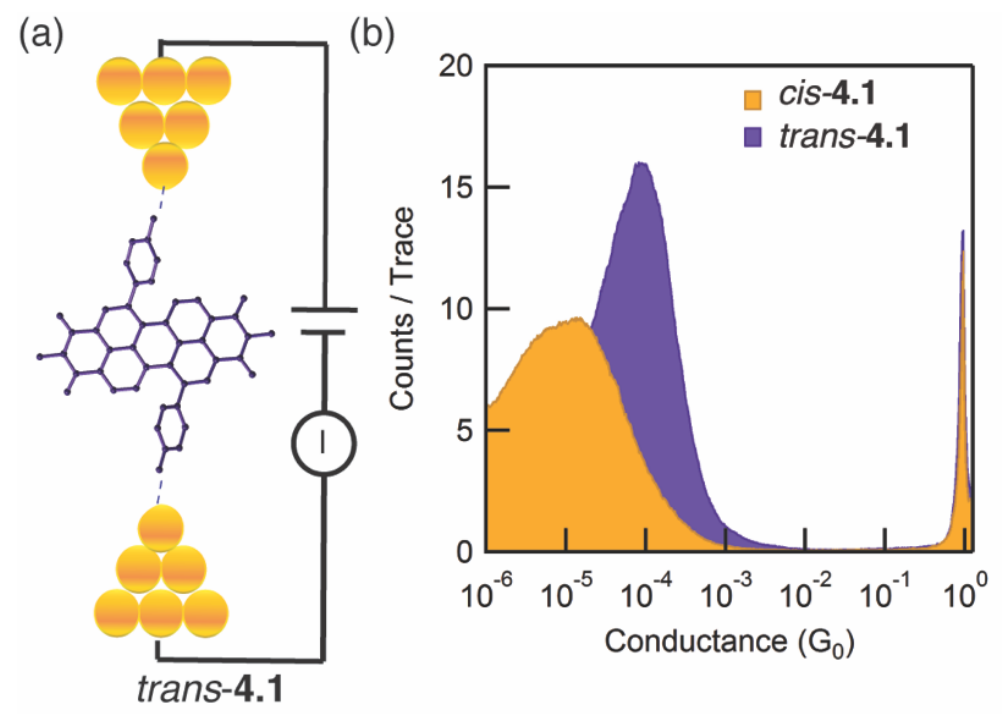

Figure 4.7. (a) Schematic of a single-molecule junction showing trans-4.1 in the break junction. The diphenyl PDI contains two aurophilic amino groups on the aryl rings to bind the gold electrodes in the junction; (b) Logarithm conductance histograms for cis-4.1 (yellow) and trans4.1 (purple) measured with an applied bias of $450 \mathrm{mV}$ in a 1,2,4-trichlorobenzene solution.

Because the STM-BJ studies determined that trans-substituted PDI molecular junctions are better conductors than cis-substituted PDI junctions, we hypothesized that the difference in the mobilities seen for trans- and cis-PBPB based OFETs is due to the trans/cis substitution patterns. The experiments described next find that the substitution patterns in the acyclic subunits do not 
explain the differences in mobility between the two three dimensional macrocyclic semiconductors.

\subsection{Acyclic Controls Possess Analogous Electron Mobility in OFETs}

We synthesized the acyclic relatives of trans- and cis-PBPB, cis- and trans-4.2, which comprise a diphenyl PDI substituted in a cis and trans orientation (Figure 4.1d). Trans-4.2 was called $\mathbf{1 . 1}$ in the preceding chapters, but renamed here for ease of comparison to the cis-version. We made OFETs using cis- and trans-4.2, and find that the two have similar averaged electron mobilities: $1.9 \times 10^{-4} \mathrm{~cm}^{2} / \mathrm{V} \cdot \mathrm{s}$ and $1.5 \times 10^{-4} \mathrm{~cm}^{2} / \mathrm{V} \cdot \mathrm{s}$ for cis-4.2 and trans-4.2, respectively (see Table 4.1, Table 4.1 and Figure 4.9 for details). We also studied the film morphology using AFM, and both films were smooth, with a root mean square roughness of 0.43 and $0.45 \mathrm{~nm}$ for cis- and trans-4.2, respectively (Figure 4.9). Since cis-4.2 and trans $\mathbf{- 4 . 2}$ show similar mobilities in OFETs, the cis and trans substitution pattern alone is not the reason for the difference in the performance found in the macrocyclic systems.

Table 4.1. Comparison of trans-PBPB and cis-PBPB Electronic Data

\begin{tabular}{|l|c|c|c|}
\hline & $\begin{array}{c}\text { Mobility } \mu \\
\left(\mathrm{cm}^{2} \mathrm{~V}^{-1} \mathrm{~s}^{-1}\right)\end{array}$ & $\begin{array}{c}\text { LUMO level }^{a} \\
(\mathrm{eV})\end{array}$ & Optical gap $^{b}(\mathrm{eV})$ \\
\hline trans-PBPB & $(1.2 \pm 0.1) \times 10^{-3}$ & -3.82 & 1.78 \\
\hline cis-PBPB & $(0.4 \pm 0.1) \times 10^{-3}$ & -3.79 & 1.85 \\
\hline trans-4.2 & $(1.5 \pm 0.3) \times 10^{-4}$ & -3.74 & 2.10 \\
\hline cis-4.2 & $(1.9 \pm 0.3) \times 10^{-4}$ & -3.74 & 2.10 \\
\hline
\end{tabular}

Table 4.1: CV, optical gap and FET performance for the two macrocycles and acyclic controls. ${ }^{a}$ LUMO levels were estimated from onset of the first reduction peaks. ${ }^{b}$ Optical band gaps were estimated from the onset of absorption.

Both trans-PBPB and trans-4.2 possess a trans linkage, suggesting higher intramolecular conductivity than the cis analogues from the STM-BJ measurements. Yet OFET devices from 
either trans- or cis-4.2 show similar electron mobilities, while electron mobilities from transPBPB or cis-PBPB macrocycles show marked differences in their mobilities.

From this data, we conclude that the substitution pattern in the subunits is not responsible for the difference in charge transport in the acyclic controls, but influences charge transport for the relatively complex three dimensional semiconducting macrocycles. Trans-PBPB is more conjugated than cis-PBPB, as reflected in the UV-vis and electrochemistry data, suggesting that the intramolecular conductivity is higher in the trans-based macrocycle. Together, the acyclic control data, STM-BJ measurements, and spectroscopy support that intramolecular carrier pathways govern charge transport as the complexity of the molecule increases in molecular solids. We also found that film morphology and molecular strain are not the source of the difference in mobility for the macrocycles. This study reveals the importance of not just intermolecular interactions and reorganization energy as conditions for electrical conduction in OFETs but also shows the importance of intramolecular conduction.

\subsection{Appendix - Supplementary Figures}

Table 4.2. OFET Characteristics for Semiconductors

\begin{tabular}{lcccc}
\hline & cis-PBPB & trans-PBPB & cis-4.2 & trans-4.2 \\
\hline Mobility & $0.4 \times 10^{-3}$ & $1.3 \times 10^{-3}$ & $2.1 \times 10^{-4}$ & $1.8 \times 10^{-4}$ \\
& $\left(0.4 \pm 0.1 \times 10^{-3}\right)$ & $\left(1.2 \pm 0.1 \times 10^{-3}\right)$ & $\left(1.9 \pm 0.3 \times 10^{-4}\right)$ & $\left(1.5 \pm 0.3 \times 10^{-4}\right)$ \\
\hline
\end{tabular}

Table 4.2: Parentheses indicates average mobility and error. Mobility is in units of $\mathrm{cm}^{2} \mathrm{~V}^{-1} \mathrm{~s}^{-1}$. 


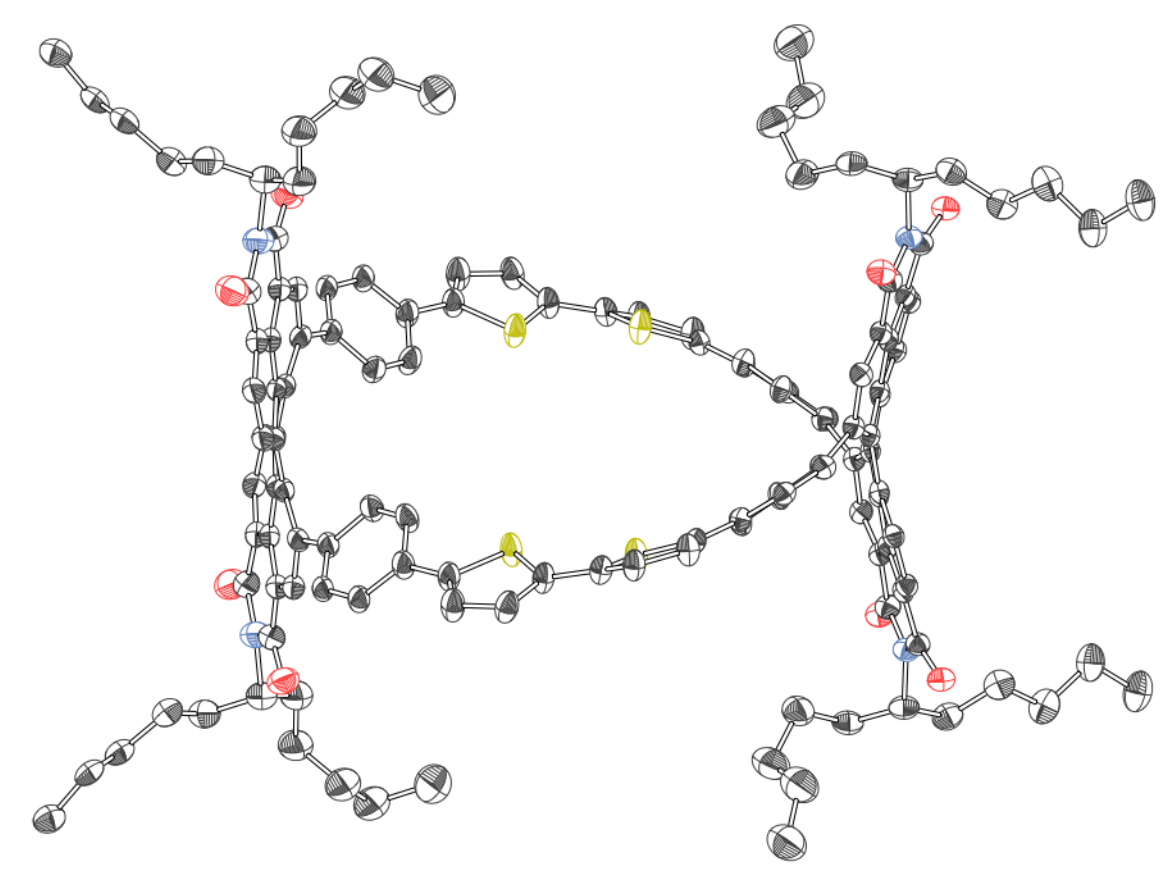

Figure 4.8. Molecular structure of trans-PBPB. One of the two independent molecules is shown. Thermal ellipsoids are rendered at the $20 \%$ probability level. Black, carbon; red, oxygen; blue, nitrogen; yellow, sulfur. Hydrogen atoms and the minor positions of disordered atoms are omitted. Crystallographic data is provided in Chapter 2.

(a)

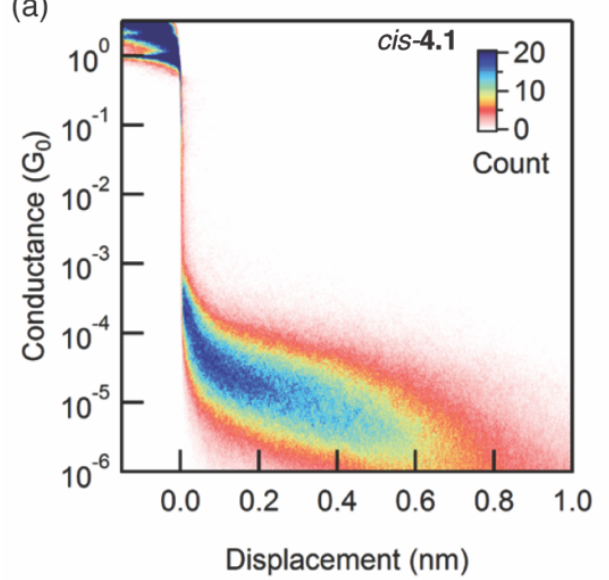

(b)

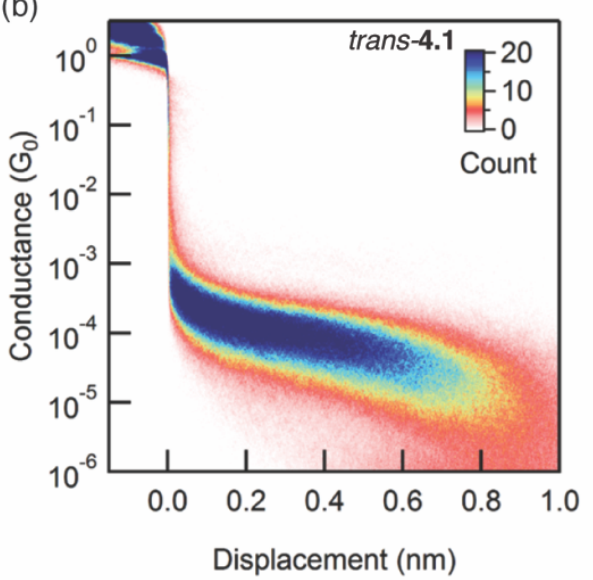

Figure 4.9. The two-dimensional conductance-displacement histograms of (a) cis-4.1 and (b) trans-4.1. 
(a)

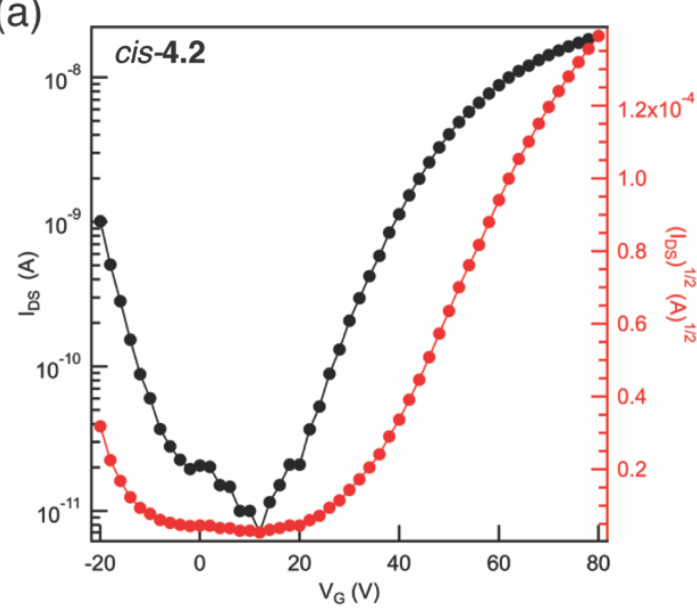

(b)

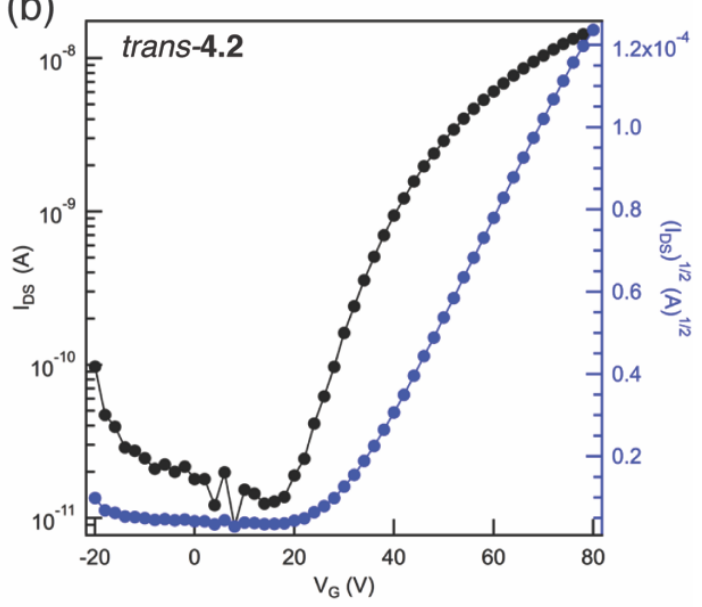

Figure 4.10. Transfer characteristics for (a) cis-4.2 and (b) trans-4.2. The mobilities are similar: $2.1 \times 10^{-4} \mathrm{~cm}^{2} \mathrm{~V}^{-1} \mathrm{~s}^{-1}$ and $1.8 \times 10^{-4} \mathrm{~cm}^{2} \mathrm{~V}^{-1} \mathrm{~s}^{-1}$ for cis-4.2 and trans-4.2, respectively.

(a)

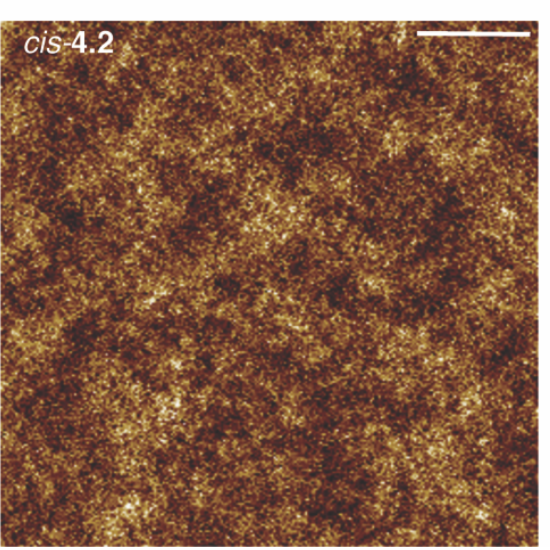

(b)

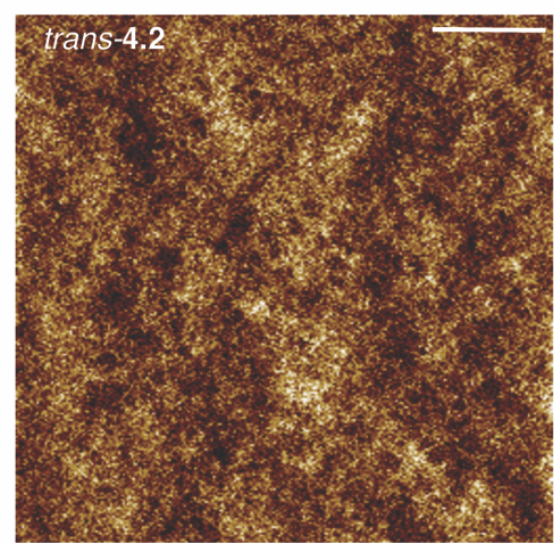

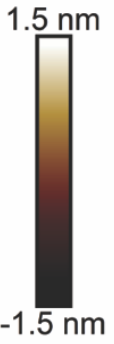

Figure 4.11. AFM micrograph height image for $c i s-\mathbf{4 . 2}$ and (b) trans-4.2. Both films are continuous and smooth and have a root mean square roughness of 0.43 and $0.45 \mathrm{~nm}$ for the cis and trans-based films, respectively. The scale bar is $1.0 \mu \mathrm{m}$.

\subsection{General Experimental Information}

Synthesis. All reactions were performed in oven-dried or flame-dried round bottom flasks, unless otherwise noted. The flasks were fitted with rubber septa and reactions were conducted under a positive pressure of nitrogen or argon, unless otherwise noted. Anhydrous and anaerobic solvents were obtained from a Glass Contour solvent system consisting of a Schlenk manifold with purification columns packed with activated alumina and supported copper catalyst. Reaction monitoring by TLC was performed on J.T. Baker Baker-flex Silica Gel IB2-F (25 mm x $75 \mathrm{~mm}$ ) 
TLC plates. TLC visualization was accomplished by visible observation and irradiation with a UV lamp. Commercial reagents were used without further purification. $\mathrm{Pt}(\mathrm{COD}) \mathrm{Cl}_{2}$ was purchased from Strem Chemicals, and all other reagents were purchased from Sigma-Aldrich.

Purification. Automated flash chromatography was performed using a Teledyne Isco Combiflash Rf200 and Redisep Rf Silica columns. Preparative HPLC was performed on a Waters Prep150 instrument equipped with a UV-vis detector, an automated fraction collector, and a Nacalai Tesque COSMOSIL Buckyprep column (20 mm I.D. x 250 mm, $5 \mu \mathrm{m})$.

Spectrometers. ${ }^{1} \mathrm{H}$ NMR spectra were recorded on a Bruker 400 or $500 \mathrm{MHz}$ spectrometer. ${ }^{13} \mathrm{C}$ NMR spectra were recorded on a Bruker 125 or $100 \mathrm{MHz}$ spectrometer with complete proton decoupling. NMR spectra were recorded at $300 \mathrm{~K}$ unless otherwise noted. Chemical shifts are reported in parts per million (ppm) Data are represented as follows: chemical shift, multiplicity (s $=$ singlet, $\mathrm{d}=$ doublet, $\mathrm{dd}=$ doublet of doublets, $\mathrm{t}=$ triplet, $\mathrm{m}=$ multiplet $)$, coupling constants in $\mathrm{Hz}$, and integration. Broadening (br) of peaks in the 1H NMR spectrum is due to rotational isomers about the imide side chains. Multiple peaks for the same carbon in the ${ }^{13} \mathrm{C}$ NMR spectrum arise due to rotational isomers about the imide side chains that have been seen previously. ${ }^{42,61}$ Trans4.2 was previously published and it represented as compound $\mathbf{1 . 1}$ in Chapters 1 and $3 .{ }^{29}$ HRMS was performed on a (1) Waters XEVO G2-XS QTOF instrument equipped with a UPC SFC inlet, and ESI and APCI ionization sources; or (2) a Bruker UltrafleXtreme MALDI TOF/TOF instrument using a dithranol matrix. UV-vis absorption spectra were recorded on a Shimadzu UV1800 spectrophotometer using a $1.0 \mathrm{~cm}$ quartz cell. IR spectra were recorded on a Perkin Elmer Spectrum400 FTIR spectrometer using a PIKE ATR attachment.

Atomic force microscopy. AFM measurements were carried out in tapping mode on a Bruker Multi-Mode AFM at ambient conditions. A commercial silicon cantilever (RTESPA, MPP- 
$11120-10$, Bruker) was used in this study with a typical radius of curvature of $\sim 8 \mathrm{~nm}$ and a nominal spring constant of $\sim 40 \mathrm{Nm}^{-1}$.

Cyclic Voltammetry. CVs were recorded on a CH166 electrochemical workstation using an $\mathrm{Ag} / \mathrm{AgCl}$ electrode as the reference electrode at room temperature. Experiments were performed in $\mathrm{CH}_{2} \mathrm{Cl}_{2}$ with $\mathrm{NBu}_{4} \mathrm{PF}_{6}$ as the supporting electrolyte at a scan rate of $0.1 \mathrm{~V} / \mathrm{s}^{52}$

Thin film transistors. To create the devices, we first silanize the substrate $\left(300 \mathrm{~nm}\right.$ of $\mathrm{SiO}_{2}$ on a Si wafer) with OTS. Au is deposited onto the substrate as bottom-contact source and drain electrodes $(40 \mathrm{~nm})$ with a width of $105 \mu \mathrm{m}$ and length of $20 \mu \mathrm{m}$. Next, we spin-cast organic films onto the surface at 1,000 r.p.m. for $1 \mathrm{~min}$, to form transistors using the silicon wafer as the global back gate for the device. Finally, the samples were annealed under inert atmosphere at $160^{\circ} \mathrm{C}$ for 10 minutes to optimize device. The thin film transistors were tested on the Agilent $4155 \mathrm{C}$ semiconductor parameter analyzer.

STM-Break Junction Measurements. We measure the single-molecule conductance using the STM-BJ technique with a custom-built setup described previously. ${ }^{58}$ Briefly, we drive a $\mathrm{Au}$ tip in and out of contact with an Au-on-mica substrate and record the conductance (current/voltage) of the junction as the tip is withdrawn. Upon rupture of the Au contact, a molecule may bridge the gap as evidenced by an additional plateau in the conductance versus displacement trace. We collect 10,000 such traces, which contain 2000 data points per nanometer of extension (40 kHz sampling rate) and construct the 1D and 2D conductance histograms without data selection from these data. The conductance histogram is binned logarithmically, with 100 bins per decade along the conductance axis. For two-dimensional histograms, traces are aligned along the displacement axis at the point when the conductance crosses $0.5 \mathrm{G}_{0}$ and then overlaid in $2 \mathrm{D}$ (see Figure 4.9). The histograms are normalized by the number of traces used to construct them. The 
PDIs studied here were introduced into the setup in a 1,2,4-trichlorobenzene solution with $0.1 \sim 1$ $\mathrm{mM}$ concentration.

\subsection{Synthetic Procedures \& Characterization}

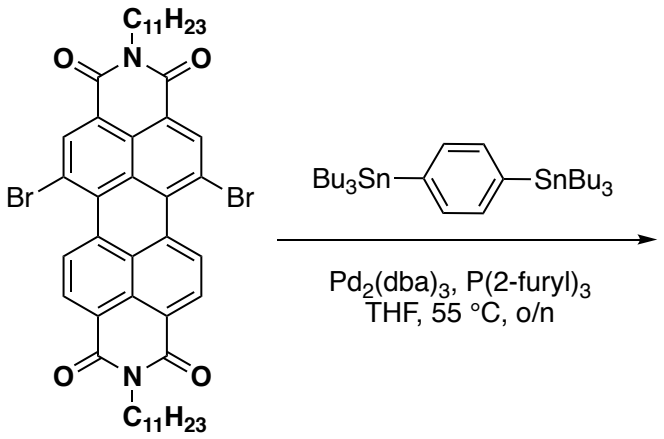

1,6-PDI cis-PDI

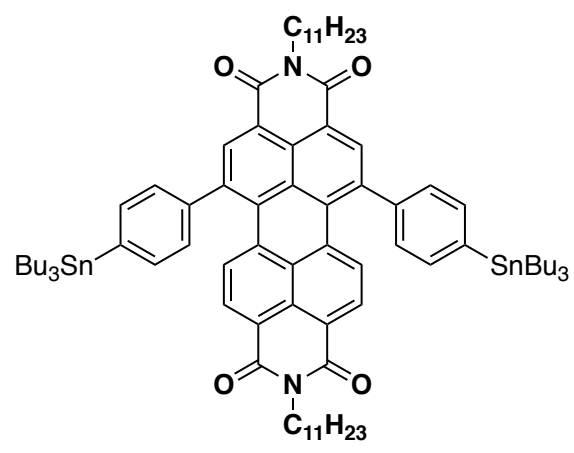

SI-4.1

Synthesis of 1,6-Bis[4-(tributylstannyl)-phenyl]-PDI (SI-4.1). Pure 1,6-dibromo PDI (1.00 equiv, $0.199 \mathrm{mMol}, 0.171 \mathrm{~g})$, 1,4-bis(tributylstannyl)benzene (4.00 equiv, $0.799 \mathrm{mMol}, 0.524 \mathrm{~g})$, THF (9.95 mL), and tri(2-furyl)phosphine (0.400 equiv, $0.0796 \mathrm{mMol}, 0.0180 \mathrm{~g})$ were added to an ovendried $25.0 \mathrm{~mL}$ round bottom flask under nitrogen and equipped with a stir bar. Solution was sparged with $\mathrm{N}_{2}$ for 30 minutes. Tris(dibenzylidenacetone)dipalladium ( 0.100 equiv, $0.018 \mathrm{mMol}$, $0.0199 \mathrm{~g}$ ) was added to the solution which was then degassed for an additional 30 minutes. Mixture was then placed in a $55^{\circ} \mathrm{C}$ oil bath overnight. The crude mixture was concentrated and purified by column chromatography (40 g Redisep Rf Silica) with a gradient of $0 \%$ to $80 \% \mathrm{CH}_{2} \mathrm{Cl}_{2} / \mathrm{Hexanes}$ flow to yield the 1,6-isomer as a magenta pink solid $(0.083 \mathrm{mMol}, 0.071 \mathrm{~g})$. By-products were resubmitted to the same conditions described above to yield $0.119 \mathrm{~g}$ for a combined total yield of 42\%. ${ }^{1} \mathbf{H}$ NMR (400 MHz, 300K, $\left.\mathrm{CDCl}_{3}\right) \delta 8.59$ (br s, 2H), 8.09 (br d, 2H), 7.84 (d, J = 8.1 Hz, 2H), 7.57* (d, J = 7.7 Hz, 4H), 7.39 (d, J = 7.9 Hz, 4H), 5.20 (br m, 1H), 5.11 (br m, 1H), 2.25 (br m, 2H), 2.16 (br m, 2H), 1.82 (br m, 4H), 1.61* (m, 12H), 1.40 (m, 12H), 1.26 (br m, 24H), 1.14* (m, 12H), $0.94(\mathrm{t}, 18 \mathrm{H}), 0.83$ (br t, 12H). ${ }^{13} \mathbf{C ~ N M R}\left(100 \mathrm{MHz}, 300 \mathrm{~K}, \mathrm{CDCl}_{3}\right) \delta 164.78(\mathrm{br}), 163.73$ 
(br), 142.83, 142.22, 142.18, 142.13, 138.05*, 135.97, 135.32, 134.34, 132.70, 130.00, 129.70, $129.50,129.25,128.70,128.47,128.06^{*}, 127.25,122.87,122.03,121.84,54.68,54.55,32.34$, $31.78,31.76,31.59,29.13 *, 27.35 *, 26.65,26.56,22.65,22.57,22.54,14.11,14.04,13.72,13.75$, 9.74*. IR $\left(\mathrm{cm}^{-1}\right)$ 2954, 2928, 2870, 2856, 1697, 1658, 1589, 1587, 1465, 1459, 1421, 1414, 1344 , $1325,1262,1250,813$. HRMS $\left(\right.$ APCI + ) calculated $\mathrm{m} / \mathrm{z}$ for $\left[\mathrm{C}_{82} \mathrm{H}_{114} \mathrm{~N}_{2} \mathrm{O}_{4} \mathrm{Sn}_{2}+\mathrm{H}\right]^{+}$is 1431.6924 , found 1431.6901. * Tin satellite peaks.

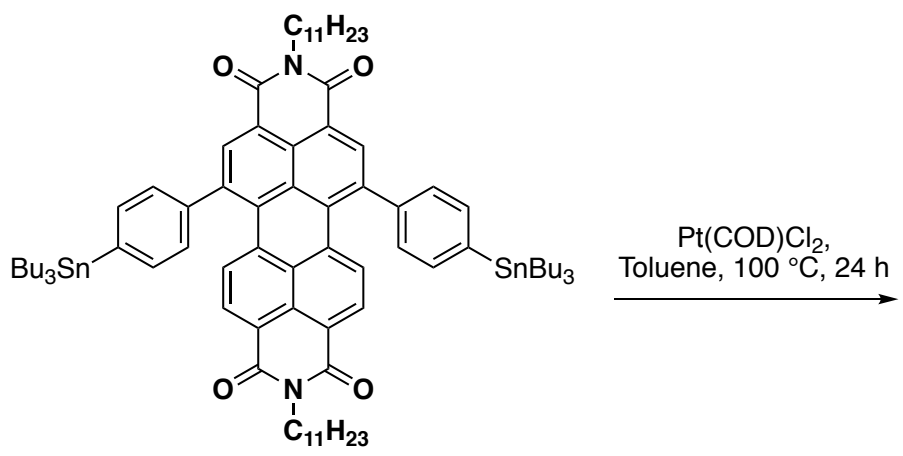

SI-4.1

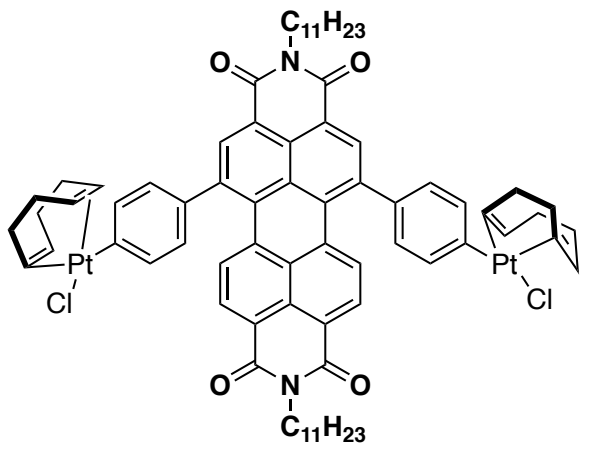

SI-4.2

Synthesis of 1,6-Bis[4-(Pt(COD)Cl)-phenyl]-PDI (SI-4.2): 1,6-Bis[4-(tributylstannyl)-phenyl]PDI (SI-4.1) (0.206 mMol, 0.295 g, 1 eq), Dichloro(1,5-cyclooctadiene)platinum(II) (0.433 mmol, $0.162 \mathrm{~g}, 2.1 \mathrm{eq})$ and toluene $(21 \mathrm{~mL})$ were added to an oven-dried two-neck, $50-\mathrm{mL}$ round bottom flask equipped with a stir bar. The mixture was degassed for 30 minutes then placed in a $100{ }^{\circ} \mathrm{C}$ oil bath and allowed to stir for 24 hours. The crude mixture was then concentrated and purified by column chromatography (24 g Redisep RF Silica) using a gradient from $0 \%$ to $80 \%$ $\mathrm{CH}_{2} \mathrm{Cl}_{2} /$ hexanes at $60 \mathrm{~mL} / \mathrm{min}$. Product was collected and concentrated as a purple solid $(0.123 \mathrm{~g}$, $0.0771 \mathrm{mMol}, 38 \%$ yield). ${ }^{1} \mathrm{H}$ NMR $\left(500 \mathrm{MHz}, 300 \mathrm{~K}, \mathrm{CDCl}_{3}\right) \delta 8.56$ (br s, $\left.1 \mathrm{H}\right), 8.54$ (br s, $\left.1 \mathrm{H}\right)$, 8.09 (br d, 1H), 8.06 (br d, 1H), $7.85(\mathrm{~d}, \mathrm{~J}=8.2 \mathrm{~Hz}, 2 \mathrm{H}), 7.36(\mathrm{~d}, \mathrm{~J}=7.8 \mathrm{~Hz}, 4 \mathrm{H}), 7.16(\mathrm{~d}, \mathrm{~J}=8.2$ $\mathrm{Hz}, 4 \mathrm{H}), 5.88(\mathrm{~s}, 4 \mathrm{H}), 5.17$ (br m, 1H), 5.11 (br m, 1H), 4.71 (s, 4H), 2.74 (br m, 4H), 2.60 (br m, 4H), 2.44 (br m, 8H), 2.24 (br m, 2H), 2.15 (br m, 2H), 1.82 (br m, 4H), 1.25 (br m, 24H), 0.85 (br t, $12 \mathrm{H}) .{ }^{13} \mathbf{C}$ NMR $\left(100 \mathrm{MHz}, 300 \mathrm{~K}, \mathrm{CDCl}_{3}\right) \delta 164.80,163.84,145.29,142.26,138.67,135.92 * *$, 
$135.58,135.21^{* *}, 134.65,129.99^{* *}, 129.51,129.45,129.25^{* *}, 128.75,128.50,128.39,126.98$ 122.80**, 122.29**, 121.94**, 121.43**, 115.91, 87.78, 54.58, 54.48, 32.41, 32.32, 32.28, 31.81, 31.76, 28.01, 26.68, 26.55, 22.57, 14.09, 14.05. IR $\left(\mathrm{cm}^{-1}\right) 2958,2925,2857,1695,1656,1583$, $1410,1325,1275,1262,763,749$. HRMS $(E S I+)$ calculated $\mathrm{m} / \mathrm{z}$ for $\left[\mathrm{C}_{74} \mathrm{H}_{84} \mathrm{Cl}_{2} \mathrm{~N}_{2} \mathrm{O}_{4} \mathrm{Pt}_{2}+\mathrm{Na}\right]^{+}$is 1546.4923, found 1546.4922 .

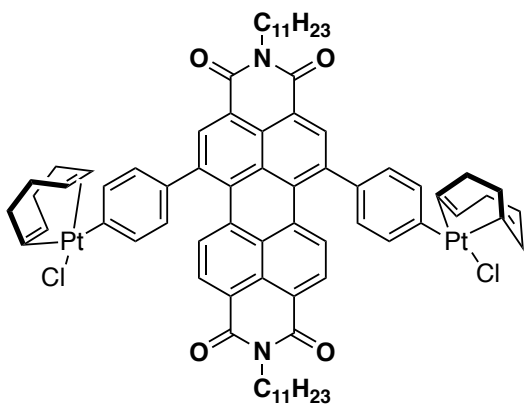

SI-4.2

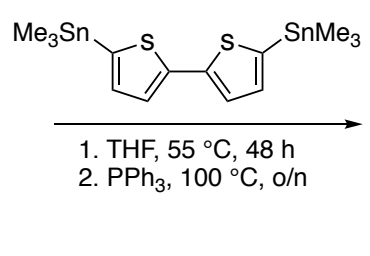

-

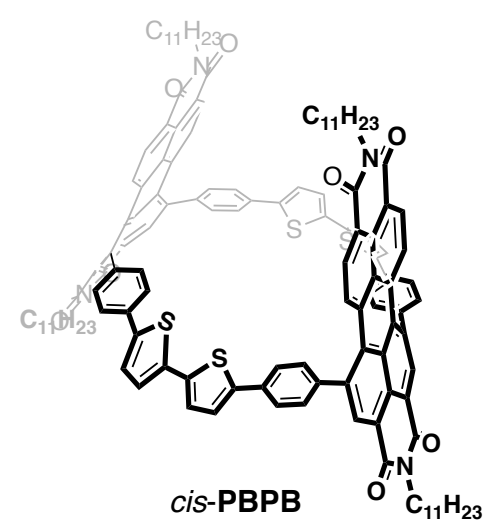

Synthesis of cis-PBPB: Compound SI-4.2 (0.105 mMol, 0.160 g), commercially available 5,5'(bistrimethylstannyl)-2,2'-bithiophene ( $0.105 \mathrm{mMol}, 0.0516 \mathrm{~g})$, and THF were added to a $250 \mathrm{~mL}$ oven dried round bottom flask equipped with a stir bar. The mixture was sparged with nitrogen for $30 \mathrm{~min}$ then added to an oil bath at $55^{\circ} \mathrm{C}$ and allowed to stir for $48 \mathrm{~h}$. Crude mixture was then removed from oil bath and concentrated. Triphenylphosphine $(2.10 \mathrm{mMol}, 0.613 \mathrm{~g})$ and toluene $(40.0 \mathrm{~mL})$ were added to the flask. Mixture was sparged with nitrogen for 15 min then placed in a $100{ }^{\circ} \mathrm{C}$ oil bath and allowed to stir overnight. The crude mixture was first washed with hexanes, followed by methanol and then purified by column chromatography (24 g Redisep Rf Silica) using a gradient from $0 \%$ to $85 \% \mathrm{CH}_{2} \mathrm{Cl}_{2} /$ hexanes at $35 \mathrm{~mL} / \mathrm{min}$. The polar fractions were further purified with prepatory TLC. Product was a dark purple solid $\left(0.009\right.$ g, 8.5\%). ${ }^{1} \mathbf{H}$ NMR (400 MHz, $\left.360 \mathrm{~K}, \mathrm{C}_{2} \mathrm{D}_{2} \mathrm{Cl}_{4}\right) \delta 8.75(\mathrm{~s}, 4 \mathrm{H}), 8.18(\mathrm{~d}, J=8.3 \mathrm{~Hz}, 4 \mathrm{H}), 7.81(\mathrm{~d}, J=8.2 \mathrm{~Hz}, 8 \mathrm{H}), 7.57$ (d, $J=8.2 \mathrm{~Hz}, 8 \mathrm{H}), 7.47(\mathrm{~d}, J=8.2 \mathrm{~Hz}, 4 \mathrm{H}), 7.41(\mathrm{~d}, J=3.7 \mathrm{~Hz}, 4 \mathrm{H}), 7.28(\mathrm{~d}, J=3.7 \mathrm{~Hz}, 4 \mathrm{H}), 5.23$ (br m, 2H), 5.05 (br m, 2H), 2.30 (br m, 4H), 2.09 (br m, 4H), 1.95 (br m, 4H), 1.36 (br m, 28H)*, 
1.05 (br m, 8H), 0.90 (br m, 28H), 0.58 (br m, $12 \mathrm{H}) .{ }^{13} \mathbf{C} \mathbf{N M R}\left(100 \mathrm{MHz}, 328 \mathrm{~K}, \mathrm{CDCl}_{3}\right) \delta 166.17$, $165.49,165.07,164.43,144.44,142.15,141.98,140.08,136.12,134.71,134.16,132.63,131.73$, $131.44,130.73,130.03,129.62,129.45,128.57,124.85,124.44,124.21,123.96,123.27,56.23$, 55.16, 33.74, 33.56, 33.10, 32.56, 31.05, 27.97, 27.58, 23.90, 23.49, 15.46, 15.15. IR $\left(\mathrm{cm}^{-1}\right) 2972$, 2954, 2926, 2855, 1695, 1655, 1586, 1426, 1405, 1322, 1260, 1250, 1103, 1126, 811, 794, 751. HRMS (ESI+) calculated $\mathrm{m} / \mathrm{z}$ for $\left[\mathrm{C}_{132} \mathrm{H}_{128} \mathrm{~N}_{4} \mathrm{O}_{8} \mathrm{~S}_{4}+\mathrm{H}\right]^{+}$is 2025.8693 , found 2025.8676. *There is a peak underneath that corresponds to four protons that are one of the methylenes of the side chains.

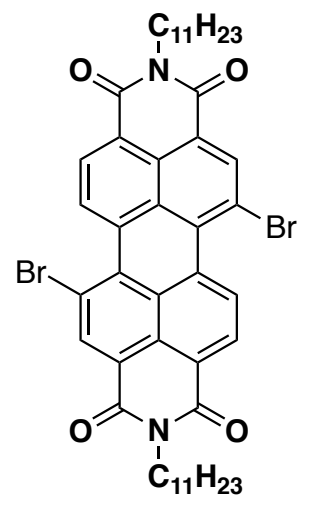

1,7-PDI trans-PDI

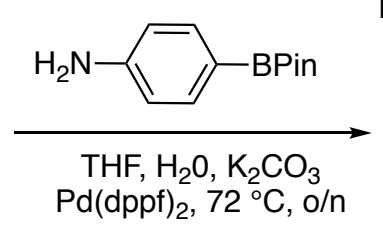

$\mathrm{Pd}(\mathrm{dppf})_{2}, 72{ }^{\circ} \mathrm{C}, \mathrm{o} / \mathrm{n}$

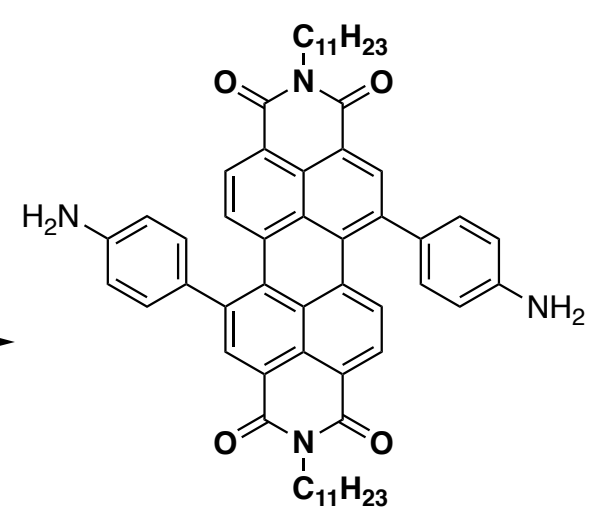

trans-4.1

A solution of 1,7-dibromo PDI (100 mg, $0.117 \mathrm{mmol}, 1.00$ equiv) and 4-aminophenylboronic acid pinacol ester ( $150 \mathrm{mg}, 0.685 \mathrm{mmol}, 5.85$ equiv) in THF $(6 \mathrm{~mL})$ was degassed under nitrogen for 30 mins. In a separate reaction vial, an aqueous solution of potassium phosphate (500 mg, 2.355 mmol, 20.13 equiv) in $2 \mathrm{~mL}$ water was degassed under nitrogen for $30 \mathrm{mins}$, and syringed into the degassing orange THF solution. The resultant reaction mixture was degassed for another 15 mins followed by addition of solid [1,1'-bis(diphenylphosphino)ferrocene]dichloropalladium (II) (10 $\mathrm{mg}, 0.012 \mathrm{mmol}, 10 \mathrm{~mol} \%$ ). The orange solution was degassed for an additional $15 \mathrm{mins}$. It was placed in oil bath set at $72{ }^{\circ} \mathrm{C}$ under refluxing conditions for overnight. The blue solution was concentrated under reduced pressure to remove THF. The blue solution was diluted with brine (30 
$\mathrm{mL})$ and extracted with $\mathrm{CH}_{2} \mathrm{Cl}_{2}(50 \mathrm{~mL})$ twice. The combined organic layer was dried $\left(\mathrm{MgSO}_{4}\right)$, filtered and concentrated under reduced pressure to a blue powdery residue. The solid was washed with methanol, and the residual solid was purified by silica gel chromatography ( 24 g Redisep Rf Silica) using a gradient from $100 \% \mathrm{CH}_{2} \mathrm{Cl}_{2}$ to $5 \%$ ethyl acetate $/ \mathrm{CH}_{2} \mathrm{Cl}_{2}$ to yield trans-4.1 as dark blue solid (92 mg, $0.104 \mathrm{mmol}, 89 \%$ yield). ${ }^{1} \mathrm{H}$ NMR (500 MHz, $\left.\mathrm{C}_{2} \mathrm{D}_{2} \mathrm{Cl}_{4}, 350 \mathrm{~K}\right): \delta 8.61(\mathrm{~s}, 2 \mathrm{H})$, $8.16(\mathrm{~d}, \mathrm{~J}=8.2 \mathrm{~Hz}, 2 \mathrm{H}), 8.04$ (d, J=8.2 Hz, 2H), 7.40 (d, J=8.5 Hz, 4H), 6.82 (d, J=8.5 Hz, 4H), 5.19-5.13 (m, 2H), 3.92 (br s, 4H), 2.27-2.20 (m, 4H), 1.94-1.88 (m, 4H), 1.40-1.31 (m, 24H), 0.90 (t, J=6.8 Hz, 12H). ${ }^{13} \mathbf{C}$ NMR (100 MHz, $\left.\mathrm{CDCl}_{3}, 323 \mathrm{~K}\right): \delta 163.92,147.03,141.21,135.44,132.29$, $132.16,130.33,129.60,129.42,127.74,122.34,116.42,54.59,32.43,31.75,26.60,22.50,13.94$. IR (ATR-ZnSe) $\left[\mathrm{cm}^{-1}\right]$ 3371, 2925, 2857, 1688, 1648, 1622, 1607, 1582, 1519, 1406, 1324, 1265, 1240, 1180. HRMS (ESI+) calculated $\mathrm{m} / \mathrm{z}$ for $\left[\mathrm{C}_{58} \mathrm{H}_{64} \mathrm{~N}_{4} \mathrm{O}_{4}+\mathrm{H}\right]^{+} 881.5006$; found 881.5009 .

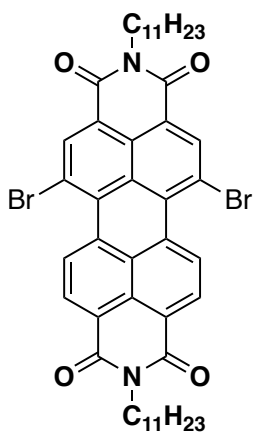

1,6-PDI cis-PDI

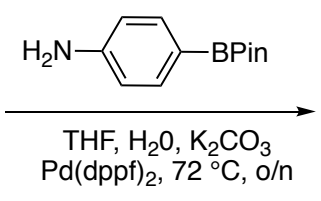

THF, $\mathrm{H}_{2} \mathrm{O}, \mathrm{K}_{2} \mathrm{CO}_{3}$
$\mathrm{Pd}(\mathrm{dppf})_{2}, 72{ }^{\circ} \mathrm{C}, \mathrm{o} / \mathrm{n}$

A solution of 1,6-dibromoPDI (90 mg, $0.105 \mathrm{mmol}, 1.00$ equiv) and 4-aminophenylboronic acid pinacol ester (90 mg, $0.411 \mathrm{mmol}, 3.91$ equiv) in THF ( $8 \mathrm{~mL}$ ) was degassed under nitrogen for 30 mins. In a separate reaction vial, an aqueous solution of potassium phosphate $(250 \mathrm{mg}, 1.178$ mmol, 11.22 equiv) in $2 \mathrm{~mL}$ water was degassed under nitrogen for $30 \mathrm{mins}$, and syringed into the orange THF solution. The resultant reaction mixture was degassed for another 15 mins followed by addition of solid [1,1'-bis(diphenylphosphino)ferrocene]dichloropalladium (II) (10 mg, 0.012 
mmol, $11 \mathrm{~mol} \%)$. The orange solution was degassed for another 15 mins. It was placed in oil bath set at $72{ }^{\circ} \mathrm{C}$ under refluxing conditions for overnight. The blue solution was concentrated under reduced pressure to remove THF. The blue solution was diluted with brine $(30 \mathrm{~mL})$ and extracted with $\mathrm{CH}_{2} \mathrm{Cl}_{2}(50 \mathrm{~mL})$ twice. The combined organic layer was dried $\left(\mathrm{MgSO}_{4}\right)$, filtered and concentrated under reduced pressure to a blue powdery residue. The solid was washed with methanol, and the residual solid was purified by silica gel chromatography (24 g Redisep Rf Silica) using a gradient from $100 \% \mathrm{CH}_{2} \mathrm{Cl}_{2}$ to $5 \%$ ethyl acetate/ $\mathrm{CH}_{2} \mathrm{Cl}_{2}$ to yield cis-4.1 as dark blue solid (77 mg, $0.087 \mathrm{mmol}, 83 \%$ yield). ${ }^{1} \mathbf{H}$ NMR (500 MHz, $\left.\mathrm{C}_{2} \mathrm{D}_{2} \mathrm{Cl}_{4}, 350 \mathrm{~K}\right): \delta 8.56$ (s, $2 \mathrm{H}$ ), 8.17 (d, $\mathrm{J}=8.2 \mathrm{~Hz}, 2 \mathrm{H}), 8.00(\mathrm{~d}, \mathrm{~J}=8.2 \mathrm{~Hz}, 2 \mathrm{H}), 7.26(\mathrm{~d}, \mathrm{~J}=8.4 \mathrm{~Hz}, 4 \mathrm{H}), 6.80(\mathrm{~d}, \mathrm{~J}=8.4 \mathrm{~Hz}, 4 \mathrm{H}), 5.20-5.11$ (m, 2H), $3.90($ br s, 4H), 2.28-2.16 (m, 4H), 1.93-1.89 (m, 4H), 1.36-1.32 (m, 24H), 0.91-0.88 (m, 12H). ${ }^{13} \mathrm{C}$ NMR $\left(100 \mathrm{MHz}, \mathrm{CDCl}_{3}, 323 \mathrm{~K}\right): \delta 164.90,164.80,163.75,146.79,142.35,135.59$, $134.85,132.27,129.97,129.57,128.96,128.85,128.42,126.66,122.63,121.97,121.20,116.29$, $54.53,54.42,32.29,31.73,31.69,26.58,26.50,22.51,22.49,14.01,13.99$. IR (ATR-ZnSe) [cm $\left.{ }^{-1}\right] 3360,2953,2924,2856,1691,1642,1604,1583,1504,1436,1325,1247,1181$. HRMS (ESI+) calculated $\mathrm{m} / \mathrm{z}$ for $\left[\mathrm{C}_{58} \mathrm{H}_{64} \mathrm{~N}_{4} \mathrm{O}_{4}+\mathrm{H}\right]^{+}$881.5006; found 881.4988 .
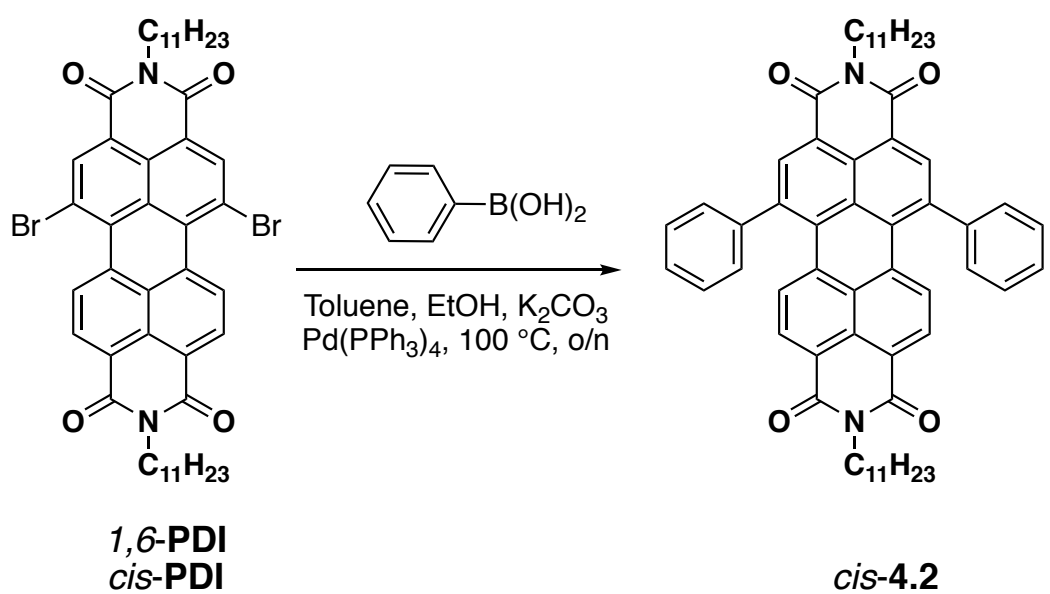

cis-4.2

Synthesis of cis-4.2: $N, N^{\prime}$-Di(6-undecyl)-1,6-dibromoperylene-3,4:9,10-tetracarboxylic diimide (0.0500 g, $0.0584 \mathrm{mmol}, 1.00$ equiv), phenyl boronic acid ( $0.0290 \mathrm{~g}, 0.234 \mathrm{mmol}, 4.00$ equiv), 
aqueous $\mathrm{K}_{2} \mathrm{CO}_{3}(2 \mathrm{M}, 1.00 \mathrm{~mL}), \mathrm{EtOH}(0.200 \mathrm{~mL})$ and toluene $(3.00 \mathrm{~mL})$ were added to a $20 \mathrm{ml}$ scintillation vial equipped with a stir bar. The mixture was sparged with $\mathrm{N}_{2}$ for thirty minutes. While under $\mathrm{N}_{2}$, a spatula tip of tetrakis(triphenylphosphine)palladium(0) was added. The mixture was further sparged for ten minutes before placed in a $100{ }^{\circ} \mathrm{C}$ oil bath under $\mathrm{N}_{2}$ for overnight. The crude mixture was extracted with ethyl acetate and brine, concentrated, and purified by column chromatography (24 g Redisep Rf Silica) using a gradient from $0 \%$ to $100 \% \mathrm{CH}_{2} \mathrm{Cl}_{2} /$ hexanes to yield cis-4.2 (0.0480 g, 0.0565 mmol, 97\%). ${ }^{1} \mathrm{H}$ NMR (400 MHz, CDCl3, 300K): $\delta 8.61$ (br s, 2H), 8.12 (br s, 2H), $7.83(\mathrm{~d}, 2 \mathrm{H}), 7.59-7.55(\mathrm{~m}, 4 \mathrm{H}), 7.54-7.47$ (br d, 6H), 5.15 (br, 2H), 2.20 (m, 4H), $1.83(\mathrm{~m}, 4 \mathrm{H}), 1.34(\mathrm{~m}, 24 \mathrm{H}), 0.81(\mathrm{t}, 12 \mathrm{H}) .{ }^{13} \mathbf{C ~ N M R}\left(100 \mathrm{MHz}, \mathrm{CDCl}_{3}\right) \delta$ 164.70, 163.60, $142.26,141.10,135.82,135.19,134.84,132.50,130.24,130.17,129.92,129.25,129.05,128.61$, 127.92, 122.86, 122.52, 122.16, 122.81, 54.65, 32.33, 31.76, 26.59, 22.56, 14.04. IR $\left(\mathrm{cm}^{-1}\right) 2953$, 2928, 2868, 2861, 2856, 1697, 1659, 1598, 1589, 1408, 1326, 1240, 814. HRMS (ESI+) calculated $\mathrm{m} / \mathrm{z}$ for $\left[\mathrm{C}_{58} \mathrm{H}_{62} \mathrm{~N}_{2} \mathrm{O}_{4}+\mathrm{H}\right]^{+} 851.4788$, found 851.4780 . 


\subsection{NMR Spectra of Compounds}

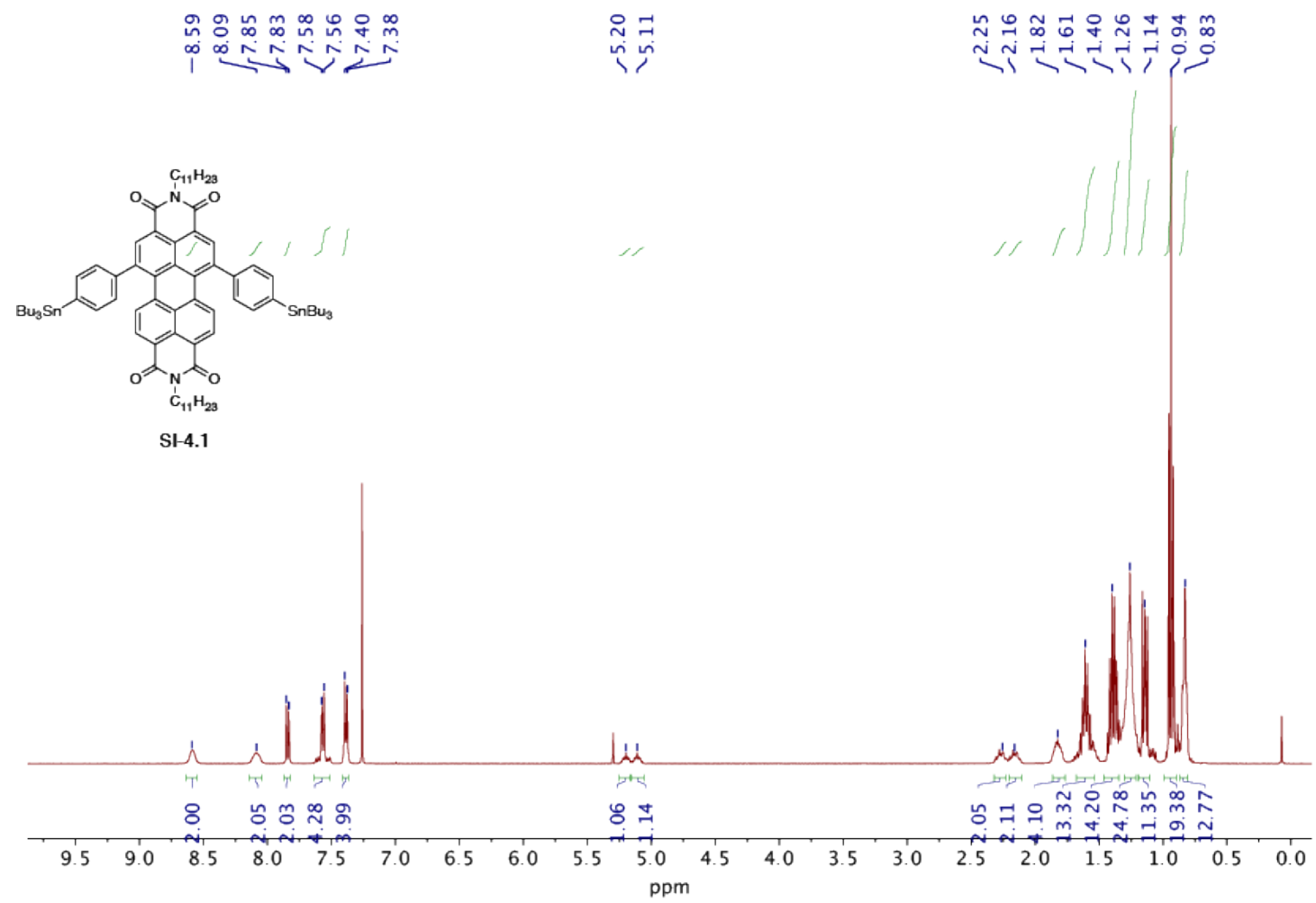

$\infty m \infty \approx ⿻ 上 丨$

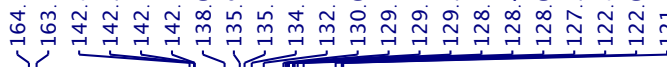

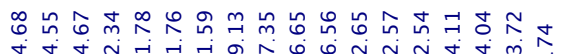

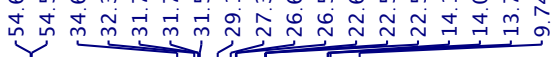
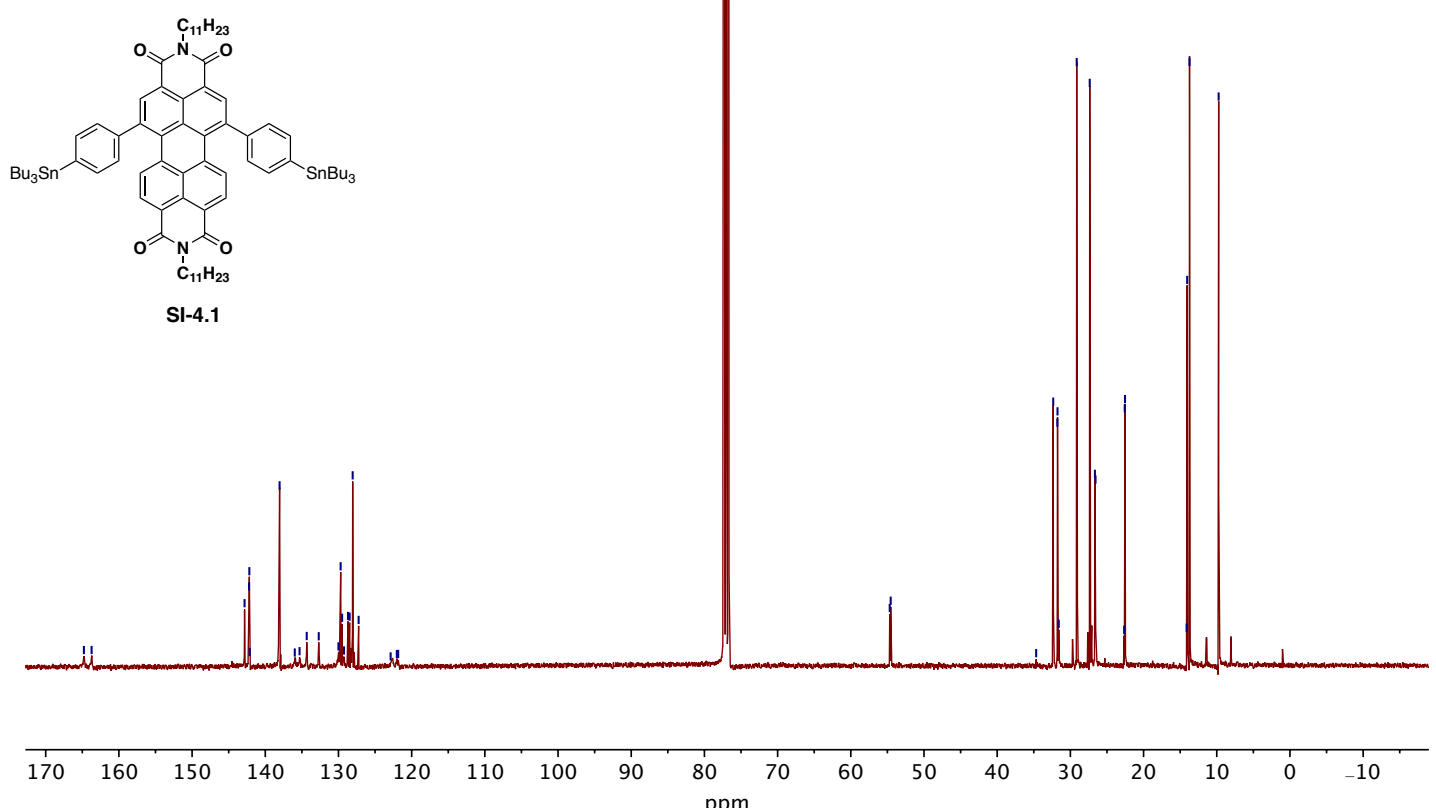


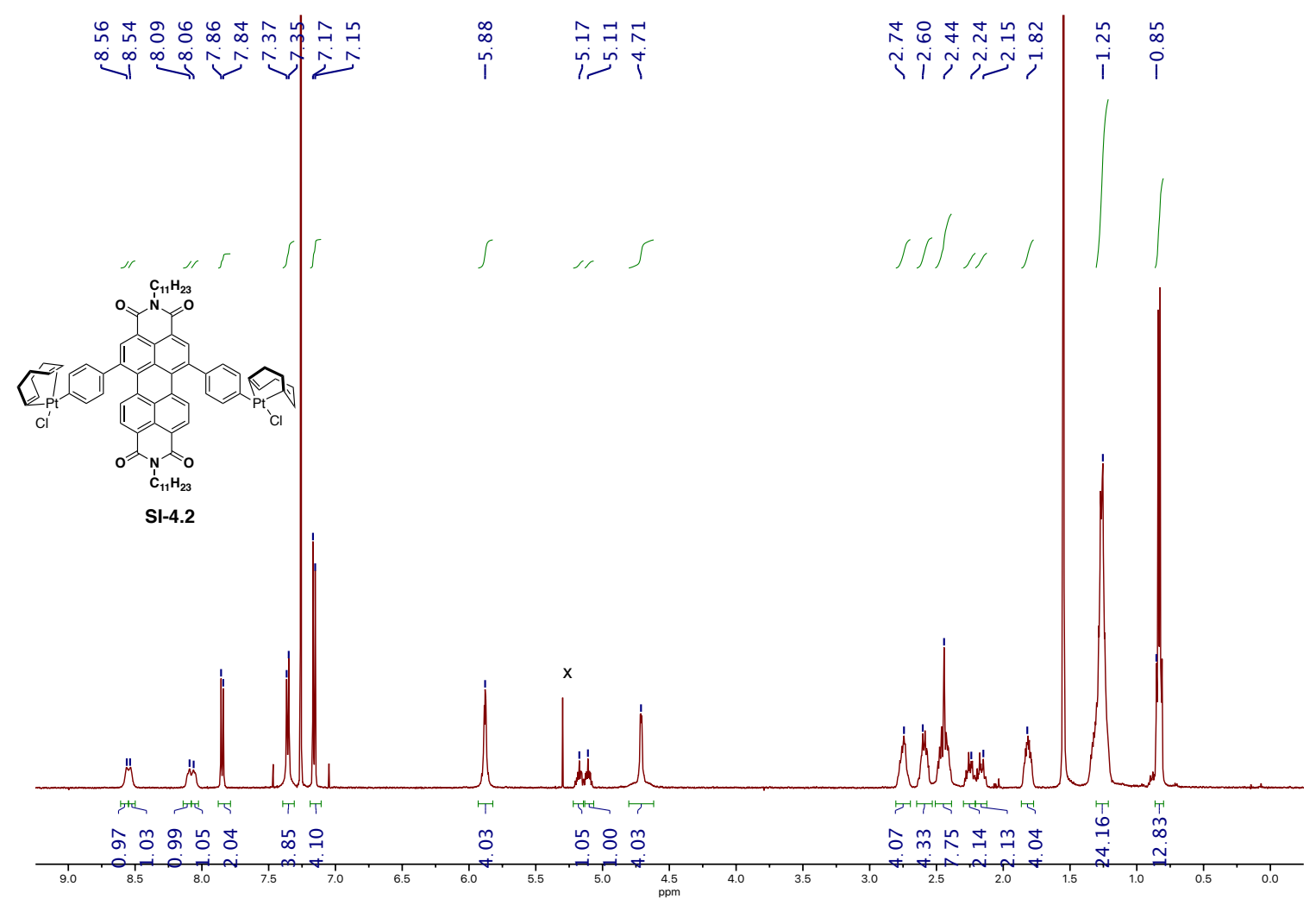

○

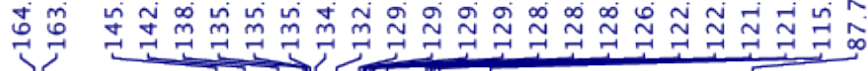

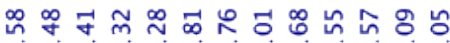

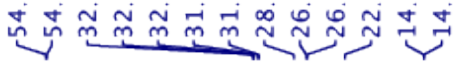
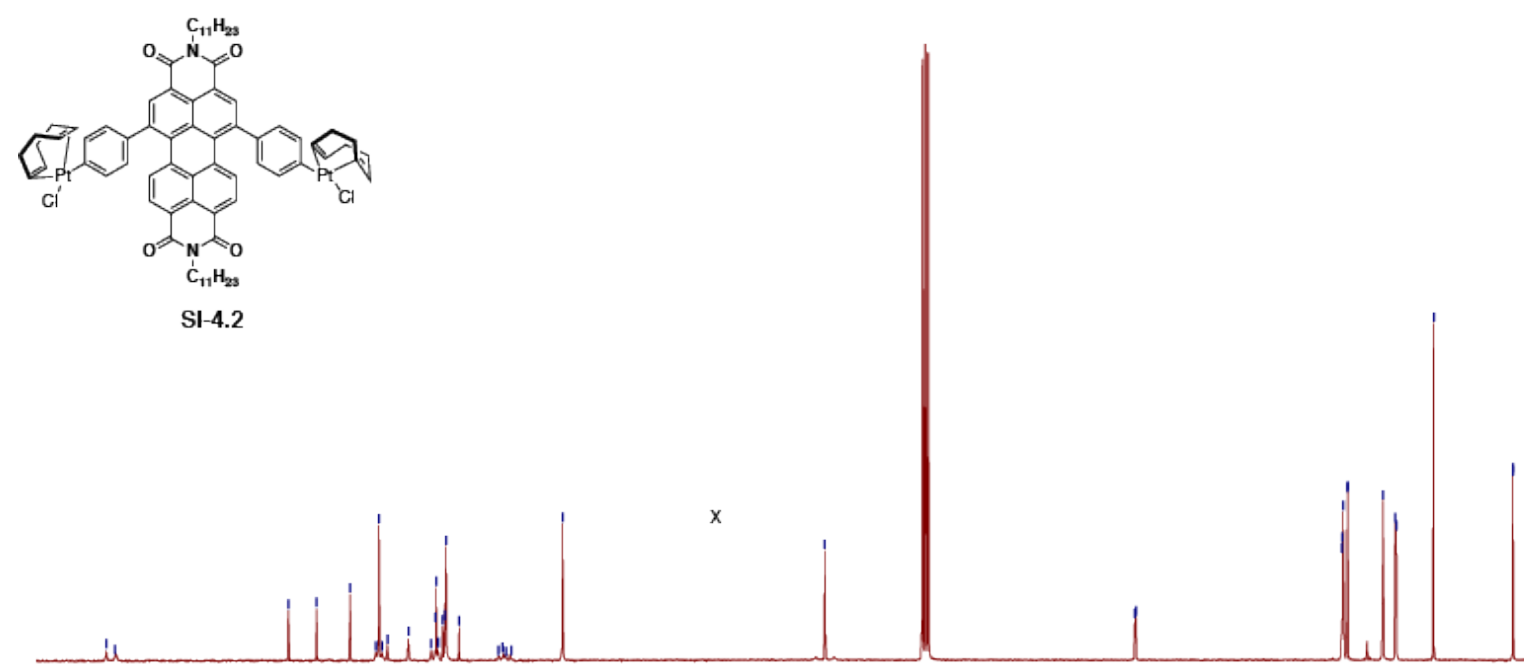

$\prod_{170}^{1}$
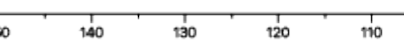


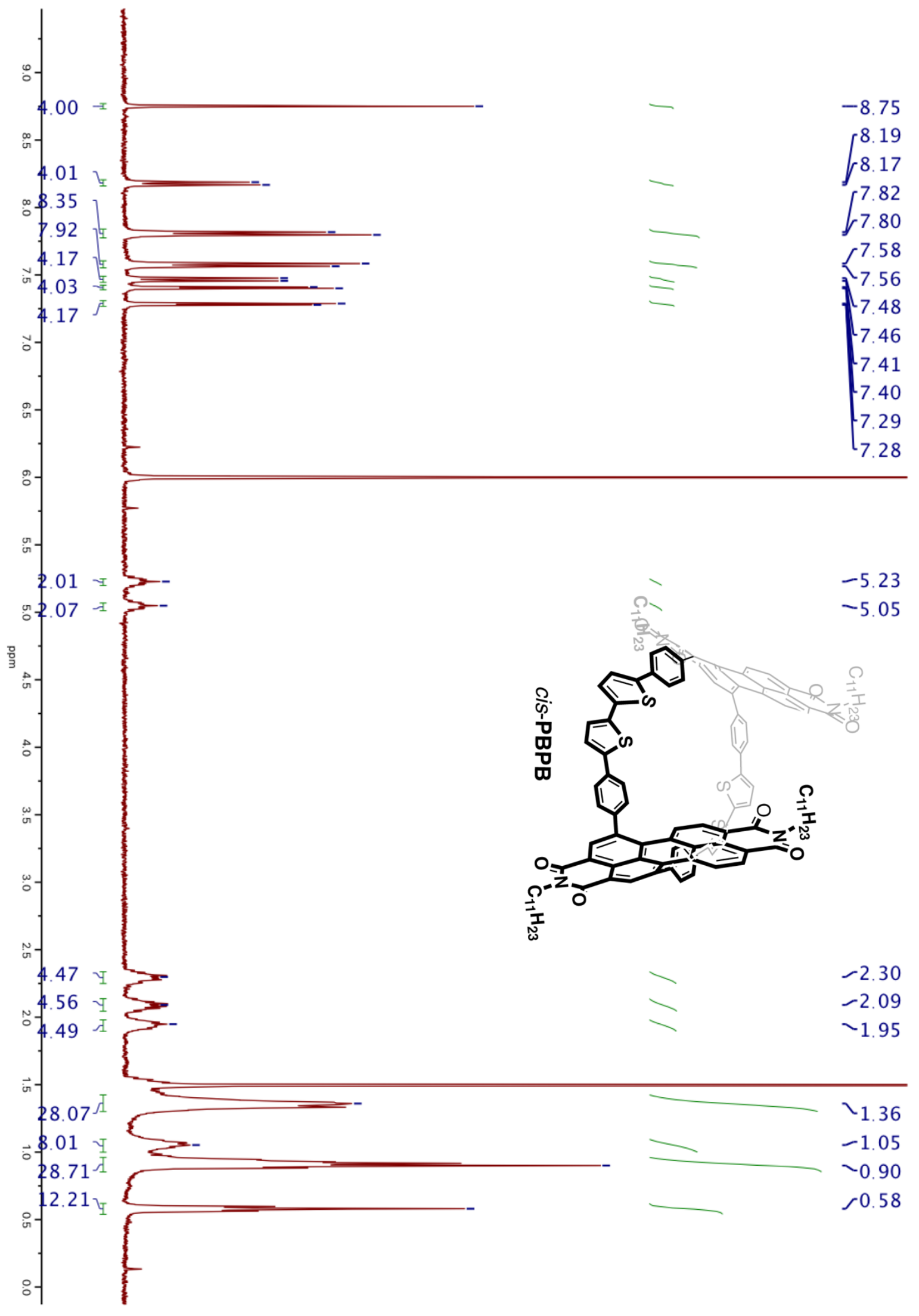




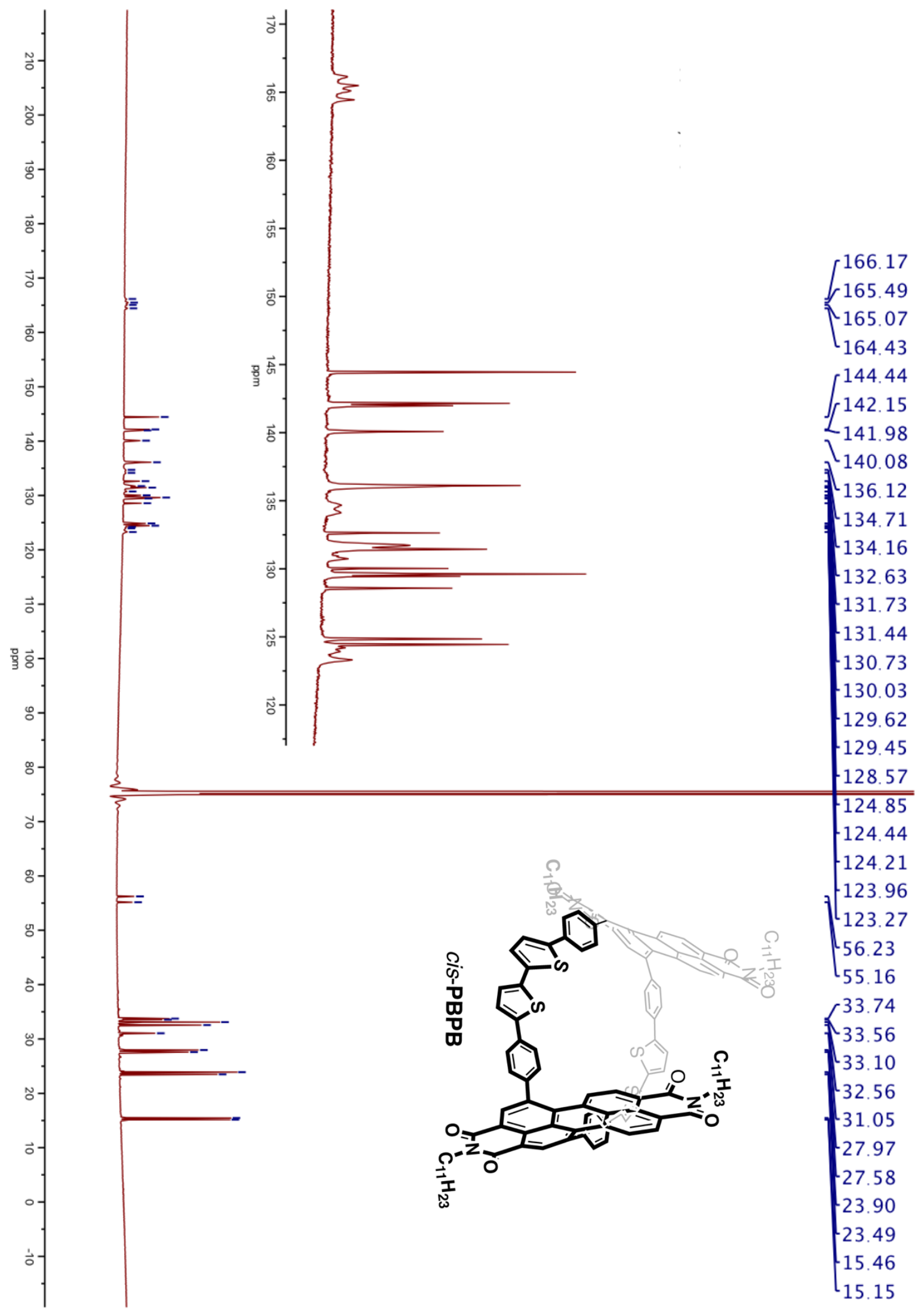



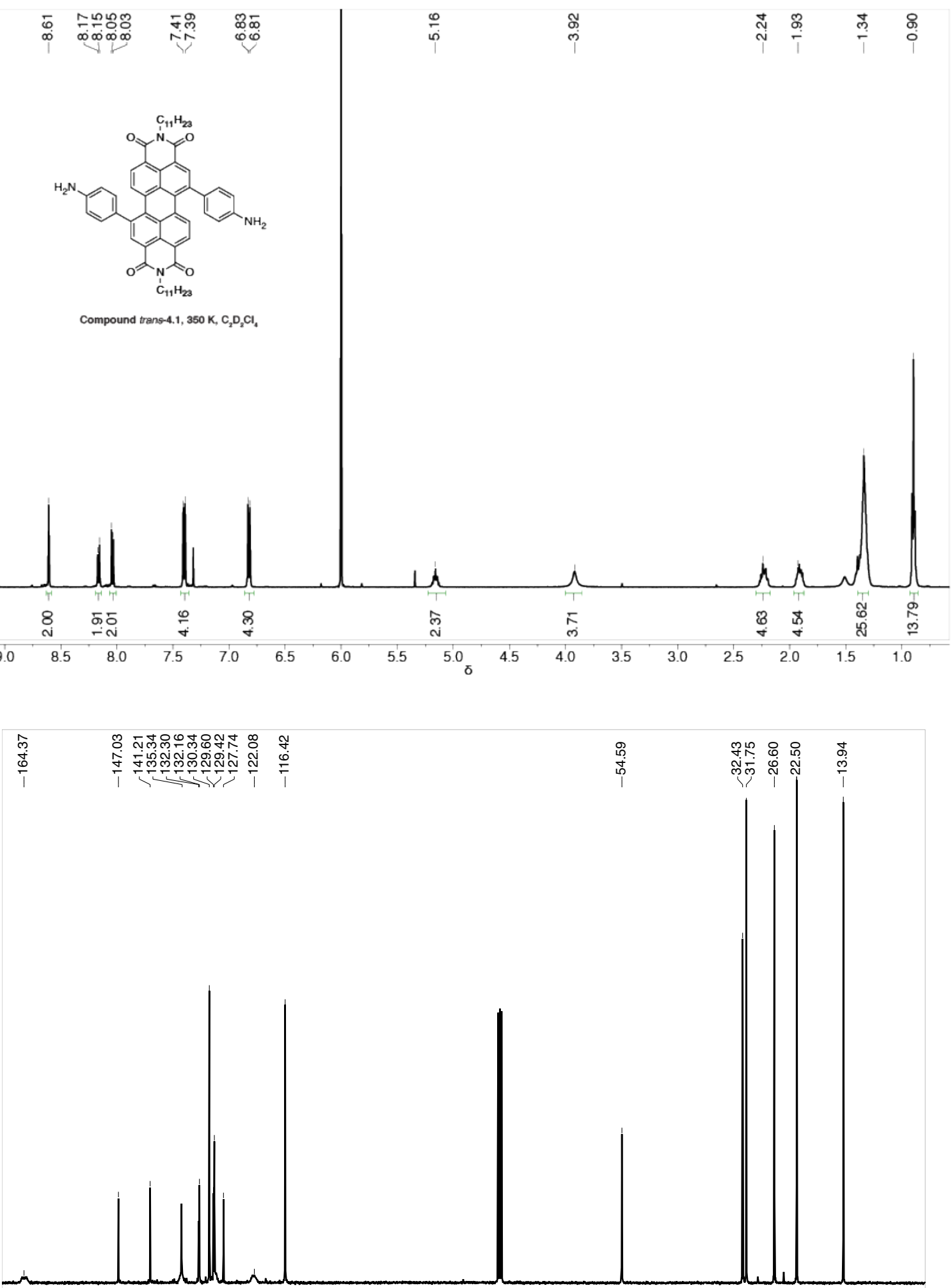

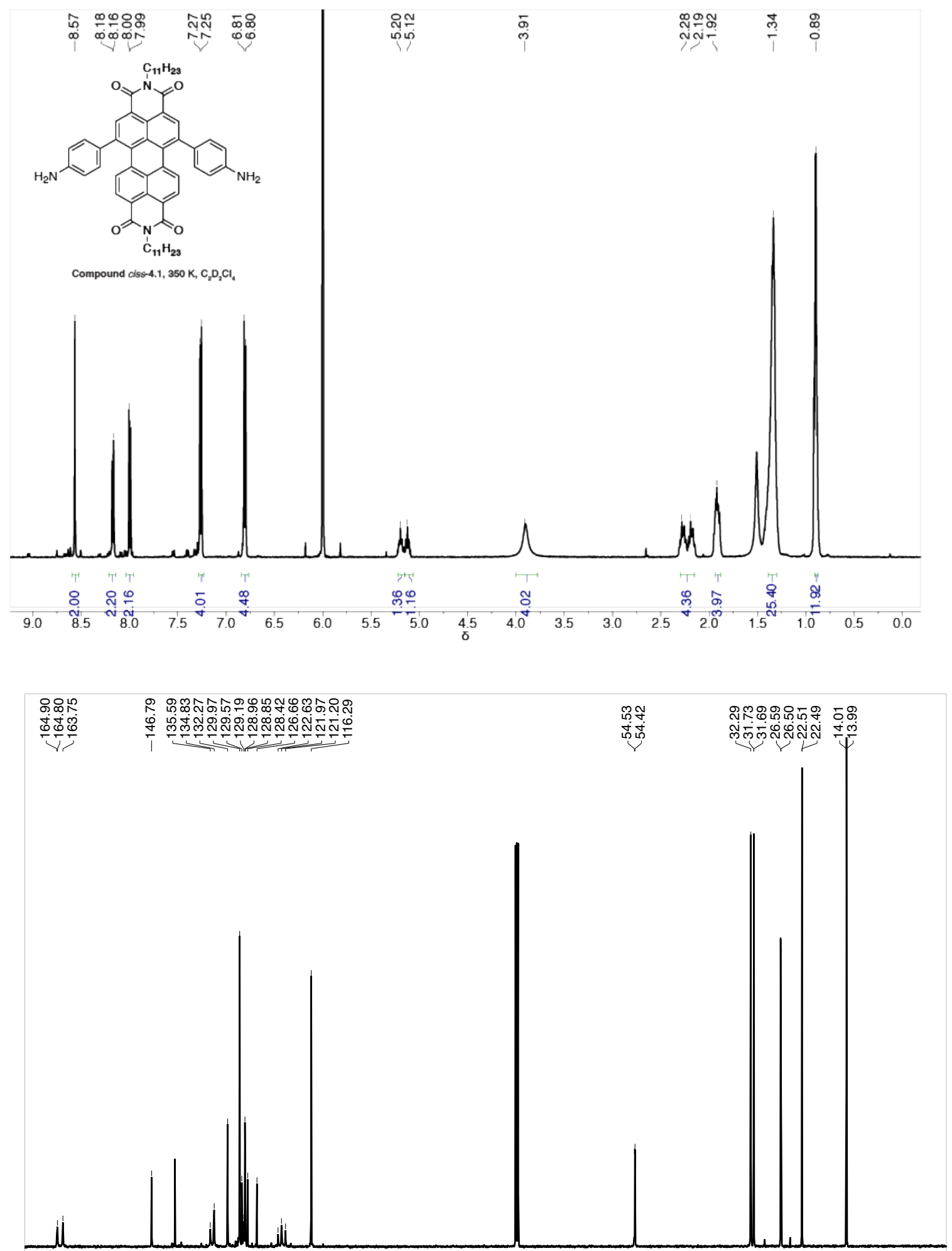

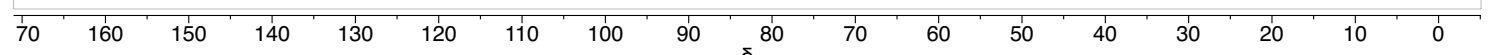




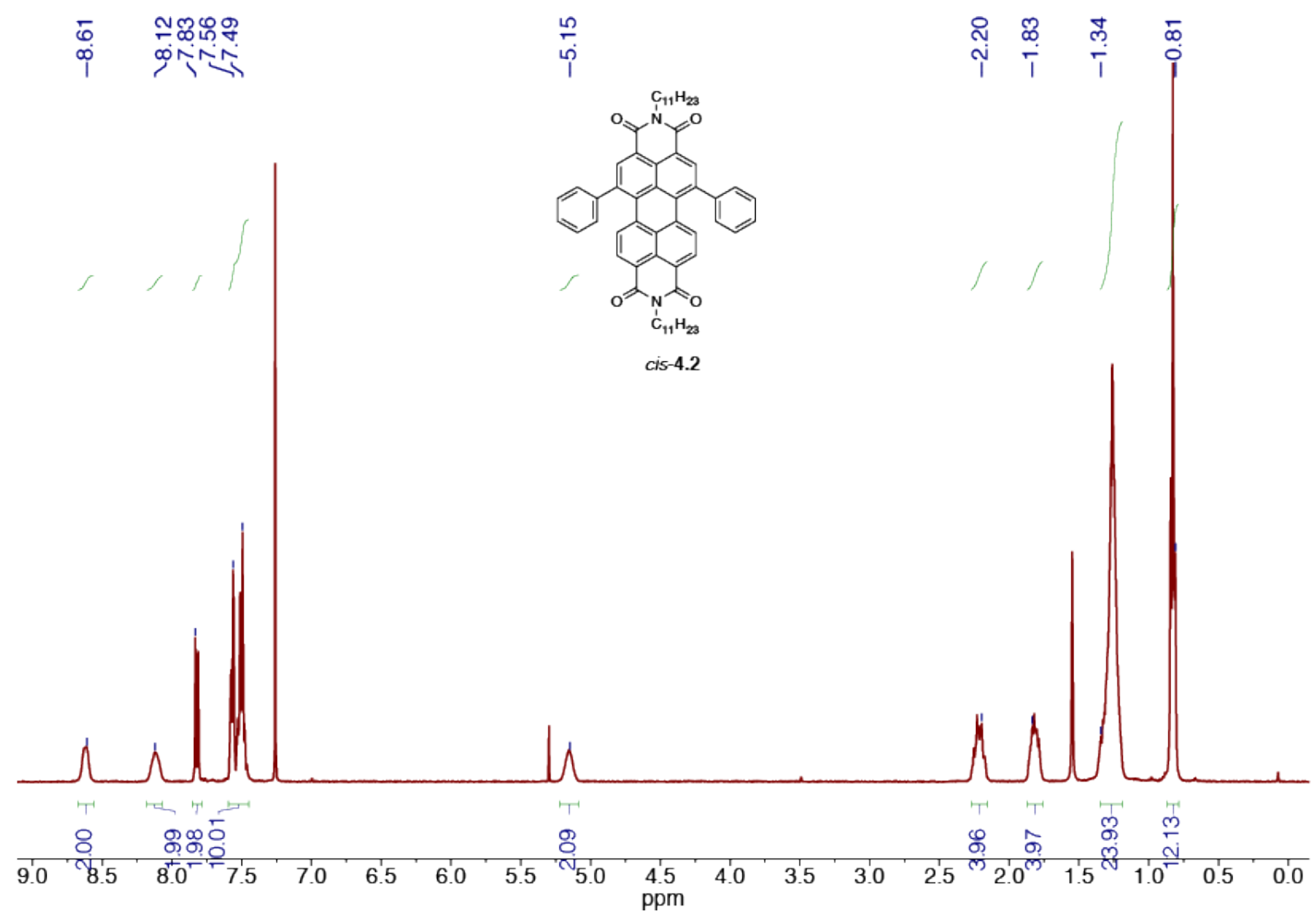

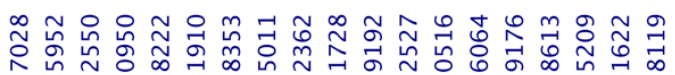

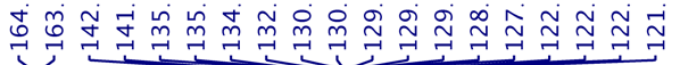
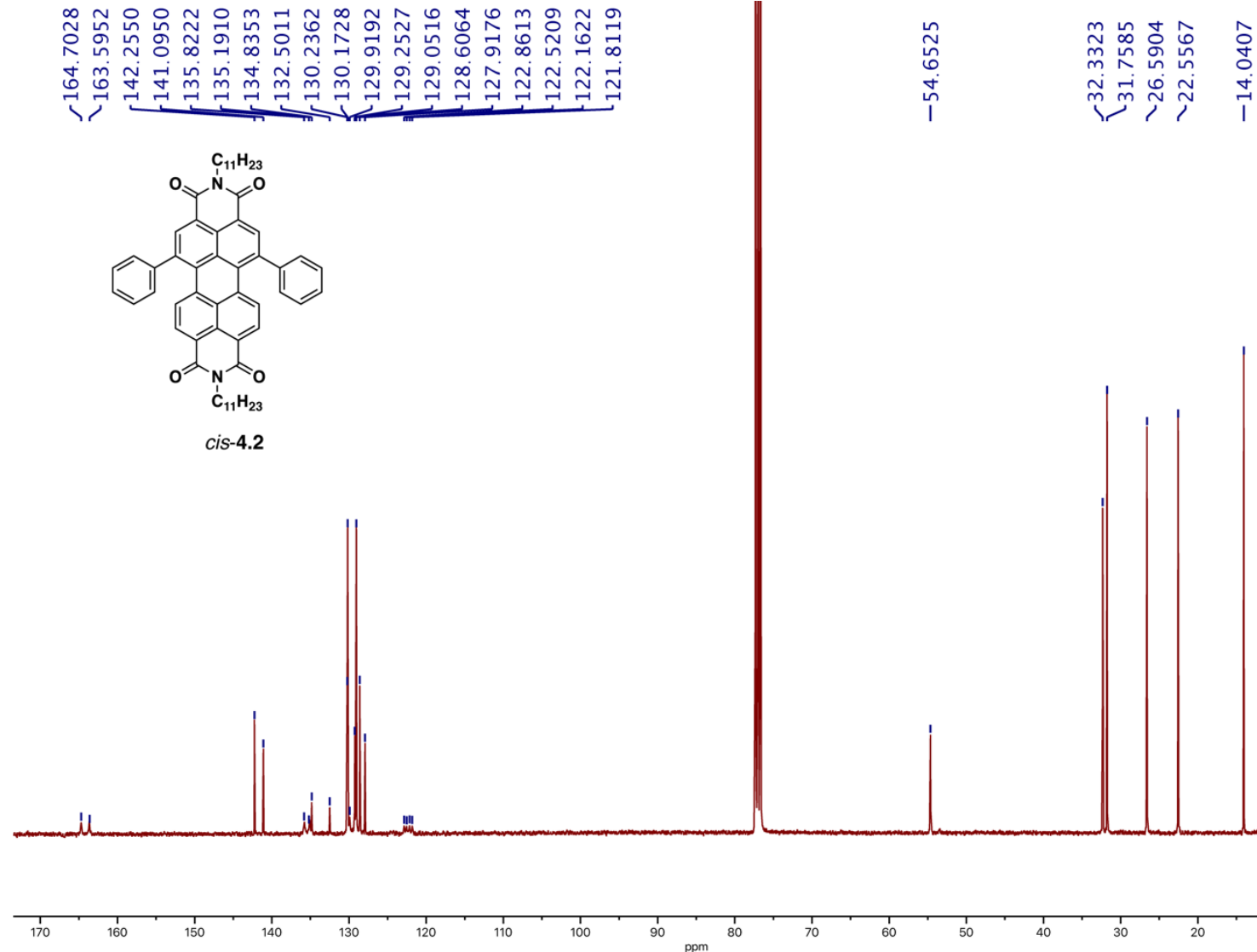


\subsection{DFT Output}

All quantum chemical calculations were performed using Jaguar, version 8.3, Schrodinger, Inc., New York, NY, 2013. (See A. D. Bochevarov, E. Harder, T. F. Hughes, J. R. Greenwood, D. A. Braden, D. M. Philipp, D. Rinaldo, M. D. Halls, J. Zhang, R. A. Friesner, "Jaguar: A High Performance Quantum Chemistry Software Program with Strengths in Life and Materials Sciences", Int. J. Quantum Chem., 2013, 113(18), 2110-2142). All geometries were optimized using the B3LYP functional and the 6-31G basis set. In the following pages, we include for each molecule its optimized geometry and total energy. The TD-DFT excited state calculations for cisPBPB present the fifteen lowest energy roots. We also provide the results of the homodesmotic reaction employed in order to calculate strain within the two macrocycles. Computations for transPBPB were previously published. ${ }^{42}$

\section{Minimized Geometries for Compounds}

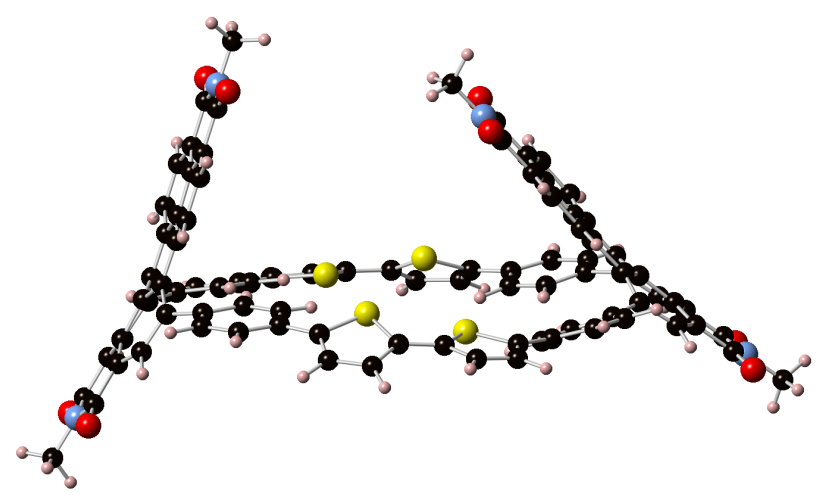

Cis-PBPB

Final Heat of Formation $=-5948.571806$

$\begin{array}{rrrr}\mathrm{H} & 7.658252 & -4.281555 & 1.179927 \\ \mathrm{H} & -8.874982 & -1.420517 & 2.190158 \\ \mathrm{H} & -8.107008 & 3.709119 & 1.433691 \\ \mathrm{H} & 7.719802 & 3.217096 & 2.202196 \\ \mathrm{H} & 8.368280 & 0.696616 & 2.148717 \\ \mathrm{H} & 8.550660 & -1.838906 & 1.345177 \\ \mathrm{H} & 1.503125 & -5.313964 & 0.283514 \\ \mathrm{H} & 4.795645 & -8.075281 & 0.283243\end{array}$




\begin{tabular}{|c|c|c|c|}
\hline $\mathrm{H}$ & 6.359200 & -6.194807 & \\
\hline $\mathrm{H}$ & 3.043003 & -3.451240 & \\
\hline & -3.638890 & 346 & \\
\hline $\mathrm{H}$ & -8.959234 & 1.245785 & \\
\hline & -8.210788 & -3.931177 & \\
\hline & -2.21 & 1265 & \\
\hline & -1.305559 & & \\
\hline $\mathrm{C}$ & -1.20 & 919 & \\
\hline $\mathrm{C}$ & 0.0 & & \\
\hline 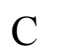 & 1.18 & 7.0 & \\
\hline C & -2.28 & & \\
\hline & -0.99 & 02 & \\
\hline C & $1.2^{\prime}$ & & \\
\hline C & -2.5 & & \\
\hline$C$ & 1.4 & & \\
\hline C & -0.1 & & \\
\hline C & 2.5 & 02 & \\
\hline $\mathrm{C}$ & 0.8 & & \\
\hline $\mathrm{C}$ & -2.1 & 92 & \\
\hline $\mathrm{C}$ & 2.48 & & \\
\hline 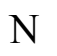 & -0.3 & 60 & \\
\hline C & 2.2 & & \\
\hline $\mathrm{C}$ & 2.3 & & \\
\hline $\mathrm{C}$ & $0.9^{\prime}$ & & \\
\hline $\mathrm{C}$ & -1.5 & & \\
\hline $\mathrm{C}$ & $0.1^{\prime}$ & & 01 \\
\hline$C$ & -0.9 & & \\
\hline$C$ & -0.3 & -9. & 80 \\
\hline$C$ & 0.8 & 58 & \\
\hline $\mathrm{C}$ & 1.76 & -6.3 & \\
\hline$C$ & 5.21 & & \\
\hline $\mathrm{C}$ & 3.50 & & \\
\hline$C$ & 3.8 & & \\
\hline $\mathrm{C}$ & 5.72 & -3.5 & \\
\hline I & 2.56 & & \\
\hline $\mathrm{C}$ & -0.21 & 88 & -2 . \\
\hline ( & -1.46 & 79 & \\
\hline 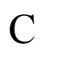 & -1.90 & -5.9 & -4 \\
\hline $\mathrm{C}$ & 0.427694 & 10.4 & \\
\hline $\mathrm{O}$ & 2.6 & & \\
\hline $\mathrm{C}$ & -2.617401 & 637 & 6937 \\
\hline $\mathrm{N}$ & -0.78 & -5.0 & \\
\hline $\mathrm{C}$ & -1.995878 & -5.3 & -5.576284 \\
\hline 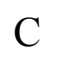 & 6.65 & & \\
\hline $\mathrm{S}$ & 5.076212 & -1.890935 & 0.32 \\
\hline 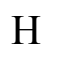 & 3.093576 & 6.510555 & -3.696890 \\
\hline
\end{tabular}




\begin{tabular}{|c|c|c|c|}
\hline & & & \\
\hline $\mathrm{O}$ & -2.674023 & 760384 & 5.444848 \\
\hline $\mathrm{C}$ & 0846 & & \\
\hline 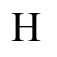 & 334 & & \\
\hline $\mathrm{O}$ & & & \\
\hline & 0.4 & -5.34 & \\
\hline C & & & \\
\hline & -0.446169 & & \\
\hline C & -5.1 & & \\
\hline $\mathrm{C}$ & -3.00 & -6.8 & \\
\hline $\mathrm{C}$ & & & \\
\hline 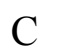 & & -7.9 & \\
\hline $\mathrm{C}$ & -0.6 & -6.2 & \\
\hline C & -1.5 & -10 . & \\
\hline 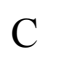 & -2.9 & & \\
\hline $\mathrm{C}$ & & -8.8 & \\
\hline $\mathrm{C}$ & -3.0 & -6.2 & \\
\hline $\mathrm{C}$ & & & \\
\hline $\mathrm{C}$ & 5.2 & -5.5 & \\
\hline $\mathrm{C}$ & -4.7 & & \\
\hline $\mathrm{C}$ & & & \\
\hline $\mathrm{C}$ & -1.7 & -7.1 & \\
\hline $\mathrm{C}$ & -3.8 & & \\
\hline $\mathrm{C}$ & 1.8 & -6.8 & -2 \\
\hline $\mathrm{C}$ & 0.5 & -5.9 & \\
\hline $\mathrm{C}$ & 7.0 & -3.4 & \\
\hline 0 & -3.0 & -5. & \\
\hline $\mathrm{O}$ & -1.9 & & \\
\hline $\mathrm{N}$ & 0 & & \\
\hline $\mathrm{O}$ & -2.52 & -10 . & \\
\hline $\mathrm{C}$ & -3.25 & & \\
\hline $\mathrm{C}$ & & & \\
\hline $\mathrm{C}$ & 0.9 & -10. & \\
\hline$C$ & -6.0 & 5 & \\
\hline $\mathrm{N}$ & -0.2 & -10 . & \\
\hline $\mathrm{C}$ & -5.2 & 60 & \\
\hline $\mathrm{O}$ & & & -6 \\
\hline $\mathrm{C}$ & 3.0 & & \\
\hline $\mathrm{C}$ & & & \\
\hline $\mathrm{C}$ & -5.5 & & \\
\hline $\mathrm{C}$ & -0.22 & -11. & \\
\hline $\mathrm{S}$ & & & \\
\hline $\mathrm{C}$ & 3.006959 & & \\
\hline O & & 2.4 & \\
\hline $\mathrm{O}$ & 2.040622 & 10.9 & 3.2 \\
\hline$C$ & -7.443537 & 2.851734 & 1.452753 \\
\hline
\end{tabular}




\begin{tabular}{|c|c|c|c|}
\hline & 20520 & 13260 & \\
\hline C & -4.102038 & & \\
\hline & 7.910592 & & \\
\hline$u$ & -6.899136 & & \\
\hline & 6615 & & \\
\hline & 4.4 & -7.0 & \\
\hline & -6.951369 & -0.8 & \\
\hline $\mathrm{C}$ & -0.4 & -8.2 & \\
\hline C & -6.0 & & \\
\hline$\checkmark$ & -7.5 & -3. & \\
\hline C & & & \\
\hline $\mathrm{C}$ & -6.3 & -3.3 & \\
\hline S & -5.3 & & \\
\hline$\gamma$ & -7.9 & -1. & \\
\hline$S$ & -5.5 & -1.7 & \\
\hline $\mathrm{C}$ & & & \\
\hline C & 7.5 & 1.1 & \\
\hline $\mathrm{C}$ & -2.8 & -7.9 & \\
\hline n & -3.0 & & \\
\hline H & -3.5 & & \\
\hline$\Pi$ & 3.5 & 8.1 & \\
\hline H & 5.3 & & \\
\hline 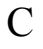 & -1.7 & -7.7 & \\
\hline C & -2.7 & -8.8 & \\
\hline 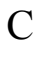 & -0.5 & -6. & \\
\hline $\mathrm{H}$ & -3.4 & & \\
\hline $\mathrm{C}$ & -1.5 & -9.5 & \\
\hline $\mathrm{H}$ & 3.3 & 2.9 & \\
\hline $\mathrm{C}$ & 0.70 & -7.1 & 36 \\
\hline $\mathrm{H}$ & 1.9 & & \\
\hline & -3.6 & -9. & \\
\hline $\mathrm{H}$ & -6.6 & & \\
\hline 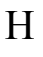 & -4.0 & -6.0 & \\
\hline $\mathrm{H}$ & -5.0 & & \\
\hline $\mathrm{T}$ & 6.7 & 5.4 & \\
\hline H & 2.9 & $-9 .($ & \\
\hline & 2.6633 & -6.1 & \\
\hline 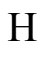 & -5.6 & -7.5 & 13 \\
\hline & -7.11 & -5.9 & \\
\hline 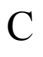 & -0.8 & -4.4 & -7.5 \\
\hline-7 & -1.492304 & 5.205793 & -7.273610 \\
\hline & 0.00 & & -7.639107 \\
\hline & 0.114290 & 4.417737 & -7.153124 \\
\hline & 1.46 & 10.7 & \\
\hline & -0.204846 & 11.328638 & 6.18 \\
\hline & 0.058753 & 9.805052 & 7.044161 \\
\hline
\end{tabular}




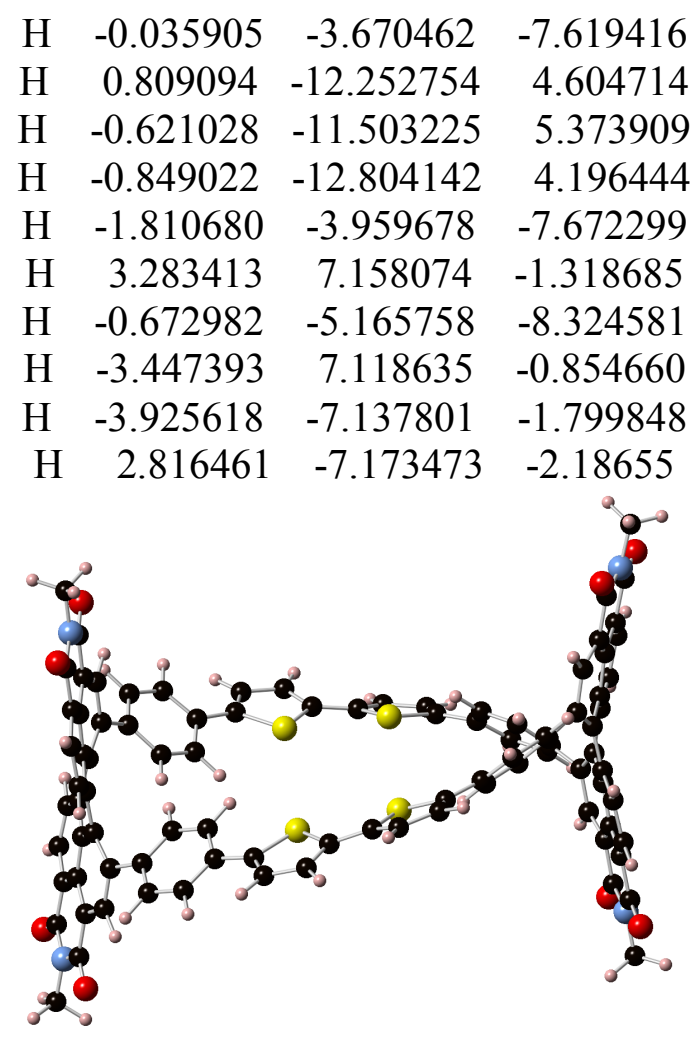

Trans-PBPB

Final Heat of Formation $=-5948.582452$

$\begin{array}{rrrr}\mathrm{H} & 7.378979 & -4.061255 & -1.403716 \\ \mathrm{H} & -8.552342 & -1.130130 & 0.960989 \\ \mathrm{H} & -7.808430 & 3.698092 & -0.499800 \\ \mathrm{H} & 7.723016 & 3.176686 & 0.618561 \\ \mathrm{H} & 8.257131 & 0.651206 & 0.244544 \\ \mathrm{H} & 8.121079 & -1.589498 & -1.014161 \\ \mathrm{H} & 1.549809 & -5.996235 & -0.110919 \\ \mathrm{H} & 4.264289 & -7.363391 & -3.155524 \\ \mathrm{H} & 5.796426 & -5.538855 & -2.596380 \\ \mathrm{H} & 3.101928 & -4.215162 & 0.485313 \\ \mathrm{H} & -3.557266 & -3.764780 & -0.518083 \\ \mathrm{H} & -8.545919 & 1.229471 & -0.107770 \\ \mathrm{H} & -7.951011 & -3.655420 & 1.212110 \\ \mathrm{H} & -2.189449 & -5.752749 & -0.237154 \\ \mathrm{C} & -1.156063 & 7.863026 & -0.595819 \\ \mathrm{C} & -1.240975 & 7.962287 & 0.863294 \\ \mathrm{C} & -0.035351 & 7.873851 & 1.625341 \\ \mathrm{C} & 1.311428 & 8.099306 & -0.421930 \\ \mathrm{C} & -2.443363 & 8.199950 & 1.535777 \\ \mathrm{C} & -1.345102 & 8.135796 & 3.686849 \\ \mathrm{C} & 1.225487 & 7.683485 & 0.981719 \\ \mathrm{C} & -2.221768 & 7.496082 & -1.450187\end{array}$




$\begin{array}{rrrr}\text { C } & 1.053574 & 7.613966 & 3.800389 \\ \mathrm{C} & 0.101314 & 8.198406 & -1.178366 \\ \mathrm{C} & 2.197979 & 7.189738 & 3.152480 \\ \mathrm{C} & 1.421477 & 9.480075 & -4.509042 \\ \mathrm{C} & -2.493746 & 8.310023 & 2.929405 \\ \mathrm{C} & 2.290310 & 7.148587 & 1.741085 \\ \mathrm{~N} & 0.210341 & 9.461060 & -5.219887 \\ \mathrm{C} & 2.545462 & 8.936712 & -2.355348 \\ \mathrm{C} & 2.513136 & 8.466821 & -1.036156 \\ \mathrm{C} & 1.384550 & 8.981980 & -3.115281 \\ \mathrm{C} & -1.007695 & 8.944574 & -4.744943 \\ \mathrm{C} & -0.111891 & 7.887293 & 3.044701 \\ \mathrm{C} & -1.415547 & 8.196847 & 5.163853 \\ \mathrm{C} & -0.165691 & -9.052789 & 2.175949 \\ \mathrm{C} & 1.089090 & -9.526138 & 2.621649 \\ \mathrm{C} & 1.414272 & -7.558929 & -3.635819 \\ \mathrm{C} & 4.929477 & 4.016460 & 0.462769 \\ \mathrm{C} & 3.335836 & 6.268971 & 1.153821 \\ \mathrm{C} & 3.696590 & 4.257347 & -0.169802 \\ \mathrm{C} & 5.367256 & -3.506402 & -0.824260 \\ \mathrm{C} & 2.495495 & -5.927797 & -0.636083 \\ \mathrm{C} & 0.158635 & 8.565944 & -2.548963 \\ \mathrm{C} & -1.017600 & 8.486300 & -3.334723 \\ \mathrm{C} & -2.208465 & -8.299149 & -3.901923 \\ \mathrm{C} & -0.307612 & 8.029891 & 7.327945 \\ \mathrm{O} & 2.001625 & 7.410603 & 5.969473 \\ \mathrm{C} & -2.149391 & 7.888691 & -2.807137 \\ \mathrm{~N} & -1.278891 & -8.012767 & -6.158693 \\ \mathrm{C} & -2.413230 & -8.274304 & -5.369769 \\ \mathrm{C} & 6.150159 & 0.300998 & -0.126483 \\ \mathrm{~S} & 4.597653 & -2.021262 & -0.293849 \\ \mathrm{H} & 3.474185 & 9.266054 & -2.808193 \\ \mathrm{C} & 1.016225 & 7.647524 & 5.283179 \\ \mathrm{O} & -2.001069 & 8.896858 & -5.458539 \\ \mathrm{C} & -3.247528 & 6.511953 & -1.020909 \\ \mathrm{H} & -1.782595 & 5.406030 & 0.114289 \\ \mathrm{O} & 2.437735 & 9.910639 & -5.037677 \\ \mathrm{C} & 0.023518 & -7.821747 & -5.672234 \\ \mathrm{C} & 0.793222 & -8.624118 & -0.049522 \\ \mathrm{C} & 0.257672 & 9.990555 & -6.586232 \\ \mathrm{C} & -4.942190 & 4.288860 & -0.461791 \\ \mathrm{C} & -3.115921 & -8.425502 & -1.663325 \\ \mathrm{C} & -5.403865 & 5.390176 & -1.208318 \\ \mathrm{C} & 2.022814 & -9.080113 & 0.434201 \\ \mathrm{C} & -0.912883 & -8.147992 & -3.360556 \\ \mathrm{C} & -1.103828 & -9.344304 & 4.486025\end{array}$




\begin{tabular}{|c|c|c|c|}
\hline & -2.805736 & 5.424842 & \\
\hline$C$ & 2.164114 & -9.551533 & \\
\hline & -3.292850 & -8.460161 & 1526 \\
\hline 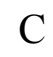 & 5.396772 & 581 & \\
\hline & 4.883721 & -5.655206 & -2.021698 \\
\hline C & -4.573671 & 576 & 4108 \\
\hline $\mathrm{C}$ & 4.615365 & 93 & 7630 \\
\hline 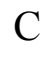 & -1.847975 & -8.290602 & -1 . \\
\hline$C$ & -3.62 & 01 & \\
\hline$c$ & 1.631272 & 7001 & -2.2 \\
\hline$C$ & 0.19 & -7.8 & -4.2 \\
\hline $\mathrm{C}$ & 6.70 & -3.2 & -1. \\
\hline O & -3.50 & -8. & \\
\hline 0 & -2.45 & & 5.7 \\
\hline$N$ & -0.2 & & \\
\hline $\mathrm{O}$ & -2.00 & -9.2 & \\
\hline $\mathrm{C}$ & $-3.1 c$ & -5. & \\
\hline $\mathrm{C}$ & 4.5 & $-4{ }^{\prime}$ & -1. \\
\hline $\mathrm{C}$ & 1.26 & -9.9 & 4.0 \\
\hline $\mathrm{C}$ & -5.6 & -5.5 & \\
\hline $\mathbf{N}$ & 0.13 & -9. & 4.8 \\
\hline $\mathrm{C}$ & -4.85 & -6. & \\
\hline $\mathrm{O}$ & 0.9 & -7.6 & -6. \\
\hline $\mathrm{C}$ & & & -1. \\
\hline $\mathrm{C}$ & 6.0 & -1.1 & -0 . \\
\hline $\mathrm{C}$ & -5.17 & -4. & \\
\hline C & 0.2 & -10 & \\
\hline S & 4.71 & 1.3 & -0.1 \\
\hline $\mathrm{C}$ & 2.92 & & \\
\hline $\mathrm{C}$ & 6.96 & 2.4 & \\
\hline $\mathrm{O}$ & 2.32 & -10 & \\
\hline $\mathrm{C}$ & -7.10 & 2.8 & -0.2 \\
\hline $\mathrm{C}$ & -3.5 & -6.8 & \\
\hline $\mathrm{C}$ & -3.93 & -4.5 & \\
\hline $\mathrm{C}$ & -7.51 & & -0. \\
\hline $\mathrm{C}$ & -6.46 & 0.6 & \\
\hline 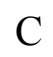 & 3.36 & -4.9 & -0 . \\
\hline $\mathrm{C}$ & 4.00 & -6.6 & -2.3 \\
\hline $\mathrm{C}$ & -6.492063 & -0.7 & \\
\hline $\mathrm{C}$ & -0.342396 & -8.6 & \\
\hline $\mathrm{C}$ & -5.745970 & 3.085545 & -0.2 \\
\hline $\mathrm{C}$ & -7.228508 & -2.8 & 56 \\
\hline $\mathrm{C}$ & 7.110144 & 289 & -0.850733 \\
\hline $\mathrm{C}$ & -5.905096 & -3.1 & \\
\hline $\mathrm{S}$ & -4.946352 & 1.563098 & 0.108522 \\
\hline 4 & -7.560627 & -1.528190 & 0.781948 \\
\hline
\end{tabular}




$\begin{array}{rrrr}\text { S } & -5.050567 & -1.713822 & 0.243775 \\ \mathrm{C} & 5.632654 & 2.750703 & 0.250552 \\ \mathrm{C} & 7.259848 & 1.067242 & 0.170126 \\ \mathrm{C} & -2.586073 & -7.857098 & 1.320055 \\ \mathrm{H} & -3.428076 & 8.509312 & 3.442435 \\ \mathrm{H} & -2.980052 & 7.690456 & -3.476168 \\ \mathrm{H} & 3.022092 & 6.830193 & 3.758593 \\ \mathrm{H} & 4.983361 & 6.788306 & 2.453730 \\ \mathrm{C} & -1.628147 & -8.258887 & 0.360921 \\ \mathrm{C} & -2.398289 & -8.259977 & 2.663276 \\ \mathrm{C} & -0.711874 & -8.224294 & -1.954879 \\ \mathrm{H} & -3.237357 & 3.534143 & 0.635062 \\ \mathrm{C} & -1.249508 & -8.911113 & 3.076065 \\ \mathrm{H} & 3.323487 & 3.552430 & -0.906718 \\ \mathrm{C} & 0.608421 & -8.137935 & -1.419943 \\ \mathrm{H} & 1.940504 & 5.460379 & -0.280465 \\ \mathrm{H} & -3.143673 & -8.015725 & 3.412958 \\ \mathrm{H} & 2.887520 & -9.068311 & -0.218566 \\ \mathrm{H} & -6.410104 & 5.379493 & -1.615123 \\ \mathrm{H} & -4.278303 & -8.585496 & -3.486358 \\ \mathrm{H} & -4.960551 & 7.296303 & -2.081765 \\ \mathrm{H} & 6.348009 & 4.840491 & 1.870574 \\ \mathrm{H} & 3.117400 & -9.922927 & 2.103997 \\ \mathrm{H} & 2.198226 & -7.200088 & -4.293774 \\ \mathrm{H} & -3.983376 & -8.501508 & -1.020115 \\ \mathrm{H} & -5.224000 & -7.521995 & 2.243050 \\ \mathrm{H} & -6.575282 & -5.491669 & 2.020933 \\ \mathrm{C} & -1.465338 & -7.952602 & -7.612388 \\ \mathrm{H} & -0.757612 & 9.998668 & -6.974604 \\ \mathrm{H} & 0.678198 & 10.997504 & -6.569986 \\ \mathrm{H} & 0.896112 & 9.363261 & -7.212833 \\ \mathrm{H} & 0.702342 & 7.963657 & 7.724491 \\ \mathrm{H} & -0.785249 & 8.964626 & 7.624361 \\ \mathrm{H} & -0.913098 & 7.201230 & 7.704026 \\ \mathrm{H} & -0.751046 & -7.241437 & -8.023064 \\ \mathrm{H} & 1.251676 & -10.750354 & 6.374781 \\ \mathrm{H} & 0.106132 & -9.489760 & 6.926877 \\ \mathrm{H} & -0.509098 & -11.080434 & 6.455336 \\ \mathrm{H} & -2.490520 & -7.648993 & -7.813184 \\ \mathrm{H} & -3.358636 & 8.297589 & 0.965425 \\ \mathrm{H} & 3.436168 & 8.414110 & -0.471374 \\ \mathrm{H} & -1.288423 & -8.933121 & -8.065543\end{array}$




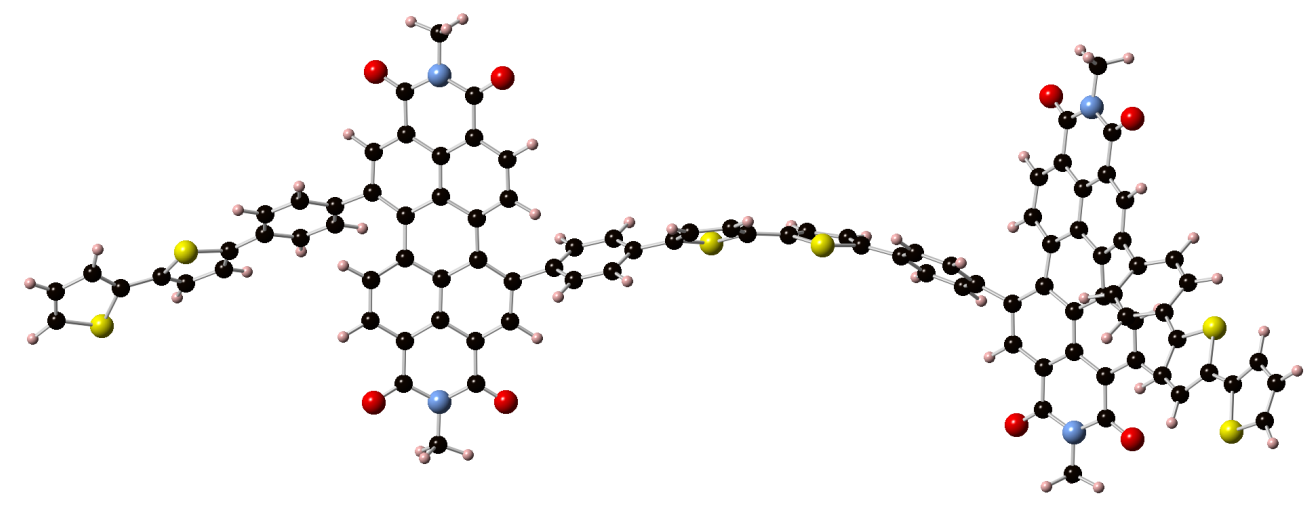

Trans-PBPB-Acyclic for Homodesmotic Calculations

Final Heat of Formation $=-7053.442019$

$\begin{array}{crcc}\mathrm{H} & -3.233960 & -6.432410 & -14.857947 \\ \mathrm{H} & -2.058853 & 0.684038 & -18.424512 \\ \mathrm{H} & -3.776636 & -1.236636 & -17.972620 \\ \mathrm{H} & -1.775695 & -4.273604 & -14.788876 \\ \mathrm{H} & -8.024836 & -7.371139 & -18.749207 \\ \mathrm{H} & -5.305647 & -10.121626 & -16.880256 \\ \mathrm{H} & -3.946001 & -8.246249 & -16.082889 \\ \mathrm{H} & -6.668862 & -5.489172 & -17.956364 \\ \mathrm{H} & -13.087586 & -10.481150 & -23.675002 \\ \mathrm{H} & -12.127208 & -9.956352 & -21.492947 \\ \mathrm{C} & 5.143194 & 6.940450 & -17.056760 \\ \mathrm{C} & 5.884552 & 6.228233 & -18.113325 \\ \mathrm{C} & 5.492925 & 4.894972 & -18.464409 \\ \mathrm{C} & 3.349923 & 5.188678 & -17.229018 \\ \mathrm{C} & 6.929674 & 6.821555 & -18.827061 \\ \mathrm{C} & 7.429917 & 4.785823 & -20.005890 \\ \mathrm{C} & 4.275089 & 4.303227 & -17.970355 \\ \mathrm{C} & 5.620588 & 8.096059 & -16.392319 \\ \mathrm{C} & 6.031192 & 2.819424 & -19.665553 \\ \mathrm{C} & 3.832739 & 6.450572 & -16.733042 \\ \mathrm{C} & 4.921123 & 2.232647 & -19.105361 \\ \mathrm{C} & 0.732859 & 7.667324 & -14.827264 \\ \mathrm{C} & 7.690846 & 6.119120 & -19.764944 \\ \mathrm{C} & 4.021882 & 2.946163 & -18.288108 \\ \mathrm{~N} & 1.272043 & 8.836722 & -14.276367 \\ \mathrm{C} & 1.160672 & 5.677884 & -16.237118 \\ \mathrm{C} & 2.009082 & 4.864450 & -16.994992 \\ \mathrm{C} & 1.635865 & 6.841485 & -15.664527 \\ \mathrm{C} & 2.588477 & 9.282185 & -14.465736 \\ \mathrm{C} & 6.326323 & 4.167719 & -19.372049 \\ \mathrm{C} & 8.282043 & 4.033311 & -20.957340 \\ \mathrm{C} & -12.074580 & -9.280565 & -16.711511 \\ \mathrm{C} & -11.577682 & -8.637659 & -15.554208\end{array}$




\begin{tabular}{|c|c|c|c|}
\hline 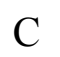 & & & \\
\hline $\mathrm{C}$ & & & \\
\hline $\mathrm{C}$ & & & \\
\hline $\mathrm{C}$ & & & \\
\hline$C$ & & & \\
\hline$C$ & & & \\
\hline $\mathrm{C}$ & & & \\
\hline $\mathrm{C}$ & & & \\
\hline G & -9.0 & 32 & \\
\hline $\mathrm{C}$ & & & \\
\hline $\mathrm{O}$ & & & \\
\hline $\mathrm{C}$ & 4.7 & & \\
\hline $\mathrm{N}$ & & & \\
\hline $\mathrm{C}$ & -8.1 & -14 & \\
\hline $\mathrm{C}$ & -2.2 & & \\
\hline $\mathrm{S}$ & -4.2 & & \\
\hline $\mathrm{H}$ & & & \\
\hline $\mathrm{C}$ & & & \\
\hline $\mathrm{O}$ & & & \\
\hline C & & & \\
\hline $\mathrm{H}$ & & & \\
\hline $\mathrm{O}$ & -0. & & \\
\hline & & 40 & \\
\hline $\mathrm{C}$ & & & \\
\hline $\mathrm{C}$ & & & \\
\hline $\mathrm{C}$ & & & \\
\hline & 1 & -12 & \\
\hline $\mathrm{C}$ & & & \\
\hline $\mathrm{C}$ & -9.3 & & \\
\hline $\mathrm{C}$ & -8.5 & -12 & \\
\hline $\mathrm{C}$ & -14 & & \\
\hline $\mathrm{C}$ & & & \\
\hline$C$ & & & \\
\hline $\mathrm{C}$ & 8 & -13 & \\
\hline $\mathrm{C}$ & & & -18 \\
\hline $\mathrm{C}$ & -4.8 & & \\
\hline $\mathrm{C}$ & & & \\
\hline $\mathrm{C}$ & & & \\
\hline $\mathrm{C}$ & 94 & 44 & \\
\hline $\mathrm{C}$ & & & \\
\hline $\mathrm{C}$ & -7.4 & -10 . & \\
\hline $\mathrm{C}$ & -7.1 & -11. & -20 \\
\hline $\mathrm{C}$ & -3.3 & -5.6 & -15 \\
\hline $\mathrm{O}$ & -8.5 & -14 & -22 \\
\hline $\mathrm{O}$ & & & -21.5 \\
\hline $\mathrm{N}$ & 7.958248 & 2.687707 & -21.17355 \\
\hline
\end{tabular}




\begin{tabular}{|c|c|c|c|}
\hline $\mathrm{O}$ & -15.626919 & -8.633649 & -16.210995 \\
\hline $\mathrm{C}$ & -13.049704 & -10.526006 & -21.544543 \\
\hline $\mathrm{C}$ & -5.195437 & -6.712889 & -16.953446 \\
\hline $\mathrm{C}$ & -12.497823 & -8.000937 & -14.579116 \\
\hline $\mathrm{C}$ & -15.446708 & -11.926922 & -21.709548 \\
\hline $\mathrm{N}$ & -13.867466 & -8.048558 & -14.888736 \\
\hline $\mathrm{C}$ & -14.911334 & -11.598454 & -20.464091 \\
\hline $\mathrm{O}$ & -4.988410 & -12.462879 & -20.979185 \\
\hline $\mathrm{C}$ & -6.774221 & -8.895046 & -17.872623 \\
\hline $\mathrm{C}$ & -2.889284 & -3.518990 & -16.486858 \\
\hline $\mathrm{C}$ & -14.789265 & -11.565744 & -22.897308 \\
\hline $\mathrm{C}$ & -14.819465 & -7.425670 & -13.964311 \\
\hline $\mathrm{S}$ & -0.586799 & -1.921334 & -16.385091 \\
\hline $\mathrm{C}$ & 2.636147 & 1.519266 & -16.676236 \\
\hline $\mathrm{C}$ & -1.825681 & -0.207032 & -17.853206 \\
\hline $\mathrm{O}$ & -12.095833 & -7.461282 & -13.556754 \\
\hline $\mathrm{C}$ & -13.689460 & -10.917339 & -20.354153 \\
\hline $\mathrm{C}$ & -13.589692 & -10.831630 & 1083 \\
\hline $\mathrm{C}$ & -6.365956 & -6.502038 & -17.705945 \\
\hline $\mathrm{C}$ & -5.619681 & -9.105446 & -17.101649 \\
\hline $\mathrm{C}$ & -11.186290 & -9.917448 & -17.627051 \\
\hline $\mathrm{C}$ & -2.563809 & -4.439620 & -15.514879 \\
\hline $\mathrm{C}$ & -0.593469 & -0.410086 & -17.274378 \\
\hline $\mathrm{C}$ & -2.759601 & -1.239152 & -17.597431 \\
\hline $\mathrm{C}$ & -13.097361 & -10.519150 & -19.046735 \\
\hline $\mathrm{H}$ & 8.501506 & 6.602018 & -20.299187 \\
\hline $\mathrm{H}$ & 5.116606 & 9.714331 & -15.054369 \\
\hline $\mathrm{H}$ & 4.719112 & 1.192776 & -19.336400 \\
\hline $\mathrm{H}$ & 2.031556 & 2.286950 & -19.924127 \\
\hline $\mathrm{C}$ & -11.725150 & -10.655689 & -18.729726 \\
\hline $\mathrm{C}$ & -13.946428 & -9.860130 & -18.131282 \\
\hline $\mathrm{C}$ & -9.389290 & -11.346354 & -19.317099 \\
\hline $\mathrm{H}$ & 8.715936 & 11.430415 & -17.359321 \\
\hline $\mathrm{C}$ & -13.467143 & -9.295062 & -16.967251 \\
\hline $\mathrm{H}$ & 1.410826 & 0.238987 & -15.458636 \\
\hline $\mathrm{C}$ & -8.847174 & -10.354992 & -18.429175 \\
\hline $\mathrm{H}$ & 3.349067 & 1.706438 & -15.878350 \\
\hline $\mathrm{H}$ & -15.005371 & -9.769872 & -18.346998 \\
\hline $\mathrm{H}$ & -8.271392 & -9.112091 & -16.043419 \\
\hline $\mathrm{H}$ & 10.220599 & 7.693628 & -15.825425 \\
\hline $\mathrm{H}$ & -10.745515 & -14.329644 & -21.452266 \\
\hline $\mathrm{H}$ & 7.936256 & 6.837203 & -15.687988 \\
\hline $\mathrm{H}$ & 0.110797 & 0.804154 & -19.517140 \\
\hline $\mathrm{H}$ & -9.846430 & -8.142161 & -14.417545 \\
\hline $\mathrm{H}$ & -5.582111 & -10.606240 & -19.419771 \\
\hline $\mathrm{H}$ & -12.305081 & -12.893407 & -20.188982 \\
\hline
\end{tabular}




\begin{tabular}{|c|c|c|c|}
\hline $\mathrm{H}$ & -15.435347 & -11.908580 & -19.564404 \\
\hline $\mathrm{H}$ & -16.372090 & -12.493649 & -21.754443 \\
\hline $\mathrm{C}$ & -5.816329 & -14.491842 & -22.516572 \\
\hline $\mathrm{H}$ & 0.925256 & 10.501424 & -13.089299 \\
\hline $\mathrm{H}$ & -0.497763 & 9.946419 & -14.027586 \\
\hline $\mathrm{H}$ & 0.016630 & 9.036247 & -12.600264 \\
\hline $\mathrm{H}$ & 8.449619 & 0.934420 & -22.167438 \\
\hline $\mathrm{H}$ & 8.793066 & 2.433237 & -23.087939 \\
\hline $\mathrm{H}$ & 9.850643 & 1.970492 & -21.747498 \\
\hline $\mathrm{H}$ & -5.312220 & -13.834470 & -23.228329 \\
\hline $\mathrm{H}$ & -14.250864 & -6.995900 & -13.143554 \\
\hline $\mathrm{H}$ & -15.388833 & -6.653726 & -14.486091 \\
\hline $\mathrm{H}$ & -15.521410 & -8.175998 & -13.594369 \\
\hline $\mathrm{H}$ & -6.389517 & -15.253876 & -23.038713 \\
\hline $\mathrm{H}$ & 7.170860 & 7.858140 & -18.662184 \\
\hline $\mathrm{H}$ & 1.594026 & 3.961550 & -17.407899 \\
\hline $\mathrm{H}$ & -5.057059 & -14.950241 & -21.879594 \\
\hline $\mathrm{C}$ & -15.265772 & -11.931290 & -24.234574 \\
\hline $\mathrm{C}$ & -14.501453 & -12.038111 & -25.376890 \\
\hline $\mathrm{C}$ & -15.229742 & -12.405567 & -26.529472 \\
\hline $\mathrm{C}$ & -16.574803 & -12.595063 & -26.300577 \\
\hline $\mathrm{S}$ & -16.943360 & -12.310567 & -24.604618 \\
\hline $\mathrm{H}$ & -13.429975 & -11.879955 & -25.383939 \\
\hline $\mathrm{H}$ & -14.778035 & -12.528283 & -27.507372 \\
\hline $\mathrm{C}$ & -17.590847 & -12.973175 & -27.260133 \\
\hline $\mathrm{C}$ & -18.961639 & -13.010824 & -27.12 \\
\hline $\mathrm{C}$ & -19.636894 & -13.425681 & -28.310068 \\
\hline $\mathrm{C}$ & -18.782956 & -13.704403 & -29.338830 \\
\hline $\mathrm{S}$ & -17.126249 & -13.476729 & -28.880570 \\
\hline $\mathrm{H}$ & -19.471608 & -12.734404 & -26.211814 \\
\hline $\mathrm{H}$ & -20.714586 & -13.507354 & -28.390304 \\
\hline $\mathrm{C}$ & 11.043176 & 10.088055 & -16.806316 \\
\hline $\mathrm{C}$ & 12.213975 & 9.454434 & -16.449800 \\
\hline $\mathrm{C}$ & 13.386538 & 10.158255 & -16.805777 \\
\hline $\mathrm{C}$ & 13.139325 & 11.348257 & -17.451992 \\
\hline $\mathrm{S}$ & 11.412620 & 11.598582 & -17.622670 \\
\hline $\mathrm{H}$ & 12.239505 & 8.501847 & -15.938141 \\
\hline $\mathrm{H}$ & 14.387755 & 9.808455 & -16.582166 \\
\hline $\mathrm{C}$ & 14.095592 & 12.299548 & -17.972307 \\
\hline $\mathrm{C}$ & 13.929660 & 13.634723 & -18.262454 \\
\hline $\mathrm{C}$ & 15.109959 & 14.258654 & -18.749761 \\
\hline $\mathrm{C}$ & 16.170845 & 13.399446 & -18.833180 \\
\hline $\mathrm{S}$ & 15.742411 & 11.797992 & -18.325320 \\
\hline $\mathrm{H}$ & 12.991952 & 14.155616 & -18.104750 \\
\hline $\mathrm{H}$ & 15.168047 & 15.306800 & -19.017837 \\
\hline $\mathrm{H}$ & 17.179502 & 13.606874 & -19.161314 \\
\hline
\end{tabular}




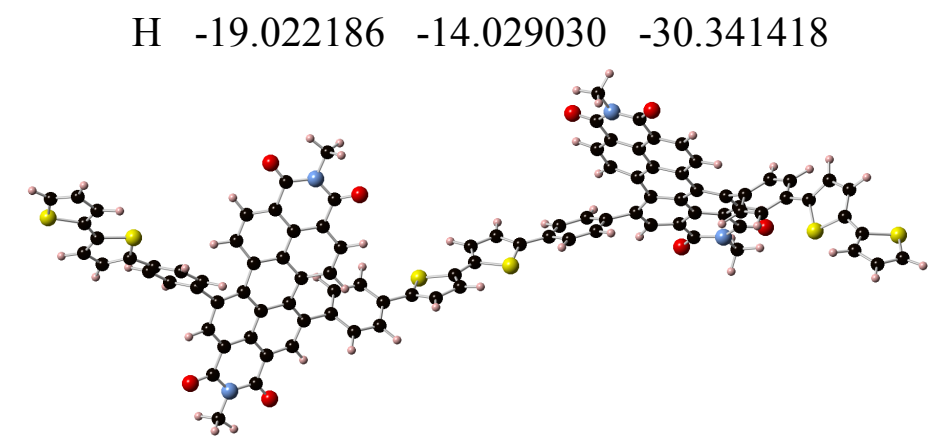

Cis-PBPB-Acyclic for Homodesmotic Calculations

Final Heat of Formation $=-7053.435400$

$\begin{array}{rrrr}\mathrm{H} & -1.834122 & -7.849178 & -15.386708 \\ \mathrm{H} & 0.585078 & -2.296806 & -20.657102 \\ \mathrm{H} & -1.203638 & -4.109005 & -20.081153 \\ \mathrm{H} & 0.032051 & -6.075792 & -15.801352 \\ \mathrm{H} & -6.716251 & -8.403724 & -19.252300 \\ \mathrm{H} & -5.384920 & -10.947647 & -16.057199 \\ \mathrm{H} & -3.303308 & -9.656397 & -15.896592 \\ \mathrm{H} & -4.649469 & -7.115692 & -19.100859 \\ \mathrm{H} & -13.650605 & -8.662737 & -23.571423 \\ \mathrm{H} & -11.832841 & -9.157944 & -21.991861 \\ \mathrm{C} & 5.184765 & 5.150000 & -17.279303 \\ \mathrm{C} & 6.384775 & 4.873091 & -18.091168 \\ \mathrm{C} & 6.476311 & 3.581346 & -18.723017 \\ \mathrm{C} & 4.054602 & 3.169999 & -18.298256 \\ \mathrm{C} & 7.450856 & 5.783429 & -18.257328 \\ \mathrm{C} & 8.761521 & 4.118088 & -19.470327 \\ \mathrm{C} & 5.368750 & 2.662793 & -18.733857 \\ \mathrm{C} & 5.121259 & 6.163265 & -16.319804 \\ \mathrm{C} & 7.852765 & 1.900306 & -19.891748 \\ \mathrm{C} & 4.017512 & 4.350963 & -17.494047 \\ \mathrm{C} & 6.825900 & 0.992858 & -19.766042 \\ \mathrm{C} & 0.295540 & 4.469725 & -16.608928 \\ \mathrm{C} & 8.612994 & 5.384702 & -18.954948 \\ \mathrm{C} & 5.591631 & 1.341826 & -19.181767 \\ \mathrm{~N} & 0.331967 & 5.533863 & -15.698172 \\ \mathrm{C} & 1.621575 & 3.008471 & -18.110790 \\ \mathrm{C} & 2.840697 & 2.562884 & -18.635836 \\ \mathrm{C} & 1.587250 & 4.055848 & -17.207503 \\ \mathrm{C} & 1.487878 & 6.236659 & -15.320929 \\ \mathrm{C} & 7.696681 & 3.199154 & -19.359493 \\ \mathrm{C} & 10.022948 & 3.754300 & -20.163558 \\ \mathrm{C} & -10.567710 & -6.646406 & -17.405416 \\ \mathrm{C} & -9.689138 & -6.001871 & -16.499859 \\ \mathrm{C} & -7.332012 & -11.930861 & -18.015977\end{array}$




$\begin{array}{crrr}\text { C } & 2.785555 & -1.927669 & -18.933712 \\ \mathrm{C} & 4.622606 & 0.215598 & -19.075930 \\ \mathrm{C} & 3.280766 & -1.325949 & -17.763988 \\ \mathrm{C} & -2.603969 & -7.453500 & -17.372487 \\ \mathrm{C} & -5.969983 & -8.682193 & -18.516351 \\ \mathrm{C} & 2.783230 & 4.740896 & -16.887640 \\ \mathrm{C} & 2.755707 & 5.819241 & -15.971028 \\ \mathrm{C} & -10.633270 & -12.872562 & -19.369603 \\ \mathrm{C} & 11.372758 & 2.096795 & -21.331978 \\ \mathrm{O} & 9.262822 & 0.352228 & -21.014658 \\ \mathrm{C} & 3.928096 & 6.484370 & -15.662178 \\ \mathrm{~N} & -9.282591 & -14.900990 & -19.103070 \\ \mathrm{C} & -10.503840 & -14.344090 & -19.509726 \\ \mathrm{C} & -0.017443 & -4.664935 & -18.354864 \\ \mathrm{~S} & -2.127713 & -6.403790 & -18.693989 \\ \mathrm{H} & 0.690218 & 2.522599 & -18.380282 \\ \mathrm{C} & 9.108570 & 1.480081 & -20.564831 \\ \mathrm{O} & 1.440999 & 7.146828 & -14.503609 \\ \mathrm{C} & 7.523600 & 7.203878 & -17.794913 \\ \mathrm{H} & 5.966205 & 7.995279 & -19.059764 \\ \mathrm{O} & -0.767899 & 3.925813 & -16.878061 \\ \mathrm{C} & -8.202585 & -14.173278 & -18.588426 \\ \mathrm{C} & -9.068398 & -8.598456 & -17.465697 \\ \mathrm{C} & -0.951677 & 5.923567 & -15.107074 \\ \mathrm{C} & 8.007721 & 9.914547 & -17.105107 \\ \mathrm{C} & -11.920401 & -10.845043 & -19.819211 \\ \mathrm{C} & 8.761562 & 8.872812 & -16.534668 \\ \mathrm{C} & -8.274651 & -7.953230 & -16.509242 \\ \mathrm{C} & -9.611009 & -12.093513 & -18.788301 \\ \mathrm{C} & -12.049287 & -4.602380 & -17.349511 \\ \mathrm{C} & 6.739220 & 8.233262 & -18.334905 \\ \mathrm{C} & -8.572387 & -6.674105 & -16.035693 \\ \mathrm{C} & -11.746339 & -12.244643 & -19.880366 \\ \mathrm{C} & 3.288590 & -1.489007 & -20.170759 \\ \mathrm{C} & -4.046741 & -9.372808 & -16.634185 \\ \mathrm{C} & 8.531747 & 7.550321 & -16.882241 \\ \mathrm{C} & 4.194679 & -0.439992 & -20.239009 \\ \mathrm{C} & -11.008126 & -10.057842 & -19.074383 \\ \mathrm{C} & 6.976653 & 9.564103 & -17.995954 \\ \mathrm{C} & -7.473431 & -10.542383 & -17.832568 \\ \mathrm{C} & -8.392041 & -12.711034 & -18.427853 \\ \mathrm{C} & -1.728623 & -7.306896 & -16.318620 \\ \mathrm{O} & -11.405422 & -15.039530 & -19.957630 \\ \mathrm{O} & 10.954524 & 4.535657 & -20.303349 \\ \mathrm{~N} & 10.117490 & 2.449379 & -20.661465 \\ \mathrm{O} & -13.046343 & -3.985674 & -17.703726\end{array}$




\begin{tabular}{|c|c|c|c|}
\hline & & & \\
\hline & & & \\
\hline & & & \\
\hline & -15.3 & & \\
\hline & & & \\
\hline & & & \\
\hline & -7.1 & -14 & \\
\hline & & & \\
\hline & & & \\
\hline & -15 . & & \\
\hline & -11 & & \\
\hline & & -4.1 & \\
\hline & & & \\
\hline & & & \\
\hline & & & \\
\hline & -13. & -10 & \\
\hline & -13 . & & \\
\hline 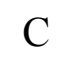 & & & \\
\hline & -5.2 & -10 & \\
\hline & -10 . & & \\
\hline $\mathrm{C}$ & & & \\
\hline Y & & & \\
\hline 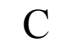 & & & \\
\hline & -12 & & \\
\hline $\mathrm{H}$ & & & \\
\hline & & & \\
\hline I & & & \\
\hline $\mathrm{H}$ & & & \\
\hline & -11 . & & \\
\hline & -12 . & & \\
\hline & -9.8 & 10 & \\
\hline$\pi$ & & & \\
\hline $\mathrm{C}$ & -11. & & \\
\hline $\mathrm{H}$ & & & \\
\hline $\mathrm{C}$ & -8. & -9 & \\
\hline $\mathrm{H}$ & & & \\
\hline & -13 . & & \\
\hline 11 & & -8 . & \\
\hline $\mathrm{H}$ & & & \\
\hline & -12. & & 71 \\
\hline 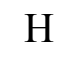 & & & \\
\hline 11 & 2.9 & -1.9 & -21 \\
\hline 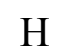 & -7.9 & -6.1 & \\
\hline & & -12 . & \\
\hline & -14. & -11 . & \\
\hline & -16.3 & -11.060093 & -21 \\
\hline
\end{tabular}




$\begin{array}{cccc}\mathrm{C} & -9.089729 & -16.348093 & -19.235164 \\ \mathrm{H} & -0.765619 & 6.752007 & -14.428143 \\ \mathrm{H} & -1.646841 & 6.219527 & -15.895619 \\ \mathrm{H} & -1.385030 & 5.076604 & -14.570894 \\ \mathrm{H} & 11.296563 & 1.060949 & -21.653227 \\ \mathrm{H} & 11.532335 & 2.754916 & -22.188746 \\ \mathrm{H} & 12.209300 & 2.225239 & -20.641995 \\ \mathrm{H} & -8.244844 & -16.549913 & -19.896938 \\ \mathrm{H} & -10.633168 & -2.325210 & -15.375494 \\ \mathrm{H} & -11.523095 & -1.989809 & -16.895629 \\ \mathrm{H} & -12.392353 & -2.640254 & -15.498787 \\ \mathrm{H} & -10.005731 & -16.767521 & -19.643907 \\ \mathrm{H} & 2.827812 & 1.722332 & -19.312284 \\ \mathrm{H} & -8.869843 & -16.782619 & -18.257759 \\ \mathrm{C} & -16.237349 & -9.626155 & -23.470185 \\ \mathrm{C} & -17.592301 & -9.650874 & -23.220557 \\ \mathrm{C} & -18.391200 & -9.538525 & -24.386580 \\ \mathrm{C} & -17.659044 & -9.412686 & -25.544526 \\ \mathrm{~S} & -15.942209 & -9.442812 & -25.187318 \\ \mathrm{H} & -18.002136 & -9.733576 & -22.220623 \\ \mathrm{H} & -19.475189 & -9.532744 & -24.381177 \\ \mathrm{C} & -18.134743 & -9.352472 & -26.917683 \\ \mathrm{C} & -17.747531 & -8.521885 & -27.942625 \\ \mathrm{C} & -18.428127 & -8.779503 & -29.166907 \\ \mathrm{C} & -19.312763 & -9.818528 & -29.076828 \\ \mathrm{~S} & -19.356947 & -10.482731 & -27.476143 \\ \mathrm{H} & -17.004663 & -7.743168 & -27.812392 \\ \mathrm{H} & -18.258937 & -8.219726 & -30.079514 \\ \mathrm{C} & 8.375129 & 11.296850 & -16.790046 \\ \mathrm{C} & 9.629450 & 11.746143 & -16.441798 \\ \mathrm{C} & 9.692895 & 13.134325 & -16.184319 \\ \mathrm{C} & 8.486360 & 13.781805 & -16.334189 \\ \mathrm{~S} & 7.238944 & 12.634305 & -16.802332 \\ \mathrm{H} & 10.495107 & 11.095526 & -16.407888 \\ \mathrm{H} & 10.605623 & 13.649539 & -15.908999 \\ \mathrm{C} & 8.194712 & 15.180950 & -16.108235 \\ \mathrm{C} & 7.046902 & 15.892950 & -16.376437 \\ \mathrm{C} & 7.106161 & 17.250093 & -15.952368 \\ \mathrm{C} & 8.291357 & 17.569772 & -15.351598 \\ \mathrm{~S} & 9.373735 & 16.214711 & -15.308595 \\ \mathrm{H} & 6.186834 & 15.455380 & -16.871055 \\ \mathrm{H} & -13.294520 & 17.956560 & -16.081422 \\ \mathrm{H} & -18518 & -8.437356 & -19.608203 \\ \mathrm{H} & -10.732670 & -16.079736 \\ \mathrm{H} & -10.213751 & -29.844783\end{array}$




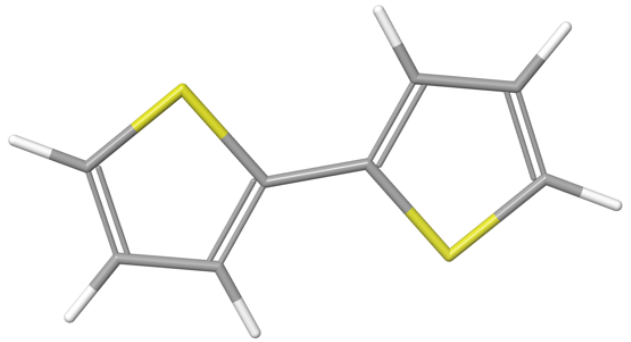

Bithiophene

Total Energy: -1104.826802 hartrees

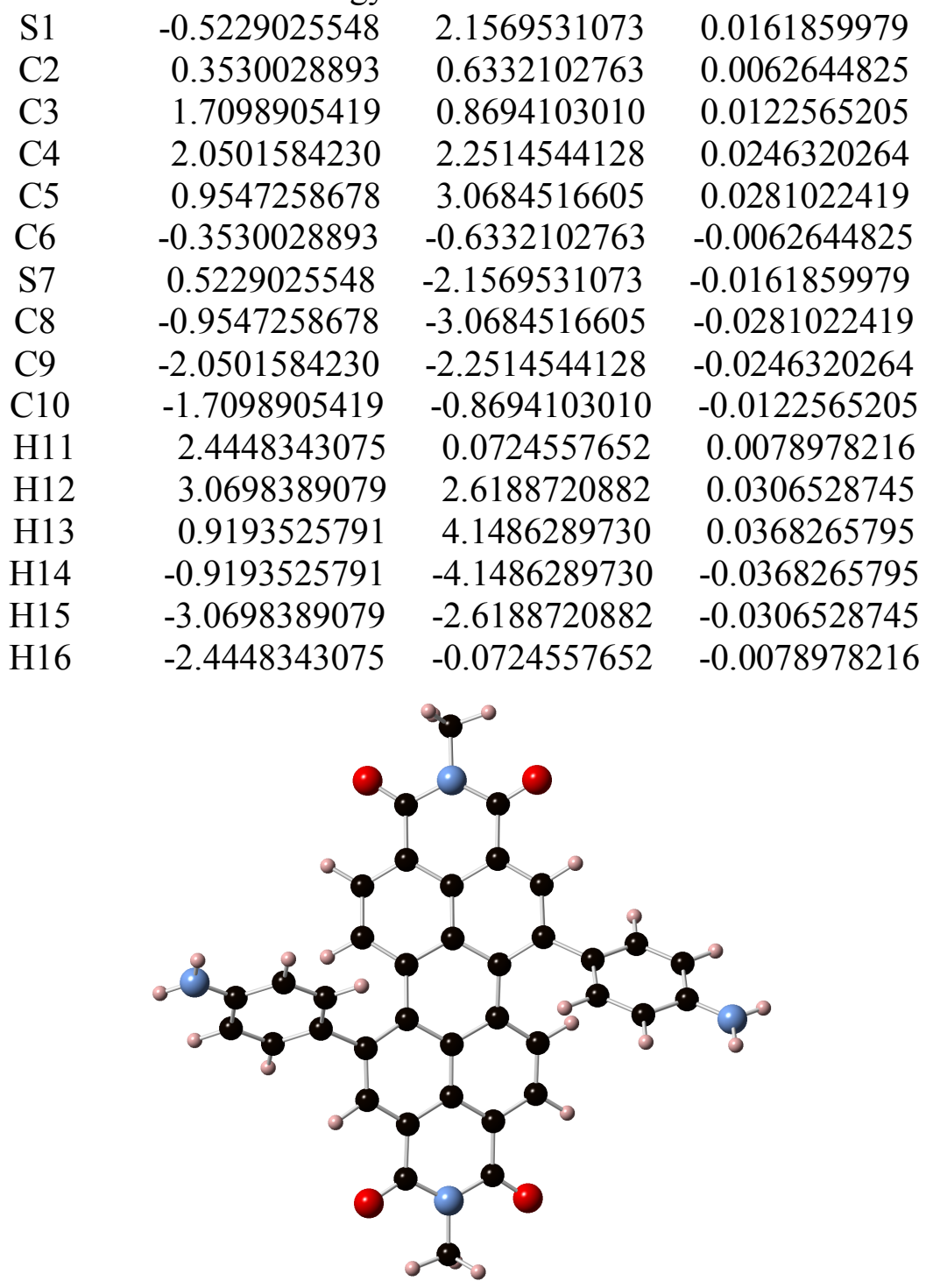

Trans-4.1

Final Heat of Formation $=-1982.587675$ $\begin{array}{llll}\text { C } & 5.032948 & 6.225155 & -17.363312\end{array}$ 


\begin{tabular}{|c|c|c|c|}
\hline & & & \\
\hline & & & \\
\hline & .237958 & 92861 & \\
\hline & & & \\
\hline & 855 & & \\
\hline & & & \\
\hline & & 17 & \\
\hline & 5.6 & 37 & \\
\hline & & & \\
\hline & 4.663 & 2 & \\
\hline & & & \\
\hline & 7.2 & & \\
\hline & & & \\
\hline & 1.2 & & \\
\hline & & & \\
\hline & & & \\
\hline & & & \\
\hline & & & \\
\hline & & & \\
\hline & & & \\
\hline & & 0 & \\
\hline & & & \\
\hline & & & \\
\hline & 2.8 & & \\
\hline & & & \\
\hline & 8.0 & & \\
\hline & & & \\
\hline & & & \\
\hline & & & \\
\hline & & & \\
\hline & 2.9 & & 19 \\
\hline & 7.002 & & \\
\hline & 6.6 & & \\
\hline & $-0.4 S$ & & \\
\hline & & & \\
\hline & 9.7 & & \\
\hline & & & \\
\hline & 7.39 & 9.0 & -16 \\
\hline & & -0.41 & \\
\hline $\mathrm{C}$ & 8.021 & 6.7 & -16 \\
\hline & & & \\
\hline . & 8.72 & 9.42 & \\
\hline & 8.562403 & 4.27 & 04581 \\
\hline 10 & 7.327963 & 2.39 & -22 . \\
\hline & 3.023314 & 1.298025 & -16.491150 \\
\hline & 8.045957 & 6.194203 & -20.94554 \\
\hline
\end{tabular}




$\begin{array}{ccrl}\mathrm{H} & 5.116518 & 8.863013 & -15.191477 \\ \mathrm{H} & 4.491123 & 0.626203 & -19.912058 \\ \mathrm{H} & 2.076760 & 0.529741 & -19.653740 \\ \mathrm{H} & 8.995080 & 10.475625 & -16.780836 \\ \mathrm{H} & 2.296104 & 0.346795 & -14.711873 \\ \mathrm{H} & 3.690640 & 1.948406 & -15.934685 \\ \mathrm{H} & 10.118396 & 6.351931 & -16.245429 \\ \mathrm{H} & 7.756851 & 5.701079 & -16.352040 \\ \mathrm{H} & 0.668303 & -1.068415 & -18.439677 \\ \mathrm{H} & 0.914920 & 9.696799 & -13.238721 \\ \mathrm{H} & -0.521409 & 9.181358 & -14.180010 \\ \mathrm{H} & -0.006954 & 8.229938 & -12.780725 \\ \mathrm{H} & 7.670499 & 0.745039 & -23.369271 \\ \mathrm{H} & 7.901176 & 2.327457 & -24.178676 \\ \mathrm{H} & 9.122932 & 1.742354 & -23.041207 \\ \mathrm{H} & 6.952601 & 7.272600 & -19.007703 \\ \mathrm{H} & 1.491733 & 3.260078 & -17.747457 \\ \mathrm{~N} & 11.063884 & 8.828903 & -16.469162 \\ \mathrm{~N} & 0.581945 & -1.376527 & -15.797400 \\ \mathrm{H} & 11.786475 & 8.140303 & -16.353334 \\ \mathrm{H} & 11.339296 & 9.792017 & -16.548012 \\ \mathrm{H} & 0.607789 & -1.430199 & -14.794383 \\ \mathrm{H} & -0.028594 & -2.007096 & -16.286587\end{array}$

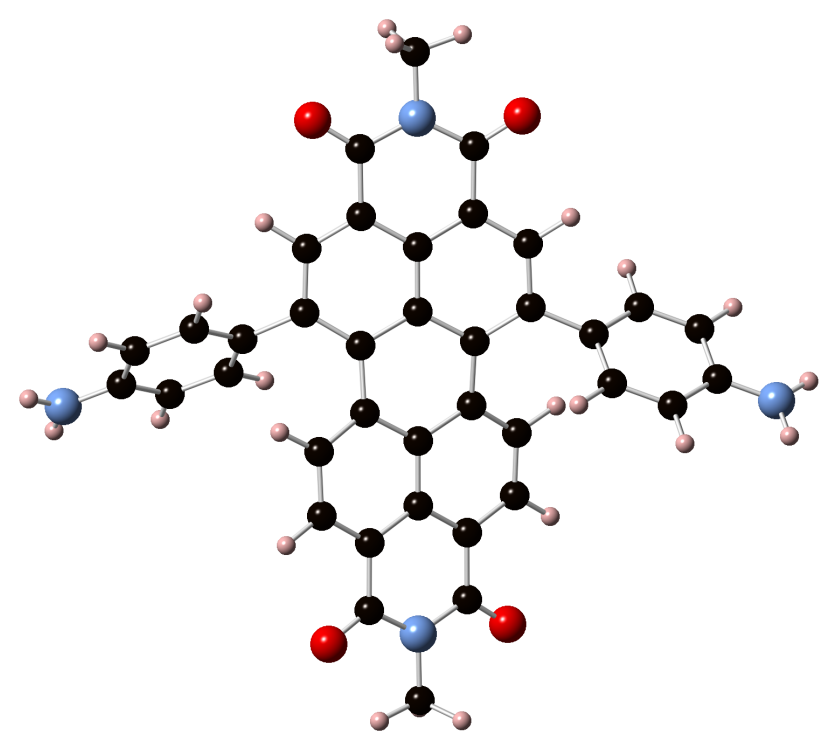

\section{Cis-4.1}

Final Heat of Formation $=-1982.586248$
C 4.546200
$3.491118-17.334244$
C $\quad 5.817619 \quad 3.993776 \quad-17.881884$
$\begin{array}{lllll}\text { C } & 6.692704 & 3.034674 & -18.502647\end{array}$
C $\quad 4.815958 \quad 1.416413 \quad-18.691877$ 


\begin{tabular}{|c|c|c|c|}
\hline & & & \\
\hline & & & \\
\hline & 258 & & \\
\hline & & & \\
\hline & & & \\
\hline & & & \\
\hline & & & \\
\hline & & & \\
\hline & & & \\
\hline & 7.2 & & \\
\hline & & & \\
\hline & 2.8 & & \\
\hline & & & \\
\hline & 2.1 & & \\
\hline & & & \\
\hline & 8.0 & & \\
\hline & & & \\
\hline & & & \\
\hline & 6.9 & & \\
\hline & & & \\
\hline & & & \\
\hline C & & & \\
\hline & & & \\
\hline & & & \\
\hline 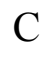 & & & \\
\hline & & & \\
\hline & & & \\
\hline & & & \\
\hline $\mathrm{C}$ & & & \\
\hline & & & \\
\hline & & 0 & \\
\hline & & & \\
\hline$\gamma$ & & & \\
\hline 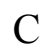 & & & \\
\hline G & & & \\
\hline 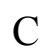 & & & \\
\hline 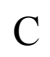 & & & \\
\hline 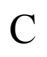 & 7.3 & -1. & 90 \\
\hline $\mathrm{C}$ & & & \\
\hline & 10.2 & & \\
\hline & 10.6 & & \\
\hline . & & -1.47 & \\
\hline 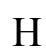 & & & \\
\hline & & & \\
\hline $\mathrm{H}$ & 9.257248 & 3554 & -19 \\
\hline & 7.736418 & -0.853774 & -21.39874 \\
\hline
\end{tabular}




$\begin{array}{crrr}\mathrm{H} & 2.382092 & 7.891319 & -18.534840 \\ \mathrm{H} & 5.998260 & -3.419006 & -17.538482 \\ \mathrm{H} & 6.218103 & -0.981002 & -17.383350 \\ \mathrm{H} & 5.167731 & 9.318406 & -15.575729 \\ \mathrm{H} & 6.602664 & 7.372679 & -15.998010 \\ \mathrm{H} & 7.502928 & -3.290673 & -21.572020 \\ \mathrm{H} & -1.705737 & 1.355121 & -15.790766 \\ \mathrm{H} & -1.938251 & 0.749297 & -17.462267 \\ \mathrm{H} & -1.323958 & -0.343571 & -16.215285 \\ \mathrm{H} & 12.589086 & 3.761450 & -20.157304 \\ \mathrm{H} & 12.026637 & 5.423638 & -20.519409 \\ \mathrm{H} & 12.533321 & 5.012851 & -18.874991 \\ \mathrm{H} & 4.719046 & -0.235670 & -20.063061 \\ \mathrm{H} & 4.226303 & 5.034943 & -15.871474 \\ \mathrm{~N} & 6.592868 & -4.907601 & -19.663347 \\ \mathrm{~N} & 2.849441 & 9.837752 & -16.780963 \\ \mathrm{H} & 6.855294 & -5.397410 & -20.500525 \\ \mathrm{H} & 6.239814 & -5.447817 & -18.893298 \\ \mathrm{H} & 3.105590 & 10.539386 & -16.108998 \\ \mathrm{H} & 1.984343 & 9.955241 & -17.278311\end{array}$

\section{Fifteen Lowest Roots for cis-PBPB}

Restricted Singlet Excited State 1:

$$
\begin{aligned}
& \text { Excitation energy }=0.0610906005 \text { hartrees } \quad 1.66235982 \mathrm{eV} \quad 745.83 \mathrm{~nm} \\
& \text { excitation X coeff. } \\
& 377=>379 \quad 0.33699 \\
& 378=>379-0.92552 \\
& 378=>380 \quad-0.11201 \\
& \mathrm{X}=-0.7055 \mathrm{Y}=3.6338 \mathrm{Z}=-1.2782 \mathrm{Tot}=3.9161
\end{aligned}
$$

Oscillator strength, $f=0.0967$

Restricted Singlet Excited State 2:

Excitation energy $=0.0625676576$ hartrees $\quad 1.70255259 \mathrm{eV} \quad 728.23 \mathrm{~nm}$ excitation X coeff. 


$$
\begin{aligned}
& 377=>379 \quad-0.90608 \\
& 377=>380 \quad-0.17843 \\
& 378=>379 \quad-0.35791
\end{aligned}
$$

Transition dipole moment (debye):

$$
\mathrm{X}=1.0060 \mathrm{Y}=1.8391 \quad \mathrm{Z}=-1.2889 \mathrm{Tot}=2.4608
$$

Oscillator strength, $\mathrm{f}=0.0391$

Restricted Singlet Excited State 3:

Excitation energy $=0.0638476678$ hartrees $\quad 1.73738344 \mathrm{eV} \quad 713.63 \mathrm{~nm}$

excitation X coeff.

$377=>379 \quad 0.11622$

$378=>380 \quad 0.98095$

Transition dipole moment (debye):

$$
\mathrm{X}=-1.6218 \mathrm{Y}=-1.3297 \mathrm{Z}=-3.3019 \mathrm{Tot}=3.9116
$$

\begin{tabular}{|c|c|}
\hline $377=>379$ & 0.18899 \\
\hline $377=>380$ & -0.97047 \\
\hline $78=>380$ & -0.11217 \\
\hline
\end{tabular}

Oscillator strength, $\mathrm{f}=\quad 0.1008$

Restricted Singlet Excited State 4:

Excitation energy $=0.0659836862$ hartrees $\quad 1.79550746 \mathrm{eV} \quad 690.52 \mathrm{~nm}$

excitation $\mathrm{X}$ coeff.

Transition dipole moment (debye):

$$
\mathrm{X}=-3.5429 \mathrm{Y}=0.4476 \quad \mathrm{Z}=1.2379 \text { Tot }=3.7795
$$

Oscillator strength, $\mathrm{f}=\quad 0.0973$

Restricted Singlet Excited State 5: 
Excitation energy $=0.0759310259$ hartrees $\quad 2.06618834 \mathrm{eV} \quad 600.06 \mathrm{~nm}$ excitation $\mathrm{X}$ coeff.

$376=>379 \quad-0.99402$

Transition dipole moment (debye):

$\mathrm{X}=-0.5525 \mathrm{Y}=-0.2414 \mathrm{Z}=-5.4369 \mathrm{Tot}=5.4702$

Oscillator strength, $\mathrm{f}=\quad 0.2345$

Restricted Singlet Excited State 6:

Excitation energy $=0.0777532387$ hartrees $\quad 2.11577327 \mathrm{eV} \quad 586.00 \mathrm{~nm}$

excitation $\mathrm{X}$ coeff.

$376=>380-0.98776$

Transition dipole moment (debye):

$\mathrm{X}=-0.2331 \mathrm{Y}=0.6236 \quad \mathrm{Z}=-3.2380$ Tot $=3.3057$

Oscillator strength, $\mathrm{f}=\quad 0.0877$

Restricted Singlet Excited State 7:

Excitation energy $=0.0839091082$ hartrees $\quad 2.28328301 \mathrm{eV} \quad 543.01 \mathrm{~nm}$

excitation X coeff.

$375=>379-0.99157$

Transition dipole moment (debye):

$$
\mathrm{X}=-0.2253 \mathrm{Y}=-2.1072 \mathrm{Z}=-2.3713 \mathrm{Tot}=3.1803
$$

Oscillator strength, $\mathrm{f}=0.0876$

Restricted Singlet Excited State 8: 


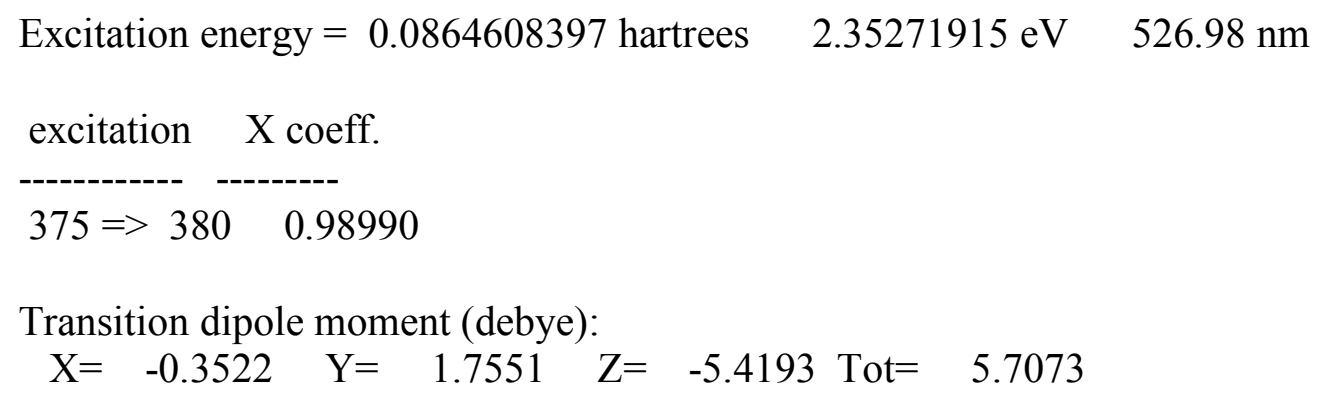

Transition dipole moment (debye):

$$
\mathrm{X}=-0.3522 \mathrm{Y}=1.7551 \quad \mathrm{Z}=-5.4193 \mathrm{Tot}=5.7073
$$

Oscillator strength, $\mathrm{f}=0.2906$

Restricted Singlet Excited State 9:

Excitation energy $=0.0948538603$ hartrees $\quad 2.58110486 \mathrm{eV} \quad 480.35 \mathrm{~nm}$

excitation X coeff.

$\begin{array}{rr}372=>379 & 0.16804 \\ 374=>379 & -0.85580 \\ 374=>380 & 0.25715 \\ 377=>379 & 0.11098 \\ 377=>382 & 0.15426 \\ 378=>381 & -0.33998\end{array}$

Transition dipole moment (debye):

$$
\mathrm{X}=2.2358 \mathrm{Y}=-0.6118 \mathrm{Z}=-0.2839 \mathrm{Tot}=2.3353
$$

Oscillator strength, $\mathrm{f}=0.0534$

Restricted Singlet Excited State 10:

$$
\begin{aligned}
& \text { Excitation energy }=0.0963328717 \text { hartrees } \quad 2.62135081 \mathrm{eV} \quad 472.98 \mathrm{~nm} \\
& \begin{array}{ll}
\text { excitation } & \text { X coeff. } \\
-372=>380 & -0.14821 \\
374=>379 & -0.44565 \\
374=>380 & -0.58636 \\
377=>382 & -0.18366 \\
378=>381 & 0.60161
\end{array}
\end{aligned}
$$

Transition dipole moment (debye): 


$$
\mathrm{X}=1.8757 \mathrm{Y}=0.9499 \mathrm{Z}=-0.1500 \mathrm{Tot}=2.1079
$$

Oscillator strength, $\mathrm{f}=\quad 0.0442$

Restricted Singlet Excited State 11:

Excitation energy $=0.0988396557$ hartrees $\quad 2.68956388 \mathrm{eV} \quad 460.98 \mathrm{~nm}$ excitation $\mathrm{X}$ coeff.

$\begin{array}{rl}374=>380 & 0.71487 \\ 375=>381 & 0.10446 \\ 376=>383 & -0.15476 \\ 378=>381 & 0.63986\end{array}$

Transition dipole moment (debye):

$$
\mathrm{X}=-1.4208 \mathrm{Y}=1.2562 \mathrm{Z}=0.2145 \mathrm{Tot}=1.9086
$$

Oscillator strength, $\mathrm{f}=\quad 0.0372$

Restricted Singlet Excited State 12:

Excitation energy $=0.1056867332$ hartrees $\quad 2.87588233 \mathrm{eV} \quad 431.12 \mathrm{~nm}$ excitation X coeff.

$377=>381 \quad 0.97270$

Transition dipole moment (debye):

$$
\mathrm{X}=-0.7305 \mathrm{Y}=-10.9434 \mathrm{Z}=-0.5560 \text { Tot }=10.9818
$$

Oscillator strength, $\mathrm{f}=\quad 1.3153$

Restricted Singlet Excited State 13:

Excitation energy $=0.1097775026$ hartrees $\quad 2.98719783 \mathrm{eV} \quad 415.05 \mathrm{~nm}$ excitation X coeff.

$373=>379-0.11909$ 


$$
378=>382 \quad-0.97130
$$

Transition dipole moment (debye):

$$
\mathrm{X}=0.0965 \quad \mathrm{Y}=-7.8524 \quad \mathrm{Z}=-0.3643 \quad \mathrm{Tot}=7.8615
$$

Oscillator strength, $\mathrm{f}=0.7001$

Restricted Singlet Excited State 14:

Excitation energy $=0.1099973014$ hartrees $\quad 2.99317886 \mathrm{eV} \quad 414.22 \mathrm{~nm}$

\begin{tabular}{|c|c|c|}
\hline $372=>?$ & 379 & 0.11890 \\
\hline $374=>$ & 379 & 0.13804 \\
\hline $375 \Rightarrow$ & 383 & -0.17290 \\
\hline $376=>$ & 381 & 0.73838 \\
\hline $376=>$ & 386 & 0.15620 \\
\hline $377=>$ & 382 & 0.12515 \\
\hline $378=>$ & 383 & -0.54733 \\
\hline
\end{tabular}

excitation $\mathrm{X}$ coeff.

Transition dipole moment (debye):

$$
\mathrm{X}=-4.6623 \quad \mathrm{Y}=-0.0028 \quad \mathrm{Z}=0.0219 \mathrm{Tot}=4.6624
$$

Oscillator strength, $\mathrm{f}=\quad 0.2467$

Restricted Singlet Excited State 15:

Excitation energy $=0.1117001572$ hartrees $\quad 3.03951593 \mathrm{eV} \quad 407.91 \mathrm{~nm}$

$$
\begin{array}{rr}
\text { excitation } & \multicolumn{1}{c}{\text { X coeff. }} \\
\hdashline------- & ----- \\
368=>379 & 0.11729 \\
373=>379 & 0.94633 \\
376=>382 & 0.12027 \\
378=>382 & -0.11004
\end{array}
$$

Transition dipole moment (debye):

$$
\mathrm{X}=0.4579 \quad \mathrm{Y}=-1.1293 \quad \mathrm{Z}=-1.7251 \text { Tot }=2.1121
$$

Oscillator strength, $f=0.0514$ 


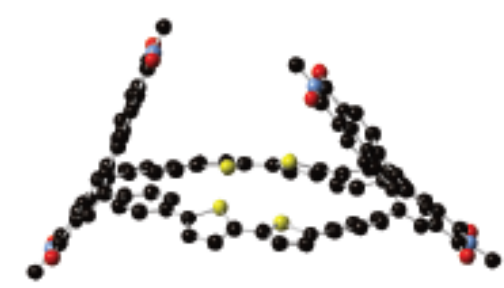

cis-PBPB

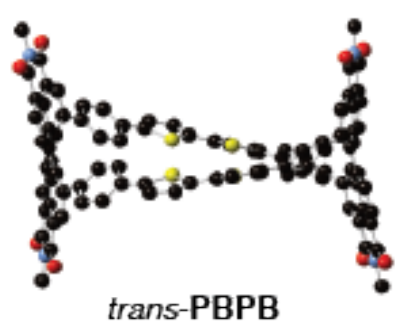

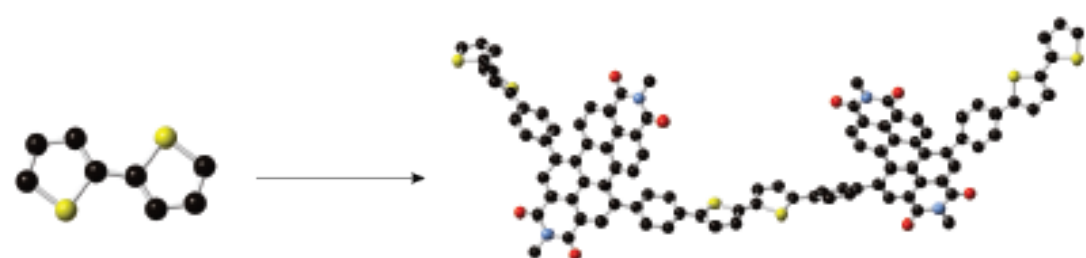

bithiophene

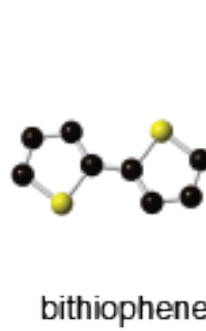

cis-PBPB-Acyclic

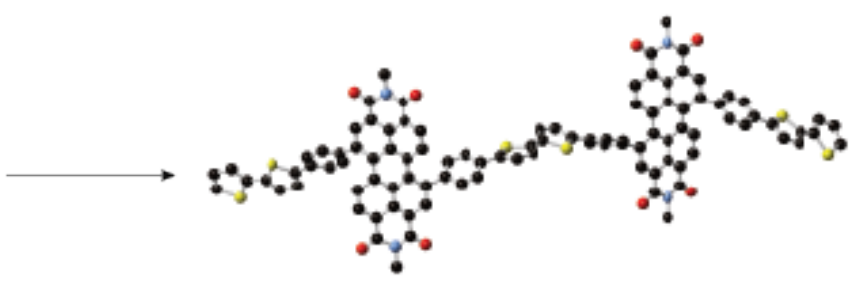

trans-PBPB-Acyclic

Table 4.3. Homodesmotic Calculations for Strain

\begin{tabular}{|c|c|c|c|}
\hline Compound & $\begin{array}{c}\text { Total energy } \\
\text { (hartree) }\end{array}$ & $\begin{array}{c}\text { Strain energy } \\
\text { (hartree) }\end{array}$ & $\begin{array}{c}\text { Strain energy } \\
\text { (kcal/mol) }\end{array}$ \\
\hline trans-PBPB & -5948.58245 & 0.033 & 21 \\
\hline cis-PBPB & -5948.57180 & 0.037 & 23 \\
\hline Bithiophene & -1104.82680 & - & - \\
\hline Trans-PBPB-Acyclic & -7053.44201 & - & \\
\hline Cis-PBPB-Acyclic & -7053.43540 & \multicolumn{2}{|}{} \\
\hline
\end{tabular}

Table 4.3: Table of energies for the Homodesmotic Reaction (enthalpy)

\subsection{References}

1. Brédas, J. L. et al. Organic semiconductors: A theoretical characterization of the basic parameters governing charge transport. Proc. Natl. Acad. Sci. 99, 5804 LP - 5809 (2002).

2. Bendikov, M. et al. Tetrathiafulvalenes, Oligoacenenes, and Their Buckminsterfullerene Derivatives: The Brick and Mortar of Organic Electronics. Chem. Rev. 104, 4891-4946 (2004).

3. Newman, C. R. et al. Introduction to Organic Thin Film Transistors and Design of nChannel Organic Semiconductors. Chem. Mater. 16, 4436-4451 (2004).

4. Anthony, J. E. Functionalized Acenes and Heteroacenes for Organic Electronics. Chem. Rev. 106, 5028-5048 (2006).

5. Murphy, A. R. et al. Organic Semiconducting Oligomers for Use in Thin Film Transistors. Chem. Rev. 107, 1066-1096 (2007).

6. Onwubiko, A. et al. Fused electron deficient semiconducting polymers for air stable electron transport. Nat. Commun. 9, 416 (2018).

7. Lin, Y. et al. Stacked pentacene layer organic thin-film transistors with improved characteristics. IEEE Electron Device Lett. 18, 606-608 (1997). 
8. Gundlach, D. J. et al. Thin-film transistors based on well-ordered thermally evaporated naphthacene films. Appl. Phys. Lett. 80, 2925-2927 (2002).

9. Coropceanu, V. et al. Charge Transport in Organic Semiconductors. Chem. Rev. 107, 926952 (2007).

10. Kroto, H. W. et al. C60: Buckminsterfullerene. Nature 318, 162-163 (1985).

11. Haddon, R. C. et al. C60 thin film transistors. Appl. Phys. Lett. 67, 121-123 (1995).

12. Li, H. et al. High-Mobility Field-Effect Transistors from Large-Area Solution-Grown Aligned C60 Single Crystals. J. Am. Chem. Soc. 134, 2760-2765 (2012).

13. Zhang, Y. et al. Development of fullerenes and their derivatives as semiconductors in fieldeffect transistors: exploring the molecular design. J. Mater. Chem. C 6, 3514-3537 (2018).

14. Dou, L. et al. 25th Anniversary Article: A Decade of Organic/Polymeric Photovoltaic Research. Adv. Mater. 25, 6642-6671 (2013).

15. Savoie, B. M. et al. Mesoscopic Features of Charge Generation in Organic Semiconductors. Acc. Chem. Res. 47, 3385-3394 (2014).

16. Savoie, B. M. et al. Unequal Partnership: Asymmetric Roles of Polymeric Donor and Fullerene Acceptor in Generating Free Charge. J. Am. Chem. Soc. 136, 2876-2884 (2014).

17. Gong, X. et al. High-Detectivity Polymer Photodetectors with Spectral Response from 300 $\mathrm{nm}$ to $1450 \mathrm{~nm}$. Science. 325, 1665-1667 (2009).

18. Nielsen, C. B. et al. Non-Fullerene Electron Acceptors for Use in Organic Solar Cells. Acc. Chem. Res. 48, 2803-2812 (2015).

19. Ball, M. et al. Contorted Polycyclic Aromatics. Acc. Chem. Res. 48, 267-276 (2015).

20. Yan, C. et al. Non-fullerene acceptors for organic solar cells. Nat. Rev. Mater. 3, 18003 (2018).

21. Zhang, J. et al. Material insights and challenges for non-fullerene organic solar cells based on small molecular acceptors. Nat. Energy 3, 720-731 (2018).

22. Hou, J. et al. Organic solar cells based on non-fullerene acceptors. Nat. Mater. 17, 119 (2018).

23. Ball, M. et al. Conjugated Macrocycles in Organic Electronics. Acc. Chem. Res. (2019). doi:10.1021/acs.accounts.9b00017

24. Mandoc, M. M. et al. Trap-limited electron transport in disordered semiconducting polymers. Phys. Rev. B 75, 193202 (2007).

25. Kaake, L. G. et al. Intrinsic Charge Trapping in Organic and Polymeric Semiconductors: A Physical Chemistry Perspective. J. Phys. Chem. Lett. 1, 628-635 (2010).

26. Arias, A. C. et al. Materials and Applications for Large Area Electronics: Solution-Based Approaches. Chem. Rev. 110, 3-24 (2010).

27. Nicolai, H. T. et al. Unification of trap-limited electron transport in semiconducting polymers. Nat. Mater. 11, 882-887 (2012).

28. Iwamoto, T. et al. Selective and Random Syntheses of n Cycloparaphenylenes $(n=8-13)$ and 
Size Dependence of Their Electronic Properties. J. Am. Chem. Soc. 133, 8354-8361 (2011).

29. Ball, M. et al. Macrocyclization in the Design of Organic n-Type Electronic Materials. $J$. Am. Chem. Soc. 138, 12861-12867 (2016).

30. Iyoda, M. et al. Conjugated Macrocycles: Concepts and Applications. Angew. Chem. Int. Ed. 50, 10522-10553 (2011).

31. Iwamoto, T. et al. Size- and Orientation-Selective Encapsulation of C70 by Cycloparaphenylenes. Chem. Eur. J. 19, 14061-14068 (2013).

32. Nakanishi, Y. et al. Size-Selective Complexation and Extraction of Endohedral Metallofullerenes with Cycloparaphenylene. Angew. Chem. Int. Ed. 53, 3102-3106 (2014).

33. Zhang, B. et al. Hollow organic capsules assemble into cellular semiconductors. Nat. Commun. 9, 1957 (2018).

34. Huang, Q. et al. Photoconductive Curved-Nanographene/Fullerene Supramolecular Heterojunctions. Angew. Chem. Int. Ed. 58, 6244 (2019).

35. Nakamura, T. et al. Double-Circularly Connected Saloph-Belt Macrocycles Generated from a Bis-Armed Bifunctional Monomer. J. Am. Chem. Soc. 141, 6462-6467 (2019).

36. Darzi, E. R. et al. Synthesis, Properties, and Design Principles of Donor-Acceptor Nanohoops. ACS Cent. Sci. 1, 335-342 (2015).

37. Zhang, B. et al. Rigid, Conjugated Macrocycles for High Performance Organic Photodetectors. J. Am. Chem. Soc. 138, 16426-16431 (2016).

38. Van Raden, J. M. et al. Synthesis and characterization of a highly strained donor-acceptor nanohoop. Org. Biomol. Chem. 14, 5721 (2016).

39. Kayahara, E. et al. Gram-Scale Syntheses and Conductivities of $\{[\} 10]$ Cycloparaphenylene and Its Tetraalkoxy Derivatives. J. Am. Chem. Soc. 139, 18480-18483 (2017).

40. Nishigaki, S. et al. Synthesis, Structures, and Photophysical Properties of Alternating Donor-Acceptor Cycloparaphenylenes. Chem. Eur. J. 23, 7227-7231 (2017).

41. Ball, M. L. et al. Influence of Molecular Conformation on Electron Transport in Giant, Conjugated Macrocycles. J. Am. Chem. Soc. 140, 10135-10139 (2018).

42. Ball, M. et al. Chiral Conjugated Corrals. J. Am. Chem. Soc. 137, 9982-9987 (2015).

43. Zaumseil, J. et al. Electron and Ambipolar Transport in Organic Field-Effect Transistors. Chem. Rev. 107, 1296-1323 (2007).

44. Dimitrakopolous, C.D. et al. Organic Thin Film Transistors for Large Area Electronics. Adv. Mater. 14, 99-117 (2002).

45. Anthony, J. E. et al. n-Type Organic Semiconductors in Organic Electronics. Adv. Mater. 22, 3876-3892 (2010).

46. Chen, Z. et al. Effect of Core Twisting on Self-Assembly and Optical Properties of Perylene Bisimide Dyes in Solution and Columnar Liquid Crystalline Phases. Chem. Eur. J. 13, 450465 (2007).

47. Kaiser, T. E. et al. Fluorescent J-Aggregates of Core-Substituted Perylene Bisimides: 
Studies on Structure-Property Relationship, Nucleation-Elongation Mechanism, and Sergeants-and-Soldiers Principle. J. Am. Chem. Soc. 131, 6719-6732 (2009).

48. Würthner, F. et al. Perylene Bisimide Dye Assemblies as Archetype Functional Supramolecular Materials. Chem. Rev. 116, 962-1052 (2016).

49. George, P. et al. An alternative approach to the problem of assessing stabilization energies in cyclic conjugated hydrocarbons. Theor. Chim. Acta 38, 121-129 (1975).

50. Bachrach, S. M. et al. DFT Study of Cycloparaphenylenes and Heteroatom-Substituted Nanohoops. J. Org. Chem. 75, 6595-6604 (2010).

51. Segawa, Y. et al. Theoretical Studies on the Structures and Strain Energies of Cycloparaphenylenes. Org. Lett. 12, 2262-2265 (2010).

52. You, J. B. et al. A polymer tandem solar cell with $10.6 \%$ power conversion efficiency. Nat. Commun. 4, 10 (2013).

53. Ahrens, M. J. et al. Bis(n-octylamino)perylene-3,4:9,10-bis(dicarboximide)s and Their Radical Cations: Synthesis, Electrochemistry, and ENDOR Spectroscopy. J. Org. Chem. 71, 2107-2114 (2006).

54. Goretzki, G. et al. Building Multistate Redox-Active Architectures Using Metal-Complex Functionalized Perylene Bis-imides. Inorg. Chem. 48, 10264-10274 (2009).

55. Dubey, R. K. et al. 1,7- And 1,6-Regioisomers of Diphenoxy and Dipyrrolidinyl Substituted Perylene Diimides: Synthesis, Separation, Characterization, and Comparison of Electrochemical and Optical Properties. Chem. Mater. 23, 778-788 (2011).

56. Dubey, R. K. et al. Direct Evidence of Significantly Different Chemical Behavior and Excited-State Dynamics of 1,7- and 1,6-Regioisomers of Pyrrolidinyl-Substituted Perylene Diimide. Chem. Eur. J. 19, 6791-6806 (2013).

57. $\mathrm{Xu}, \mathrm{B}$. et al. Measurement of Single-Molecule Resistance by Repeated Formation of Molecular Junctions. Science. 301, 1221-1223 (2003).

58. Venkataraman, L. et al. Dependence of single-molecule junction conductance on molecular conformation. Nature 442, 904 (2006).

59. Hybertsen, M. S. et al. Amine-linked single-molecule circuits: systematic trends across molecular families. J. Phys. Condens. Matter 20, 374115 (2008).

60. Su, T. A. et al. Chemical principles of single-molecule electronics. Nat. Rev. Mater. 1, 16002 (2016).

61. Rajasingh, P. et al. Selective Bromination of Perylene Diimides under Mild Conditions. $J$. Org. Chem. 72, 5973-5979 (2007).

62. Blanc, E. et al. The evaluation of transmission factors and their first derivatives with respect to crystal shape parameters. J. Appl. Crystallogr. 24, 1035-1041 (1991).

63. Clark, R. C. et al. The analytical calculation of absorption in multifaceted crystals. Acta Crystallogr. Sect. A 51, 887-897 (1995).

64. Oxford Diffraction/Agilent Technologies UK Ltd. Version 1.171.37.35. (2014).

65. Sheldrick, G. M. Crystal structure refinement with $\{$ it SHELXL\}. Acta Crystallogr. Sect. 
C 71, 3-8 (2015).

66. Dolomanov, O. V et al. \{lit OLEX2\}: A complete structure solution, refinement and analysis program. J. Appl. Crystallogr. 42, 339-341 (2009).

67. Spek, A. L. Structure validation in chemical crystallography. Acta Crystallogr. Sect. D 65 , 148-155 (2009).

68. van der Sluis, P et al. BYPASS: an effective method for the refinement of crystal structures containing disordered solvent regions. Acta Crystallogr. Sect. A 46, 194-201 (1990).

69. Crystalmaker Software Ltd. 


\section{Chapter 5. Rigid, Conjugated Macrocycles for High Performance Organic Photodetectors}

\subsection{Preface}

Chapter 5 is reproduced with permission from the authors: Boyuan Zhang, M. Tuan Trinh, Brandon Fowler, Melissa Ball, Qizhi Xu, Fay Ng, Michael L. Steigerwald, X.-Y. Zhu, Colin Nuckolls, and Yu Zhong published in the Journal of the American Chemical Society. ${ }^{1}$ Copyright 2016 American Chemical Society. Brandon Fowler and I synthesized all the compounds. I performed DFT calculations, with essential input from Michael L. Steigerwald. Boyuan Zhang and $\mathrm{Yu}$ Zhong fabricated and characterized all the devices with assistance from Qizhi Xu. M. Tuan Trinh conducted TA measurements and data analysis.

\subsection{Introduction}

Chapter 5 describes a new molecular design for creating OPDs with unparalleled ability to detect photons. The use of organic materials as the active component in photodetectors is attractive because of the potential ease of their fabrication as light-weight and mechanically flexible devices. ${ }^{2-5}$ One critical parameter that limits OPDs performance is high dark current, because it determines the noise current level and sensitivity of an OPD. The current state-of-the-art for OPDs employs a number of modifications such as, carrier blocking layers, ${ }^{6}$ vertical phase separation ${ }^{7}$ and thick active layers, ${ }^{8}$ to lower the dark current. An alternative approach is to use reaction chemistry to build the desired properties into the structure of the active molecule to minimize the charge carriers in the active layers.

The dark current in organic, electronic materials is multi-faceted, and its origins are not completely understood. Covalent defects formed upon photo/thermo-activation ${ }^{9-11}$ and mechanically deformed $s p^{2}$ carbon-carbon bonds in $\pi$-conjugated molecules are known to produce charged defects that introduce carriers. ${ }^{12-14}$ Fullerenes, which are one of the most ubiquitous organic electronic materials, undergo a facile dimerization when irradiated. ${ }^{9,10}$ This also 
contributes to the dark current. ${ }^{12}$ To address these issues, we designed a macrocycle that consists of PDI and biphenyl moieties wrapped into a tetrameric structure and call this $\left(\mathbf{P P h}_{2}\right)_{4}($ Figure 1B. 1 and 5.1). ${ }^{15}$
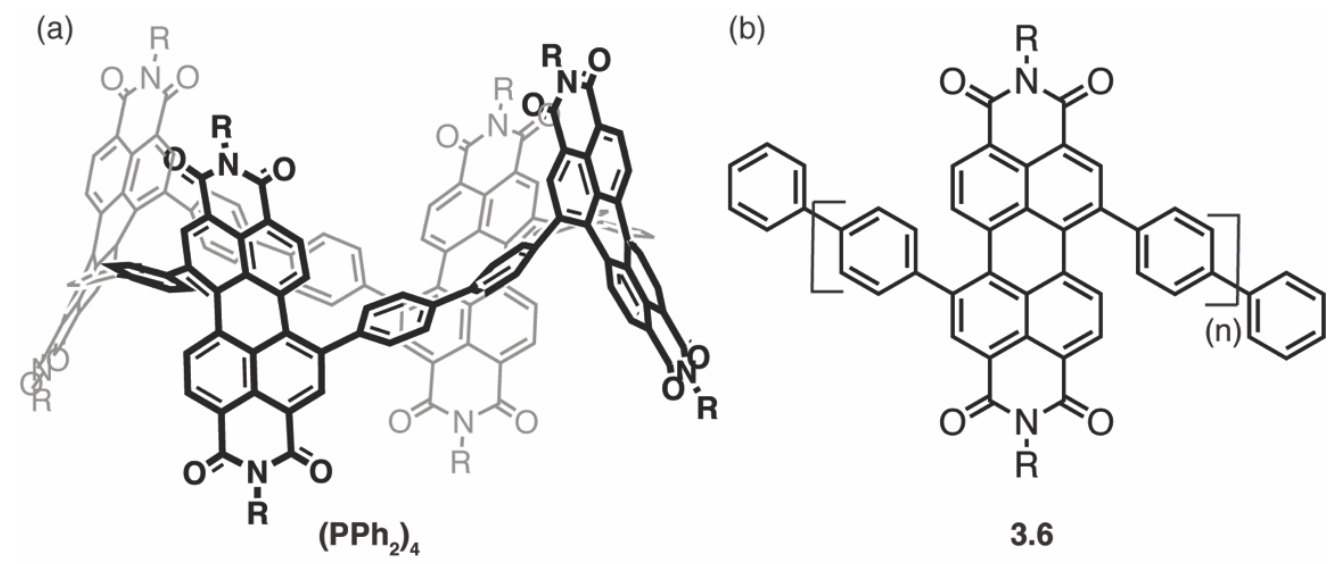

Figure 5.1. (a) Molecular structure of $\left(\mathbf{P P h}_{2}\right)_{4}$. (b) Energy minimized structures from DFT for $(\mathbf{P P h})_{4}$. Carbon $=$ gray, nitrogen $=$ blue, oxygen $=$ red. Hydrogen atoms have been removed to clarify the view. A methyl group substitutes the sidechains in the calculations. The methyl group, too, has been removed to clarify the view in the structures presented here.

We find $\left(\mathbf{P P h}_{2}\right)_{4}$-based OPDs have high sensitivity for visible light detection in a simple device structure. The macrocycle contains several key design elements to yield the highly sensitive OPD result: (1) the rigid structure, constrained in a ring, minimizes the number of charged defects originating from deformed $s p^{2}$ carbons; (2) no covalent defects are formed upon photo/thermoactivation; (3) it is efficacious at transporting electrons; (4) and it has high visible light absorption that yields significant photocurrent in a bulk heterojunction photodiode. ${ }^{15}$ Using this design we are able to suppress the dark current density while retaining high responsivity in an ultra-sensitive non-fullerene OPD. Without the need for extra carrier blocking layers, the highest detectivity in our device approaches $10^{14}$ Jones at near zero bias voltage. This detectivity is comparable to the best fullerene-based photodetectors, and the sensitivity at low working voltages $(<0.1 \mathrm{~V})$ is a record for non-fullerene OPDs. A direct comparison between $(\mathbf{P P h})_{4}$ and an acyclic, polymeric 
control molecule (Compound 3.6) reveals that $\left(\mathbf{P P h}_{2}\right)_{4}$ 's superior performance originates from its rigid, conjugated, and macrocyclic structure (Figure 5.1).

\subsection{OPD Results for $\left(\mathrm{PPh}_{2}\right)_{4}$}

Chapter 3 described $\left(\mathbf{P P h}_{2}\right)_{4}$ as an n-type semiconductor that transports electrons in both field effect transistors and solar cells. (PPh $)_{4}$ 's $\sim 2 \mathrm{~nm}$ diameter interior cavity is large enough to thread donor polymers (Figure 1B.4); its branched sidechains impart high solubility in common organic solvents. In solution, the lowest-energy absorption peak at $585 \mathrm{~nm}$ has an extinction coefficient of $7.9 \times 10^{4} \mathrm{M}^{-1} \mathrm{~cm}^{-1}$ (Figure 1B.6). The active layer of the photodetector is a blended film of $\left(\mathbf{P P h}_{2}\right)_{4}$ and the commercially available polymer donor PTB7-Th. ${ }^{16,17}$ The absorption of PTB7-Th is shifted to lower energy relative to $\left(\mathbf{P P h}_{2}\right)_{4}$ (Figure 5.2a). Figure 5.2b shows a model of a simple device structure that was used in this study. Figure 5.2c contains the energy diagram of the materials used in this device. We note the energy offset between the donor's LUMO and the acceptor's LUMO is well-matched (Figure 5.2c); energy levels were estimated from CV. ${ }^{15,16}$ The thickness of the active film is $\sim 150 \mathrm{~nm}$.

Figure 5.2d displays the current-voltage curves for a typical OPD under dark conditions and simulated AM 1.5G irradiation $\left(100 \mathrm{mWcm}^{-2}\right)$. One of the exciting findings is that the OPD 

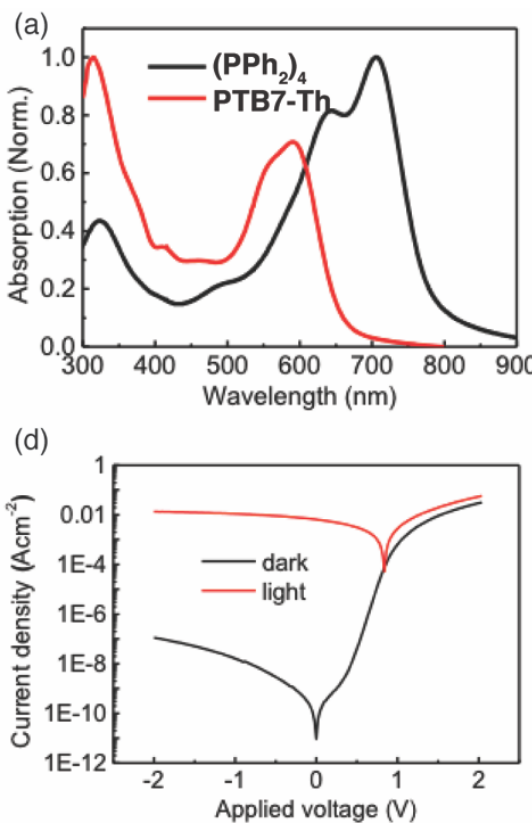

(b)

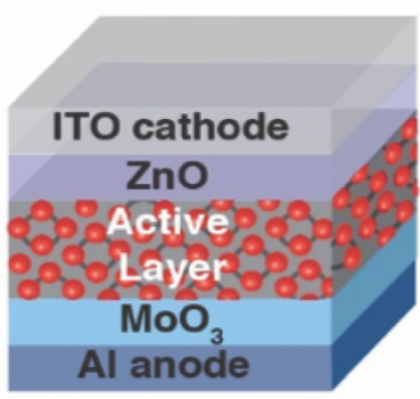

(e)

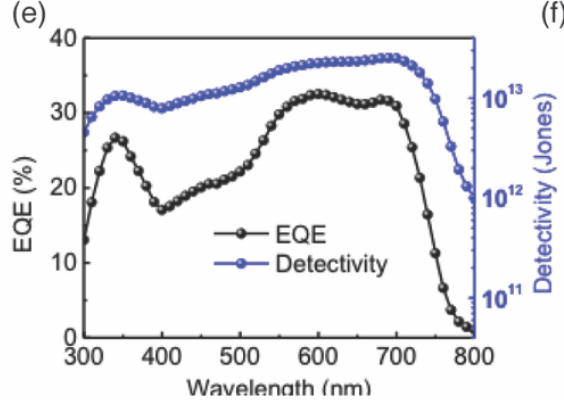

(c)

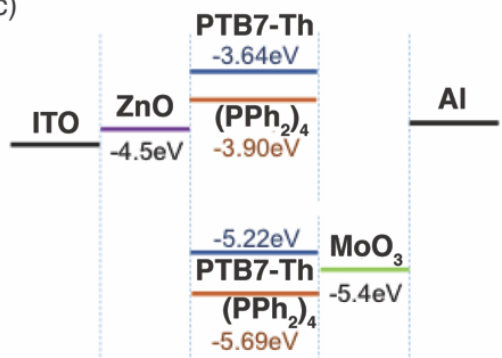

(f)

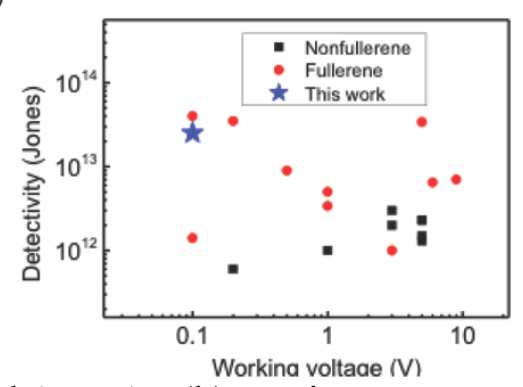

Figure 5.2. (a) Normalized film absorption spectra of PTB7-Th and (PPh $\mathbf{P h}_{4}$. (b) Device structure for the inverted organic photodetector. (c) Schematic of the energy levels of ITO, ZnO, PTB7-Th, $\left(\mathbf{P P h}_{2}\right)_{4}, \mathrm{MoO}_{3}$ and Al. Energy levels of PTB7-Th and $\left(\mathbf{P P h}_{2}\right)_{4}$ were estimated from cyclic voltammetry measurements in solution and adopted from ref. 16 and ref. 15, respectively. (d) Current density-voltage curves under dark condition and simulated AM $1.5 \mathrm{G}$ irradiation $\left(100 \mathrm{mWcm}^{-2}\right)$. (e) External quantum efficiency and specific detectivity spectra calculated at -0.1 $\mathrm{V}$ bias voltage. (f) Comparison of detectivity and working voltage in reported organic photodetectors and this work.

possesses extremely small dark current at reverse bias voltage. The dark $J-V$ curve shows a high rectification ratio of $>10^{5}$ at $\pm 2 \mathrm{~V}$ and a dark current density as small as $1.4 \times 10^{-10} \mathrm{Acm}^{-2}$ at $-0.1 \mathrm{~V}$. This dark current density is one order of magnitude lower than the best fullerene-free OPDs ${ }^{8}$ and comparable with fullerene-based OPDs and perovskite photodetectors. ${ }^{5,6}$ Notably, both fullerenebased OPDs and perovskite photodetectors need extra electron or hole blocking layers in order to achieve a low level of the dark current. ${ }^{6,17}$ Equally important is the large photocurrent that is generated upon photoexcitation at small reverse bias voltages. The on/off ratio is $>10^{7}$ calculated at $-0.1 \mathrm{~V}$ under simulated AM 1.5G irradiation $\left(100 \mathrm{mWcm}^{-2}\right)$. The OPD device described here can operate at small bias voltages and even at zero bias. This compares favorably with previously reported non-fullerene OPDs; they require much larger reverse bias voltages (typically $-1 \mathrm{~V} \sim-3$ V) to operate due to the poor carrier extraction. ${ }^{18-20}$ 
Ultralow dark current could result from poor carrier transport ability. This is not the case for $(\mathbf{P P h})_{4}$. As discussed above, $\left(\mathbf{P P h}_{2}\right)_{4}$ effectively transports electrons generated upon photoexcitation. This allows the OPD to operate at a small bias voltage. We inherently achieve high photocurrent and low dark current simultaneously in the $\left(\mathbf{P P h} \mathbf{h}_{\mathbf{4}} \mathbf{O P D}\right.$ with a thin active layer and a simple device structure. $(\mathbf{P P h})_{4}$ is an ideal design for an electron acceptor to create a highly sensitive non-fullerene OPD.

Table 5.1. A Summary of Responsivity, Detectivity, and EQE.

\begin{tabular}{|c|c|c|c|c|}
\hline & $\mathrm{J}_{\mathrm{d}}\left(\mathrm{Acm}^{-2}\right)$ & EQE $(\%)$ & $\mathrm{R}\left(\mathrm{AW}^{-1}\right)$ & $\mathrm{D}^{*}(\mathrm{Jones})$ \\
\hline $\left.\mathbf{( P P h}_{2}\right)_{4}$ & $1.5 \times 10-10$ & $33,600 \mathrm{~nm}$ & $0.18,690 \mathrm{~nm}$ & $2.5 \times 10^{13}, 690 \mathrm{~nm}$ \\
\hline $\mathbf{P C}_{\mathbf{7 1}} \mathbf{B M}$ & $5.6 \times 10-7$ & $70,640 \mathrm{~nm}$ & $0.39,710 \mathrm{~nm}$ & $9.2 \times 10^{11}, 710 \mathrm{~nm}$ \\
\hline $\mathbf{3 . 6}$ & $1.0 \times 10-9$ & $22,350 \mathrm{~nm}$ & $0.09,680 \mathrm{~nm}$ & $4.8 \times 10^{12}, 680 \mathrm{~nm}$ \\
\hline
\end{tabular}

Table 5.1: A summary of responsivity, detectivity, and EQE.

Table 5.1 summarizes the responsivity $(R)$, specific detectivity $\left(D^{*}\right)$, EQE and dark current $\left(J_{\mathrm{d}}\right)$ for the OPD device data for the PTB7-Th: $(\mathbf{P P h})_{4}$ blended films. The devices have a linear dynamic range $(\mathrm{LDR})>140 \mathrm{~dB}$ (Figure 5.5) and cutoff frequency of $467 \mathrm{kHz}$ (Figure 5.6). Overall the device characteristics are excellent. We find that the specific detectivity is more than $10^{13}$ Jones over the whole visible light region at $-0.1 \mathrm{~V}$. The highest $D^{*}$ was calculated to be $2.5 \times 10^{13}$ Jones at 700nm (Figure 5.2e). At zero bias, the calculated specific detectivity is as high as $1 \times 10^{14}$ Jones. These values are among the highest detectivities for the state-of-the-art fullerene

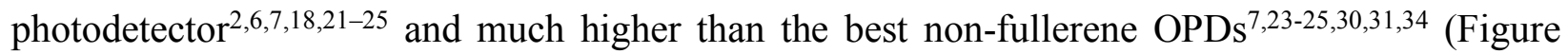
5.2f). Moreover, the ( $\left.\mathbf{P} \mathbf{P h}_{2}\right)_{4}$-based OPD requires much smaller working voltage compared with

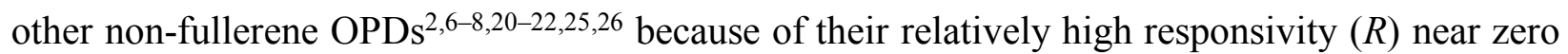
bias voltage (Figure 5.2f). 


\subsection{Transient Absorption Shows Fast Charge Separation}
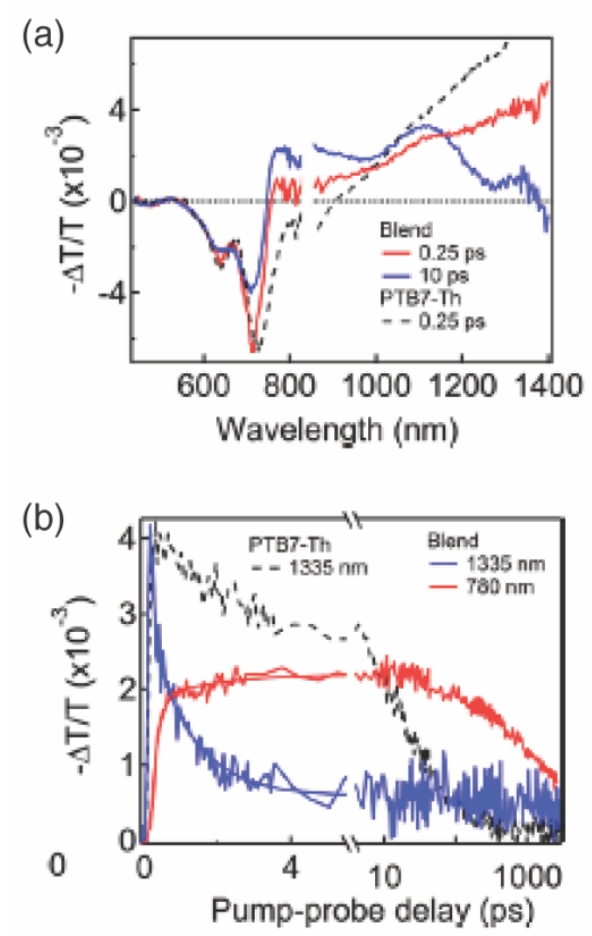

Figure 5.3. Transient absorption spectra (b) and dynamics (b) for the neat film of PTB7-Th and blended film of $\left(\mathbf{P P h}_{2}\right)_{4}$ and PTB7-Th pumped at $710 \mathrm{~nm}$. The dashed-black curves are from PTB7-Th which were scaled for comparison.

One of the two key parameters responsible for high responsivity $(R)$ is efficient charge generation from photo-excitation in the active layer. Extensive research on molecular donor/acceptor interfaces has pointed to the critical role of electronic delocalization in facilitating efficient charge separation at the donor/acceptor interfaces. ${ }^{27,28}$ In particular, the spherical fullerene based electron acceptors are believed to transport charge efficiently due to their three dimensional connectivity in ensuring electronic delocalization on the acceptor side. ${ }^{29,30}$ Can $\left(\mathbf{P P h}_{2}\right)_{4}$ provide similar connectivity and electronic delocalization that is necessary for charge separation? While the high responsivity near zero bias suggests the answer above is affirmative, we further support this by studying the efficient charge separation at the PTB7-Th/(PPh $)_{4}$ interface using transient absorption (TA) spectroscopy (Figure 5.3). 
The linear absorption spectra in Figure 5.2a show that the donor material, PTB7-Th can be selectively excited at $710 \mathrm{~nm}$ in the blended film; subsequently, we can monitor electron transfer from PTB7-Th to $\left(\mathbf{P P h}_{2}\right)_{4}$. At a short pump-probe delay (0.25 ps), we observe features arising from PTB7-Th (red curve in Figure 5.3a) with the bleaching at 630 and $715 \mathrm{~nm}$ and excited state absorption (ESA) in the near IR range. This is similar to the TA spectrum from the neat PTB7-Th film (black dashed curve in Figure 5.3a). The bleaching in PTB7-Th is reduced and new ESA transitions from 770 to $1300 \mathrm{~nm}$ evolve as the delay time increases. These new ESA features centered at 840 and $1120 \mathrm{~nm}$ are attributed to the charge (polaron) absorptions in the PDI and PTB7-Th moieties, respectively. ${ }^{13,31,32}$ While the ESA of the PTB7-Th singlet dominates in the long probe wavelength range $(>1300 \mathrm{~nm})$, the ESA of charges in either donor or acceptor materials is negligible. Thus, dynamics at $1335 \mathrm{~nm}$ are a good measure of exciton dissociation at the donor/acceptor interface. Figure 5.3b shows that the biexponential fit (blue curve) to the dynamics at $1335 \mathrm{~nm}$ (blue dots) yields time constants of $0.11 \pm 0.04 \mathrm{ps}$ ( $42 \%$ weight) and $1.2 \pm 0.1 \mathrm{ps}(58 \%$ weight). The charge buildup monitored by polaron ESA at $780 \mathrm{~nm}$ (red dots) gives nearly identical time constants (red curve). These time constants indicate ultrafast electron transfer from PTB7-Th to $\left(\mathbf{P P h}_{2}\right)_{4}$. The short time constant can be attributed to the instantaneous charge transfer near the interface upon photoexcitation, while the longer time constant is attributed to exciton diffusion in PTB7-Th prior to the dissociation event. ${ }^{13,32}$ For comparison, the singlet exciton lifetime in neat PTB7-Th is of the order of nanoseconds (black dashed curve in Figure 5.3b). This is much longer than that in the blend. We also observe similarly ultrafast hole transfer from $\left(\mathbf{P P h}_{2}\right)_{4}$ to PTB7-Th when both donor and acceptor are excited at $560 \mathrm{~nm}$. These measurements confirm that $\left(\mathbf{P P h}_{2}\right)_{4}$ photodetector performance is comparable, if not better than that of a fullerene, for exciton dissociation in blend films. ${ }^{33-35}$ 
The second important parameter that determines the high detectivity in these devices is the dark current density. To gain insight into the origin of the device characteristics, we analyze the $J$ $V$ curve under dark conditions to calculate the reverse saturation dark current $J_{0}$. The fitting method

is detailed in the Appendix. The saturation dark current density is as low as $7.7 \times 10^{-13} \mathrm{Acm}^{-2}$ (Figure 5.8). The intrinsic conductivity is determined by the intrinsic free carrier density and the mobility. Photocurrent and thin film field effect transistor measurements reveal efficacious electron transporting ability. ${ }^{36}$ Therefore, such a low level of $J_{0}$ indicates a small amount of intrinsic free carrier density. To verify that the intrinsic conductivity of the thin films is dominated by the charged defects, we measured the dependence of the dark current density on temperature. The activation energies are calculated to be $(0.15 \pm 0.01) \mathrm{eV}$ and $(0.20 \pm 0.01) \mathrm{eV}$ at $-2 \mathrm{~V}$ and -0.5 $\mathrm{V}$, respectively (Figure 5.9). These values are much smaller than the band gaps of the active organic components (Figure 5.2c) and are consistent with thermal activation energy of locally bound charged defects. ${ }^{12,37}$

\subsection{PC 71 BM Shows High Dark Current Density}

To better understand the origin of the low density of charged defects in $\left(\mathbf{P P h} \mathbf{~}_{\mathbf{4}}\right.$, we made a direct comparison between a $\mathrm{PC}_{71} \mathrm{BM}$ and $\left(\mathbf{P P h}_{2}\right)_{4}$-based OPD with the same device structure as shown in Figure 5.2b. Although the responsivity $(R)$ of the $\mathrm{PC}_{71} \mathrm{BM}$-based OPD is approximately two fold higher than that of the ( $\mathbf{P P h} \mathbf{P}_{4}$-based device (Table 5.1 and Figure 5.11), the dark current of the $\mathrm{PC}_{71} \mathrm{BM}$-based device is $2-3$ orders of magnitude higher than that of the $\left(\mathbf{P P h} \mathbf{~}_{\mathbf{4}}\right.$-based one (Figure 5.4a). It is obvious that the dark current level dominates the detectivity in this type of OPD. Thus, the detectivity of the $\left(\mathbf{P P h}_{2}\right)_{4}$-based OPD is more than one order of magnitude higher than that of the $\mathrm{PC}_{71} \mathrm{BM}$-based one (Figure 5.4b). One of the origins of the high dark current is from the chemistry of fullerenes. $\mathrm{PC}_{71} \mathrm{BM}$ is known to undergo dimerization that is initiated by electron 
donors, metals, and photons..$^{9-11,38,39}$ In each of these cases, in a solid state film, the partners need to be unencumbered and in the correct orientation for the reaction to occur. In the absence of these conditions, the films will contain carriers that contribute to the relatively high dark current. ${ }^{9-11}$ Fullerene-based OPDs made with fullerenes require extra blocking layers to minimize this relatively high dark current. Compared with fullerenes, PDIs are known to exhibit exceptional chemical, thermal and photochemical stability. ${ }^{40-43}$ They have also been widely used as building blocks to construct macrocycles. ${ }^{44-53}$ The PDI units in $(\mathbf{P P h})_{4}$ have no readily accessible pathway to introduce these same type of covalent defects. This accounts for the low intrinsic conductivity in $\left(\mathbf{P P h}_{2}\right)_{4}$ OPDs. ${ }^{12}$
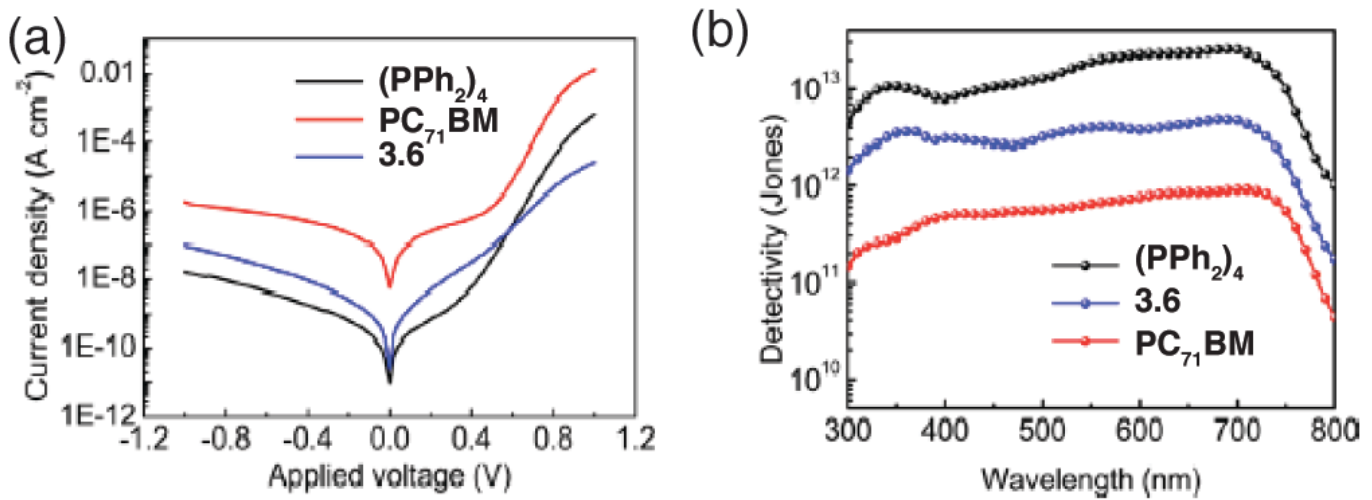

Figure 5.4. (a) Dark current density-voltage curves for $\mathrm{PC}_{71} \mathrm{BM}, \mathbf{3 . 6}$, and $\left(\mathbf{P P h _ { 2 }}\right)_{4}$ based photodetectors with the same device structure as shown in Figure 5.2b. (b) Specific detectivity spectra for $\left(\mathbf{P P h}_{2}\right)_{4}, \mathbf{3 . 6}$ and $\mathrm{PC}_{71} \mathrm{BM}$ based OPDs calculated at $-0.1 \mathrm{~V}$ bias voltage.

\subsection{Rigid Macrocyclic Design Impedes Charge Defects}

In order to assess the importance of the cyclic, rigid structure of $\left(\mathbf{P P h} \mathbf{~}_{\mathbf{4}}\right.$, we fabricated a 3.6-based OPD (Figure 5.1b). Overall, the 3.6 photodetector performs very well and shows high sensitivity. Although the responsivity of the 3.6 OPD is about one fourth of that in the $\mathrm{PC}_{71} \mathrm{BM}$ OPD, the dark current density in the 3.6 OPD is one order of magnitude lower than that in the $\mathrm{PC}_{71} \mathrm{BM}$ OPD (Figure 5.4a). As a result, the 3.6 OPD shows a doubling of the detectivity compared to the $\mathrm{PC}_{71} \mathrm{BM}$ OPD (Figure 5.4b). These results, once again, suggest that dark current dominates 
the sensitivity and confirm the importance of incorporating chemically stable PDIs into electron acceptors in OPDs. Even so, the 3.6 OPD is still not as effective as the (PPh $)_{4}$ OPD in terms of detectivity. As a comparison, the 3.6-based OPD has a dark current $1.0 \times 10^{-9} \mathrm{Acm}^{-2}$ at $-0.1 \mathrm{~V}$, which is about ten-fold higher than that of the $(\mathbf{P P h})_{4}$-based device (see Table 5.1 and Figure 5.4a). In addition, the 3.6-based OPD also shows lower responsivity compared to $\left(\mathbf{P P h} \mathbf{~}_{\mathbf{4}}\right.$ devices (Table 5.1 and Figure 5.11). The resulting peak $D^{*}$ for the 3.6-based OPD is $4.8 \times 10^{12}$ Jones at 680 $\mathrm{nm}$, only about one third of the peak value in the (PPh $)_{4}$-based OPD. This is because intrinsic, charged defects in linear polymer semiconductors originate from endgroups ${ }^{13,54,55}$ and deformed $s p^{2}$ carbons near the rotatable C-C single bonds. ${ }^{12-14,56}$ The torsional effect is inevitable in linear molecules with flexible backbones. These problems are reduced by winding linear molecules into rigid, conjugated macrocycles with no endgroups. ${ }^{15,36,57}$ The macrocyclic $\left(\mathbf{P P h}_{\mathbf{2}} \mathbf{4}_{\mathbf{4}}\right.$ possesses a locked conformation with higher rigidity (Figure 5.1a) and is expected to create fewer locally charged defects relative to the linear polymeric counterpart.

\subsection{Conclusion}

The results described above show that the rigid, cyclic molecular structure is an important design criterion to achieve ultralow intrinsic conductivity in OPDs. We found that the rigid, conjugated macrocycle is able to act as the electron acceptor in high performance OPDs. Using this molecular design, we suppress dark current density while retaining high responsivity in an ultra-sensitive non-fullerene OPD. Without the need for extra carrier blocking layers, this detectivity is comparable to the best fullerene-based photodetectors, and the sensitivity at low working voltages is a record for non-fullerene OPDs. It is clear from this study that the devices can be further improved by designing the electron donating material to form a shape and electronic match for these macrocyclic electron acceptors. 


\subsection{Appendix - Supplementary Figures}

The linear dynamic range (LDR) is expressed as $\mathrm{LDR}=20 \log \left(J_{\text {high }} / J_{\mathrm{d}}\right)$, where $J_{\text {high }}$ is the highest measurable current density in the linear response range and $J_{d}$ is the dark current density.

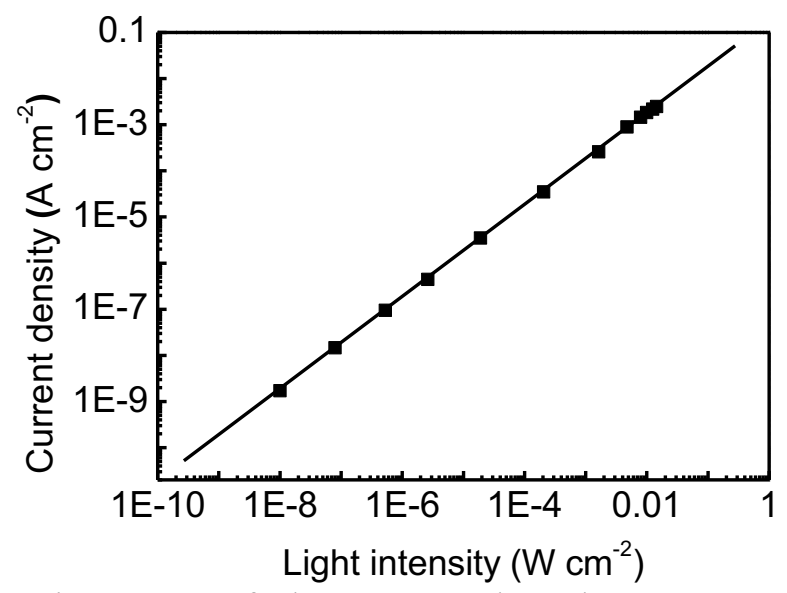

Figure 5.5. Linear dynamic range of the $(\mathbf{P P h})_{4}$-based OPD. A red light emitting diode $(\lambda=625 \mathrm{~nm})$ was used as the light source.

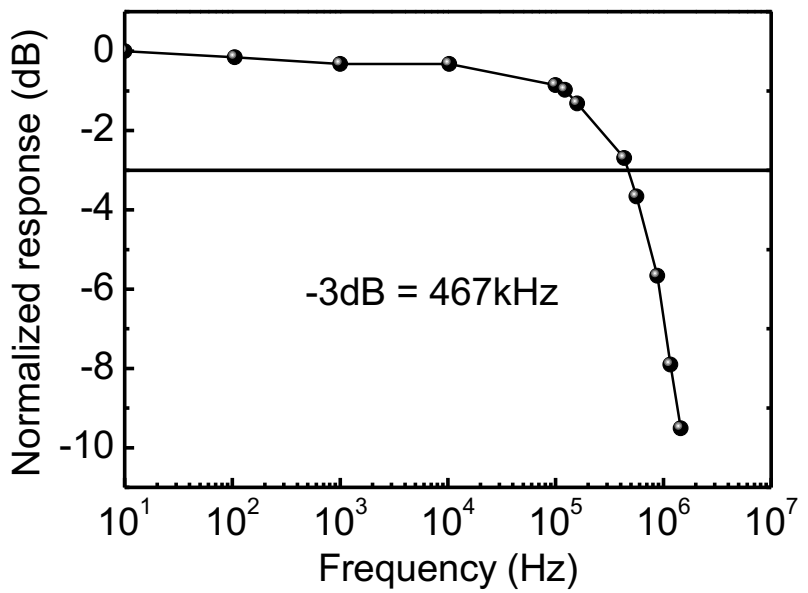

Figure 5.6. Frequency response of the $\left(\mathbf{P P h}_{2}\right)_{4}$-based OPD. The device area is $0.09 \mathrm{~cm}^{2}$. 

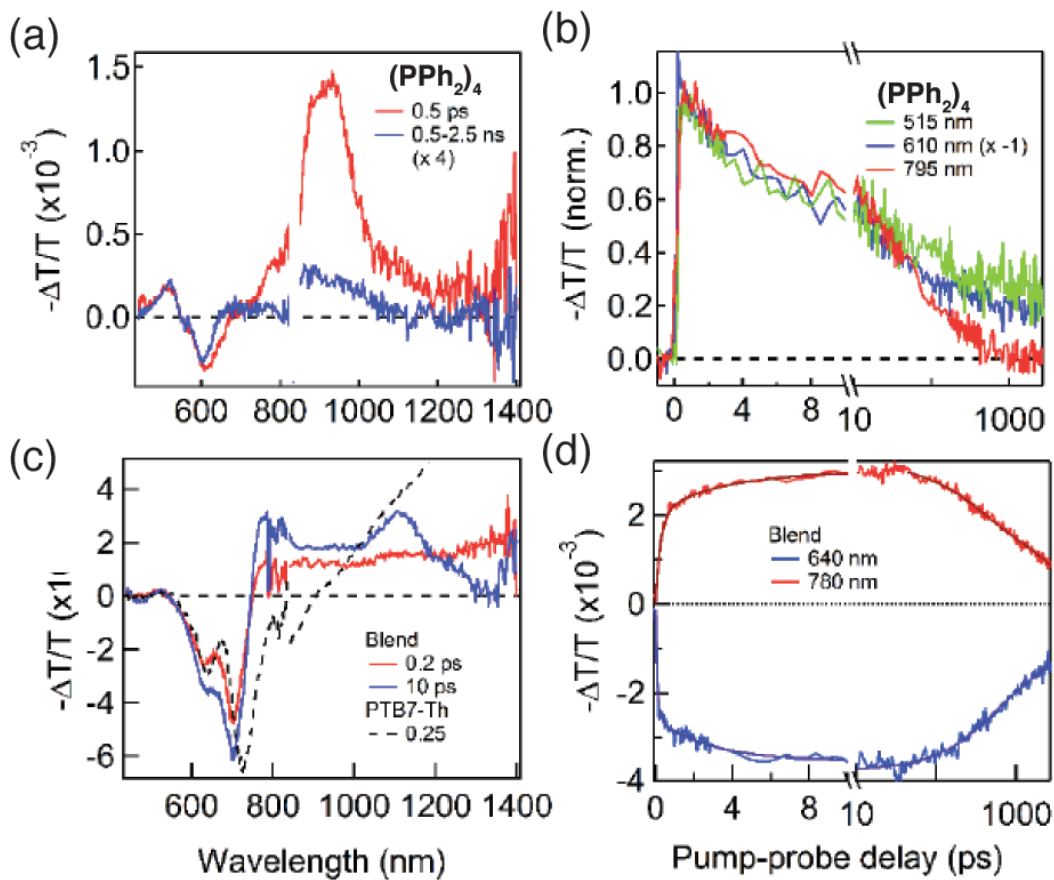

Figure 5.7. Transient absorption spectra and dynamics for the neat $\left(\mathbf{P P h}_{2}\right)_{4}(\mathrm{a}, \mathrm{b})$ and the blended film of $\left(\mathbf{P P h}_{2}\right)_{4}$ and PTB7-Th (c,d) upon $560 \mathrm{~nm}$ excitation. The dashed-black curve in (c) is the spectrum from PTB7-Th upon the excitation of $710 \mathrm{~nm}$. The signal from PTB7-th was scaled for comparison.

Figure 5.7a,b show the differential transmission spectra and dynamics for the neat film $\left(\mathbf{P P h}_{2}\right)_{4}$ upon $560 \mathrm{~nm}$ excitation. The negative feature centered at $600 \mathrm{~nm}$ is attributed to a bleaching of the ground state absorption under the presence of photo-excited excitons/charges. The positive features centered at $500 \mathrm{~nm}$ and from $700 \mathrm{~nm}$ to near IR are the excited state absorption (ESA) of the photo-excited excitons or charges. The ESA feature at $785 \mathrm{~nm}$ decays biexponentially with the time constants of $8 \pm 0.7$ ps $(52 \%)$ and $122 \pm 8$ ps (48\%) (red curve, Figure $5.7 \mathrm{~b})$. This feature is assigned to the singlet ESA, $\mathrm{S}_{1} \rightarrow \mathrm{S}_{\mathrm{N}}$ transition, similar to that of other PDI derivative compounds.

The ESA dynamics at $500 \mathrm{~nm}$ and ground state bleaching, however, behave differently. More than $50 \%$ of the ESA signal at $500 \mathrm{~nm}$ decays with a time constant that is similarly to the dynamics at $785 \mathrm{~nm}$. The remaining signal (20\%) lasts for a long time, ns time scale (green and 
blue curves). The long living signal can either come from photo-excited charges or from triplet excitons.

At the same excitation wavelength, $560 \mathrm{~nm}$, for the blend of $\left(\mathbf{P P h}_{2}\right)_{4}$ and PTB7-Th, we observed the dominance of ground state bleaching from PTB7-Th at the range of 575-750 nm (Figure 5.7c, the dashed-black curve is from the neat PTB7-Th pumping at $710 \mathrm{~nm}$ ). Note that at this excitation wavelength, we excite both donor and acceptor materials. The ESA cross section and the ground state bleaching from the polymer are much stronger than that of $\left(\mathbf{P P h} \mathbf{~}_{4}\right.$ resulting in a dominant feature from the polymer even when we excited both materials at $560 \mathrm{~nm}$. At the longer time (10 ps, Figure S4 C), the bleaching from PTB7-Th increases and the hole ESA feature in PTB7-Th at $1100 \mathrm{~nm}$ appears. The increase of bleaching feature from PTB7-Th indicates the hole transfers from $\left(\mathbf{P P h}_{2}\right)_{4}$ to PTB7-Th. Since the ESA cross session at $780 \mathrm{~nm}$ for singlet in $(\mathbf{P P h})_{4}$ is very small compared to that of charge ESA (compare Figure 5.7a,c), the ESA signal at this wavelength reflects the dynamics of charge generation from exciton dissociation. The fit to the dynamics at $780 \mathrm{~nm}$ gives two time constants, $0.2 \pm 0.02 \mathrm{ps}$ and $3.3 \pm 0.4 \mathrm{ps}$ (Figure $5.7 \mathrm{~d}$ ). These time constants reflect the electron and hole transfer upon $560 \mathrm{~nm}$ excitation. In addition, we observed a much longer charge lifetime in the blends (on the nanosecond time scale) when compared to that of the exciton dynamics in the neat films (Figure 5.7b,d). The longer charge lifetime is due to exciton dissociated into separating charges rather than charge transfer excitons. 


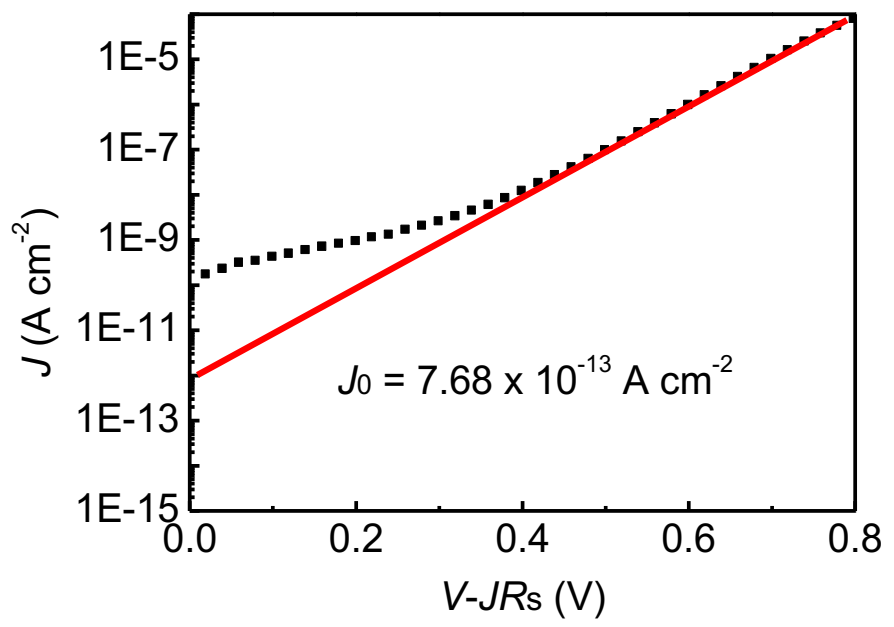

Figure 5.8. The device structure is ITO/ZnO/PBDTT-TT:(PPh $)_{4} / \mathrm{MoO}_{3} / \mathrm{Al}$. The original $J-V$ curve is shown in Figure 5.2c Plot of d $V / \mathrm{d} J$ vs $J^{-1}$ and linear fitting. (b) $\ln (J)$ vs $\left(V-J R_{\mathrm{s}}\right)$ and linear fitting. The $J_{0}$ is calculated to be $7.68 \times 10^{-13} \mathrm{Acm}^{-2}$.

The dark current-voltage characteristics are described by $J_{\mathrm{d}}=J_{0}\left\{\exp \left[\mathrm{e}\left(V-J R_{\mathrm{S}}\right) / \mathrm{nk} \mathrm{B}_{\mathrm{B}} \mathrm{T}\right]-1\right\}$, where $J_{\mathrm{d}}$ is the dark current density, $J_{0}$ is the dark saturate current density, $V$ is the applied voltage, $\mathrm{n}$ is the ideality factor, $\mathrm{k}_{\mathrm{B}}$ is the Boltzmann constant, $\mathrm{T}$ is the temperature and $\mathrm{e}$ is the electron charge. The fitting method is described in ref. $17 .{ }^{17}$

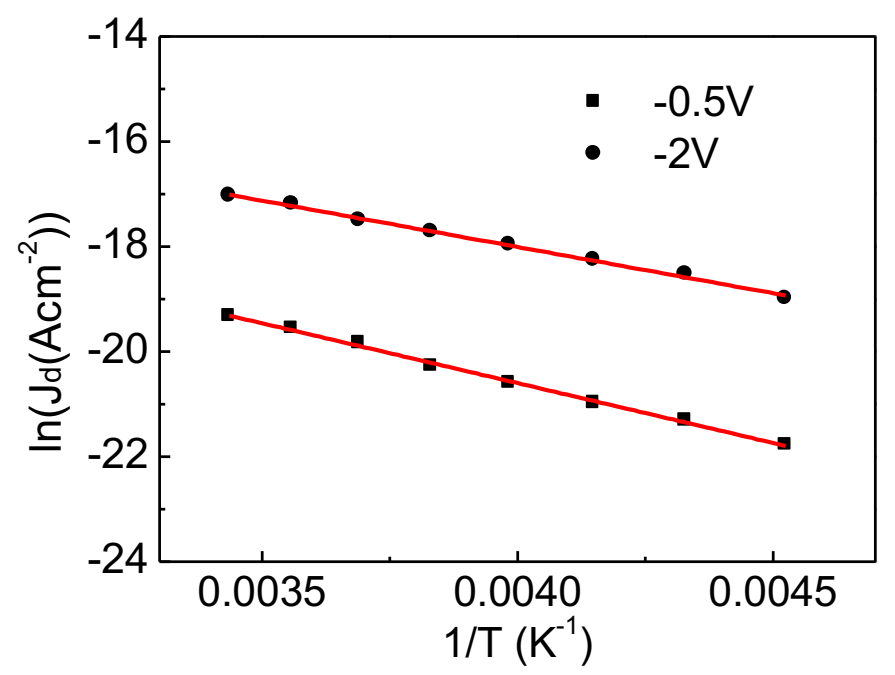

Figure 5.9. The dependence of the dark current density of the $\left(\mathbf{P P h}_{2}\right)_{4}$-based OPD on temperature measured at $-0.5 \mathrm{~V}$ and $-2.0 \mathrm{~V}$. 


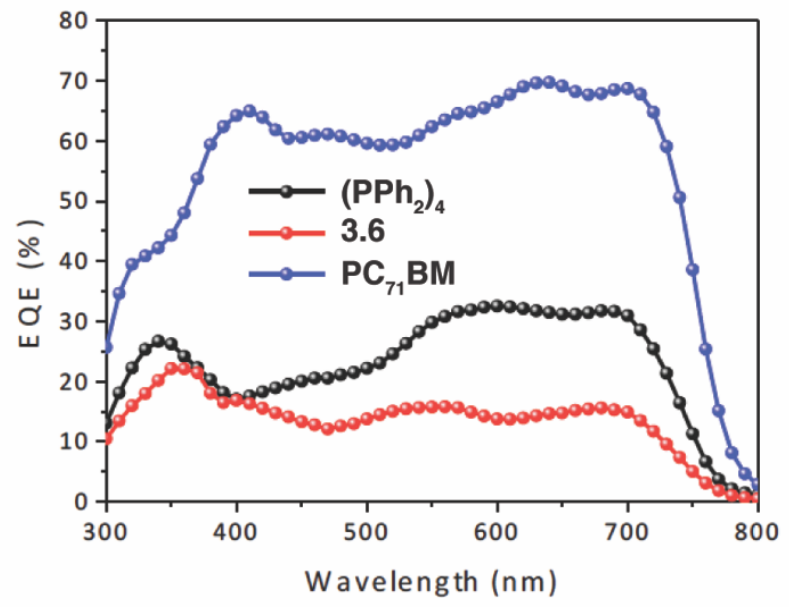

Figure 5.10. External quantum efficiency at different wavelength with inverted OPD structure at $-0.1 \mathrm{~V}$.

The responsivity $R$ was calculated from the following equation: $R=\lambda q / h c \times E Q E$, where $E Q E$ is external quantum efficiency, $\lambda$ the wavelength, $q$ the electron charge, $h$ the Planck constant, $c$ the speed of light. When the noise current is dominated by the shot noise from the dark current, the specific detectivity $\left(D^{*}\right)$ is given by $D^{*}=R /\left(2 q J_{\mathrm{d}}\right)^{1 / 2}$, where $R$ is the responsivity, $q$ the electron charge and $J_{d}$ the dark current density.

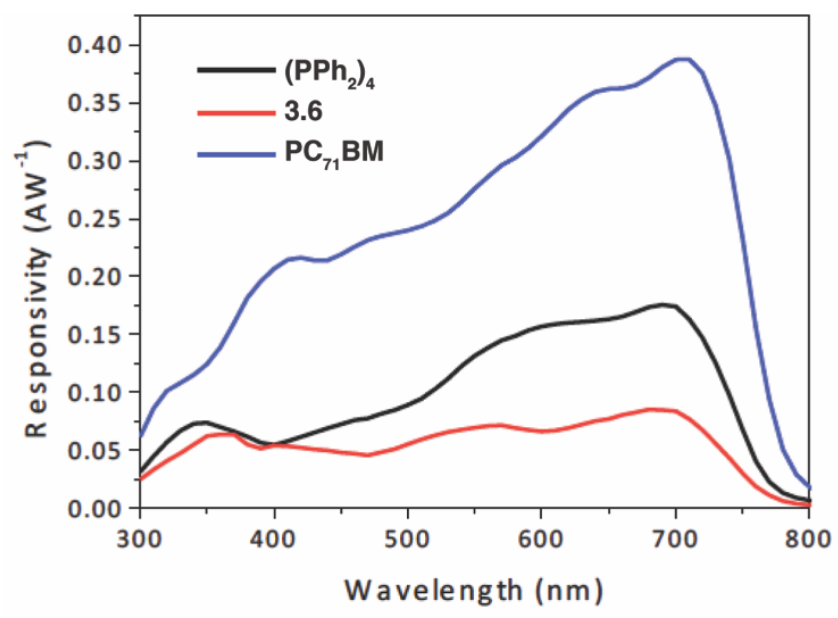

Figure 5.11. Calculated responsivity $\left(R\right.$ in $\left.\mathrm{AW}^{-1}\right)$ spectra for different OPDs.

\subsection{General Experimental Information}

Device Fabrication. The synthesis of $\mathrm{ZnO}$ precursor was described elsewhere. ${ }^{58}$ Prepatterned ITO-coated glass with a sheet resistance of $\sim 15 \Omega /$ sq was cleaned with detergent, ultrasonicated in 
DI water, acetone and isopropanol for $30 \mathrm{~min}$, respectively. Subsequently, we treated the substrates by UV-Ozone for $10 \mathrm{~min}$. The prepared $\mathrm{ZnO}$ precursor was spin-cast onto the ITO substrate at $3000 \mathrm{rpm}$ for $1 \mathrm{~min}$, followed by annealing at $200^{\circ} \mathrm{C}$ for $1 \mathrm{~h}$ in air, to form a thin film with approximate thickness of $20 \mathrm{~nm}$. The BHJ active layer was prepared by spin-coating a mixed solution containing polymer and acceptor in chlorobenzene. The thickness of the prepared active layers is about $150 \mathrm{~nm}$. Finally, a $10 \mathrm{~nm} \mathrm{MoO}$ layer was deposited first and then a $100 \mathrm{~nm} \mathrm{Al}$ electrode were subsequently deposited through a shadow mask by thermal evaporation under a vacuum about $1 \times 10^{-6}$ torr. The active area of the device was $9 \mathrm{~mm}^{2}$.

Characterization. The synthesis for $\left(\mathbf{P P h}_{2}\right)_{4}$ and $\mathbf{3 . 6}$ is provided in Chapter 3. Absorption spectra were obtained on Shimadzu UV 1800 UV-Vis. The current-density-voltage (J-V) curves were measured by a Keithley 2635A source measure unit. EQE measurements were performed using a QEXL system from PV Measurements Inc. For linear dynamic range measurement, a lightemitting diode $(625 \mathrm{~nm})$ was used as light source. For cutoff frequency measurement, the LED was modulated by a function generator (Tektronix CFG253). The photodetectors were connected to an oscilloscope (Tektronix TDS 224) with an input impedance of $50 \Omega$.

Temperature-dependent current-voltage measurements were realized in a vacuum cryogenic probe station (Lakeshore TTP4). Computer-controlled source units were used to apply DC potentials (Yokogawa 7651). Current measurements were obtained using a current preamplifier (Stanford Research System SR570) and a digital multimeter (Keysight 34401A). All device measurements were performed in vacuum $\left(\mathrm{P}<1 \times 10^{-4}\right.$ Torr $)$ at different temperatures. In the transient absorption experiments, the pump pulse comes from an optical parametric amplifier (tunable from UV to the near IR, $100 \mathrm{fs}$ pulse width, $1 \mathrm{KHz}$ rep-rate). The probe pulse is a white-light supercontinuum (from 450 to $900 \mathrm{~nm}$ and from 850 to $1600 \mathrm{~nm}$ for the visible and 
near IR range, respectively). The detection consists of a pair of high resolution multichannel detector arrays coupled to a high-speed data acquisition system (HELIOS, Ultrafast System Inc.). In order to avoid photo degradation, we kept samples in the inert gas both in storing and during measurements.

\subsection{DFT Output}

All quantum chemical calculations were performed using Jaguar, version 8.3, Schrodinger, Inc., New York, NY, 2013. (See A. D. Bochevarov, E. Harder, T. F. Hughes, J. R. Greenwood, D. A. Braden, D. M. Philipp, D. Rinaldo, M. D. Halls, J. Zhang, R. A. Friesner, "Jaguar: A High Performance Quantum Chemistry Software Program with Strengths in Life and Materials Sciences", Int. J. Quantum Chem., 2013, 113(18), 2110-2142). All geometries were optimized using the B3LYP functional and the 6-31G basis set. The geometry optimizations can be found in reference $1 .^{59}$

\subsection{References}

1. Zhang, B. et al. Rigid, Conjugated Macrocycles for High Performance Organic Photodetectors. J. Am. Chem. Soc. 138, 16426-16431 (2016).

2. Baeg, K.-J. et al. Organic Light Detectors: Photodiodes and Phototransistors. Adv. Mater. 25, 4267-4295 (2013).

3. Sekitani, T. et al. A Rubberlike Stretchable Active Matrix Using Elastic Conductors. Science. 321, 1468-1472 (2008).

4. Rogers, J. A. et al. Materials and Mechanics for Stretchable Electronics. Science. 327, 1603-1607 (2010).

5. Ball, M. et al. Contorted Polycyclic Aromatics. Acc. Chem. Res. 48, 267-276 (2015).

6. Gong, X. et al. High-Detectivity Polymer Photodetectors with Spectral Response from 300 $\mathrm{nm}$ to $1450 \mathrm{~nm}$. Science. 325, 1665-1667 (2009).

7. Zhang, L. et al. Toward Highly Sensitive Polymer Photodetectors by Molecular Engineering. Adv. Mater. 27, 6496-6503 (2015).

8. Kim, I. K. et al. High-Performance, Fullerene-Free Organic Photodiodes Based on a Solution-Processable Indigo. Adv. Mater. 27, 6390-6395 (2015).

9. Segura, J. L. et al. [60]fullerene dimers. Chem. Soc. Rev. 29, 13-25 (2000).

10. Tsetseris, L. et al. Intermolecular bridges and carrier traps in defective C60 crystals. Phys. 
Rev. B 84, (2011).

11. Sheka, E. F. Donor-acceptor interaction and fullerene C-60 dimerization. Chem. Phys. Lett. 438, 119-126 (2007).

12. Gregg, B. A. Charged defects in soft semiconductors and their influence on organic photovoltaics. Soft Matter 5, 2985-2989 (2009).

13. Kaake, L. G. et al. Intrinsic Charge Trapping in Organic and Polymeric Semiconductors: A Physical Chemistry Perspective. J. Phys. Chem. Lett. 1, 628-635 (2010).

14. Darling, S. B. Isolating the Effect of Torsional Defects on Mobility and Band Gap in Conjugated Polymers. J. Phys. Chem. B 112, 8891-8895 (2008).

15. Ball, M. et al. Macrocyclization in the Design of Organic n-Type Electronic Materials. $J$. Am. Chem. Soc. 138, 12861-12867 (2016).

16. Liao, S.-H. et al. Fullerene Derivative-Doped Zinc Oxide Nanofilm as the Cathode of Inverted Polymer Solar Cells with Low-Bandgap Polymer (PTB7-Th) for High Performance. Adv. Mater. 25, 4766-4771 (2013).

17. Dou, L. et al. Solution-processed hybrid perovskite photodetectors with high detectivity. Nat. Commun. 5, 5404 (2014).

18. Kim, I. K. et al. High-Performance, Solution-Processed Non-polymeric Organic Photodiodes. Adv. Opt. Mater. 3, 50-56 (2015).

19. Leem, D.S. et al. Low dark current small molecule organic photodetectors with selective response to green light. Appl. Phys. Lett. 103, 43305 (2013).

20. Lim, S.-J. et al. Organic-on-silicon complementary metal-oxide-semiconductor colour image sensors. Sci. Rep. 5, 7708 (2015).

21. Armin, A. et al. Thick junction broadband organic photodiodes. Laser Photon. Rev. 8, 924932 (2014).

22. Pierre, A. et al. High Detectivity All-Printed Organic Photodiodes. Adv. Mater. 27, 64116417 (2015).

23. Qi, J. et al. Optimization of Solubility, Film Morphology and Photodetector Performance by Molecular Side-Chain Engineering of Low-Bandgap Thienothiadiazole-Based Polymers. Adv. Funct. Mater. 24, 7605-7612 (2014).

24. Ramuz, M. et al. High sensitivity organic photodiodes with low dark currents and increased lifetimes. Org. Electron. 9, 369-376 (2008).

25. Saracco, E. et al. Work Function Tuning for High-Performance Solution-Processed Organic Photodetectors with Inverted Structure. Adv. Mater. 25, 6534-6538 (2013).

26. Lee, K.H. et al. Green-Sensitive Organic Photodetectors with High Sensitivity and Spectral Selectivity Using Subphthalocyanine Derivatives. ACS Appl. Mater. Interfaces 5, 1308913095 (2013).

27. Zhu, X.-Y. et al. Charge Transfer Excitons at van der Waals Interfaces. J. Am. Chem. Soc. 137, 8313-8320 (2015).

28. Banerji, N. Sub-picosecond delocalization in the excited state of conjugated homopolymers 
and donor-acceptor copolymers. J. Mater. Chem. C 1, 3052-3066 (2013).

29. Savoie, B. M. et al. Mesoscopic Features of Charge Generation in Organic Semiconductors. Acc. Chem. Res. 47, 3385-3394 (2014).

30. Savoie, B. M. et al. Unequal Partnership: Asymmetric Roles of Polymeric Donor and Fullerene Acceptor in Generating Free Charge. J. Am. Chem. Soc. 136, 2876-2884 (2014).

31. Zhong, Y. et al. Efficient Organic Solar Cells with Helical Perylene Diimide Electron Acceptors. J. Am. Chem. Soc. 136, 15215 (2014).

32. Zhong, Y. et al. Molecular helices as electron acceptors in high-performance bulk heterojunction solar cells. Nat. Commun. 6, 8242 (2015).

33. Hwang, I. et al. Ultrafast relaxation of charge-transfer excitons in low-bandgap conjugated copolymers. Chem. Sci. 3, 2270-2277 (2012).

34. Hwang, I.-W. et al. Ultrafast Electron Transfer and Decay Dynamics in a Small Band Gap Bulk Heterojunction Material. Adv. Mater. 19, 2307-2312 (2007).

35. Grancini, G. et al. Hot exciton dissociation in polymer solar cells. Nat. Mater. 12, 29 (2012).

36. Ball, M. et al. Conjugated Macrocycles in Organic Electronics. Acc. Chem. Res. (2019). doi:10.1021/acs.accounts.9b00017

37. Gregg, B. A. Transport in Charged Defect-Rich $\pi$-Conjugated Polymers. J. Phys. Chem. C 113, 5899-5901 (2009).

38. Wang, G.-W. et al. Synthesis and X-ray structure of dumb-bell-shaped C120. Nature 387, 583-586 (1997).

39. Komatsu, K. et al. Mechanochemical Synthesis and Characterization of the Fullerene Dimer C120. J. Org. Chem. 63, 9358-9366 (1998).

40. Huang, C. et al. Perylene-3,4,9,10-tetracarboxylic Acid Diimides: Synthesis, Physical Properties, and Use in Organic Electronics. J. Org. Chem. 76, 2386-2407 (2011).

41. Würthner, F. Perylene bisimide dyes as versatile building blocks for functional supramolecular architectures. Chem. Commun. 1564-1579 (2004). doi:10.1039/B401630K

42. Würthner, F. et al. Perylene Bisimide Dye Assemblies as Archetype Functional Supramolecular Materials. Chem. Rev. 116, 962-1052 (2016).

43. Kozma, E. et al. Perylene diimides based materials for organic solar cells. Dye. Pigment. 98, 160-179 (2013).

44. Langhals, H. et al. Cyclophanes as Model Compounds for Permanent, Dynamic Aggregates - Induced Chirality with Strong CD Effects. European J. Org. Chem. 1998, 1915-1917 (1998).

45. Wang, W. et al. Cyclization and Catenation Directed by Molecular Self-Assembly. J. Am. Chem. Soc. 128, 11150-11159 (2006).

46. Feng, J. et al. Cyclophanes of Perylene Tetracarboxylic Diimide with Different Substituents at Bay Positions. Chem. Eur. J. 14, 7000-7010 (2008).

47. Shaller, A. D. et al. Tunable Molecular Assembly Codes Direct Reaction Pathways. Angew. 
Chem. Int. Ed. 47, 7705-7709 (2008).

48. Wang, W. et al. Twisted Perylene Stereodimers Reveal Chiral Molecular Assembly Codes. J. Am. Chem. Soc. 130, 8271-8279 (2008).

49. Schlosser, F. et al. Perylene bisimide macrocycles and their self-assembly on HOPG surfaces. Chem. Commun. 46, 8350-8352 (2010).

50. Lee, J.-E. et al. Structure-Property Relationship of Perylene Bisimide Macrocycles Probed by Atomic Force Microscopy and Single-Molecule Fluorescence Spectroscopy. ACS Nano 7, 5064-5076 (2013).

51. Schlosser, F. et al. Redox-switchable Intramolecular $\pi-\pi$-Stacking of Perylene Bisimide Dyes in a Cyclophane. Adv. Mater. 25, 410-414 (2013).

52. Brown, K. E. et al. Direct Observation of Ultrafast Excimer Formation in Covalent Perylenediimide Dimers Using Near-Infrared Transient Absorption Spectroscopy. J. Phys. Chem. Lett. 5, 2588-2593 (2014).

53. Ham, S. et al. Reconstruction of the Molecular Structure of a Multichromophoric System Using Single-Molecule Defocused Wide-Field Imaging. J. Phys. Chem. Lett. 5, 2830-2835 (2014).

54. Nicolai, H. T. et al. Unification of trap-limited electron transport in semiconducting polymers. Nat. Mater. 11, 882-887 (2012).

55. Mandoc, M. M. et al. Trap-limited electron transport in disordered semiconducting polymers. Phys. Rev. B 75, 193202 (2007).

56. Gregg, B. A. et al. Coulomb forces and doping in organic semiconductors. Chem. Mater. 16, 4586-4599 (2004).

57. Ball, M. et al. Chiral Conjugated Corrals. J. Am. Chem. Soc. 137, 9982-9987 (2015).

58. Jo, J. et al. Enhanced Efficiency of Single and Tandem Organic Solar Cells Incorporating a Diketopyrrolopyrrole-Based Low-Bandgap Polymer by Utilizing Combined $\mathrm{ZnO}$ /Polyelectrolyte Electron-Transport Layers. Adv. Mater. 25, 4783-4788 (2013).

59. Ball, M. L. et al. Influence of Molecular Conformation on Electron Transport in Giant, Conjugated Macrocycles. J. Am. Chem. Soc. 140, 10135-10139 (2018). 


\section{Chapter 6. Hollow Organic Capsules Assemble into Cellular Semiconductors}

\subsection{Preface}

Chapter 6 is reproduced with permission from the authors: Boyuan Zhang, Raúl Hernández Sánchez, Yu Zhong, Melissa Ball, Maxwell W. Terban, Daniel Paley, Simon J. L. Billinge, Fay Ng, Michael L. Steigerwald, and Colin Nuckolls, published in Nature Communications. ${ }^{1}$ Boyuan Zhang and $\mathrm{Yu}$ Zhong performed all device fabrication and characterization. Fay Ng synthesized all compounds. Raúl Hernández Sánchez performed XRD analysis. I performed DFT calculations and developed the HPLC method to separate the PDI isomers. Maxwell W. Terban performed PDF analysis.

\subsection{Introduction}

There is a growing class of electroactive, conjugated cyclic molecules that are being applied in several areas of materials science. ${ }^{2-28}$ These cyclic, conjugated organic semiconductors have interior spaces that should be useful as a locus for guest inclusion to tune the electronic and optoelectronic properties. ${ }^{12,25,27-29}$ Conjugated, cyclic semiconductors that incorporate PDIs have many benefits as the active elements in organic field effect transistors OFETs, ${ }^{30}$ OPVs, ${ }^{11,31}$ and OPDs. ${ }^{10}$ It has been challenging to synthesis rigid PDI-based macrocycles that interconvert as the previous chapters have demonstrated. ${ }^{5}$

Chapter 6 focuses on a new electronic material whose molecular components are shape persistent and self-assemble into semiconducting films that are capable of detecting nuanced differences in small molecule guests. The macrocycle comprises a tetra-brominated bithiophene $\left(\mathrm{BBr}_{4}\right)$ and a PDI wound into a trimeric structure called $\left(\mathbf{P B B r}_{4}\right)_{3}$. Figure 6.1a provides the structure of $\left(\mathbf{P B B r}_{4}\right)_{3}$. These macrocycles exist as a single pair of enantiomers and are shape persistent to temperatures above $160^{\circ} \mathrm{C}$. Their capsular structure is capped on the ends by the alkyl sidechains and on the equator by the electronic components (the PDI and $\mathrm{BBr}_{4}$ subunits). This is 
shown in Figure 6.1b-g. We found that $\left(\mathbf{P B B r}_{4}\right)_{3}$ 's cellular films act as the active layer in OFETs, and the electrical response depends on the guest that occupies the interior space.

(a)

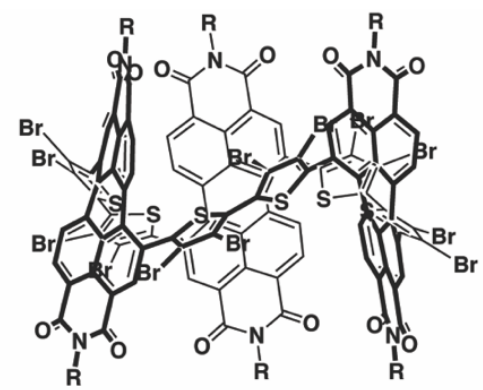

(b)

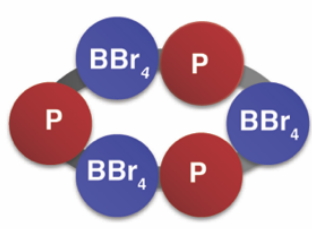

(d)

$\left(\mathrm{PBBr}_{4}\right)_{3}$

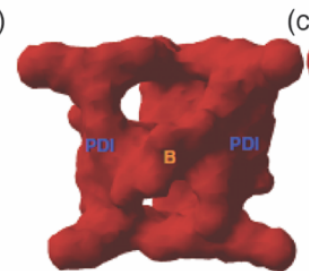

(e)
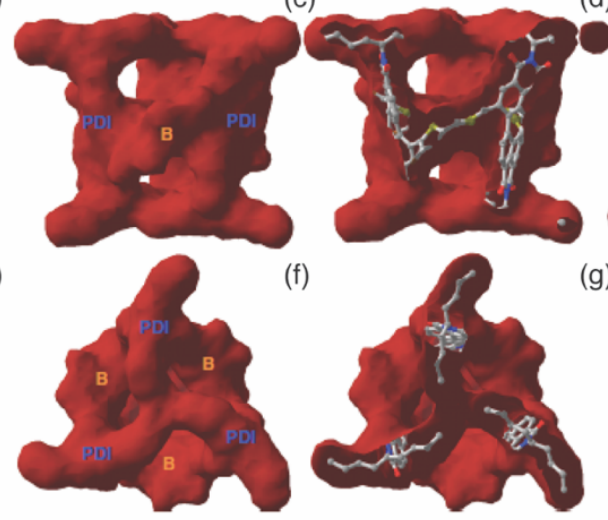

(f)

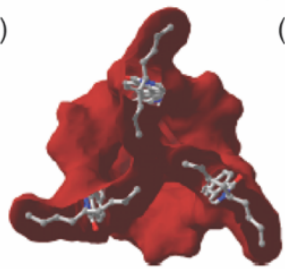

(g)

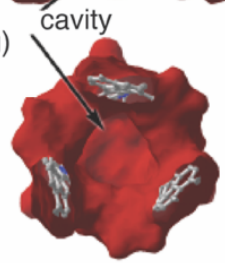

Figure 6.1. (a) Structure of $\left(\mathbf{P B B r}_{4}\right)_{3}$; (b) - (g) van der Waals Surface of $\left(\mathbf{P B B r}_{4}\right)_{3}$ seen from the side and top, trimmed to reveal the cavity $(\mathrm{g}) . \mathrm{R}=\mathrm{C}_{11} \mathrm{H}_{23}$ side chains.

\subsection{Capsule Construction}

We developed a synthesis of $\left(\mathbf{P B B r}_{4}\right)_{3}$ based on our own previous studies to make PDIbased macrocycles ${ }^{5,11}$ that builds from the methodology originally pioneered for cyclothiophenes ${ }^{2}$ and later for cycloparaphenylenes. ${ }^{21,32}$ Our earlier studies suggested the bithiophenes adopt a syn geometry when incorporated into a macrocycle, which provides strain relief (Chapter 1B.6 and Chapter 2.6). With three sets of bithiophenes, $\left(\mathbf{P B B r}_{4}\right)_{3}$ possesses little strain (Chapter 1B.5). Figure 6.2 contains the scheme to synthesis $\left(\mathbf{P B B r}_{4}\right)_{3}$. Using regio-pure 1,7-dibromo PDI, we form 6.1 via a Stille coupling, and react 6.1 with $\mathrm{PtCODCl}_{2}$, and after a ligand exchange and reductive elimination, afford intermediate $\mathbf{6 . 2}$ in a $15 \%$ yield. In addition to $\mathbf{6 . 2}$, the reaction produces several different sized macrocycles raning from the trimeric species to a hexameric structure (Section 6.9). 
We post-synthetically modify $\mathbf{6 . 2}$ with bromine atoms in the thiophene rings to encourage selfassembly through halogen bonding interactions to form $\left(\mathbf{P B B r}_{4}\right){ }^{33}$

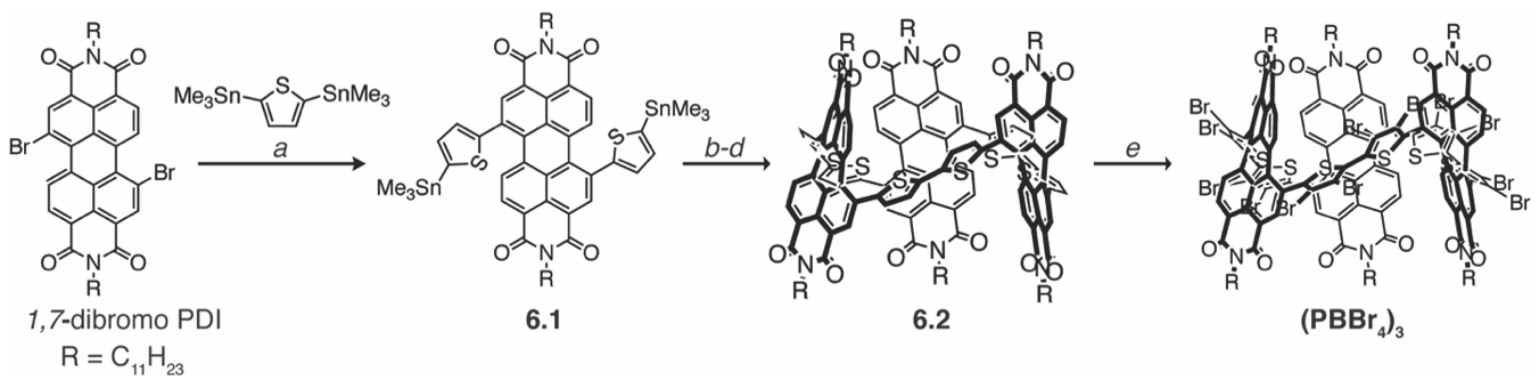

Figure 6.2. (a) $\mathrm{Pd}\left(\mathrm{PPh}_{3}\right)_{4}$, Toluene, $110{ }^{\circ} \mathrm{C}, 13 \mathrm{~h}, 69 \%$ yield; (b) $\mathrm{PtCODCl}_{2}$, 1,2-dichloroethane, $85^{\circ} \mathrm{C}, 72 \mathrm{~h}$; (c) 1,1'-ferrocenediyl-bis(diphenylphophine), $\mathrm{CH}_{2} \mathrm{Cl}_{2}, \mathrm{rt}, 24 \mathrm{~h}$; (d) $\mathrm{PPh}_{3}$, Toluene, $110{ }^{\circ} \mathrm{C}, 48 \mathrm{~h}, 15 \%$ yield for $\left(\mathbf{P B B r}_{4}\right)_{3}$; (e) $\mathrm{Br}_{2}, \mathrm{I}_{2}, \mathrm{CH}_{2} \mathrm{Cl}_{2}, \mathrm{rt}, 80 \%$ yield.

The 1,7-dithienyl-PDI subunit (6.1) introduces an element of chirality: ${ }^{5}$ it can exist in either a $P$ - or $M$-helical conformations. This allows for the possibility of two pairs of enantiomers $(P, P, P / M, M, M$ and $P, P, M / M, M, P)$. However, in the reaction to form 6.2, we only observe the $(P, P, P / M, M, M)$ pair. We separate the two enantiomers of $\mathbf{6 . 2}$, using a chiral stationary phase for HPLC, and monitor their interconversion as the samples are heated. The two enantiomers of 6.2 do not interconvert, even when heated up to $160{ }^{\circ} \mathrm{C}$, and both 6.2 and $\left(\mathbf{P B B r}_{4}\right)_{3}$ exhibit an intense (and opposite) chiroptic response in their CD spectra (Figure 6.3).
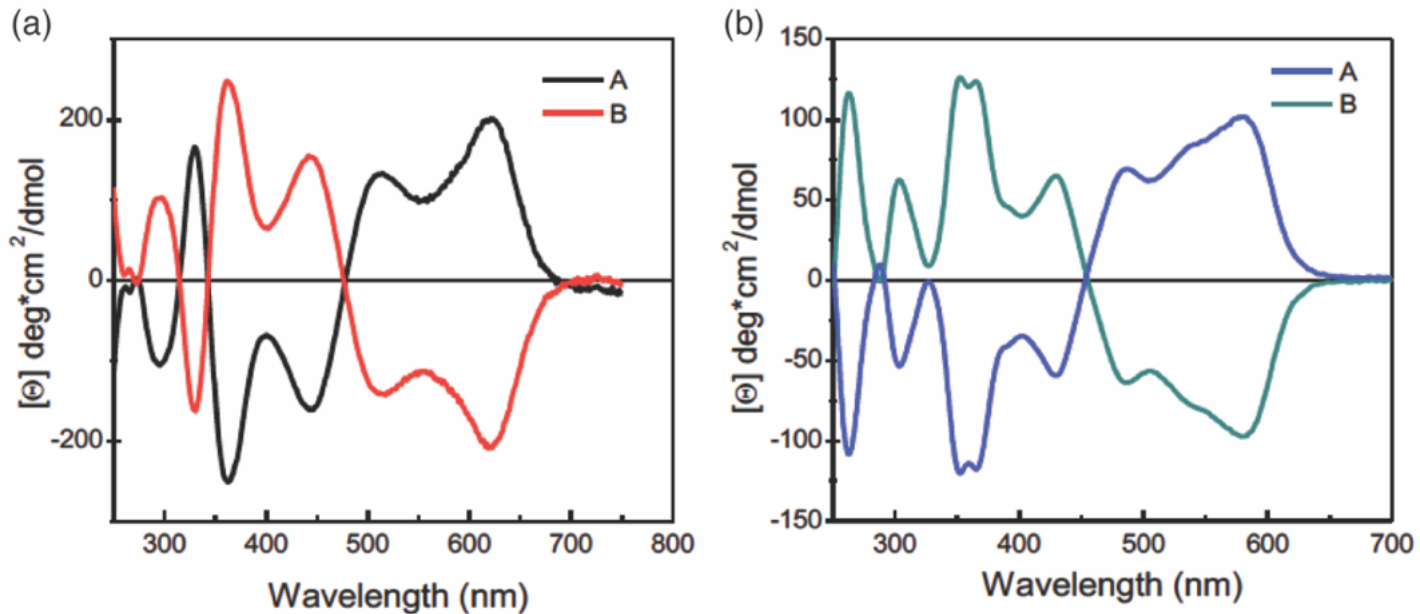

Figure 6.3. (a) $\mathrm{CD}$ of 6.2; (b) $\mathrm{CD}$ of $\left(\mathrm{PBBr}_{4}\right)_{3}$. Both spectra show each enantiomer preferentially absorbs left or right-handed circularly polarized light. 


\subsection{Capsule Structure.}

We were able to grow single crystals of sufficient quality to yield the structures of $\left(\mathbf{P B B r}_{4}\right)_{3}$. Figure 6.4 displays the structure of one of the two enantiomers of $\left(\mathbf{P B B r}_{4}\right)_{3}$ present in the solid-state: $(S S S)-\left(\mathbf{P B B r}_{4}\right)_{3}$. The structure of $\left(\mathbf{P B B r}_{4}\right)_{3}$ is cylindrical with the three sets of bithiophenes and three PDIs forming the walls at the equator. The ends of the cylinder are capped with branched alkyl chains (Figure 6.4 and highlighted in green in Figure 6.5a). This creates windows on the side of the structure, displayed in Figure 6.1b,e. We estimate the interior volume of the capsule in $\left(\mathbf{P B B r}_{4}\right)_{3}$ (shown in Figure 6.1d,g) to be approximately $415 \AA^{3} .{ }^{34}$
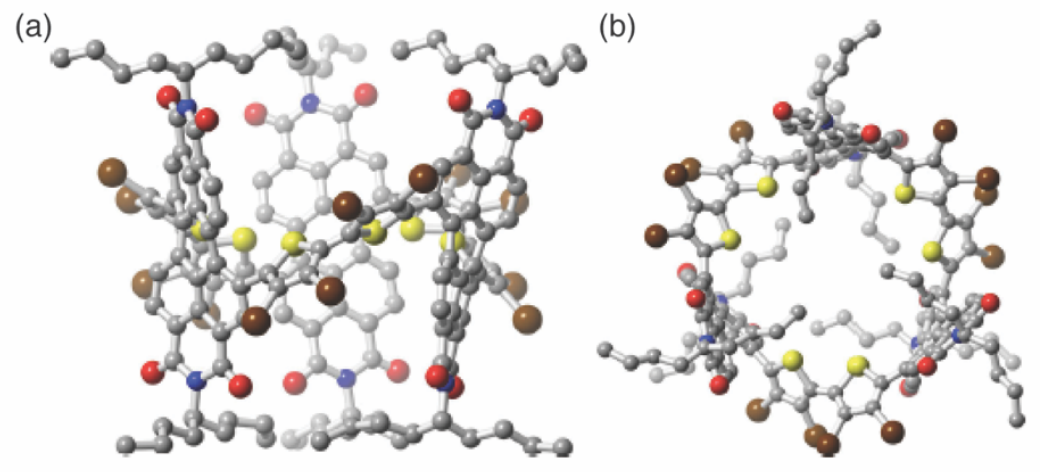

Figure 6.4. Molecular structure from SCXRD of (PBBr $)_{3}$. (a) Side and b, top view of (SSS)$\left(\mathrm{PBBr}_{4}\right)_{3} . \mathrm{C}, \mathrm{N}, \mathrm{O}, \mathrm{S}$, and $\mathrm{Br}$ atoms are colored in grey, blue, red, yellow, and brown, respectively. Hydrogen atoms have been removed to clarify the view. The alkyl chains on the imide are refined to only nine of the eleven carbon atoms due to disorder.

\subsection{Cellular Solids from Capsular Nanostructures.}

The packing structure in the solid state of $\left(\mathbf{P B B r}_{4}\right)_{3}$ (Figure 6.5) reveals why the bromines were necessary for long range crystallinity. The structure is composed of sheets of a honeycomblike arrangement in the a,b plane (Figure 6.5a). The interactions that bind the cylinders within the a,b plane are from neighboring brominated thiophene rings and the adjacent carbonyl groups of an adjacent macrocycle (Figure 6.5c). The PDIs do not $\pi$-stack with each other, and halogen bonding from the functionalized thiophenes drives the self-assembly process. The cavity of $\left(\mathbf{P B B r}_{4}\right)_{3}$ 
(a)

(b)

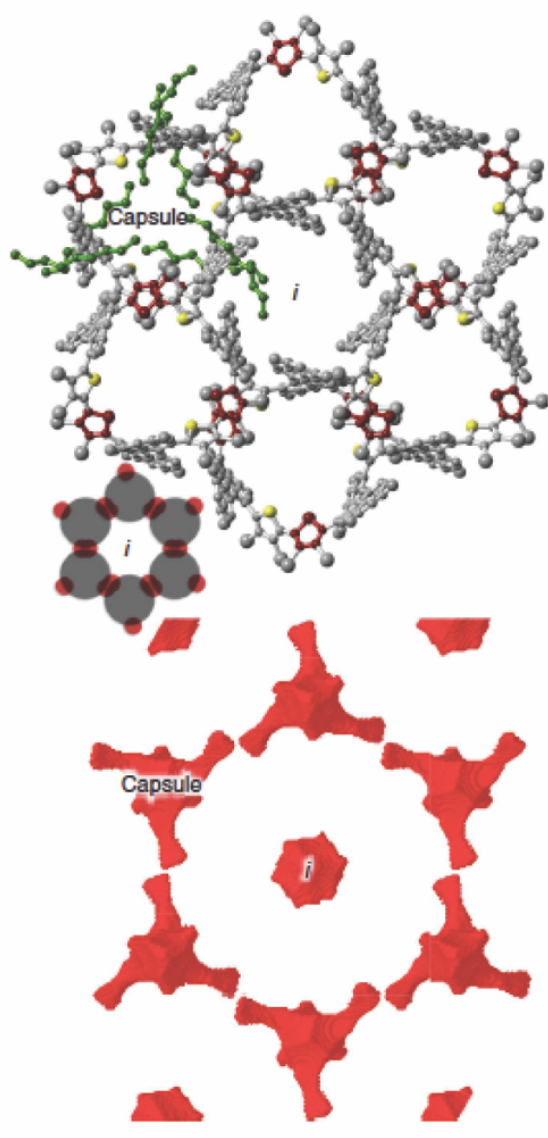

(c)

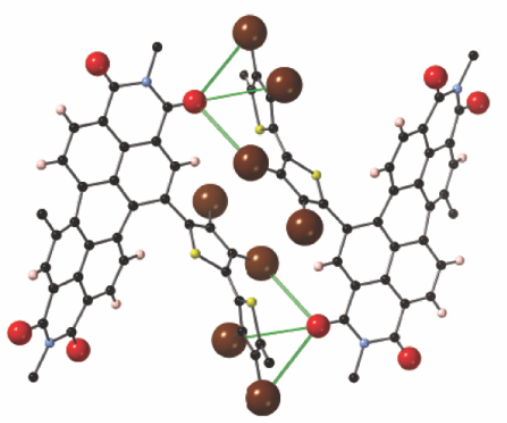

(d)
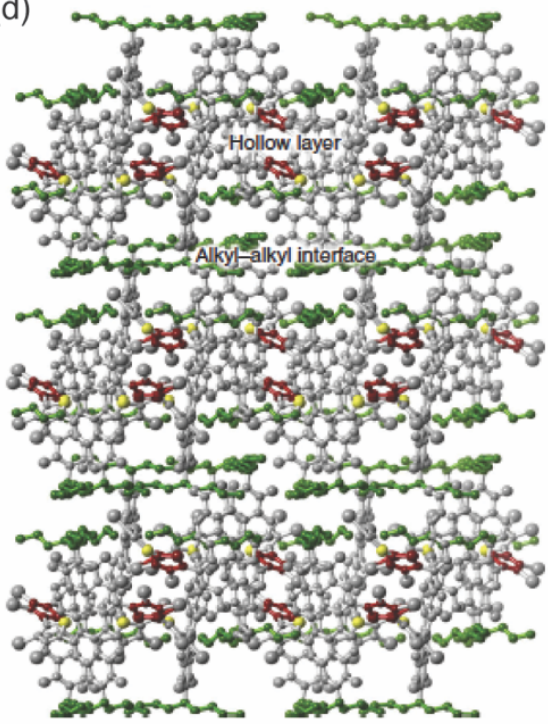

Figure 6.5. Structural packing of $\left(\mathbf{P B B r}_{4}\right)_{3}$. (a) view of the honeycomb structure in the ab plane for $\left(\mathbf{P B B r}_{4}\right)_{3}$. The capsule and $i$ corresponds to the internal cavity of $\left(\mathbf{P B B r}_{4}\right)_{3}$ and the cavity formed by the packing of $\left(\mathbf{P B B r}_{4}\right)_{3}$, respectively. The remaining sulfur atoms are colored in yellow to provide a marker to identify the macrocycle cavities. See bottom left cartoon. Highlighted in green are the imide side chains (some of the sidechains have been removed to clarify the view of the cavity). In red are the thiophene rings. (b) Surface map of the void space in the ab plane of (PBBr $)_{3}$. (c) Two molecules of $\left(\mathbf{P B B r}_{4}\right)_{3}$ showing the $\mathrm{C}=\mathrm{O} \ldots \mathrm{Br}-\mathrm{C}$ halogen interaction responsible for the 2D assembly. (d) View of the packing of $\left(\mathbf{P B B r}_{4}\right)_{3}$. As shown, the vertical stacking follows the c axis. The alkyl sidechains of the imide are shown in green. Hydrogen atoms have been removed from all structures to clarify the view.

(labeled "capsule" in Figure $6.5 \mathrm{a}, \mathrm{b}$ ) is $\sim 11.4 \AA$ in diameter and is a three-fold symmetric chiral nanoenvironment for guest incorporation within the two-dimensional layer. Due to the packing of the subunits of $\left(\mathbf{P B B r}_{4}\right)_{3}$ into a hexameric cyclic structure, a second cavity forms at the center of each hexagon (labeled $i$ in Figure 6.5a,b). These honeycomb two-dimensional, cellular sheets then stack through the packing of the alkyl side chains of the imides, (shown in green in Figure 6.5). 
This packing arrangement propagates along the c-axis, hinting that these materials could likely be exfoliated to yield molecularly thin sheets of $\left(\mathbf{P B B r}_{4}\right)_{3}$.

We find that $\left(\mathbf{P B B r}_{4}\right)_{3}$ self-assembly in cast thin films and powder samples is analogous to what we described above for the single crystal. Figure 6.6 compares the thin film and powder diffraction data for $\left(\mathbf{P B B r}_{4}\right)_{3}$ with the simulated pattern calculated from the SCXRD data. No other reflections are present in the films or powders indicating that the self-assembly motif using the halogen bonding is robust.

(a)

(b)

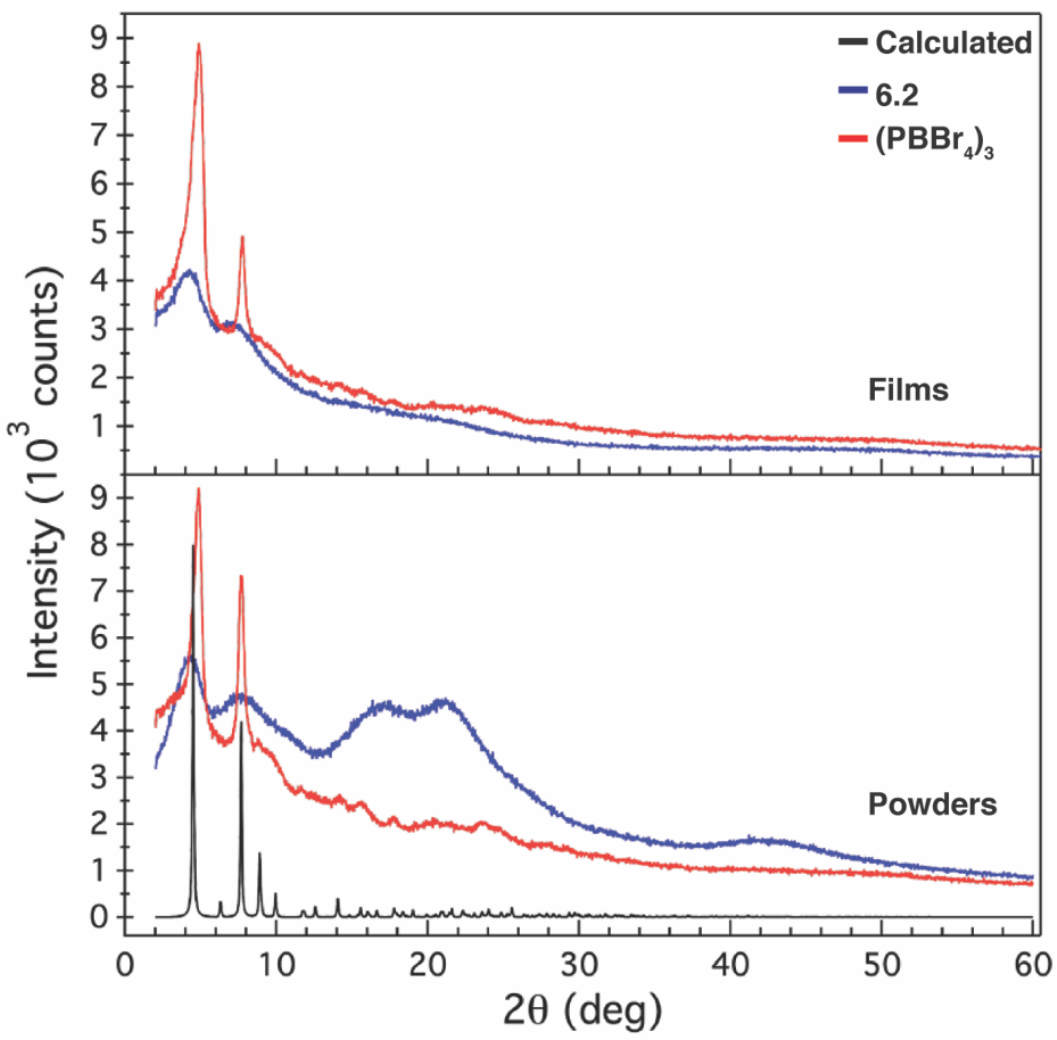

Figure 6.6. $P X R D$ of $\left(\mathrm{PBBr}_{4}\right)_{3}$ and 6.1. (a) Films drop cast from a chloroform solution; (b) data obtained from powder samples. The black line represents the predicted pattern for $\left(\mathbf{P B B r}_{4}\right)_{3}$.

We performed pair distribution function (PDF) analyses on powders of 6.1 and $\left(\mathbf{P B B r}_{4}\right)_{3}$ to extract quantitative information about the long-range crystallinity of these new hollow semiconductors. We found that the crystalline domains for $\left(\mathbf{P B B r}_{4}\right)_{3}$ contain on average up to 23 to 27 capsules arranged in one direction relative to just a few capsules for 6.1 (Figure 6.9). 


\subsection{Electron Transport through Cellular Films.}

Figure 6.7 displays transfer curves from an OFET constructed using a self-assembled thin film of $\left(\mathbf{P B B r}_{4}\right)_{3}$. Details for the device dimensions and its properties can be found in the Appendix. The device exhibits electron transporting character and has a mobility of $\sim 1.5 \times 10^{-2}$ $\mathrm{cm}^{2} \mathrm{~V}^{-1} \mathrm{~s}^{-1}$. The mobility of $\left(\mathbf{P B B r}_{4}\right)_{3}$ is more than 20 times greater than that of $6.1\left(\sim 6.8 \times 10^{-4}\right.$ $\mathrm{cm}^{2} \mathrm{~V}^{-1} \mathrm{~s}^{-1}$ ) (Figure 6.10). We attribute this to the robust self-assembly process for $\left(\mathbf{P B B r}_{4}\right)_{3}$ described in Section 6.4. From the AFM height images, films of 6.1 display a smooth surface with RMS roughness of $0.347 \mathrm{~nm}$; in contrast, $\left(\mathbf{P B B r}_{4}\right)_{3}$ displays a larger RMS roughness of $3.2 \mathrm{~nm}$, presumably due to its more crystalline nature and better self-assembly properties (Figure 6.11).
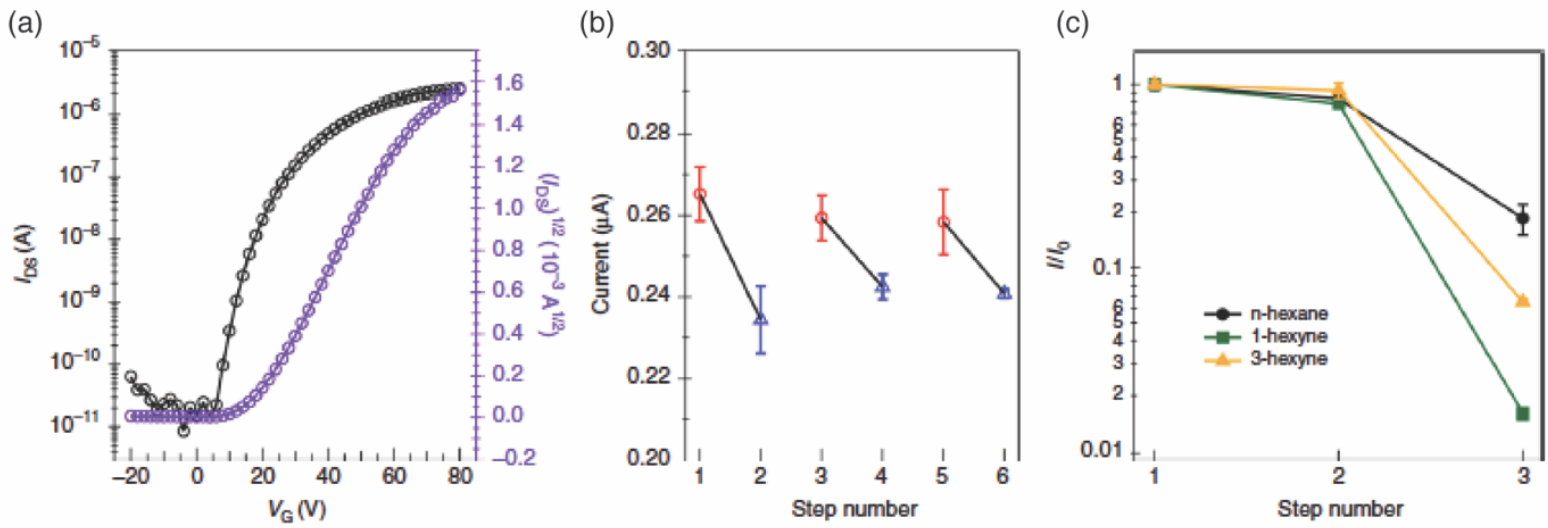

Figure 6.7. Charge transport for $\left(\mathrm{PBBr}_{4}\right)_{3}$. (a) Transfer characteristics of OFET device for (PBBr 4$)_{3}$; (b) device cycling response under vacuum (red circles) and $\mathrm{N}_{2}$ atmosphere (blue triangles); and (c) normalized behavior of the device response under vacuum (step 1), $\mathrm{N}_{2}$ (step 2), and different analytes atmosphere (step 3: n-hexane, 3-hexyne and 1-hexyne). Error bars represent the standard error obtained in three measurements.

The exciting finding is that the devices show modulation in the drain current depending on what, if anything, is within the cavities of the macrocycles. Every potential guest tested had a measurable effect on the drain current in the device, but the absolute levels of the drain current varied depending on the guest. Figure $6.7 \mathrm{~b}$ is a representative data set for a device measured sequentially in steps as the atmosphere is changed between vacuum and $\mathrm{N}_{2}$. In certain cases, traditional OFETs show a differential response to nitrogen and vacuum that is from extrinsic 
effects (moisture, oxygen, or dielectric effects). ${ }^{35}$ We were curious whether the response to $\mathrm{N}_{2}$ for $\left(\mathbf{P B B r}_{4}\right)_{3}$ was due to an extrinsic effect or due to the macrocycle's ability to sense the gas, and made a control FET device from 6.2, which does not self-organize into cellular semiconducting films or crystals. We observe no response when comparing its response to nitrogen and vacuum (Figure 6.12a).

Incorporating more polarizable and functional guests causes more pronounced changes in the drain current. Figure $6.12 \mathrm{~b}$ compares the effects of several different guests with a variety of functional groups such as ketones, alcohols, nitriles, alkynes, and alkanes. In each case we are able to differentiate the guest by the current in the device. Figure $6.7 \mathrm{c}$ highlights one striking example of how this material responds to a series of closely related hydrocarbons (3-hexyne, 1-hexyne, and n-hexanes). These hydrocarbons were chosen so that their length, size, and polarity were roughly similar to that of n-hexane, while having an additional functional group in them. We compare the transistor output for three devices exposed to n-hexane, 3-hexyne, or 1-hexyne, carried by nitrogen under their saturated vapor pressure $\left(148,99\right.$, and $138 \mathrm{mmHg}$ at $25^{\circ} \mathrm{C}$, respectively).$^{36} \mathrm{In}$ each case, the devices can both easily detect and distinguish each hydrocarbon. There is also specificity towards particular analytes; the trend in the device responses does not simply follow the vapor pressure.

For n-hexane and 3-hexyne the data was collected after the OFET was in contact with the vapor for $\sim 70$ minutes (Figure $6.8 \mathrm{a}$ ). After this time, the drain current no longer decreased. The original current levels for n-hexane and 3-hexyne could be restored by removing the analyte by placing the devices in vacuum (Figure 6.8b) During these long exposures, we speculate that the guests infiltrate the films to reside in the active part of the films at the gate dielectric interface..$^{37,38}$ 

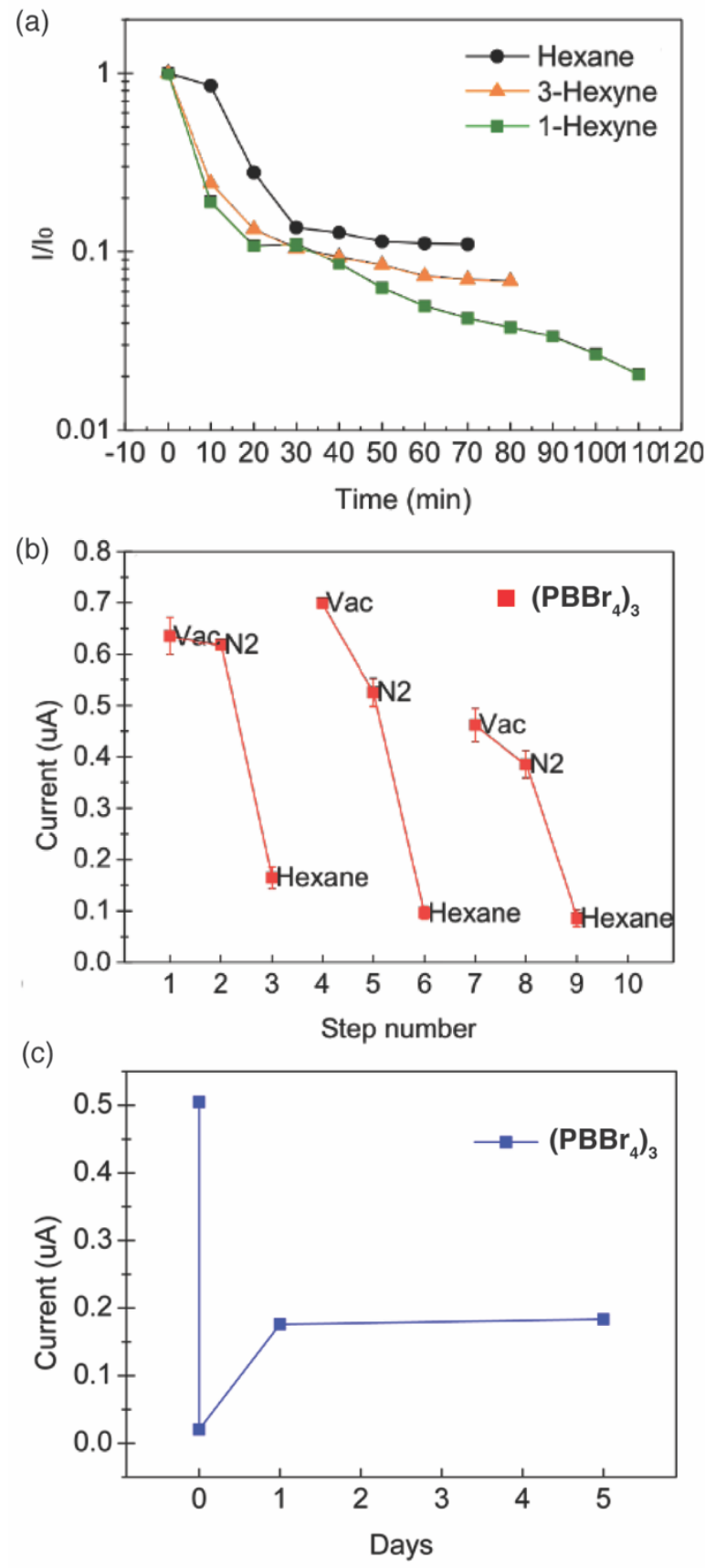

Figure 6.8. (a) $\mathrm{I} / \mathrm{I}_{0}$ for $\left(\mathrm{PBBr}_{4}\right)_{3}$ under $n$-hexane, 3-hexyne and 1-hexyne. (b) Device cycling response for $\left(\mathbf{P B B r}_{4}\right)_{3}$ under vacuum, $\mathrm{N}_{2}$ and hexane atmosphere. Error bars represent the standard error obtained in three measurements. (c) Recovery response for $\left(\mathbf{P B B r}_{4}\right)_{3}$ under vacuum after exposure to 1-hexyne. The films do not recover their original current levels.

We tested the sensitivity of $\left(\mathbf{P B B r}_{4}\right)_{3}$ films and find that there is a linear response between vacuum and $20 \mathrm{ppt}$ of the analyte in the atmosphere, after we observed a plateau region as the concentration reaches saturation (Figure 6.13). The guests can infiltrate the porous network 
through the capsule's interior $\left(\sim 415 \AA^{3}\right)$ or the $i$-sites $\left(\sim 110 \AA^{3}\right)$. Note that n-hexane has a van der Waals molecular volume of $\sim 113 \AA^{3} \cdot{ }^{39}$ The implication is that $\mathrm{n}$-hexane would not fit into the $i$ site. In addition, most of the cellular nature of self-assembled $\left(\mathbf{P B B r}_{4}\right)_{3}$ comes from the capsule interior which is present in a 2:1 numerical ratio relative to the $i$-site. Given the 8 -fold difference in volume between these two cavities we speculate that guests can be accommodated most feasibly at the capsules' interior. Using BET, we find that powders of $\left(\mathbf{P B B r}_{4}\right)_{3}$ have a surface area of 20 $\mathrm{m}^{2} \mathrm{~g}^{-1}$ (versus $1.2 \mathrm{~m}^{2} \mathrm{~g}^{-1}$ for 6.2)

1-hexyne behaves differently than each of the other guests tested (Figure 6.8c). For the devices in an atmosphere of 1-hexyne, the current continues to drop and does not reach a plateau even at times that exceed 2 hours of exposure. In addition, the 1-hexyne devices do not recover to their original levels when placed in vacuum. We speculate that the terminal alkyne is undergoing a reaction under the device conditions that is not possible with the internal alkyne or the alkane. This offers the intriguing possibility that in addition to sensing, these nanoenvironments in these hollow semiconductors ${ }^{40,41}$ can be used as nanoreactors.

\subsection{Conclusion}

We have described here a shape persistent, hollow macrocycle that self-assembles both in the solid state and thin films to form cellular organic semiconductors. The macrocycle is chiral and conformationally locked into a capsular structure, with $\sim 415 \AA^{3}$ volume on its interior. The self-assembly of a brominated derivative of the trimer into cellular films forms the active layer in an organic field effect transistor device. Once assembled, these films have periodic, nanoscopic, cellular voids in them. Because the macrocyclic component in the film is conformationally locked, the self-assembled films maintain their interior, open spaces. The hollow films of $\left(\mathbf{P B B r}_{\mathbf{4}}\right)_{3}$ are 
responsive to the atmosphere in which the OFETs are measured. These studies chart a clear path to using the interior of the cellular organic semiconductors as gas sensors and nanoreactors.

\subsection{Appendix - Supplementary Figures}

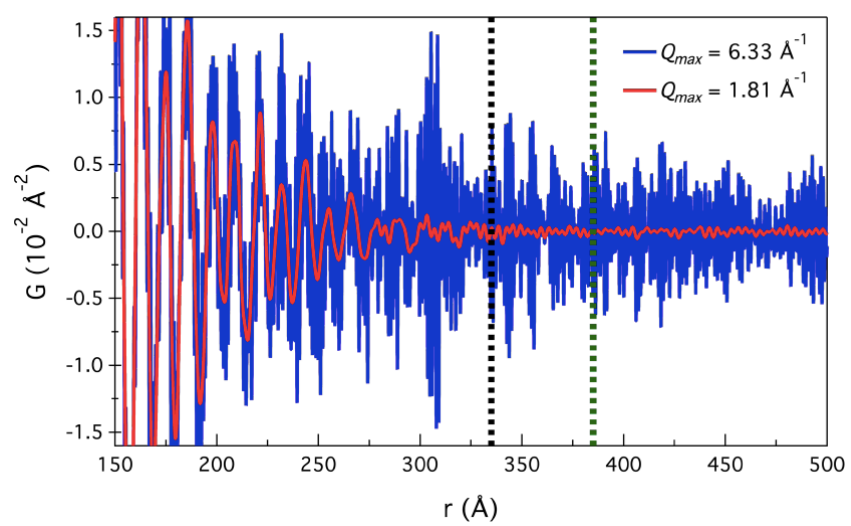

Figure 6.9. Coherence length for $\left(\mathrm{PBBr}_{4}\right)_{3}$. We can approximate the coherence length lower bound at $335 \AA$ (black dotted line) and upper bound at $385 \AA$ (green dotted line). ${ }^{42}$ The online Supporting Information contains the additional PDF analysis details and spectra for $\mathbf{6 . 2}$ and for $\left(\operatorname{PBBr}_{4}\right)_{3}$. 1

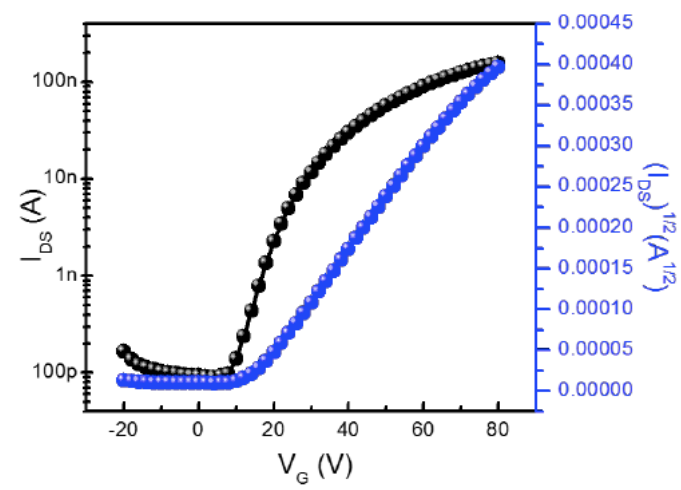

Figure 6.10. Transfer characteristics for 6.2.
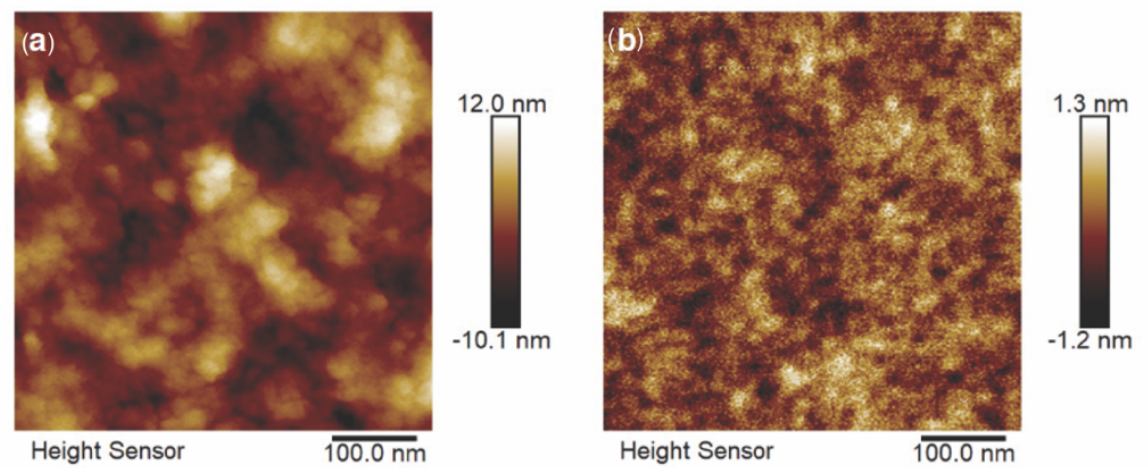

Figure 6.11. (a) AFM height images of spin-cast films for (a) $\left(\mathbf{P B B r}_{4}\right)_{3}$ and (b) 6.2. The root mean square roughness are $3.2 \mathrm{~nm}$ for $\left(\mathbf{P B B r}_{4}\right)_{3}$ and $0.347 \mathrm{~nm}$ for 6.2, respectively. 

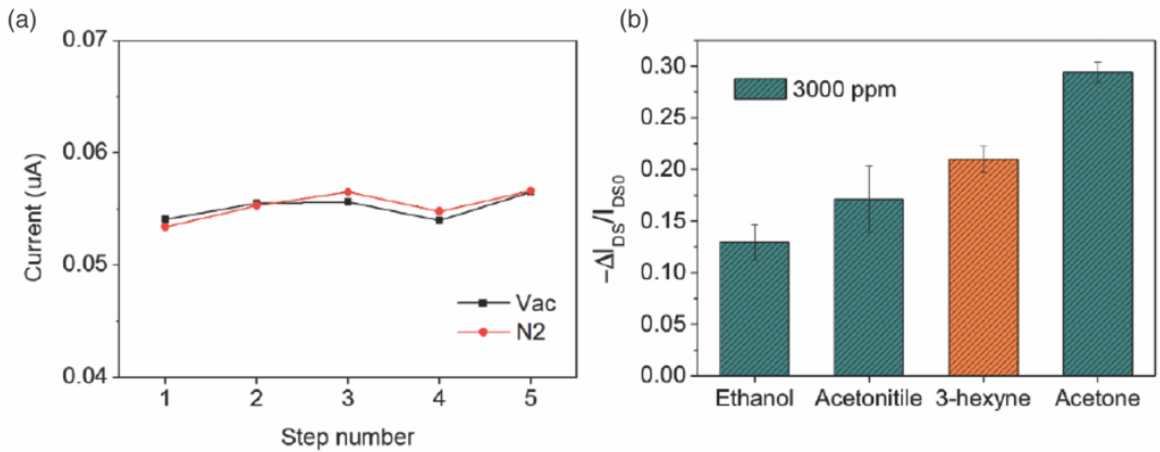

Figure 6.12. (a) Device cycling response for 6.2 under vacuum and $\mathrm{N}_{2}$ atmosphere. (b) The average current of response for $\left(\mathbf{P B B r}_{4}\right)_{3}$ OFET to series of analytes at a concentration of 3000 PPM. Error bars represent the standard error obtained in three measurements.

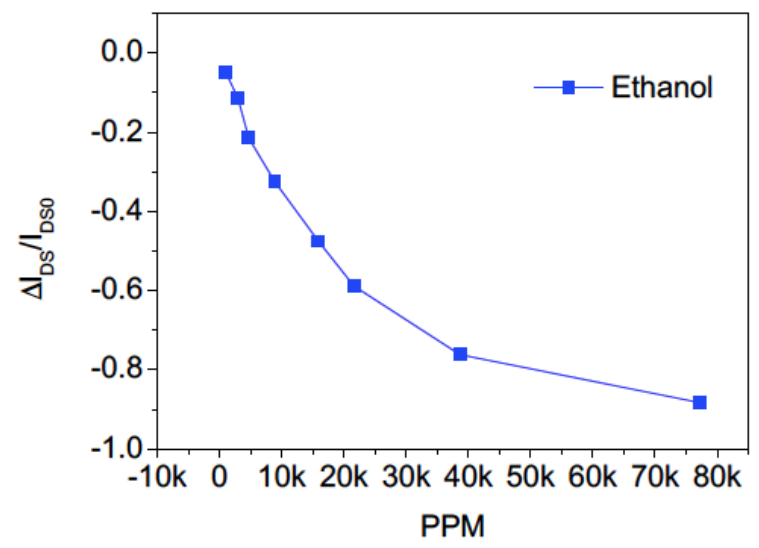

Figure 6.13. Sensitivity for $\left(\mathrm{PBBr}_{4}\right)_{3}$ OFET under different concentrations of ethanol.

\subsection{General Experimental Information}

All reactions were performed in oven-dried or flame-dried round bottom flasks, unless otherwise noted. The flasks were fitted with Teflon magnetic stir bar, rubber septa and reactions were conducted under a positive pressure of nitrogen, unless otherwise noted. Anhydrous and anaerobic solvents were obtained from Schlenk manifold with purification columns packed with activated alumina and supported copper catalyst (Glass Contour, Irvine, CA). Automated flash chromatography was performed using a Teledyne Isco Combiflash $\mathrm{R}_{\mathrm{f}} 200$ and Redisep $\mathrm{R}_{\mathrm{f}}$ Silica columns. Preparative HPLC purification was performed on a Waters Prep150 instrument equipped with a UV-vis detector, an automated fraction collector with either a Nacalai Tesque COSMOSIL 
Buckyprep column (20 mm ID x $250 \mathrm{~mm}, 5 \mu \mathrm{m}$ ) or a COSMOSIL 5PBB column (20 mm ID x $250 \mathrm{~mm}, 5 \mu \mathrm{m})$.

Chiral Analyses and Purification. Racemic trimer 6.2 samples were resolved by an Agilent 1200 Series analytical HPLC equipped with a diode array detector (300 $\mathrm{nm}$ to $900 \mathrm{~nm}$ ) and a CHIRALPAK IA-3 column (4.6 mm ID x $250 \mathrm{~mm}, 3 \mu \mathrm{m}$ ) from Chiral Technologies; preparative purification was performed using CHIRALPAK IA-3 column (21 mm ID x $250 \mathrm{~mm}, 5 \mu \mathrm{m})$ on Waters Prep150 instrument.

Materials. Pure regioisomeric 1,7-dibromoperylene-3,4,9,10-tetracarboxylicbisimides was synthesized (as a mixture of 1,7- and 1,6-regioisomers) according to known procedures ${ }^{43}$ and separated using COSMOSIL Buckyprep column (20 mm ID x $250 \mathrm{~mm}, 5 \mu \mathrm{m}$ ) on Waters Prep150 instrument. All chemicals were purchased from commercial sources and used without further purification unless otherwise specified.

Instrumentation. ${ }^{1} \mathrm{H}$ and ${ }^{13} \mathrm{C}$ NMR spectra were recorded on Bruker DRX400 (400 MHz) or a Bruker DMX500 (500 MHz) spectrometer. Chemical shifts for protons are reported in parts per million downfield from tetramethylsilane and are referenced to residual protium in the NMR solvent $\left(\mathrm{CHCl}_{3}: \delta 7.26 ; \mathrm{CH}_{2} \mathrm{Cl}_{2}: \delta 5.32 ; \mathrm{C}_{2} \mathrm{H}_{2} \mathrm{Cl}_{4}: \delta 6.00\right)$. Chemical shifts for carbon are reported in parts per million downfield from tetramethylsilane and are referenced to the carbon resonances of the solvent $\left(\mathrm{CDCl}_{3} \delta 77.0 ; \mathrm{C}_{2} \mathrm{H}_{2} \mathrm{Cl}_{4} \delta 73.78\right)$. Data are represented as follows: chemical shift, multiplicity $(\mathrm{s}=$ singlet, $\mathrm{d}=$ doublet, $\mathrm{t}=$ triplet, $\mathrm{m}=$ multiplet, $\mathrm{br}=$ broad $)$, coupling constants in Hertz, and integration. Some ${ }^{1} \mathrm{H}$ NMR and ${ }^{13} \mathrm{C}$ NMR were recorded at elevated temperatures (in $\mathrm{K})$ to enhance peak resolution in the aromatic region. Resonances corresponding to the numerous aromatic carbon atoms in the reported compound sometimes overlap, thereby reducing the number of observed resonances. HRMS was performed on (1) a Waters XEVO G2-XS QTOF instrument 
equipped with a UPC2 SFC inlet, and electrospray (ESI) and atmospheric pressure chemical (APCI) ionization sources; or (2) a Bruker UltrafleXtreme MALDI TOF instrument using dithranol matrix. Absorption spectra were obtained on Shimadzu UV 1800 UV-Vis spectrophotometer and emission spectra were recorded in a Fluorolog-3 spectrophotometer.

Cyclic voltammograms: CVs were recorded on a CHI600C electrochemical workstation using $\mathrm{Ag} / \mathrm{AgCl}$ electrode as the reference electrode. $0.1 \mathrm{M}$ solution tetrabutylammonium hexafluorophosphate, $\left[\mathrm{Bu}_{4} \mathrm{~N}\right]\left[\mathrm{PF}_{6}\right]$, in dichloromethane was used as the supporting electrolyte. The thin film transistors were tested on the Agilent $4155 \mathrm{C}$ semiconductor parameter analyzer. CD spectra were recorded by a Jasco J-810 spectropolarimeter. It is assumed that the absolute energy level for $\mathrm{Fc} / \mathrm{Fc}^{+}$redox potential is $-4.80 \mathrm{eV}$ with respect to vacuum level. The energy levels of the LUMO $)$ and HOMO are calculated according to the following equation: $\mathrm{E}_{\mathrm{LUMO}}=-\mathrm{e}\left(\mathrm{E}_{\mathrm{red}}+4.80\right)$ $(\mathrm{eV}), \mathrm{E}_{\text {номо }}=-\mathrm{e}\left(\mathrm{E}_{\text {oxi }}+4.80\right)(\mathrm{eV}) .^{44}$

AFM was performed with a PSIA XE100.

PXRD was collected on a PANalytical X'Pert ${ }^{3}$ Powder Diffractometer. Data was collected on powder samples and films drop-cast from chloroform solution and p-xylene solution. For all data collection a Si zero-background holder was used.

Single crystal data for $\left(\mathrm{PBBr}_{4}\right)_{3}$ was collected on an Agilent SuperNova diffractometer using a mirror-monochromated $\mathrm{Cu} \mathrm{K} \mathrm{K}_{\alpha}$ radiation. The hexagonal-shaped crystals were mounted on a MiTeGen Kapton loop (polyimide). These were cooled to $100 \mathrm{~K}$ with an Oxford Cryosystems nitrogen flow apparatus. Data integration, scaling (ABSPACK) and absorption correction were perfomed in CrysAlisPro. ${ }^{45}$ Structure solution was performed using ShelXS ${ }^{46}$ ShelXT, ${ }^{47}$ or SuperFlip. ${ }^{48}$ Subsequent refinement was performed by full-matrix least-squares on $\mathrm{F}^{2}$ in ShelXL. Olex $2^{34}$ was used for viewing and to prepare CIF files. PLATON ${ }^{49}$ was used for SQUEEZE, ${ }^{50}$ 
ADDSYM $^{51}$ and TwinRotMat. Details of crystallographic data and refinement parameters are given in Table 6.2. Due to heavy disorder of the alkyl imide chains in $\left(\mathbf{P B B r}_{4}\right)_{3}$, only nine carbons (out of eleven) in each alkyl fragment were modeled. The cavity size of $\left(\mathbf{P B B r}_{4}\right)_{3}$ was calculated from the solvent accessible volume calculator in Olex2. By employing this functionality we found discrete pockets within the structure of $\left(\mathbf{P B B r}_{4}\right)_{3}$ which match the cavities of these molecules. Thus, the calculated cavity size of $(M, M, M / P, P, P)-\left(\mathbf{P B B r}_{4}\right)_{3}$ is $414.9 \AA^{3}$ (CalcSolv $3.0 \AA$ probe, grid step $0.2 \AA$ ).

Table 6.1. Crystallographic data for $\left(\mathrm{PBBr}_{4}\right)_{3}$.

\begin{tabular}{|c|c|}
\hline & $\left(\mathbf{P B B r}_{4}\right)_{3}$ \\
\hline Chemical formula & $\mathrm{C}_{150} \mathrm{H}_{132} \mathrm{Br}_{12} \mathrm{~N}_{6} \mathrm{O}_{12} \mathrm{~S}_{6}$ \\
\hline Formula weight & 3361.89 \\
\hline Space group & $P-3$ \\
\hline$a(\AA)$ & $23.028(4)$ \\
\hline$b(\AA)$ & $23.028(4)$ \\
\hline$c(\AA)$ & $19.626(3)$ \\
\hline$\alpha(\mathrm{deg})$ & 90 \\
\hline$\beta(\mathrm{deg})$ & 90 \\
\hline$\gamma(\mathrm{deg})$ & 120 \\
\hline$V\left(\AA^{3}\right)$ & $9013(3)$ \\
\hline$Z$ & 2 \\
\hline$\mu\left(\mathrm{mm}{ }^{-1}\right)$ & 4.21 \\
\hline $\mathrm{T}(\mathrm{K})$ & 100 \\
\hline
\end{tabular}




\begin{tabular}{|c|c|}
\hline$R 1^{\mathrm{a}}\left(w R 2^{\mathrm{b}}\right)$ & $0.157(0.476)$ \\
\hline Reflections & 3155 \\
\hline Radiation type & $\mathrm{Cu} \mathrm{K} \alpha$ \\
$\left.{ }^{\mathrm{a}} R 1=\left[\sum w\left(F_{\mathrm{o}}-F_{\mathrm{c}}\right)^{2} / \Sigma w F_{\mathrm{o}}{ }^{2}\right]^{1 / 2} ;{ }^{\mathrm{b}} w R 2=\left[\Sigma\left[w\left(F_{\mathrm{o}}{ }^{2}-F_{\mathrm{c}}{ }^{2}\right)^{2}\right] / \Sigma w\left(F_{\mathrm{o}}{ }^{2}\right)^{2}\right]^{1 / 2}\right], w$ \\
$\left.+(\mathrm{a} P)^{2}+\mathrm{b} P\right]$, where $P=\left[\max \left(F_{\mathrm{o}}{ }^{2}, 0\right)+2\left(F_{\mathrm{c}}{ }^{2}\right)\right] / 3$
\end{tabular}$=1 /\left[\sigma^{2}\left(F_{\mathrm{o}}{ }^{2}\right)\right.$

\section{$\mathrm{X}$-ray total scattering experiments (PDF analysis)}

X-ray total scattering experiments were conducted on beamline 28 -ID-2 at the National Synchrotron Light Source II at Brookhaven National Laboratory. An X-ray beam of energy 67.756 $\operatorname{keV}(\lambda=0.18299 \AA)$ was focused on samples loaded into Kapton capillaries. Scattered intensities were collected at room temperature, in rapid acquisition mode ${ }^{52}$ on a Perkin-Elmer 2D flat panel detector ( 2048 × 2048 pixels and $200 \times 200 \mu \mathrm{m}$ pixel size) mounted orthogonal to the beam path. Data collection was carried out with a short $(239.9734 \mathrm{~mm})$ and a large (1552.689 mm) sampleto-detector distance to obtain improved Q-space resolution. Q is the magnitude of the scattering momentum transfer where for elastic scattering is defined as: $\mathrm{Q}=(4 \pi \sin (\theta)) \lambda$, for scattering angle $2 \theta$ and wavelength $\lambda$. A Ni standard sample was measured in both cases to calibrate the detector geometry. 2D intensities were azimuthally integrated to $1 \mathrm{D}$ intensities versus $\mathrm{Q}$ using Fit2D. ${ }^{53}$ Scattering from an empty Kapton tube was measured for background subtraction. The PDF gives the scaled probability of finding atom-pairs in the material at a distance $r$ apart. The program xPDFsuite with PDFGetX3 $3^{54,55}$ was used to obtain the PDFs from the experimental scattering intensities. The coherent scattering $\mathrm{I}(\mathrm{Q})$ was extracted through background subtraction and corrections to the raw intensities, then normalized by the atomic scattering factors to give the total scattering structure function $\mathrm{S}(\mathrm{Q})$ which is converted to the real-space pair distribution function (PDF), G(r), by 


$$
G(r)=\frac{2}{\pi} \int_{Q_{\min }}^{Q_{\max }} Q[S(Q)-1](Q) \sin (Q r) d Q
$$

where $Q_{\min }$ and $Q_{\max }$ are the minimum and maximum values of the scattering momentum transfer considered. These limits were determined by the $Q$-range accessible for the different detector configurations, $0.29-22.0 \AA^{-1}$ for the short sample-to-detector distance which gives a wide range of momentum transfer in order to achieve a high real-space resolution in the PDFs. The far sampleto-detector distance gives a smaller $Q$-range of $0.20-6.33 \AA^{-1}$, which gives a lower real space resolution, but provides a much better $Q$-resolution of the scattering which allows the resulting PDFs to be analyzed over longer real-space distances. ${ }^{56,57} \mathrm{In}$ this case, the $Q_{\max }$ was reduced further to decrease the noise level below any signal observable at high distances. The structural coherence of the sample was estimated from visual observation of the distance at which the structural signal became indistinguishable from the average atomic density, $G(r)=0$.

It is important to note the following for the coherence length estimation: (1) all approximated coherence lengths are well below the resolution limit determined from fitting $\mathrm{Ni}$ measured with the same experimental setup, and (2) the approximated lengths may be underestimated as the presence of noise may still obscure some high-r signals.

Electron Transport. We first treat the substrate $\left(300 \mathrm{~nm}\right.$ of $\mathrm{SiO}_{2}$ on a $\mathrm{Si}$ wafer) with OTS in order to passivate traps on the $\mathrm{SiO}_{2}$ surface. Au source and drain electrodes are deposited on the film to make a bottom-contact configuration. We then spin-cast films of 6.2 and $\left(\mathbf{P B B r}_{4}\right)_{3}$ onto this surface at 1000 r.p.m. for $1 \mathrm{~min}$, to form transistors using the silicon wafer as the global back gate for the device. The thickness of the organic films is $15-20 \mathrm{~nm}$. OFETs made from thicker films $(40-60 \mathrm{~nm})$ exhibit nonlinear characteristic at low bias voltage. Finally, the film of $(\mathbf{P B B r})_{3}$ was annealed under p-xylene vapor for $10 \mathrm{~min}$ and then annealed under inert atmosphere at $160{ }^{\circ} \mathrm{C}$ for 10 mins to optimize the device performance. The film of 6.2 was annealed under inert atmosphere 
at $200{ }^{\circ} \mathrm{C}$ for 10 mins to optimize the device performance. Vapor annealing of p-xylene didn't show enhanced performance for $\mathbf{6 . 2}$.

The mobility is calculated in the saturation regime using $I_{\mathrm{DS}}=(W / 2 L) C \mathrm{i} \mu\left(V_{\mathrm{G}}-V_{\mathrm{T}}\right)^{2}$, where $W$ and $L$ are the width and length of the channel, $C i\left(11.5 \mathrm{nFcm}^{-2}\right), \mu$, and $V_{\mathrm{T}}$ correspond to the capacitance per unit area of the gate insulator, the field effect mobility, and the threshold voltage, respectively. $W=105 \mu \mathrm{m}$ and $L=20 \mu \mathrm{m}$ for transistor devices. $W=2 \mathrm{~mm}$ and $L=10 \mu \mathrm{m}$ for sensor devices.

The crystalline material $\left(\mathbf{P B B r}_{4}\right)_{3}$ provide a unique opportunity to study how thin film assembly and charge transport are affected by the dynamics of the semiconducting subunits. This is important because, if films can be made to maintain the open spaces on the interior of the hollow semiconductor, they could be used as a locus for guest incorporation to modulate the semiconducting properties. The crystalline material made here, $\left(\mathbf{P B B r}_{4}\right)_{3}$, possesses an enormous amount of space within their interiors.

A saturated vapor of the analyte or odor is delivered to the thin film transistor using a bubbler. Nitrogen was used as the carrier gas. A sealed exposure chamber with a volume of $1.7 \mathrm{~L}$ was used; the flow rate of $\mathrm{N}_{2}$ through the chamber was $0.2 \mathrm{Lmin}^{-1}$. Responsing experiments were conducted by fixing the drain voltage at the determined minimum saturation value and conducting gate sweeps. The gate was applied in a pulsed manner in order to reduce device hysteresis. The responses of these devices to analytes were investigated by plotting the percent change in drain current, $I_{\mathrm{DS}} / I_{\mathrm{DS}, 0}\left(\right.$ measured at $\mathrm{V}_{\mathrm{G}}=80 \mathrm{~V}, \mathrm{~V}_{\mathrm{DS}}=80 \mathrm{~V}$ ) versus time of exposure to analytes. For concentration dependent measurement, the analyte concentration was set via a saturated vapor of the analyte or odor using a bubbler, subsequently diluted through a series of gas mass-flow 
controllers (FMA-5506A and FMA-5514A). During the course of experiment, the total fow rate of the $\mathrm{N}_{2}$ gas was set to a constant 500 standard cubic centimeter (SCCM).

\subsection{Synthetic Procedures and Characterizations}

Synthesis of 6.1: A solution of 1,7-dibromoperylene-3,4,9,10-tetracarboxylicbisimides (1.145 g, $1.34 \mathrm{mmol}, 1.00$ equiv) in toluene $(15 \mathrm{~mL})$ was degassed under nitrogen for 20 minutes. In a separate flask, a solution of 2,5-bis(trimethylstannyl)thiophene (2.64g, 6.44 mmol, 4.81 equiv) in toluene $(20 \mathrm{~mL})$ was degassed under nitrogen for 20 minutes. The 2,5bis(trimethylstannyl)thiophene solution was transferred under nitrogen to the dibromoperylene solution, and degassed for 40 minutes. Tetrakis(triphenylphosphine)palladium(0) (70 mg, 0.06 mmol, 4 mol\%) was added, and the resultant solution was degassed for 20 minutes. The mixture was placed in oil bath set at $115^{\circ} \mathrm{C}$ for $13 \mathrm{~h}$ under nitrogen. The resultant blue reaction mixture was concentrated via rotoevaporation under reduced pressure. The product was purified using $\mathrm{C}_{18}$ reverse phase column chromatography (DCM:acetonitrile 1:1) to yield blue-purple solid 6.1 (1.10 g, 0.93 mmol, 69\%). ${ }^{1} \mathbf{H}$ NMR (500 MHz, $\left.\mathrm{CDCl}_{3}\right): \delta 8.65$ (br, 2H); $8.21(\mathrm{br}, 2 \mathrm{H}) ; 8.12(\mathrm{~d}, \mathrm{~J}=8$ $\mathrm{Hz}, 2 \mathrm{H}) ; 7.38(\mathrm{~d}, \mathrm{~J}=3 \mathrm{~Hz}, 2 \mathrm{H}) ; 7.22(\mathrm{~d}, \mathrm{~J}=3 \mathrm{~Hz}, 2 \mathrm{H}) ; 5.16(\mathrm{~m}, 2 \mathrm{H}) ; 2.26-2.22(\mathrm{~m}, 4 \mathrm{H}) ; 1.83(\mathrm{~m}$, 4H); $1.28(\mathrm{~m}, 24 \mathrm{H}) ; 0.85(\mathrm{~m}, 12 \mathrm{H}) ; 0.46^{*}(\mathrm{~s}, 18 \mathrm{H}) .{ }^{13} \mathbf{C}$ NMR $\left(100 \mathrm{MHz}, \mathrm{CDCl}_{3}\right): \delta 164.59 ; 163.47$; $149.25 ; 141.92 * ; 136.82 ; 136.16 ; 135.47 ; 134.83 ; 133.61 ; 132.95 ; 130.01 ; 129.41 ; 129.20 ; 128.39$; $128.04 ; 122.71 ; 122.57 ; 122.01 ; 121.83 ; 54.57 ; 32.28 ; 31.70 ; 26.53 ; 22.51 ; 14.00 ;-8.02 *$. IR (ATR-ZnSe) $\left[\mathrm{cm}^{-1}\right]$ 3025, 2966, 2929, 2909, 2842, 2723, 2244, 1628, 1427, 1371. HRMS (APCI+) calculated $\mathrm{m} / \mathrm{z}$ for $\left[\mathrm{C}_{60} \mathrm{H}_{74} \mathrm{~N}_{2} \mathrm{O}_{4} \mathrm{~S}_{2} \mathrm{Sn}_{2}+\mathrm{H}\right]^{+}$1189.3225; found 1189.3237. *Tin satellite peaks visible.

Synthesis of Trimer 6.2: A solution of stannane 6.1 (833 mg, $0.70 \mathrm{mmol}, 1.00$ equiv) and dichloro(1,5-cyclooctadiene)platinum (262 mg, $0.70 \mathrm{mmol}, 1.00$ equiv) in 1,2-dichloroethane (180 
$\mathrm{mL}, 3.9 \mathrm{mM}$ ) was placed in oil bath set at $85{ }^{\circ} \mathrm{C}$ under $\mathrm{N}_{2}$ for 3 days. The blue solution was concentrated to a dark blue solid that was washed with hexane. The resultant solid was dried under vacuum. It was combined with 1,1'-ferrocenediyl-bis(diphenylphosphine) (550 mg, $1.00 \mathrm{mmol}$, 1.43 equiv) in $\mathrm{CH}_{2} \mathrm{Cl}_{2}(100 \mathrm{~mL})$. The solution was set to stir under $\mathrm{N}_{2}$ for 2 days. The solution was concentrated, and redissolved in toluene $(100 \mathrm{~mL})$ and combined with triphenylphosphine $(2.01 \mathrm{~g}$, 7.12 mmol, 10.17 equiv). The resultant solution was set to reflux under $\mathrm{N}_{2}$ for 2 days. The reaction mixture was concentrated under reduced pressure, the resultant dark blue solid was subjected to Soxhlet extraction: hexane, methanol, acetone, and $\mathrm{CH}_{2} \mathrm{Cl}_{2}$. The $\mathrm{CH}_{2} \mathrm{Cl}_{2}$ extract contained the cyclic and acyclic compounds. The resultant solid was first purified by alumina chromatography using gradient of $50 \% \mathrm{CH}_{2} \mathrm{Cl}_{2} /$ hexane to $80 \% \mathrm{CH}_{2} \mathrm{Cl}_{2} /$ hexane. The resultant fractions with cyclic and acyclic compounds were purified into its individual cyclic components by HPLC with a 5PBB column eluting with $27 \% \mathrm{CH}_{2} \mathrm{Cl}_{2} /$ hexane. Trimer 6.2 (90 mg, $0.035 \mathrm{mmol}, 15 \%$ yield); tetramer (62 mg, $0.018 \mathrm{mmol}, 10 \%$ yield); pentamer (32 mg, $0.007 \mathrm{mmol}, 5 \%$ yield); hexamer (26 mg, $0.005 \mathrm{mmol}, 4 \%$ yield). The remaining mass balance is higher order cyclic and acyclic oligomers.

Trimer 6.2: ${ }^{1} \mathbf{H}$ NMR $\left(500 \mathrm{MHz}, \mathrm{CD}_{2} \mathrm{Cl}_{2}\right): \delta 8.48-8.47(\mathrm{br}, 6 \mathrm{H}) ; 8.37(\mathrm{~d}, \mathrm{~J}=8.1 \mathrm{~Hz}, 6 \mathrm{H})$; 8.23 (br, 6H); 7.57 (d, J = 3.7 Hz, 6H); 7.43 (d, J = 3.7 Hz, 6H); 5.07-5.04 (m, 6H); 2.12 (br, 12H); $1.74(\mathrm{br}, 12 \mathrm{H}) ; 1.26-1.21(\mathrm{~m}, 72 \mathrm{H}) ; 0.80-0.71(\mathrm{~m}, 36 \mathrm{H}) .{ }^{13} \mathbf{C ~ N M R}\left(100 \mathrm{MHz}, \mathrm{CDCl}_{3}\right): \delta 164.21$; $163.17 ; 144.32 ; 139.55 ; 135.94 ; 135.23 ; 134.17 ; 133.18 ; 131.89 ; 130.60 ; 129.87 ; 129.38 ; 128.77$; $128.05 ; 127.79 ; 125.16 ; 125.16 ; 122.84 ; 122.22 ; 54.65 ; 32.19 ; 31.59 ; 26.49 ; 22.42 ; 13.92$. IR (ATR-ZnSe) $\left[\mathrm{cm}^{-1}\right] 2924.4 ; 2856.6 ; 1696.9 ; 1655.7 ; 1584.9 ; 1455.0 ; 1400.9 ; 1321.2 ; 1243.0 ;$ 1180.2; 1118.0; 974.9; 911.8; 859.2; 832.8; 810.1; 756.7; 713.5. HRMS (MALDI, dithranol matrix) calculated $\mathrm{m} / \mathrm{z}$ for $\left[\mathrm{C}_{162} \mathrm{H}_{168} \mathrm{~N}_{6} \mathrm{O}_{12} \mathrm{~S}_{6}\right]^{-2} 2581.1039$; found 2581.1036. 
Tetramer: ${ }^{1} \mathbf{H}$ NMR $\left(500 \mathrm{MHz}, \mathrm{C}_{2} \mathrm{D}_{2} \mathrm{Cl}_{4}, 333 \mathrm{~K}\right): \delta 8.60(\mathrm{~s}, 8 \mathrm{H}) ; 8.41(\mathrm{~d}, \mathrm{~J}=8.2 \mathrm{~Hz}, 8 \mathrm{H})$; 8.31(d, J = 8.2 Hz, 8H); $7.51(\mathrm{~d}, \mathrm{~J}=3.7 \mathrm{~Hz}, 8 \mathrm{H})$; 7.33 (d, J = 3.7 Hz, 8H); 5.14-5.09 (m, 8H); 2.19$2.16(\mathrm{~m}, 16 \mathrm{H}) ; 1.90-1.87(\mathrm{~m}, 16 \mathrm{H}) ; 1.31-1.27(\mathrm{~m}, 96 \mathrm{H}) ; 0.83-0.82(\mathrm{~m}, 48 \mathrm{H}) .{ }^{13} \mathrm{C}$ NMR $(100 \mathrm{MHz}$, $\left.\mathrm{CDCl}_{3}\right): \delta 164.31 ; 163.22 ; 144.16 ; 139.22 ; 135.91 ; 135.19 ; 134.04 ; 132.55 ; 132.21 ; 130.24 ;$ $129.69 ; 128.99 ; 128.41 ; 128.01 ; 125.69 ; 122.82 ; 122.24 ; 54.69 ; 32.22 ; 31.63 ; 26.50 ; 22.46 ; 13.93$. IR (ATR-ZnSe) $\left[\mathrm{cm}^{-1}\right] 2923.3 ; 2855.9 ; 1696.9 ; 1655.9 ; 1584.6 ; 1454.6 ; 1401.4 ; 1320.7 ; 1241.7$; 1179.6; 1118.9; 973.8; 926.6; 860.3; 832.7; 809.9; 755.6; 715.7. HRMS (MALDI, dithranol matrix) calculated $\mathrm{m} / \mathrm{z}$ for $\left[\mathrm{C}_{216} \mathrm{H}_{224} \mathrm{~N}_{8} \mathrm{O}_{16} \mathrm{~S}_{8}\right]^{-} 3441.4721$; found 3441.4648 .

Pentamer: ${ }^{1} \mathrm{H}$ NMR (500 MHz, $\left.\mathrm{CD}_{2} \mathrm{Cl}_{2}\right): \delta 8.59-8.57(\mathrm{br}, 10 \mathrm{H}) ; 8.35(\mathrm{~d}, \mathrm{~J}=8.2 \mathrm{~Hz}, 10 \mathrm{H}) ; 8.25$ (br, 10H); $7.40(\mathrm{~d}, \mathrm{~J}=3.6 \mathrm{~Hz}, 10 \mathrm{H}) ; 7.35(\mathrm{~d}, \mathrm{~J}=3.6 \mathrm{~Hz}, 10 \mathrm{H}) ; 5.08(\mathrm{br}, 10 \mathrm{H}) ; 2.16-2.13(\mathrm{~m}, 20 \mathrm{H})$; 1.76-1.75 (m, 20H); 1.26-1.17 (m, 120H); 0.76-0.73 (m, 60H). $\left.{ }^{13} \mathbf{C ~ N M R ~ ( 1 0 0 ~ M H z , ~ C D C l} 3\right): \delta$ $164.37 ; 163.28 ; 143.59 ; 139.25 ; 135.98 ; 135.26 ; 134.15 ; 132.69 ; 132.27 ; 130.33 ; 129.70 ; 129.09$; $128.70 ; 128.10 ; 125.60 ; 123.02 ; 122.83 ; 122.30 ; 122.06 ; 54.63 ; 32.18 ; 31.63 ; 26.48 ; 22.45 ; 13.96$. IR (ATR-ZnSe) $\left[\mathrm{cm}^{-1}\right] 2925.7 ; 2856.1 ; 1699.1 ; 1658.4 ; 1586.2 ; 1403.5 ; 1323.6 ; 1275.6 ; 1260.9$; 750.7. HRMS (MALDI, dithranol matrix) calculated $\mathrm{m} / \mathrm{z}$ for $\left[\mathrm{C}_{270} \mathrm{H}_{280} \mathrm{~N}_{10} \mathrm{O}_{20} \mathrm{~S}_{10}\right]^{-} 4031.8402$; found 4031.8465 .

Hexamer: ${ }^{1} \mathbf{H}$ NMR (500 MHz, $\left.\mathrm{CDCl}_{3}\right): \delta 8.62$ (br, $\left.12 \mathrm{H}\right) ; 8.36(\mathrm{~d}, \mathrm{~J}=8.2 \mathrm{~Hz}, 12 \mathrm{H}) ; 8.28$ (br, 12H); 7.37-7.33 (m, 24H); $5.10(\mathrm{br}, 12 \mathrm{H}) ; 2.18-2.16(\mathrm{~m}, 24 \mathrm{H}) ; 1.79-1.77(\mathrm{~m}, 24 \mathrm{H}) ; 1.52-1.19$ (m, 144H); 0.78-0.77 (m, 72H). ${ }^{13}$ C NMR (100 MHz, $\left.\mathrm{CDCl}_{3}\right): \delta 164.41 ; 163.28 ; 143.56 ; 139.26$; $136.04 ; 135.35 ; 134.28 ; 132.89 ; 132.37 ; 131.20 ; 130.41 ; 129.74 ; 129.17 ; 128.74 ; 128.20 ; 127.95 ;$ $125.81 ; 122.93 ; 122.34 ; 54.72 ; 32.24 ; 31.67 ; 26.53 ; 22.48 ; 13.98$. IR (ATR-ZnSe) $\left[\mathrm{cm}^{-1}\right] 2952.0$; $2923.1 ; 2856.0 ; 1696.9 ; 1655.9 ; 1585.2 ; 1455.7 ; 1402.7 ; 1321.6 ; 1246.6 ; 1180.0 ; 1121.2 ; 862.4$; 
833.4; 757.8. HRMS (MALDI, dithranol matrix) calculated $\mathrm{m} / \mathrm{z}$ for $\left[\mathrm{C}_{324} \mathrm{H}_{336} \mathrm{~N}_{12} \mathrm{O}_{24} \mathrm{~S}_{12}\right]^{-}$ 5162.2084; found 5162.2105.

Interconversion study of both enantiomers of trimer 6.2 : The enantiomers of trimer 6.2 was separated on CHIRALPAK IA-3 column (21 mm ID x $250 \mathrm{~mm}, 5 \mu \mathrm{m})$ on Waters Prep150 instrument eluting with $24 \%$ dichloromethane in hexane. Each enantiomer ( $1 \mathrm{mg}$ each) labeled trimer-entA (shorter retention time, $\mathrm{t}=14.7 \mathrm{~min}$ ) and trimer-entB (longer retention time, $\mathrm{t}=$ $28.0 \mathrm{~min}$ ) was dissolved in $1 \mathrm{~mL}$ of anhydrous 1,2-dichlorobenzene, sparged under $\mathrm{N}_{2}$ for 30 minutes. It was placed in oil bath set at $160{ }^{\circ} \mathrm{C}$ for 19 hours. The solvent was distilled off under high vacuum reduced pressure. ${ }^{1} \mathrm{HNMR}$ was identical to starting enantiomer and the chiral HPLC trace exhibited same retention time.

Synthesis of $\left(\mathbf{P B B r}_{4}\right)_{3}$ : A solution of trimer $6.2(60 \mathrm{mg}, 0.023 \mathrm{mmol})$ in $\mathrm{CH}_{2} \mathrm{Cl}_{2}(5 \mathrm{~mL})$ was set stirring in a vial. Excess bromine $(0.5 \mathrm{~mL})$ was added, followed by a crystal of iodine. The reaction was capped and left to stir for 3 days. The solvent and bromine was purged under air. The resultant red solid was dissolved in chloroform and purified by small silica gel column chromatography, eluting with $100 \%$ chloroform to yield dark violet solids $(65 \mathrm{mg}, 0.018 \mathrm{mmol}$, $80 \%$ yield). ${ }^{1} \mathbf{H}$ NMR (400 MHz, $\left.\mathrm{CDCl}_{3}\right): \delta 8.77-8.74(\mathrm{br}, 6 \mathrm{H}) ; 8.36-8.33(\mathrm{br}, 6 \mathrm{H}) ; 8.07$ (d, J = 8 $\mathrm{Hz}, 6 \mathrm{H}) ; 5.09-5.06(\mathrm{~m}, 6 \mathrm{H}) ; 2.14-2.06(\mathrm{~m}, 12 \mathrm{H}) ; 1.83(\mathrm{br}, 12 \mathrm{H}) ; 1.25$ (br, 72H); 0.80-0.77 (m, 36H). ${ }^{13} \mathbf{C}$ NMR $\left(100 \mathrm{MHz}, \mathrm{CDCl}_{3}\right): \delta 163.98 ; 163.61 ; 162.89 ; 162.48 ; 140.20 ; 135.36 ; 134.61$; $133.31 ; 132.95 ; 130.86 ; 130.37 ; 129.52 ; 128.47 ; 128.07 ; 123.48 ; 122.78 ; 117.27 ; 116.26 ; 54.86$; $32.07 ; 31.56 ; 26.51 ; 22.45 ; 13.94$. IR (ATR-ZnSe) $\left[\mathrm{cm}^{-1}\right] 3005.9 ; 2986.5 ; 2923.4 ; 2851.9 ; 1696.9$; $1656.4 ; 1584.9 ; 1394.5 ; 1320.0 ; 1260.8 ; 862.9 ; 811.9 ; 750.5$. HRMS (MALDI, dithranol matrix) calculated $\mathrm{m} / \mathrm{z}$ for $\left[\mathrm{C}_{162} \mathrm{H}_{156} \mathrm{Br}_{12} \mathrm{~N}_{6} \mathrm{O}_{12} \mathrm{~S}_{6}\right]^{-} 3516.0306$; found 3516.0356 


\subsection{NMR Spectra of Compounds}

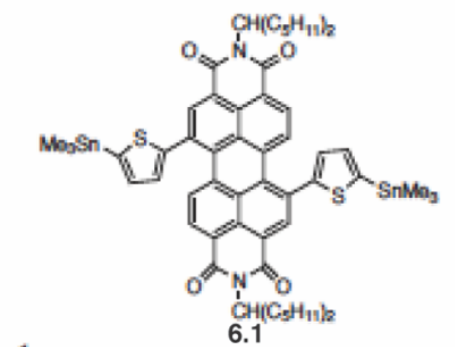

${ }^{1} \mathrm{H}$ NMR (500 MHz, $\left.{ }^{6.1} \mathrm{CDCl}_{3}\right)$

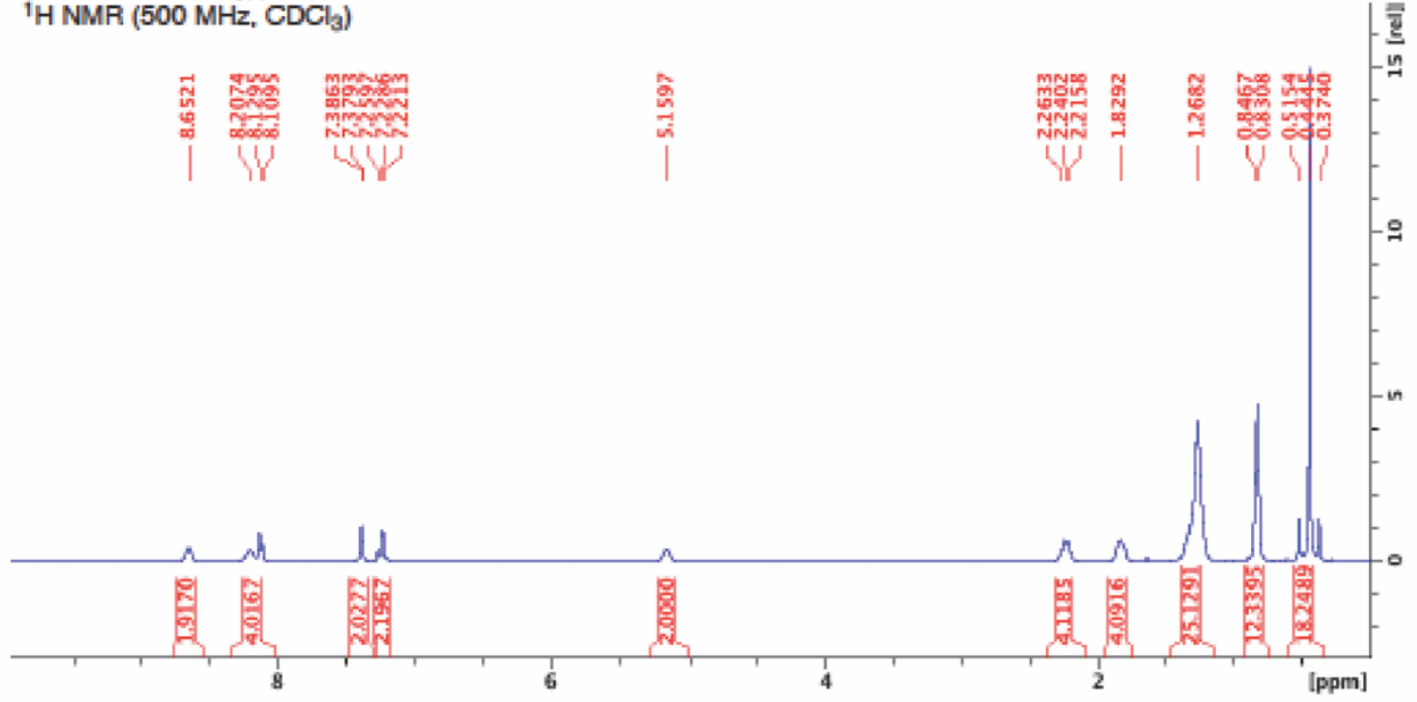

${ }^{13} \mathrm{C}$ NMR (100 MHz, $\left.\mathrm{CDCl}_{3}\right)$
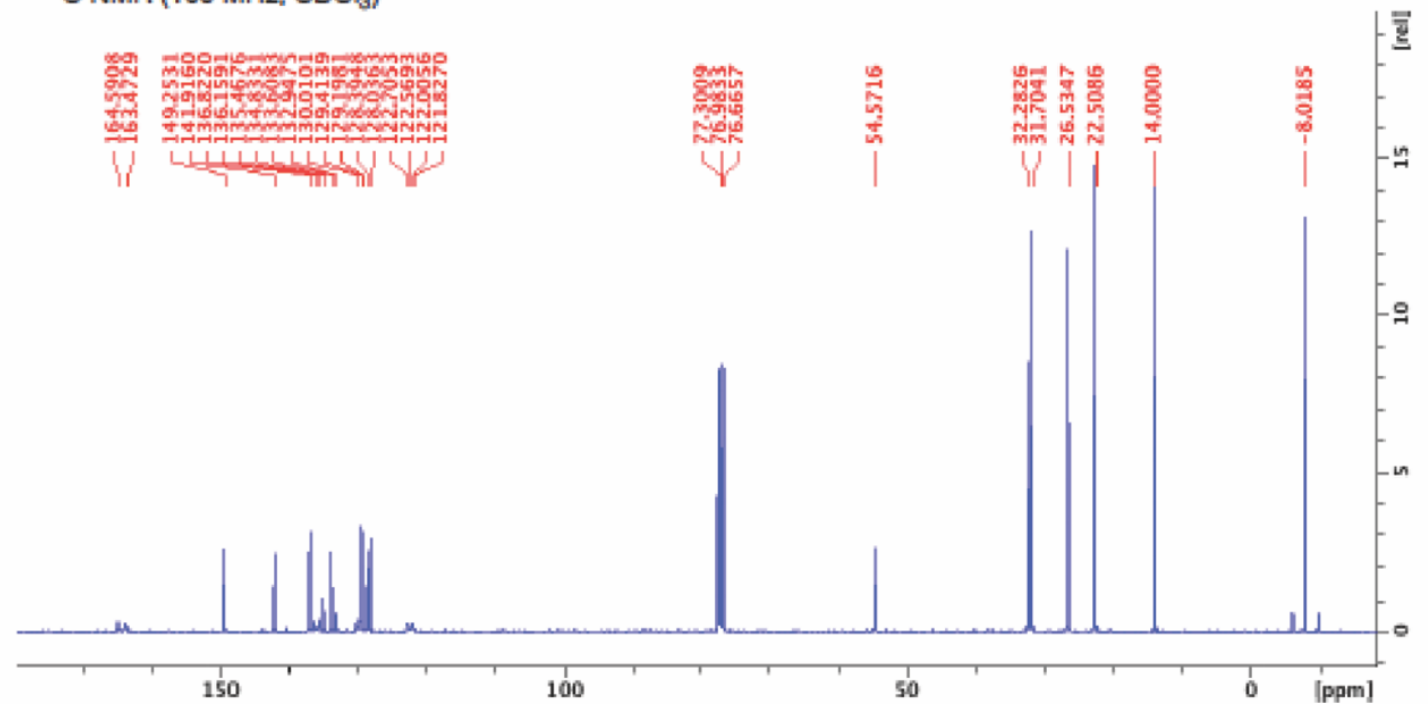

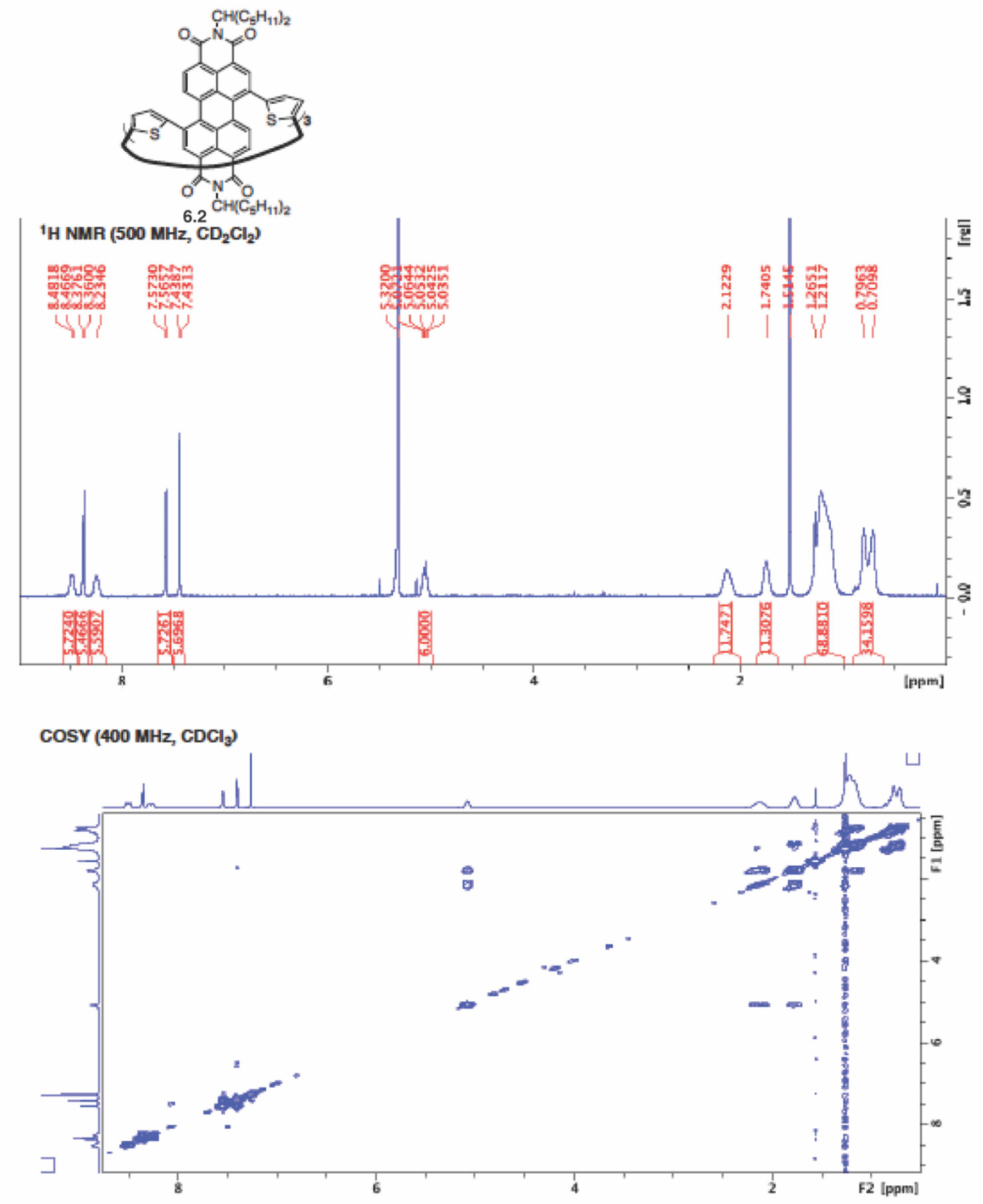


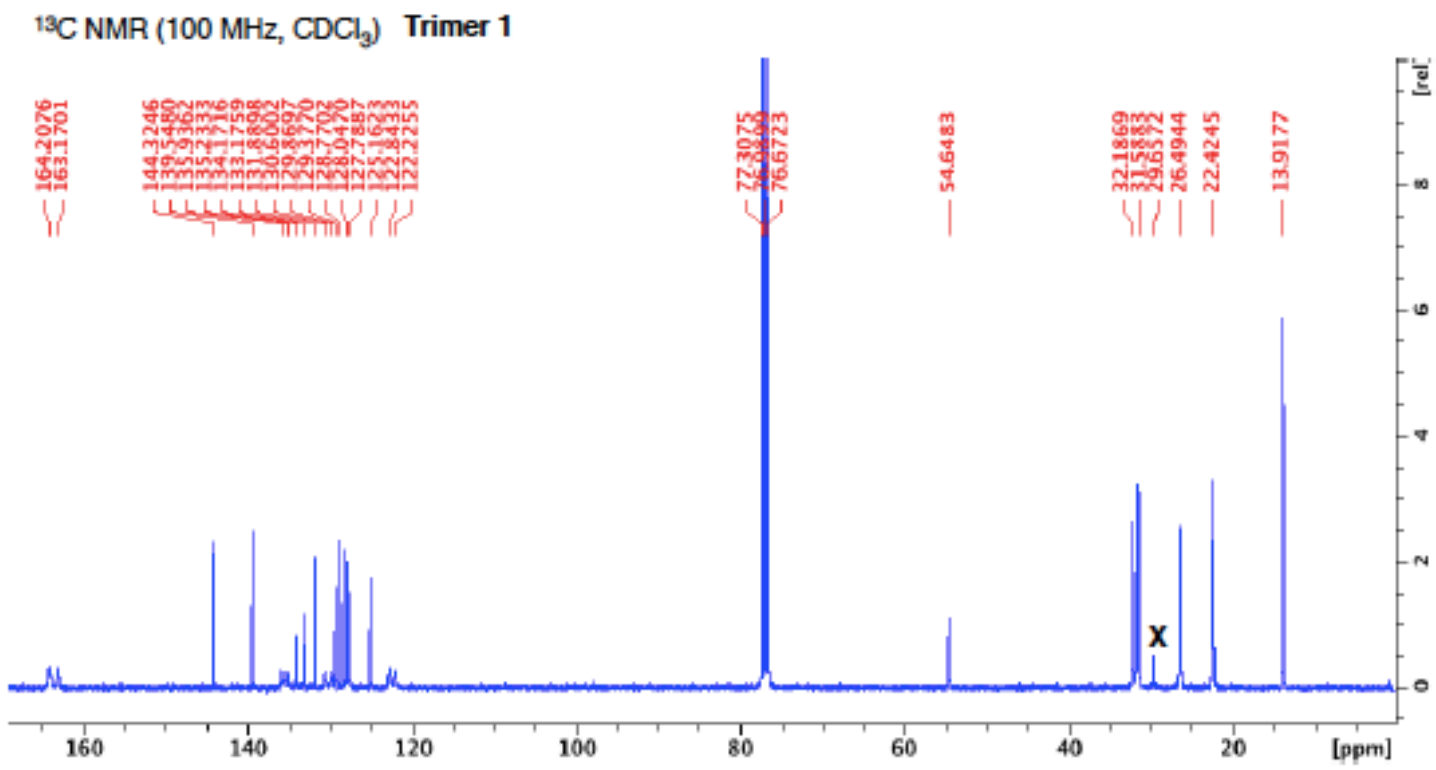

\section{Chiral HPLC trace of trimer 1}
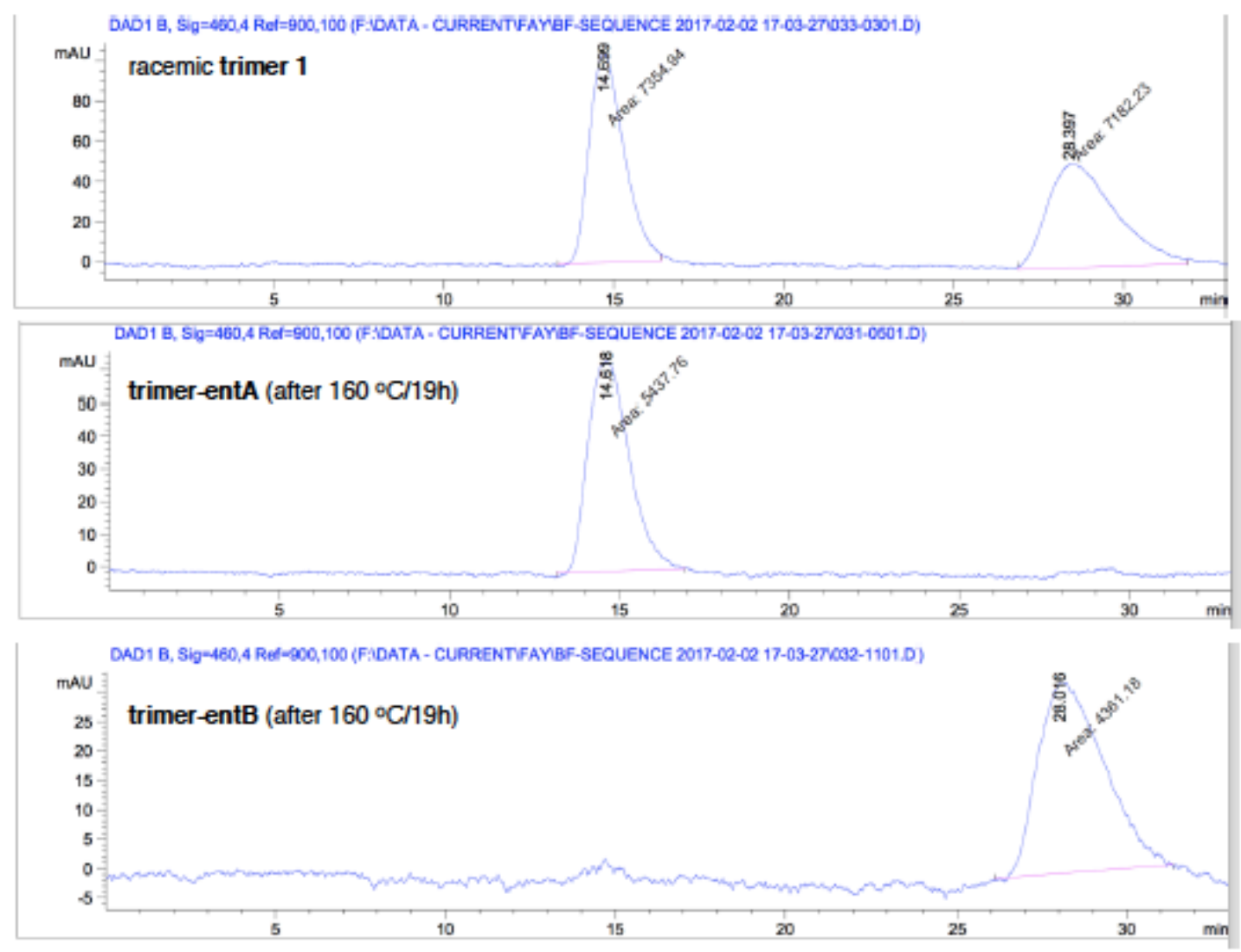


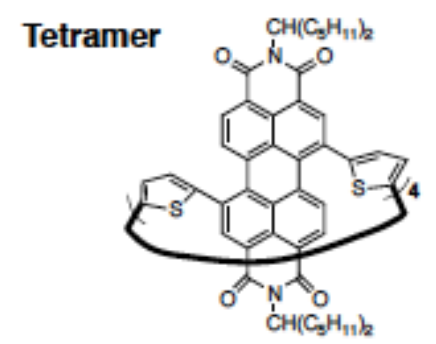

${ }^{1} \mathrm{H}$ NMR $\left(500 \mathrm{MHz}, \mathrm{C}_{2} \mathrm{D}_{2} \mathrm{Cl}_{4}, 333 \mathrm{~K}\right)$

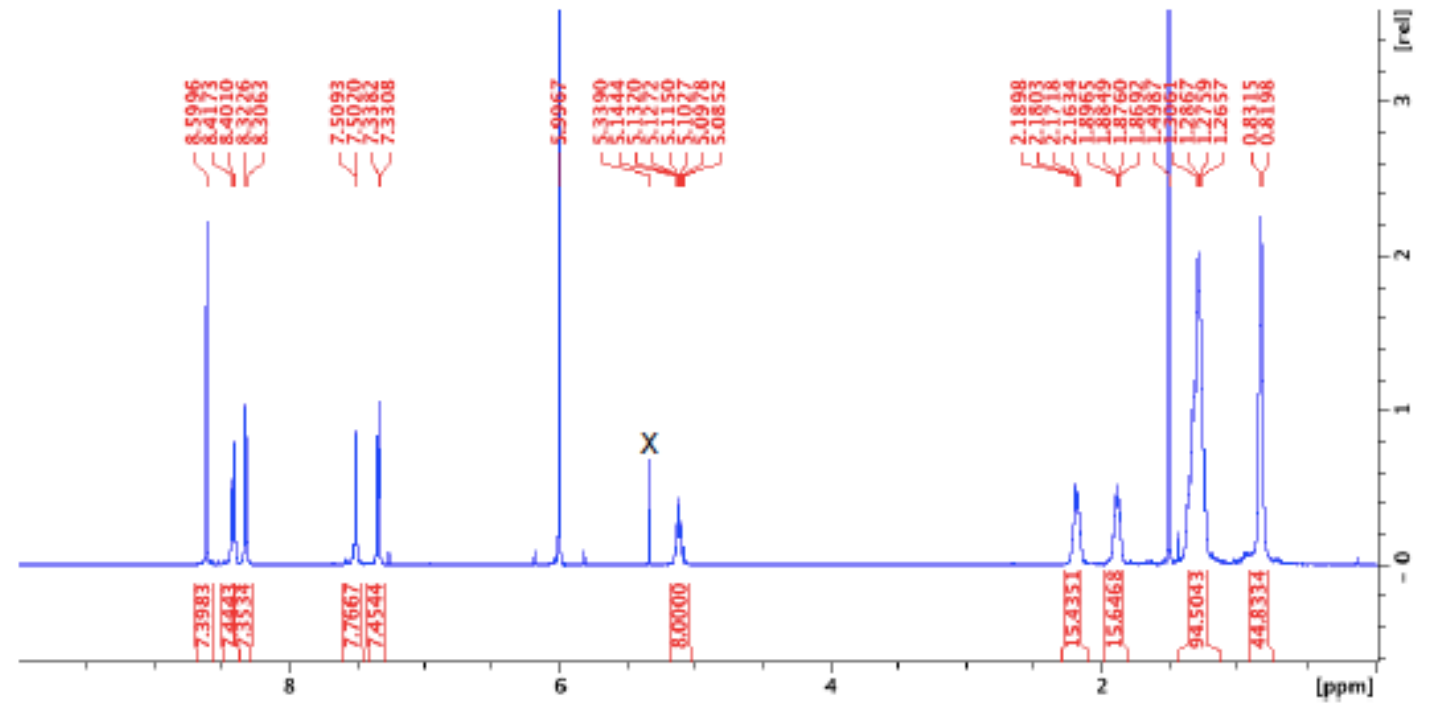

${ }^{13} \mathrm{C}$ NMR $\left(100 \mathrm{MHz}, \mathrm{CDCl}_{3}\right)$

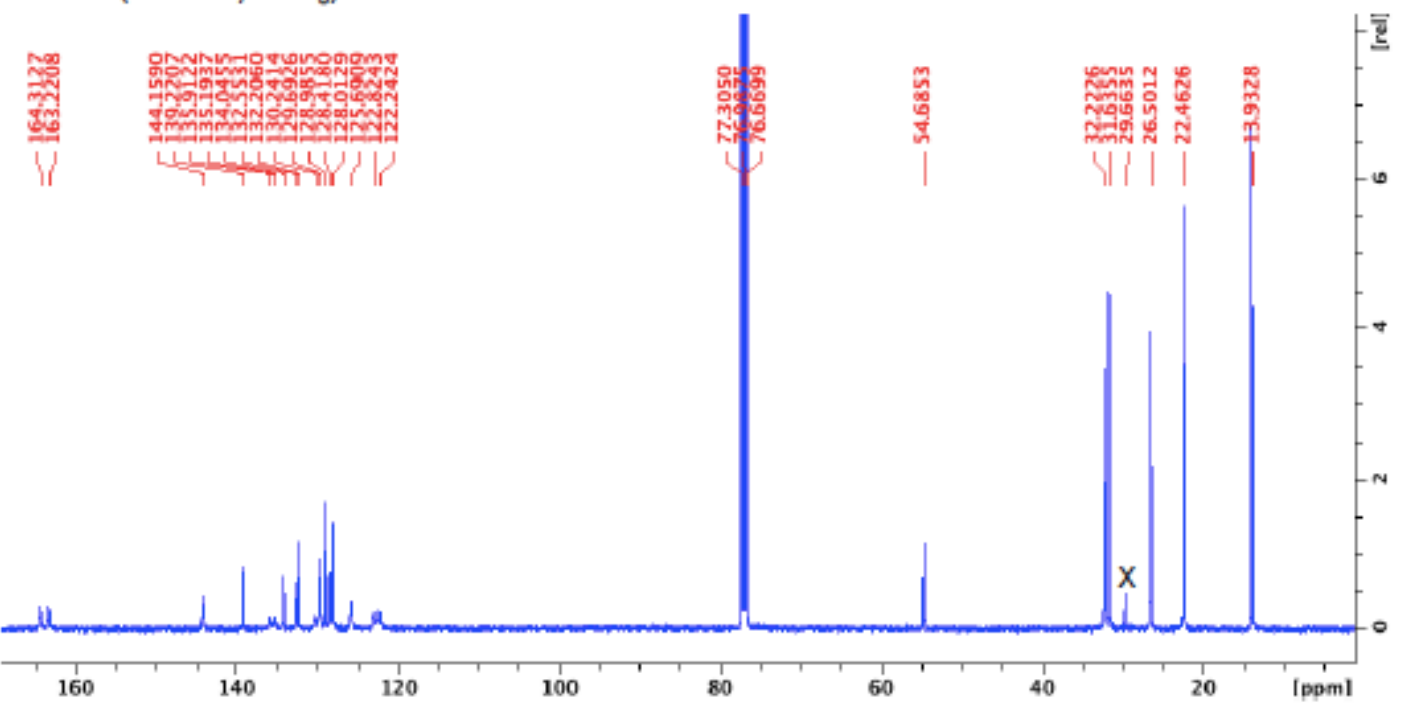


Pentamer

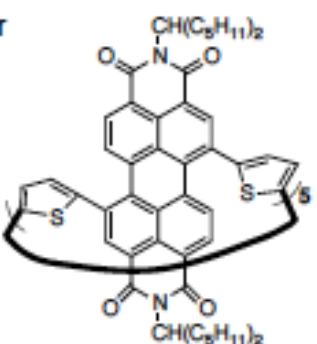

${ }^{1} \mathrm{H}$ NMR $\left(500 \mathrm{MHz}, \mathrm{CD}_{2} \mathrm{Cl}_{2}\right)$

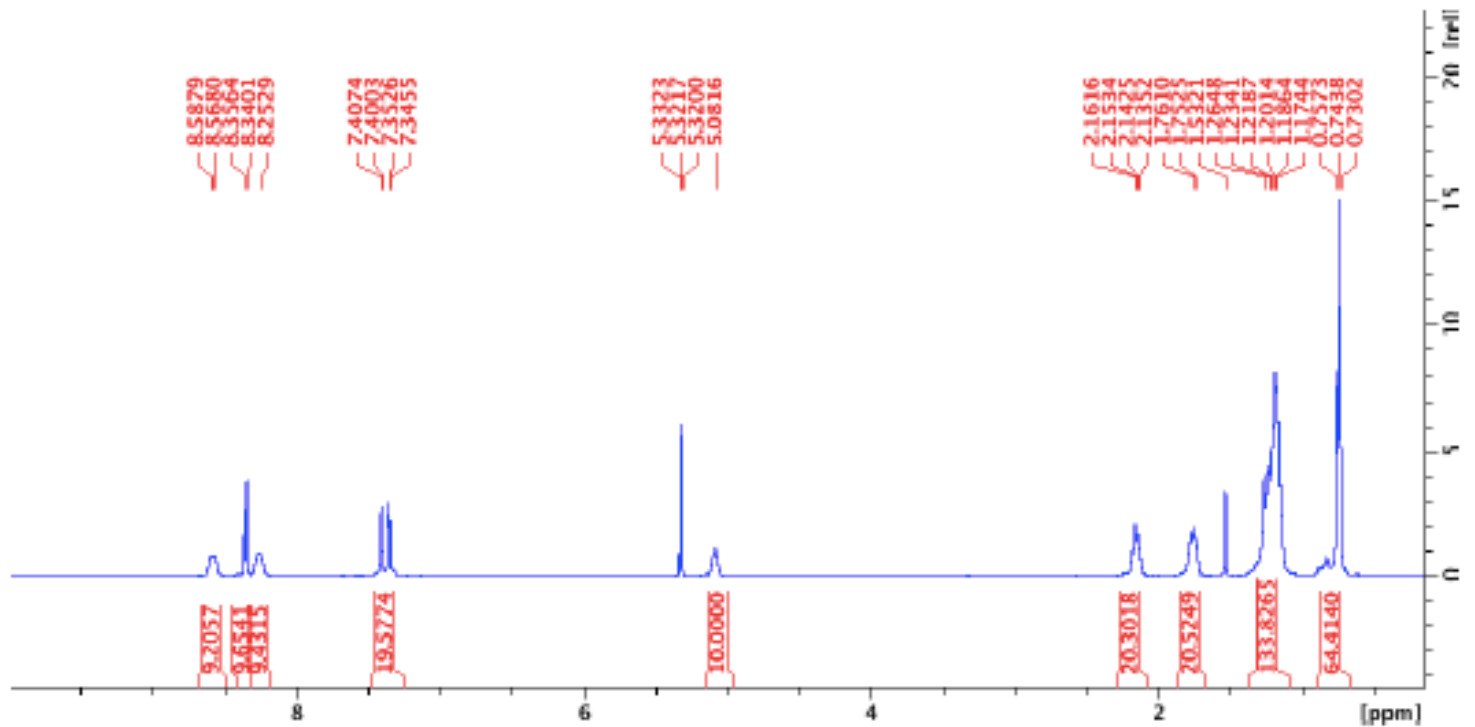

${ }^{13} \mathrm{C}$ NMR $\left(100 \mathrm{MHz}, \mathrm{CDCl}_{3}\right)$

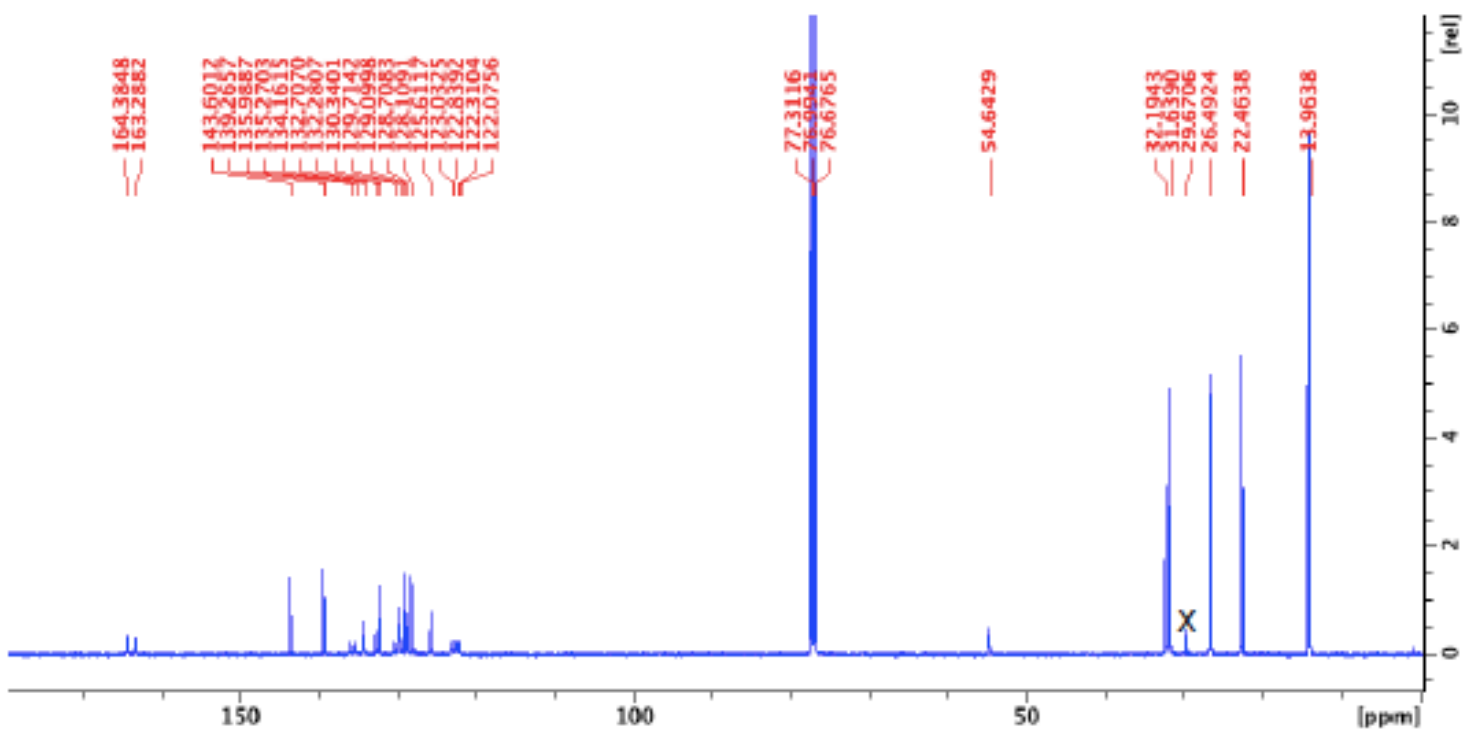




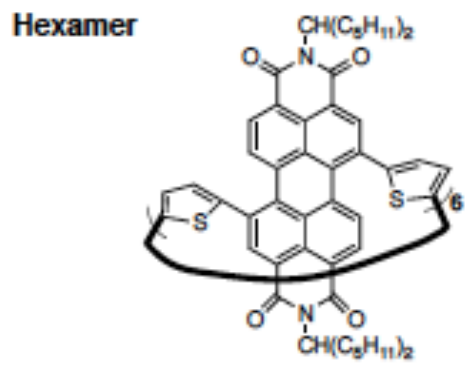

${ }^{1} \mathrm{H}$ NMR (500 MHz, $\mathrm{CDCl}_{3}$ )

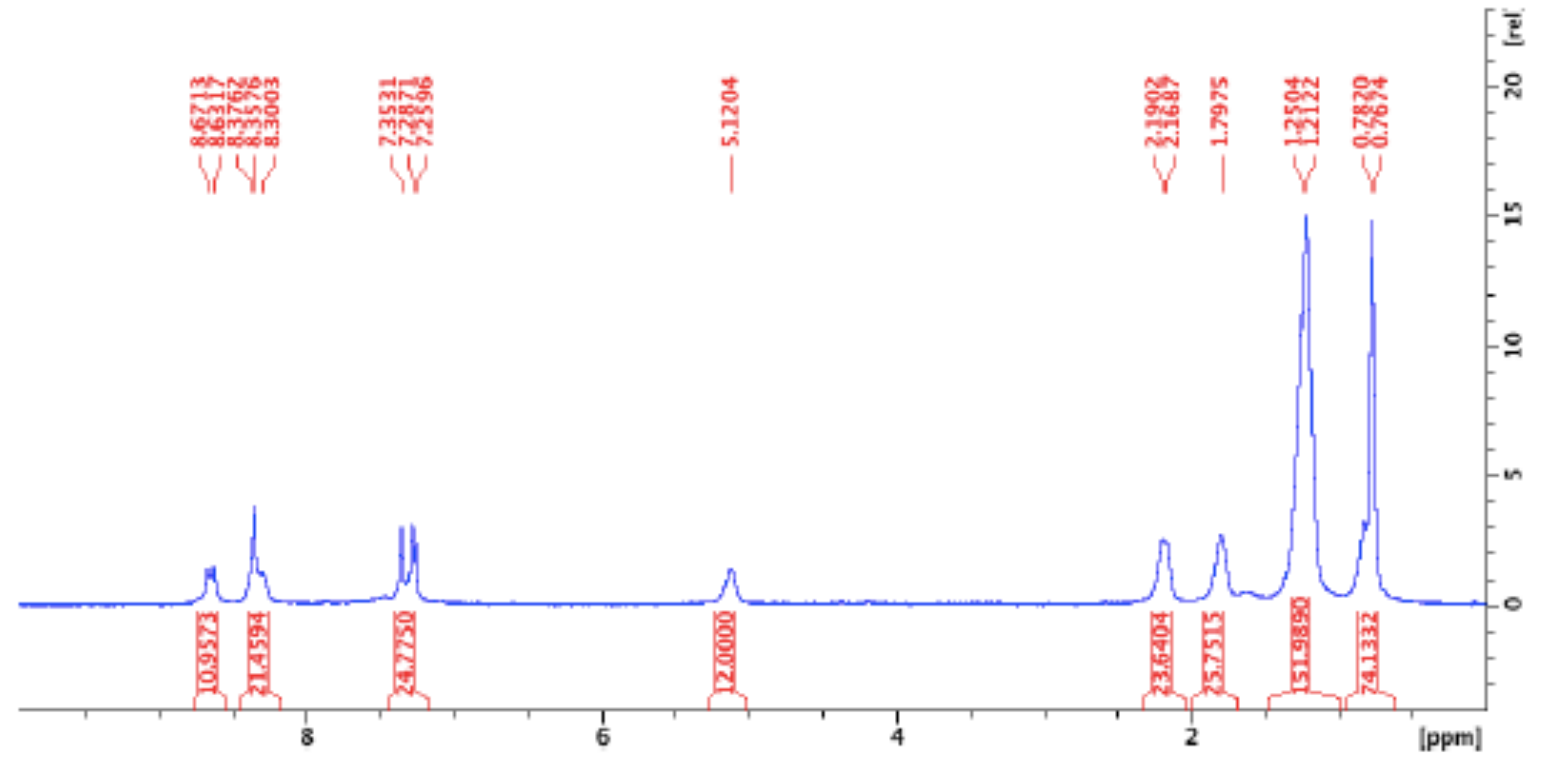

$\left.{ }^{13} \mathrm{C} \mathrm{NMR} \mathrm{(100} \mathrm{MHz,} \mathrm{CDCl} 3\right)$
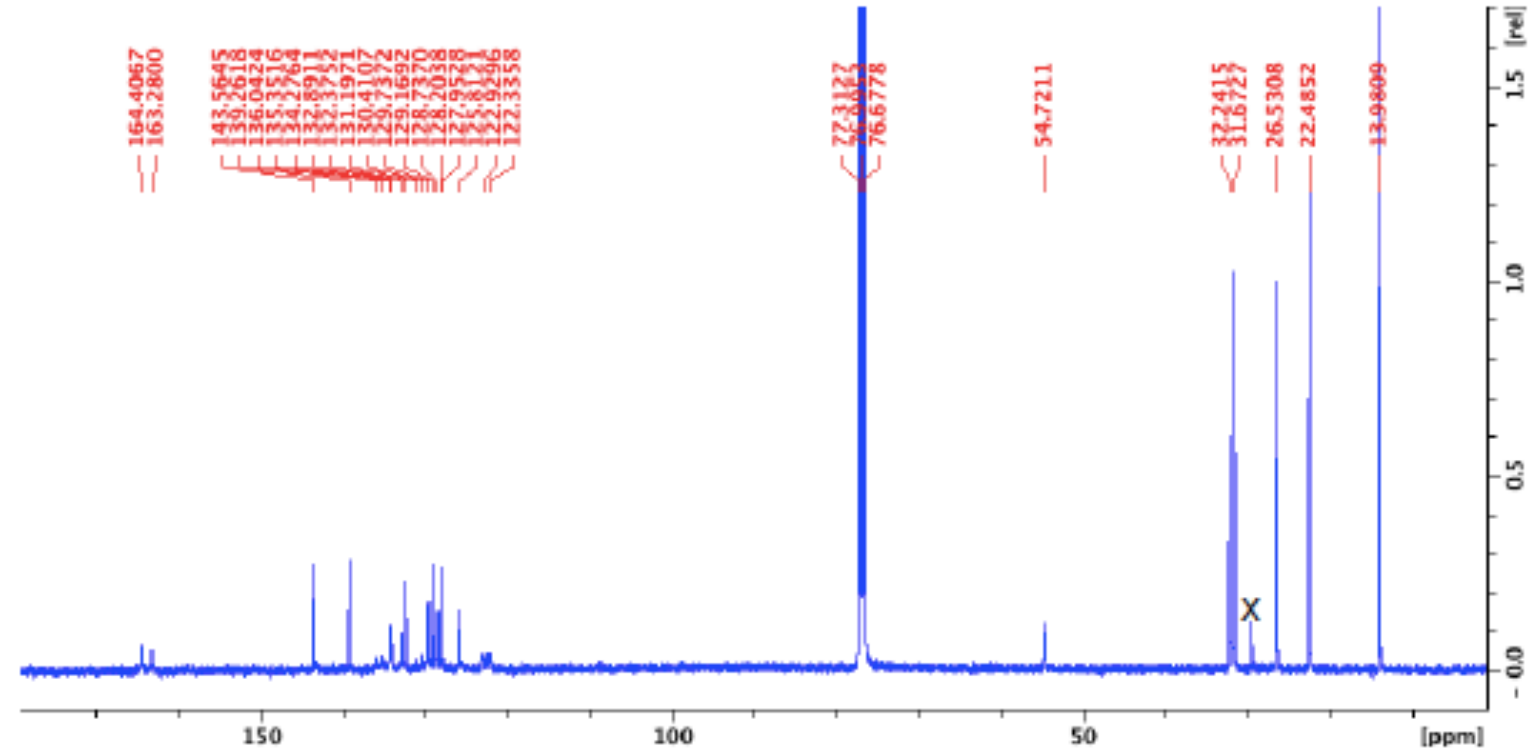


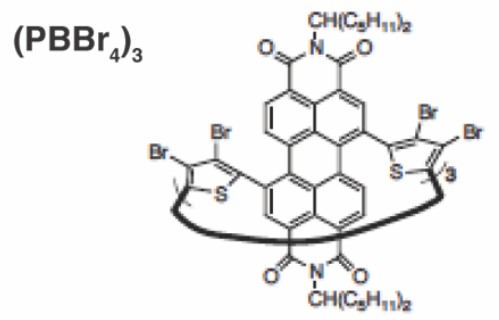

1'H NMR (400 MHz, $\left.\mathrm{CDCl}_{3}\right)$
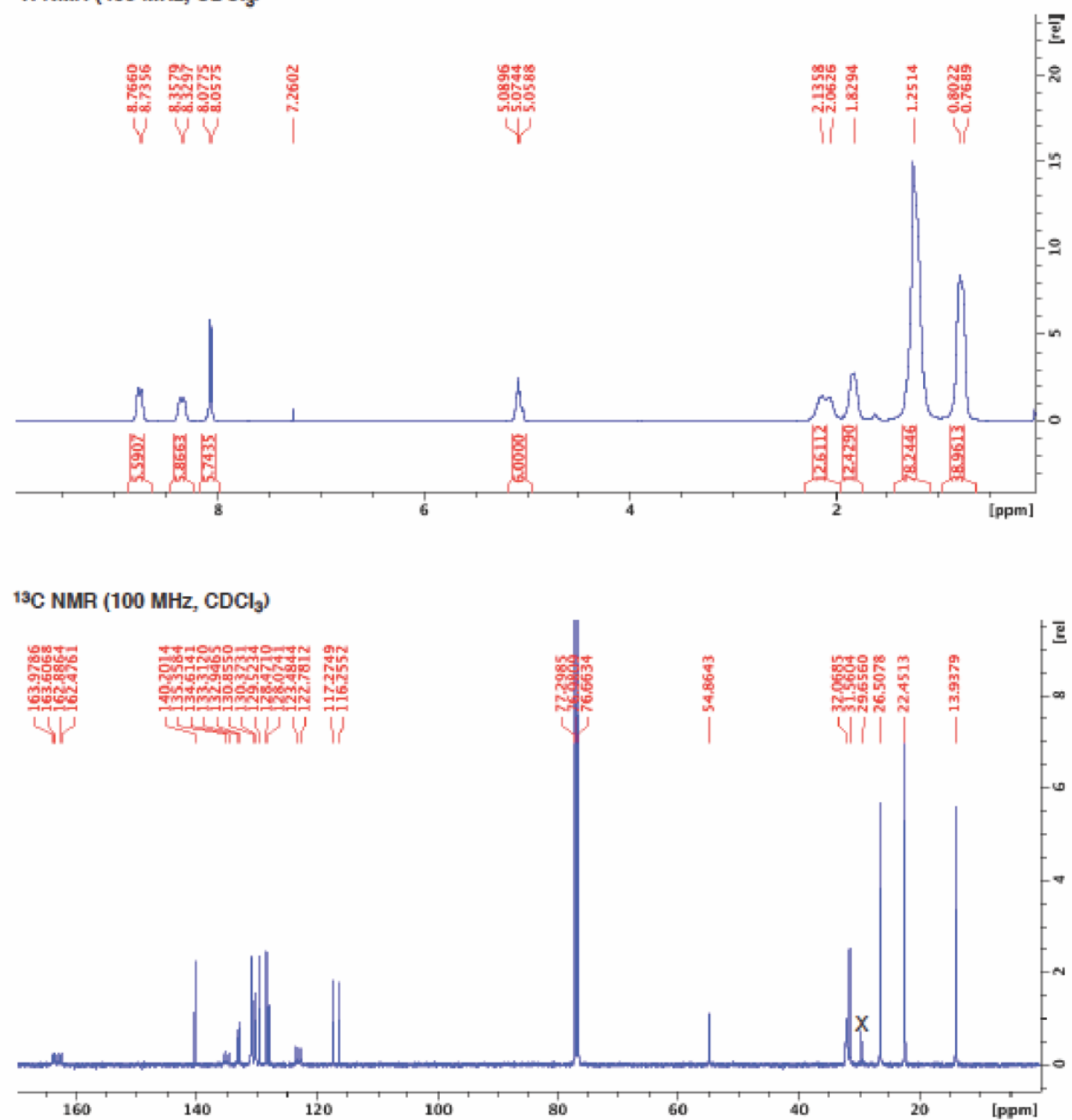

\subsection{DFT Output}

All quantum chemical calculations were performed using Jaguar, version 8.3, Schrodinger, Inc., New York, NY, 2013. (See A. D. Bochevarov, E. Harder, T. F. Hughes, J. R. Greenwood, D. A. 
Braden, D. M. Philipp, D. Rinaldo, M. D. Halls, J. Zhang, R. A. Friesner, "Jaguar: A High Performance Quantum Chemistry Software Program with Strengths in Life and Materials Sciences", Int. J. Quantum Chem., 2013, 113(18), 2110-2142). All geometries were optimized using the B3LYP functional and the LACVP** basis set.

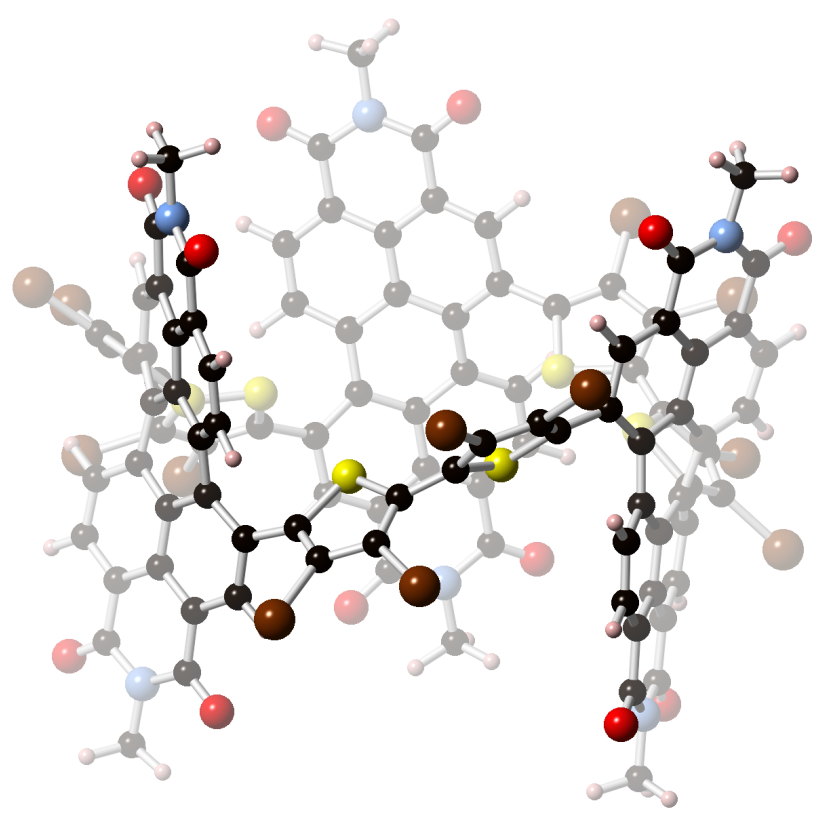

$\left(\mathrm{PBBr}_{4}\right)_{3}$

Final Heat of Formation: -7687.0083

$\begin{array}{rrrr}\mathrm{Br} & -11.07353 & 3.05844 & -0.23637 \\ \mathrm{Br} & -11.93869 & 3.46266 & 3.15296 \\ \mathrm{Br} & -8.48656 & -0.88369 & -4.04970 \\ \mathrm{Br} & -10.80890 & -0.40223 & -1.44883 \\ \mathrm{Br} & 4.23672 & 3.23274 & -0.88615 \\ \mathrm{Br} & 1.79296 & 5.04959 & -2.67498 \\ \mathrm{Br} & 5.30854 & -2.57089 & 0.97605 \\ \mathrm{Br} & 4.31135 & -0.46729 & -1.68291 \\ \mathrm{Br} & -3.05497 & -3.40898 & 11.33508 \\ \mathrm{Br} & -6.38367 & -4.16710 & 10.51682 \\ \mathrm{Br} & 0.87547 & 1.11359 & 11.50628 \\ \mathrm{Br} & -2.44482 & 0.17504 & 12.15510 \\ \mathrm{C} & -9.33767 & 3.24094 & 5.42546 \\ \mathrm{C} & -9.40297 & 1.92425 & 4.90928 \\ \mathrm{C} & -9.16817 & 0.83267 & 5.77151 \\ \mathrm{C} & -8.62842 & 1.08765 & 7.07171 \\ \mathrm{C} & -8.64150 & 2.41378 & 7.58827 \\ \mathrm{C} & -9.03450 & 3.48441 & 6.74887 \\ \mathrm{C} & -8.06639 & 0.03691 & 7.86304\end{array}$




\begin{tabular}{|c|c|c|c|}
\hline & & & \\
\hline & -7.82682 & 50905 & \\
\hline & 14 & 005 & \\
\hline & -9.49938 & & 7 \\
\hline & & & \\
\hline & -7.93528 & & \\
\hline & 31 & & \\
\hline & -10.7 & & \\
\hline & 10 & & \\
\hline $\mathrm{C}$ & -9.02 & -2. & 78 \\
\hline 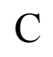 & -8.2 & -3 & \\
\hline $\mathrm{C}$ & -7.2 & & \\
\hline $\mathrm{C}$ & -7.0 & -2.3 & \\
\hline $\mathrm{C}$ & -5.8 & & \\
\hline 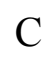 & -9.5 & & \\
\hline$c$ & -9. & & \\
\hline & 10 & & \\
\hline & & & \\
\hline $\mathrm{C}$ & -8 & & \\
\hline $\mathrm{C}$ & -6. & & \\
\hline C & -8.0 & $-0 .($ & \\
\hline $\mathrm{C}$ & -8 & & \\
\hline & -5 & -1.2 & \\
\hline & -3. & -1. & \\
\hline & -2.5 & -0 . & \\
\hline $\mathrm{C}$ & -3 . & & \\
\hline$C$ & -4.4 & & \\
\hline $\mathrm{C}$ & & & \\
\hline & -1 & -1. & \\
\hline & -0 & -0 . & -4 \\
\hline & -0.7 & & \\
\hline$T$ & -2.0 & & -3 \\
\hline & -2.2 & & -2 \\
\hline$C$ & -3.5 & & \\
\hline & -4.6 & & -2 \\
\hline $\mathrm{C}$ & -3.7 & & -2 . \\
\hline & -5.0 & & \\
\hline O & -6.1 & & -2 . \\
\hline 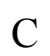 & -5.9 & & -2 \\
\hline & -1.2 & & -1 . \\
\hline 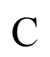 & -1.4 & 4.8 & -1 \\
\hline 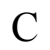 & -2.7 & & -1 . \\
\hline 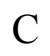 & & & \\
\hline$C$ & 1.8 & 1.5 & -0 . \\
\hline $\mathrm{C}$ & & & \\
\hline 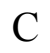 & 1.37777 & 3.48278 & -1.6127 \\
\hline
\end{tabular}




\begin{tabular}{|c|c|c|c|}
\hline & & & \\
\hline & 3326 & & \\
\hline & & -1.270 & \\
\hline & +261 & -0.35674 & \\
\hline$C$ & 794 & & \\
\hline & 2.54861 & & \\
\hline 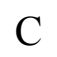 & 1556 & 707 & \\
\hline C & 5047 & -2.1 & \\
\hline & 97364 & 075 & \\
\hline C & 3 & -1.9 & \\
\hline & 1.9 & -4.3 & \\
\hline C & & -3.7 & \\
\hline $\mathrm{C}$ & & -2.3 & \\
\hline $\mathrm{C}$ & & -1.5 & \\
\hline G & & -0.0 & \\
\hline C & & & \\
\hline $\mathrm{C}$ & & -0. & \\
\hline $\mathrm{C}$ & & & \\
\hline $\mathrm{C}$ & & & \\
\hline $\mathrm{C}$ & 2.6 & 2.6 & \\
\hline $\mathrm{C}$ & & & \\
\hline $\mathrm{C}$ & 4.2 & 2.6 & \\
\hline $\mathrm{C}$ & 4.8 & & \\
\hline C & & & \\
\hline 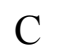 & 6 & & \\
\hline & & -2.4 & \\
\hline C & & -2.7 & \\
\hline $\mathrm{C}$ & & -0.7 & \\
\hline 4 & & 0.1 & \\
\hline $\mathrm{C}$ & 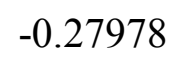 & & \\
\hline & -1.5 & -0.1 & 69 \\
\hline $\mathrm{C}$ & 37 & -3 & -4 \\
\hline $\mathrm{C}$ & -1.4 & -2.4 & -4.77158 \\
\hline $\mathrm{C}$ & -2.9 & 2 & 38 \\
\hline $\mathrm{C}$ & -5.3 & 6.69 & -2.02938 \\
\hline $\mathrm{C}$ & & & \\
\hline $\mathrm{C}$ & 4.6 & & \\
\hline $\mathrm{C}$ & 2.40 & -5.6 & \\
\hline $\mathrm{C}$ & 1.8 & -5.8 & 324 \\
\hline $\mathrm{C}$ & 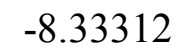 & 4.0 & \\
\hline $\mathrm{C}$ & -9.06 & 4.8 & 7.25847 \\
\hline & 0.24907 & -4.6 & 4.42997 \\
\hline $\mathrm{C}$ & -8.40455 & -5.39201 & 5.95374 \\
\hline $\mathrm{C}$ & -2.23912 & -4.69109 & -5.18263 \\
\hline 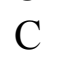 & -9.67 & -7.0 & 4.62499 \\
\hline $\mathrm{C}$ & 1.97632 & -7.81610 & 5.2295 \\
\hline
\end{tabular}




$\begin{array}{crrr}\mathrm{C} & -4.39942 & 8.83668 & -1.20927 \\ \mathrm{C} & 4.39916 & 6.02908 & 7.43356 \\ \mathrm{C} & -8.80226 & 6.40309 & 9.18526 \\ \mathrm{H} & -9.52884 & 4.08924 & 4.77898 \\ \mathrm{H} & -7.34300 & -0.47703 & 9.81971 \\ \mathrm{H} & -7.56185 & 1.81008 & 10.75173 \\ \mathrm{H} & -11.05295 & -0.14969 & 4.03882 \\ \mathrm{H} & -11.51876 & -2.52652 & 3.50823 \\ \mathrm{H} & -6.57095 & -4.42231 & 7.58373 \\ \mathrm{H} & 0.34330 & -0.43066 & -4.68372 \\ \mathrm{H} & 0.03865 & 1.81593 & -3.67921 \\ \mathrm{H} & -7.08857 & 4.91509 & -2.91565 \\ \mathrm{H} & -6.77047 & 2.48092 & -3.27181 \\ \mathrm{H} & -0.70049 & 5.49879 & -1.00347 \\ \mathrm{H} & 1.40657 & -4.41402 & 8.85473 \\ \mathrm{H} & 1.44406 & -1.94916 & 9.11446 \\ \mathrm{H} & 1.04943 & 2.63701 & 9.02027 \\ \mathrm{H} & 5.64571 & 2.43905 & 4.16718 \\ \mathrm{H} & 4.90250 & 0.15106 & 3.55118 \\ \mathrm{H} & -3.14412 & -5.27438 & -5.03015 \\ \mathrm{H} & -8.96361 & -7.67496 & 5.15982 \\ \mathrm{H} & 2.19965 & -8.07365 & 4.19690 \\ \mathrm{H} & -5.42885 & 9.09733 & -1.44347 \\ \mathrm{H} & 3.78378 & 6.39086 & 8.25397 \\ \mathrm{H} & -9.14251 & 7.07841 & 8.40381 \\ \mathrm{H} & -5.92932 & -2.05243 & -3.01117 \\ \mathrm{H} & 2.90329 & -3.77953 & 2.36086 \\ \mathrm{H} & -1.39929 & -5.13655 & -4.64477 \\ \mathrm{H} & -1.98239 & -4.64902 & -6.24359 \\ \mathrm{H} & -9.53579 & -7.14759 & 3.54580 \\ \mathrm{H} & -10.69792 & -7.32567 & 4.86908 \\ \mathrm{H} & 0.96584 & -8.13656 & 5.49382 \\ \mathrm{H} & 2.67826 & -8.30312 & 5.90990 \\ \mathrm{H} & -3.70579 & 9.44527 & -1.79371 \\ \mathrm{H} & -4.19218 & 9.00359 & -0.14978 \\ \mathrm{H} & 4.26076 & 6.65201 & 6.54707 \\ \mathrm{H} & 5.45667 & 6.05069 & 7.70605 \\ -7.81568 & 6.70218 & 9.54654 \\ \mathrm{~N} & -9.49355 & 6.41084 & 10.03083 \\ \mathrm{~N} & -4.21114 & 7.41845 & -1.53547 \\ \mathrm{H} & -5.65526 & 5.02730 \\ \mathrm{H} & -5.02182 & 3.63506\end{array}$




\subsection{References}

$\begin{array}{lrrr}\mathrm{O} & -7.70685 & -6.28815 & 6.40827 \\ \mathrm{O} & -8.06324 & 4.28705 & 10.65152 \\ \mathrm{O} & -9.36645 & 5.83001 & 6.54729 \\ \mathrm{O} & -4.63313 & -3.88657 & -4.07512 \\ \mathrm{O} & -0.35634 & -2.78958 & -5.26623 \\ \mathrm{O} & 1.58015 & -6.58510 & 7.57349 \\ \mathrm{O} & 2.56485 & -6.12826 & 3.12941 \\ \mathrm{O} & 2.37256 & 4.72116 & 8.76817 \\ \mathrm{O} & 5.56854 & 4.59148 & 5.49820 \\ \mathrm{O} & -2.04493 & 7.60896 & -0.85323 \\ \mathrm{O} & -6.39595 & 7.22019 & -2.20797 \\ \mathrm{~S} & -8.37950 & 0.85963 & 2.54020 \\ \mathrm{~S} & -6.71582 & 1.02359 & -0.43922 \\ \mathrm{~S} & 0.14310 & 1.53685 & -0.49714 \\ \mathrm{~S} & 1.99070 & 0.19344 & 2.05421 \\ \mathrm{~S} & -4.67061 & -0.87885 & 8.04886 \\ \mathrm{~S} & -1.18671 & -0.62416 & 7.96230\end{array}$

1. Zhang, B. et al. Hollow organic capsules assemble into cellular semiconductors. Nat. Commun. 9, 1957 (2018).

2. Krömer, J. et al. Synthesis of the First Fully $\alpha$-Conjugated Macrocyclic Oligothiophenes: Cyclo[n]thiophenes with Tunable Cavities in the Nanometer Regime. Angew. Chem. Int. Ed. 39, 3481-3486 (2000).

3. Nakao, K. et al. Giant Macrocycles Composed of Thiophene, Acetylene, and Ethylene Building Blocks. J. Am. Chem. Soc. 128, 16740-16747 (2006).

4. Darzi, E. R. et al. Synthesis, Properties, and Design Principles of Donor-Acceptor Nanohoops. ACS Cent. Sci. 1, 335-342 (2015).

5. Ball, M. et al. Chiral Conjugated Corrals. J. Am. Chem. Soc. 137, 9982-9987 (2015).

6. Ito, H. et al. Thiophene-Based, Radial pi-Conjugation: Synthesis, Structure, and Photophysical Properties of Cyclo-1,4-phenylene-2 ',5'-thienylenes. Angew. Chem. Int. Ed. 54, 159-163 (2015).

7. Xue, J. Y. et al. Aromatic hydrocarbon macrocycles for highly efficient organic lightemitting devices with single-layer architectures. Chem. Sci. 7, 896-904 (2016).

8. Kayahara, E. et al. Synthesis and Characterization of [n]CPP ( $\mathrm{n}=5,6,8,10$, and 12) Radical Cation and Dications: Size-Dependent Absorption, Spin, and Charge Delocalization. J. Am. Chem. Soc. 138, 338-344 (2016).

9. Kayahara, E. et al. Size Dependence of $\{[\} n]$ Cycloparaphenylenes $(n=5-12)$ in Electrochemical Oxidation. Chem. Asian J. 11, 1793-1797 (2016).

10. Zhang, B. et al. Rigid, Conjugated Macrocycles for High Performance Organic Photodetectors. J. Am. Chem. Soc. 138, 16426-16431 (2016).

11. Ball, M. et al. Macrocyclization in the Design of Organic n-Type Electronic Materials. $J$. 
Am. Chem. Soc. 138, 12861-12867 (2016).

12. Van Raden, J. M. et al. Synthesis and characterization of a highly strained donor-acceptor nanohoop. Org. Biomol. Chem. 14, 5721 (2016).

13. Peeks, M. D. et al. Aromatic and antiaromatic ring currents in a molecular nanoring. Nature 541, 200-203 (2017).

14. Zang, L. et al. One-Dimensional Self-Assembly of Planar $\pi$-Conjugated Molecules: Adaptable Building Blocks for Organic Nanodevices. Acc. Chem. Res. 41, 1596-1608 (2008).

15. Izumi, T. et al. Efficient Blue Electroluminescence from a Single-layer Organic Device Composed Solely of Hydrocarbons. Chem. Asian J. 12, 730-733 (2017).

16. Nishigaki, S. et al. Synthesis, Structures, and Photophysical Properties of Alternating Donor-Acceptor Cycloparaphenylenes. Chem. Eur. J. 23, 7227-7231 (2017).

17. Thakellapalli, H. et al. Synthesis and Properties of Conjugated Macrocycles Containing 2,7Bis(2-thienyl)-9H-fluoren-9-one Units. Org. Lett. 19, 2674-2677 (2017).

18. Li, T. et al. Synthesis, solvent-dependent emission and two- photon absorption of a triangular -[D-pi-A](3)- macrocycle. Org. Chem. Front. 4, 737-742 (2017).

19. Van Raden, J. M. et al. 2,2'-Bipyridyl-Embedded Cycloparaphenylenes as a General Strategy To Investigate Nanohoop-Based Coordination Complexes. J. Am. Chem. Soc. 139, 2936-2939 (2017).

20. Li, C. et al. A diketopyrrolopyrrole-based macrocyclic conjugated molecule for organic electronics. J. Mater. Chem. C 7, 3802-3810 (2019).

21. Kayahara, E. et al. Gram-Scale Syntheses and Conductivities of $\{[\}$ 10]Cycloparaphenylene and Its Tetraalkoxy Derivatives. J. Am. Chem. Soc. 139, 18480-18483 (2017).

22. Zhang, F. et al. Giant Cyclo n thiophenes with Extended pi Conjugation. Angew. Chem. Int. Ed. 48, 6632-6635 (2009).

23. Fenwick, O. et al. Linear and Cyclic Porphyrin Hexamers as Near-Infrared Emitters in Organic Light-Emitting Diodes. Nano Lett. 11, 2451-2456 (2011).

24. Zhang, F. et al. Molecular and electronic structure of cyclo[10]thiophene in various oxidation states: polaron pair vs. bipolaron. Chem. Sci. 2, 781-784 (2011).

25. Iyoda, M. et al. Conjugated Macrocycles: Concepts and Applications. Angew. Chem. Int. Ed. 50, 10522-10553 (2011).

26. Jiang, H.-W. et al. Cyclic 2,12-Porphyrinylene Nanorings as a Porphyrin Analogue of Cycloparaphenylenes. J. Am. Chem. Soc. 137, 2219-2222 (2015).

27. Kuwabara, T. et al. Curved Oligophenylenes as Donors in Shape-Persistent DonorAcceptor Macrocycles with Solvatofluorochromic Properties. Angew. Chem. Int. Ed. 54, 9646-9649 (2015).

28. Chen, Q. et al. Strain-Induced Stereoselective Formation of Blue-Emitting Cyclostilbenes. J. Am. Chem. Soc. 137, 12282-12288 (2015).

29. Iwamoto, T. et al. Size-Selective Encapsulation of C60 by [10]Cycloparaphenylene: 
Formation of the Shortest Fullerene-Peapod. Angew. Chem. Int. Ed. 50, 8342-8344 (2011).

30. Ball, M. L. et al. Influence of Molecular Conformation on Electron Transport in Giant, Conjugated Macrocycles. J. Am. Chem. Soc. 140, 10135-10139 (2018).

31. Ball, M. et al. Conjugated Macrocycles in Organic Electronics. Acc. Chem. Res. (2019). doi:10.1021/acs.accounts.9b00017

32. Iwamoto, T. et al. Selective and Random Syntheses of n Cycloparaphenylenes $(\mathrm{n}=8-13)$ and Size Dependence of Their Electronic Properties. J. Am. Chem. Soc. 133, 8354-8361 (2011).

33. Cavallo, G. et al. The Halogen Bond. Chem. Rev. 116, 2478-2601 (2016).

34. Dolomanov, O. V, et al. \{lit OLEX2\}: A complete structure solution, refinement and analysis program. J. Appl. Crystallogr. 42, 339-341 (2009).

35. Su, W.-C. et al. Characterization of intrinsic hysteresis of pentacene-based organic thinfilm transistor through in-situ real-time electrical measurement. Jpn. J. Appl. Phys. 53, $03 \mathrm{CC03}$ (2014).

36. Yaws, C. L. et al. Chapter 1 - Vapor Pressure - Organic Compounds. in (ed. Yaws, C. L. B. T.-T. Y. H. of V. P. (Second E.) 1-314 (Gulf Professional Publishing, 2015).

37. Hsieh, J. C. et al. Response characteristics of lead phthalocyanine gas sensor: effects of film thickness and crystal morphology. Thin Solid Films 322, 98-103 (1998).

38. Sakai, G. et al. Theory of gas-diffusion controlled sensitivity for thin film semiconductor gas sensor. Sensors Actuators B Chem. 80, 125-131 (2001).

39. Zhao, Y. H. et al. Fast Calculation of van der Waals Volume as a Sum of Atomic and Bond Contributions and Its Application to Drug Compounds. J. Org. Chem. 68, 7368-7373 (2003).

40. Crone, B. et al. Electronic sensing of vapors with organic transistors. Appl. Phys. Lett. 78, 2229-2231 (2001).

41. Huang, W. et al. Highly Sensitive NH3 Detection Based on Organic Field-Effect Transistors with Tris(pentafluorophenyl)borane as Receptor. J. Am. Chem. Soc. 134, 14650-14653 (2012).

42. Farrow, C. L. et al. PDFfit2 and PDFgui: computer programs for studying nanostructure in crystals. J. Phys. Condens. Matter 19, 335219 (2007).

43. Rajasingh, P. et al. Selective Bromination of Perylene Diimides under Mild Conditions. $J$. Org. Chem. 72, 5973-5979 (2007).

44. You, J. B. et al. A polymer tandem solar cell with $10.6 \%$ power conversion efficiency. Nat. Commun. 4, 10 (2013).

45. CrysAlis Pro. (2013).

46. Sheldrick, G. M. A short history of $\{$ lit SHELX $\}$. Acta Crystallogr. Sect. A 64, 112-122 (2008).

47. Sheldrick, G. M. \{lit SHELXT $\}\{--\}$ Integrated space-group and crystal-structure determination. Acta Crystallogr. Sect. A 71, 3-8 (2015). 
48. Palatinus, L. et al. \{lit SUPERFLIP $\}\{--\}$ a computer program for the solution of crystal structures by charge flipping in arbitrary dimensions. J. Appl. Crystallogr. 40, 786-790 (2007).

49. Spek, A. L. Structure validation in chemical crystallography. Acta Crystallogr. Sect. D 65 , 148-155 (2009).

50. Sheldrick, G. M. Phase annealing in $\{$ lit SHELX\}-90: direct methods for larger structures. Acta Crystallogr. Sect. A 46, 467-473 (1990).

51. Le Page, Y. \{\it MISSYM $1.1\{--\}$ a flexible new release. J. Appl. Crystallogr. 21, 983984 (1988).

52. Betteridge, P. W. et al. \{lit CRYSTALS \} version 12: software for guided crystal structure analysis. J. Appl. Crystallogr. 36, 1487 (2003).

53. Hammersley, A. P. et al. Two-dimensional detector software: From real detector to idealised image or two-theta scan. High Press. Res. 14, 235-248 (1996).

54. Yang, X. et al. PDFgetXgui: a program for pair distribution function transformation and visualization. (2014).

55. Juhás, P. et al. \{\it PDFgetX3\}: a rapid and highly automatable program for processing powder diffraction data into total scattering pair distribution functions. J. Appl. Crystallogr. 46, 560-566 (2013).

56. Toby, B. H. et al. Accuracy of pair distribution function analysis applied to crystalline and non-crystalline materials. Acta Crystallogr. Sect. A 48, 336-346 (1992).

57. Qiu, X. et al. Reciprocal-space instrumental effects on the real-space neutron atomic pair distribution function. J. Appl. Crystallogr. 37, 110-116 (2004). 


\section{Chapter 7. Chiral PDI as Electron Deficient Moiety in Locked Cycloparaphenylene}

\subsection{Preface}

Chapter 7 contains work from a collaboration between the Nuckolls Laboratory and the Yamago Laboratory at Kyoto University. I synthesized PPh6-PhHex while in Kyoto University with invaluable assistance from Sun Liansheng. I performed all compound characterizations. Nathanial Schuster performed the CD analysis. I performed the DFT calculations, with valuable input from Dr. Michael Steigerwald.

\subsection{Introduction}

This manuscript describes a new donor/acceptor macrocycle that incorporates PDI and is shape persistent and chiral. Conformationally rigid and chiral macrocycles remain a challenging synthetic target as they require the design of a rigid scaffold in order to impede isomerization between stereoisomers. Chapter 6 described $\left(\mathbf{P B B r}_{4}\right)_{3}$ as a sensor and nanoreactor ${ }^{1}$ with small molecule guests, and we envision additional potential uses within catalysis, ${ }^{2}$ sensing, ${ }^{1,3,4}$ and applications requiring preferential absorption of light; such as, circularly polarized light (CPL) emitters $^{5-8}$ with macrocyclic host with larger cavities. Isobe and coworkers too have synthesized conformationally rigid arene-based macrocycles. ${ }^{9-11}$ Here we show the design and synthesis of the first chiral, donor/acceptor nanobelt that incorporates a PDI as the electron acceptor moiety (part of the system that has low energy empty space available to accept an electron), and an alkoxysubstituted CPP belt as the donor moiety (part of the molecule that possesses a large density of high energy electrons). 
(a)

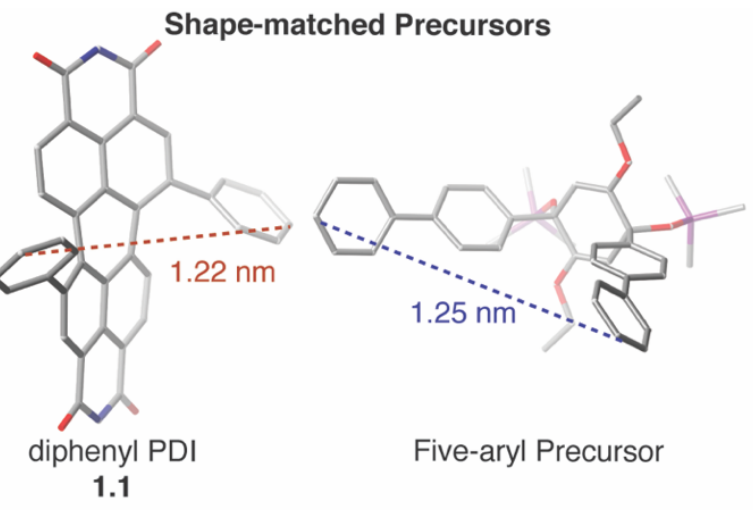

(b)

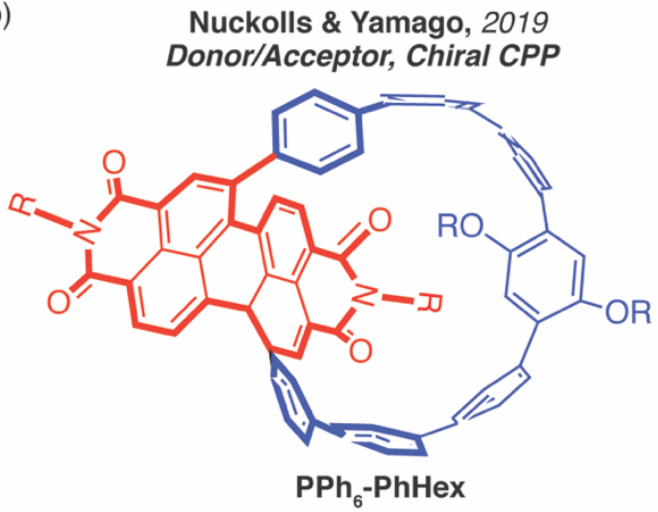

Figure 7.1. (a) The two pieces that were joined to make PPh $\mathbf{h}_{\mathbf{6}} \mathbf{P h H e x}$ : a diphenyl PDI (Compound 1.1) and five-aryl precursor that possess analogous distance between substituents; (b) a PDIsubstituted CPP called $\mathbf{P P h} \mathbf{p h}_{\mathbf{6}}-\mathbf{P h H e x}$, where the PDI is the electron poor and the belt is the electron rich portion of the molecule. Red = relative electron poor subunit = lower energy available space to accept an electron and blue $=$ relative electron rich subunit e.g. larger density of high energy electrons. $\mathrm{R}=\mathrm{C}_{11} \mathrm{H}_{23}$ for $\mathrm{PDI}$ and $\mathrm{R}=\mathrm{OC}_{6} \mathrm{H}_{13}$ for aryl ring.

\subsection{Design of Conformationally Restricted Macrocycle}

We used DFT to calculate the aryl-aryl distance in $\mathbf{1 . 1}(1.22 \mathrm{~nm})$, and chose a five-aryl CPP precursor (7.2) that was best matched to form a one-to-one complex (Figure 7.1a). ${ }^{12}$ We call this molecule $\mathbf{P P h}_{6}$-PhHex. Relative to unsubstituted CPPs, ${ }^{13-15}$ the addition of a PDI molecule results in strong absorption in the visible range up to $700 \mathrm{~nm}$ for $\mathbf{P P h}$ - $\mathbf{P h H e x}$. Using HPLC and CD experiments, we find $\mathbf{P} \mathbf{P h}_{\mathbf{6}}-\mathbf{P h H e x}$ exists as a pair of enantiomers that are conformationally rigid and do not racemize up to temperatures of $100{ }^{\circ} \mathrm{C}$.

Our previous work consisted of the design and synthesis of chiral donor/acceptor macrocycles, with a PDI as the relative electron deficient moiety and a phenyl-bithiophene-phenyl 
or phenyl moiety as the electron rich part of the molecule. ${ }^{16-20}$ PDI has many advantages as electronic materials. ${ }^{21-25}$ Due to their n-type behavior and broad absorption of visible light, we have used these macrocycles in electronic applications such as OFETs, OPVs, OPDs, and sensors. The motivation for the design of $\mathbf{P} \mathbf{P h} \mathbf{h}_{\mathbf{6}}-\mathbf{P h H e x}$ was to create a chiral, conformationally rigid molecule.

PPh6-PhHex was a challenging synthetic target due to the large amount of strain in the macrocycle. We performed homodesmotic calculations to assess the strain energy in $\mathbf{P} \mathbf{P h} \mathbf{h}_{\mathbf{6}}-\mathbf{P h H e x}$, and found $\mathbf{P P h}$-PhHex possesses $63 \mathrm{kcal} / \mathrm{mol}$ of strain energy (Section 7.13.3). This is similar to an [8]- and [10]CPP with similar sized cavities. ${ }^{15}$ Homodesmotic calculations compute the enthalpy difference between the macrocycle and an acyclic analog. The design of $\mathbf{P P h} \mathbf{h}_{\mathbf{6}} \mathbf{- P h H e x}$ was inspired by the Yamago ${ }^{12}$ and Jasti ${ }^{6,26}$ laboratories who use an unstrained borylated precursor as a means to access conformationally strained and rigid CPPs (Section 1B.3). Previous syntheses in the Nuckolls' laboratory used a bis-platinated PDI (2.2) in the macrocyclization step ${ }^{19}$ that is formed from a double transmetalation of 1,7-Bis[4-(tributylstannyl)-phenyl]-PDI.

\subsection{Synthesis of $\mathrm{PPh}_{6}-\mathrm{PhHex}$}

We use a cross coupling strategy in the synthesis of $\mathbf{P} \mathbf{P h}_{\mathbf{6}}-\mathbf{P h H e x}$. Figure 7.2 shows the synthesis of $\mathbf{P P h} \mathbf{h}_{6}-\mathbf{P h H e x}$. Compounds $\mathbf{2 . 2}$ and $\mathbf{7 . 2}$ were reacted with $\mathrm{K}_{3} \mathrm{PO}_{4}$ in tetrahydrofuran (THF) at $55^{\circ} \mathrm{C}$ for 48 hours. We isolated intermediate compound 7.3 in a $6 \%$ yield, and proceeded to the reductive aromatization of $\mathbf{7 . 3}$ using $\mathrm{SnCl}_{2}$ and $\mathrm{HCl}$ to yield our target compound $\mathbf{P} \mathbf{P h}_{6-}$ PhHex as a teal solid. We also isolate the precursor to SI-7.1 also in a 6\% yield (Figure 7.6 and 7.7). This byproduct suggests there is some degree of reversibility in the transmetalation step of the reaction sequence. Section 7.13 contains the details of the calculations to assess the strain energy, synthesis, and characterization of $\mathbf{P} \mathbf{P h} \mathbf{h}_{\mathbf{6}}-\mathbf{P h H e x}$. 


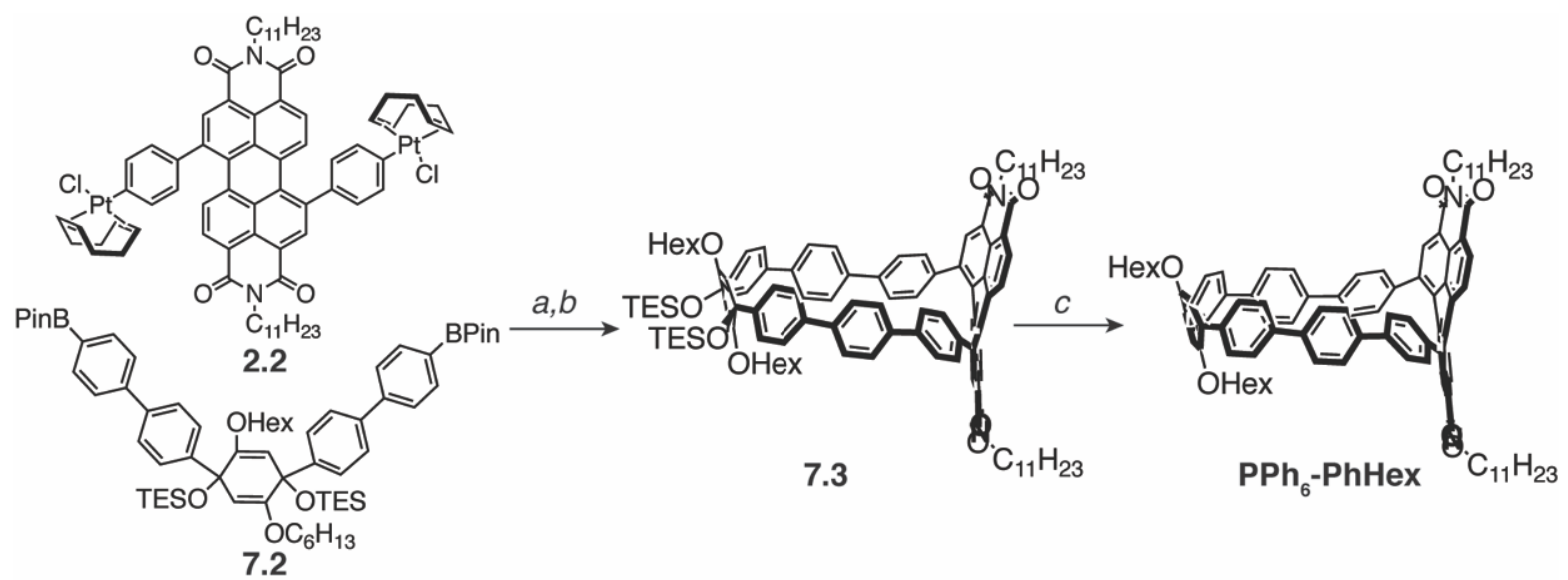

Figure 7.2. Synthesis of $\mathbf{P} \mathbf{P h}_{\mathbf{6}}-\mathbf{P h H e x}$. Compounds $\mathbf{2 . 2}$ and $\mathbf{7 . 2}$ are reacted in equimolar ratios to afford intermediate $\mathbf{7 . 3}$ in a 6\% yield. $\mathbf{P P h} \mathbf{h}_{6} \mathbf{P h H e x}$ is obtained after an aromatization step. key: ${ }^{\mathrm{a}} \mathrm{K}_{3} \mathrm{PO}_{4}, \mathrm{THF}, 55{ }^{\circ} \mathrm{C}, 48 \mathrm{~h}$; ${ }^{\mathrm{b}} \mathrm{PPh}_{3}$, toluene, $100{ }^{\circ} \mathrm{C}, 22 \mathrm{~h}, 6 \%$ yield $(2$ steps $)$; ${ }^{\mathrm{c}} \mathrm{SnCl}_{2}, \mathrm{HCl}(50 \%$ yield).

\subsection{Strain Originates from Aryl-PDI Dihedral}

Figure 7.3 shows the lowest energy geometry calculated using the 6-31G/B3LYP basis set.

PPh6-PhHex possesses an elliptiform cavity that measures $12.3 \AA$ along its long axis from the PDI to the alkoxy-substituted aryl ring (Figure 7.3). The aryl rings are arranged with their $\pi$-surfaces directed to the cavity, and form a concave surface for the perimeter of the macrocycle. Analogous to other bay-substituted PDI molecules, the two phenyl rings attached to the PDI are on the same face of the PDI molecule: this is an important design feature as it facilitates macrocyclization. ${ }^{19}$ We observe two important features about the PDI-aryl bond once the diphenyl PDI is constricted into a macrocyclic framework: 1) the torsional angle between the phenyl group and the PDI moiety is reduced for $\mathbf{P P h}$ - $\mathbf{P h H e x}$ relative to diphenyl PDI, at $26^{\circ}$ and $54^{\circ}$, respectively; and 2) the arylaryl distance (highlighted in orange circles in Figure 7.3b) decreases once the diphenyl PDI is restricted within a macrocycle. DFT measurements show this is $0.97 \mathrm{~nm}$ instead of $12.2 \mathrm{~nm}$ (Figure 7.1a) in a relatively unstrained diphenyl PDI. The relatively more coplanar linkage between the PDI and aromatic belt increases electronic coupling between the two moieties. 


\subsection{Size-Dependent Isomerization}

Due to the substitution in the bay position of the PDI, PPh $\mathbf{6}_{\mathbf{6}} \mathbf{P h H e x}$ is chiral, and exists as a $(P)$ - $\mathbf{P} \mathbf{P h} \mathbf{h}_{\mathbf{6}}$-PhHex and $(M)-\mathbf{P} \mathbf{P h} \mathbf{h}_{\mathbf{6}}-\mathbf{P h H e x}$ in solution. We have synthesized chiral PDI-based macrocycles that have been conformationally locked ${ }^{1}$ or exhibit dynamic stereochemistry. ${ }^{19}$ Chiral PDI-based macrocycles isomerize through an 'intramolecular somersault,' that is, where a chiral $(P$ or $M$ ) PDI molecule rotates its diimide head around its transverse axis through the plane of the cavity to convert to the opposite enantiomer $(M$ or $P)$. The ability to isomerize is dependent upon the rigidity of the macrocycle and the size of the cavity (Chapter 1B.5). If the diameter of the cavity is large enough, then the macrocycle exhibits dynamic stereochemistry using the intramolecular somersault mechanism, yet when the cavity is smaller, the macrocycle is conformationally locked and shape persistent. Our studies suggest that cavities with the long axis measuring at least $15 \AA$ can isomerize in solution, while cavities at $11 \AA$ or shorter are conformationally restricted.

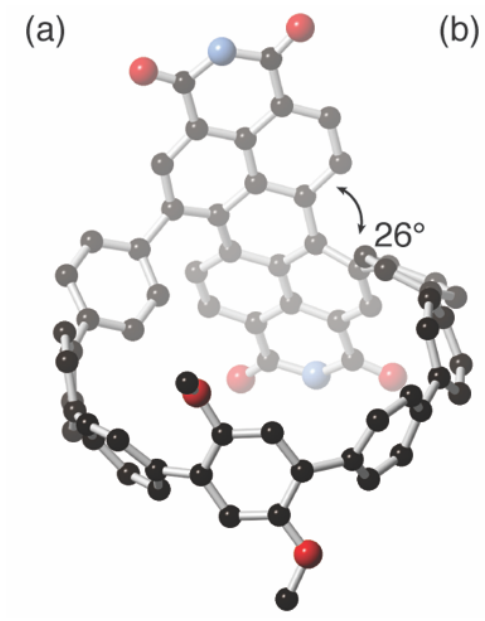

(b)

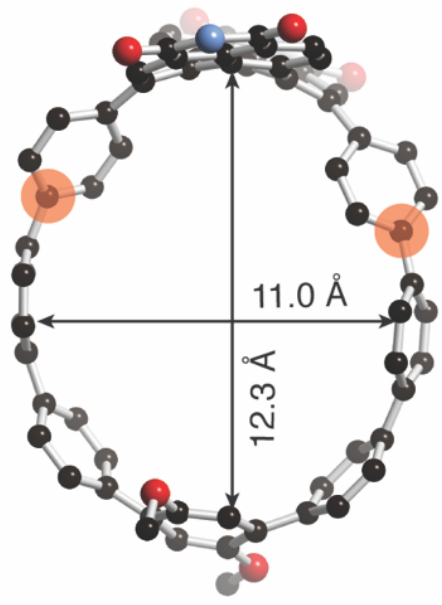

Figure 7.3. DFT calculations showing (a) the twisting of $(P)-\mathbf{P P h}_{6}-\mathbf{P h H e x}$. The torsional angle is reduced in $\mathbf{P P h} \mathbf{h}_{\mathbf{6}}$-PhHex. (b) The cavity measures $12.3 \AA$ along the long axis from PDI to alkoxysubstituted aryl ring. Orange circles indicate the distance between the PDI phenyl appendages. Red $=$ oxygen, blue $=$ nitrogen, black $=$ carbon, and yellow $=$ sulfur. Hydrogens and side chains have been removed for clarity. Computations at the using 6-31G/B3LYP level of theory. 


\subsection{Measurements Show $\mathrm{PPh}_{6}-\mathrm{PhHex}$ is Conformationally Locked}

We employed chiral HPLC to study the dynamics of $\mathbf{P P h}$-PhHex. Using a CHIRALPAK® IA-3 column (4.6 mm I.D. x $250 \mathrm{~mm}, 3 \mu \mathrm{m})$, we isolate two peaks in a 1:1 ratio (Figure 7.4a), and use CD spectroscopy to confirm that the two peaks are $(P)$ - $\mathbf{P P h}$-PhHex and (M)- $\mathbf{P P h}$ 6-PhHex. Figure 7.4b shows each enantiomer of $\mathbf{P} \mathbf{P h}_{6}-\mathbf{P h H e x}$ preferentially absorbs leftor right-handed circularly polarized light.

We also performed a variable temperature $\mathrm{CD}$ experiment, where the sample is heated and monitored by CD spectroscopy, to study the isomerization process in $\mathbf{P} \mathbf{P h} \mathbf{h}_{\mathbf{6}} \mathbf{- P h H e x}$. We heated an optically pure sample of Enantiomer 2 of $\mathbf{P P h} 6-\mathbf{P h H e x}$ to temperatures to $100{ }^{\circ} \mathrm{C}$ (over three hours) and saw no evidence of isomerization. This suggests $\mathbf{P P h}_{\mathbf{6}}-\mathbf{P h H e x}$ is conformationally restricted and unable to perform an intramolecular somersault.

Both the CD spectrum and UV-Vis spectrum show three broad groups of electronic transitions: alkoxy-substituted aryl $\rightarrow$ PDI, aryl belt $\rightarrow$ PDI, and PDI $\rightarrow$ PDI at lower wavelengths. Figure $7.4 \mathrm{c}$ contains the UV-Vis spectrum. The lowest energy transition is characterized by the promotion of an electron from the alkoxy-substituted aryl ring (HOMO) to a PDI-centered orbital (LUMO). Figure 4a,b shows the HOMO and LUMO molecular orbitals for $\mathbf{P P h}$-PhHex. The Appendix contains the calculated absorption spectrum without thermal broadening to best visualize the electronic states (Figure 7.8) and the TD-DFT information used for the assignment of the electronic states. 

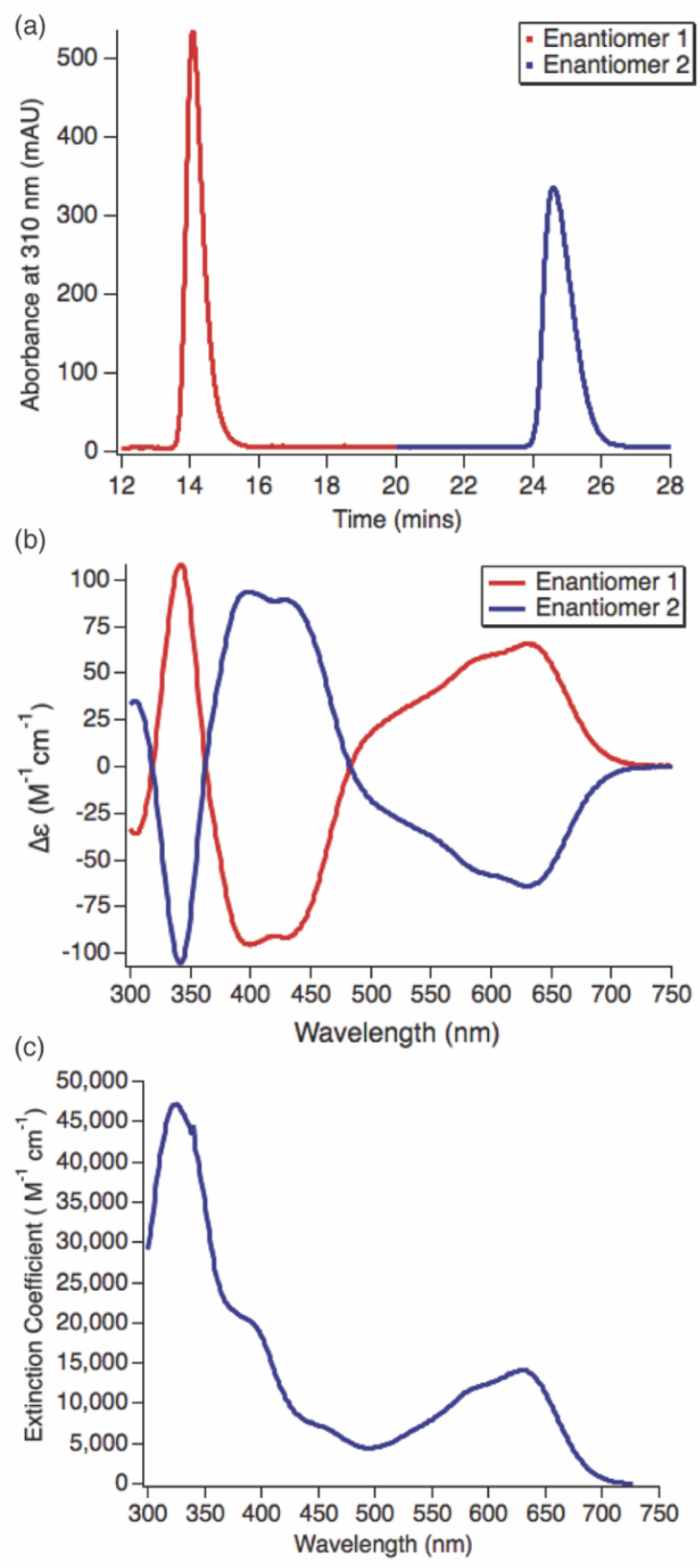

Figure 7.4. (a) HPLC chromatogram showing the retention times of both $(P)$ - PPh6-PhHex and $(M)$ - $\mathbf{P P h}_{6}$-PhHex with a mobile phase of $18 \%$ DCM/82\% Hexanes using an IA-3 Chiralpak column. We call them Enantiomer 1 and 2, arbitrarily assigned; (b) CD chromatogram $(P)$ - $\mathbf{P P h}_{6-}$ PhHex and $(M)$ - $\mathbf{P P h}_{\mathbf{6}}-\mathbf{P h H e x}$ in toluene $\left(1 \mathrm{~cm}\right.$ path length, $10^{-6} \mathrm{M}$ for Enantiomer 1 and $10^{-5} \mathrm{M}$ for Enantiomer 2); and (c) UV-Vis absorption spectrum of $\mathbf{P P h} \mathbf{P h}_{\mathbf{6}} \mathbf{P h H e x}$ (dichloromethane, $1.0 \mathrm{~cm}$ path length, $\left.1.37 \times 10^{-5} \mathrm{M}\right)$.

\subsection{Incorporation of PDI Alters Electronic Structure of $\mathrm{PPh}_{6}-\mathrm{PhHex}$}

The incorporation of a diphenyl PDI moiety into a CPP nanobelt has a profound effect on the electronic structure. While [8]- and [10]CPP are yellow in solution, $\mathbf{P P h}$-PhHex is teal 
reflecting its ability to absorb visible light: the onset of absorption is at $680 \mathrm{~nm}$ for $\mathbf{P P h}_{6}-\mathbf{P h H e x}$. The narrowing of the energy gap for $\mathbf{P P h}_{6}-\mathbf{P h H e x}$ reflects the incorporation of a PDI moiety into the nanobelt and a lowering in the LUMO energy level, as both the LUMO/LUMO+1/LUMO+2 reside on a PDI-centered orbital. Other donor/acceptor CPPs too showed a narrowing of the HOMO/LUMO level due to the incorporation of strong electron withdrawing subunits, ${ }^{27-29}$ but the effect from PDI incorporation greatly enhances this effect.

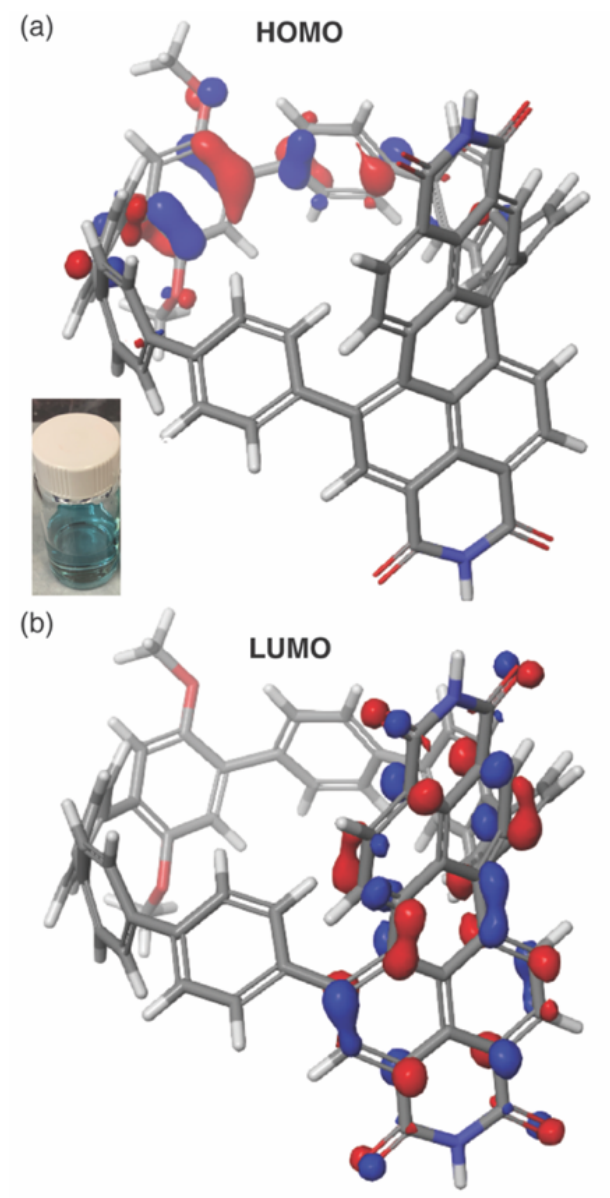

Figure 7.5. (a) HOMO energy level showing a high density of high energy electrons on the hexoxy-substituted aryl ring and (b) LUMO energy level showing unoccupied low energy space on the PDI. Red $=$ oxygen, blue $=$ nitrogen, black $=$ carbon, and yellow $=$ sulfur. Hydrogens and side chains have been removed for clarity.

It is also interesting to compare the UV-Vis spectrum of an $\mathbf{1 . 1}$ with $\mathbf{P P h} \mathbf{h}_{\mathbf{6}} \mathbf{- P h H e x}$ (Figures 7.8 and 7.9). From TDDFT, we assign the second excited state as a promotion of an electron from a PDI-centered orbital to a PDI-centered orbital for $\mathbf{P P h} \mathbf{h}_{6}-\mathbf{P h H e x}$, which is analogous to the lowest 
energy transition for 1.1. We believe this transition is shifted towards lower energy in $\mathbf{P P h}_{\mathbf{6}} \mathbf{-} \mathbf{P h H e x}$ because of the orientation of the PDI with respect to the adjacent phenyls: the phenyls pi-surfaces are more conjugated to the PDI core in $\mathbf{P P h _ { 6 }}-\mathbf{P h H e x}$ as the torsional angle is less relative to 1.1.

\subsection{Conclusion}

In conclusion, we have synthesized a chiral donor/acceptor CPP nanobelt called $\mathbf{P P h}$ PhHex. The nanobelt comprises a PDI moiety as the relatively electron deficient subunit and an alkoxy-substituted aryl belt as the electron rich portion. We have shown that incorporation of a PDI into the belt causes a profound effect on the electronic structure of the macrocycle relative to CPPs. $\mathbf{P P h}_{6}-\mathbf{P h H e x}$ is teal in solution and has an onset of absorption into the visible. The macrocycle, too, is conformationally locked, and does not show evidence of racemization at elevated temperatures. This study reveals the importance of substituting CPPs with chiral, electroactive groups, and highlights the effects on the electronic structure. Future studies will include host/guest chemistry with chiral analytes and sensing applications.

\subsection{Appendix - Supplementary Figures}

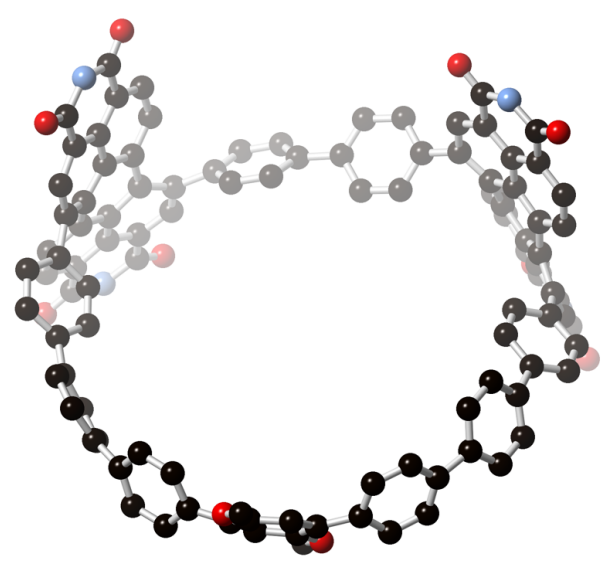

Figure 7.6. DFT minimized geometry for SI-7.1 Red $=$ oxygen, blue $=$ nitrogen, black $=$ carbon, and yellow $=$ sulfur. Hydrogens and side chains have been removed for clarity. 


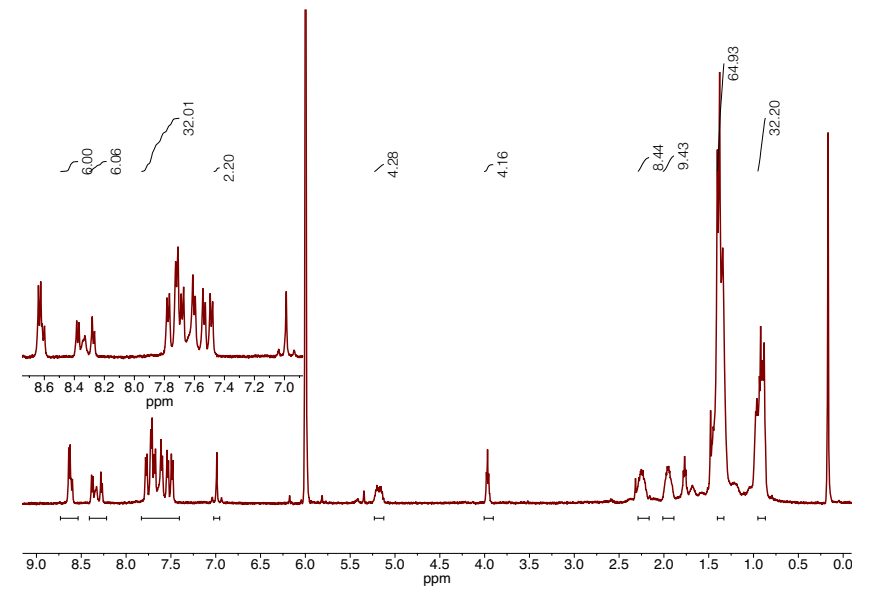

Figure 7.7. Proton NMR and chemical structure of the major byproduct from the reaction SI-7.1. This byproduct suggests there is some degree of reversibility in the reaction. It contains two bisplatinum, diphenyl-PDIs and one belt linker. ${ }^{1} \mathrm{H}$ NMR taken in $\mathrm{C}_{2} \mathrm{D}_{2} \mathrm{Cl}_{4}$ at $410 \mathrm{~K}$. HRMS (MALDI ${ }^{-}$ ) calculated $\mathrm{m} / \mathrm{z}$ for $\left[\mathrm{C}_{158} \mathrm{H}_{164} \mathrm{~N}_{4} \mathrm{O}_{10}\right]^{-}$is 2276.873 , found 2277.274 .

(a)

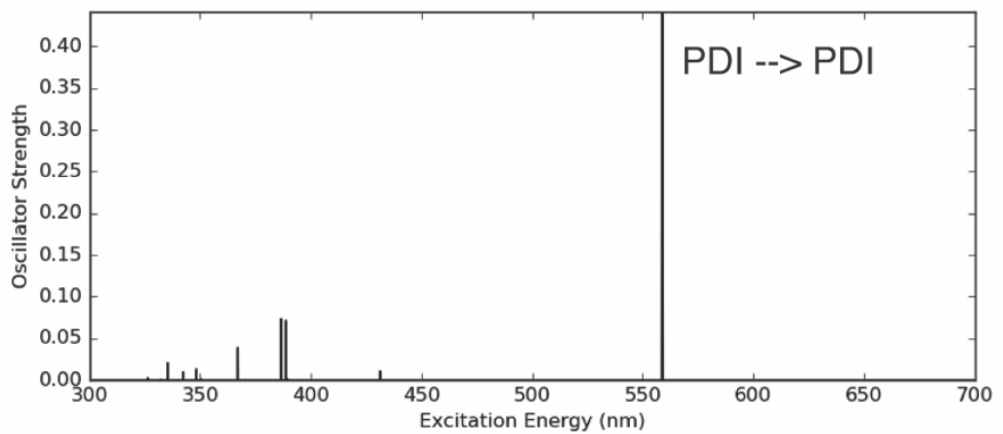

(b)

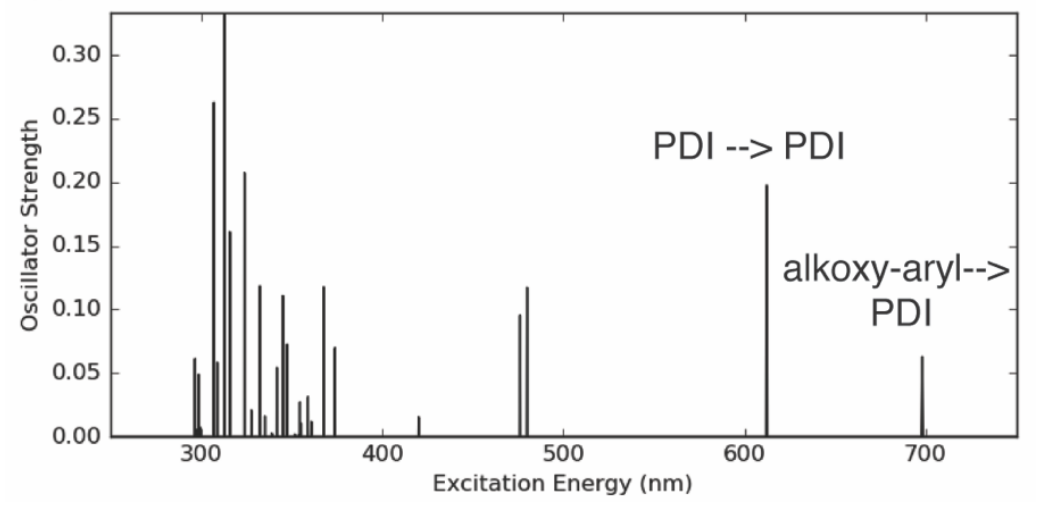

Figure 7.8. (a) The calculated UV-Vis for $\mathbf{1 . 1}$ (b) and $\mathbf{P P h}_{6}-\mathbf{P h H e x}$. The PDI $\rightarrow$ PDI transition is shifted to lower energy in the macrocycle as a result of a higher energy HOMO. 

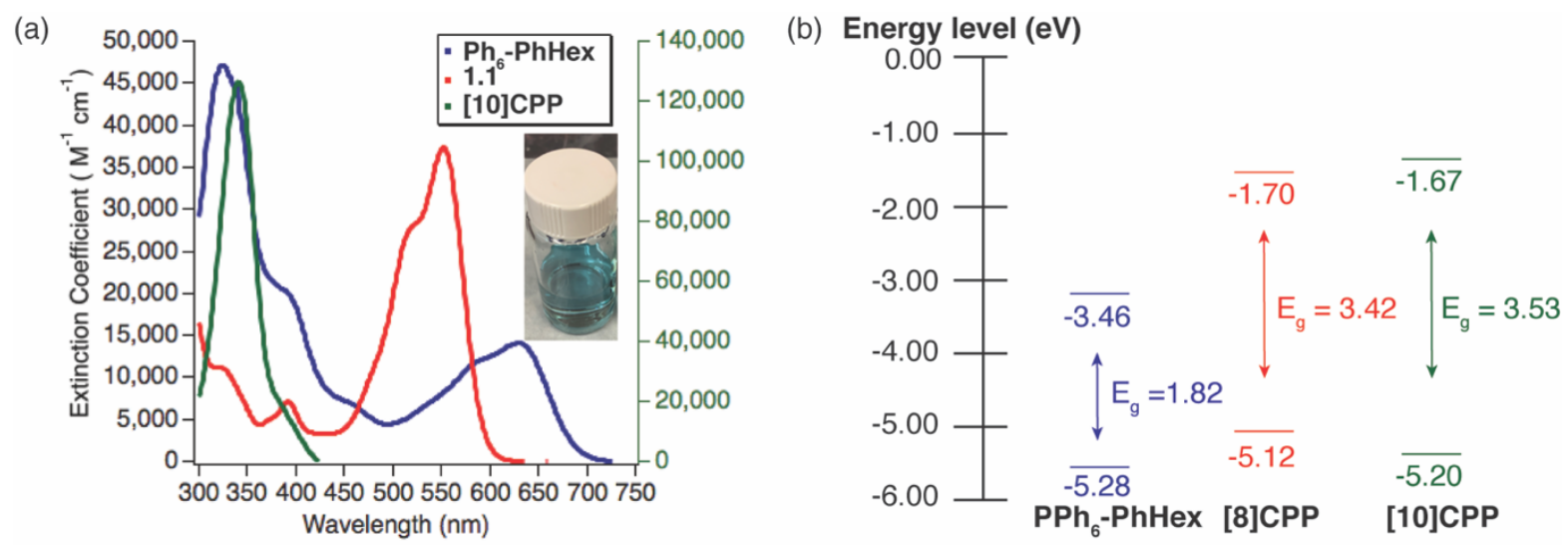

Figure 7.9. (a) UV-vis absorption spectrum of PPh $\mathbf{P h}_{6} \mathbf{P h H e x}$ (DCM, $1.0 \mathrm{~cm}$ path length, $1.37 \mathrm{x} 10^{-}$ ${ }^{5} \mathrm{M}$ ), diphenyl-PDI (1.1), and [10]CPP; (b) HOMO-LUMO energy gaps for the three compounds showing the electronic effect of incorporating a PDI moiety into a CPP nanobelt.

\subsection{General Experimental Information}

Synthesis. All reactions were performed in oven-dried or flame-dried round bottom flasks, unless otherwise noted. The flasks were fitted with rubber septa and reactions were conducted under a positive pressure of nitrogen or argon, unless otherwise noted. Anhydrous and anaerobic solvents were obtained from a Glass Contour solvent system consisting of a Schlenk manifold with purification columns packed with activated alumina and supported copper catalyst. Reaction monitoring by TLC was performed on J.T. Baker Baker-flex Silica Gel IB2-F (25 mm x $75 \mathrm{~mm}$ ) TLC plates. TLC visualization was accomplished by visible observation and irradiation with a UV lamp. Commercial reagents were used without further purification. $\mathrm{Pt}(\mathrm{COD}) \mathrm{Cl}_{2}$ was purchased from Strem Chemicals, and all other reagents were purchased from Sigma-Aldrich.

Purification. Automated flash chromatography was performed using a Teledyne Isco Combiflash Rf200 and Redisep Rf Silica and Alumina columns.

Spectrometers. ${ }^{1} \mathrm{H}$ NMR spectra were recorded on a Bruker 400 or $500 \mathrm{MHz}$ spectrometer. ${ }^{13} \mathrm{C}$ NMR spectra were recorded on a Bruker 125 or $100 \mathrm{MHz}$ spectrometer with complete proton decoupling. NMR spectra were recorded at $300 \mathrm{~K}$ unless otherwise noted. Chemical shifts are reported in parts per million (ppm) Data are represented as follows: chemical shift, multiplicity (s 
$=$ singlet, $\mathrm{d}=$ doublet, $\mathrm{dd}=$ doublet of doublets, $\mathrm{t}=$ triplet, $\mathrm{m}=$ multiplet $)$, coupling constants in $\mathrm{Hz}$, and integration. Broadening (br) of peaks in the ${ }^{1} \mathrm{H}$ NMR spectrum is due to rotational isomers about the imide side chains. Multiple peaks for the same carbon in the ${ }^{13} \mathrm{C}$ NMR spectrum arise due to rotational isomers about the imide side chains that have been seen previously. ${ }^{19,30} \mathbf{1 . 1}$ was previously presented in Chapters 1,3 , and $4 .{ }^{16}$ HRMS was performed on a (1) Waters XEVO G2XS QTOF instrument equipped with a UPC SFC inlet, and ESI and APCI ionization sources; or (2) a Bruker UltrafleXtreme MALDI TOF/TOF instrument using a dithranol matrix. UV-vis absorption spectra were recorded on a Shimadzu UV-1800 spectrophotometer using a $1.0 \mathrm{~cm}$ quartz cell. IR spectra were recorded on a Perkin Elmer Spectrum400 FTIR spectrometer using a PIKE ATR attachment.

Chirality Analysis: Analytical HPLC was performed on an Agilent 1200 Series instrument equipped with a diode array detector (300 $\mathrm{nm}$ to $900 \mathrm{~nm}$ ) and a CHIRALPAK ${ }^{\circledR}$ IA-3 column (4.6 mm I.D. x $250 \mathrm{~mm}, 3 \mu \mathrm{m})$ from Chiral Technologies. The mobile phase was $28 \%$ DCM and $78 \%$ hexanes.

\subsection{Synthetic Procedures and Characterization}
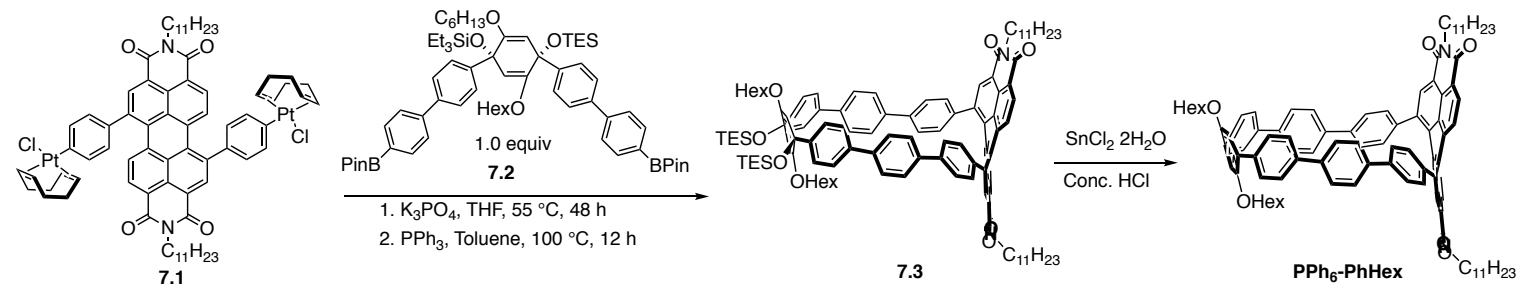

Synthesis of $\mathbf{P P h}_{6}-\mathbf{P h H e x}$ 1,7-Bis[4-(Pt(COD)Cl)-phenyl]-PDI (2.2) ${ }^{19}$ (0.261 mMol, 0.415 g) and

$7.2(0.261 \mathrm{mMol}, 0.286 \mathrm{~g})$ are added to an oven dried round bottom flask equipped with a stir bar in equimolar amounts. Oven-dried $\mathrm{K}_{3} \mathrm{PO}_{4}(0.276,1.31 \mathrm{mmol})$ was added and the solids were exposed to three cycles of purging with $\mathrm{N}_{2}$ /vacuum. $87.0 \mathrm{ml}$ of THF was added and the mixture was sparged with nitrogen for 30 min before being added to an oil bath at $55^{\circ} \mathrm{C}$ and allowed to stir 
for 48 hours. Crude mixture was then removed from oil bath and concentrated. The crude was extracted with ethyl acetate and brine to remove the salts and then triphenylphosphine $(5.21 \mathrm{mmol}$, $1.52 \mathrm{~g})$ and toluene $(87.0 \mathrm{~mL})$ were added to the flask. Mixture was sparged with nitrogen for 15 min then placed in a $100{ }^{\circ} \mathrm{C}$ oil bath and allowed to stir overnight. The crude mixture was concentrated, followed by re-precipitation from dichloromethane using methanol. and then purified by column chromatography (48 g Redisep Rf Alumina) using a gradient from $0 \%$ to $100 \%$ $\mathrm{CH}_{2} \mathrm{Cl}_{2} /$ hexanes. The polar fractions were further purified with prepatory TLC. Product was a dark green solid $(0.0280 \mathrm{~g}, 6.3 \%)$. Due to the stability of the intermediate, we proceeded to the reductive aromatization step immediately. Intermediate $\mathbf{7 . 3}$ sparged for one hour in $1.5 \mathrm{ml}$ THF while a separate flask containing $\mathrm{SnCl}_{2}(0.010 \mathrm{~g}, 0.044 \mathrm{mmol})$ and Conc. $\mathrm{HCl}(0.007 \mu \mathrm{L})$ in $3 \mathrm{ml}$ of THF was also sparged. A stock solution was made by scaling up the $\mathrm{SnCl}_{2}$ and $\mathrm{HCl}$ by three $(0.030$ $\mathrm{SnCl}_{2}$ and $0.022 \mu \mathrm{L} \mathrm{HCl} \mathrm{in} 3 \mathrm{ml}$ of THF). Using a syringe, $1 \mathrm{ml}$ of the stock solution was added to the reaction and allowed to stir overnight. Reaction was quenched with $2 \mathrm{M} \mathrm{NaOH}$ and extracted with ethyl acetate. A prepatory TLC was ran (70\%DCM/30\%hexanes) and isolated a teal solid (0.006 g, 42\% yield). ${ }^{1} \mathrm{H}$ NMR $\left(400 \mathrm{MHz}, 380 \mathrm{~K}, \mathrm{C}_{2} \mathrm{D}_{2} \mathrm{Cl}_{4}\right) \delta 8.74(\mathrm{~s}, 2 \mathrm{H}), 8.58(\mathrm{~d}, J=7.9 \mathrm{~Hz}$, 2H), $8.37(\mathrm{~d}, J=7.9 \mathrm{~Hz}, 2 \mathrm{H}), 7.70(\mathrm{~d}, J=8.7 \mathrm{~Hz}, 4 \mathrm{H}), 7.64(\mathrm{~d}, J=8.5 \mathrm{~Hz}, 4 \mathrm{H}), 7.55(\mathrm{~d}, J=8.5$ $\mathrm{Hz}, 4 \mathrm{H}), 7.50(\mathrm{~d}, J=8.7 \mathrm{~Hz}, 4 \mathrm{H}), 7.31(\mathrm{~d}, J=8.5 \mathrm{~Hz}, 4 \mathrm{H}), 7.15(\mathrm{~d}, J=8.5 \mathrm{~Hz}, 4 \mathrm{H}), 6.91(\mathrm{~s}, 2 \mathrm{H})$, 5.19 (br m, 2H), 3.98 (t, $J=6.5 \mathrm{~Hz}, 4 \mathrm{H}), 2.25$ (br m, 4H), 1.97 (br m, 4H), 1.82 (br m, 4H), 1.39 (br m, 36H)*, 0.96 (br m, 18H)*. ${ }^{13} \mathbf{C}$ NMR $\left(100 \mathrm{MHz}, 380 \mathrm{~K}, \mathrm{C}_{2} \mathrm{D}_{2} \mathrm{Cl}_{4}\right) \delta 164.42,164.08,152.27$, $142.18,140.07,139.62,139.20,138.40,138.14,138.74,135.25,134.77,132.83,130.54,129.64$, $129.60,129.43,129.16,129.07,128.93,128.61,127.13,126.67,122.86,122.42,116.54,70.42$, $55.04,53.47,32.73,32.66,31.91,31.80,31.60,29.66,26.76,25.92,22.62,22.53,22.48,22.46$, 13.97, 13.91, 13.86. HRMS (MALDI-) calculated $\mathrm{m} / \mathrm{z}$ for $\left[\mathrm{C}_{100} \mathrm{H}_{104} \mathrm{~N}_{2} \mathrm{O}_{6}\right]^{-}$is 1428.789 , found 
1428.706. *Integrations are high in the aliphatic region due to $\mathrm{H}$ grease overlapping with spectral peaks. 


\subsection{NMR Spectra of Compounds}

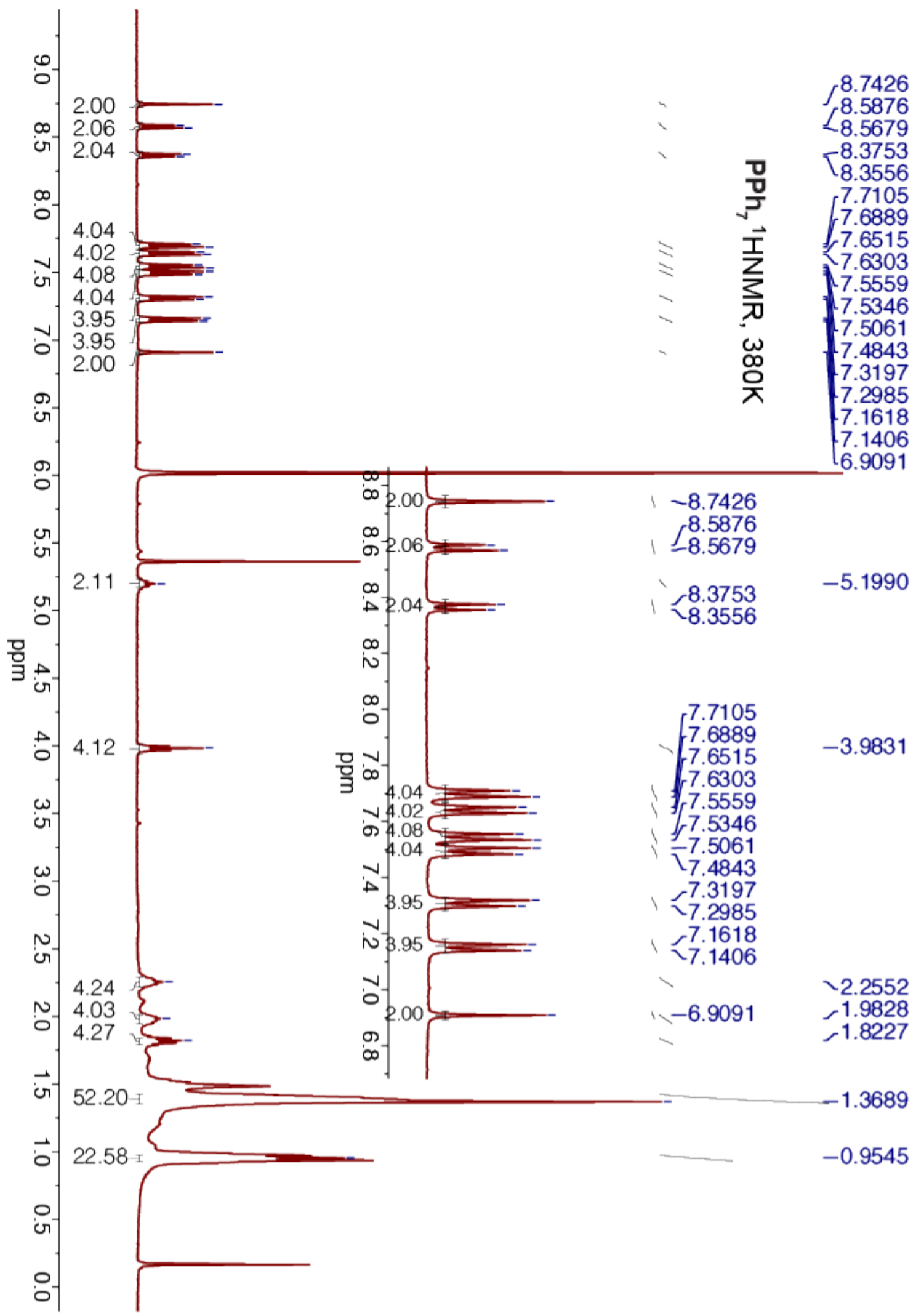



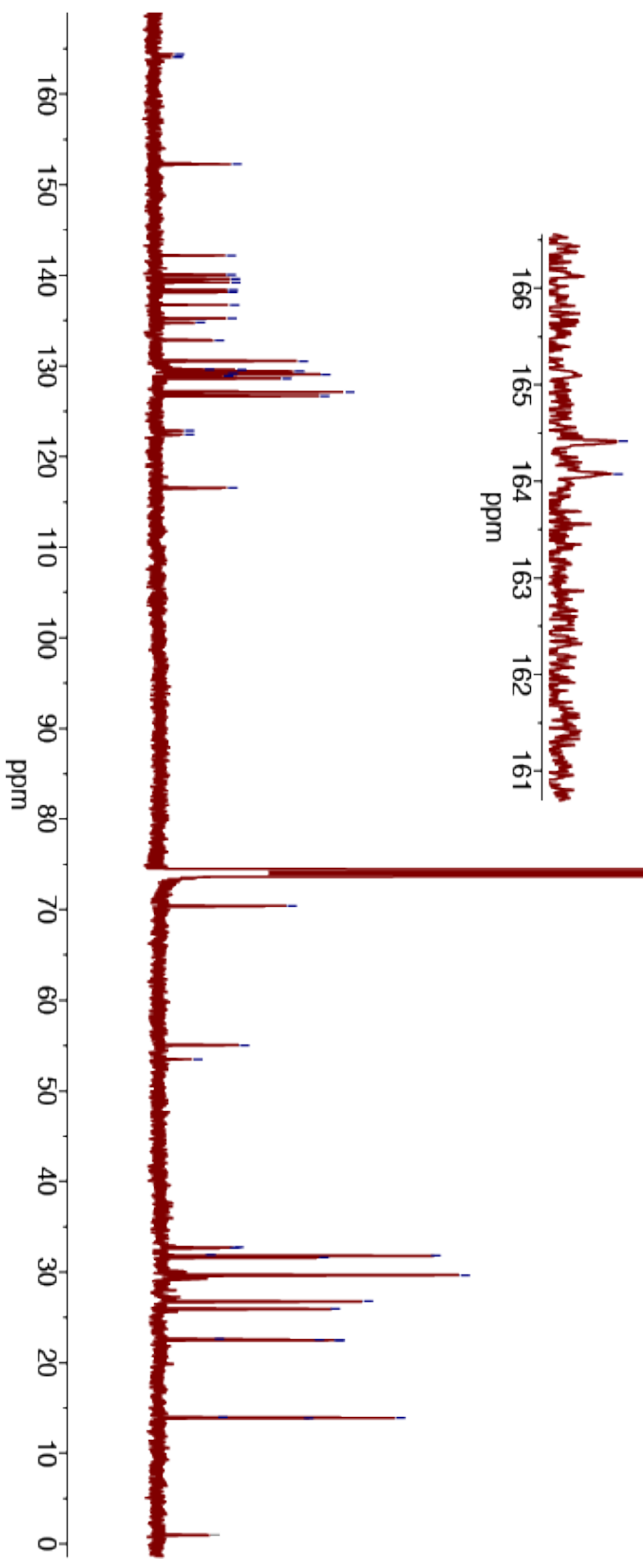

$-152.27$

142.18

140.07

139.62

139.20

138.40

138.14

136.74

135.25

134.77

$-164.42$

132.83

130.54

129.64

129.60

129.43

129.16

$-129.07$

128.93

128.61

127.13

126.67

122.86

122.42

116.54

74.33

73.78

70.42

55.04

53.47

32.73

32.66

31.91

31.80

31.60

29.66

26.76

25.92

22.62

22.53

$-22.48$

22.46

13.97
-13.91

-13.91
13.86 


\subsection{DFT Output}

All quantum chemical calculations were performed using Jaguar, version 8.3, Schrodinger, Inc., New York, NY, 2013. (See A. D. Bochevarov, E. Harder, T. F. Hughes, J. R. Greenwood, D. A. Braden, D. M. Philipp, D. Rinaldo, M. D. Halls, J. Zhang, R. A. Friesner, "Jaguar: A High Performance Quantum Chemistry Software Program with Strengths in Life and Materials Sciences", Int. J. Quantum Chem., 2013, 113(18), 2110-2142). All geometries were optimized using the B3LYP functional and the 6-31G basis set. In the following pages, we include for each molecule its optimized geometry and total energy. The geometry optimization for $\mathbf{1 . 1}$ is provided in Chapter 1B. The TDDFT excited state calculations for $\mathbf{P P h}$-PhHex present the thirty lowest energy roots and 15 lowest energy roots for 1.1. We also provide the results of the homodesmotic

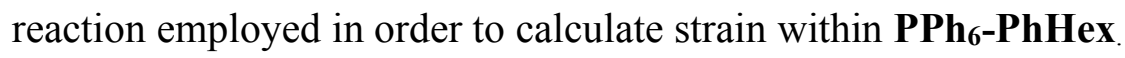

\section{Minimized Geometries for Compounds}

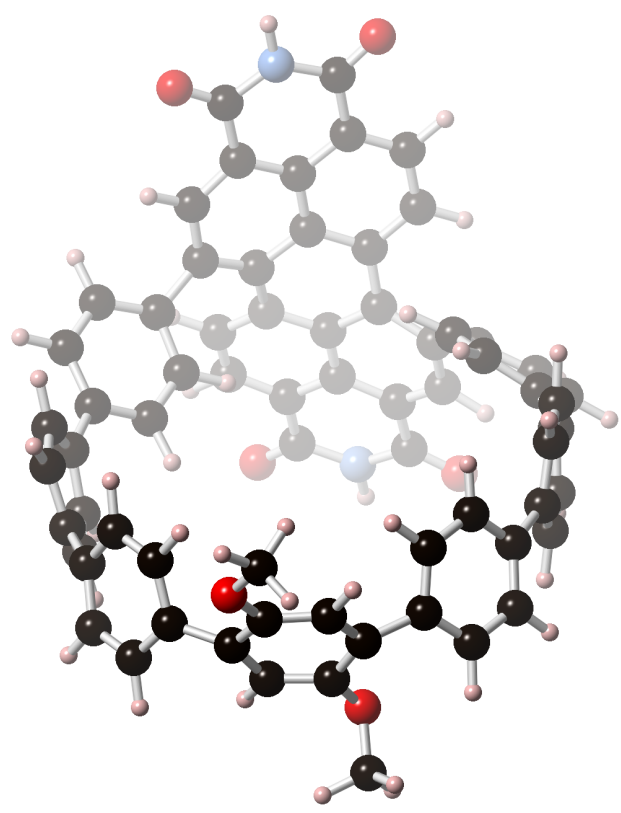

\section{$\mathbf{P P h}_{6}$-PhHex}

Final Heat of Formation $=-3176.308485$

$\begin{array}{llll}\text { C } & -1.21660 & -0.70690 & 1.58610\end{array}$

$\begin{array}{llll}\text { C } & -1.65860 & -1.72350 & 0.73350\end{array}$ 


\begin{tabular}{|c|c|c|c|}
\hline $\mathrm{C}$ & -1.93590 & -1.51390 & -0.61980 \\
\hline $\mathrm{C}$ & -1.85370 & -0.21620 & -1.15630 \\
\hline $\mathrm{C}$ & -1.18550 & 0.74810 & -0.36770 \\
\hline $\mathrm{C}$ & -0.83320 & 0.50990 & 0.95860 \\
\hline $\mathrm{C}$ & -2.76910 & 0.21170 & -2.25090 \\
\hline $\mathrm{C}$ & -3.52230 & 1.37650 & -2.00750 \\
\hline $\mathrm{C}$ & -4.70180 & 1.63500 & -2.68820 \\
\hline $\mathrm{C}$ & -5.19500 & 0.74270 & -3.65530 \\
\hline $\mathrm{C}$ & -4.34420 & -0.31170 & -4.03490 \\
\hline $\mathrm{C}$ & -3.15940 & -0.57600 & -3.34970 \\
\hline $\mathrm{C}$ & -6.64330 & 0.79120 & -3.99090 \\
\hline $\mathrm{C}$ & -7.39920 & 1.96990 & -3.84240 \\
\hline $\mathrm{C}$ & -8.77940 & 1.92550 & -3.68050 \\
\hline $\mathrm{C}$ & -9.47160 & 0.70380 & -3.70230 \\
\hline $\mathrm{C}$ & -8.74520 & -0.44420 & -4.05860 \\
\hline $\mathrm{C}$ & -7.35970 & -0.40240 & -4.19020 \\
\hline $\mathrm{C}$ & -10.80200 & 0.53710 & -3.05770 \\
\hline $\mathrm{C}$ & -10.92730 & 870 & -1.73540 \\
\hline $\mathrm{C}$ & -11.94370 & 0.55340 & -0.90680 \\
\hline $\mathrm{C}$ & -12.92240 & -0.34990 & -1.36340 \\
\hline $\mathrm{C}$ & -12.89100 & -0.67490 & -2.73530 \\
\hline $\mathrm{C}$ & -11.84380 & -0.25880 & -3.55870 \\
\hline $\mathrm{C}$ & -13.68950 & -1.15940 & -0.37200 \\
\hline $\mathrm{C}$ & -13.94240 & -2.51380 & -0.70760 \\
\hline $\mathrm{C}$ & -14.26200 & -3.46750 & 0.23650 \\
\hline $\mathrm{C}$ & -14.52220 & -4.86440 & -0.18420 \\
\hline $\mathrm{C}$ & -14.62390 & -5.53330 & 2.23970 \\
\hline $\mathrm{C}$ & -14.33740 & -4.13030 & 2.61810 \\
\hline $\mathrm{C}$ & -14.20630 & -3.13850 & 1.61540 \\
\hline $\mathrm{C}$ & -13.97620 & -1.78340 & 1.97930 \\
\hline $\mathrm{C}$ & -13.72440 & -1.46990 & 3.35180 \\
\hline $\mathrm{C}$ & -13.85660 & -2.47750 & 4.31130 \\
\hline $\mathrm{C}$ & -14.18430 & -3.78830 & 3.95370 \\
\hline $\mathrm{C}$ & -13.91780 & -0.76790 & 0.97130 \\
\hline $\mathrm{C}$ & -13.83150 & 0.91930 & 2.80530 \\
\hline $\mathrm{C}$ & -13.37690 & -0.09330 & 3.70760 \\
\hline $\mathrm{C}$ & -12.65630 & 0.29410 & 4.86340 \\
\hline $\mathrm{C}$ & -12.77480 & 1.64250 & 5.28280 \\
\hline $\mathrm{C}$ & -13.45050 & 2.59210 & 4.54430 \\
\hline $\mathrm{C}$ & -13.92400 & 2.26810 & 3.24600 \\
\hline $\mathrm{C}$ & -14.44440 & 3.26100 & 2.38030 \\
\hline $\mathrm{C}$ & -14.80330 & 2.92420 & 1.08270 \\
\hline $\mathrm{C}$ & -14.61780 & 1.62000 & 0.61010 \\
\hline $\mathrm{C}$ & -14.59130 & 4.65650 & 2.85910 \\
\hline $\mathrm{C}$ & -13.56000 & 3.98000 & 5.05130 \\
\hline $\mathrm{C}$ & -11.56330 & -0.51530 & 5.47480 \\
\hline
\end{tabular}




\begin{tabular}{|c|c|c|c|}
\hline $\mathrm{C}$ & -11.04060 & -0.23210 & 6.75250 \\
\hline $\mathrm{C}$ & -9.76080 & -0.64230 & 7.12370 \\
\hline $\mathrm{C}$ & -8.94520 & -1.37110 & 6.24070 \\
\hline $\mathrm{C}$ & -9.53160 & -1.78460 & 5.02870 \\
\hline $\mathrm{C}$ & -10.79870 & -1.36730 & 4.65270 \\
\hline $\mathrm{C}$ & -7.47470 & -1.51840 & 6.38890 \\
\hline $\mathrm{C}$ & -6.69580 & -0.44700 & 6.86090 \\
\hline $\mathrm{C}$ & -5.32460 & -0.39310 & 6.62460 \\
\hline $\mathrm{C}$ & -4.67000 & -1.40180 & 5.89570 \\
\hline $\mathrm{C}$ & -5.42170 & -2.55140 & 5.58690 \\
\hline $\mathrm{C}$ & -6.78880 & -2.60960 & 5.82790 \\
\hline $\mathrm{C}$ & -3.37150 & -1.18290 & 5.20480 \\
\hline $\mathrm{C}$ & -2.56020 & -2.23730 & 4.74570 \\
\hline $\mathrm{C}$ & -3.07420 & 0.09920 & 4.70830 \\
\hline $\mathrm{C}$ & -2.18730 & 0.28070 & 3.65570 \\
\hline $\mathrm{C}$ & -1.56490 & -0.81710 & 3.03070 \\
\hline $\mathrm{C}$ & -1.67390 & -2.05830 & 3.68540 \\
\hline $\mathrm{C}$ & -14.12010 & 0.60860 & 1.43800 \\
\hline $\mathrm{H}$ & -1.97610 & -2.67090 & 1.15600 \\
\hline $\mathrm{O}$ & -0.21010 & 1.43130 & 1.75470 \\
\hline $\mathrm{H}$ & -1.02300 & 1.72960 & -0.79690 \\
\hline $\mathrm{O}$ & -2.51410 & -2.54310 & -1.33860 \\
\hline $\mathrm{H}$ & -2.57390 & -1.44270 & -3.62980 \\
\hline $\mathrm{H}$ & -4.64200 & -0.98060 & -4.83670 \\
\hline $\mathrm{H}$ & -3.25550 & 2.01700 & -1.17370 \\
\hline $\mathrm{H}$ & -5.31000 & 2.47310 & -2.36530 \\
\hline $\mathrm{H}$ & -6.82110 & -1.33580 & -4.31940 \\
\hline $\mathrm{H}$ & -9.25240 & -1.40410 & -4.09660 \\
\hline $\mathrm{H}$ & -9.32160 & 2.84370 & -3.47420 \\
\hline $\mathrm{H}$ & -6.89500 & 2.92860 & -3.77640 \\
\hline $\mathrm{H}$ & -13.66000 & -1.31320 & -3.15990 \\
\hline $\mathrm{H}$ & -11.81680 & -0.59300 & -4.59230 \\
\hline $\mathrm{H}$ & -11.91270 & 0.83370 & 0.13800 \\
\hline $\mathrm{H}$ & -10.14080 & 1.61680 & -1.31590 \\
\hline $\mathrm{H}$ & -13.83280 & -2.85200 & -1.73110 \\
\hline $\mathrm{H}$ & -14.88620 & -6.72760 & 0.59250 \\
\hline $\mathrm{H}$ & -14.29880 & -4.56200 & 4.70550 \\
\hline $\mathrm{H}$ & -13.69240 & -2.23800 & 5.35460 \\
\hline $\mathrm{H}$ & -12.28450 & 1.97670 & 6.18980 \\
\hline $\mathrm{H}$ & -15.20690 & 3.69870 & 0.43930 \\
\hline $\mathrm{H}$ & -14.86170 & 1.38940 & -0.42040 \\
\hline $\mathrm{H}$ & -14.23470 & 5.83620 & 4.49840 \\
\hline $\mathrm{H}$ & -11.14830 & -1.61450 & 3.65780 \\
\hline $\mathrm{H}$ & -8.93460 & -2.34220 & 4.31430 \\
\hline $\mathrm{H}$ & -9.37670 & -0.35330 & 8.09820 \\
\hline $\mathrm{H}$ & -11.61730 & 0.36460 & 7.45300 \\
\hline
\end{tabular}




$\begin{array}{lrrr}\mathrm{H} & -4.94980 & -3.36790 & 5.04990 \\ \mathrm{H} & -7.34520 & -3.48610 & 5.50790 \\ \mathrm{H} & -4.77210 & 0.48560 & 6.94200 \\ \mathrm{H} & -7.18360 & 0.40500 & 7.32620 \\ \mathrm{H} & -3.64680 & 0.95450 & 5.05330 \\ \mathrm{H} & -2.07970 & 1.26840 & 3.22590 \\ \mathrm{H} & -1.13180 & -2.91780 & 3.30280 \\ \mathrm{H} & -2.67580 & -3.23080 & 5.16980 \\ \mathrm{~N} & -14.70360 & -5.76780 & 0.86430 \\ \mathrm{~N} & -14.14670 & 4.88300 & 4.16380 \\ \mathrm{O} & -13.17830 & 4.34470 & 6.15310 \\ \mathrm{O} & -15.06180 & 5.56710 & 2.19480 \\ \mathrm{O} & -14.57800 & -5.23560 & -1.34680 \\ \mathrm{O} & -14.78460 & -6.44450 & 3.03770 \\ \mathrm{C} & 0.18520 & 2.66820 & 1.18160 \\ \mathrm{C} & -1.58380 & -3.51730 & -1.80390 \\ \mathrm{H} & 0.71220 & 3.20820 & 1.96980 \\ \mathrm{H} & 0.86060 & 2.51890 & 0.32980 \\ \mathrm{H} & -0.67790 & 3.26200 & 0.85360 \\ \mathrm{H} & -2.16910 & -4.27470 & -2.33280 \\ \mathrm{H} & -0.84700 & -3.07270 & -2.49010 \\ \mathrm{H} & -1.04300 & -3.99600 & -0.97450\end{array}$

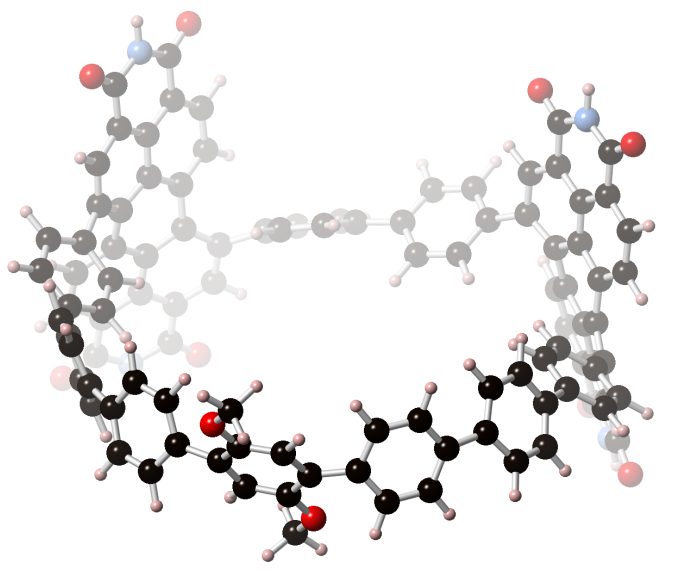

SI-7.1

Final Heat of Formation $=-4968.401876$
$\begin{array}{llll}\text { C } & -0.329708 & -0.069824 & 3.476209\end{array}$
$\begin{array}{llll}\text { C } & -0.368715 & -1.142230 & 2.576126\end{array}$
$\begin{array}{llll}\text { C } & 0.257980 & -1.107857 & 1.331319\end{array}$
$\begin{array}{llll}\text { C } & 0.924808 & 0.067533 & 0.909940\end{array}$
$\begin{array}{llll}\text { C } & 1.076249 & 1.088120 & 1.867116\end{array}$
$\begin{array}{llll}\text { C } & 0.483501 & 1.031359 & 3.129684\end{array}$
$\begin{array}{llll}\text { C } & 1.199353 & 0.323001 & -0.529092\end{array}$ 


$\begin{array}{rrrr}\mathrm{C} & 0.919302 & 1.595898 & -1.064854 \\ \mathrm{C} & 0.794475 & 1.801405 & -2.433132 \\ \mathrm{C} & 0.914139 & 0.736459 & -3.345460 \\ \mathrm{C} & 1.370940 & -0.485563 & -2.821343 \\ \mathrm{C} & 1.523437 & -0.686521 & -1.453899 \\ \mathrm{C} & 0.346782 & 0.809361 & -4.718102 \\ \mathrm{C} & 0.582980 & -0.177874 & -5.692848 \\ \mathrm{C} & -0.245109 & -0.313254 & -6.803900 \\ \mathrm{C} & -1.336990 & 0.545936 & -7.008216 \\ \mathrm{C} & -1.460443 & 1.637993 & -6.132191 \\ \mathrm{C} & -0.646987 & 1.761277 & -5.015461 \\ \mathrm{C} & -13.730676 & -1.243864 & -3.947456 \\ \mathrm{C} & -13.087752 & -0.820838 & -2.770830 \\ \mathrm{C} & -13.548130 & -1.199636 & -1.518129 \\ \mathrm{C} & -14.666476 & -2.040790 & -1.378840 \\ \mathrm{C} & -15.339513 & -2.424795 & -2.549248 \\ \mathrm{C} & -14.888629 & -2.021211 & -3.808993 \\ \mathrm{C} & -14.921714 & -2.645258 & -0.038204 \\ \mathrm{C} & -14.950580 & -4.057226 & 0.027207 \\ \mathrm{C} & -14.852861 & -4.741028 & 1.223451 \\ \mathrm{C} & -14.913390 & -6.224292 & 1.234076 \\ \mathrm{C} & -14.437530 & -6.185929 & 3.701177 \\ \mathrm{C} & -14.358476 & -4.708168 & 3.643314 \\ \mathrm{C} & -14.596572 & -4.028253 & 2.422339 \\ \mathrm{C} & -14.561553 & -2.604026 & 2.383357 \\ \mathrm{C} & -14.145119 & -1.884997 & 3.549892 \\ \mathrm{C} & -13.923991 & -2.597857 & 4.732052 \\ \mathrm{C} & -14.050070 & -3.989435 & 4.786796 \\ \mathrm{C} & -14.891645 & -1.915416 & 1.172318 \\ \mathrm{C} & -14.797274 & 0.225800 & 2.463597 \\ \mathrm{C} & -14.033266 & -0.422214 & 3.485802 \\ \mathrm{C} & -13.290723 & 0.369448 & 4.392424 \\ \mathrm{C} & -13.597283 & 1.747444 & 4.476219 \\ \mathrm{C} & -14.518772 & 2.350864 & 3.644599 \\ \mathrm{C} & -15.091095 & 1.615442 & 2.575510 \\ \mathrm{C} & -15.932969 & 2.241000 & 1.622127 \\ \mathrm{C} & -16.418530 & 1.511338 & 0.548983 \\ \mathrm{C} & -16.054294 & 0.169716 & 0.382342 \\ \mathrm{C} & -16.297271 & 3.670775 & 1.776014 \\ \mathrm{C} & -14.067930 & -0.097476 & 5.104851 \\ \mathrm{C} & -1.12569 & -0.898072 & 4.411173\end{array}$




\begin{tabular}{|c|c|c|c|}
\hline C & & & \\
\hline $\mathrm{C}$ & & & \\
\hline & -5.9 & & \\
\hline 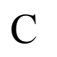 & & & \\
\hline & -5.8 & & \\
\hline C & -7.2 & & \\
\hline 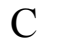 & -3.737 & -0.3 & \\
\hline C & -2.8 & -1.3 & \\
\hline $\mathrm{C}$ & -3.2 & & \\
\hline $\mathrm{C}$ & -2.1 & & \\
\hline $\mathrm{C}$ & -1.3 & & \\
\hline $\mathrm{C}$ & -1.6 & -1.2 & \\
\hline & 15. & & \\
\hline $\mathrm{H}$ & -0.9 & & \\
\hline & & & \\
\hline U & 1.6 & & \\
\hline & & & \\
\hline & & & \\
\hline & 1.5 & -1. & \\
\hline 11 & & & \\
\hline $\mathrm{H}$ & 0.5 & & \\
\hline $\mathrm{H}$ & -0.8 & & \\
\hline $\mathrm{H}$ & -2.2 & & \\
\hline & -0.0 & 1 & \\
\hline $\mathrm{H}$ & & & \\
\hline & & & \\
\hline & & & \\
\hline & -13.0 & -0 & \\
\hline $\mathrm{H}$ & & & \\
\hline & -15.0 & & \\
\hline $\mathrm{H}$ & -14. & -7 & \\
\hline $\mathrm{H}$ & -13.8 & & \\
\hline $\mathrm{H}$ & -13.6 & & \\
\hline $\mathrm{H}$ & -13.0 & & \\
\hline $\mathrm{H}$ & -17.0 & & \\
\hline $\mathrm{H}$ & -16.4 & -0 . & \\
\hline $\mathrm{H}$ & -15 & & \\
\hline $\mathrm{H}$ & -11.3 & -1 . & \\
\hline $\mathrm{H}$ & -9.1 & -1. & \\
\hline $\mathrm{H}$ & -10.1 & & \\
\hline $\mathrm{H}$ & -12.3 & & \\
\hline $\mathrm{H}$ & & -2.5 & \\
\hline $\mathrm{H}$ & -7.70 & -2.6 & \\
\hline $\mathrm{H}$ & & & \\
\hline $\mathrm{H}$ & -7.902027 & 1.3 & 89 \\
\hline I & -3.94789 & 1.79615 & 5.62141 \\
\hline
\end{tabular}




\begin{tabular}{|c|c|c|c|}
\hline & & & \\
\hline & -1.0 & & \\
\hline & 44 & -2. & \\
\hline & & & \\
\hline & -15.7 & & \\
\hline & & & \\
\hline & -17.0 & & \\
\hline & & & \\
\hline O & -14.2 & & 066 \\
\hline & & & \\
\hline & -0.6 & -3.2 & \\
\hline & 1.4 & & \\
\hline & & & \\
\hline & 1.0 & & \\
\hline & -0.6 & & \\
\hline & -0.3 & -3 & \\
\hline & & & \\
\hline & -2.4 & & \\
\hline & & & \\
\hline & -4.8 & -0. & \\
\hline & -4.6 & -0. & \\
\hline & & & \\
\hline & -2.2 & -0 . & \\
\hline & & & \\
\hline & & & \\
\hline & -6.8 & & \\
\hline & & & \\
\hline & & & \\
\hline & & & \\
\hline & -8.1 & & \\
\hline & & & \\
\hline C & -9.6 & -1.2 & \\
\hline & -10 . & -2 . & \\
\hline & -10.5 & -3. & \\
\hline 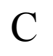 & & 4 & 88 \\
\hline $\mathrm{C}$ & -8.7 & & \\
\hline $\mathrm{C}$ & & & \\
\hline $\mathrm{C}$ & -10 & & \\
\hline 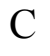 & -11 . & & \\
\hline $\mathrm{C}$ & -10.1 & 3.0 & 438 \\
\hline & & & \\
\hline 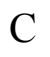 & -7.9 & 3.3 & -10 \\
\hline & -6.7 & & \\
\hline 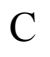 & -6.45 & & -10 \\
\hline & -8.2 & 4.8 & -10.2 \\
\hline & 10 & 4.46031 & -9.1932 \\
\hline
\end{tabular}




$\begin{array}{cccc}\mathrm{C} & -11.695466 & 0.003098 & -7.560119 \\ \mathrm{C} & -13.077501 & 0.214985 & -7.419324 \\ \mathrm{C} & -13.770426 & -0.241195 & -6.295157 \\ \mathrm{C} & -13.101103 & -0.902079 & -5.255309 \\ \mathrm{C} & -11.731583 & -1.169176 & -5.430026 \\ \mathrm{C} & -11.045872 & -0.737506 & -6.557122 \\ \mathrm{C} & -7.435277 & 0.606618 & -10.087627 \\ \mathrm{H} & -3.207476 & -1.486692 & -10.805191 \\ \mathrm{H} & -1.274951 & -0.674917 & -9.531164 \\ \mathrm{H} & -5.847415 & 0.156777 & -7.836584 \\ \mathrm{H} & -3.927192 & 0.940260 & -6.560170 \\ \mathrm{H} & -4.777237 & -3.245307 & -10.354270 \\ \mathrm{H} & -7.732816 & -6.723052 & -11.294878 \\ \mathrm{H} & -11.416036 & -4.059155 & -10.173999 \\ \mathrm{H} & -11.687320 & -1.702648 & -9.452085 \\ \mathrm{H} & -11.922656 & 2.572814 & -8.078543 \\ \mathrm{H} & -6.026090 & 3.567577 & -11.079454 \\ \mathrm{H} & -5.532490 & 1.141421 & -10.927805 \\ \mathrm{H} & -9.628491 & 6.247199 & -9.768735 \\ \mathrm{H} & -9.977650 & -0.915771 & -6.633489 \\ \mathrm{H} & -14.830827 & -0.024533 & -6.199503 \\ \mathrm{H} & -13.613363 & 0.779917 & -8.177211 \\ \mathrm{~N} & -7.845657 & -5.751816 & -11.026244 \\ \mathrm{~N} & -9.448254 & 5.249381 & -9.748931 \\ \mathrm{O} & -11.477959 & 4.964759 & -8.756541 \\ \mathrm{O} & -7.431711 & 5.662880 & -10.720346 \\ \mathrm{O} & -5.583174 & -5.527619 & -11.129365 \\ \mathrm{O} & -10.089370 & -6.114498 & -10.915339 \\ \mathrm{H} & -11.193658 & -1.701681 & -4.651356\end{array}$

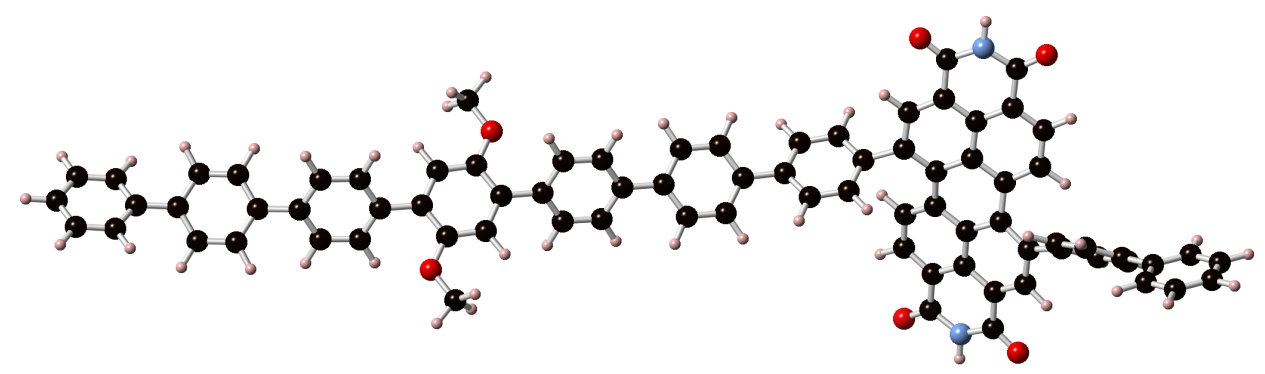

$\mathbf{P P h}_{6}$-PhHex Acyclic Analog for Homodesmotic Calculations

Final Heat of Formation $=-3639.730299$
C $\quad 3.120776 \quad-1.376616 \quad 1.091177$
$\begin{array}{llll}\text { C } & 3.489349 & -2.116950 & -0.042196\end{array}$
C $\quad 4.821942 \quad-2.355211 \quad-0.382253$
$\begin{array}{llll}\text { C } & 5.857653 & -1.848930 & 0.430197\end{array}$ 


\begin{tabular}{|c|c|c|c|}
\hline & 7466 & . & \\
\hline & & -0.854601 & \\
\hline & 415 & & \\
\hline & 8.225832 & & \\
\hline & 2549 & & \\
\hline & 83 & -2 & \\
\hline & 43 & -3.5 & \\
\hline & 7.80 & 93 & -0 \\
\hline & 11.5 & & \\
\hline & 12.4 & -2. & \\
\hline & & -2.2 & \\
\hline & 12 & -3.1 & \\
\hline & & & \\
\hline & 12.1 & -3 & \\
\hline & & & \\
\hline & $-1^{\prime}$ & & \\
\hline & & & \\
\hline & & & \\
\hline & & -0 . & \\
\hline & & & \\
\hline & & -0 . & \\
\hline & & -1 & \\
\hline & -14 & -2 & \\
\hline & & & \\
\hline & & & \\
\hline & -12.6 & -3 & \\
\hline & & -2 & \\
\hline & -13. & -1. & \\
\hline & -12. & -0 & \\
\hline & -11.2 & -1 & \\
\hline & -11.4 & -2. & \\
\hline & -14 . & -0 & \\
\hline & -13.1 & & \\
\hline & & & \\
\hline & & & \\
\hline & -10 & & \\
\hline & & & \\
\hline & -13 . & 2. & \\
\hline & -14.2 & & \\
\hline $\mathrm{C}$ & -15.4 & & \\
\hline & & & \\
\hline & -14.0 & & \\
\hline 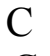 & -11.6 & 1607 & 261 \\
\hline & & & \\
\hline & -8.422190 & 0.739086 & 109 \\
\hline & -7.111649 & 0.358039 & 4.52022 \\
\hline
\end{tabular}




\begin{tabular}{|c|c|c|c|}
\hline & -6.703304 & -0.015224 & \\
\hline & -7684905 & & \\
\hline & -9.002807 & & \\
\hline & -5.277643 & -0.293073 & 830 \\
\hline & -4.269474 & & \\
\hline & -2.927374 & & \\
\hline $\mathrm{C}$ & -2.5 & -0.6 & \\
\hline & -3.5 & -1. & \\
\hline C & -4.8 & -1.2 & \\
\hline 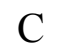 & -1.0 & -0.8 & \\
\hline C & -0.5 & $-2 .($ & \\
\hline & -0.1 & & \\
\hline & 1.1 & & \\
\hline $\mathrm{C}$ & 1.6 & -1 & \\
\hline$\zeta$ & 0.7 & -2.2 & \\
\hline $\mathrm{C}$ & -14.4 & & \\
\hline $\mathrm{H}$ & 2.6 & -2.4 & \\
\hline 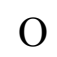 & 3.7 & -0. & \\
\hline & $6.2^{\prime}$ & -0.7 & \\
\hline ) & 5.20 & $-3 .($ & \\
\hline & 7.1 & -4 & \\
\hline 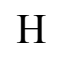 & & & \\
\hline $\mathrm{H}$ & 7.8 & -0. & \\
\hline & 10.2 & -0 & \\
\hline & 11. & -4 . & \\
\hline & 13.9 & -4 & \\
\hline $\mathrm{H}$ & 14.4 & -1. & \\
\hline $\mathrm{H}$ & 12.0 & -1.2 & \\
\hline $\mathrm{H}$ & -18.3 & -0 & \\
\hline & -20.1 & & \\
\hline $\mathrm{H}$ & -15.4 & & \\
\hline I & -17.1 & & \\
\hline & -16.6 & -2.3 & \\
\hline I & -14. & -6. & \\
\hline & -10.71 & -3.7 & \\
\hline 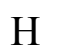 & -10.3 & -1. & \\
\hline $\mathrm{H}$ & -9.689670 & & \\
\hline $\mathrm{H}$ & -16.3 & & 49 \\
\hline $\mathrm{H}$ & -16.5 & & \\
\hline $\mathrm{H}$ & -12.7 & & \\
\hline $\mathrm{H}$ & -9.728391 & 0.3 & 1.6 \\
\hline $\mathrm{H}$ & -7.40 & -0.3 & \\
\hline $\mathrm{H}$ & -6.392577 & 0.3 & 5.33 \\
\hline & -8.70 & & \\
\hline $\mathrm{H}$ & -3.259249 & -2.09 & 0.86 \\
\hline & -5.623655 & -1.786495 & 1.41394 \\
\hline
\end{tabular}




$\begin{array}{crrr}\mathrm{H} & -2.179413 & 0.762244 & 3.884073 \\ \mathrm{H} & -4.541456 & 1.144627 & 4.366096 \\ \mathrm{H} & -0.541101 & 1.165774 & 2.462994 \\ \mathrm{H} & 1.842437 & 0.879779 & 1.998074 \\ \mathrm{H} & 1.142925 & -3.220432 & 0.942438 \\ \mathrm{H} & -1.234062 & -2.956958 & 1.462605 \\ \mathrm{~N} & -13.990157 & -5.194631 & 2.062052 \\ \mathrm{~N} & -12.808717 & 5.554459 & 5.312501 \\ \mathrm{O} & -10.554998 & 5.326012 & 5.529205 \\ \mathrm{O} & -15.049727 & 5.896706 & 5.120593 \\ \mathrm{O} & -16.027799 & -4.776763 & 1.139577 \\ \mathrm{O} & -11.967280 & -5.725735 & 2.959773 \\ \mathrm{C} & 4.797578 & 0.337249 & 3.874074 \\ \mathrm{C} & 4.194965 & -3.500837 & -2.392586 \\ \mathrm{H} & 4.275174 & 0.844587 & 4.686669 \\ \mathrm{H} & 5.405873 & -0.475828 & 4.290534 \\ \mathrm{H} & 5.457465 & 1.053440 & 3.367188 \\ \mathrm{H} & 4.721358 & -3.998282 & -3.208919 \\ \mathrm{H} & 3.522048 & -4.220588 & -1.908604 \\ \mathrm{H} & 3.599923 & -2.671930 & -2.797591 \\ \mathrm{C} & 18.651526 & -3.608669 & -0.522242 \\ \mathrm{C} & 17.877167 & -3.630134 & -1.681791 \\ \mathrm{C} & 16.492605 & -3.498679 & -1.604955 \\ \mathrm{C} & 15.847983 & -3.348775 & -0.366422 \\ \mathrm{C} & 16.643861 & -3.324552 & 0.790851 \\ \mathrm{H} & 18.027798 & -3.455486 & 0.715370 \\ \mathrm{H} & -19.731264 & -3.707142 & -0.582839 \\ \mathrm{H} & 18.353964 & -3.734909 & -2.651905 \\ \mathrm{H} & 15.902860 & -3.483842 & -2.516164 \\ \mathrm{H} & 16.167214 & -3.233357 & 1.762348 \\ \mathrm{H} & 18.619584 & -3.441244 & 1.626130 \\ \mathrm{C} & -21.885539 & 3.402820 & -2.376425 \\ \mathrm{C} & -22.133505 & 3.071022 & -1.044719 \\ \mathrm{C} & -21.135116 & 2.487451 & -0.268911 \\ \mathrm{C} & -19.866423 & 2.223678 & -0.808253 \\ \mathrm{C} & -19.631624 & 2.562212 & -2.151047 \\ \mathrm{H} & -20.629735 & 3.146517 & -2.926160 \\ & -22.664948 & 3.856033 & -2.981421 \\ \mathrm{H} & -23.105972 & 3.273106 & -0.605835 \\ \mathrm{H} & -2.3310 & 2.254944 & 0.773134 \\ \mathrm{H} & 3.390846 & -3.965213\end{array}$




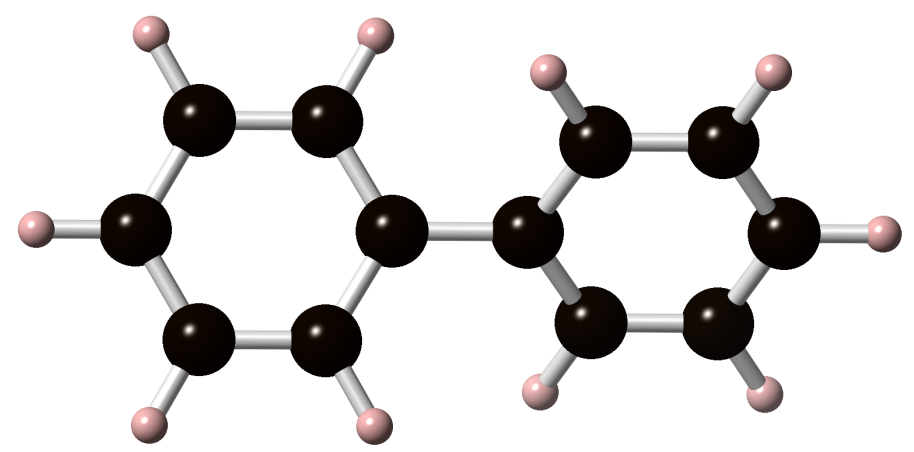

Biphenyl

FINAL HEAT OF FORMATION $=-463.321476$

$\begin{array}{rrrr}\mathrm{C} & -1.140470 & -0.387961 & 1.464750 \\ \mathrm{C} & -1.141173 & -0.387029 & 2.857959 \\ \mathrm{C} & 0.000000 & 0.000000 & 3.561339 \\ \mathrm{C} & 1.141173 & 0.387029 & 2.857959 \\ \mathrm{C} & 1.140470 & 0.387961 & 1.464750 \\ \mathrm{C} & 0.000000 & 0.000000 & 0.742532 \\ \mathrm{C} & 0.000000 & 0.000000 & -0.742532 \\ \mathrm{C} & 1.140470 & -0.387961 & -1.464750 \\ \mathrm{C} & 1.141173 & -0.387029 & -2.857959 \\ \mathrm{C} & 0.000000 & 0.000000 & -3.561339 \\ \mathrm{C} & -1.141173 & 0.387029 & -2.857959 \\ \mathrm{C} & -1.140470 & 0.387961 & -1.464750 \\ \mathrm{H} & -2.025300 & -0.716471 & 0.927586 \\ \mathrm{H} & -2.032468 & -0.698615 & 3.395283 \\ \mathrm{H} & 0.000000 & 0.000000 & 4.647453 \\ \mathrm{H} & 2.032468 & 0.698615 & 3.395283 \\ \mathrm{H} & 2.025300 & 0.716471 & 0.927586 \\ \mathrm{H} & 2.025300 & -0.716471 & -0.927586 \\ \mathrm{H} & 2.032468 & -0.698615 & -3.395283 \\ \mathrm{H} & 0.000000 & 0.000000 & -4.647453 \\ \mathrm{H} & -2.032468 & 0.698615 & -3.395283 \\ \mathrm{H} & -2.025300 & 0.716471 & -0.927586\end{array}$




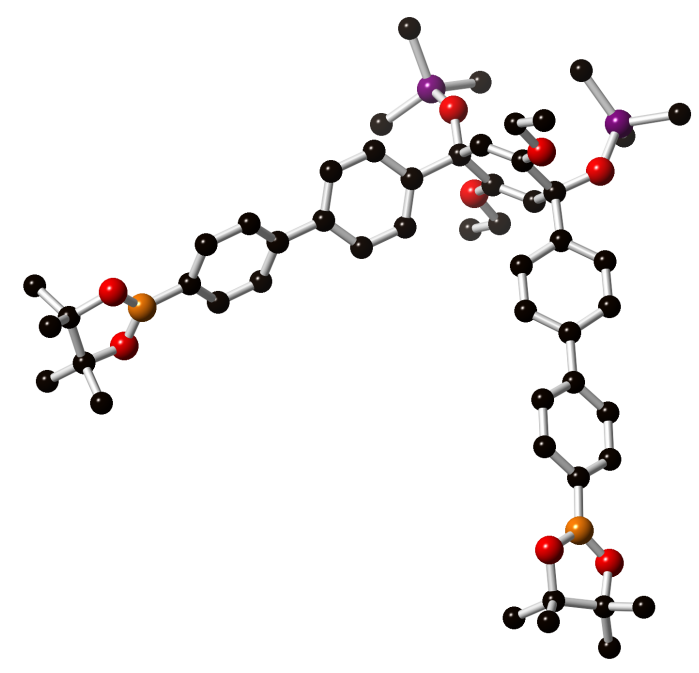

Five-Aryl Precursor (7.2)

Final Heat of Formation $=-3254.65918$

$\begin{array}{rrrr}\text { B } & 10.47009 & -1.01958 & -0.13630 \\ \text { B } & 1.46007 & -11.90187 & 0.42225 \\ \text { C } & 0.26168 & 0.07333 & -0.12649 \\ \text { C } & -0.15369 & -0.45579 & -1.48140 \\ \text { C } & -1.02572 & -1.45046 & -1.69135 \\ \text { C } & -1.71240 & -2.21774 & -0.57138 \\ \text { C } & -1.32557 & -1.66790 & 0.78211 \\ \text { C } & -0.45789 & -0.66891 & 0.98799 \\ \text { C } & -1.29766 & -3.70270 & -0.60631 \\ \text { C } & 1.79418 & -0.06824 & -0.02873 \\ \text { C } & -1.52745 & 2.90150 & 1.98740 \\ \text { C } & 1.56415 & 2.73334 & 2.03354 \\ \text { C } & 0.11625 & 4.25394 & -0.22018 \\ \text { C } & -5.79411 & -1.60109 & -1.35089 \\ \text { C } & -4.56119 & -0.50946 & 1.24741 \\ \text { C } & -3.69037 & 0.66404 & -1.46473 \\ \text { C } & -2.23596 & -4.72992 & -0.49145 \\ \text { C } & -1.82789 & -6.06091 & -0.40266 \\ \text { C } & -0.47052 & -6.41355 & -0.42709 \\ \text { C } & 0.46401 & -5.37358 & -0.57116 \\ \text { C } & 0.05881 & -4.04706 & -0.65571 \\ \text { C } & 2.60716 & 0.81230 & -0.75393 \\ \text { C } & 3.99061 & 0.67197 & -0.76603 \\ \text { C } & 4.62448 & -0.36142 & -0.05697 \\ \text { C } & 3.80536 & -1.24573 & 0.66145 \\ \text { C } & 2.41932 & -1.10361 & 0.67431 \\ \text { C } & 6.10016 & -0.51560 & -0.07387 \\ \text { C } & -0.02149 & -7.81884 & -0.26908 \\ \text { C } & 6.79324 & -0.99965 & 1.04898\end{array}$




$\begin{array}{lrrr}\mathrm{C} & 8.17470 & -1.15786 & 1.02643 \\ \mathrm{C} & 8.92823 & -0.83195 & -0.11339 \\ \mathrm{C} & 8.23486 & -0.33940 & -1.23163 \\ \mathrm{C} & 6.85217 & -0.18774 & -1.21565 \\ \mathrm{C} & 1.13485 & -8.29179 & -0.91444 \\ \mathrm{C} & 1.59134 & -9.58934 & -0.70843 \\ \mathrm{C} & 0.91133 & -10.47762 & 0.14241 \\ \mathrm{C} & -0.25850 & -10.01034 & 0.76510 \\ \mathrm{C} & -0.71480 & -8.71139 & 0.56692 \\ \mathrm{C} & 12.54508 & -1.75458 & 0.46940 \\ \mathrm{C} & 12.64447 & -0.83551 & -0.80960 \\ \mathrm{C} & 1.55881 & -14.05742 & 1.16978 \\ \mathrm{C} & 2.94128 & -13.63468 & 0.53677 \\ \mathrm{C} & 1.64707 & -14.71042 & 2.54749 \\ \mathrm{C} & 0.69813 & -14.91474 & 0.23221 \\ \mathrm{C} & 3.52674 & -14.62259 & -0.46960 \\ \mathrm{C} & 3.99943 & -13.25870 & 1.58349 \\ \mathrm{C} & 13.46335 & -1.41131 & -1.96283 \\ \mathrm{C} & 13.10663 & 0.59389 & -0.49596 \\ \mathrm{C} & 13.52192 & -1.41465 & 1.59281 \\ \mathrm{C} & 12.61285 & -3.25370 & 0.14704 \\ \mathrm{C} & -0.63923 & -0.81304 & 3.37978 \\ \mathrm{C} & -0.82614 & -1.34196 & -4.07224 \\ \mathrm{C} & -0.02531 & -0.15073 & 4.59938 \\ \mathrm{H} & -1.38674 & -2.07170 & -5.27853 \\ \mathrm{H} & -3.54352 & 0.47772 & -2.53366 \\ \mathrm{H} & 0.31312 & 0.06387 & -2.30862 \\ \mathrm{H} & -1.80885 & -2.17234 & 1.61038 \\ \mathrm{H} & -1.50795 & 3.81623 & 2.59161 \\ \mathrm{H} & -2.42297 & 2.93890 & 1.35792 \\ \mathrm{H} & -1.62961 & 2.05136 & 2.66748 \\ \mathrm{H} & 1.53720 & 3.58982 & 2.71844 \\ \mathrm{H} & 1.61159 & 1.82015 & 2.63111 \\ \mathrm{H} & 2.48675 & 2.80021 & 1.44979 \\ \mathrm{H} & 0.12033 & 5.19096 & 0.34836 \\ \mathrm{H} & 1.02307 & 4.23417 & -0.83391 \\ \mathrm{H} & -0.74404 & 4.27757 & -0.89710 \\ & -6.61620 & -0.87806 & -1.30066 \\ \mathrm{H} & -6.11993 & -1.85176 & -2.40325 \\ \mathrm{H} & -2.51247 & -0.83891 \\ \mathrm{H} & -4.48034 & -0.47650\end{array}$




$\begin{array}{rrrr}\mathrm{H} & -2.58134 & -6.83981 & -0.32793 \\ \mathrm{H} & 1.52510 & -5.60410 & -0.57601 \\ \mathrm{H} & 0.80911 & -3.26718 & -0.74259 \\ \mathrm{H} & 2.14682 & 1.62498 & -1.30654 \\ \mathrm{H} & 4.59110 & 1.39070 & -1.31556 \\ \mathrm{H} & 4.25553 & -2.07417 & 1.20026 \\ \mathrm{H} & 1.82266 & -1.81811 & 1.23140 \\ \mathrm{H} & 6.24427 & -1.23289 & 1.95572 \\ \mathrm{H} & 8.68502 & -1.53387 & 1.90909 \\ \mathrm{H} & 8.79084 & -0.08413 & -2.12977 \\ \mathrm{H} & 6.34272 & 0.16295 & -2.10835 \\ \mathrm{H} & 1.67371 & -7.63626 & -1.59234 \\ \mathrm{H} & 2.49392 & -9.92488 & -1.21219 \\ \mathrm{H} & -0.80447 & -10.67247 & 1.43177 \\ \mathrm{H} & -1.59892 & -8.36798 & 1.09614 \\ \mathrm{H} & 2.20573 & -15.65122 & 2.49952 \\ \mathrm{H} & 2.12939 & -14.05517 & 3.27451 \\ \mathrm{H} & 0.64047 & -14.93396 & 2.91191 \\ \mathrm{H} & 1.10177 & -15.92597 & 0.12410 \\ \mathrm{H} & -0.31052 & -14.98926 & 0.64741 \\ \mathrm{H} & 0.62133 & -14.46286 & -0.76064 \\ \mathrm{H} & 3.74938 & -15.58225 & 0.00886 \\ \mathrm{H} & 2.84751 & -14.79857 & -1.30518 \\ \mathrm{H} & 4.46087 & -14.22221 & -0.87354 \\ \mathrm{H} & 4.36961 & -14.13824 & 2.11895 \\ \mathrm{H} & 4.84402 & -12.78320 & 1.07746 \\ \mathrm{H} & 3.60198 & -12.54888 & 2.31426 \\ \mathrm{H} & 14.50794 & -1.55487 & -1.66675 \\ \mathrm{H} & 13.06349 & -2.36721 & -2.30481 \\ \mathrm{H} & 13.44474 & -0.71695 & -2.80752 \\ \mathrm{H} & 14.16834 & 0.62667 & -0.23338 \\ \mathrm{H} & 12.95151 & 1.21816 & -1.38001 \\ \mathrm{H} & 12.53161 & 1.02647 & 0.32742 \\ \mathrm{H} & 14.55791 & -1.54539 & 1.26242 \\ \mathrm{H} & 13.39451 & -0.38923 & 1.94314 \\ \mathrm{H} & 13.35154 & -2.08391 & 2.44077 \\ \mathrm{H} & 13.62088 & -3.55808 & -0.15029 \\ \mathrm{H} & 12.33139 & -3.82061 & 1.03854 \\ \mathrm{H} & 11.91881 & -3.52029 & -0.65489 \\ \mathrm{H} & -0.40662 & -1.88636 & 3.35580 \\ \mathrm{H} & -1.73246 & -0.70799 & 3.37888 \\ \mathrm{H} & 0.26726 & -1.44123 & -4.02109 \\ \mathrm{H} & -1.06286 & -0.26932 & -4.11004 \\ \mathrm{H} & -0.41447 & -0.61723 & 5.50968 \\ \mathrm{H} & 1.06257 & -0.26079 & 4.59302 \\ \mathrm{H} & -0.26455 & 0.91583 & 4.62995 \\ & & & \end{array}$




$\begin{array}{rrrr}\mathrm{H} & -0.96016 & -1.65895 & -6.19802 \\ \mathrm{H} & -1.14520 & -3.13688 & -5.22984 \\ \mathrm{H} & -2.47422 & -1.96710 & -5.32229 \\ \mathrm{O} & -0.10447 & 1.46094 & -0.12746 \\ \mathrm{O} & -3.12933 & -2.16862 & -0.74005 \\ \mathrm{O} & 11.19267 & -1.48759 & 0.93669 \\ \mathrm{O} & 11.25434 & -0.74355 & -1.23196 \\ \mathrm{O} & 0.87952 & -12.77828 & 1.30951 \\ \mathrm{O} & 2.59550 & -12.40908 & -0.16720 \\ \mathrm{O} & -1.41141 & -1.92193 & -2.90599 \\ \mathrm{O} & -0.09611 & -0.18617 & 2.21345 \\ \mathrm{Si} & 0.03253 & 2.76439 & 0.93359 \\ \mathrm{Si} & -4.23031 & -0.90433 & -0.57122\end{array}$

Fifteen Lowest Roots for $\mathbf{P P h}_{6}-\mathbf{P h H e x}$

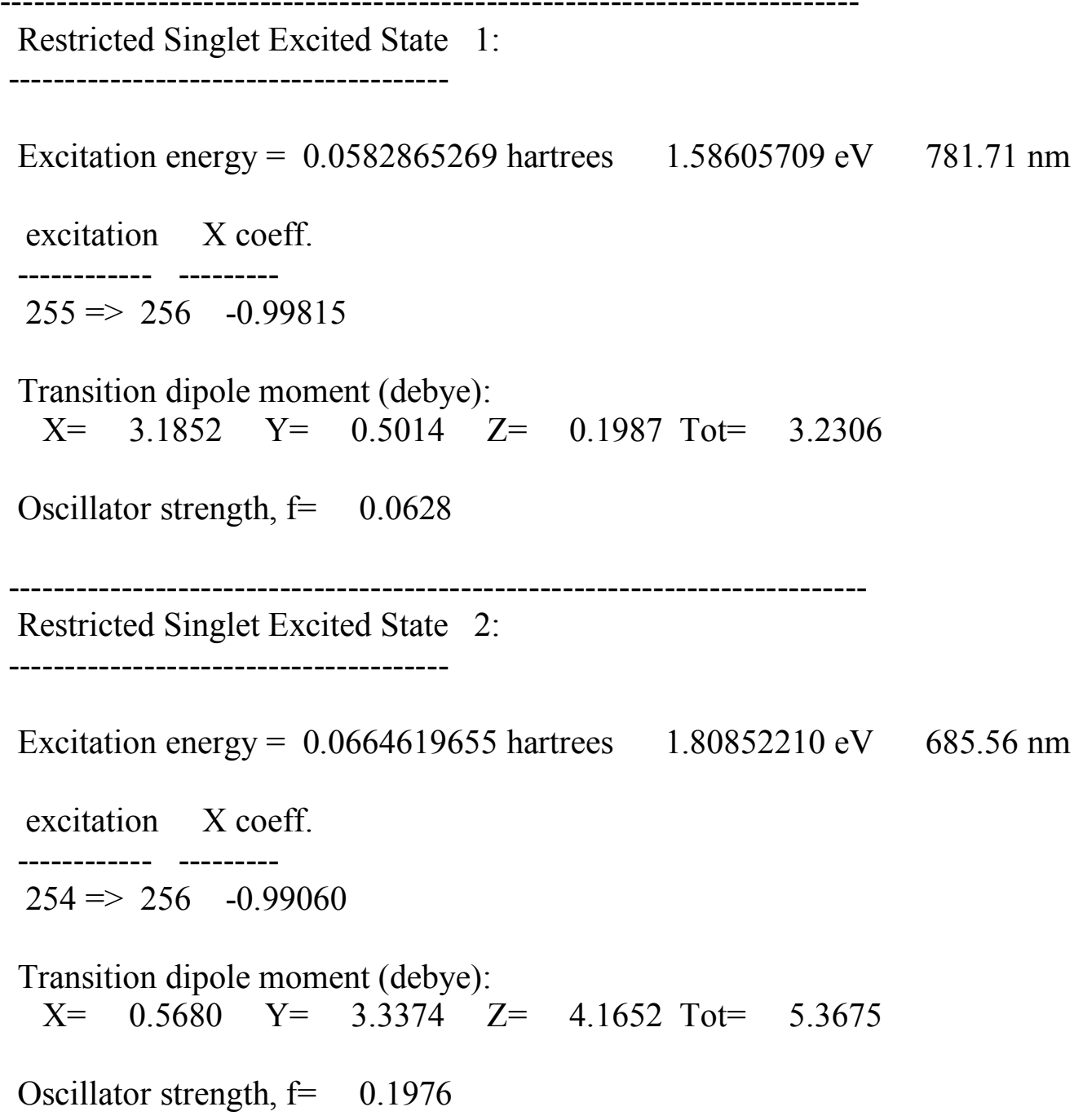


Restricted Singlet Excited State 3:

Excitation energy $=0.0847408081$ hartrees $\quad 2.30591471 \mathrm{eV} \quad 537.68 \mathrm{~nm}$

excitation X coeff.

$251=>256 \quad-0.15475$

$252=>256 \quad-0.48188$

$253=>256 \quad 0.85385$

Transition dipole moment (debye):
$\mathrm{X}=1.812$
$\mathrm{Y}=-2.9241$
$Z=-1.2539$ Tot $=3.6617$

Oscillator strength, $f=0.1172$

Restricted Singlet Excited State 4:

Excitation energy $=0.0854358977$ hartrees $\quad 2.32482906 \mathrm{eV} \quad 533.30 \mathrm{~nm}$

excitation X coeff.

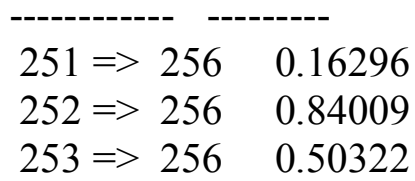

Transition dipole moment (debye):

$\mathrm{X}=-2.0862 \quad \mathrm{Y}=-2.5268 \quad \mathrm{Z}=-0.2840$ Tot $=3.2890$

Oscillator strength, $\mathrm{f}=\quad 0.0954$

Restricted Singlet Excited State 5:

Excitation energy $=0.0968336355$ hartrees $\quad 2.63497729 \mathrm{eV} \quad 470.53 \mathrm{~nm}$

excitation X coeff.

$247=>256 \quad-0.11134$

$251=>256 \quad-0.95230$

$252=>256 \quad 0.23462$ 
Transition dipole moment (debye):

$$
\mathrm{X}=1.2199 \mathrm{Y}=0.0436 \quad \mathrm{Z}=-0.2270 \mathrm{Tot}=1.2416
$$

Oscillator strength, $\mathrm{f}=\quad 0.0154$

Restricted Singlet Excited State 6:

Excitation energy $=0.1087785382$ hartrees $\quad 2.96001463 \mathrm{eV} \quad 418.86 \mathrm{~nm}$

excitation $\mathrm{X}$ coeff.

\begin{tabular}{|c|c|c|}
\hline $248=$ & 25 & -0.18354 \\
\hline $250=$ & 25 & -0.79 \\
\hline 255 & 257 & -0.37365 \\
\hline 255 & 258 & 0.32656 \\
\hline $255=>$ & 259 & -0.10935 \\
\hline $255=>$ & 260 & 0.104 \\
\hline
\end{tabular}

Transition dipole moment (debye):

$$
\mathrm{X}=0.8321 \quad \mathrm{Y}=-2.3136 \quad \mathrm{Z}=-0.4138 \text { Tot }=2.4933
$$

Oscillator strength, $\mathrm{f}=\quad 0.0698$

Restricted Singlet Excited State 7:

Excitation energy $=0.1106178912$ hartrees $\quad 3.01006597 \mathrm{eV} \quad 411.90 \mathrm{~nm}$

\begin{tabular}{|c|c|c|}
\hline $250=>$ & 256 & 0.48080 \\
\hline $254=>$ & & -0.11400 \\
\hline $254=>$ & 259 & -0.11414 \\
\hline $254=>$ & 260 & -0.17503 \\
\hline $255=>$ & 257 & -0.70542 \\
\hline $255=>$ & 258 & 0.38129 \\
\hline $255=>$ & 259 & -0.17280 \\
\hline
\end{tabular}
excitation X coeff.

Transition dipole moment (debye):

$$
\mathrm{X}=0.9845 \mathrm{Y}=1.1220 \quad \mathrm{Z}=-2.8430 \mathrm{Tot}=3.2110
$$

Oscillator strength, $f=0.1177$ 
Restricted Singlet Excited State 8:

Excitation energy $=0.1127214092$ hartrees $\quad 3.06730561 \mathrm{eV} \quad 404.21 \mathrm{~nm}$ excitation $\mathrm{X}$ coeff.

\begin{tabular}{|c|c|}
\hline $239=>256$ & 0.12110 \\
\hline $244=>256$ & 0.10394 \\
\hline $247=>256$ & -0.32382 \\
\hline $248=>256$ & -0.14972 \\
\hline $249=>256$ & -0.54087 \\
\hline $250=>256$ & 0.10494 \\
\hline $251=>256$ & 0.12693 \\
\hline $254=>257$ & 0.52293 \\
\hline $255=>257$ & 0.19326 \\
\hline $255=>258$ & 0.38200 \\
\hline $255=>259$ & -0.13434 \\
\hline
\end{tabular}

Transition dipole moment (debye):
$\mathrm{X}=-0.6730 \quad \mathrm{Y}=0.0377$
$Z=-0.7526 \mathrm{Tot}=1.0103$

Oscillator strength, $\mathrm{f}=\quad 0.0119$

Restricted Singlet Excited State 9:

Excitation energy $=0.1133028915$ hartrees $\quad 3.08312854 \mathrm{eV} \quad 402.14 \mathrm{~nm}$ excitation $\mathrm{X}$ coeff.

\begin{tabular}{|c|c|c|}
\hline $239=>2$ & 256 & -0.14503 \\
\hline $247=>2$ & & -0.23631 \\
\hline $248=>2$ & 256 & -0.12056 \\
\hline $249=>2$ & 256 & -0.58068 \\
\hline $254=>2$ & & -0.21000 \\
\hline $254=>2$ & 258 & -0.20761 \\
\hline $254=>2$ & 260 & 0.10566 \\
\hline $255 \Rightarrow>2$ & 257 & -0.36310 \\
\hline $255=>2$ & 258 & -0.53300 \\
\hline $255=>$ & 259 & 0.12869 \\
\hline
\end{tabular}

Transition dipole moment (debye): 


$$
\mathrm{X}=-0.7894 \mathrm{Y}=-0.5668 \mathrm{Z}=1.3186 \mathrm{Tot}=1.6381
$$

Oscillator strength, $\mathrm{f}=\quad 0.0314$

Restricted Singlet Excited State 10:

Excitation energy $=0.1144901191$ hartrees $\quad 3.11543465 \mathrm{eV} \quad 397.97 \mathrm{~nm}$

excitation $\mathrm{X}$ coeff.

$$
\begin{aligned}
& 239=>256 \quad-0.13464 \\
& 241=>256-0.51302 \\
& 242=>256 \quad-0.10266 \\
& 243=>256 \quad-0.51893 \\
& 244=>256 \quad-0.27751 \\
& 249=>256 \quad-0.27508 \\
& 254=>257 \quad-0.36486 \\
& 255=>257 \quad 0.23149 \\
& 255=>258 \quad 0.21378
\end{aligned}
$$

\begin{tabular}{|c|c|}
\hline $239 \Rightarrow 256$ & -0 \\
\hline$=>2$ & \\
\hline$=>2$ & 0 . \\
\hline$=>2$ & \\
\hline $244=>$ & 09 \\
\hline 249 & -0 \\
\hline $254=>$ & -0.5 \\
\hline $255=>$ & 0.3 \\
\hline $255=>$ & 0.31268 \\
\hline
\end{tabular}

Transition dipole moment (debye):
$\mathrm{X}=0.8609 \quad \mathrm{Y}=$
$0.1565 \mathrm{Z}=-0.3385$ Tot $=0.9382$

Oscillator strength, $\mathrm{f}=\quad 0.0104$

Restricted Singlet Excited State 11:

Excitation energy $=0.1147216476$ hartrees $\quad 3.12173486 \mathrm{eV} \quad 397.16 \mathrm{~nm}$

excitation $\mathrm{X}$ coeff.

Transition dipole moment (debye): 


$$
\mathrm{X}=1.3765 \mathrm{Y}=0.2586 \quad \mathrm{Z}=-0.5778 \text { Tot }=1.5151
$$

Oscillator strength, $\mathrm{f}=\quad 0.0272$

Restricted Singlet Excited State 12:

Excitation energy $=0.1149342514$ hartrees $\quad 3.12752011 \mathrm{eV} \quad 396.43 \mathrm{~nm}$

excitation X coeff.

\begin{tabular}{|c|c|}
\hline . & \\
\hline $241=>2$ & 0 \\
\hline$>$ & -0 \\
\hline (ר) & \\
\hline$\sigma^{\prime}$ & -0 . \\
\hline-7 & \\
\hline $54=>$ & -0.1 \\
\hline
\end{tabular}

Transition dipole moment (debye):

$$
\mathrm{X}=0.3434 \mathrm{Y}=0.0392 \quad \mathrm{Z}=-0.0600 \mathrm{Tot}=0.3508
$$

Oscillator strength, $\mathrm{f}=\quad 0.0015$

Restricted Singlet Excited State 13:

Excitation energy $=0.1156296372$ hartrees $\quad 3.14644252 \mathrm{eV} \quad 394.05 \mathrm{~nm}$

excitation $\mathrm{X}$ coeff.

$$
\begin{aligned}
& 245=>256-0.10525 \\
& 247=>256 \quad-0.25270 \\
& 248=>256 \quad-0.84940 \\
& 249=>256 \quad 0.32228 \\
& 250=>256 \quad 0.19302 \\
& 254=>257 \quad-0.11443
\end{aligned}
$$

Transition dipole moment (debye):

$$
\mathrm{X}=-0.1518 \mathrm{Y}=-0.2555 \mathrm{Z}=0.2542 \mathrm{Tot}=0.3911
$$

Oscillator strength, $\mathrm{f}=\quad 0.0018$

Restricted Singlet Excited State 14: 
Excitation energy $=0.1170384589$ hartrees $\quad 3.18477851 \mathrm{eV} \quad 389.30 \mathrm{~nm}$ excitation $\mathrm{X}$ coeff.

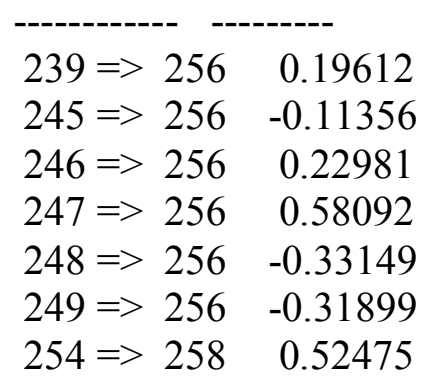

Transition dipole moment (debye):

$$
\mathrm{X}=2.4451 \mathrm{Y}=-0.0311 \mathrm{Z}=-0.1510 \mathrm{Tot}=2.4500
$$

Oscillator strength, $\mathrm{f}=\quad 0.0725$

Restricted Singlet Excited State 15:

Excitation energy $=0.1178521197$ hartrees $\quad 3.20691934 \mathrm{eV} \quad 386.61 \mathrm{~nm}$ excitation X coeff.

$$
\begin{aligned}
& 241=>256 \quad-0.19450 \\
& 244=>256 \quad 0.47240 \\
& 245=>256 \quad 0.16076 \\
& 247=>256 \quad-0.46261 \\
& 248=>256 \quad 0.15593 \\
& 249=>256 \quad 0.12112 \\
& 254=>257 \quad-0.15347 \\
& 254=>258 \quad 0.60073 \\
& 254=>259 \quad 0.13593
\end{aligned}
$$

Transition dipole moment (debye):

$$
\mathrm{X}=3.0120 \mathrm{Y}=-0.0284 \mathrm{Z}=0.1510 \mathrm{Tot}=3.0159
$$

Oscillator strength, $\mathrm{f}=0.1106$

Restricted Singlet Excited State 16: 
Excitation energy $=0.1189447489$ hartrees $\quad 3.23665130 \mathrm{eV} \quad 383.06 \mathrm{~nm}$

excitation $\mathrm{X}$ coeff.

$$
\begin{aligned}
& 238=>256 \quad 0.40710 \\
& 239=>256 \quad-0.21382 \\
& 241=>256 \quad 0.12301 \\
& 242=>256 \quad-0.10295 \\
& 243=>256 \quad 0.15780 \\
& 244=>256 \quad-0.47246 \\
& 245=>256 \quad-0.20207 \\
& 246=>256 \quad-0.48059 \\
& 247=>256 \quad-0.15802 \\
& 254=>258 \quad 0.39657 \\
& 254=>259 \quad 0.12360 \\
& 254=>261 \quad 0.10464
\end{aligned}
$$

Transition dipole moment (debye):

$$
\mathrm{X}=2.0911 \mathrm{Y}=0.0086 \mathrm{Z}=0.2063 \mathrm{Tot}=2.1013
$$

Oscillator strength, $\mathrm{f}=\quad 0.0542$

Restricted Singlet Excited State 17:

Excitation energy $=0.1198773413$ hartrees $\quad 3.26202843 \mathrm{eV} \quad 380.08 \mathrm{~nm}$

excitation X coeff.

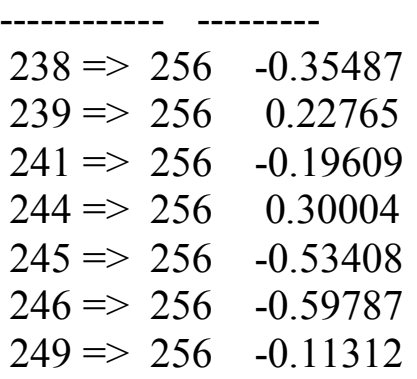

Transition dipole moment (debye):

$$
\mathrm{X}=-0.2772 \quad \mathrm{Y}=0.0406 \quad \mathrm{Z}=0.1330 \text { Tot }=0.3101
$$

Oscillator strength, $\mathrm{f}=\quad 0.0012$

Restricted Singlet Excited State 18: 
Excitation energy $=0.1199662091$ hartrees $\quad 3.26444664 \mathrm{eV} \quad 379.80 \mathrm{~nm}$

excitation X coeff.

$241=>256 \quad-0.18733$

$243=>256 \quad 0.18536$

$245=>256 \quad 0.73759$

$246=>256-0.49579$

$247=>256 \quad 0.23577$

$248=>256 \quad-0.21589$

Transition dipole moment (debye):

$\mathrm{X}=0.1117 \mathrm{Y}=0.4384 \mathrm{Z}=-0.0359 \mathrm{Tot}=0.4539$

Oscillator strength, $\mathrm{f}=0.0026$

Restricted Singlet Excited State 19:

Excitation energy $=0.1213525740$ hartrees $\quad 3.30217155 \mathrm{eV} \quad 375.46 \mathrm{~nm}$

excitation $\mathrm{X}$ coeff.

\begin{tabular}{|c|c|}
\hline $240=>25$ & -0.11159 \\
\hline $241=>256$ & 0.51286 \\
\hline $242=>256$ & 0.26817 \\
\hline $243=>256$ & -0.64587 \\
\hline $244 \Rightarrow 256$ & 0.13079 \\
\hline $245 \Rightarrow 256$ & 0.14921 \\
\hline $246=>2$ & -0.28715 \\
\hline $255=>2$ & 0.116 \\
\hline $255=>2$ & 0.230 \\
\hline
\end{tabular}

Transition dipole moment (debye):

$\mathrm{X}=-0.6209 \mathrm{Y}=-0.9309 \quad \mathrm{Z}=0.1742$ Tot $=1.1324$

Oscillator strength, $f=0.0161$

Restricted Singlet Excited State 20:

Excitation energy $=0.1223193303$ hartrees $\quad 3.32847833 \mathrm{eV} \quad 372.50 \mathrm{~nm}$ 


$$
\begin{array}{rr}
\text { excitation } & \text { X coeff. } \\
-------- & ------ \\
241=>256 & 0.16791 \\
243=>256 & -0.22451 \\
254=>258 & 0.14036 \\
254=>259 & -0.14804 \\
254=>260 & -0.18663 \\
255=>258 & -0.36045 \\
255=>259 & -0.74717 \\
255=>260 & 0.30477
\end{array}
$$

Transition dipole moment (debye):

$$
\mathrm{X}=1.3481 \mathrm{Y}=-0.8463 \mathrm{Z}=-2.6178 \mathrm{Tot}=3.0637
$$

Oscillator strength, $\mathrm{f}=\quad 0.1185$

Restricted Singlet Excited State 21:

\begin{tabular}{|c|c|}
\hline $235=>256$ & -0.11338 \\
\hline $236=>2$ & 0.1 \\
\hline $238=>256$ & -0.54146 \\
\hline $239=>256$ & 34 \\
\hline $241=>256$ & 0.22544 \\
\hline $244=>256$ & -0.51755 \\
\hline $245=>256$ & 0.14115 \\
\hline $247=>256$ & -0.29066 \\
\hline $254=>257$ & -0.12488 \\
\hline $254 \Rightarrow$ & 0.115 \\
\hline
\end{tabular}

Excitation energy $=0.1240850806$ hartrees $\quad 3.37652684 \mathrm{eV} \quad 367.19 \mathrm{~nm}$ excitation $\mathrm{X}$ coeff.

Transition dipole moment (debye):

$$
\mathrm{X}=1.2049 \mathrm{Y}=-0.1719 \mathrm{Z}=-0.3823 \mathrm{Tot}=1.2757
$$

Oscillator strength, $\mathrm{f}=0.0208$

Restricted Singlet Excited State 22:

Excitation energy $=0.1255336538$ hartrees $\quad 3.41594452 \mathrm{eV} \quad 362.96 \mathrm{~nm}$ 
excitation X coeff.

\begin{tabular}{|c|c|}
\hline$\Rightarrow 25$ & -0.1455 \\
\hline$\Rightarrow 25$ & 0.4334 \\
\hline $255=>260$ & \\
\hline
\end{tabular}

Transition dipole moment (debye):

$$
\mathrm{X}=-3.9252 \quad \mathrm{Y}=0.3219 \quad \mathrm{Z}=-0.7051 \mathrm{Tot}=4.0010
$$

Oscillator strength, $\mathrm{f}=\quad 0.2074$

Restricted Singlet Excited State 23:

Excitation energy $=0.1288198393$ hartrees $\quad 3.50536618 \mathrm{eV} \quad 353.70 \mathrm{~nm}$

excitation X coeff.

\begin{tabular}{|c|c|}
\hline $231=>2$ & -0.12222 \\
\hline $237=>$ & 0.13934 \\
\hline $239=>2$ & 0.18586 \\
\hline $240=>2$ & 0.61824 \\
\hline $250=>2$ & -0.19456 \\
\hline $254=>2$ & -0.42351 \\
\hline $254=>2$ & -0.37166 \\
\hline $254=>2$ & 0.11659 \\
\hline $255=>$ & 0.20630 \\
\hline $255=>$ & -0.17478 \\
\hline
\end{tabular}

Transition dipole moment (debye):

$$
\mathrm{X}=-0.0209 \quad \mathrm{Y}=-2.2297 \quad \mathrm{Z}=2.6706 \mathrm{Tot}=3.4791
$$

Oscillator strength, $\mathrm{f}=\quad 0.1609$

Restricted Singlet Excited State 24:

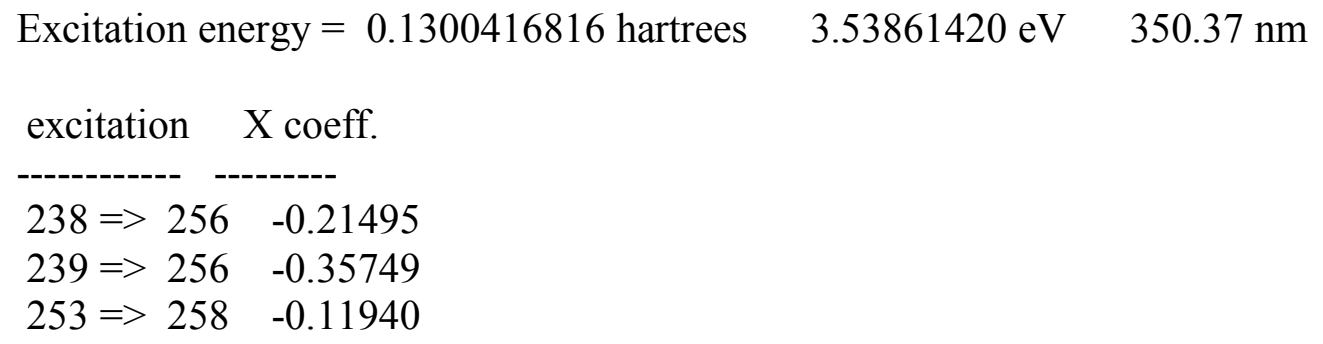




$$
\begin{aligned}
& 253=>261 \quad-0.12034 \\
& 254=>258 \quad 0.25214 \\
& 254=>259 \quad-0.54664 \\
& 254=>260 \quad 0.51780 \\
& 254=>261 \quad-0.35180 \\
& 255=>261 \quad-0.11124
\end{aligned}
$$

Transition dipole moment (debye):

\begin{tabular}{|c|c|}
\hline$=>256$ & -0 \\
\hline $239=>2$ & -0 . \\
\hline $240=>256$ & 0.2 \\
\hline$=>257$ & -0 \\
\hline $254=>257$ & 0.3 \\
\hline $254=>2$ & 0.2 \\
\hline $254=>$ & -0.1 \\
\hline $254=>$ & 0.15 \\
\hline $255=>$ & 0.10 \\
\hline
\end{tabular}

$$
\mathrm{X}=4.9750 \mathrm{Y}=-0.2687 \mathrm{Z}=0.0166 \mathrm{Tot}=4.9823
$$

Oscillator strength, $\mathrm{f}=\quad 0.3331$

Restricted Singlet Excited State 25:

Excitation energy $=0.1316641678$ hartrees $\quad 3.58276430 \mathrm{eV} \quad 346.06 \mathrm{~nm}$ excitation $\mathrm{X}$ coeff.

Transition dipole moment (debye):

$$
\mathrm{X}=-1.9442 \mathrm{Y}=0.4324 \mathrm{Z}=-0.5733 \mathrm{Tot}=2.0725
$$

Oscillator strength, $\mathrm{f}=0.0584$

Restricted Singlet Excited State 26:

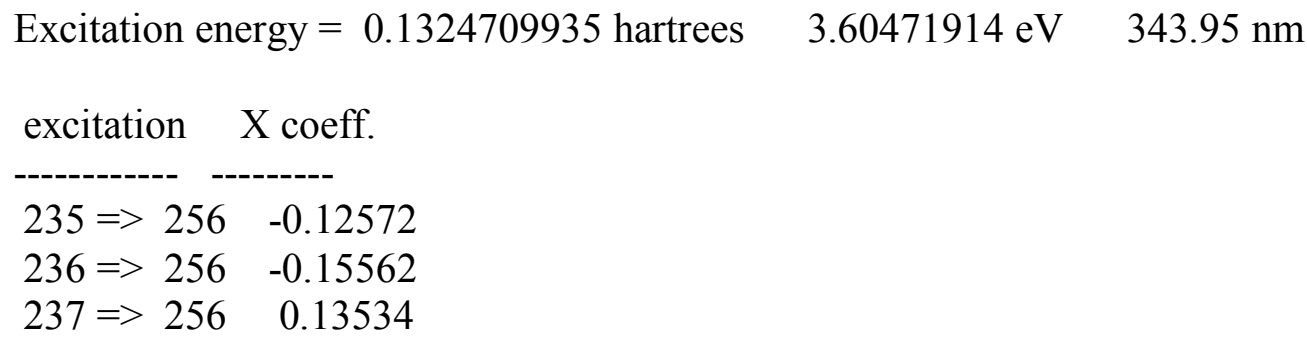




$$
\begin{aligned}
& 238=>256 \quad 0.12727 \\
& 239=>256 \quad 0.19132 \\
& 240=>256 \quad 0.56340 \\
& 252=>258 \quad-0.13145 \\
& 254=>259 \quad 0.41430 \\
& 254=>260 \quad 0.49172 \\
& 255=>259 \quad-0.17697 \\
& 255=>260 \quad 0.17755
\end{aligned}
$$

Transition dipole moment (debye):

$$
\mathrm{X}=0.0105 \mathrm{Y}=2.1344 \mathrm{Z}=-3.8284 \mathrm{Tot}=4.3832
$$

Oscillator strength, $\mathrm{f}=\quad 0.2626$

Restricted Singlet Excited State 27:

Excitation energy $=0.1357698237$ hartrees $\quad 3.69448488 \mathrm{eV} \quad 335.59 \mathrm{~nm}$ excitation X coeff.

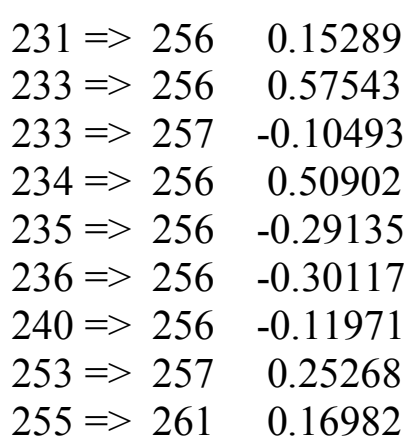

Transition dipole moment (debye):

$$
\mathrm{X}=0.5689 \mathrm{Y}=-0.4352 \mathrm{Z}=0.0853 \text { Tot }=0.7213
$$

Oscillator strength, $\mathrm{f}=\quad 0.0073$

Restricted Singlet Excited State 28:

Excitation energy $=0.1362057533$ hartrees $\quad 3.70634712 \mathrm{eV} \quad 334.52 \mathrm{~nm}$ excitation X coeff.

$232=>256-0.28325$ 


$$
\begin{aligned}
& 233=>256 \quad-0.11462 \\
& 234=>256 \quad-0.22779 \\
& 235=>256 \quad 0.10114 \\
& 236=>256 \quad 0.13164 \\
& 238=>256-0.14321 \\
& 239=>256 \quad-0.14843 \\
& 240=>256 \quad 0.17804 \\
& 252=>257 \quad-0.11286 \\
& 253=>257 \quad 0.72385 \\
& 253=>258 \quad-0.21098 \\
& 254=>261 \quad 0.10141 \\
& 255=>261 \quad 0.30217
\end{aligned}
$$

Transition dipole moment (debye):

$$
\mathrm{X}=1.5741 \mathrm{Y}=0.4449 \mathrm{Z}=-0.8893 \text { Tot }=1.8619
$$

Oscillator strength, $\mathrm{f}=\quad 0.0487$

Restricted Singlet Excited State 29:

Excitation energy $=0.1365059830$ hartrees $\quad 3.71451679 \mathrm{eV} \quad 333.78 \mathrm{~nm}$

excitation X coeff.

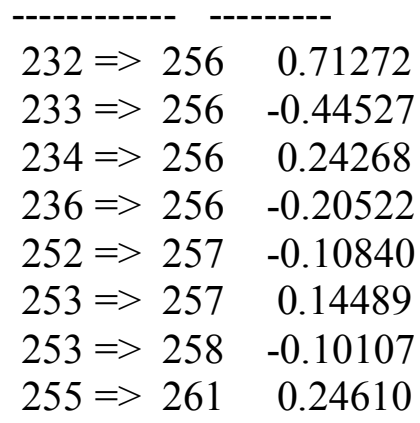

Transition dipole moment (debye):

$$
\mathrm{X}=0.4408 \mathrm{Y}=0.1291 \mathrm{Z}=-0.4373 \mathrm{Tot}=0.6342
$$

Oscillator strength, $\mathrm{f}=0.0057$

Restricted Singlet Excited State 30:

Excitation energy $=0.1370926065$ hartrees $\quad 3.73047963 \mathrm{eV} \quad 332.35 \mathrm{~nm}$ 


$$
\begin{array}{rr}
\text { excitation } & \text { X coeff. } \\
--------- & ------- \\
232=>256 & -0.14594 \\
238=>256 & 0.10924 \\
239=>256 & 0.13092 \\
252=>257 & -0.27395 \\
253=>257 & -0.36456 \\
253=>258 & 0.12363 \\
254=>259 & -0.12783 \\
254=>260 & 0.28502 \\
254=>261 & 0.12759 \\
255=>261 & 0.74361
\end{array}
$$

Transition dipole moment (debye):

$$
\mathrm{X}=-1.1523 \mathrm{Y}=1.0473 \mathrm{Z}=-1.3823 \mathrm{Tot}=2.0821
$$

Oscillator strength, $\mathrm{f}=0.0613$

\section{Diphenyl PDI: 15 roots}

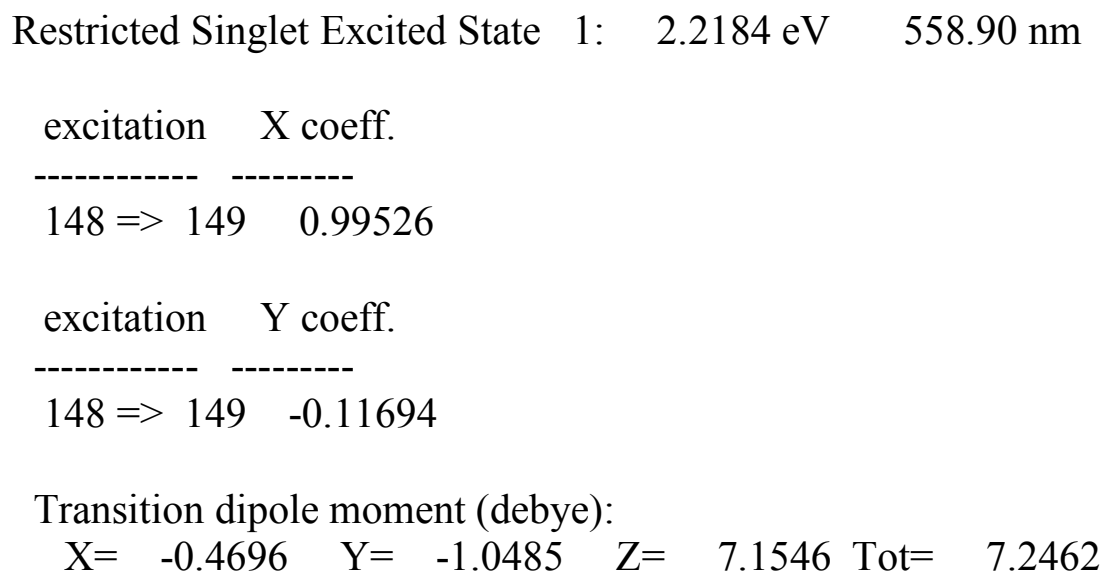

Oscillator strength, $\mathrm{f}=\quad 0.4417$

Restricted Singlet Excited State 2: $2.8743 \mathrm{eV} \quad 431.35 \mathrm{~nm}$

excitation $\mathrm{X}$ coeff.

$147=>149 \quad 0.98872$

$148=>152 \quad 0.10632$

Transition dipole moment (debye): 


$$
\mathrm{X}=-0.1041 \quad \mathrm{Y}=-0.9812 \quad \mathrm{Z}=-0.1883 \text { Tot }=1.0045
$$

Oscillator strength, $\mathrm{f}=\quad 0.0110$

Restricted Singlet Excited State $3: \quad 3.1824 \mathrm{eV} \quad 389.59 \mathrm{~nm}$

excitation X coeff.

\begin{tabular}{|c|c|}
\hline $142 \Rightarrow>149$ & -0.31441 \\
\hline $142=>150$ & 0.14324 \\
\hline $143=>149$ & 0.635 \\
\hline $143=>150$ & 0.11 \\
\hline $144=>149$ & 0.21 \\
\hline $145=>149$ & 0.62 \\
\hline
\end{tabular}

Transition dipole moment (debye):

$\mathrm{X}=-0.2710 \mathrm{Y}=-0.1021 \quad \mathrm{Z}=-0.2166$ Tot $=0.3616$

Oscillator strength, $\mathrm{f}=0.0016$

Restricted Singlet Excited State $\quad$ 4: $\quad 3.1891 \mathrm{eV} \quad 388.77 \mathrm{~nm}$

excitation X coeff.

\begin{tabular}{|c|c|}
\hline $142=>14$ & 0.564 \\
\hline$>1$ & \\
\hline $144=>1$ & $0.1^{\prime}$ \\
\hline $146=>1$ & -0.7 \\
\hline $148 \Rightarrow>1$ & \\
\hline
\end{tabular}

Transition dipole moment (debye):

$$
\mathrm{X}=2.0350 \mathrm{Y}=-0.3321 \mathrm{Z}=1.2756 \text { Tot }=2.4246
$$

Oscillator strength, $\mathrm{f}=\quad 0.0711$

Restricted Singlet Excited State 5: $\quad 3.2070 \mathrm{eV} \quad 386.60 \mathrm{~nm}$

excitation $\mathrm{X}$ coeff.

$$
\begin{aligned}
& 142=>149 \quad-0.63899 \\
& 143=>149 \quad-0.28563 \\
& 146=>149 \quad-0.66473 \\
& 148=>153 \quad 0.11843
\end{aligned}
$$


Transition dipole moment (debye):

$\mathrm{X}=2.1038 \mathrm{Y}=-0.3601 \quad \mathrm{Z}=1.2253 \mathrm{Tot}=2.4611$

Oscillator strength, $\mathrm{f}=\quad 0.0737$

Restricted Singlet Excited State 6: $\quad 3.3417 \mathrm{eV} \quad 371.02 \mathrm{~nm}$

excitation X coeff.

\begin{tabular}{|c|c|c|}
\hline $138=>$ & 149 & 0.2656 \\
\hline $140=>$ & 149 & 0.117 \\
\hline $141=>$ & 149 & -0.399 \\
\hline $142=>$ & 149 & -0.255 \\
\hline $143=>$ & 149 & 0.340 \\
\hline $144=>$ & 149 & 0.216 \\
\hline $145=>$ & 149 & -0.555 \\
\hline $148=>$ & 150 & -0.445 \\
\hline
\end{tabular}

Transition dipole moment (debye):

$\mathrm{X}=0.0313 \quad \mathrm{Y}=0.0092 \quad \mathrm{Z}=-0.0727$ Tot $=0.0797$

Oscillator strength, $f=0.0001$

Restricted Singlet Excited State 7: $3.3470 \mathrm{eV} \quad 370.44 \mathrm{~nm}$

excitation X coeff.

\begin{tabular}{|c|c|}
\hline $8=>14$ & 0.11717 \\
\hline $140=>14$ & 0.21502 \\
\hline $141=>14$ & -0.83478 \\
\hline $142=>14$ & 0.12451 \\
\hline $143=>1$ & -0.17352 \\
\hline $145=>$ & 0.25723 \\
\hline $48=$ & 0.3266 \\
\hline
\end{tabular}

Transition dipole moment (debye):

$\mathrm{X}=0.1328 \quad \mathrm{Y}=0.0911 \quad \mathrm{Z}=0.0482$ Tot $=0.1681$

Oscillator strength, $\mathrm{f}=\quad 0.0004$

Restricted Singlet Excited State 8: $\quad 3.3770 \mathrm{eV} \quad 367.14 \mathrm{~nm}$ 
excitation X coeff.

$\begin{array}{rr}----------- \\ 143=>149 & 0.36802 \\ 144=>149 & -0.91855\end{array}$

Transition dipole moment (debye):

$$
X=0.5227 \quad Y=-0.2921 \quad Z=1.6381 \text { Tot }=1.7441
$$

Oscillator strength, $\mathrm{f}=\quad 0.0390$

Restricted Singlet Excited State 9: $\quad 3.4073 \mathrm{eV} \quad 363.88 \mathrm{~nm}$

excitation X coeff.

\begin{tabular}{|c|c|c|}
\hline $138=>$ & 149 & -0.38444 \\
\hline $139=>$ & 149 & -0.23525 \\
\hline $142=>$ & 149 & -0.19648 \\
\hline $143=>$ & 149 & 0.29386 \\
\hline $145=>$ & 149 & -0.44634 \\
\hline $148=>$ & 150 & 0.668 \\
\hline
\end{tabular}

Transition dipole moment (debye):
$\mathrm{X}=0.0383 \quad \mathrm{Y}=0.0983 \quad \mathrm{Z}=0.0675$ Tot $=0.1253$

Oscillator strength, $\mathrm{f}=\quad 0.0002$

Restricted Singlet Excited State 10: $\quad 3.5375 \mathrm{eV} \quad 350.49 \mathrm{~nm}$

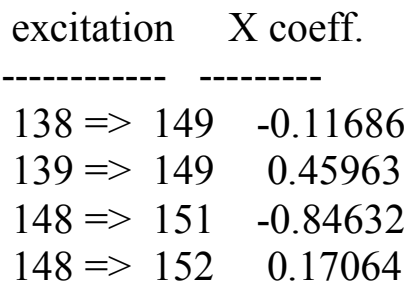

Transition dipole moment (debye):

$$
\mathrm{X}=0.1216 \quad \mathrm{Y}=-0.3065 \quad \mathrm{Z}=-0.0839 \text { Tot }=0.3403
$$

Oscillator strength, $\mathrm{f}=\quad 0.0016$

Restricted Singlet Excited State 11: $\quad 3.5599 \mathrm{eV} \quad 348.28 \mathrm{~nm}$ excitation $\mathrm{X}$ coeff. 


$\begin{array}{ll}137=>149 & -0.28905 \\ 140=>149 & 0.89672 \\ 141=>149 & 0.22500 \\ 148=>153 & 0.11724\end{array}$

Transition dipole moment (debye):

$\mathrm{X}=-0.5693 \mathrm{Y}=0.1511 \mathrm{Z}=-0.7894 \mathrm{Tot}=0.9850$

Oscillator strength, $\mathrm{f}=\quad 0.0131$

Restricted Singlet Excited State 12: $\quad 3.6205 \mathrm{eV} \quad 342.45 \mathrm{~nm}$

excitation X coeff.

$134=>149 \quad-0.25434$

$135=>149 \quad 0.26883$

$137=>149 \quad 0.25816$

$138=>149 \quad 0.32261$

$139=>149 \quad-0.67228$

$148=>151 \quad-0.43601$

Transition dipole moment (debye):
$\mathrm{X}=-0.5592 \quad \mathrm{Y}=-0.6180$
$Z=0.1435$ Tot $=0.8458$

Oscillator strength, $\mathrm{f}=\quad 0.0098$

Restricted Singlet Excited State 13: $\quad 3.6977 \mathrm{eV} \quad 335.30 \mathrm{~nm}$

excitation $\mathrm{X}$ coeff.

$\begin{array}{ll}134=>149 & 0.12970 \\ 135=>149 & 0.42122 \\ 136=>149 & 0.17323 \\ 137=>149 & 0.70094 \\ 139=>149 & 0.34950 \\ 140=>149 & 0.22810 \\ 148=>151 & 0.11886 \\ 148=>153 & -0.18693\end{array}$

Transition dipole moment (debye):

$$
\mathrm{X}=-0.9648 \mathrm{Y}=0.3198 \mathrm{Z}=0.6670 \text { Tot }=1.2157
$$

Oscillator strength, $\mathrm{f}=\quad 0.0207$ 
Restricted Singlet Excited State 14: $\quad 3.7333 \mathrm{eV} \quad 332.11 \mathrm{~nm}$

excitation X coeff.

\begin{tabular}{|c|c|}
\hline$>1$ & 0.15 \\
\hline & \\
\hline & 0 \\
\hline & \\
\hline & -0.1 \\
\hline & \\
\hline
\end{tabular}

Transition dipole moment (debye):

$\mathrm{X}=0.1581 \quad \mathrm{Y}=-0.0489 \quad \mathrm{Z}=0.2034$ Tot $=0.2623$

Oscillator strength, $\mathrm{f}=\quad 0.0010$

Restricted Singlet Excited State 15: $3.7993 \mathrm{eV} \quad 326.33 \mathrm{~nm}$

excitation X coeff.

\begin{tabular}{|c|c|}
\hline $36=>149$ & \\
\hline $138=>14$ & \\
\hline $9=>$ & 0 \\
\hline$>$ & 0.1 \\
\hline$>$ & 0.44 \\
\hline$=>$ & 0.15 \\
\hline
\end{tabular}

Transition dipole moment (debye):

$\mathrm{X}=0.1437 \quad \mathrm{Y}=0.4263 \quad \mathrm{Z}=0.0212 \mathrm{Tot}=0.4504$

Oscillator strength, $f=0.0029$ 


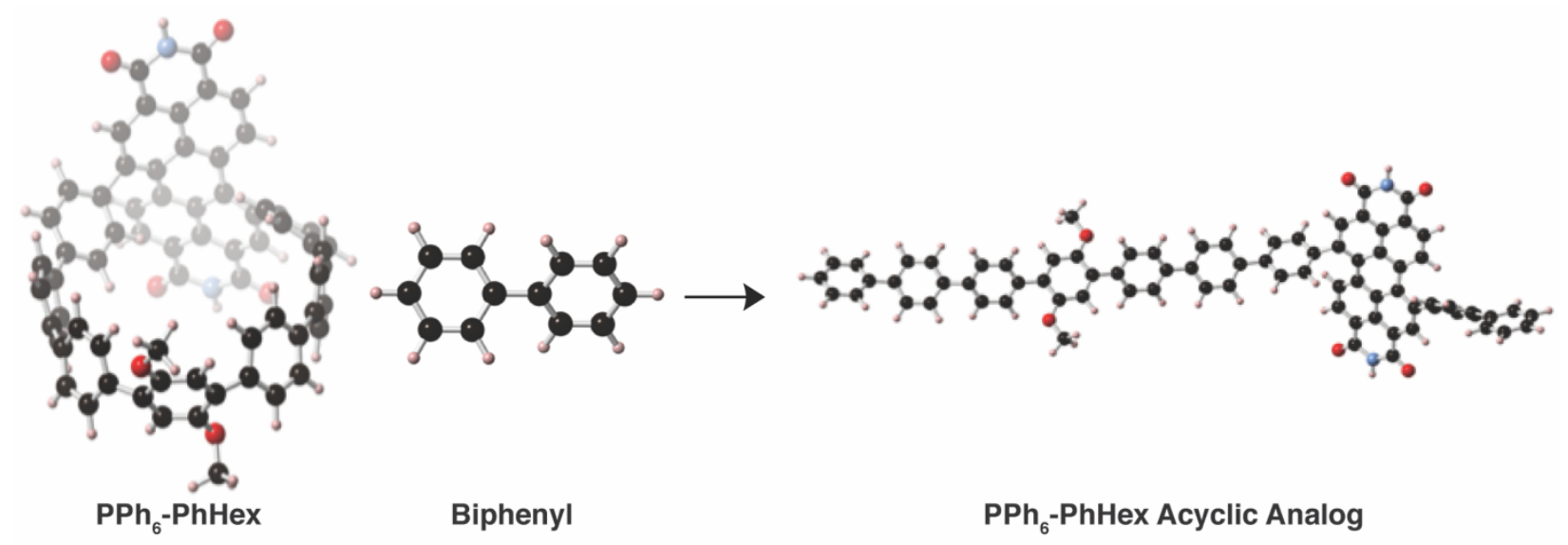

Table 7.1. Homodesmotic Calculations for Strain

\begin{tabular}{|l|c|c|c|}
\hline Compound & Total energy & $\begin{array}{c}\text { Strain energy } \\
\text { (hartree) }\end{array}$ & $\begin{array}{c}\text { Strain energy } \\
(\mathrm{kcal} / \mathrm{mol})\end{array}$ \\
\hline PPh $_{\text {6-PhHex }}$ & -3639.3085 & 0.100 & 63 \\
\hline Biphenyl & -463.3215 & - & - \\
\hline PPh $_{6}$-PhHex Acyclic Analog & -3639.7302 & - & - \\
\hline
\end{tabular}

Table 7.1: Energies for the Homodesmotic Reaction (enthalpy).

\subsection{References}

1. Zhang, B. et al. Hollow organic capsules assemble into cellular semiconductors. Nat. Commun. 9, 1957 (2018).

2. Hitosugi, S. et al. Asymmetric Autocatalysis Initiated by Finite Single-Wall Carbon Nanotube Molecules with Helical Chirality. Org. Lett. 16, 645-647 (2014).

3. Matsuno, T. et al. Self-Sorting of Two Hydrocarbon Receptors with One Carbonaceous Ligand. Angew. Chem. Int. Ed. 55, 15339-15343 (2016).

4. Matsuno, T. et al. Ratchet-free solid-state inertial rotation of a guest ball in a tight tubular host. Nat. Commun. 9, 1907 (2018).

5. Sato, S. et al. Chiral intertwined spirals and magnetic transition dipole moments dictated by cylinder helicity. Proc. Natl. Acad. Sci. 114, 13097-13101 (2017).

6. Wu, D. et al. Cycloparaphenylenes (CPPs): An Overview of Synthesis, Properties, and Potential Applications. Asian J. Org. Chem. 7, 2161-2181 (2018).

7. Nojima, Y. et al. Stereogenic cyclic oligonaphthalenes displaying ring size-dependent handedness of circularly polarized luminescence (CPL). Chem. Commun. 55, 2749-2752 (2019).

8. Xu, W. et al. Synthesis and Characterization of a Pentiptycene-Derived Dual Oligoparaphenylene Nanohoop. Angew. Chem. Int. Ed. 58, 3943-3947 (2019).

9. Nakamura, T. et al. Double-Circularly Connected Saloph-Belt Macrocycles Generated from 
a Bis-Armed Bifunctional Monomer. J. Am. Chem. Soc. (2019). doi:10.1021/jacs.9b00171

10. Hitosugi, S., et al. Bottom-up synthesis of finite models of helical (n,m)-single-wall carbon nanotubes. Nat. Commun. 2, (2011).

11. Hitosugi, S. et al. Atropisomerism in a Belt-Persistent Nanohoop Molecule: Rotational Restriction Forced by Macrocyclic Ring Strain. Chem. Asian J. 7, 1550-1552 (2012).

12. Kayahara, E. et al. Gram-Scale Syntheses and Conductivities of [10]Cycloparaphenylene and Its Tetraalkoxy Derivatives. J. Am. Chem. Soc. 139, 18480-18483 (2017).

13. Kayahara, E. et al. Synthesis and Characterization of 5 Cycloparaphenylene. J. Am. Chem. Soc. 136, 2284-2287 (2014).

14. Segawa, Y. et al. Concise Synthesis and Crystal Structure of 12 Cycloparaphenylene. Angew. Chem. Int. Ed. 50, 3244-3248 (2011).

15. Iwamoto, T. et al. Selective and Random Syntheses of n Cycloparaphenylenes $(\mathrm{n}=8-13)$ and Size Dependence of Their Electronic Properties. J. Am. Chem. Soc. 133, 8354-8361 (2011).

16. Ball, M. et al. Macrocyclization in the Design of Organic n-Type Electronic Materials. $J$. Am. Chem. Soc. 138, 12861-12867 (2016).

17. Ball, M. L. et al. Influence of Molecular Conformation on Electron Transport in Giant, Conjugated Macrocycles. J. Am. Chem. Soc. 140, 10135-10139 (2018).

18. Zhang, B. et al. Rigid, Conjugated Macrocycles for High Performance Organic Photodetectors. J. Am. Chem. Soc. 138, 16426-16431 (2016).

19. Ball, M. et al. Chiral Conjugated Corrals. J. Am. Chem. Soc. 137, 9982-9987 (2015).

20. Ball, M. et al. Conjugated Macrocycles in Organic Electronics. Acc. Chem. Res. (2019). doi:10.1021/acs.accounts.9b00017

21. Ball, M. et al. Contorted Polycyclic Aromatics. Acc. Chem. Res. 48, 267-276 (2015).

22. Nolde, F. et al. Synthesis and self-organization of core-extended perylene tetracarboxdiimides with branched alkyl substituents. Chem. Mater. 18, 3715-3725 (2006).

23. Zhong, Y. et al. Helical Ribbons for Molecular Electronics. J. Am. Chem. Soc. 136, 81228130 (2014).

24. Meng, D. et al. High-Performance Solution-Processed Non-Fullerene Organic Solar Cells Based on Selenophene-Containing Perylene Bisimide Acceptor. J. Am. Chem. Soc. 138, 375-380 (2016).

25. Wu, Q. et al. Covalently Bound Clusters of Alpha-Substituted PDI-Rival Electron Acceptors to Fullerene for Organic Solar Cells. J. Am. Chem. Soc. 138, 7248-7251 (2016).

26. Darzi, E. R. et al. An Operationally Simple and Mild Oxidative Homocoupling of Aryl Boronic Esters To Access Conformationally Constrained Macrocycles. J. Am. Chem. Soc. 139, 3106-3114 (2017).

27. Darzi, E. R. et al. Synthesis, Properties, and Design Principles of Donor-Acceptor Nanohoops. ACS Cent. Sci. 1, 335-342 (2015).

28. Nishigaki, S. et al. Synthesis, Structures, and Photophysical Properties of Alternating 
Donor-Acceptor Cycloparaphenylenes. Chem. Eur. J. 23, 7227-7231 (2017).

29. Van Raden, J. M. et al. Synthesis and characterization of a highly strained donor-acceptor nanohoop. Org. Biomol. Chem. 14, 5721 (2016).

30. Rajasingh, P. et al. Selective Bromination of Perylene Diimides under Mild Conditions. $J$. Org. Chem. 72, 5973-5979 (2007). 


\section{Chapter 8. The Influence of Molecular Conformation on Electron Transport in Giant, Conjugated Macrocycles.}

\subsection{Preface}

Chapter 8 is reproduced with permission from the authors: Melissa Ball, Boyuan Zhang, Qizhi Xu, Daniel W. Paley, Fay Ng, Michael L. Steigerwald and Colin Nuckolls published in the Journal of the American Chemical Society. ${ }^{1}$ Copyright 2018 American Chemical Society. I carried out the synthesis and characterization of all compounds. Boyuan Zhang and Qizhi Xu performed all device fabrication and characterization.

\subsection{Introduction}

This manuscript describes the direct connection between the molecular conformation of a macrocycle and its macroscopic charge transport properties. The macrocycles studied here are the merger of two classes of electronic materials: conjugated macrocycles ${ }^{2-28}$ and electron transporting hPDI ribbons. ${ }^{29-31}$ Conjugated macrocycles are emerging as efficacious materials for electron transport in OFETs, OPVs, OPD, and sensors. ${ }^{12,32-34}$ Thus far, the most successful conjugated macrocycles in devices consist of multiple copies of PDI monomers with various spacers that are wound into a macrocycle. Our goal here is to substitute monomeric PDI subunits with oligomeric hPDI subunits. The hPDI nanoribbons are known to have extraordinary properties in devices requiring efficient charge transport, such as solar cells and photodetectors, ${ }^{35-39}$ making them exciting candidates for incorporation into a conjugated macrocycle construction. 
(a) Macrocycle Building Blocks

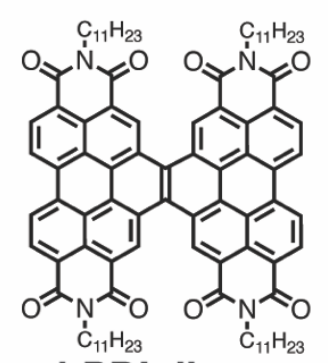
hPDI dimer

(D)

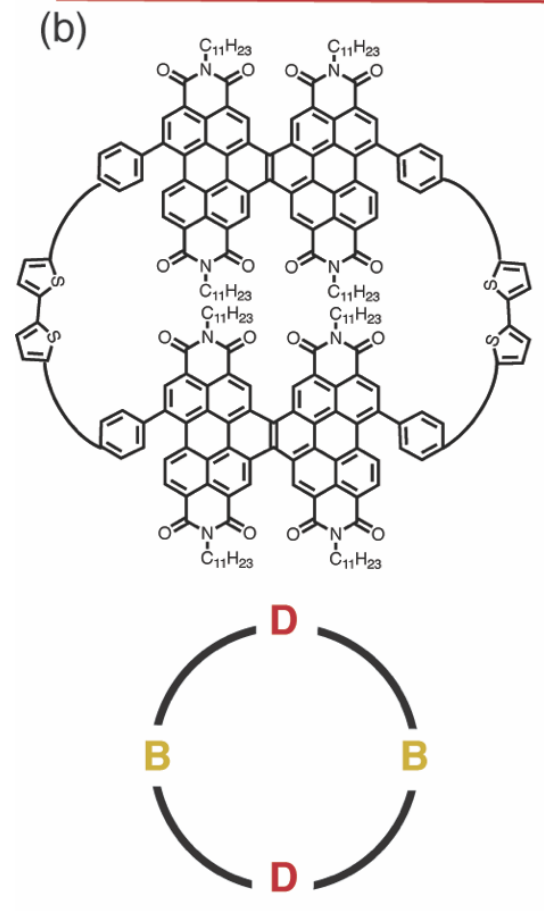

cis-DBDB<smiles>c1ccc(-c2ccc(-c3ccc(-c4ccccc4)s3)s2)cc1</smiles>

Phenyl-Bithiophene-Phenyl

(B)

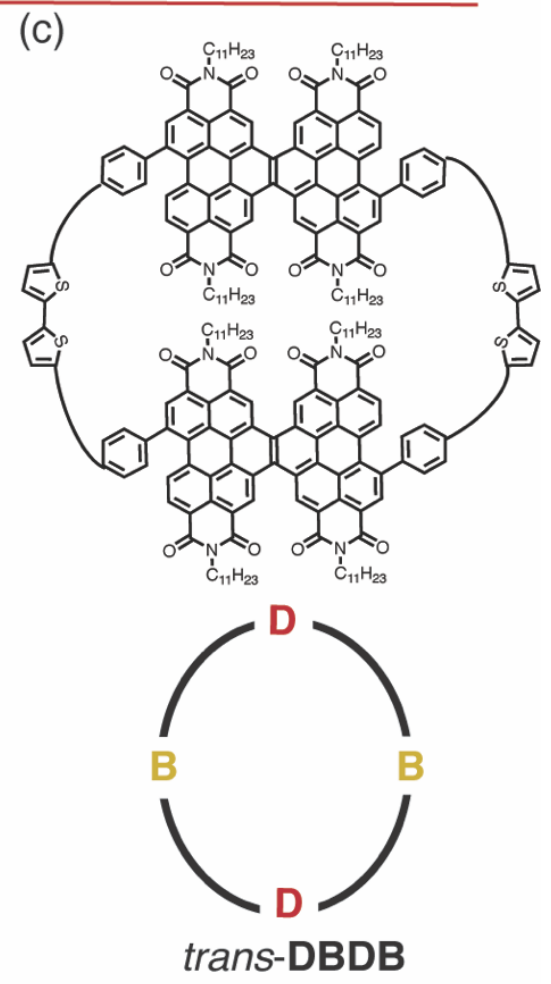

Figure 8.1. (a) Macrocycle building blocks comprising a phenyl-bithiophene-phenyl linker (B) and an hPDI dimer (D). The macrocycles are formed from these two molecular components wrapped into a-D-B-D-B- arrangement; (b) chemical structure of cis-DBDB; and (c) trans-DBDB.

Here we reveal the first of new, giant macrocycles that incorporate hPDI ribbons (Figure 8.1). Both structures comprise hPDI dimers (D) that are connected with a phenyl-bithiophenephenyl linker (B) to form the macrocycles with the structure DBDB (the "c" denoting a cyclic structure). The connectivity of the two macrocycles differ between the hPDI monomer and the linker group (B), resulting in the formation of two, giant macrocycles: cis-DBDB (Figure 8.1b) and trans-DBDB (Figure 8.1c). We create amorphous thin films from each of these macrocycles 
and compare how both the molecular structure and conformation affect charge transport. We find that both macrocycles form thin films that exhibit n-type semiconducting behavior in OFET devices. The remarkable finding is that the seemingly small change in the connectivity between hPDI dimer and the linker group results in a pronounced difference in the conductance of the films: the electron mobility of cis-DBDB is over four-times greater than that of trans-DBDB. As both films are amorphous, the difference in mobility is a direct consequence of the different molecular conformations available to the two isomers: the cis isomer's more flexible structure is better able to make intermolecular contacts than the trans isomer's more rigid structure.

\subsection{Regio-pure Synthesis To Create DBDB}

Figure 8.2 displays the scheme for cis-DBDB and trans-DBDB. Both syntheses begin from the cis and trans dibrominated dimers $\left(\mathrm{hPDI}-\mathrm{Br}_{2}\right.$ 8.1a and 8.2a) that are formed as a 1:1 mixture in the bromination of the parent hPDI dimer. ${ }^{29}$ The separation of 8.1a and 8.2a proved to be a challenging step in part because the two regioisomers are indistinguishable by ${ }^{1} \mathrm{H}-\mathrm{NMR}$ or UV/vis spectroscopy. Moreover, we were unable to find conditions using standard silica gel

chromatography to separate 8.1a and 8.2a; however, chiral HPLC proved to be successful in the separation of the two regioisomers (Figures 8.8-10 Table 8.1).

Using these regio-pure building blocks, we completed the syntheses of trans-DBDB and cis-DBDB (Figure 8.2). The approach to their syntheses utilizes square planar platinum atoms to form multi-nuclear macrocycles that form conjugated macrocycles through a reductive elimination process. We isolate the macrocycles in 6\% yield for trans-DBDB and 12\% yield for cis-DBDB. Both trans-DBDB and cis-DBDB are dark purple solids with a metallic sheen. 

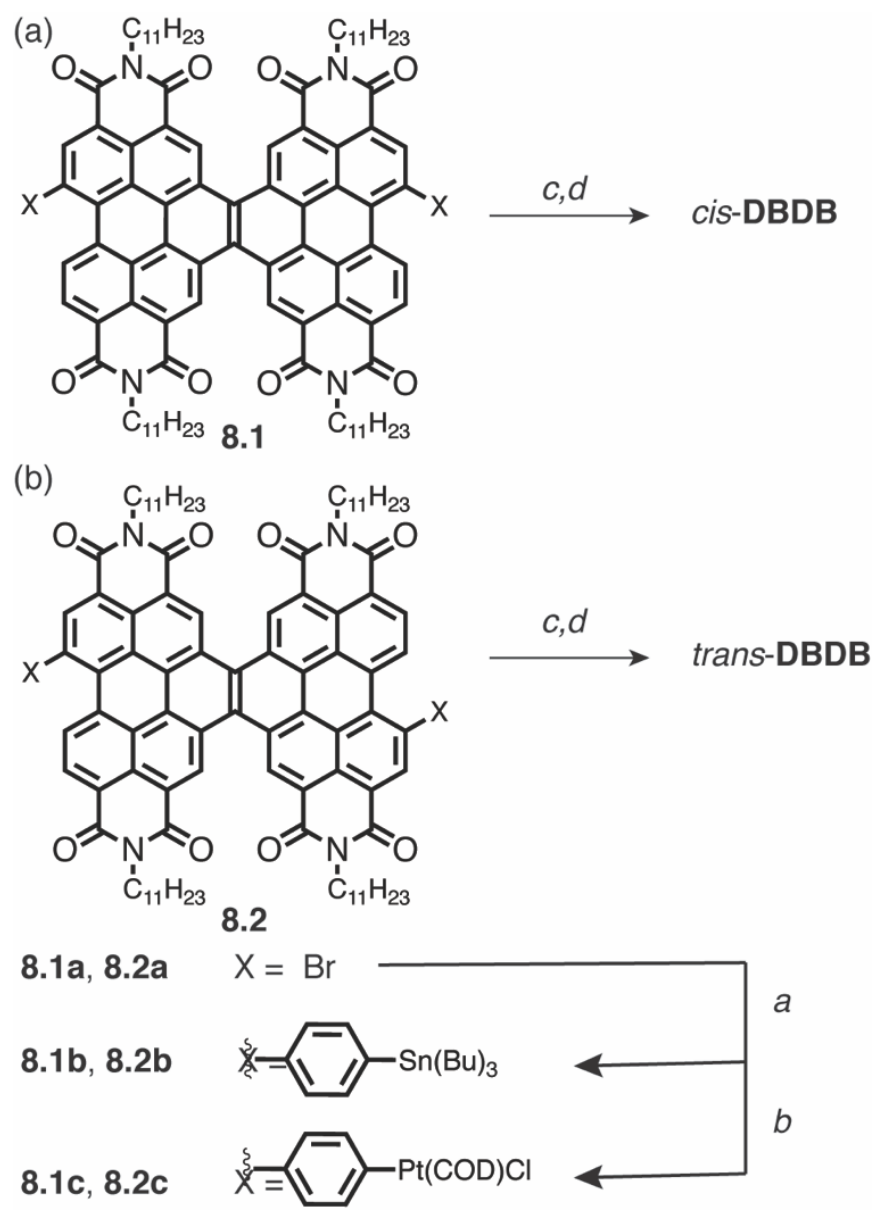

Figure 8.2. Synthesis of DBDB. key: a) 1,4-bis(tributylstannyl)benzene, P-(2-furyl $)_{3}, \mathrm{Pd}_{2} \mathrm{dba}_{3}$, THF, $55^{\circ} \mathrm{C}$, $12 \mathrm{~h}$; b) $\mathrm{Pt}(\mathrm{COD}) \mathrm{Cl}_{2}$, toluene, $100{ }^{\circ} \mathrm{C}, 12 \mathrm{~h}$; c) 5,5'-bis(tributylstannyl)-2,2'bithiophene, THF, $55^{\circ} \mathrm{C}, 40$ h. (d) $\mathrm{PPh}_{3}$, toluene, $100{ }^{\circ} \mathrm{C}, 12 \mathrm{~h}$.

\subsection{Cis-DBDB Four-fold Increase in Carrier Mobility}

We next explore how the connectivity of the macrocycles influences charge transport in thin films. From the potential of the first reduction peak in the cyclic voltammogram, we estimate the lowest unoccupied molecular orbital (LUMO) energy level to be $-3.80 \mathrm{eV}$ and $-3.82 \mathrm{eV}$ for trans-DBDB and cis-DBDB (Figure 8.11), respectively. These values are similar to the parent hPDI dimer and common n-type semiconductors like [6,6]-phenyl $\mathrm{C}_{61}$-butyric acid methyl ester $\left(\mathrm{PC}_{61} \mathrm{BM}\right) .{ }^{29}$ This data suggests both macrocycles are promising n-type semiconductors.

Figure 8.3 contains a schematic of the OFET device, and displays the gate sweep for the devices from trans-DBDB and cis-DBDB. The remarkable finding is cis-DBDB shows a four-fold 
increase in mobility relative to trans-DBDB, using the methods developed by McCulloch and Choi. ${ }^{40,41}$ The cis-DBDB based transistor has a mobility of $\sim 5.3 \times 10^{-3} \mathrm{~cm}^{2} / \mathrm{V} \bullet$ s relative to $\sim 1.3 \mathrm{x}$ $10^{-3} \mathrm{~cm}^{2} / \mathrm{V} \cdot \mathrm{s}$ for trans-DBDB (Figure 8.3). The devices in Figure 8.3 are the highest mobility for each isomer. To confirm the statistical relevance of this data, we measured seven devices and averaged the data for both trans-DBDB and cis-DBDB (Table 8.2). The averaged data, too, showed the cis isomer has a four-fold increase in electron mobility. We tested 8.1a and 8.2a, too, to see if there was a difference in mobility within the acyclic fragments, and found that the mobilities of the two are essentially identical (Figure 8.13). This suggests that the difference in mobility between cis-DBDB and trans- DBDB has to do with the molecular conformation of each macrocycle.

(a)

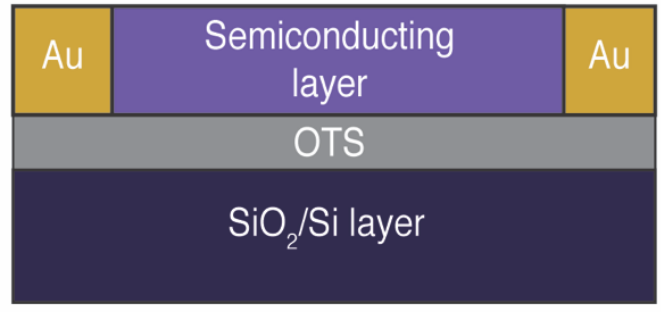

(b)

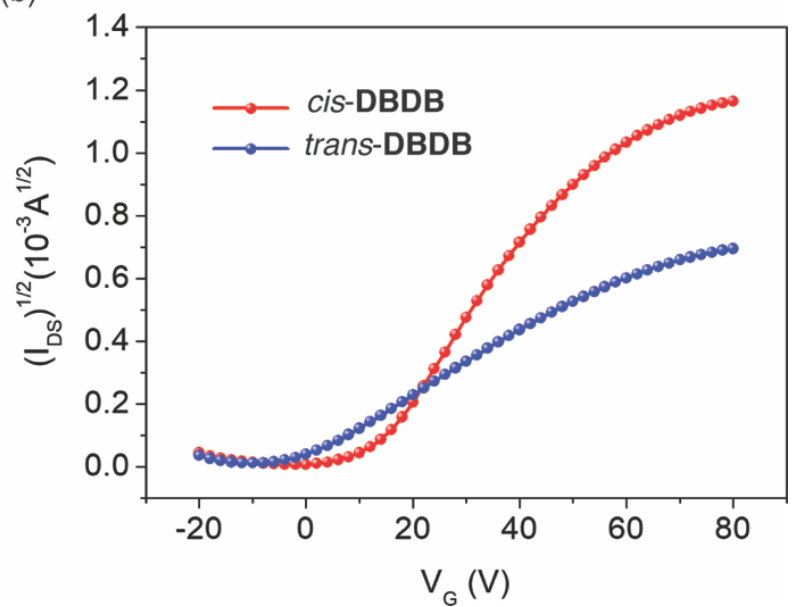

Figure 8.3. (a) Schematic of the OFET device and (b) transfer characteristics for trans-DBDB and cis-DBDB, showing a four-fold increase in electron mobility for the latter.

To understand why cis-DBDB's mobility is much greater than trans-DBDB, we consider two factors that have a significant and general impact on charge transport in thin films: (1) the film 
morphology and (2) the molecular and electronic structure of the molecule. We conclude that the films as we have prepared them are amorphous, and the electronic structures of the two macrocycles are essentially identical. These points suggest that it is the molecular structures and their associated dynamics that determine device performance.

\subsection{Both Macrocycles are Amorphous by PXRD, AFM, and DSC}

It is well-established that a thin film's crystallinity and morphology will influence the charge transport characteristics, and thus we first investigate the film morphology under device fabrication for each macrocycle. ${ }^{42}$ We used a combination of differential scanning calorimetry (DSC), PXRD, and AFM to investigate the film morphology and crystallinity (Figure 8.14). Broad and featureless transitions in the DSC occur below $160{ }^{\circ} \mathrm{C}$ for both trans-DBDB and cis-DBDB. PXRD of both films suggests that both films are amorphous, lacking any obvious signs of crystallinity for both trans-DBDB and cis-DBDB. The AFM images, too, showed both films possess a smooth surface, with a root mean square roughness of $1.39 \mathrm{~nm}$ and $1.36 \mathrm{~nm}$ for transDBDB and cis-DBDB, respectively. Given this data, the essential point is that the origin of the difference in device performance cannot be due to the film morphology. We next investigate the electronic and molecular structure of the molecules as a source of the difference.

\subsection{DFT Shows Pronounced Difference in Geometries}

DFT calculations reveal that there is a marked difference between the lowest energy structures of both macrocycles. Trans-DBDB possesses an upright conformation (Figure 8.4a), where the hPDI dimer subunits are vertically arranged with respect to the macrocyclic plane. In contrast, cis-DBDB adopts a tent shape (Figure 8.4b), where the hPDI dimer subunits collapse across the macrocycle. The two macrocycles show a pronounced difference in their shapes: while both are elliptical, the eccentricity of trans-DBDB is greater than that of cis-DBDB (Figure 8.4c). 
(a)

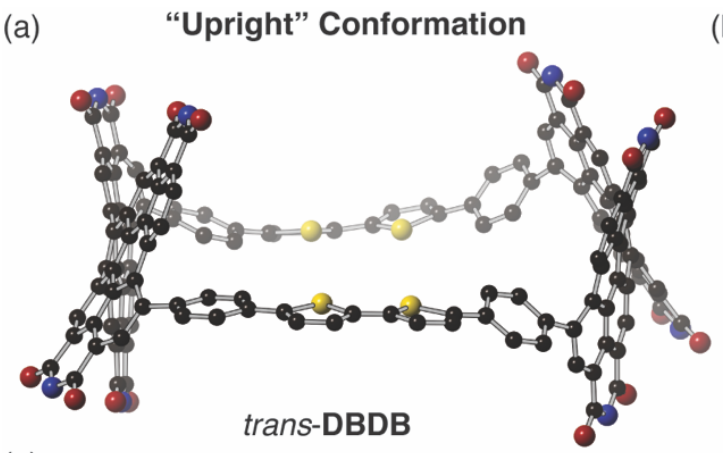

(c)

(d)

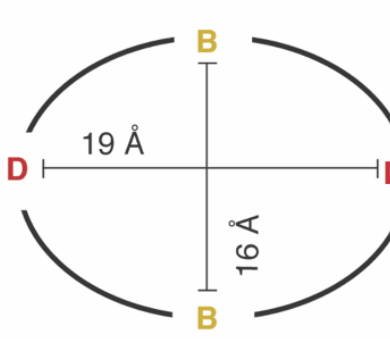

trans-DBDB

linker

oval (b)
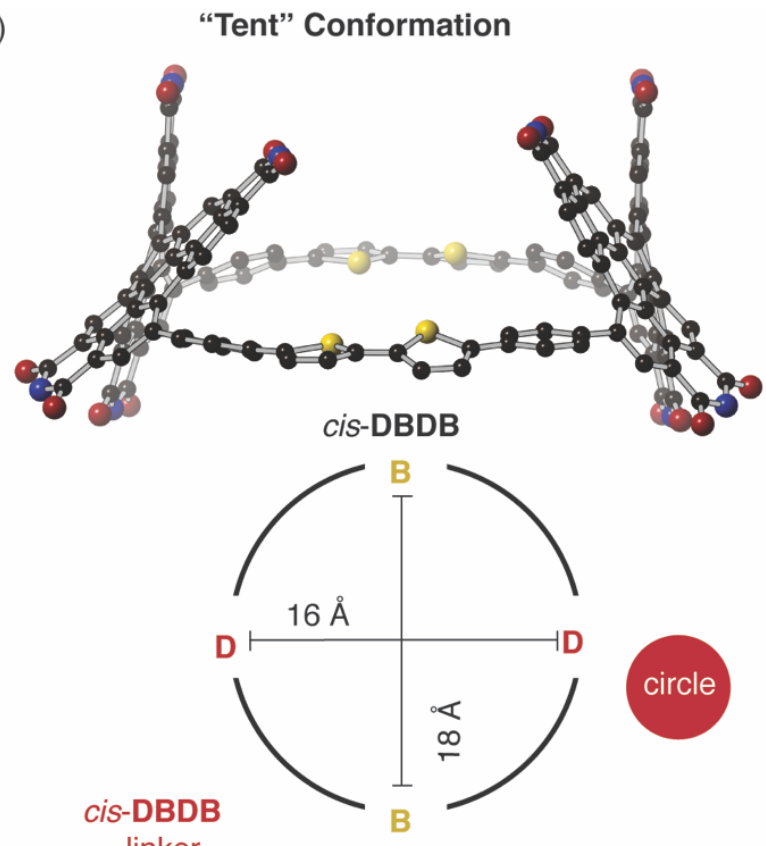

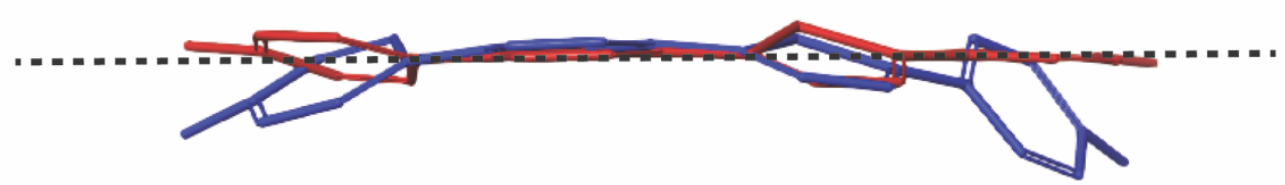

Figure 8.4. (a) DFT calculated lowest energy geometry for trans-DBDB; (b) and cis-DBDB. Red $=$ oxygen, blue $=$ nitrogen, black $=$ carbon, and yellow $=$ sulfur. Hydrogens and side chains have been removed for clarity; (c) Schematics of both trans-DBDB and cis-DBDB, respectively; (d) an overlay of the tetracyclic linker of both trans-DBDB and cis-DBDB. trans-DBDB shows a pronounced bowing relative to the cis isomer. Dashed black line indicates no bowing.

From the elongation and the upright structure for trans-DBDB, we reasoned that it was more strained than cis-DBDB. Calculations using a homodesmotic ${ }^{24,43-45}$ series reveal that the strain energy difference between trans-DBDB and cis-DBDB is $9 \mathrm{kcal} / \mathrm{mol}$, with the former structure possessing $18.6 \mathrm{kcal} / \mathrm{mol}$ of strain relative to just $9.5 \mathrm{kcal} / \mathrm{mol}$ for cis-DBDB (Table 8.3 ). To put this number into context, trans-DBDB contains a similar amount of strain to $\mathbf{P B P B}{ }^{12}$ highlighting cis-DBDB's conformational flexibility. We can visualize the strain in the two isomers from the difference in their tetracyclic linkers (phenyl-bithiophene-phenyl) connecting the hPDI subunits (Figure 8.4d). It is apparent from the over-lay that the tetracyclic linker bows in order to accommodate the rigidity of the trans-DBDB macrocycle (blue model). This is not the case for the 
cis isomer (red model) where the tetracyclic linker is essentially flat and less strained relative to trans-DBDB.

\subsection{Variable Temperature NMR Studies}

The difference in strain for the two macrocycles affects the dynamics in solution. From variable temperature ${ }^{1} \mathrm{HNMR}$ measurements, we observe trans-DBDB is much less floppy than cis-DBDB. Both macrocycles possess different conformers that are able to interconvert through an "intramolecular somersault" process, where a hPDI subunit rotates through the center of the cavity (denoted with green arrows in Figure $8.5 \mathrm{~b}, \mathrm{c}) .{ }^{12}$

(a)

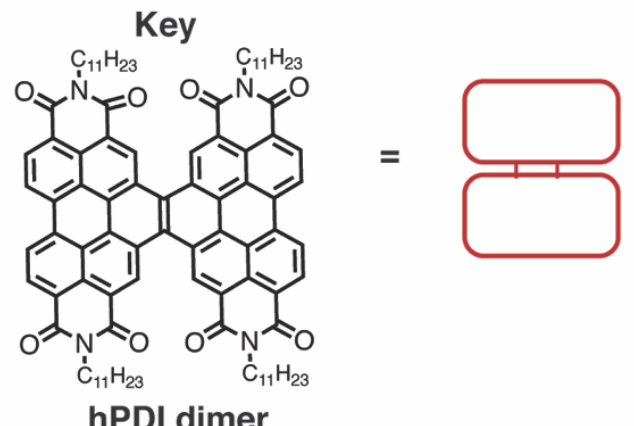

(b)

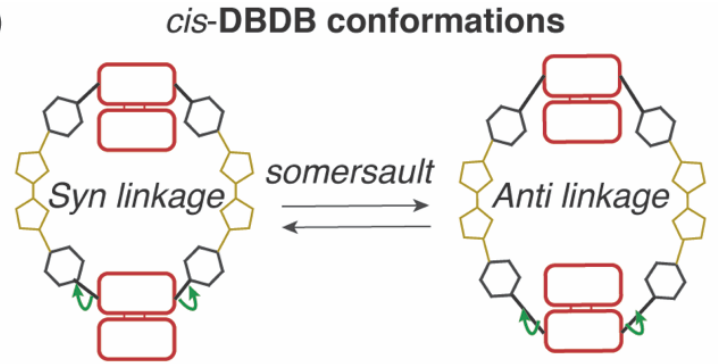

(c)

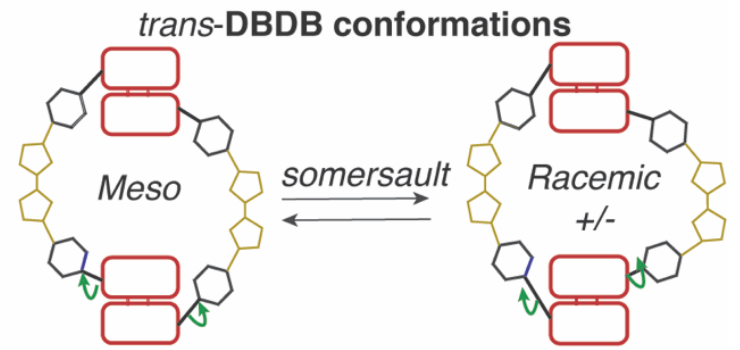

(d) trans-DBDB<smiles></smiles>

Phenyl-Bithiophene-Phenyl

(B)
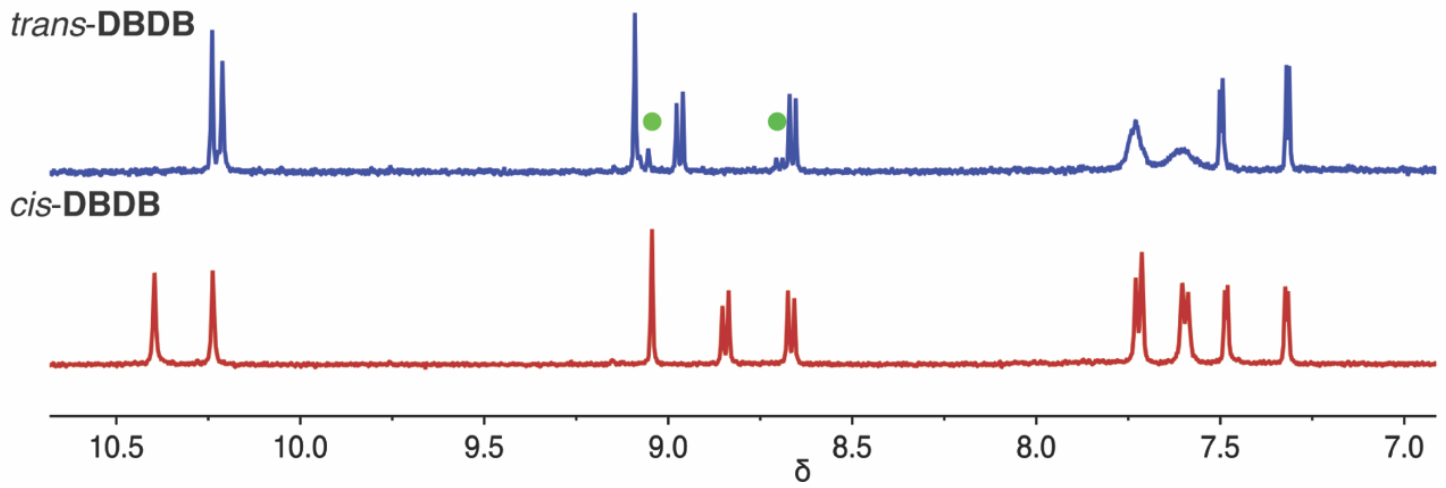

Figure 8.5. (a) Molecular building blocks for DBDB macrocycles; (b) Schematics the two conformers of cis-DBDB; (c) and trans-DBDB that interconvert through a somersault mechanism; and (d) VT-NMR of both macrocycles at $380 \mathrm{~K}$. trans-DBDB shows the presence of both conformers at $380 \mathrm{~K}$ at 9.1 and $8.7 \mathrm{ppm}$, denoted with green circles. 
Yet the intramolecular somersault dynamics differ for trans-DBDB and cis-DBDB. While the ${ }^{1} \mathrm{H}-\mathrm{NMR}$ resonances for cis-DBDB's two conformers fully coalesce at $380 \mathrm{~K}$, the NMR shows the presence of both diastereomers for trans-DBDB (denoted with green circles in Figure 8.5d). Figure 8.15 contains further coalescence details. This suggests the activation barrier for the somersault process is higher for trans-DBDB than for cis-DBDB. We believe this reflects the geometries of each macrocycle: the cis isomer's "tent" shape is less rigid, and the hPDI subunits are more able to rotate through the cavity relative to the trans isomer's upright, rigid scaffold.

\subsection{Rigidity Evident in the UV-Vis spectrum}

The floppiness of the cis-DBDB relative to trans-DBDB is evident in both their UV-Vis and fluorescence spectra. While trans-DBDB retains features of a vibrational progression, characteristic of the unsubstituted PDI dimer, ${ }^{29}$ cis-DBDB possesses a broadened spectrum. TransDBDB's lowest energy transition, too, is shifted to lower energy shifted relative to cis-DBDB by approximately $20 \mathrm{~nm}$. Both structural features reflect trans-DBDB's more rigid, strained conformation.

\subsection{VT-OFET Shows Larger Temperature Dependence for Cis}

As both films are amorphous with no crystalline features, the disparity in transport properties reflects the differences in molecular structure between trans-DBDB and cis-DBDB geometries. The cis isomer's "tent" shape is conformationally flexible, aiding its ability to transport charge more effectively than the more rigid trans isomer. cis-DBDB's less strained and floppy structure allows it to make better intermolecular contacts with neighboring molecules, resulting in higher mobility. We tested this hypothesis using temperature dependent transport measurements on films of pure $c i s$-DBDB and trans-DBDB to temperatures as low as $250 \mathrm{~K}$. We find that the mobility of cis-DBDB films is much more sensitive to temperature than is that of the 
trans isomer (Figure 8.6). The slope of the change in current in the device with decreasing temperature is an order of magnitude steeper for the cis isomer relative to a shallow slope for the trans isomer (Figure 8.6). When we plot electron mobility verses temperature and analyze the data according to McCulloch and Choi ${ }^{40,41}$ we find the slope remains more steep for cis-DBDB, albeit the difference is less stark due to the higher threshold voltage for trans-DBDB. The structure of cis-DBDB, which is quite flexible at room temperature, stiffens as the temperature decreases; in contrast, the trans isomer is already rigid, making it less susceptible to temperature changes.

(a)

(c)
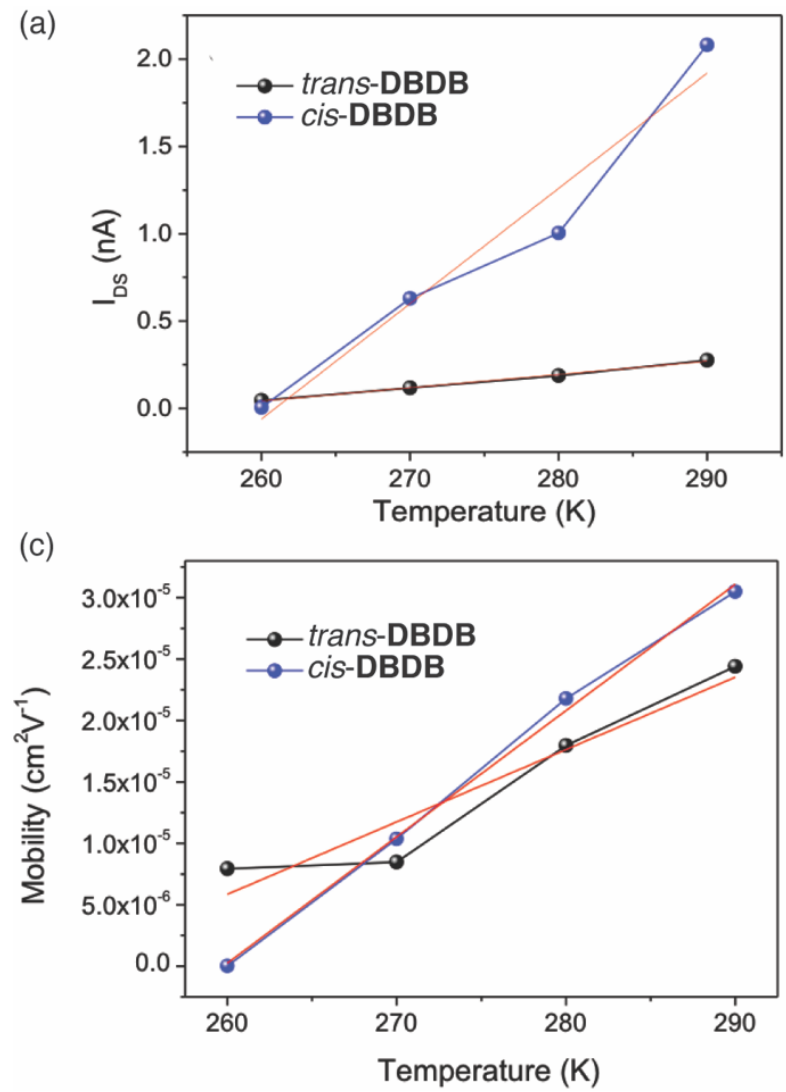

(b)

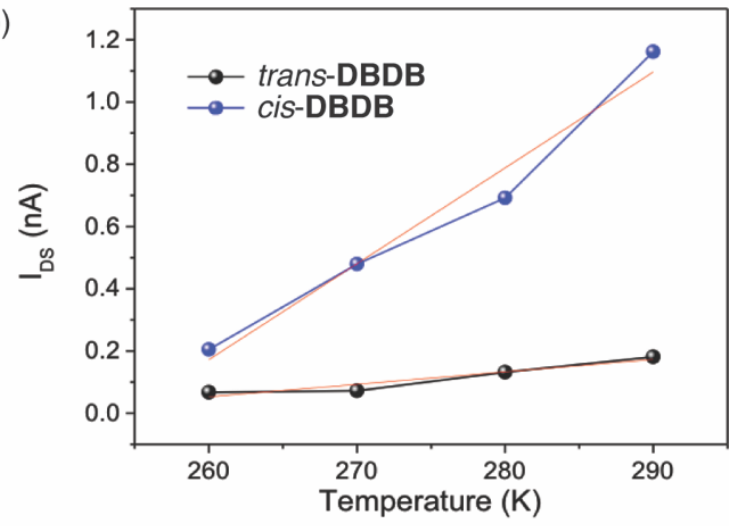

(d)

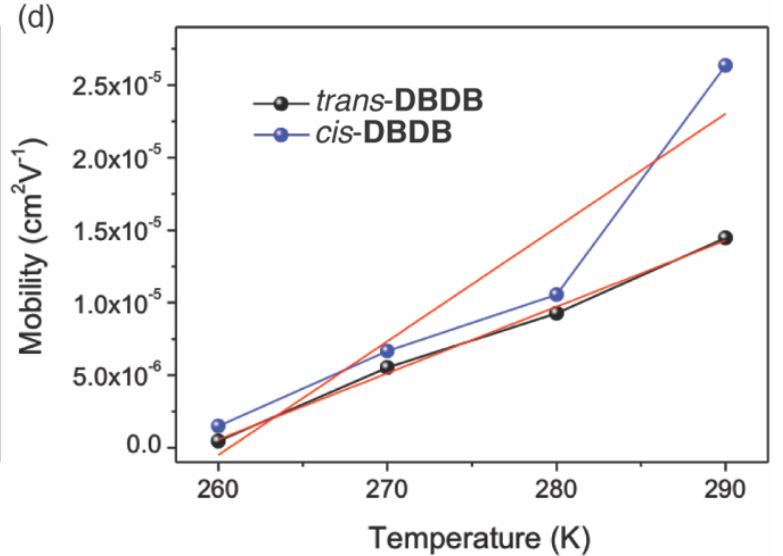

Figure 8.6. The dependence of source-drain current of the cis-DBDB and trans-DBDB-based OFET on temperature (a) from $290 \mathrm{~K}$ to $250 \mathrm{~K}$ and (b) from $250 \mathrm{~K}$ to $290 \mathrm{~K}$, measured at gate voltage of $40 \mathrm{~V}$ and bias voltage of $80 \mathrm{~V}$. The slope of the best fit line for a) is $6.6 \times 10^{-2}$ and $7.6 \mathrm{x}$ $10^{-3}$ for $\mathrm{cis}$-DBDB and trans-DBDB, suggesting the cis corral is more temperature sensitive than the trans corral. The dependence of mobility of the cis-DBDB and trans-DBDB-based OFET on temperature (c) from $290 \mathrm{~K}$ to $250 \mathrm{~K}$ and (d) from $250 \mathrm{~K}$ to $290 \mathrm{~K}$, measured at bias voltage of 80 $\mathrm{V}$. 


\subsection{Conclusion}

In summary, we describe two n-type, electronic materials made from giant conjugated macrocycles comprising hPDI dimers and bithiophenes in an alternating pattern. We find that there is a marked difference in the electron transporting properties of the two macrocycles. Creating amorphous films of both macrocycles, we were able to probe the effects of conformation on transport and find that the cis-DBDB's conformational flexibility enhances its ability to transport charge relative to trans-DBDB. These materials will serve as the electron accepting semiconducting material in organic photovoltaics, taking advantage of their ability to transport electrons and open cavities.

\subsection{Appendix - Supplementary Figures}
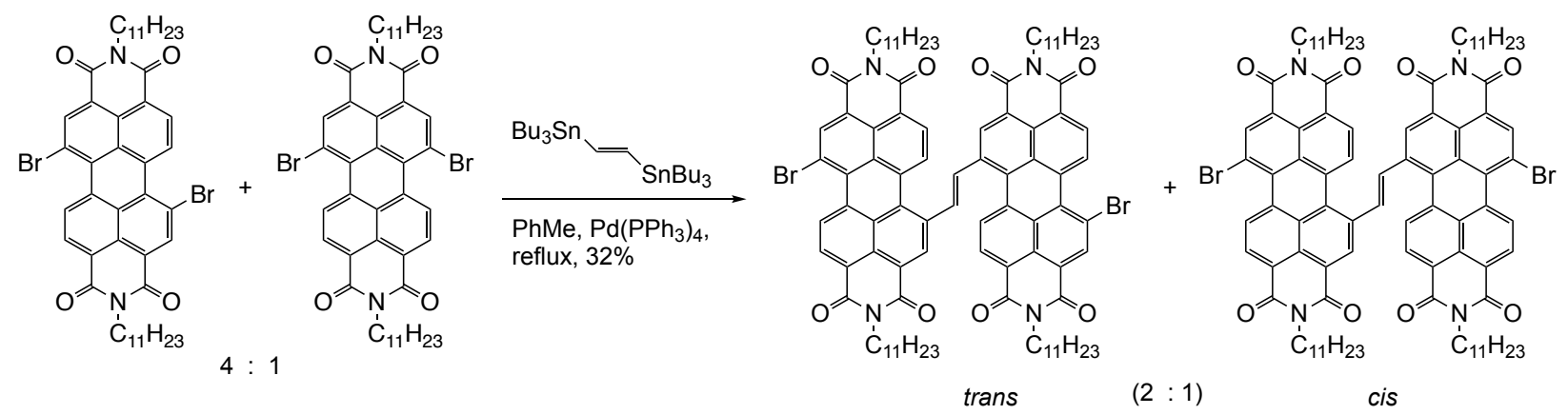

A solution of dibromoPDI (4:1 trans/cis) (850 mg, $1.00 \mathrm{mmol}, 4.00$ equiv) and trans-1,2bis(tributylstannyl)ethene (150 mg, $0.25 \mathrm{mmol}, 1.00$ equiv) in toluene (20 mL) was degassed under Argon for 30 minutes. Tetrakis(triphenylphosphine)palladium(0) (100 mg, $0.09 \mathrm{mmol}$ ) was added, and the resultant solution was degassed for 15 minutes. The mixture was refluxed for overnight under Argon. The black reaction mixture was filtered through celite. The solvent was removed under reduced pressure and the product was purified using silica gel column chromatography (DCM:hexane 6:4) to yield dark purple solid (126 mg, $0.08 \mathrm{mmol}, 32 \%)$ as an inseparable 2:1 mixture of regioisomers. See ${ }^{1} \mathrm{H}$ NMR spectrum for regioisomeric distribution. 


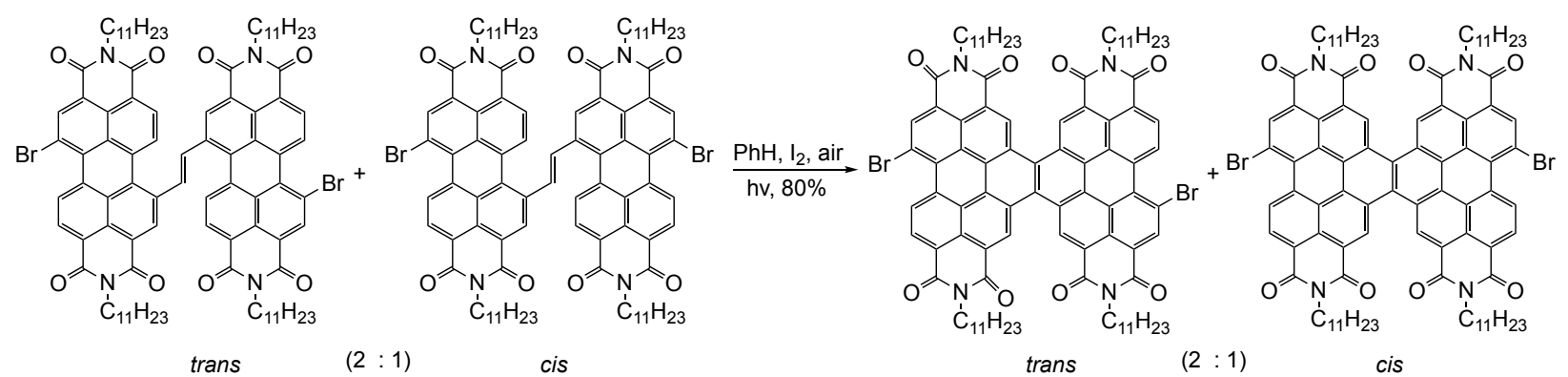

In standard photocyclization glassware, uncyclized dimer mixture $(120 \mathrm{mg}, 0.076 \mathrm{mmol}, 1.00$ equiv) was dissolved in $150 \mathrm{~mL}$ toluene and iodine $(150 \mathrm{mg}, 0.59 \mathrm{mmol}, 7.76$ equiv) was added. The resultant purple solution was photoirradiated using 450 W mercury lamp for 10 hours. The resultant pink reaction mixture extracted with saturated sodium bicarbonate $(2 \mathrm{X} 100 \mathrm{~mL})$, brine $(100 \mathrm{~mL})$ and concentrated under reduced pressure. The residue was treated with $50 \mathrm{~mL}$ methanol to crash out dark red solid. The red solid was purified using silica gel column chromatography (gradient mobile phase: DCM:hexane 2:8 to DCM:hexane 6:4) to yield dark red solid (96 mg, $0.061 \mathrm{mmol}, 80 \%$ ) that is spectroscopically identical to $\mathrm{hPDI}_{2} \mathrm{Br}_{2}$ from dibromination of $\mathrm{hPDI}_{2}$.

Figure 8.7: Synthetic details for the two dibromo $\mathrm{hPDI}_{2}$ isomers $(\mathbf{8 . 1 a}$ and 8.2a) showing an enriched mixture of $8.2 \mathrm{a}$ (2:1 trans:cis). 


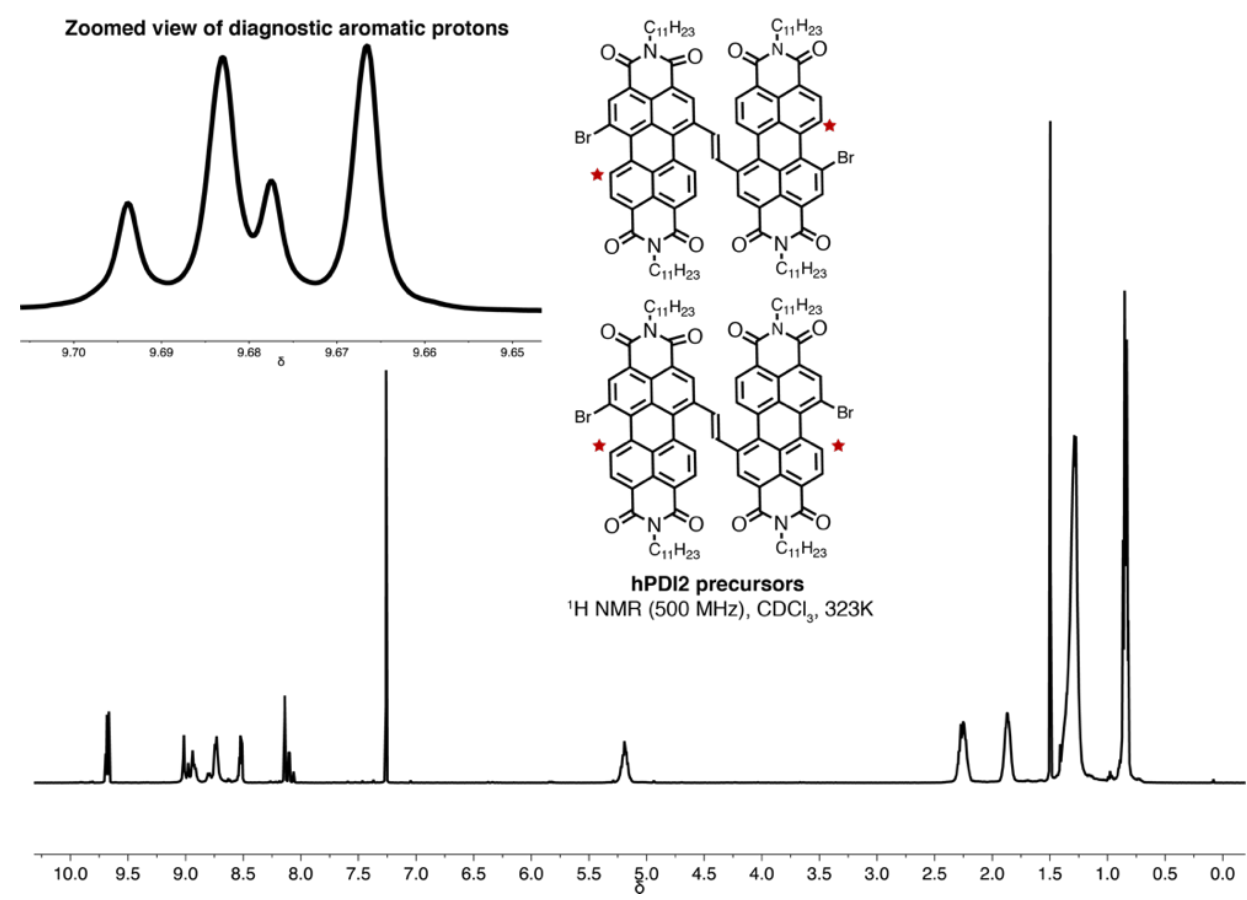

Figure 8.8. ${ }^{1} \mathrm{H}$ NMR spectrum of the enriched mixture of 8.2a:8.1a's precursors, showing an approximate $2: 1$ ratio of the downfield protons at $9.65-9.70 \mathrm{ppm}$. These peaks correspond to the red starred protons that are deshielded due to the bromine atoms.

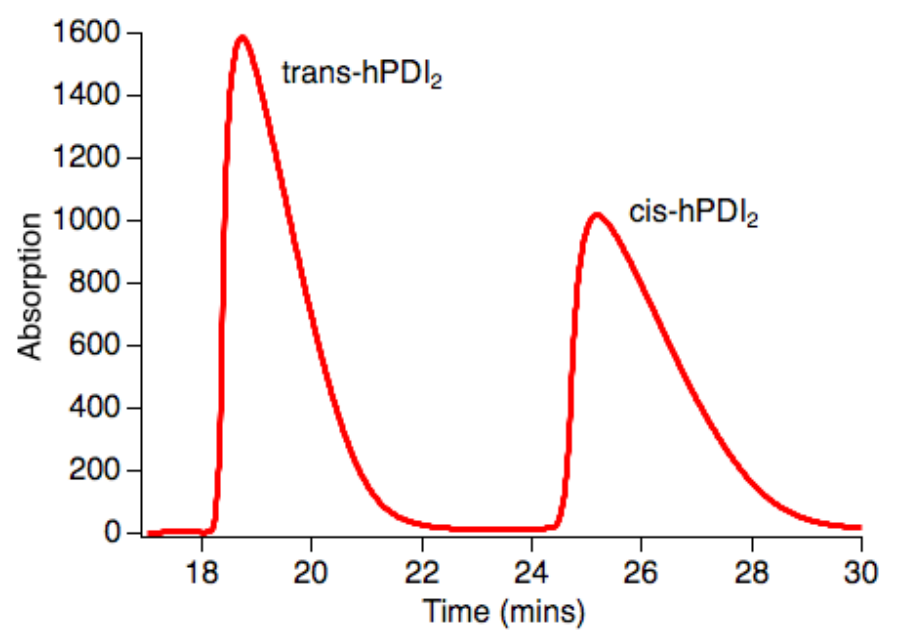

Figure 8.9: The two dibromo $\mathrm{hPDI}_{2}$ isomers are separated using a CHIRALPAK ${ }^{\circledR}$ IA-3 column (4.6 mm I.D. x $250 \mathrm{~mm}, 3 \mu \mathrm{m}$ ), using an isocratic method of $22 \%$ methylene chloride: $78 \%$ hexanes. Using an enriched mixture (2:1 trans isomer:cis isomer), we were able to confirm the first peak from HPLC is 8.2a. 


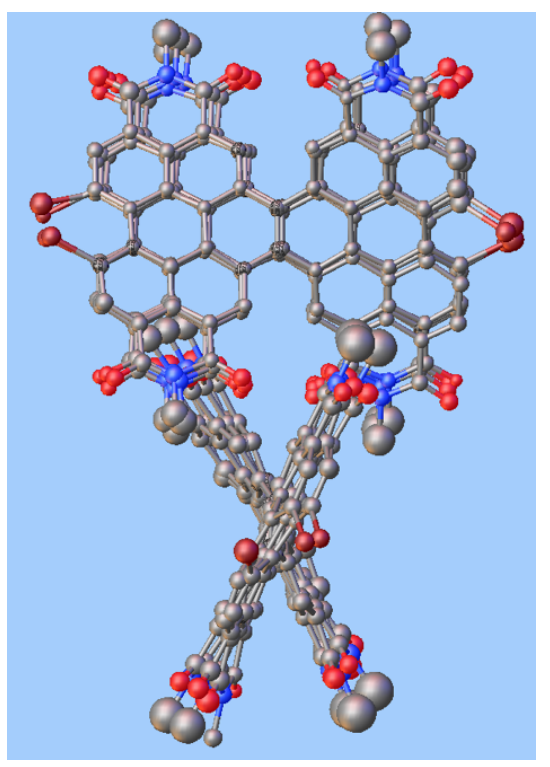

Figure 8.10. Thermal ellipsoid plot of hPDI- $\mathrm{Br}_{2}$. The two independent sites are disordered over 4 and 3 positions. All atoms were refined with isotropic ADPs due to the extensive disorder. Thermal ellipsoids are depicted at the $40 \%$ level. The $\mathrm{C}_{11} \mathrm{H}_{23}$ side chains were omitted from the refinement. Hydrogen atoms are omitted for clarity.

Table 8.1. Table of crystallographic data for Compound 8.2a

\begin{tabular}{|c|c|}
\hline Compound & Compound 8.2a \\
\hline & \\
\hline Formula & $\mathrm{C}_{54} \mathrm{H}_{22} \mathrm{Br}_{2} \mathrm{~N}_{4} \mathrm{O}_{8}+$ side chains + solvent \\
\hline $\mathrm{MW}$ & 1014.57 \\
\hline Space group & $\mathrm{P}-1$ \\
\hline $\mathrm{a}(\AA)$ & $15.4448(11)$ \\
\hline $\mathrm{b}(\AA)$ & $19.9037(13)$ \\
\hline $\mathrm{c}(\AA)$ & $26.3807(18)$ \\
\hline$\alpha\left(^{\circ}\right)$ & $86.411(5)$ \\
\hline$\beta\left(^{\circ}\right)$ & $82.181(6)$ \\
\hline$\gamma\left(^{\circ}\right)$ & $79.782(6)$ \\
\hline $\mathrm{V}(\AA 3)$ & $7900.6(9)$ \\
\hline $\mathrm{Z}$ & 4 \\
\hline$\rho \mathrm{\AA} a \mathrm{c}(\mathrm{g}$ cm- 3$)$ & 0.853 \\
\hline & \\
\hline $\mathrm{T}(\mathrm{K})$ & 100 \\
\hline$\lambda(\AA)$ & 1.54184 \\
\hline $2 \theta \mathrm{min}, 2 \theta \mathrm{max}$ & 7,90 \\
\hline Nref & 41264 \\
\hline
\end{tabular}




\begin{tabular}{|c|c|}
\hline $\mathrm{R}($ int $), \mathrm{R}(\sigma)$ & $.0679, .0809$ \\
\hline$\mu(\mathrm{mm}-1)$ & 1.600 \\
\hline Size $(\mathrm{mm})$ & $.12 \times .04 \times .03$ \\
\hline Tmax, Tmin & $.954, .831$ \\
\hline & \\
\hline Data & 12531 \\
\hline Restraints & 3167 \\
\hline Parameters & 1940 \\
\hline R1(obs) & 0.1673 \\
\hline wR2(all) & 0.5119 \\
\hline S & 1.846 \\
\hline Peak, hole (e- $\AA-3)$ & $1.03,-0.81$ \\
\hline
\end{tabular}

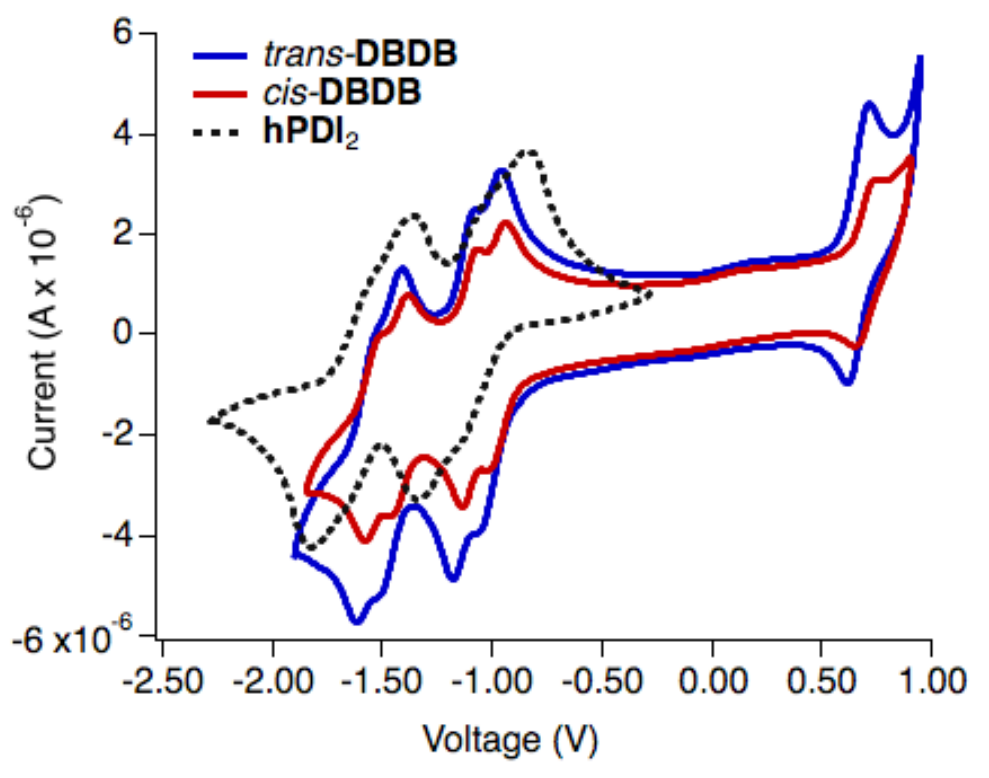

Figure 8.11. CV for cis-DBDB and trans-DBDB relative to a hPDI monomer. 
(a)

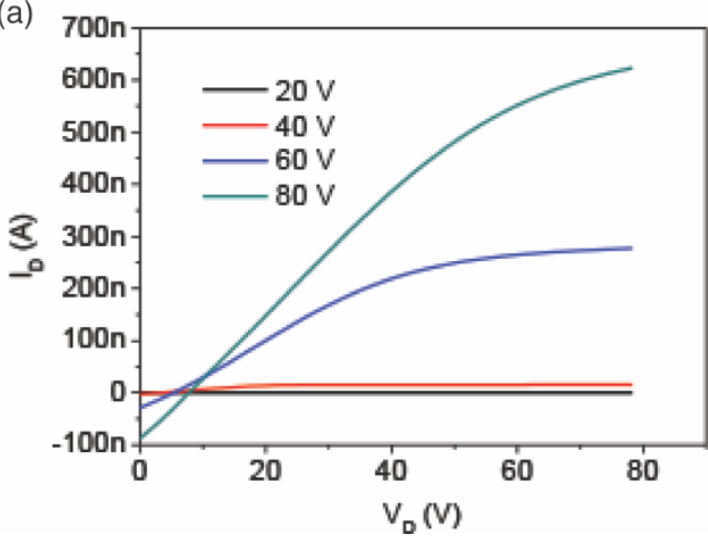

(b)

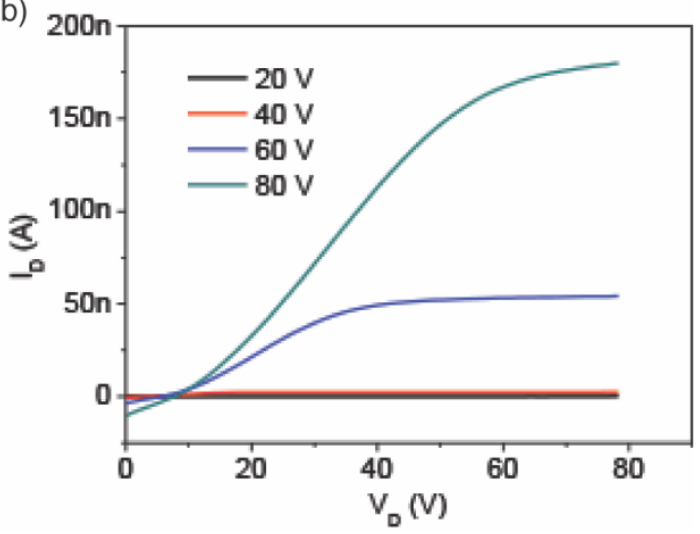

Figure 8.12. Output curves for (a) cis-DBDB and (b) trans-DBDB. Both devices show current saturation at $80 \mathrm{~V}$.

\begin{tabular}{|c|c|c|}
\hline & $\begin{array}{c}\text { cis-DBDB } \\
\text { Highest/average }\end{array}$ & $\begin{array}{c}\text { trans-DBDB } \\
\text { Highest/average }\end{array}$ \\
\hline $\begin{array}{l}\text { Mobility } \\
\left(\mathrm{cm}^{2} \mathbf{V}^{-1}\right)\end{array}$ & $\begin{array}{c}4.1 \times 10^{-3} / \\
2.7 \pm 1.2 \times 10^{-3}\end{array}$ & $\begin{array}{c}9.9 \times 10^{-4} / \\
8.2 \pm 1.4 \times 10^{-4}\end{array}$ \\
\hline
\end{tabular}

Table 8.2. Transfer characteristics for (a) cis-DBDB and (b) trans-DBDB.
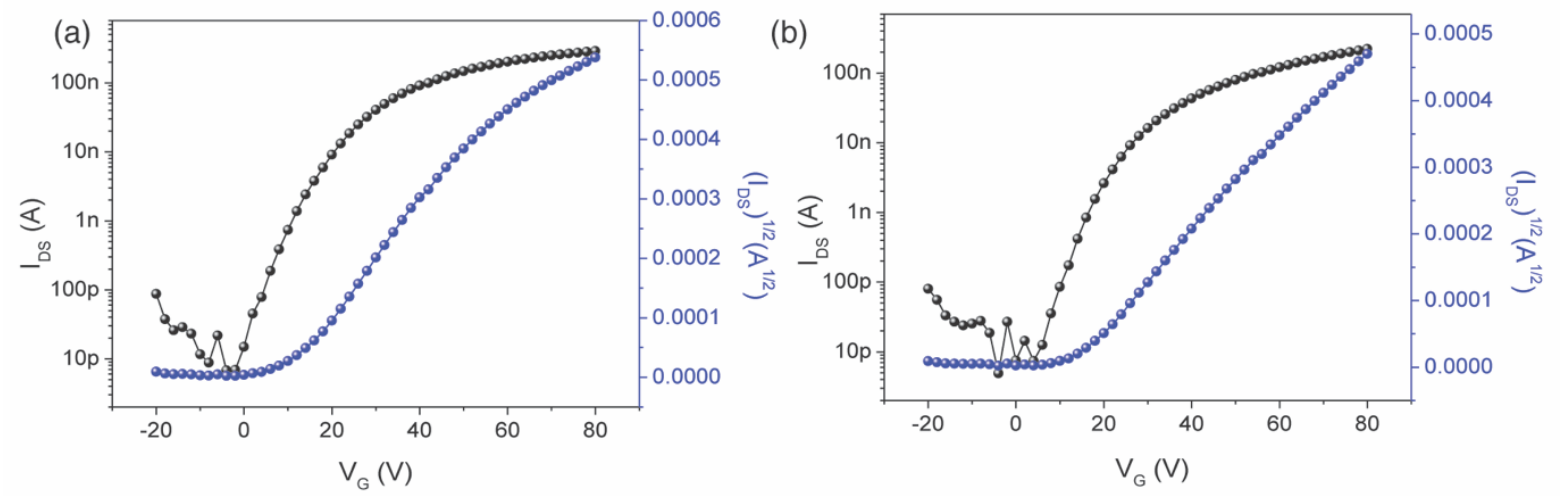

Figure 8.13. Transfer curves for (a) 8.1a and (b) 8.2a. The mobilities are essentially identical at $2.0 \times 10^{-3}$ and $1.6 \times 10^{-3}$ for 8.1a and 8.2a, respectively. 

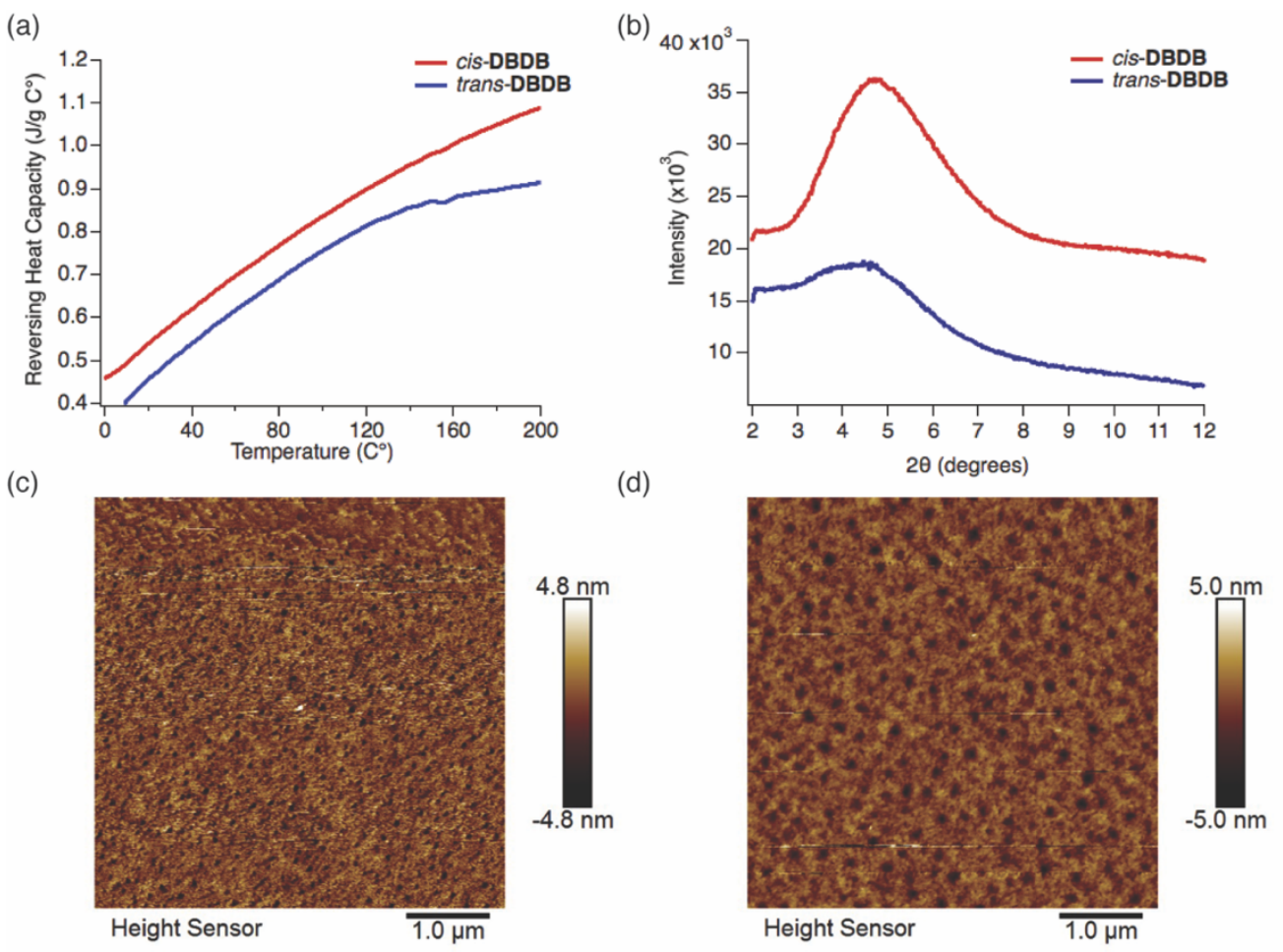

Figure 8.14. (a) DSC showing broad features at temperatures below $160{ }^{\circ} \mathrm{C}$; (b) PXRD of cisDBDB and trans-DBDB at three temperatures, showing the materials are amorphous at higher temperatures $\sim 160{ }^{\circ} \mathrm{C}$; (c) AFM of height images of cis-DBDB and (d) trans-DBDB.
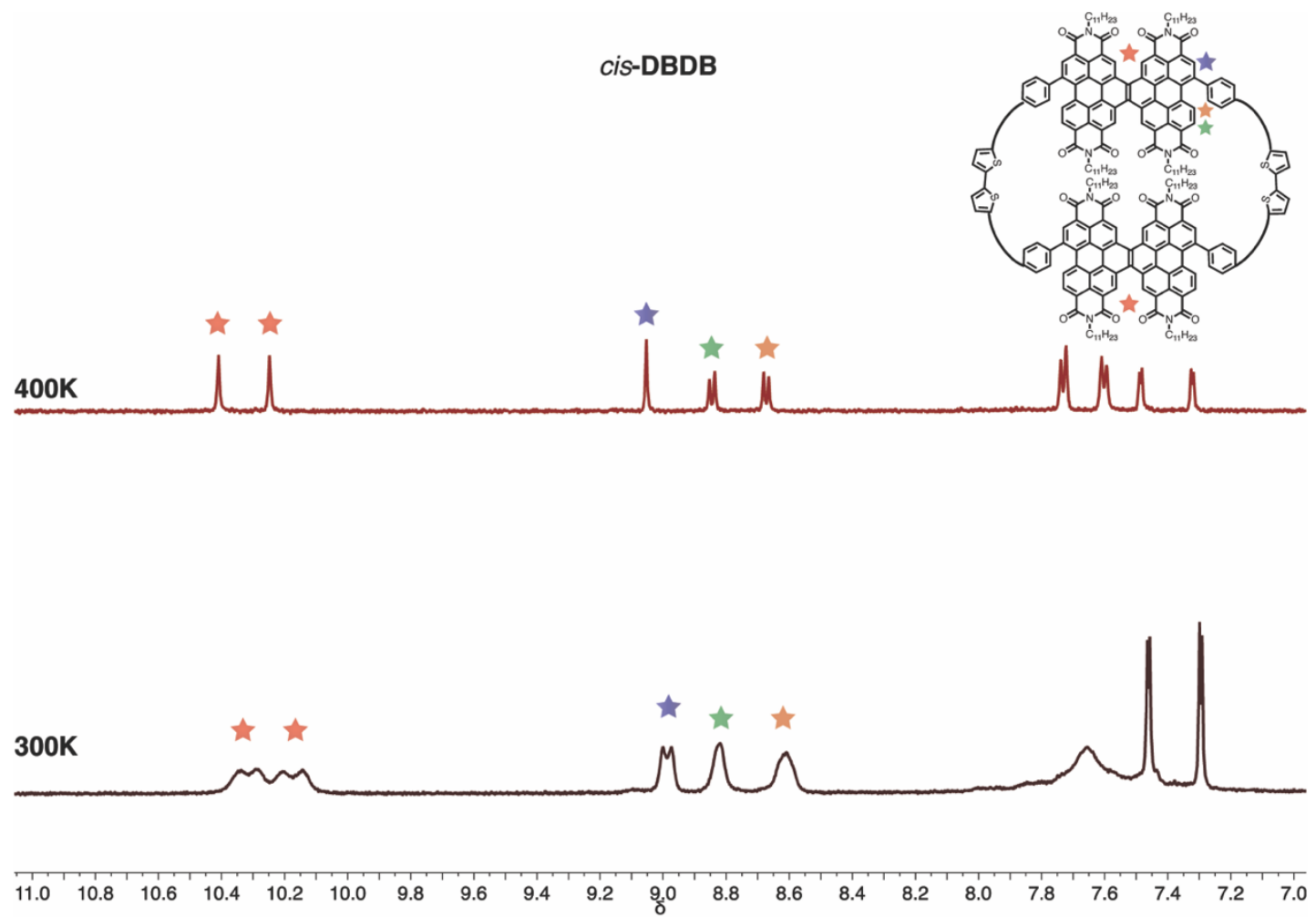

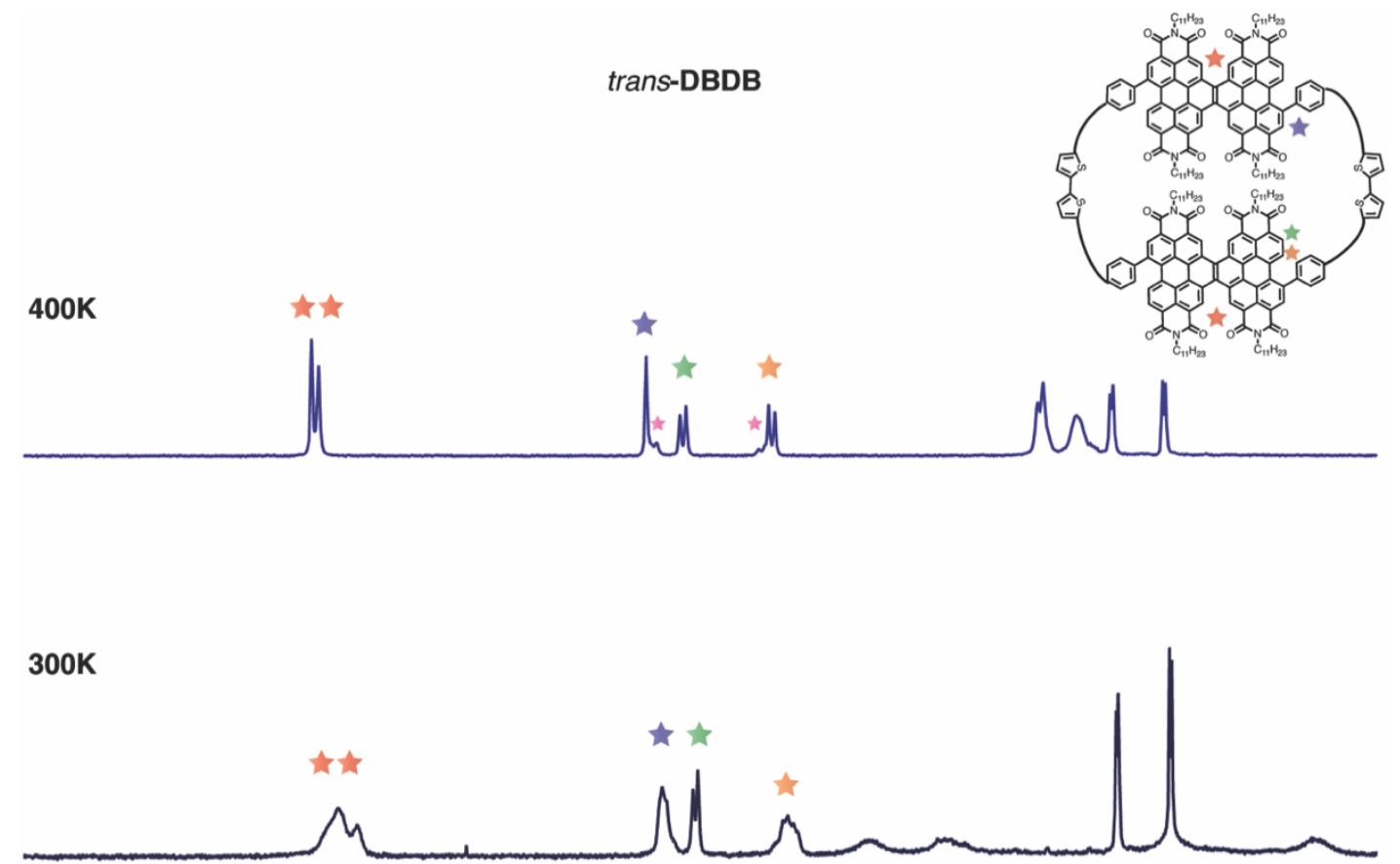

$\begin{array}{lllllllllllllllllllllllllll}1.2 & 11.0 & 10.8 & 10.6 & 10.4 & 10.2 & 10.0 & 9.8 & 9.6 & 9.4 & 9.2 & 9.0 & 8.8 & 8.6 & 8.4 & 8.2 & 8.0 & 7.8 & 7.6 & 7.4 & 7.2 & 7.0 & 6.8 & 6 .\end{array}$

Figure 8.15. VT-NMR for cis-DBDB and trans-DBDB. Both room temperature spectra show the presence of multiple conformers. Yet, at 400K, the spectrum for cis-DBDB shows coalescence, while trans-DBDB shows multiple peaks at 8.7 and $9.1 \mathrm{ppm}$, denoted with pink stars.

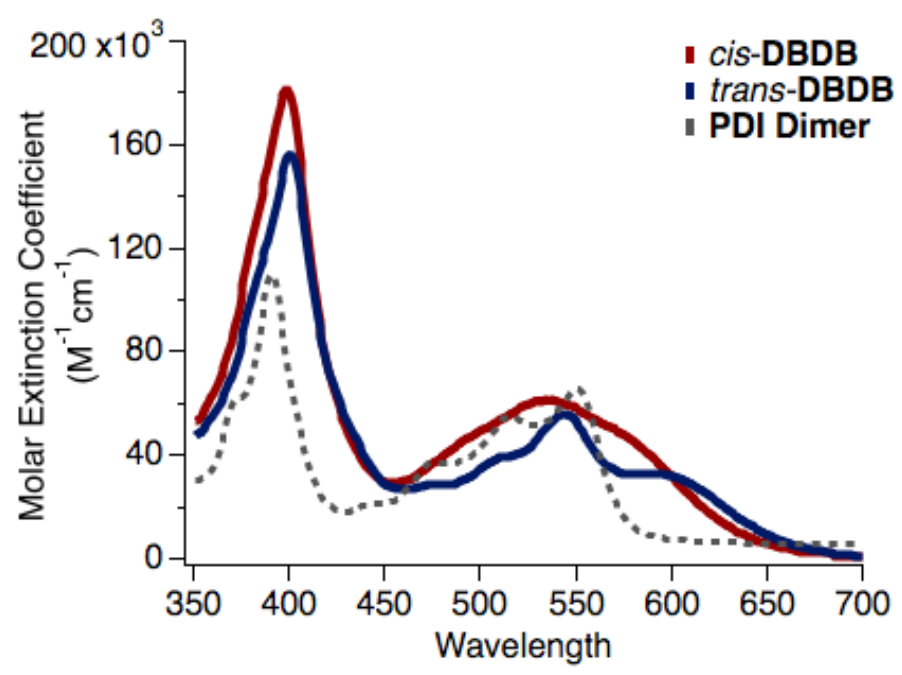

Figure 8.16. UV-Vis absorption spectra for cis-DBDB, trans-DBDB, and a hPDI 2 .

\subsection{General Experimental Information}

Synthesis. All reactions were performed in oven-dried or flame-dried round bottom flasks, unless otherwise noted. The flasks were fitted with rubber septa and reactions were conducted 
under a positive pressure of nitrogen or argon, unless otherwise noted. Anhydrous and anaerobic solvents were obtained from a Glass Contour solvent system consisting of a Schlenk manifold with purification columns packed with activated alumina and supported copper catalyst. Reaction monitoring by TLC was performed on J.T. Baker Baker-flex Silica Gel IB2-F (25 mm x $75 \mathrm{~mm}$ ) TLC plates. TLC visualization was accomplished by visible observation and irradiation with a UV lamp. Commercial reagents were used without further purification. $\mathrm{Pt}(\mathrm{COD}) \mathrm{Cl}_{2}$ was purchased from Strem Chemicals, and all other reagents were purchased from Sigma-Aldrich.

Purification. Automated flash chromatography was performed using a Teledyne Isco Combiflash Rf200 and Redisep Rf Silica and Alumina columns.

Spectrometers. ${ }^{1} \mathrm{H}$ NMR spectra were recorded on a Bruker 400 or $500 \mathrm{MHz}$ spectrometer. ${ }^{13} \mathrm{CNMR}$ spectra were recorded on a Bruker 125 or $100 \mathrm{MHz}$ spectrometer with complete proton decoupling. NMR spectra were recorded at $300 \mathrm{~K}$ unless otherwise noted. Chemical shifts are reported in parts per million (ppm) Data are represented as follows: chemical shift, multiplicity (s $=$ singlet, $\mathrm{d}=$ doublet, $\mathrm{dd}=$ doublet of doublets, $\mathrm{t}=$ triplet, $\mathrm{m}=$ multiplet), coupling constants in $\mathrm{Hz}$, and integration. Broadening (br) of peaks in the $1 \mathrm{H}$ NMR spectrum is due to rotational isomers about the imide side chains. The synthesis of 8.1a and 8.2a was previously reported. ${ }^{29}$ The separation of the two isomers is provided in Figures 8.7-8.9. Broadening (br) of peaks in the ${ }^{1} \mathrm{H}$ NMR spectrum is due to rotational isomers about the imide side chains. ${ }^{12,46}$ HRMS was performed on a (1) Waters XEVO G2-XS QTOF instrument equipped with a UPC SFC inlet, and ESI and APCI ionization sources; or (2) a Bruker UltrafleXtreme MALDI TOF/TOF instrument using a dithranol matrix. UV-vis absorption spectra were recorded on a Shimadzu UV-1800 spectrophotometer using a $1.0 \mathrm{~cm}$ quartz cell. IR spectra were recorded on a Perkin Elmer Spectrum400 FTIR spectrometer using a PIKE ATR attachment. 
Atomic force microscopy. AFM measurements were carried out in scan analysis mode on a Bruker Multi-Mode AFM at ambient conditions. A commercial silicon cantilever (SCANASYST-AIR, Bruker) was used in this study with a typical radius of curvature of $\sim 8 \mathrm{~nm}$ and a nominal spring constant of $\sim 0.4 \mathrm{Nm}^{-1}$.

Cyclic Voltammetry. CVs were recorded on a CH166 electrochemical workstation using an $\mathrm{Ag} / \mathrm{AgCl}$ electrode as the reference electrode at room temperature. Experiments were performed in $\mathrm{CH}_{2} \mathrm{Cl}_{2}$ with $\mathrm{NBu}_{4} \mathrm{PF}_{6}$ as the supporting electrolyte at a scan rate of $0.1 \mathrm{~V} / \mathrm{s}^{47}$

Thin film transistors. To create the devices, we first silanize the substrate $(300 \mathrm{~nm}$ of $\mathrm{SiO} 2$ on a Si wafer) with OTS Au is deposited onto the substrate as bottom-contact source and drain electrodes $(40 \mathrm{~nm})$ with a width of $115 \mu \mathrm{m}$ and length of $10 \mu \mathrm{m}$. Next, we spin-cast organic films onto the surface at 3,000 r.p.m. for $1 \mathrm{~min}$, to form transistors using the silicon wafer as the global back gate for the device. Finally, the samples were annealed under inert atmosphere at $160^{\circ} \mathrm{C}$ for 10 minutes to optimize device performance. The thin film transistors were tested on the Agilent 4155C semiconductor parameter analyzer.

The mobility is calculated in the saturation regime using IDS $=(\mathrm{W} / 2 \mathrm{~L}) \mathrm{C}_{\mathrm{i}} \mu\left(\mathrm{V}_{\mathrm{G}}-\mathrm{V}_{\mathrm{T}}\right)_{2}$, where $\mathrm{W}$ and $\mathrm{L}$ are the width and length of the channel, $\mathrm{C}_{\mathrm{i}}\left(11.5 \mathrm{nFcm}^{-2}\right), \mu$, and $\mathrm{V}_{\mathrm{T}}$ correspond to the capacitance per unit area of the gate insulator, the field effect mobility, and the threshold voltage, respectively. $\mathrm{W}=115 \mu \mathrm{m}$ and $\mathrm{L}=10 \mu \mathrm{m}$ for transistor devices. The data is fitted using the methods developed by McCulloch and Choi to avoid overestimated mobilities. ${ }^{40,41}$

Temperature dependence measurement. Temperature-dependent current-voltage measurements were realized in a vacuum cryogenic probe station (Lakeshore TTP4). Computercontrolled source units were used S3 to apply DC potentials (Yokogawa 7651). Current measurements were obtained using a current preamplifier (Stanford Research System SR570) and 
a digital multimeter (Keysight 34401A). All device measurements were performed in vacuum (P $<1 \times 10-4$ Torr) at different temperatures.

Single crystal X-ray diffraction. Data for all compounds was collected on an Agilent SuperNova diffractometer using mirror-monochromated $\mathrm{Cu} \mathrm{K} \alpha$ radiation. Data collection, integration, scaling (ABSPACK) and absorption correction (multi-scan) were performed in CrysAlisPro. ${ }^{48}$ Structure solution was performed using ShelXT. ${ }^{49}$ Subsequent refinement was performed by full-matrix least-squares on F4 in ShelXL. ${ }^{50} \mathrm{Olex} 2^{51}$ was used for viewing and to prepare CIF files. PLATON ${ }^{52}$ was used extensively for SQUEEZE. ${ }^{53}$

A toluene solution of hPDI2 $-\mathrm{Br}_{2}$ - trans was diluted with methanol by vapor diffusion to afford bright red prisms. Part of a crystal $(0.12 \times 0.04 \times 0.03 \mathrm{~mm})$ was separated carefully, mounted with Paratone oil, and cooled to $100 \mathrm{~K}$ on the diffractometer. Complete data $(99.7 \%)$ were collected to $1.1 \AA$, outside of which resolution there was little usable data. 41264 reflections were collected (12531 unique, 7287 observed) with R(int) $6.8 \%$ and R(sigma) $8.1 \%$ after multiscan absorption correction (Tmax 0.954 , Tmin 0.831$)$.

The structure solved readily in P-1 but showed signs of extensive disorder. Each of the two independent molecules was a mixture of PPP and PPM isomers (+ enantiomers) and was further disordered by a twofold rotation around the helical axis, which is not a point symmetry operator of the molecule but is an approximate symmetry of the van der Waals surface. For each site, these 4 possible isomers + orientations were introduced as fragments with DFT-optimized geometry and subsequently allowed to refine with all 1,2- and 1,3- distances restrained to match their DFT geometry. One site had all 4 possibilities occupied and the other had 3 of the 4 possibilities occupied. 
The $\mathrm{C}_{11} \mathrm{H}_{23}$ side chains were not possible to locate in view of the extensive disorder of the hPDI cores. Thus they were modeled as methyl groups and the rest of the chains were treated as a diffuse contribution to the overall scattering using Platon SQUEEZE.

All ADPs were modeled isotropically because the data/parameters ratio was already poor due to the low resolution of the diffraction and the numerous overlapping disordered positions. All $\mathrm{C}-\mathrm{H}$ hydrogens were placed in calculated positions and refined with riding coordinates and isotropic ADPs. The final refinement (12531 data, 3167 restraints, 1940 parameters) converged with $\mathrm{R} 1(\mathrm{Fo}>4 \sigma(\mathrm{Fo}))=16.7 \%, \mathrm{wR} 2=51.2 \%, \mathrm{~S}=1.85$. The largest Fourier features were 1.03 and -0.81 e- A-3.

\subsection{Synthetic Procedures and Characterization}

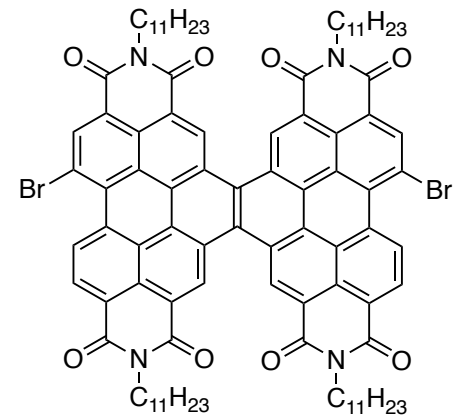

8.1a

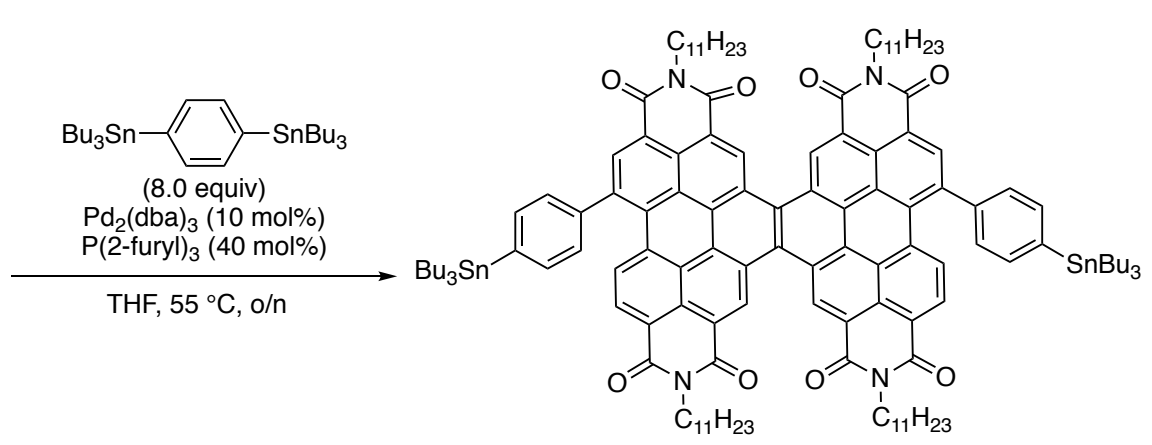

8.1b

Synthesis of Bis[4-(tributylstannyl)-phenyl]-hPDI 2 -Cis (8.1b): Regiopure 8.1a (1.00 equiv, 0.196 mmol, $0.308 \mathrm{~g})$, 1,4-bis(tributylstannyl)benzene (8.00 equiv, $1.56 \mathrm{mmol}, 0.895 \mathrm{~mL})$, tri(2furyl)phosphine (0.400 equiv, $0.0784 \mathrm{mmol}, 0.0180 \mathrm{~g})$ and THF $(24 \mathrm{~mL})$ were added to an ovendried two-neck $100 \mathrm{~mL}$ round bottom flask equipped with a stir bar. The mixture was degassed for thirty minutes. While under $\mathrm{N}_{2}$, tris(dibenzylideneacetone)dipalladium (0.100 equiv, 0.0196 mmol, $0.0180 \mathrm{~g}$ ) was added. The mixture was further degassed for ten minutes before being placed in a $55{ }^{\circ} \mathrm{C}$ oil bath and allowed to stir overnight. The crude mixture was concentrated and purified by column chromatography using a gradient from $0 \%$ to $80 \% \mathrm{CH}_{2} \mathrm{Cl}_{2} /$ hexanes to yield $\mathbf{8 . 1 b}$ as a 
magenta solid (0.246 g, $0.115 \mathrm{mmol}, 59 \%$ yield). ${ }^{1} \mathbf{H}$ NMR (400 MHz, $\left.\mathrm{CDCl}_{3}\right) \delta 10.29$ (s, br, 4H), 9.05 (s, br, 2H), 8.69 (d, J = 8.5 Hz, 2H), 8.60 (d,br, 2H), 7.78* (d, J = 7.2 Hz, 4H), 7.66 (d, J = $7.1 \mathrm{~Hz}, 4 \mathrm{H}), 5.31(\mathrm{~m}, 4 \mathrm{H}), 2.34(\mathrm{~m}, \mathrm{br}, 8 \mathrm{H}), 1.95(\mathrm{~m}, \mathrm{br}, 8 \mathrm{H}), 1.69^{*}(\mathrm{~m}, 12 \mathrm{H}), 1.45^{* *}(\mathrm{~m}, 12 \mathrm{H})$, $1.32^{* *}(\mathrm{~m}, \mathrm{br}, 48 \mathrm{H}), 1.23^{*},{ }^{* *}(\mathrm{~m}, 12 \mathrm{H}), 1.00(\operatorname{tr}, 18 \mathrm{H}), 0.84(\mathrm{~m}, \mathrm{br}, 24 \mathrm{H}){ }^{13} \mathbf{C}$ NMR (100 MHz, $\left.\mathrm{CDCl}_{3}\right) \delta 164.9,163.9,143.2,142.7,142.6,138.4 *, 135.4,134.8,134.2,133.9,133.3,131.8$ 130.0*, 128.0, 126.9, 126.8, 126.3, 126.2, 126.1, 126.0, 125.9, 125.5, 123.8, 123.5, 122.1, 121.5, 55.1, 55.0, 32.4, 31.8, 29.2*, 27.4*, 26.7, 22.6, 14.1, 14.1, 13.8, 9.8*. IR $\left(\mathrm{cm}^{-1}\right) 2957,2924,2859$, 1703, 1661, 1593, 1323, 1264, 126. HRMS (APCI+) calculated m/z for $\left[\mathrm{C}_{136} \mathrm{H}_{164} \mathrm{~N}_{4} \mathrm{O}_{8} \mathrm{Sn}_{2}+\mathrm{Na}\right]^{+}$ is 2172.0547 , found $2172.0491 .{ }^{*}$ Tin satellite peaks visible. ${ }^{* *}$ Overlapping peaks in the ${ }^{1} \mathrm{HNMR}$.

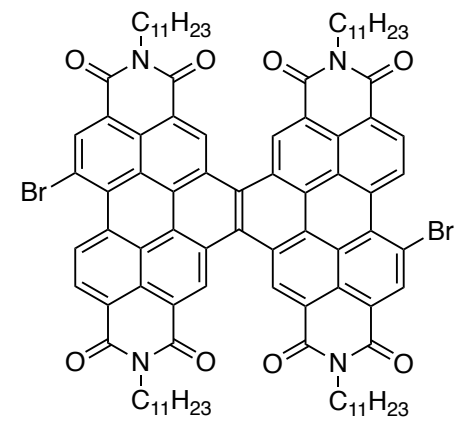

8.2a

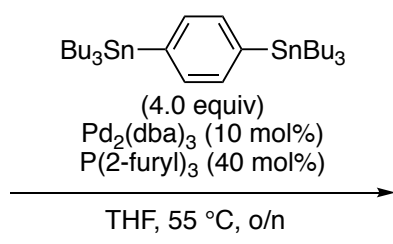

$\mathrm{THF}, 55^{\circ} \mathrm{C}, \mathrm{o} / \mathrm{n}$

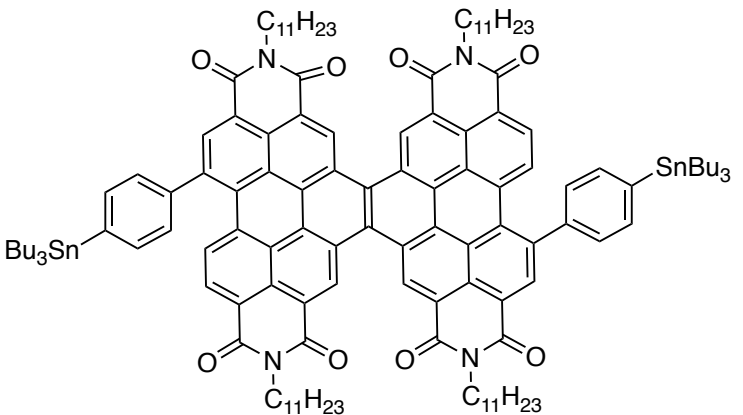

8.2b

Synthesis of Bis[4-(tributylstannyl)-phenyl]-hPDI ${ }_{2}$-Cis (8.2b): Regiopure 8.2a (1.00 equiv, 0.451 mmol, $0.710 \mathrm{~g})$, 1,4-bis(tributylstannyl)benzene (4.00 equiv, $1.80 \mathrm{mmol}, 1.03 \mathrm{~mL})$, tri(2furyl)phosphine ( 0.400 equiv, $0.180 \mathrm{mmol}, 0.0419 \mathrm{~g})$ and THF $(45 \mathrm{~mL})$ were added to an ovendried two-neck $100 \mathrm{~mL}$ round bottom flask equipped with a stir bar. The mixture was degassed for thirty minutes. While under $\mathrm{N}_{2}$, tris(dibenzylideneacetone)dipalladium (0.100 equiv, 0.0451 mmol, $0.0413 \mathrm{~g}$ ) was added. The mixture was further degassed for ten minutes before being placed in a $55{ }^{\circ} \mathrm{C}$ oil bath and allowed to stir overnight. The crude mixture was concentrated and purified by column chromatography using a gradient from $0 \%$ to $80 \%$ hexanes to $\mathrm{CH}_{2} \mathrm{Cl}_{2}$ to yield $\mathbf{8 . 2 b}$ as a magenta solid ( $0.657 \mathrm{~g}, 0.306 \mathrm{mmol}, 68 \%$ yield). ${ }^{1} \mathbf{H}$ NMR $\left(400 \mathrm{MHz}, \mathrm{CDCl}_{3}\right) \delta 10.30$ (s, br, 
4H), 9.05 (s, br, 2H), 8.69 (d, J = 8.5 Hz, 2H), 8.59 (d, br, 2H), 7.77* (d, J = 7.3 Hz, 4H), 7.66 (d, $\mathrm{J}=7.2 \mathrm{~Hz}, 4 \mathrm{H}), 5.31(\mathrm{~m}, 4 \mathrm{H}), 2.34(\mathrm{~m}, \mathrm{br}, 8 \mathrm{H}), 1.94(\mathrm{~m}, \mathrm{br}, 8 \mathrm{H}), 1.69^{*}(\mathrm{~m}, 12 \mathrm{H}), 1.47(\mathrm{~m}, 12 \mathrm{H})$, $1.32(\mathrm{~m}, \mathrm{br}, 48 \mathrm{H}), 1.23^{*}(\mathrm{~m}, 12 \mathrm{H}), 1.00(\mathrm{tr}, 18 \mathrm{H}), 0.84(\mathrm{~m}, \mathrm{br}, 24 \mathrm{H}) .{ }^{13} \mathbf{C} \mathbf{N M R}\left(100 \mathrm{MHz}, \mathrm{CDCl}_{3}\right)$ $\delta 164.9,163.9,143.2,142.7,142.5,138.4 *, 134.2,133.9,133.2,131.8,130.5,130.0,128.8$, $128.0 *, 126.9,126.8,126.7,126.3,126.3,126.1,126.0,125.9,125.5,124.0,123.4,122.2,121.5$, $55.1,55.0,32.4,31.8,29.2^{*}, 27.4^{*}, 26.7,22.6,14.1,14.1,13.8,9.8^{*}$. IR $\left(\mathrm{cm}^{-1}\right) 2955,2924,2855$ 1701, 1661, 1597, 1322, 1268. HRMS (APCI+) calculated $\mathrm{m} / \mathrm{z}$ for $\left[\mathrm{C}_{136} \mathrm{H}_{164} \mathrm{~N}_{4} \mathrm{O}_{8} \mathrm{Sn}_{2}+\mathrm{Na}\right]^{+}$is 2172.0542, found 2172.0491.*Tin satellite peaks visible.

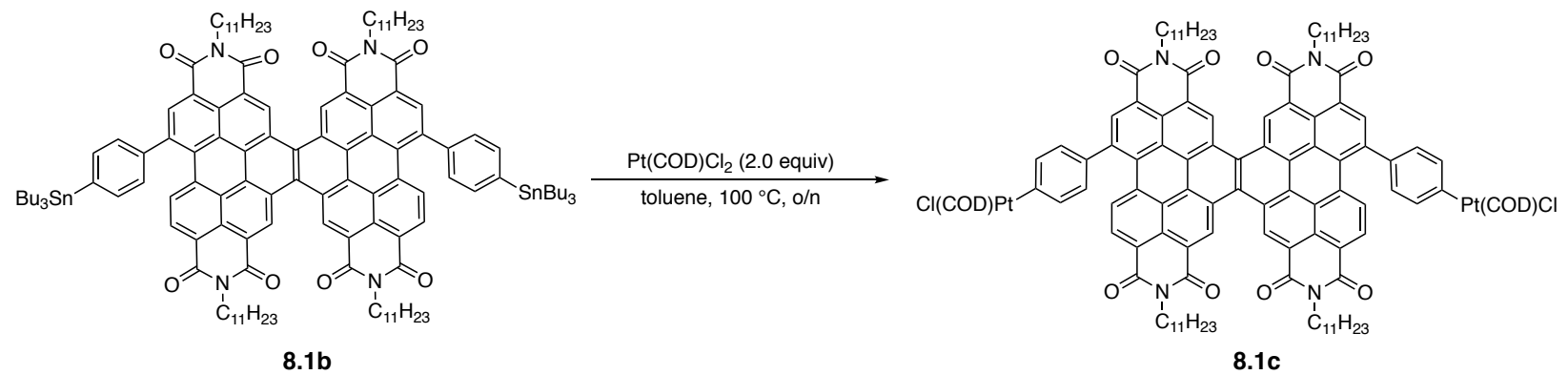

Synthesis of Bis[4-(Pt(COD)Cl)-phenyl]-hPDI ${ }_{2}$-cis (8.1c): 8.1b (1.00 equiv, 0.0700 mmol, 0.150 g) was added to an oven-dried two-neck, $100 \mathrm{~mL}$ round bottom flask equipped with a stir bar. Dichloro(1,5-cyclooctadiene)platinum(II) (2.00 equiv, $0.140 \mathrm{mmol}, 0.0522 \mathrm{~g})$ was added to the flask with anhydrous toluene $(7.00 \mathrm{~mL})$. The mixture was degassed for thirty minutes and then placed in a $100{ }^{\circ} \mathrm{C}$ oil bath and allowed to stir overnight. The crude mixture was allowed to cool to room temperature and concentrated in vacuo. The crude mixture is recrystallized from methanol before being purified by column chromatography using a gradient from $0 \%$ to $100 \%$ $\mathrm{CH}_{2} \mathrm{Cl}_{2} /$ hexanes to elute the desired product. The product is a purple solid $(0.100 \mathrm{~g}, 0.0445 \mathrm{mmol}$, 64\% yield). ${ }^{1} \mathbf{H}$ NMR (400 MHz, $\left.\mathrm{CDCl}_{3}\right) \delta 10.26$ (s, br, 4H), 9.04 (s, br, 2H), 8.68 (d, J = 8.5 Hz, 2H), 8.59 (d, br, 2H), 7.57 (d, J = 7.8 Hz, 4H), 7.44 (d, J = 7.6 Hz, 4H), $5.95(\mathrm{~s}, 4 \mathrm{H}), 5.31$ (m, br, 4H), $4.85(\mathrm{~s}, 4 \mathrm{H}), 2.82(\mathrm{~m}, \mathrm{br}, 4 \mathrm{H}), 2.65(\mathrm{~m}, \mathrm{br}, 4 \mathrm{H}), 2.51(\mathrm{~m}, \mathrm{br}, 8 \mathrm{H}), 2.33(\mathrm{~m}, \mathrm{br}, 8 \mathrm{H}), 1.94(\mathrm{~m}$, 
br, 8H), 1.31 (m, br, 48H), 0.84 (m, br, 24H). ${ }^{13} \mathbf{C}$ NMR (100 MHz, $\left.\mathrm{CDCl}_{3}\right) \delta$ 165.0, 163.9, 145.7, $142.9,139.0,136.0,134.5,133.9,133.2,131.7,129.9,128.5,128.3,126.8,126.8,126.2,126.1$, $126.1,126.0,125.9,125.5,123.9,123.4,122.0,121.4,116.0,87.9,55.0,54.9,32.4,32.3,31.8$, 28.1, 26.7, 22.6, 14.1. IR $\left(\mathrm{cm}^{-1}\right)$ 2953, 2924, 2858, 1701, 1660, 1595, 1324, 1246. HRMS (ESI+) calculated $\mathrm{m} / \mathrm{z}$ for $\left[\mathrm{C}_{122} \mathrm{H}_{134} \mathrm{Cl}_{2} \mathrm{~N}_{4} \mathrm{O}_{8} \mathrm{Pt}_{2}+\mathrm{Na}\right]^{+}$is 2265.8772 , found 2265.8774 .
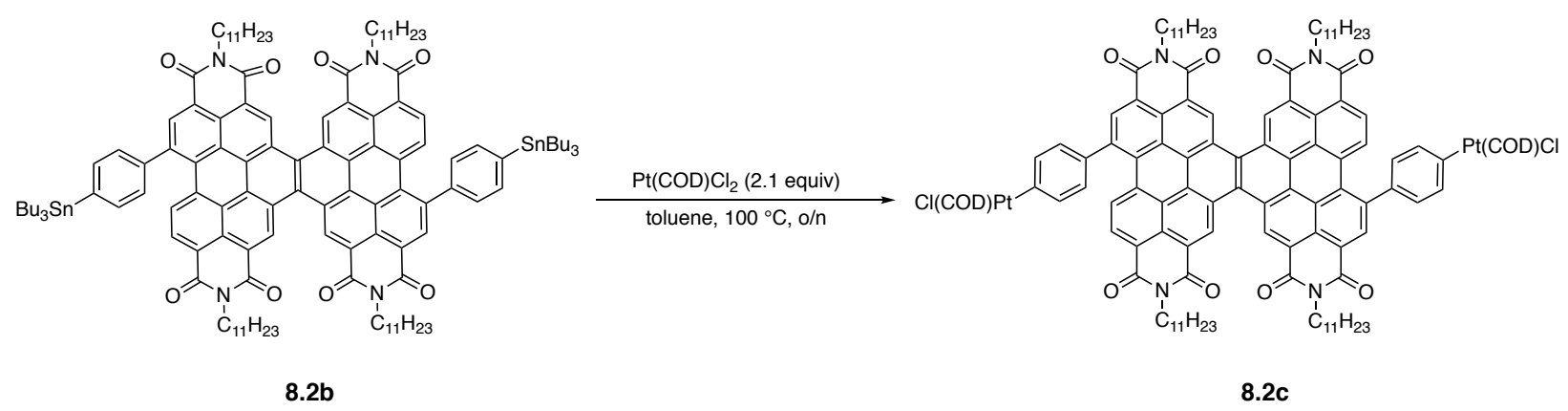

Synthesis of Bis[4-(Pt(COD)Cl)-phenyl]-hPDI 2 -trans (8.2c): 8.2b (1.00 equiv, $0.0758 \mathrm{mmol}$, $0.163 \mathrm{~g}$ ) was added to an oven-dried two-neck, $100 \mathrm{~mL}$ round bottom flask equipped with a stir bar. Dichloro(1,5-cyclooctadiene)platinum(II) (2.10 equiv, $0.159 \mathrm{mmol}, 0.0596 \mathrm{~g}$ ) was added to the flask with anhydrous toluene $(8.00 \mathrm{~mL})$. The mixture was degassed for thirty minutes and then placed in a $100{ }^{\circ} \mathrm{C}$ oil bath and allowed to stir overnight. The crude mixture was allowed to cool to room temperature and concentrated in vacuo. The crude mixture is recrystallized from methanol before being purified by column chromatography using a gradient from $0 \%$ to $100 \%$ $\mathrm{CH}_{2} \mathrm{Cl}_{2} /$ hexanes to elute the desired product. The product is a purple solid $(0.130 \mathrm{~g}, 0.0579 \mathrm{mmol}$, 76\% yield). ${ }^{1} \mathbf{H}$ NMR (400 MHz, $\left.\mathrm{CDCl}_{3}\right) \delta 10.27$ (s, br, 4H), 9.04 (s, br, 2H), 8.68 (d, J = 8.6 Hz, 2H), $8.58(\mathrm{~d}, \mathrm{br}, 2 \mathrm{H}), 7.57$ (d, J = 7.7 Hz, 4H), 7.44 (d, J = 7.7 Hz, 4H), $5.95(\mathrm{~s}, 4 \mathrm{H}), 5.30$ (m, br, 4H), $4.84(\mathrm{~s}, 4 \mathrm{H}), 2.82(\mathrm{~m}, \mathrm{br}, 4 \mathrm{H}), 2.65(\mathrm{~m}, \mathrm{br}, 4 \mathrm{H}), 2.51(\mathrm{~m}, \mathrm{br}, 8 \mathrm{H}), 2.33(\mathrm{~m}, \mathrm{br}, 8 \mathrm{H}), 1.94(\mathrm{~m}$, br, 8H), 1.31 (m, br, 48H), 0.85 (m, br, 24H). ${ }^{13} \mathbf{C}$ NMR (100 MHz, $\left.\mathrm{CDCl}_{3}\right) \delta$ 165.0, 164.0, 145.7, 
$142.9,139.0,136.0,134.5,134.0^{*}, 133.0^{*}, 131.7,129.9,129.1,128.3,126.8,126.8,126.7,126.3$, $126.2,126.0,125.9,125.9,125.5,124-121 *, 116.0,87.9,55.0,54.9,32.4,32.3,31.8,28.1,26.7$, 22.6, 14.1. IR $\left(\mathrm{cm}^{-1}\right)$ 2970, 2954, 2928, 2924, 2853, 1739, 1701, 1660, 1595, 1322, 1232. HRMS (ESI+) calculated $\mathrm{m} / \mathrm{z}$ for $\left[\mathrm{C}_{122} \mathrm{H}_{134} \mathrm{Cl}_{2} \mathrm{~N}_{4} \mathrm{O}_{8} \mathrm{Pt}_{2}+\mathrm{Na}\right]^{+}$is 2265.8772 , found $2265.8772 .{ }^{*}$ Between 134-133 ppm and 124-121 ppm, there are broad signals that likely represent the quaternary carbons of the aromatic core of the dimer. Used the corresponding cis isomer (cis-DBDB) as a guide.

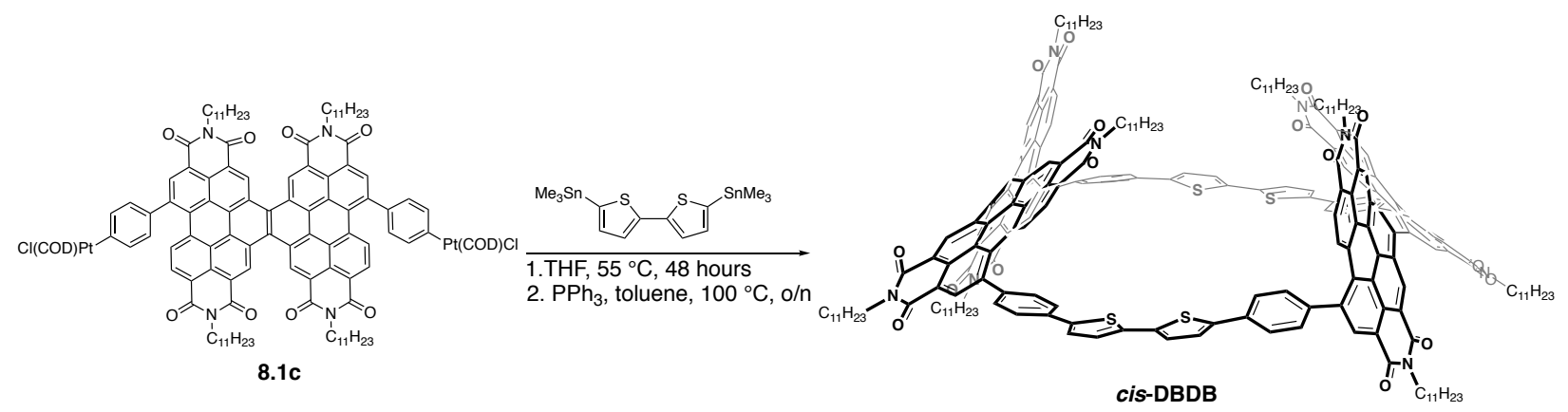

Synthesis of cis-DBDB: Bis[4-(Pt(COD)Cl)-phenyl]-hPDI 2 -cis (8.1c) (1.00 equiv, 0.0771 mmol, $0.172 \mathrm{~g}$ ), commercially available 5,5'-bis(trimethylstannyl)-2,2'-bithiophene (1.00 equivalent, $0.0771 \mathrm{mmol}, 0.0379$ grams $)$ and THF $(26.0 \mathrm{~mL})$ were added to an oven-dried $50 \mathrm{~mL}$ round bottom flask. The mixture was degassed for thirty minutes, and stirred in a $55^{\circ} \mathrm{C}$ oil bath for $48 \mathrm{~h}$. The crude mixture was concentrated and triphenylphosphine (20.0 equiv, $1.54 \mathrm{mmol}, 0.450 \mathrm{~g}$ ) was added to the flask with toluene $(26.0 \mathrm{~mL})$. The mixture was degassed for ten minutes, then stirred for overnight in a $100^{\circ} \mathrm{C}$ oil bath. The crude mixture was first purified by column chromatography (24 g Redisep Rf Silica) using a gradient from $0 \%$ to $100 \% \mathrm{CH}_{2} \mathrm{Cl}_{2} /$ hexanes at $35 \mathrm{~mL} / \mathrm{min}$. Fractions that contained cis-DBDB were collected and further purified on by preparative TLC using 70\%:30\% $\mathrm{CH}_{2} \mathrm{Cl}_{2} /$ hexanes. The solids were re-precipitated from methanol, followed by hexanes. The product is a dark purple solid $\left(0.0150 \mathrm{~g}, 0.00448 \mathrm{mmol}, 12 \%\right.$ yield). ${ }^{1} \mathbf{H}$ NMR (500 $\left.\mathrm{MHz}, 400 \mathrm{~K}, \mathrm{C}_{2} \mathrm{D}_{2} \mathrm{Cl}_{4}\right) \delta 10.41(\mathrm{~s}, 4 \mathrm{H}), 10.25(\mathrm{~s}, 4 \mathrm{H}), 9.05(\mathrm{~s}, 4 \mathrm{H}), 8.85(\mathrm{~d}, \mathrm{~J}=8.4 \mathrm{~Hz}, 4 \mathrm{H}), 8.67$ $(\mathrm{d}, \mathrm{J}=8.3 \mathrm{~Hz}, 4 \mathrm{H}), 7.73(\mathrm{~d}, \mathrm{~J}=8.1 \mathrm{~Hz}, 8 \mathrm{H}), 7.60(\mathrm{~d}, \mathrm{~J}=7.8 \mathrm{~Hz}, 8 \mathrm{H}), 7.49(\mathrm{~d}, \mathrm{~J}=4.0 \mathrm{~Hz}, 4 \mathrm{H})$ 
$7.32(\mathrm{~d}, \mathrm{~J}=4.1 \mathrm{~Hz}, 4 \mathrm{H}), 5.37(\mathrm{~m}, 4 \mathrm{H}), 5.23(\mathrm{~m}, 4 \mathrm{H}), 2.41(\mathrm{~m}, \mathrm{br}, 8 \mathrm{H}), 2.28(\mathrm{~m}, \mathrm{br}, 8 \mathrm{H}), 2.05(\mathrm{~m}$, br, 8H), 1.94 (m, br, 8H), 1.33* (m, br, 96H), 0.93 (tr, 24H), 0.80 (tr, 24H). ${ }^{13} \mathbf{C}$ NMR (100 MHz, $\left.400 \mathrm{~K}, \mathrm{C}_{2} \mathrm{D}_{2} \mathrm{Cl}_{4}\right) \delta 164.4,164.3,164.1,142.7,141.9,141.7,137.8,134.4,134.3,133.5,133.0$ $132.7,132.6,130.5,130.3,128.9,127.4,127.2,127.2,127.1,127.0,126.6,126.3,126.2,126.0$, $125.9,125.6,125.0,124.1,124.0,123.7,123.4,122.4,122.4,55.6,55.3,32.8,32.6,31.8,31.6$, 26.7, 26.6, 22.4, 22.3, 13.8, 13.7. IR $\left(\mathrm{cm}^{-1}\right)$ 2957, 2924, 2855, 1702, 1660, 1595, 1321. HRMS (MALDI-) calculated $\mathrm{m} / \mathrm{z}$ for $\left[\mathrm{C}_{228} \mathrm{H}_{228} \mathrm{~N}_{8} \mathrm{O}_{16} \mathrm{~S}_{4}\right]^{-}$is 3461.6156 , found $3461.6205 .{ }^{*}$ Integration is higher due to overlapping peak with both $\mathrm{H}_{2} 0$, and/or a small amount of grease.
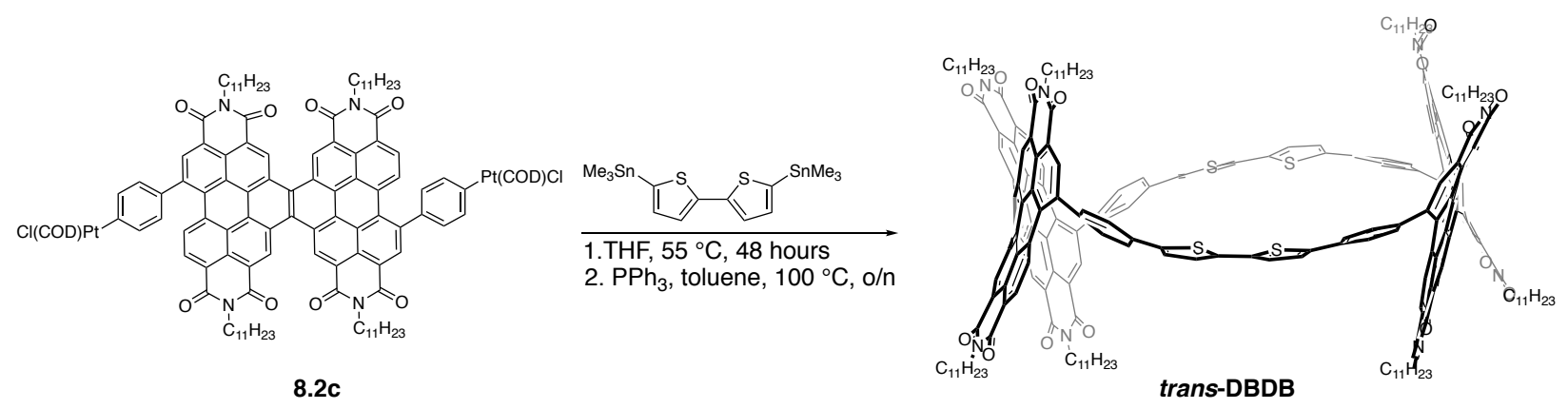

Synthesis of trans-DBDB: $\quad$ Bis[4-(Pt(COD)Cl)-phenyl]-hPDI 2 -trans (8.2c) (1.00 equiv, 0.0922 mmol, $0.207 \mathrm{~g})$, commercially available 5,5'-bis(trimethylstannyl)-2,2'-bithiophene (1.00 equivalent, $0.0922 \mathrm{mmol}, 0.0450$ grams $)$ and THF $(31.0 \mathrm{~mL})$ were added to an oven-dried 150. $\mathrm{mL}$ round bottom flask. The mixture was degassed for thirty minutes and, then, stirred in a $55^{\circ} \mathrm{C}$ oil bath for $48 \mathrm{~h}$. The crude mixture was concentrated and triphenylphosphine (20.0 equiv, 1.84 $\mathrm{mmol}, 0.538 \mathrm{~g})$ was added to the flask with toluene $(31.0 \mathrm{~mL})$. The mixture was degassed for ten minutes, then stirred for overnight in a $100{ }^{\circ} \mathrm{C}$ oil bath. The crude mixture was first purified by column chromatography (24 g Redisep Rf Silica) using a gradient from $0 \%$ to $100 \%$ $\mathrm{CH}_{2} \mathrm{Cl}_{2} /$ hexanes at $35 \mathrm{~mL} / \mathrm{min}$. Fractions that contained trans-DBDB were collected and further purified on by preparative TLC using $70 \%: 30 \% \mathrm{CH}_{2} \mathrm{Cl}_{2} /$ hexanes. To remove a small impurity, the product mixture was further purified using a COSMOSIL Buckyprep column (20 mm I.D. x 250 
$\mathrm{mm}, 5 \mu \mathrm{m}$ ), using an isocratic method of 50\% methylene chloride: $50 \%$ hexanes. The solids were re-precipitated from methanol, followed by hexanes. The product is a dark purple solid $(0.0100 \mathrm{~g}$, $0.00289 \mathrm{mmol}, 6 \%$ yield). ${ }^{1} \mathrm{H}$ NMR $\left(400 \mathrm{MHz}, 400 \mathrm{~K}, \mathrm{C}_{2} \mathrm{D}_{2} \mathrm{Cl}_{4}\right) \delta[$ major stereoisomer] 10.25 (s, 4H), $10.22(\mathrm{~s}, 4 \mathrm{H}), 9.10(\mathrm{~s}, 4 \mathrm{H}), 8.97(\mathrm{~d}, \mathrm{~J}=8.4 \mathrm{~Hz}, 4 \mathrm{H}), 8.67(\mathrm{~d}, \mathrm{~J}=8.4 \mathrm{~Hz}, 4 \mathrm{H}), 7.75(\mathrm{~d}, \mathrm{~J}=8.2$ $\mathrm{Hz}, 8 \mathrm{H}), 7.61(\mathrm{~d}, \mathrm{~J}=8.1 \mathrm{~Hz}, 8 \mathrm{H}), 7.50(\mathrm{~d}, \mathrm{~J}=4.0 \mathrm{~Hz}, 4 \mathrm{H}), 7.32(\mathrm{~d}, \mathrm{~J}=3.9 \mathrm{~Hz}, 4 \mathrm{H}), 5.39$ (m, 4H), $5.20(\mathrm{~m}, 4 \mathrm{H}), 2.45(\mathrm{~m}, \mathrm{br}, 8 \mathrm{H}), 2.24(\mathrm{~m}, \mathrm{br}, 8 \mathrm{H}), 2.05(\mathrm{~m}, \mathrm{br}, 8 \mathrm{H}), 1.96(\mathrm{~m}, \mathrm{br}, 8 \mathrm{H}), 1.37 *(\mathrm{~m}, \mathrm{br}$, 96H), 0.94* (tr, br, 24H), 0.83 (tr, br, 24H). [distinguishable minor stereoisomer peaks] 9.06, 8.71. ${ }^{13} \mathrm{C}$ NMR $\left(100 \mathrm{MHz}, 400 \mathrm{~K}, \mathrm{C}_{2} \mathrm{D}_{2} \mathrm{Cl}_{4}\right) \delta 164.4,164.1,164.0,142.6,142.0,141.3,137.4,134.4$, $134.2,133.5,132.7,132.6,132.5,130.6,130.4,130.4,130.4,130.1,128.9,127.2,127.1,127.1$, $127.0,126.8,126.5,126.1,125.2,125.1,124.9,124.5,124.2,123.4,122.8,122.4,122.1,55.6$, $55.3,33.0,32.8,32.6,32.4,31.8,31.7,31.7,26.7,26.7,26.7,22.5,22.4,22.3,13.9,13.8,13.7$. IR $\left(\mathrm{cm}^{-1}\right)$ 2956, 2924, 2854, 1703, 1661, 1594, 1320. HRMS (MALDI-) calculated m/z for $\left[\mathrm{C}_{228} \mathrm{H}_{228} \mathrm{~N}_{8} \mathrm{O}_{16} \mathrm{~S}_{4}\right]^{-}$is 3461.61516 , found 3461.6384 . *Integration is higher due to overlapping peak with both $\mathrm{H}_{2} 0$, and/or a small amount of grease. 


\subsection{NMR Spectra of Compounds}
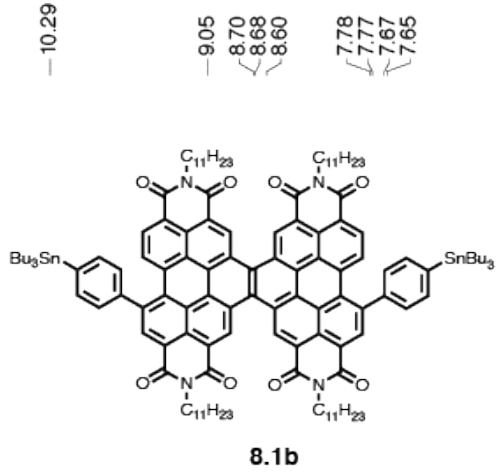

${ }^{1} \mathrm{H}$ NMR (400 MHz), $\mathrm{CDCl}_{3}, 300 \mathrm{~K}$

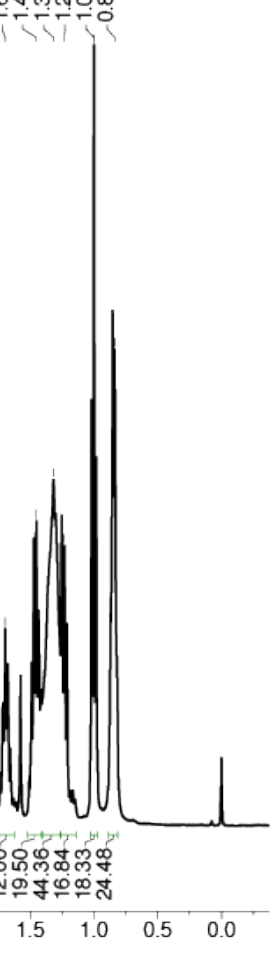

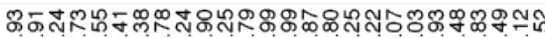

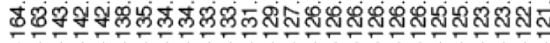

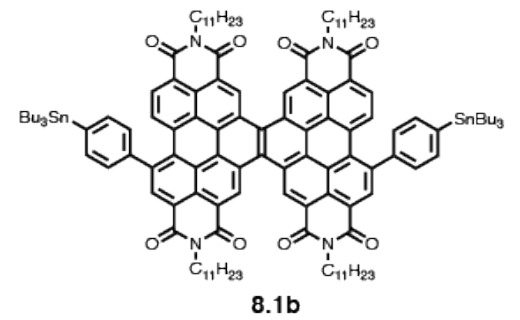

${ }^{13} \mathrm{C}$ NMR (100 MHz), $\mathrm{CDCl}_{3}, 300 \mathrm{~K}$
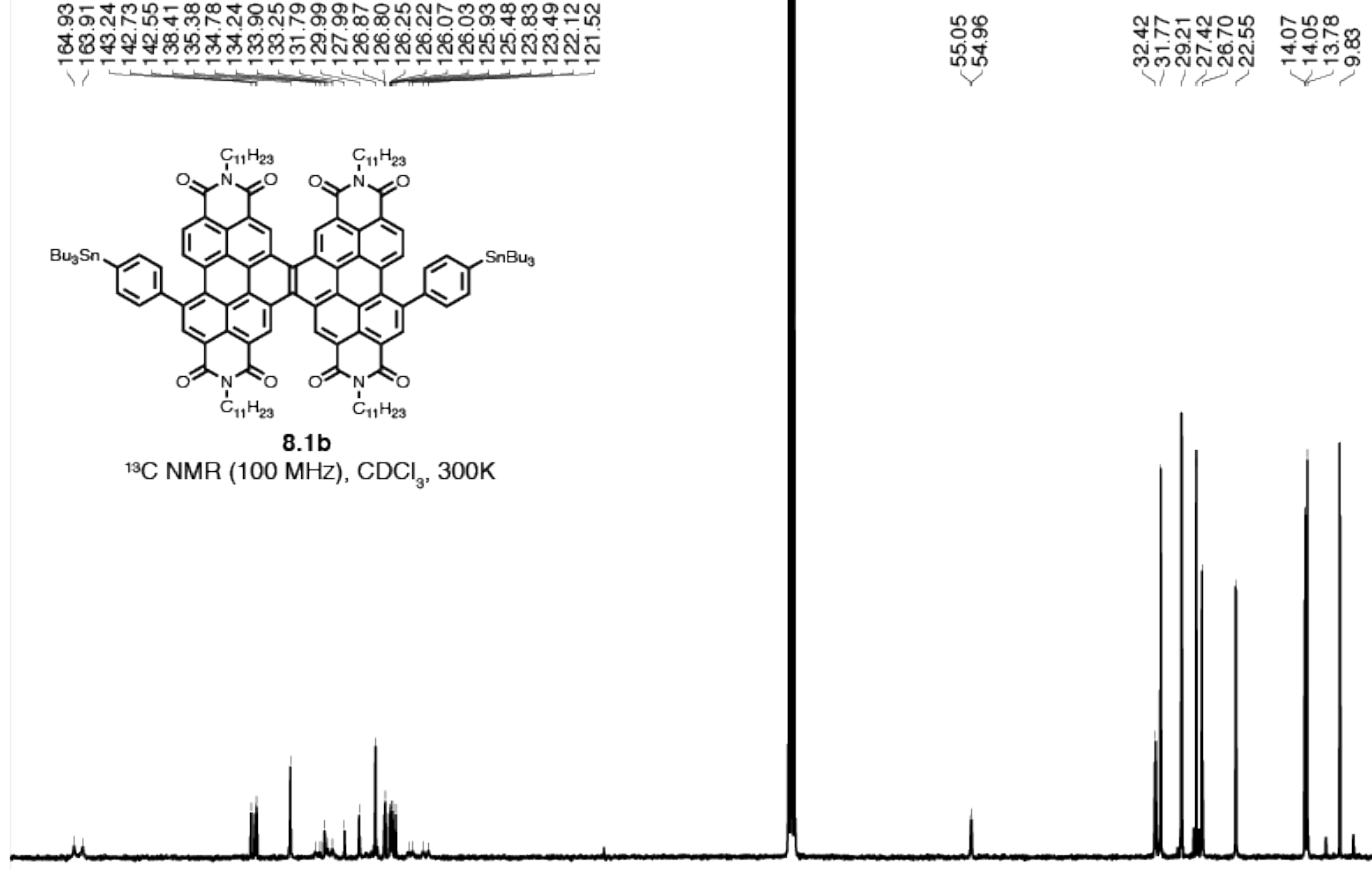

$170 \quad 160$

$160 \quad 150$

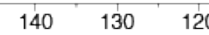

$120 \quad 110$

$\bar{\sigma}^{80}$ 


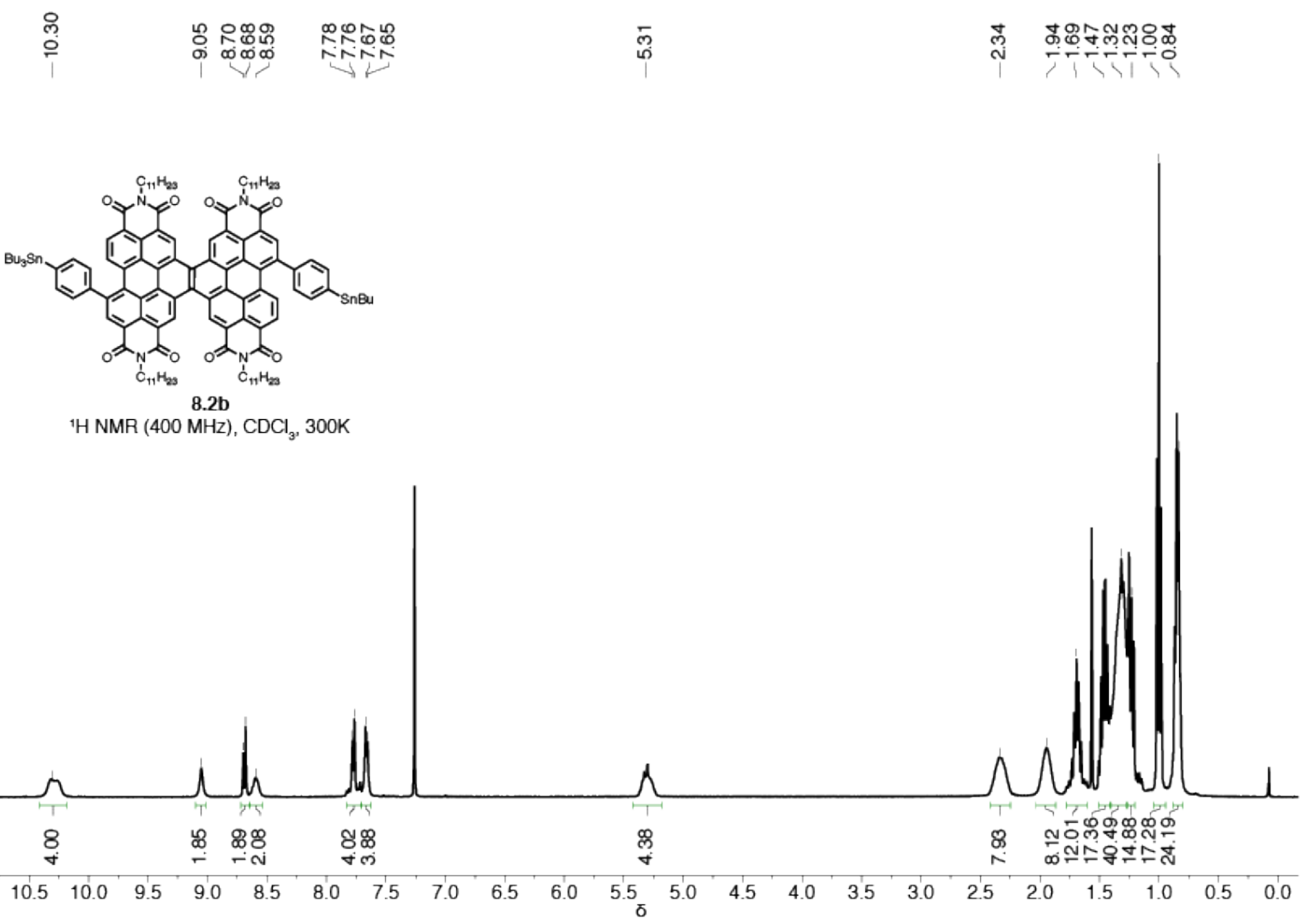

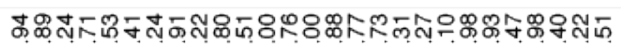

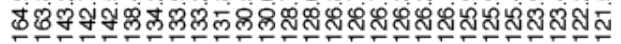

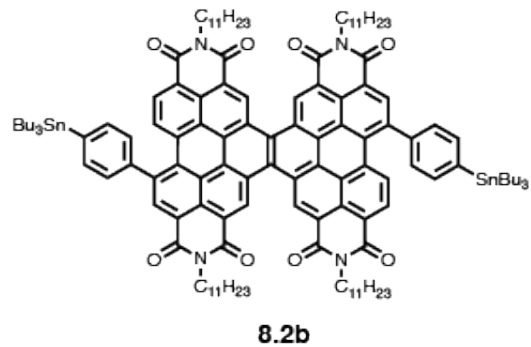

${ }^{13} \mathrm{C}$ NMR (100 MHz), $\mathrm{CDCl}_{3}, 300 \mathrm{~K}$
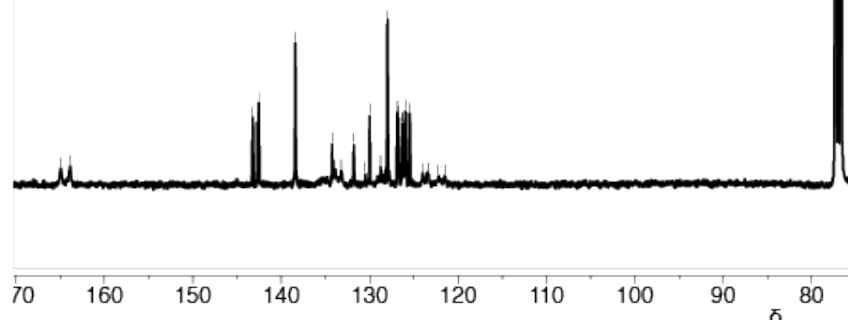

$10 \quad 100 \quad 90 \quad{ }^{8}$ 


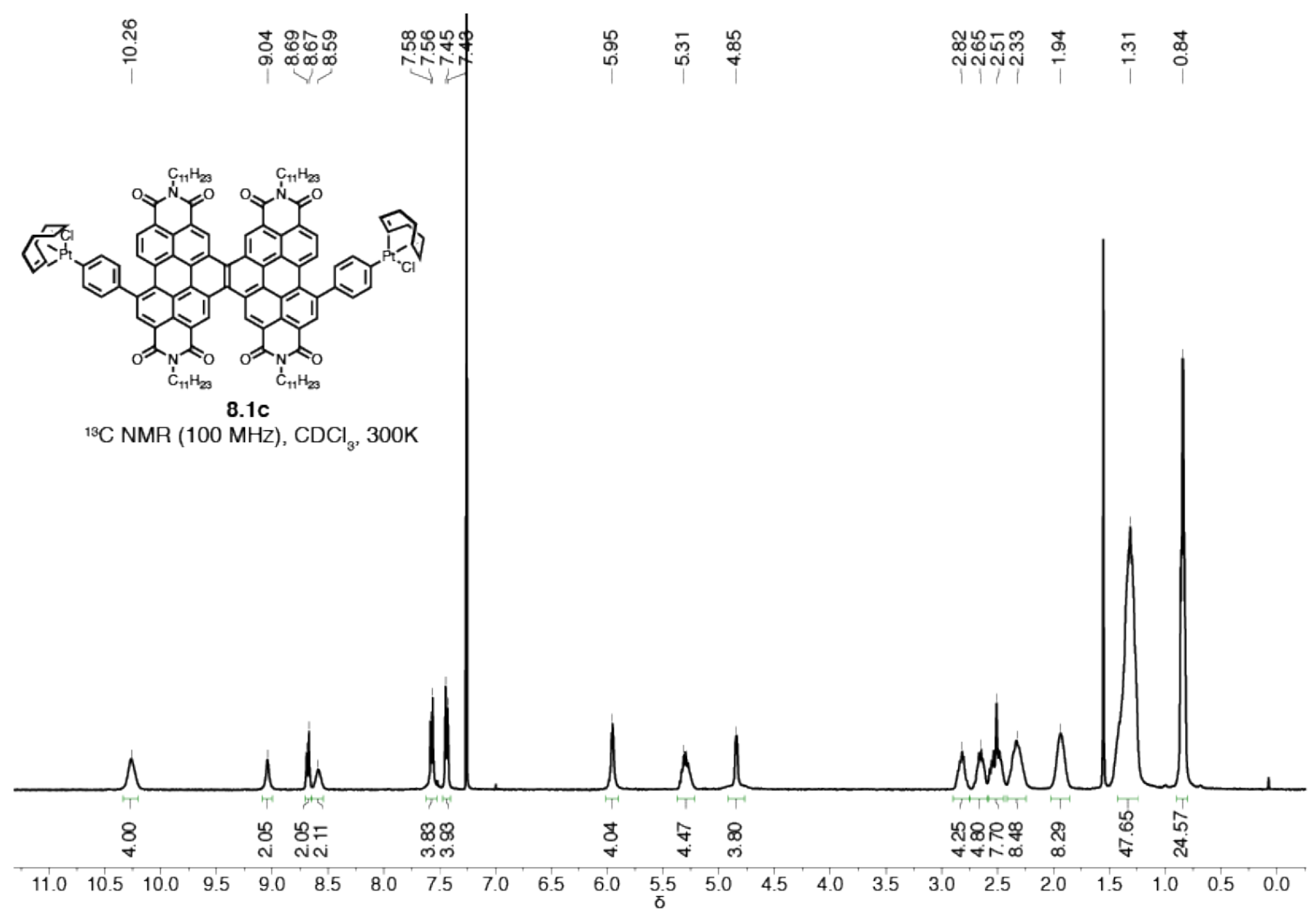

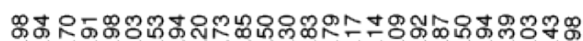

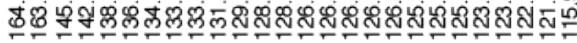

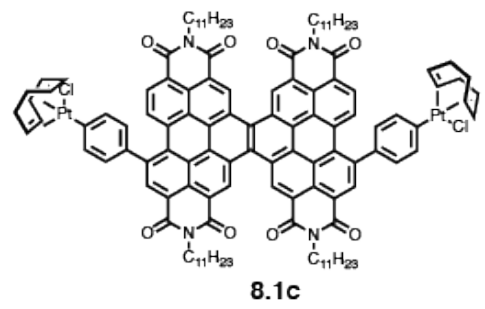

${ }^{13} \mathrm{C}$ NMR (100 MHz), $\mathrm{CDCl}_{3}, 300 \mathrm{~K}$
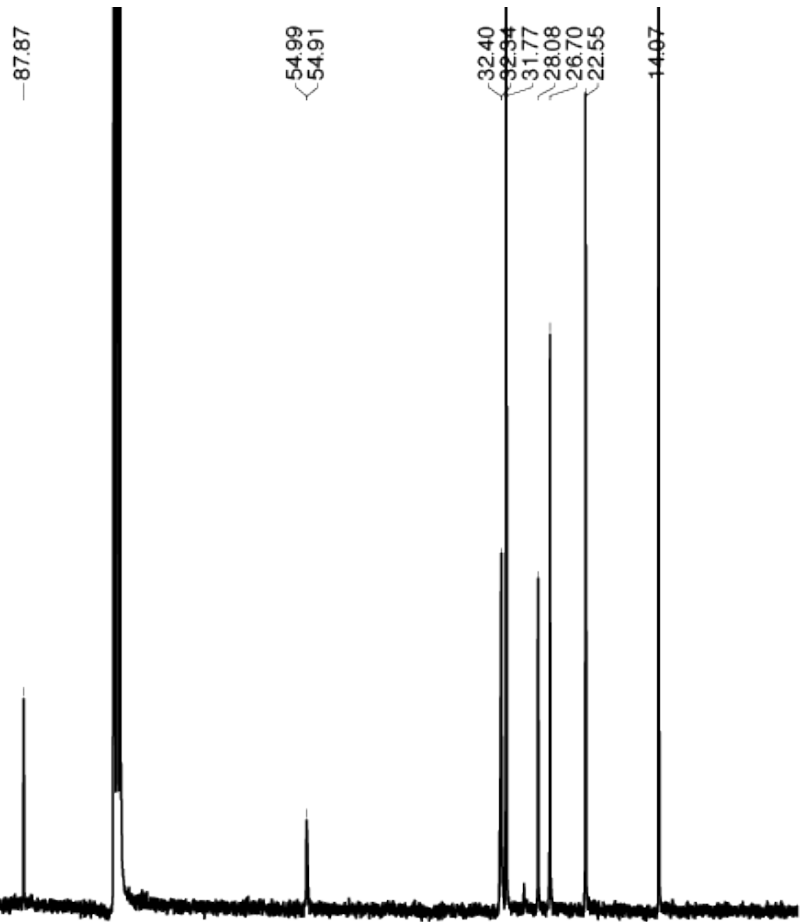

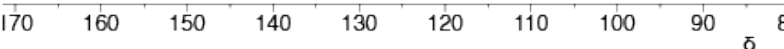




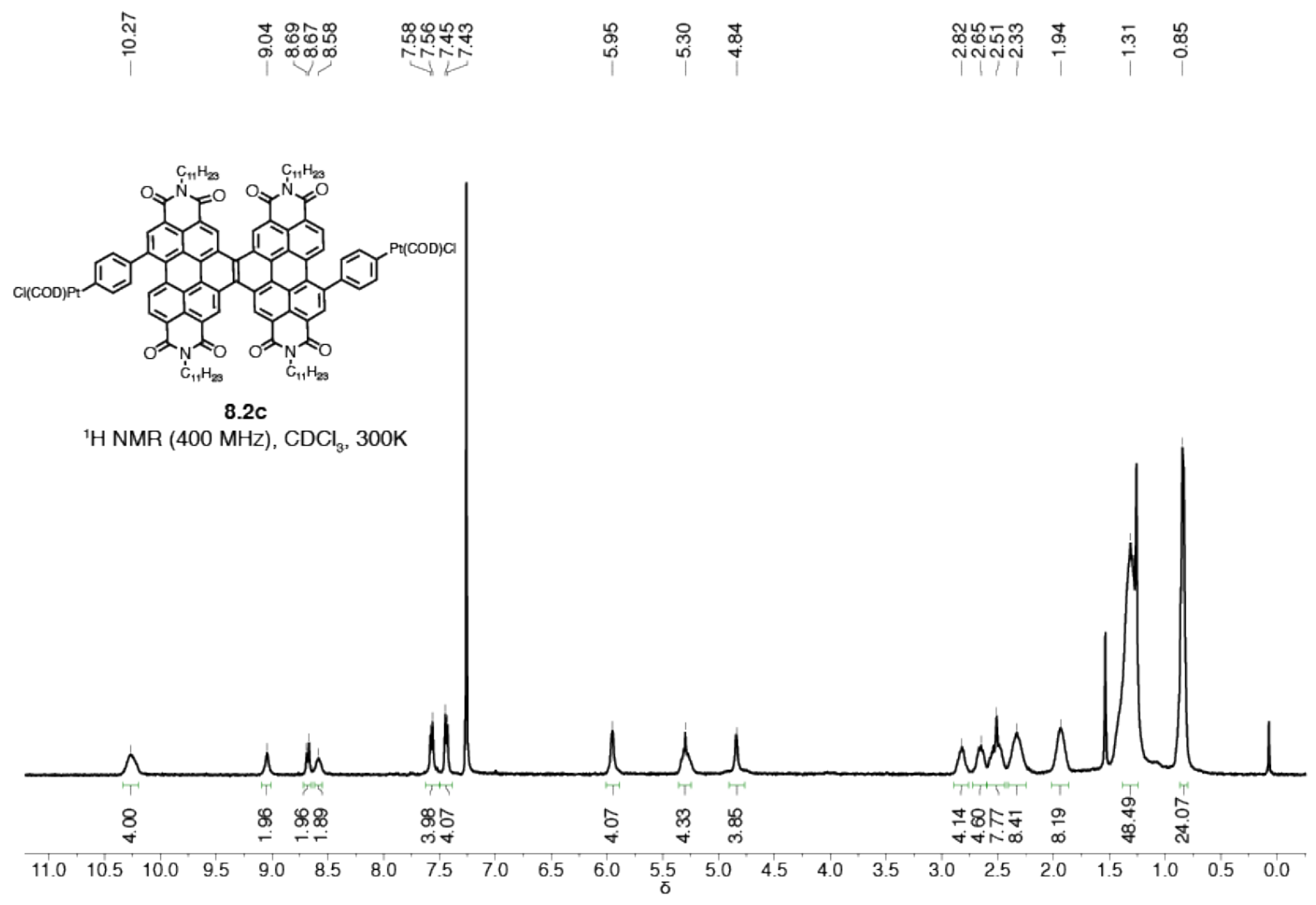

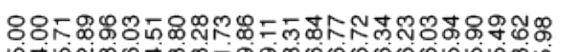

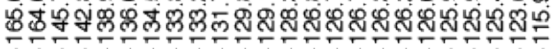
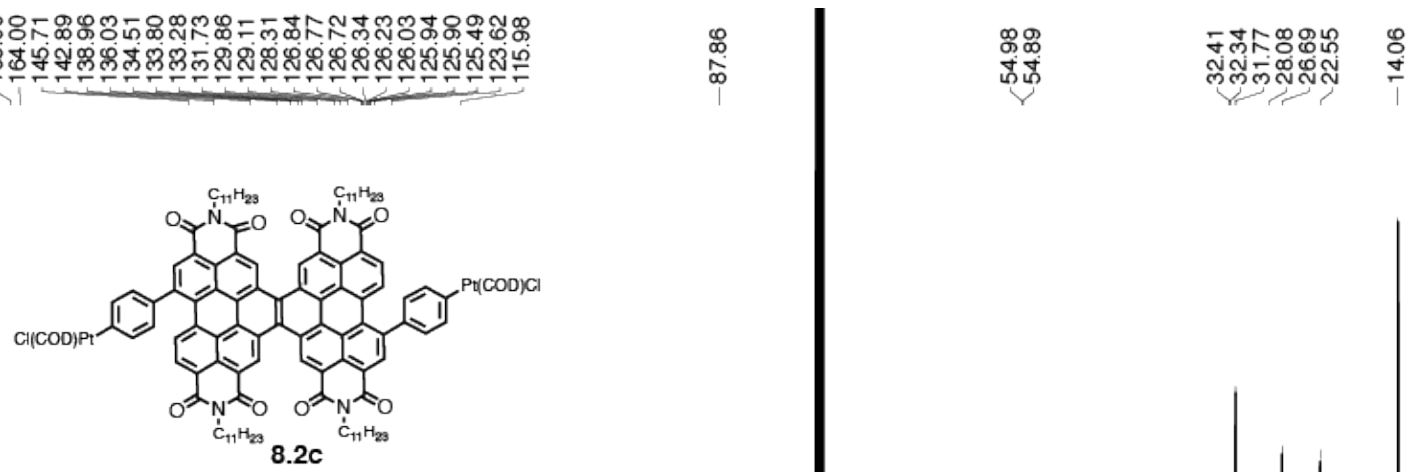

${ }^{13} \mathrm{C}$ NMR (100 MHz), $\mathrm{CDCl}_{3}, 300 \mathrm{~K}$

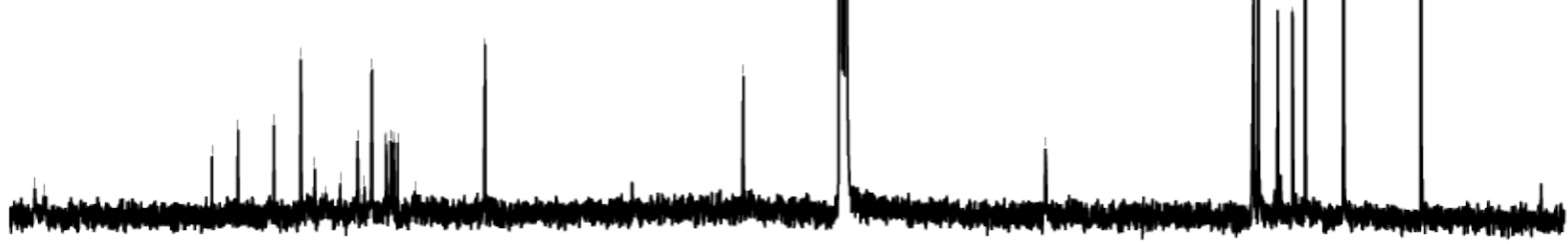

\begin{tabular}{|l|l|l|l|l|l|l|l|l|l|l|l|l|l|l|l|l|l|l|l|}
160 & 150 & 140 & 130 & 120 & 110 & 100 & 90 & 80 & 70 & 60 & 50 & 40 & 30 & 20 & 10 & 0
\end{tabular}



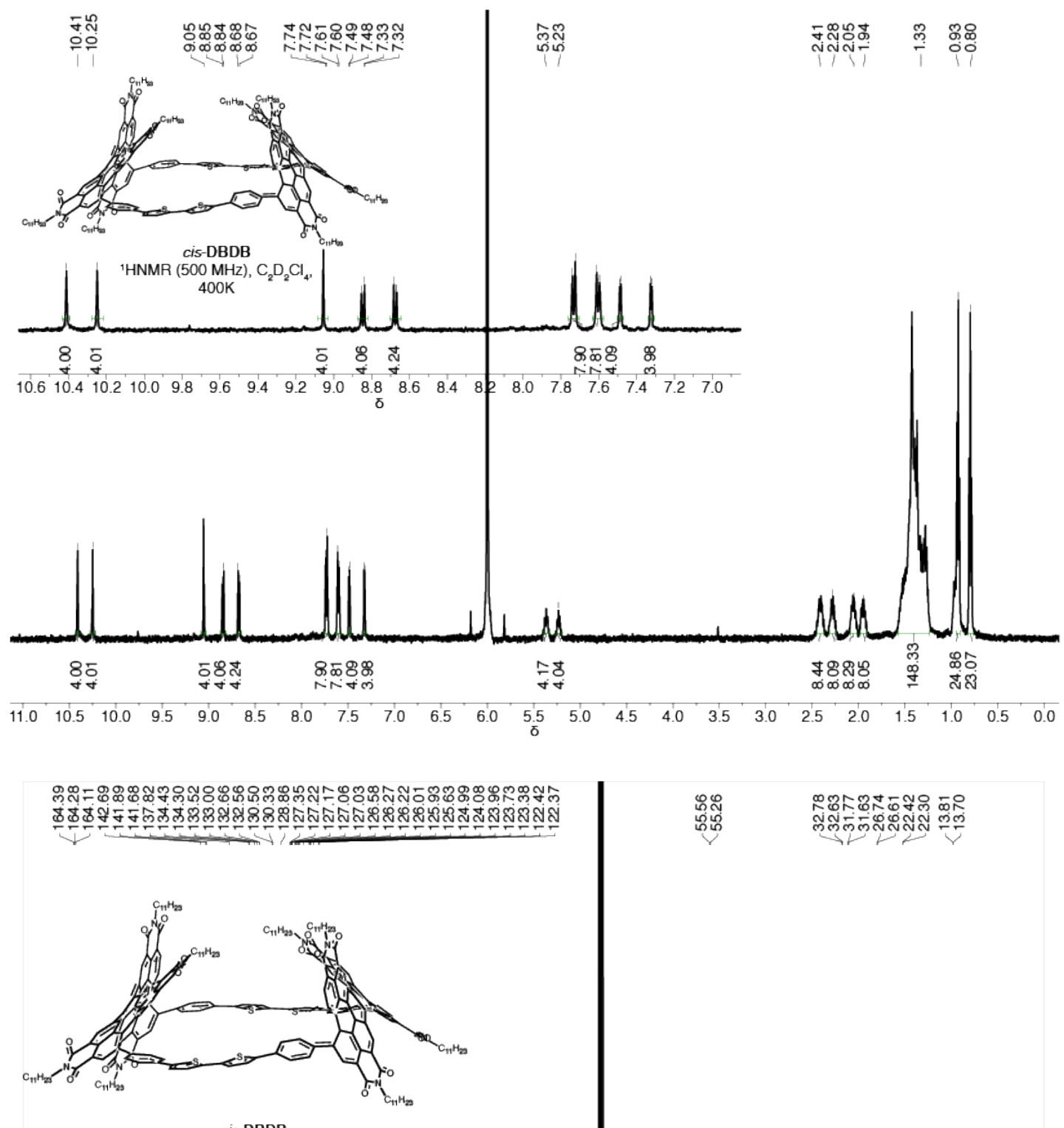

Cis-DBDB
${ }^{13} \mathrm{CNMR}(100 \mathrm{MHz}), \mathrm{C}_{2} \mathrm{D}_{2} \mathrm{Cl}_{4}, 400 \mathrm{~K}$

\section{in}

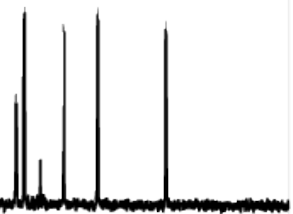

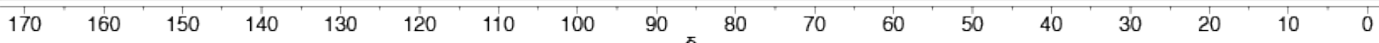




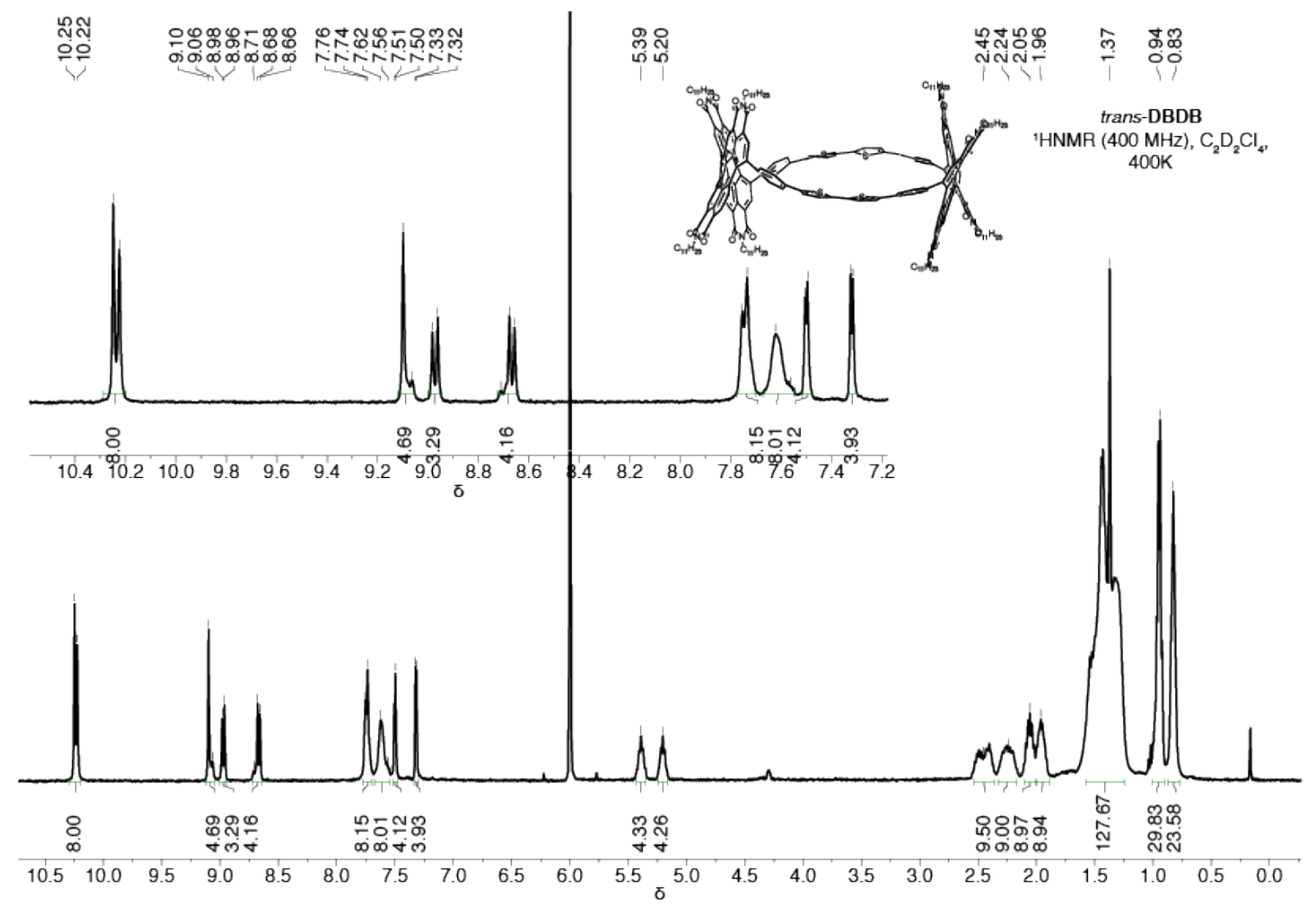

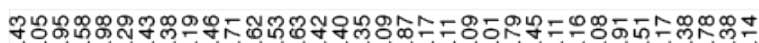

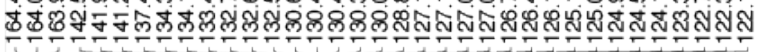

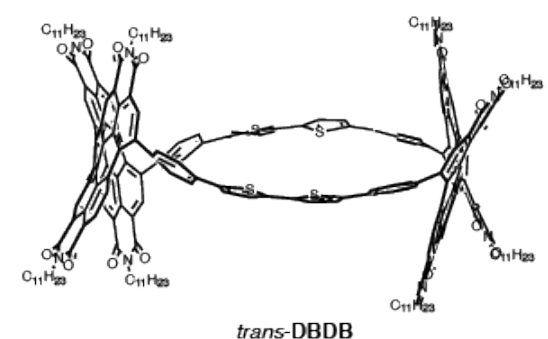

${ }^{13} \mathrm{CNMR}(100 \mathrm{MHz}), \mathrm{C}_{2} \mathrm{D}_{2} \mathrm{Cl}_{4}, 400 \mathrm{~K}$

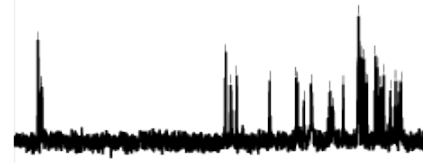

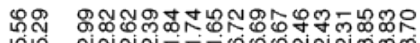

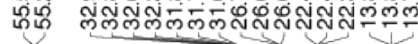

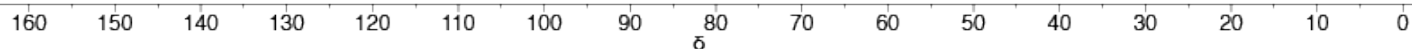




\subsection{DFT Output}

All quantum chemical calculations were performed using Jaguar, version 8.3, Schrodinger, Inc., New York, NY, 2013. (See A. D. Bochevarov, E. Harder, T. F. Hughes, J. R. Greenwood, D. A. Braden, D. M. Philipp, D. Rinaldo, M. D. Halls, J. Zhang, R. A. Friesner, "Jaguar: A High Performance Quantum Chemistry Software Program with Strengths in Life and Materials Sciences", Int. J. Quantum Chem., 2013, 113(18), 2110-2142). All geometries were optimized using the B3LYP functional and the 6-31G basis set. The geometry optimizations can be found in reference $1 .{ }^{1}$ I provide the results of the homodesmotic reaction employed in order to calculate strain within the two hPDI 2 macrocycles.

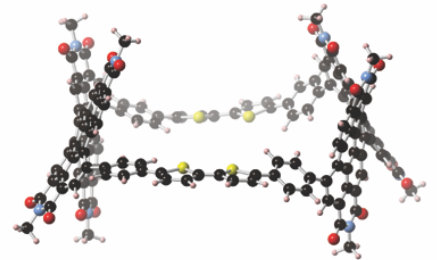

trans-DBDB

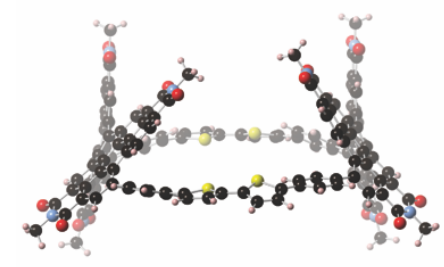

cis-DBDB

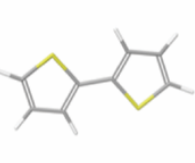

Bithiophene

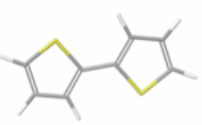

Bithiophene

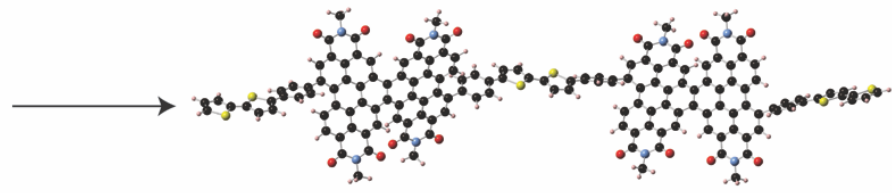

trans-DBDB-Acyclic

Table 8.3. Homodesmotic Calculations for Strain

\begin{tabular}{|c|c|c|c|}
\hline Compound & Total energy (hartree) & $\begin{array}{c}\text { Strain energy } \\
\text { (hartrees) }\end{array}$ & $\begin{array}{c}\text { Strain energy } \\
\text { (kcal/mol) }\end{array}$ \\
\hline trans-DBDB & -8915.8024 & 0.0296 & 18.575 \\
\hline trans-DBDB-Acyclic & -10020.6588 & - & - \\
\hline Bithiophene & -1104.8268 & - & - \\
\hline cis-DBDB & -8915.8154 & 0.0151 & 9.476 \\
\hline cis-DBDB-Acyclic & -10020.6573 & - & - \\
\hline
\end{tabular}

Table 8.3: Calculated enthalpy from DFT using B3LYP functional and the 6-31G basis set 


\subsection{References}

1. Ball, M. L. et al. Influence of Molecular Conformation on Electron Transport in Giant, Conjugated Macrocycles. J. Am. Chem. Soc. 140, 10135-10139 (2018).

2. Krömer, J. et al. Synthesis of the First Fully $\alpha$-Conjugated Macrocyclic Oligothiophenes: Cyclo[n]thiophenes with Tunable Cavities in the Nanometer Regime. Angew. Chem. Int. Ed. 39, 3481-3486 (2000).

3. Nakao, K. et al. Giant Macrocycles Composed of Thiophene, Acetylene, and Ethylene Building Blocks. J. Am. Chem. Soc. 128, 16740-16747 (2006).

4. Ishii, Y. et al. Size-selective synthesis of 9 - 11 and 13 cycloparaphenylenes. Chem. Sci. 3, 2340-2345 (2012).

5. Hitosugi, S. et al. Bottom-up Synthesis and Thread-in-Bead Structures of Finite (n,0)Zigzag Single-Wall Carbon Nanotubes. J. Am. Chem. Soc. 134, 12442-12445 (2012).

6. Omachi, H. et al. Synthesis of Cycloparaphenylenes and Related Carbon Nanorings: A Step toward the Controlled Synthesis of Carbon Nanotubes. Acc. Chem. Res. 45, 1378-1389 (2012).

7. Kayahara, E. et al. Selective Synthesis and Crystal Structure of [10]Cycloparaphenylene. Org. Lett. 14, 3284-3287 (2012).

8. Hitosugi, S. et al. Atropisomerism in a Belt-Persistent Nanohoop Molecule: Rotational Restriction Forced by Macrocyclic Ring Strain. Chem. Asian J. 7, 1550-1552 (2012).

9. Kayahara, E. et al. Synthesis and Characterization of [5]Cycloparaphenylene. J. Am. Chem. Soc. 136, 2284-2287 (2014).

10. Yamago, S. et al. Organoplatinum-Mediated Synthesis of Cyclic pi-Conjugated Molecules: Towards a New Era of Three-Dimensional Aromatic Compounds. Chem. Rec. 14, 84-100 (2014).

11. Evans, P. J. et al. Efficient room-temperature synthesis of a highly strained carbon nanohoop fragment of buckminsterfullerene. Nat. Chem. 6, 404-408 (2014).

12. Ball, M. et al. Chiral Conjugated Corrals. J. Am. Chem. Soc. 137, 9982-9987 (2015).

13. Chang, S.-W. et al. A donor-acceptor conjugated block copolymer of poly(arylenevinylene)s by ring-opening metathesis polymerization. Chem. Commun. 51, 9113-9116 (2015).

14. Jasti, R. et al. Synthesis, Characterization, and Theory of 9 -, 12 -, and 18 Cycloparaphenylene: Carbon Nanohoop Structures. J. Am. Chem. Soc. 130, 17646-17647 (2008).

15. Ito, H. et al. Thiophene-Based, Radial pi-Conjugation: Synthesis, Structure, and Photophysical Properties of Cyclo-1,4-phenylene-2 ',5 '-thienylenes. Angew. Chem. Int. Ed. 54, 159-163 (2015).

16. Chen, Q. et al. Strain-Induced Stereoselective Formation of Blue-Emitting Cyclostilbenes. J. Am. Chem. Soc. 137, 12282-12288 (2015).

17. Jiang, H.-W. et al. Cyclic 2,12-Porphyrinylene Nanorings as a Porphyrin Analogue of 
Cycloparaphenylenes. J. Am. Chem. Soc. 137, 2219-2222 (2015).

18. Darzi, E. R. et al. Synthesis, Properties, and Design Principles of Donor-Acceptor Nanohoops. ACS Cent. Sci. 1, 335-342 (2015).

19. Asai, K. et al. A Cyclic Octithiophene Containing B,B'-linkages. Chem. Commun. 51, 6096 (2015).

20. Kuwabara, T. et al. Curved Oligophenylenes as Donors in Shape-Persistent DonorAcceptor Macrocycles with Solvatofluorochromic Properties. Angew. Chem. Int. Ed. 54, 9646-9649 (2015).

21. Van Raden, J. M. et al. Synthesis and characterization of a highly strained donor-acceptor nanohoop. Org. Biomol. Chem. 14, 5721 (2016).

22. Zhang, F. et al. Giant Cyclo n thiophenes with Extended pi Conjugation. Angew. Chem. Int. Ed. 48, 6632-6635 (2009).

23. Omachi, H. et al. A Modular and Size-Selective Synthesis of [n]Cycloparaphenylenes: A Step toward the Selective Synthesis of n, n Single-Walled Carbon Nanotubes. Angew. Chem. Int. Ed. 49, 10202-10205 (2010).

24. Iwamoto, T. et al. Selective and Random Syntheses of n Cycloparaphenylenes $(\mathrm{n}=8-13)$ and Size Dependence of Their Electronic Properties. J. Am. Chem. Soc. 133, 8354-8361 (2011).

25. Iwamoto, T. et al. Size-Selective Encapsulation of C60 by [10]Cycloparaphenylene: Formation of the Shortest Fullerene-Peapod. Angew. Chem. Int. Ed. 50, 8342-8344 (2011).

26. Sprafke, J. K. et al. Belt-Shaped $\pi$-Systems: Relating Geometry to Electronic Structure in a Six-Porphyrin Nanoring. J. Am. Chem. Soc. 133, 17262-17273 (2011).

27. Segawa, Y. et al. Concise Synthesis and Crystal Structure of 12 Cycloparaphenylene. Angew. Chem. Int. Ed. 50, 3244-3248 (2011).

28. Segawa, Y. et al. [9]Cycloparaphenylene: Nickel-mediated Synthesis and Crystal Structure. Chem. Lett. 40, 423-425 (2011).

29. Zhong, Y. et al. Helical Ribbons for Molecular Electronics. J. Am. Chem. Soc. 136, 81228130 (2014).

30. Ball, M. et al. Contorted Polycyclic Aromatics. Acc. Chem. Res. 48, 267-276 (2015).

31. Sisto, T. J. et al. Long, Atomically Precise Donor-Acceptor Cove-Edge Nanoribbons as Electron Acceptors. J. Am. Chem. Soc. 139, 5648-5651 (2017).

32. Ball, M. et al. Macrocyclization in the Design of Organic n-Type Electronic Materials. $J$. Am. Chem. Soc. 138, 12861-12867 (2016).

33. Zhang, B. et al. Rigid, Conjugated Macrocycles for High Performance Organic Photodetectors. J. Am. Chem. Soc. 138, 16426-16431 (2016).

34. Zhang, B. et al. Hollow organic capsules assemble into cellular semiconductors. Nat. Commun. 9, 1957 (2018).

35. Zhong, Y. et al. Efficient Organic Solar Cells with Helical Perylene Diimide Electron Acceptors. J. Am. Chem. Soc. 136, 15215 (2014). 
36. Zhong, Y. et al. Molecular helices as electron acceptors in high-performance bulk heterojunction solar cells. Nat. Commun. 6, 8242 (2015).

37. Edison, C. et al. Cove-Edge Nanoribbon Materials for Efficient Inverted Halide Perovskite Solar Cells. Angew. Chem. Int. Ed. 56, 14648-14652 (2017).

38. Zhong, Y. et al. Helical Nanoribbons for Ultra-Narrowband Photodetectors. J. Am. Chem. Soc. 139, 5644-5647 (2017).

39. Peurifoy, S. R. et al. Three-Dimensional Graphene Nanostructures. J. Am. Chem. Soc. 40, 9341-9345 (2018).

40. McCulloch, I. et al. Avoid the kinks when measuring mobility. Science (80-. ). 352, 1521 LP - 1522 (2016).

41. Choi, H. H. et al. Critical assessment of charge mobility extraction in FETs. Nat. Mater. 17, 2 (2017).

42. C.D., D. et al. Organic Thin Film Transistors for Large Area Electronics. Adv. Mater. 14, 99-117 (2002).

43. George, P. et al. An alternative approach to the problem of assessing stabilization energies in cyclic conjugated hydrocarbons. Theor. Chim. Acta 38, 121-129 (1975).

44. Bachrach, S. M. et al. DFT Study of Cycloparaphenylenes and Heteroatom-Substituted Nanohoops. J. Org. Chem. 75, 6595-6604 (2010).

45. Segawa, Y. et al. Theoretical Studies on the Structures and Strain Energies of Cycloparaphenylenes. Org. Lett. 12, 2262-2265 (2010).

46. Rajasingh, P. et al. Selective Bromination of Perylene Diimides under Mild Conditions. $J$. Org. Chem. 72, 5973-5979 (2007).

47. You, J. B. et al. A polymer tandem solar cell with $10.6 \%$ power conversion efficiency. Nat. Commun. 4, 10 (2013).

48. Crysalis Pro. (2013).

49. Sheldrick, G. M. \{lit SHELXT $\} \quad\{--\}$ Integrated space-group and crystal-structure determination. Acta Crystallogr. Sect. A 71, 3-8 (2015).

50. Sheldrick, G. M. Crystal structure refinement with \{lit SHELXL\}. Acta Crystallogr. Sect. C 71, 3-8 (2015).

51. Dolomanov, O. V et al. \{lit OLEX2\}: A complete structure solution, refinement and analysis program. J. Appl. Crystallogr. 42, 339-341 (2009).

52. Spek, A. L. Structure validation in chemical crystallography. Acta Crystallogr. Sect. D 65 , 148-155 (2009).

53. van der Sluis, P. et al. BYPASS: an effective method for the refinement of crystal structures containing disordered solvent regions. Acta Crystallogr. Sect. A 46, 194-201 (1990). 


\section{Chapter 9. Conjugated PDI Macrocycles as Electronic Hosts}

\subsection{Preface}

Chapter 9 contains unpublished work on PBPB and its utility as a host system. Dr. Timothy Andrew Barendt from Oxford University was integral to this work, and performed all spectroscopy presented and I synthesized all compounds. The second half of this chapter briefly describes initial work I performed while a visiting student in Kyoto University in 2018. I believe both show the potential of these materials as chiral hosts within a range of applications and scope to design interesting structures in the future

\subsection{Introduction}

This study uses PBPB as an electronic host for fullerenes. ${ }^{1}$ Fullerenes stand out amongst the carbon nanomaterials as desirable components within organic electronic materials due to their unique electrochemical and photophysical properties. ${ }^{2-8}$ This has motivated the use of selfassembly with a rationally designed hosts as a means to incorporate fullerenes into electronically

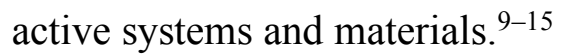

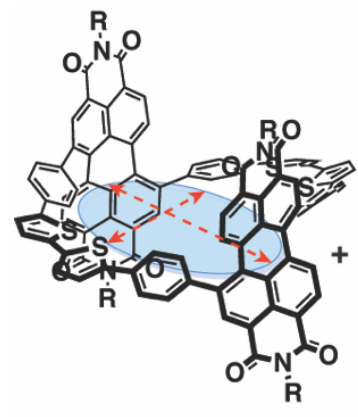

PBPB +

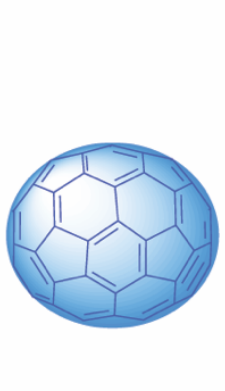

$\mathrm{C}_{70}$

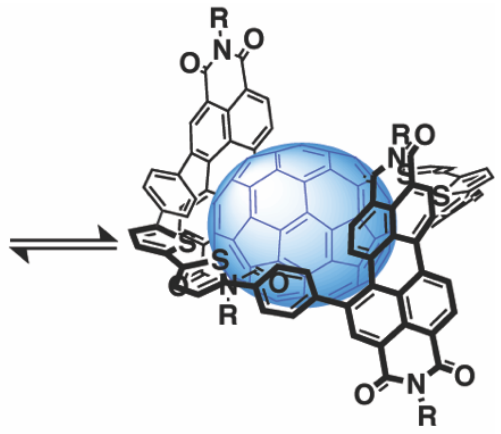

$[\mathrm{PBPB}] \supset[\mathrm{F}]$

Figure 9.1. The recognition of fullerenes $\mathbf{C}_{70}, \mathbf{C}_{60}$ or PCBM by bis-PDI macrocycle PBPB to form a supramolecular complex $\left(\mathrm{R}=\mathrm{C}_{11} \mathrm{H}_{23}\right)$.

To date, a number of fullerene receptors have been constructed, thereby establishing several principles of host design in order to maximize guest affinities..$^{9,11-29}$ These include; 1) a large, preorganized and typically macrocyclic cavity to accommodate the fullerene; 2) extensive 
$\pi$-conjugation that facilitates convex-concave intermolecular aromatic stacking interactions; and 3) the integration of electron donating heteroatoms to provide electronic complementarity with the electron deficient guest.

We have investigated the dynamics and electronic properties of PBPB, and found the macrocycle possesses many of the desirable characteristics for strong, non-covalent recognition of fullerenes, namely; 1) a large inner nano-space with a persistent elliptical geometry; 2) $\pi$ conjugation that extends throughout the concave aromatic framework; and 3) Lewis basic sulfur atoms that are directed into the cavity and available for lone pair $-\pi$ interactions. Experimental NMR, absorption and emission spectroscopies, mass spectrometry and theoretical molecular modelling are used to characterize and quantify the resulting supramolecular complexes in the solution phase. A preference for $\mathbf{C}_{70}$ fullerene is revealed. Furthermore, an insight into the electronic communication between macrocyclic host and fullerene guest is provided from UV-Vis absorption and fluorescence emission spectroscopies and supported by density functional theory calculations. As such these findings have significant implications for the generation of conducting organic electronic materials composed from supramolecular assemblies of electron donor and acceptor units.

\subsection{NMR Spectroscopy Shows Formation of Supramolecular Complex}

The ability of PBPB to form a supramolecular complex with fullerenes (F) $\mathbf{C}_{60}, \mathbf{C}_{\mathbf{7 0}}$ and [6,6]-Phenyl $\mathrm{C}_{61}$ butyric acid methyl ester (PCBM) was investigated by NMR spectroscopy in $\mathrm{d}_{8}$ toluene solution. Initial proton NMR titrations, in which up to ten equivalents of fullerene (F) guest were titrated into a $0.5 \mathrm{mM}$ solution of macrocyclic PBPB host, showed three features indicative of non-covalent interactions and formation of the supramolecular complex $[\mathbf{P B P B}] \supset[\mathbf{F}]$ with all guests (Figure 9.2a-d and Figure 9.8-Figure 9.11, NMR data provided for all complexes in the 
Appendix). Our attempts to grow single crystals of the supramolecular complexes were unsuccessful, so we employed DFT to elucidate how $\mathbf{F}$ guest rests within the cavity of PBPB (Figure 9.2a).

The largest perturbations were the downfield shifts of peaks associated with phenyl aromatic protons $\mathrm{H}_{\mathrm{d}}$ and $\mathrm{H}_{\mathrm{e}}$ (up to $\Delta \delta=0.18 \mathrm{ppm}$, Figure 9.2b). We believe this is indicative of the formation of strong intermolecular aromatic stacking $(\pi-\pi)$ interactions between the concave surface of the fullerene guest and convex belt of the macrocyclic host. Analogous effects have been observed in fullerene receptors also containing extensive $\pi$-conjugation such as CPP and porphyrin-based systems. ${ }^{16,26,28}$ Downfield shifts of the $\mathrm{H}_{\mathrm{a}-\mathrm{c}}$ proton signals (PDI-based protons), too, suggest a close approach between PBPB and the fullerene guests (Figure 9.2e).

The second interaction consists of lone pair donation from the thiophene bridges of PBPB to the fullerene (S: $\pi$ interactions), made possible by the Lewis basic sulfur atoms of the heterocycles being directed towards the guest in the cavity (Figure 9.2c). Both cyclothiophenes and sulfur-based macrocycles have shown this interaction contributes to the stabilization of the supramolecular fullerene complexes involving these hosts. ${ }^{11,12,30}$ The thiophene proton shift is much less pronounced relative to the neighboring phenyl rings $\left(\Delta \delta \mathrm{H}_{\mathrm{i}}=0.02-0.05 \mathrm{ppm}\right)$ because the sulfur lone pairs are orthogonal to the aromatic system of the macrocycle. Analogous to literature reports, we observe an upfield shift in the heterocycle's protons as they are outside the binding pocket.

Interaction 3 involves the methylene protons of the $\mathrm{C}_{11} \mathrm{H}_{23}$ side chains of PBPB. While there are significant changes in the alkyl region, the clearest occur with protons labelled $\mathrm{H}_{\mathrm{f}}, \mathrm{H}_{\mathrm{g}}$, and $H_{f}$, and $H_{g}$, in Figure 9.2d and 9.12 and 9.13. These sixteen protons exist as a diastereotopic set of resonances that, in the absence of any fullerene guest, produce two broad peaks $(\delta=2.16$ 
ppm and $1.83 \mathrm{ppm}, \mathrm{H}_{\mathrm{f}} / \mathrm{H}_{\mathrm{f}}$ and $\mathrm{H}_{\mathrm{g}} / \mathrm{H}_{\mathrm{g}}$ ' respectively) of equal integration. (Figure 9.12 and 9.13). ${ }^{1}$ However, upon fullerene recognition both of these signals are further split into two broad peaks, giving, in total, four signals that each integrate to four protons $\left(\mathrm{H}_{\mathrm{f}}, \mathrm{H}_{\mathrm{g}}, \mathrm{H}_{\mathrm{f}}, \mathrm{H}_{\mathrm{g}}\right)$

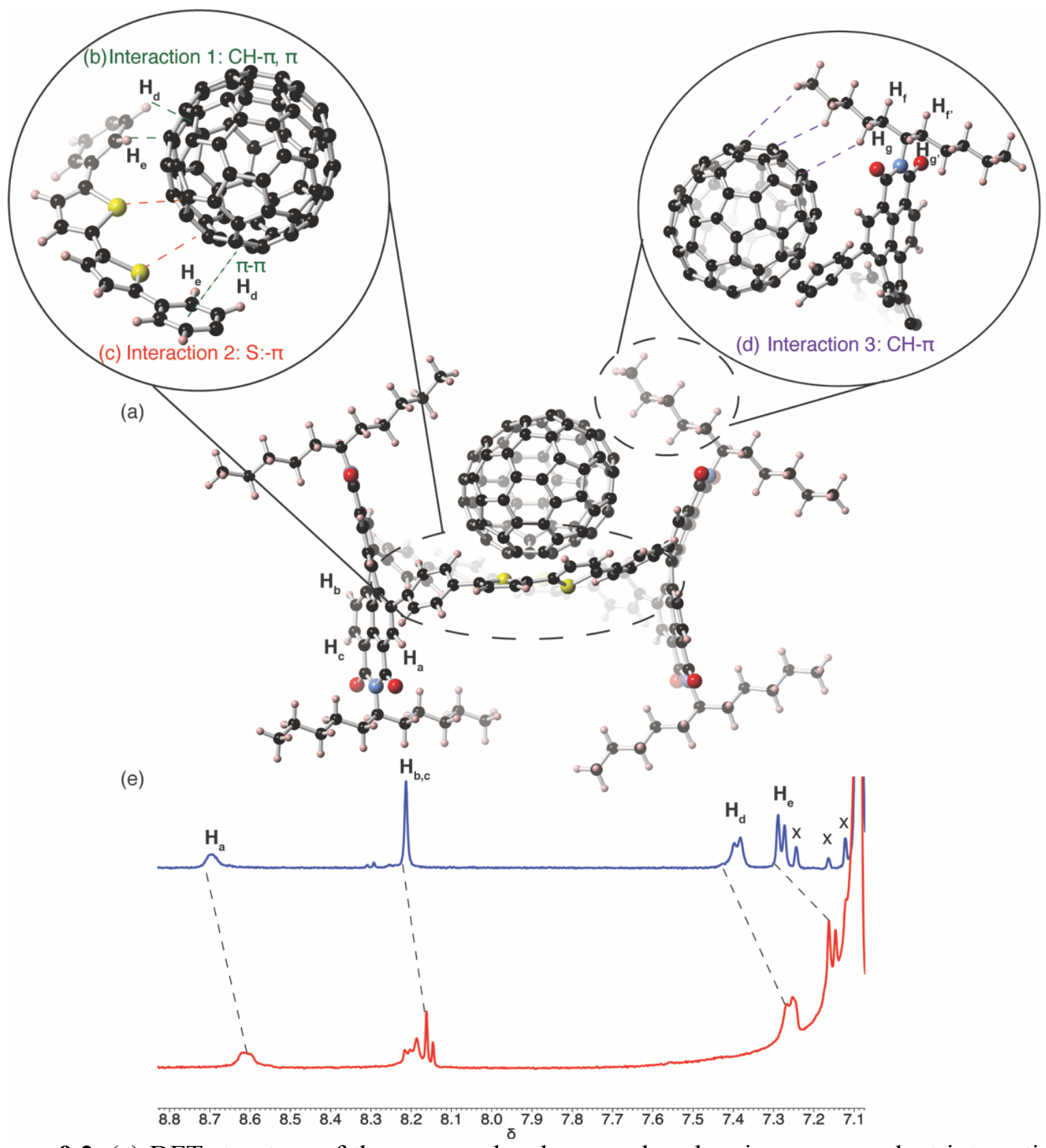

Figure 9.2. (a) DFT structure of the supramolecular complex showing non-covalent interactions present. (b) $\mathrm{CH}-\pi$ interactions from aryl rings to $\mathbf{C}_{70}$; (c) sulfur lone pair- $\pi$ interactions; (d) $\mathrm{CH}-\pi$ interaction between methylene and fullerene; and (e) Truncated ${ }^{1} \mathrm{H}$ NMR spectra from the titration of 1 eq. of $\mathbf{C}_{70}$ fullerene guest into a $0.5 \mathrm{mM}$ host solution of $\mathbf{P B P B}$ (start point $=$ red, end point $=$ blue, $\mathrm{d}_{8}$-toluene, $298 \mathrm{~K}, 500 \mathrm{MHz}$. 
Our hypothesis is that fullerene encapsulation within the macrocyclic cavity renders the internal and external environments of the macrocycle inequivalent by restricting free rotation of the sidechains, rendering $\mathrm{H}_{\mathrm{f}} / \mathrm{H}_{\mathrm{f}}$ and $\mathrm{H}_{\mathrm{g}} / \mathrm{H}_{\mathrm{g}}$, to become inequivalent.

To better understand the role of the alkyl side chains, we prepared PBPB with 2,6diisopropyl phenyl substituents (aryl-PBPB) at the imide positions and studied the binding of $\mathrm{C}_{70}$ (Figure 9.14). Fullerene recognition was shown to be over an order of magnitude weaker (Table 9.1), confirming the integral role of the flexible alkyl side chains to a) allow ease of access to the cavity and b) undergo additional $\mathrm{CH}-\pi$ non-covalent interactions to accommodate the fullerene guest. The side chains help form a macrocyclic cage around the fullerene.

We qualified the importance of the macrocyclic cavity by performing NMR titrations studies on a diphenyl PDI (Compound 1.1), ${ }^{31}$ and observe no evidence of a supramolecular complex between 1.1 and $\mathrm{C}_{70}$ (Figure 9.15). This suggest the cavity is critical to form a supramolecular complex. We also investigated the importance of size complementarity between host and guest in $[\mathbf{P B P B}] \supset[\mathbf{F}]$ by performing analogous ${ }^{1} \mathrm{H}$ NMR spectroscopic titration experiments with trans-DBDB and $\mathbf{C}_{\mathbf{7 0}}$. From DFT, the cavity for trans-DBDB measures $19 \AA$ from $\mathrm{hPDI}_{2}$ to $\mathrm{hPDI}_{2}$ and $16 \AA$ along the short axis between the bithiophenes vs. $16 \AA$ and $12 \AA$ respectively for PBPB (Figure 1B.4 and Figure 8.4). Analogous to PBPB, trans-DBDB possesses the three key interactions described: 1) four aryl rings that can participate in $\pi-\pi$ interactions; 2) $\mathrm{C}_{11} \mathrm{H}_{23}$ branched side-chains that can stabilize a supramolecular complex through $\mathrm{CH}-\pi$ interactions; and 3) four sulfur groups with their lone pairs directed to the center of the cavity. The ${ }^{1} \mathrm{H}$ NMR spectra exhibited modest shifts in the corresponding aryl groups $\left(\Delta \delta \mathrm{H}_{\mathrm{a}}=0.01 \mathrm{ppm}\right)$, indicative of weak binding and negligible formation of complex DBDB $\supset$ C70, and despite DBDB 
possessing analogous functionality responsible for strong interactions between PBPB and $\mathbf{F}$ guests (Figure 9.16).

Job's plot analysis of the titration data revealed the maximum chemical shift change occurred when the fullerene mole fraction $\chi(\mathbf{F})=0.5$, indicating 1:1 host:guest stoichiometric binding for all guests investigated in this study (Figure 9.16). The supramolecular complexes were also studied by ESI mass spectrometry (Figures 9.17). Solutions of macrocycle and fullerene mixtures revealed peaks for 1:1 and 1:2 host:guest complexes ([PBPB] $[\mathbf{F}]$ and $[\mathbf{P B P B}] \supset[\mathbf{2 F}])$, the former being of significantly higher intensity relative to the latter. An indication of the robustness of complex [PBPB] $\supset[\mathbf{F}]$ was provided by ESI MS/MS experiments; large collision energies (80-100 eV) were required to break the intermolecular interactions and observe peaks for discrete macrocycle and fullerene components (Figures 9.18).

Table 9.1. Fullerene Association Constants

\begin{tabular}{|l|c|}
\hline & $\mathrm{K}_{\mathrm{a}}\left(\mathrm{M}^{-1}\right)$ \\
\hline $\mathbf{C}_{70}$ & 9,278 \\
\hline $\mathbf{C}_{60}$ & 2,119 \\
\hline $\mathbf{P C B M}$ & $1,336^{\mathrm{a}}$ \\
\hline $\mathbf{C}_{70} /$ aryl-PBPB & 591 \\
\hline
\end{tabular}

Table 9.1: Fullerene association constants $\left(\mathrm{K}_{\mathrm{a}}, \mathrm{M}^{-1}\right)$ for macrocycle PBPB determined by ${ }^{1} \mathrm{HNMR}$ spectroscopy in d8-toluene using chemical shift data of $\mathrm{H}_{\mathrm{d}}$ unless otherwise stated $(298 \mathrm{~K}, 500$ $\mathrm{MHz}$ ). Errors $<7 \%$. Chemical shift of thiophene proton $\mathrm{H}_{\mathrm{h}}$ used due to spectral overlap of $\mathrm{H}_{\mathrm{d}}$.

We quantified fullerene recognition with macrocyclic host PBPB using Bindfit, ${ }^{32}$ following $\mathrm{H}_{\mathrm{d}}$ proton (Table 9.1). Interestingly, the association constant for $\mathrm{C}_{70}$ was over four times that of $\mathrm{C}_{60}$, at $9,278 \mathrm{M}^{-1}$ relative to $2,119 \mathrm{M}^{-1}$, respectively. This suggests the ellipsoidal cavity of PBPB provides a better size and shape complementarity for the less spherical fullerene guest. There is also potential for an enhanced electronic contribution to complexation: $\mathrm{C}_{70}$ is more 
electron deficient than $\mathrm{C}_{60}$ (vide infra for photophysics). ${ }^{33}$ Binding with PCBM, too, is also observed despite the additional steric bulk arising from covalent functionalization of the carbon cage.

Carbon NMR spectroscopy was used to further characterize fullerene complexation by macrocycle PBPB. Analysis of host solutions containing a small excess (1.5 eq.) of $\mathbf{C}_{60}$ or $\mathbf{C}_{70}$ guest in $d 8$-toluene revealed upfield shifts of the ${ }^{13} \mathrm{C}$ NMR fullerene signals, consistent with a ring current effect from the diamagnetic aromatic system of the macrocycle (Figure 9.20). We note that $\mathbf{C}_{70}$ fullerene exhibits a larger maximum perturbation than $\mathbf{C}_{60}\left(\Delta \delta_{\max }=0.18 v s .0 .09 \mathrm{ppm}\right)$. Therefore, to further probe fullerene selectivity of PBPB in solution, a competition experiment was performed by combining $\mathbf{P B P B}, \mathbf{C}_{60}$ and $\mathbf{C}_{70}$ in a 1:1.5:1.5 stoichiometric ratio (Figure 9.20b). In agreement with ${ }^{1} \mathrm{H}$ NMR calculated $K_{\mathrm{a}}$ values, a larger proportion of the maximum possible ${ }^{13} \mathrm{C}$ NMR shift was observed for $\mathbf{C}_{70}\left(\Delta \delta / \Delta \delta_{\max }=67 \%\right)$ than for $\mathbf{C}_{60}(44 \%)$.

\subsection{Photophysics Between PBPB and Fullerenes}

In order to provide insight into electronic communication between macrocyclic host and fullerene guest, UV-Vis absorption spectra were recorded for macrocycle PBPB $(\mu \mathrm{M}$ concentration) in the presence of an excess of $\mathrm{C}_{70}$ (Figure 9.3) and $\mathrm{C}_{60}$ guests in toluene (Figure 9.21). The addition of up to 100 equivalents of fullerene caused a decrease in intensity of the lowest energy absorption band and a concomitant increase in absorptions at longer wavelength generating an isosbestic point at $\lambda=650 \mathrm{~nm}$ indicative of the establishment of a thermodynamic equilibrium between host and host-guest complex. 

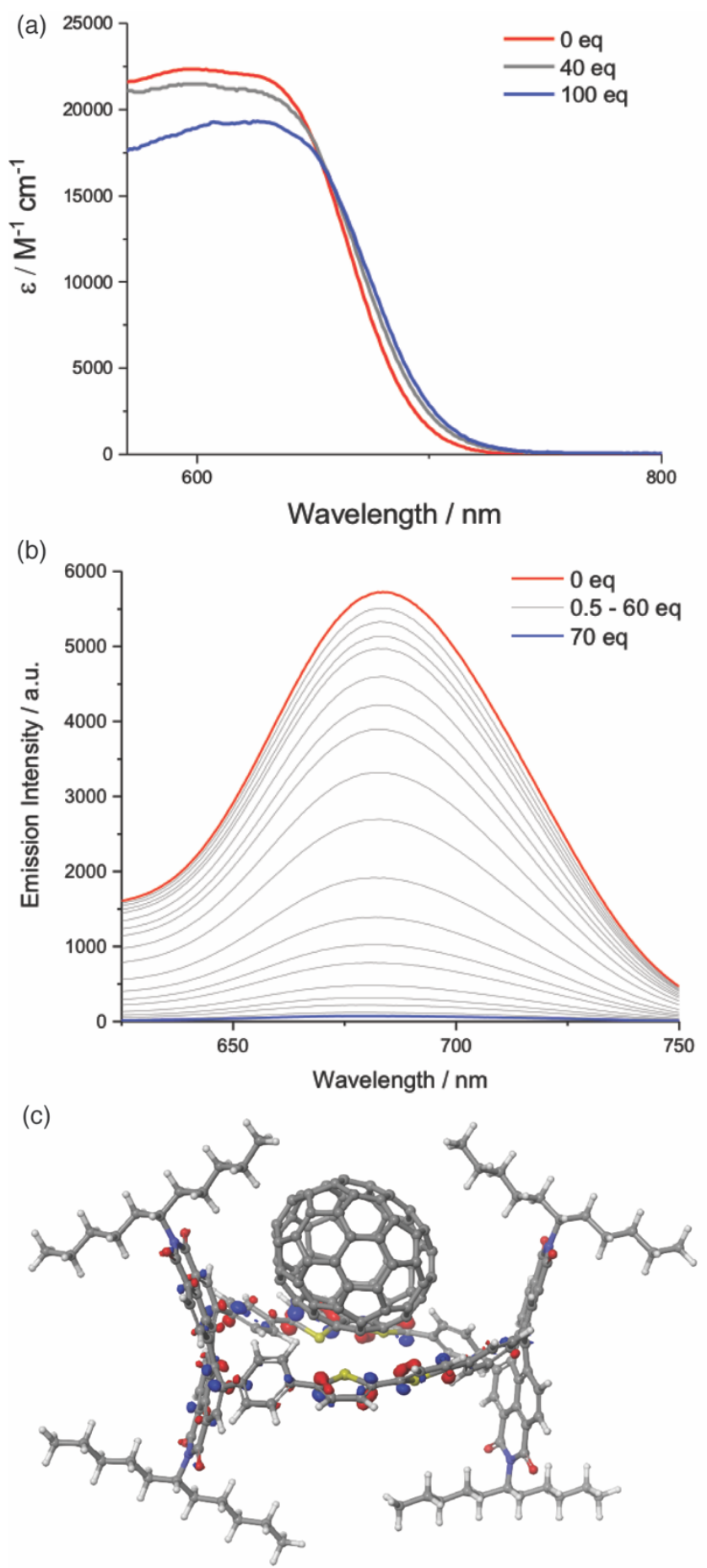

Figure 9.3. (a) Electronic absorption spectra of PBPB upon the addition of $\mathbf{C}_{70}$ (toluene, corrected for $\mathbf{C}_{70}$ absorptions); (b) Fluorescence emission spectra of PBPB upon the titration with $\mathbf{C}_{\mathbf{7 0}}$ (toluene, $4 \mu \mathrm{M}, \lambda \mathrm{ex}=600 \mathrm{~nm}$ ); and (c) HOMO energy level showing electron delocalization.

TDDFT calculations show the HOMO is PBPB-based, suggesting there is a high density of high energy electrons on the perimeter of the macrocycle. Relative to the free macrocycle, the 
complex shows a high degree of electron delocalization across the bithiophenes and PDI in the HOMO. We characterize the low energy transition in the absorption spectrum as a promotion of an electron from the HOMO (macrocyclic-based) to a purely PDI-based LUMO. Upon addition of fullerene guest, we observe a decrease in absorption at low energy wavelengths. A possible explanation for the decrease in absorption at this wavelength is that the proximal fullerene guest also possesses empty, low energy orbitals available to accept an electron. As such, this effect is most pronounced with $\mathbf{C}_{70}$ because, while it forms the strongest intermolecular complex, it is also more electron deficient than $\mathbf{C}_{60} .{ }^{33}$ The change in absorption could also reflect geometrical changes in PBPB upon fullerene encapsulation: electron delocalization is greater in the complex relative to the free macrocycle ${ }^{1}$ and could result in ground state electron transfer.

Fluorescence emission host-guest titration experiments revealed the influence of fullerene binding on the excited state of PBPB (host concentration maintained at $4 \mu \mathrm{M}$ throughout, Figure 9.3b and Figure 9.22 and Figure 9.22). Both $\mathbf{C}_{60}$ and $\mathbf{C}_{70}$ recognition induced significant quenching

of fluorescence emission due to intermolecular energy and/or electron transfer ${ }^{34-39}$ from host to guest due to their electronic complementarity. The stronger recognition of $\mathbf{C}_{70}$ by PBPB was manifested in a near depletion (99\%) of fluorescence emission after 70 equivalents of guest, while an equal molar ratio of $\mathbf{C}_{60}$ resulted in a smaller decrease (57\%).

\subsection{Potential Applications for PBPB-Fullerene Complex}

Chapter 9 describes the supramolecular complexation of fullerene (F) guests by a conjugated PDI-bithiophene-based macrocycle (PBPB) in solution (Figure 9.1). Qualitative evidence for the non-covalent interactions that promote this encapsulation is, in part, provided by a detailed analysis of perturbations to the ${ }^{1} \mathrm{H}$ and ${ }^{13} \mathrm{C}$ NMR spectra and is supported by mass spectrometry and DFT calculations. The importance of geometric and electronic complementarity 
to the formation of $[\mathbf{P B P B}] \supset[\mathbf{F}]$ is highlighted through comparisons with analogous, yet inferior, PDI-based acyclic and macrocyclic receptors. These principles also explain the $>4$-fold preference of PBPB for $\mathbf{C}_{70}$ over $\mathbf{C}_{60}$ fullerenes. Electronic absorption and fluorescence emission spectroscopies revealed electronic communication between the PBPB macrocycle (donor) and fullerene (acceptor) in the ground and excited state. This, and the fact PBPB was demonstrated to be a potent receptor for the fullerene PCBM, paves the way for these donor-acceptor supramolecular complexes to be integrated into organic electronic materials for enhancing the performance of OFET and OPV devices.

\subsection{Future Directions for Conjugated Macrocycles}

The success of both $\left(\mathbf{P B B r}_{4}\right)_{3}$ and $\mathbf{P B P B}$ as a hosts systems is exciting as one relatively under-explored area for the conjugated macrocycles is applications that utilize the cavities; for example, one can envision using the cavity as a chiral environment for catalysis. ${ }^{40}$ Each of the macrocycles created possess different electronic and physical structures, providing an opportunity to select guests that are electronically and size-matched to each host.
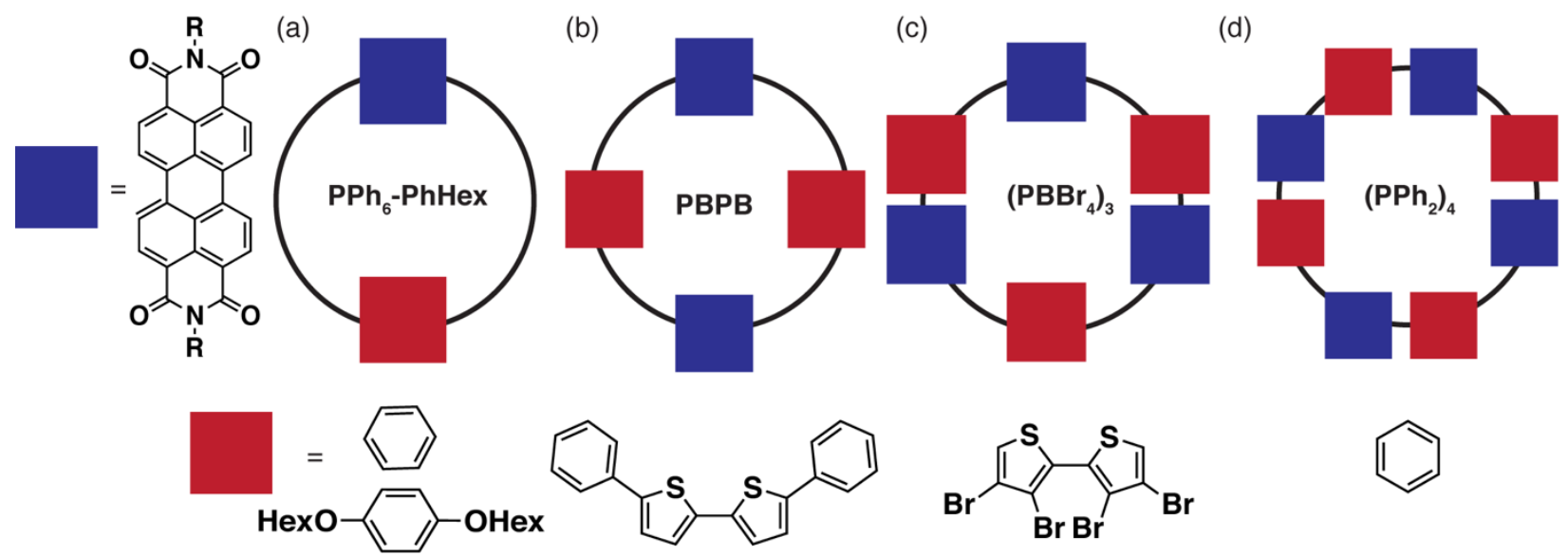

Figure 9.4. Schematic of (a) $\mathbf{P P h}_{6}-\mathbf{P h H e x}$; (b) PBPB; (c) $\left(\mathbf{P B B r}_{4}\right)_{3}$; and (d) $\left(\mathbf{P P h}_{2}\right)_{4}$.

One promising idea would be to incorporate phenanthroline into a conjugated macrocycle.

Phenanthroline moieties (PA) are ubiquitous in catalysis and host-guest applications, making this linker a strong candidate for inclusion into a macrocyclic design. ${ }^{41,42}$ The phenanthroline nitrogen 
long pair are orthogonal to the $\pi$-plane of the macrocycle and do not contribute significant electron density to the frontier orbitals. This suggests the lone pair could be available for hostguest/coordination/sensing interactions.

Figure 9.5 shows the first synthesis attempted to make a macrocycle that comprised a PDI (P) and PA moiety. I first synthesized Compound 9.1 using a literature procedure using 1,4benzenediboronic acid bis(pinacol) ester and 2,9-dichloro-1,10-phenanthroline. The macrocyclization step was inspired by our synthesis of $\mathbf{P P h}_{6}-\mathbf{P h H e x}$. Compound $\mathbf{2 . 2}$ and $\mathbf{9 . 1}$ are then reacted in equimolar ratios for two days. ${ }^{43,44}$ After extraction to remove the salts, the intermediate platinum complex is subjected to reductive elimination. Upon workup, however, the target compound 9.2 was not isolated; instead, both Compounds 9.3 and 9.4 were detected by MALDI. In addition to these two major byproducts, MALDI too showed several additional combinations of PDI and 9.1 (Figure 9.23) after the column was spiked with $\mathrm{NEt}_{3}$. Further purification using GPC was unsuccessful; the macrocycles did not appear to be stable. The multiple byproducts is a curious result; it suggests that there is reversibility in the macrocyclization step, analogous to Chapter 7.45

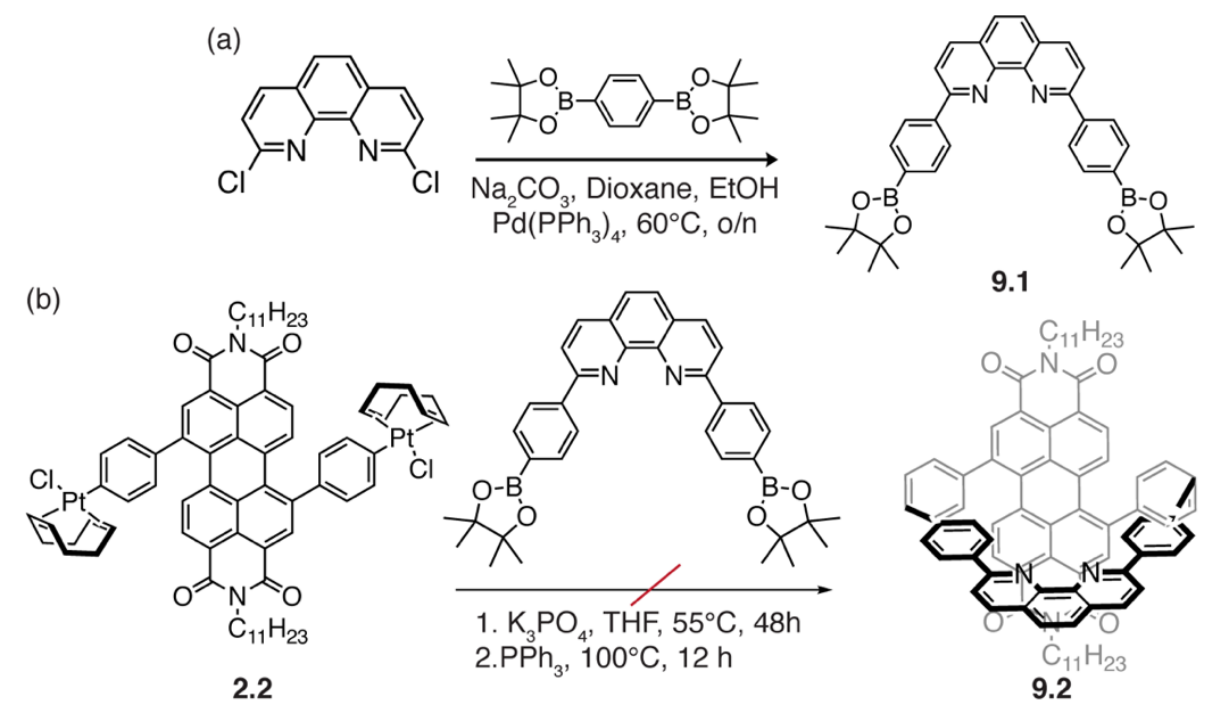

Figure 9.5. (a) Literature synthesis to make 9.1; (b) First synthesis to make macrocycle 9.2. 
One plausible reason we do not observe the targeted macrocycle could be due to strain. The aryl to aryl distance measures $8.39 \AA$ in 9.2 relative to $12.2 \AA$ in an unstrained diphenyl PDI. Chapter 1B discussed the implications a tighter distance between the two aryl rings adjacent to the PDI. The smallest conjugated macrocycle isolated has an aryl-aryl distance of $0.97 \mathrm{~nm}$. According to DFT, both 9.3 and 9.4 have much wider aryl-aryl distances, presumably relieving strain, and stabilizing the complex and formation. Figure 9.5 shows the three energy minimized structures and their aryl-aryl distances.

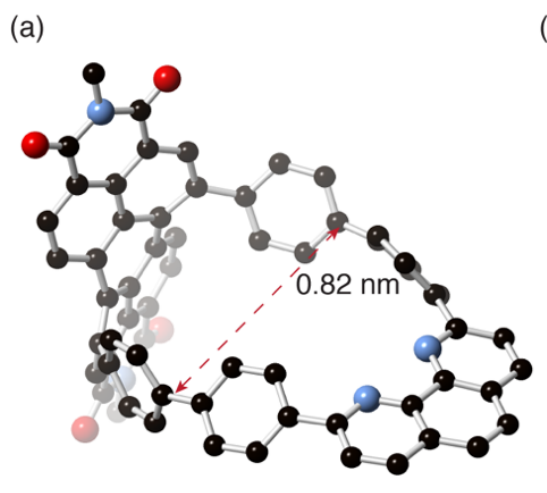

9.2

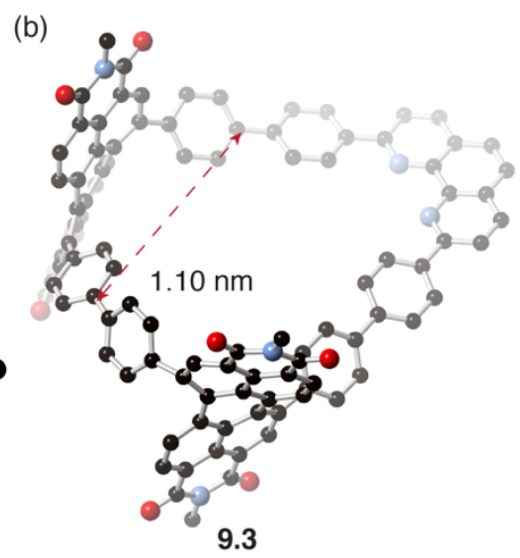

9.3

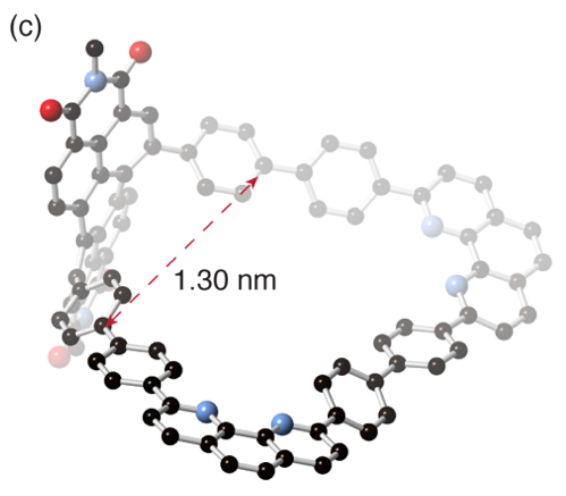

9.4

Figure 9.6. The DFT energy minimized structures for (a) 9.2; (b) 9.3; and (c) 9.4. One hypothesis for not forming $\mathbf{9 . 2}$ could be due to strain. The aryl-aryl distance serves as a quick tool to assess strain. The smallest PDI-based macrocycle synthesized has an aryl-aryl distance at $0.97 \mathrm{~nm}$.

A rational synthesis is required in order to try and isolate either product in high yield. One potential synthesis is proposed in Figure 9.6. Compound 9.1 and excess SI-3.6 could be coupled through a Suzuki reaction to form 9.5. This intermediate provides several opportunities to create interesting topologies. Using a nickel-based homocoupling approach, one could form 9.3. ${ }^{41}$ There is precedent for this approach forming a phenanthroline-incorporated CPP. ${ }^{41}$ One could also envision using Compound 9.5 to form a catenane structure by first exchanging the bromides for BPin, followed by the addition of copper(I) to coordinate two molecules of 9.5 geometry. ${ }^{42}$ This intermediate $(9.6)$ could then be subjected to a oxidative homocoupling strategy ${ }^{43}$ for catenane 
formation. This is one example of how we could incorporate different ligands to build in additional functionality utilizing the nano-space rendered by the macrocyclic design.

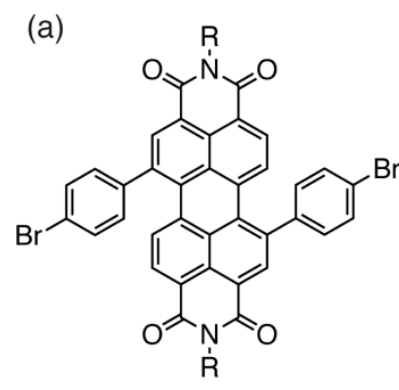

SI-3.6

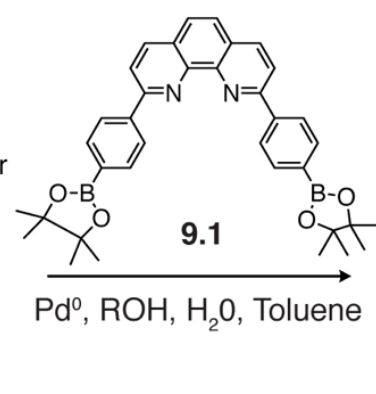

9.1
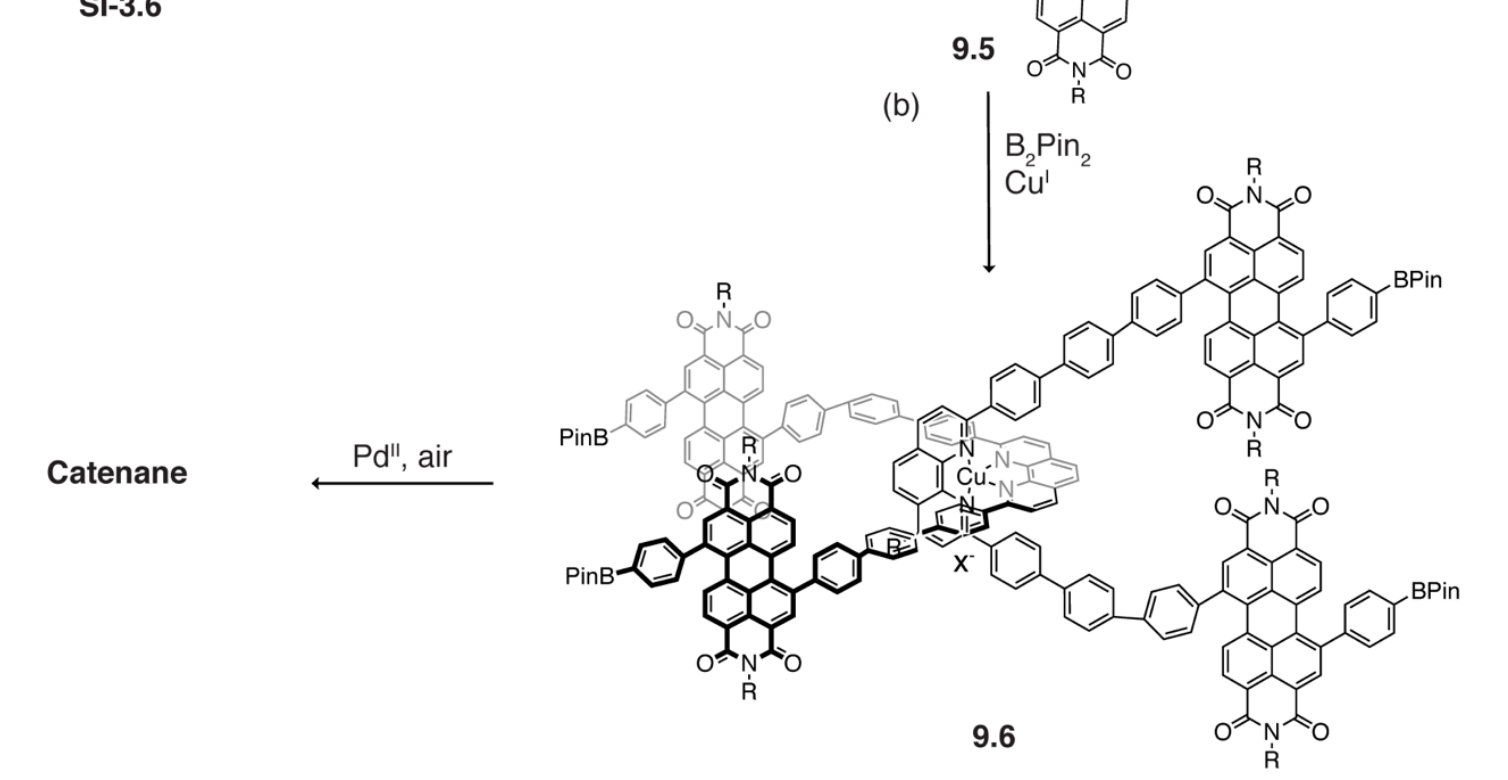

Figure 9.7. (a) A proposed synthesis to form 9.2 using a nickel-based strategy; (b) one could also employ oxidative coupling conditions to yield a catenane structure.

Over the past five years, I have asked myself the question of how fundamental physical organic concepts impact organic electronics - it has been both illuminating and fun to study classroom principles in the laboratory. In particular, the conjugated macrocycles described in this thesis allowed me to explore rigidity, strain, and chirality as they applied to device properties. We found that the cyclic design resulted in interesting properties in each application, which prompted a five-year long discovery process that is ongoing. I look forward to seeing the directions this program takes in the coming years. 


\subsection{Appendix - Supplementary Figures}

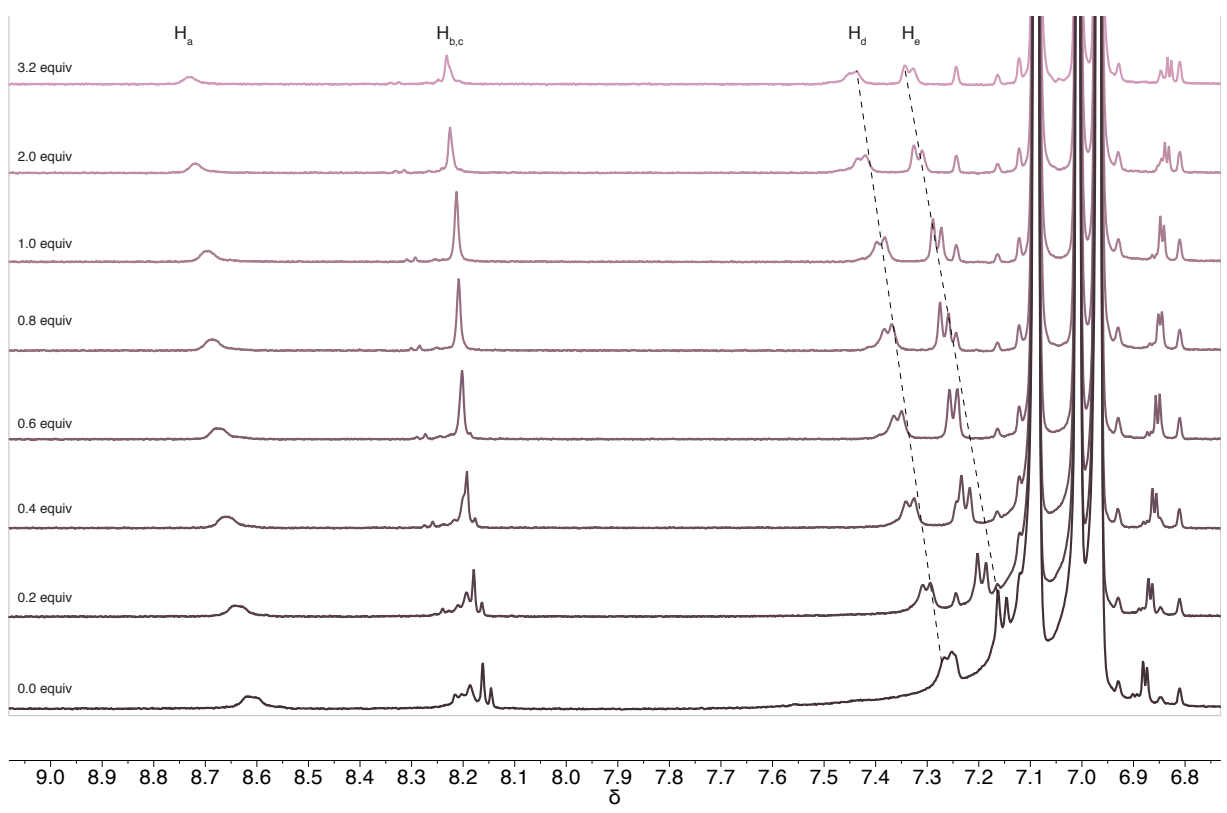

Figure 9.8. The ${ }^{1} \mathrm{H}$ NMR of $\mathbf{P B P B}$ and $\mathbf{C}_{70}$ from 0.0 to 3.2 equivalents. The aryl protons shift dramatically as the concentration of $\mathbf{C}_{70}$ increases.

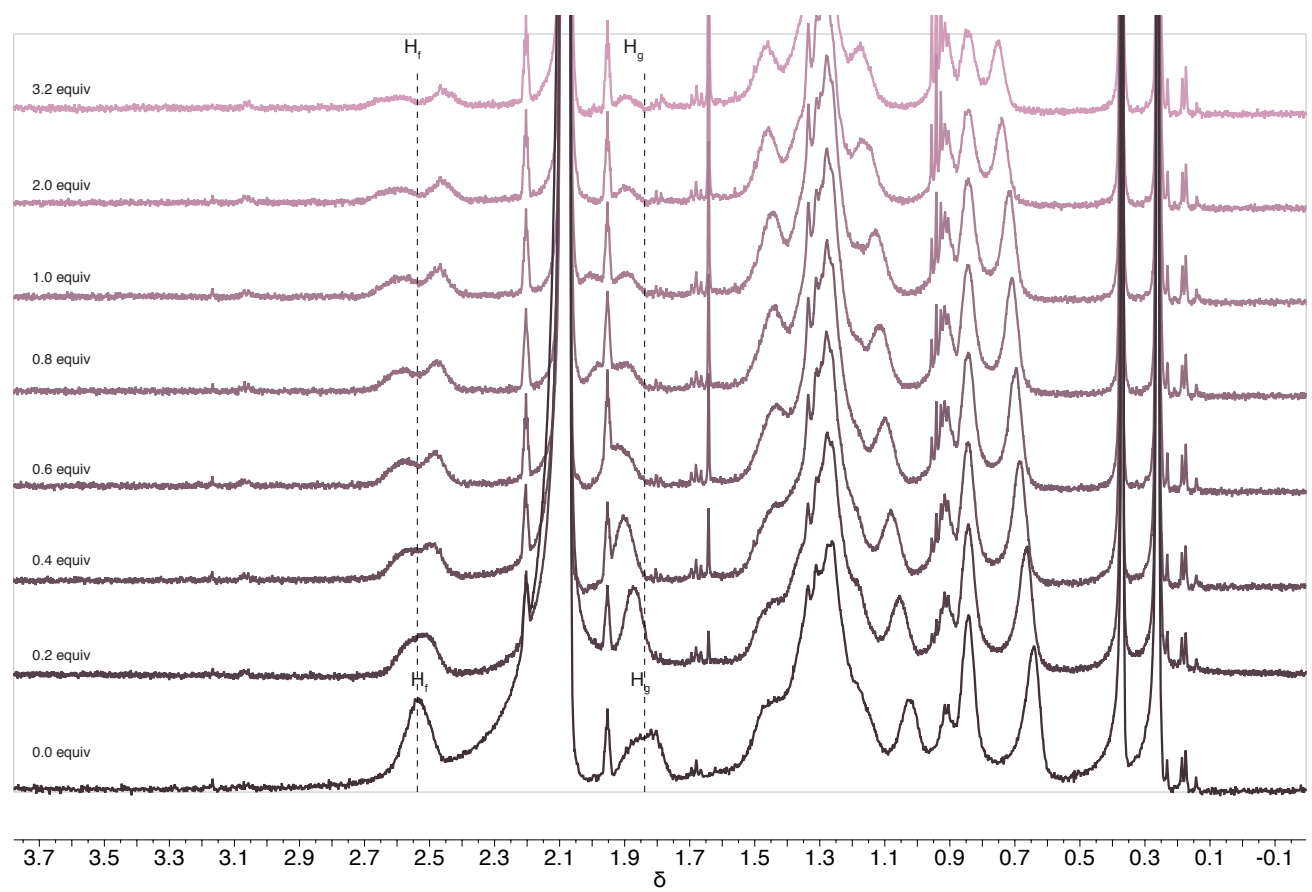

Figure 9.9. The ${ }^{1} \mathrm{H}$ NMR of PBPB and $\mathrm{C}_{70}$ from 0.0 to 3.2 equivalents. The alkyl protons split into two as the concentration of $\mathrm{C}_{70}$ increases, indicative of outside and inside environments for methylenes. 


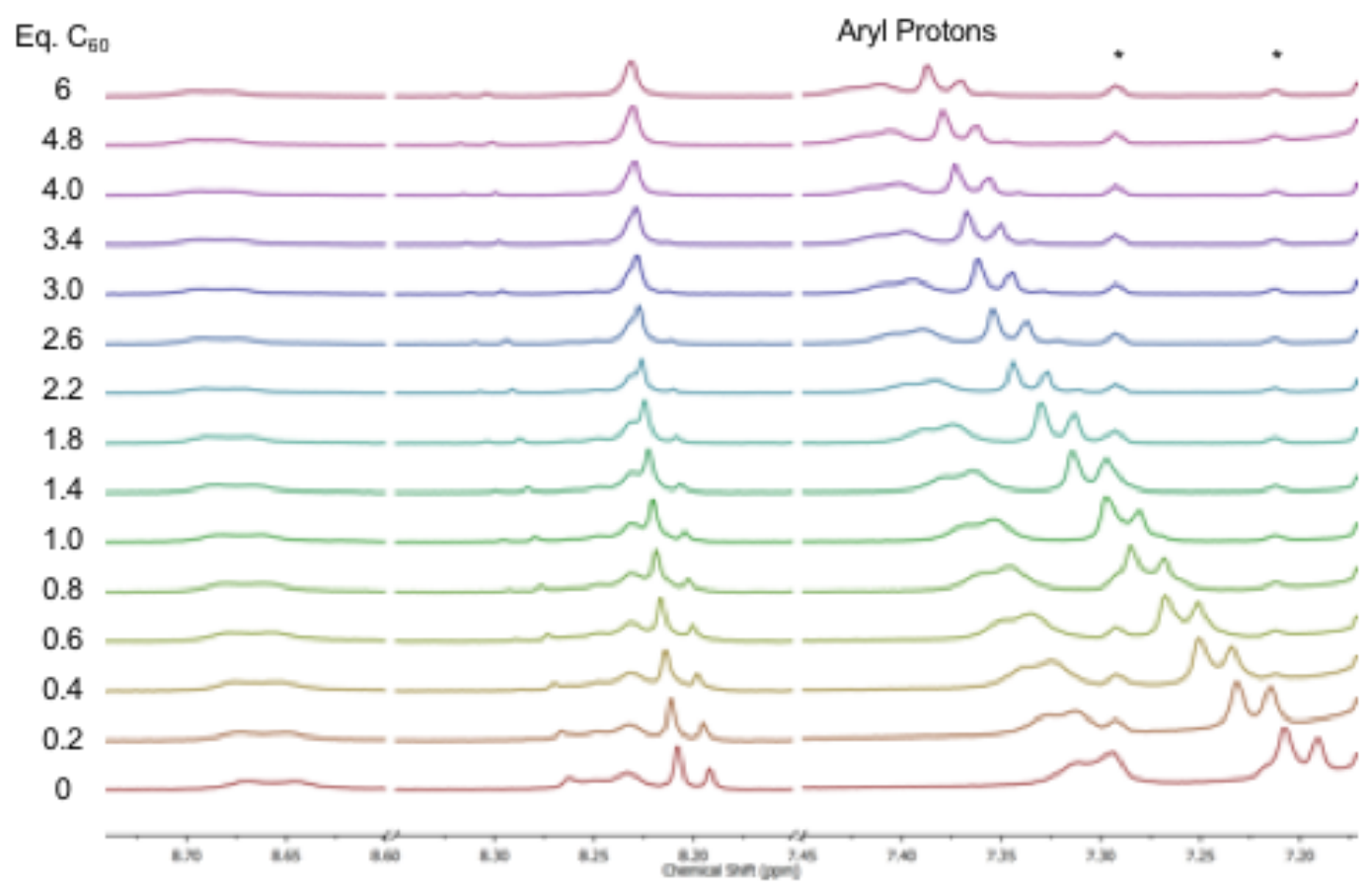

Figure 9.10. The ${ }^{1} \mathrm{H}$ NMR of PBPB and $\mathbf{C}_{60}$ from 0.0 to 6 equivalents.

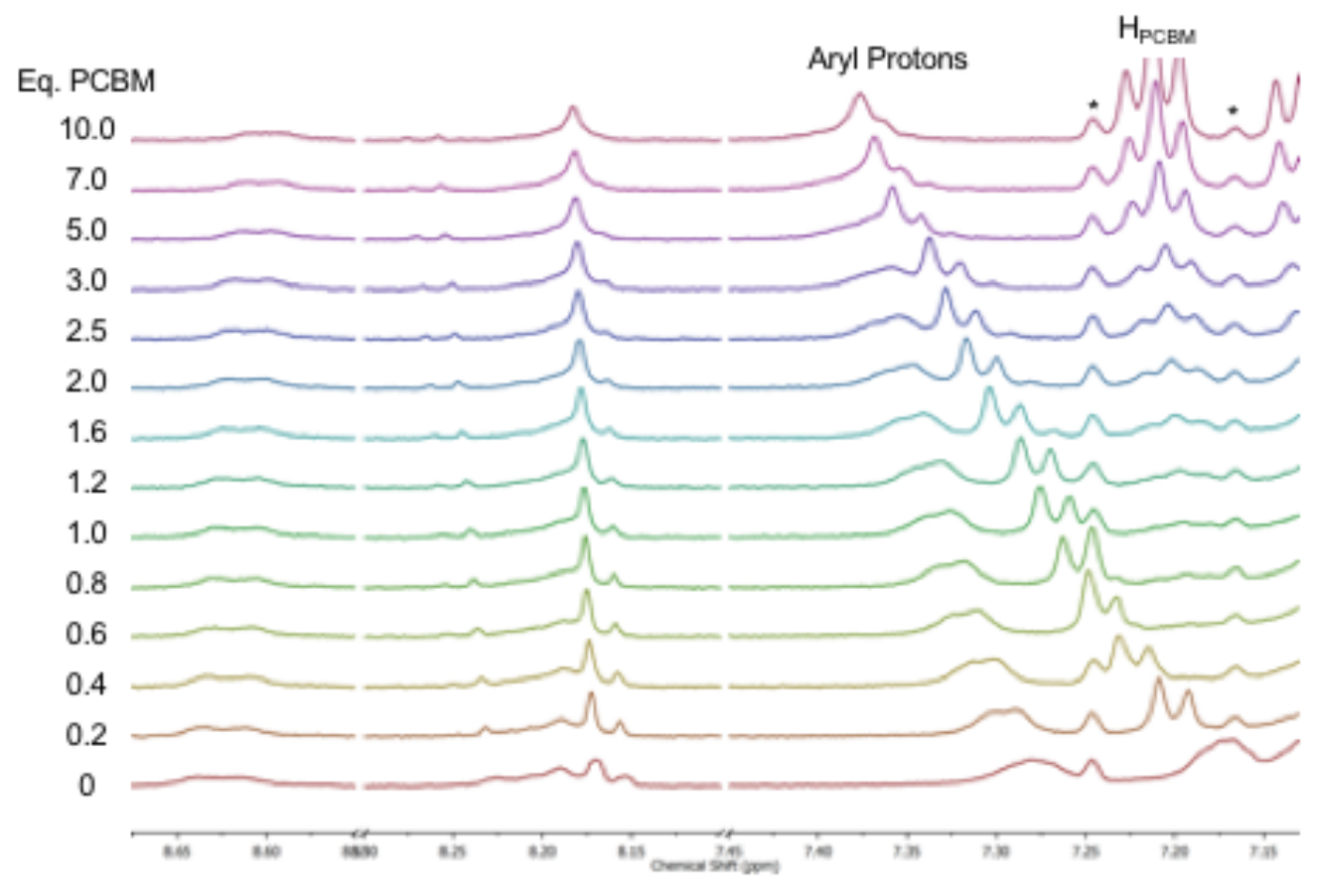

Figure 9.11. The ${ }^{1} \mathrm{H}$ NMR of PBPB and PCBM from 0.0 to 6 equivalents. 


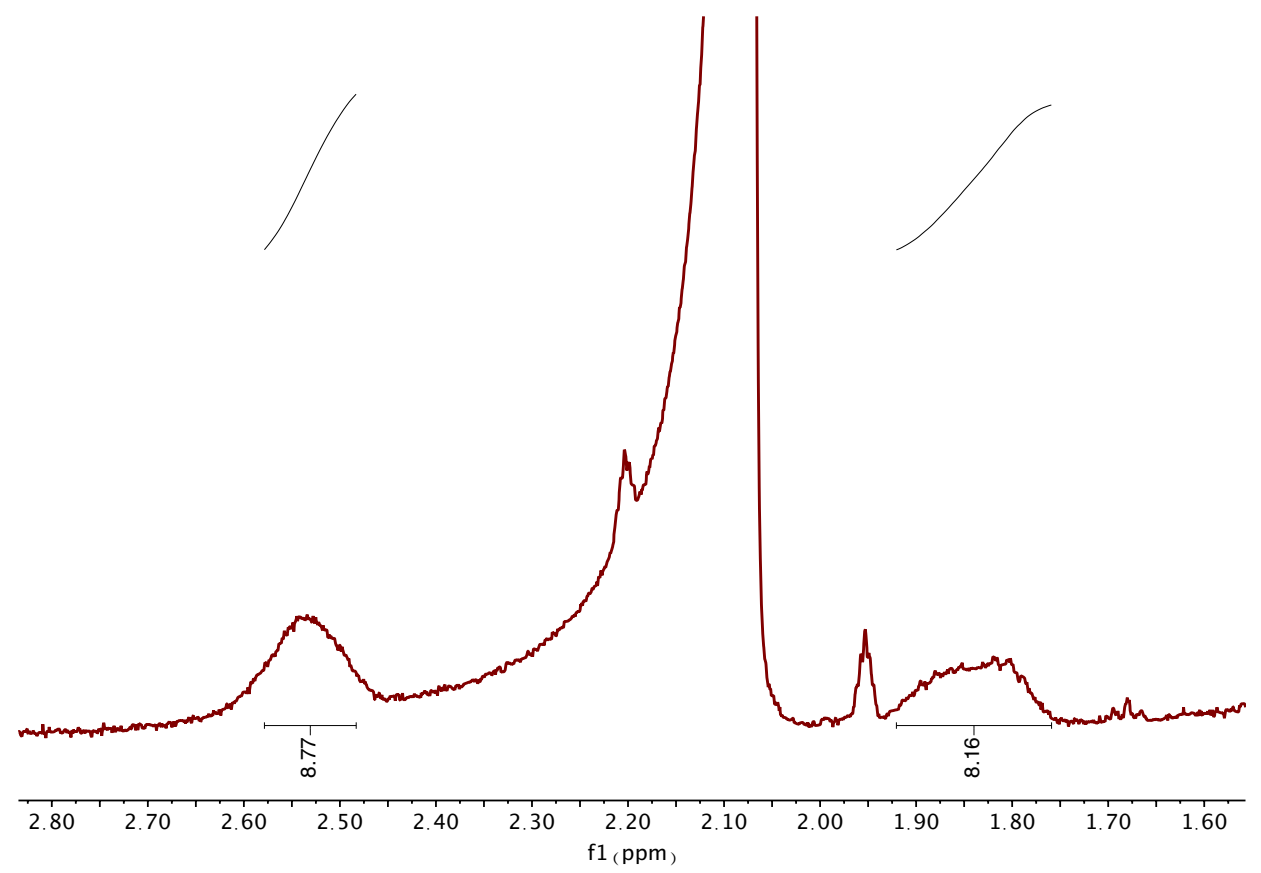

Figure 9.12. The ${ }^{1} \mathrm{H}$ NMR of PBPB alkyl region showing the methylene protons ortho to the methine $\left(\mathrm{H}_{\mathrm{f}}\right.$ and $\left.\mathrm{H}_{\mathrm{g}}\right)$ as two broad peaks as they are diastereotopic. Each integrates to eight protons.

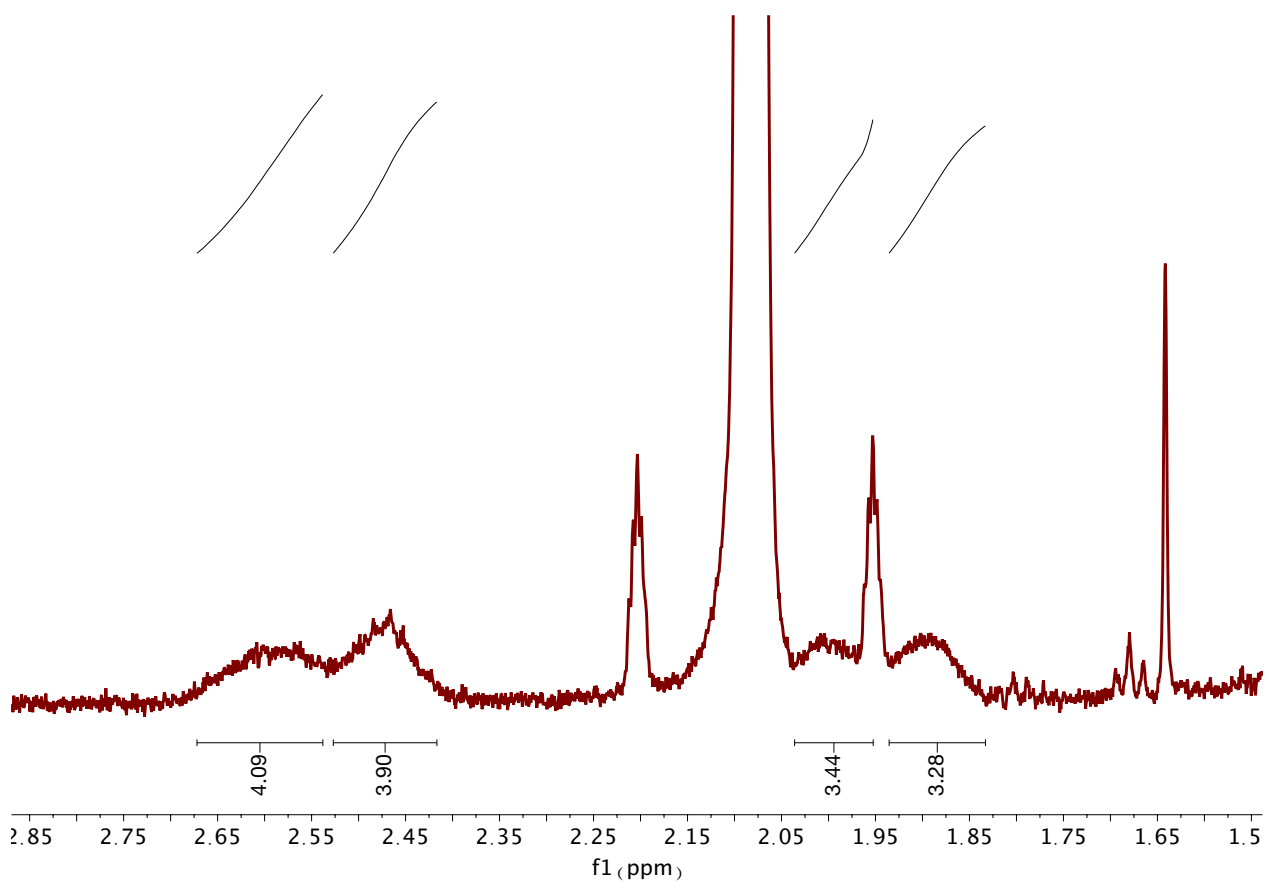

Figure 9.13. The ${ }^{1} \mathrm{H}$ NMR of $\mathbf{P B P B}$ and 1.0 equivalent of $\mathbf{C}_{\mathbf{7 0}}$. The methylene protons $\left(\mathrm{H}_{\mathrm{f}}\right.$ and $\mathrm{H}_{\mathrm{g}}$ ) ortho to the methine split into two broad peaks and are now inequivalent. 


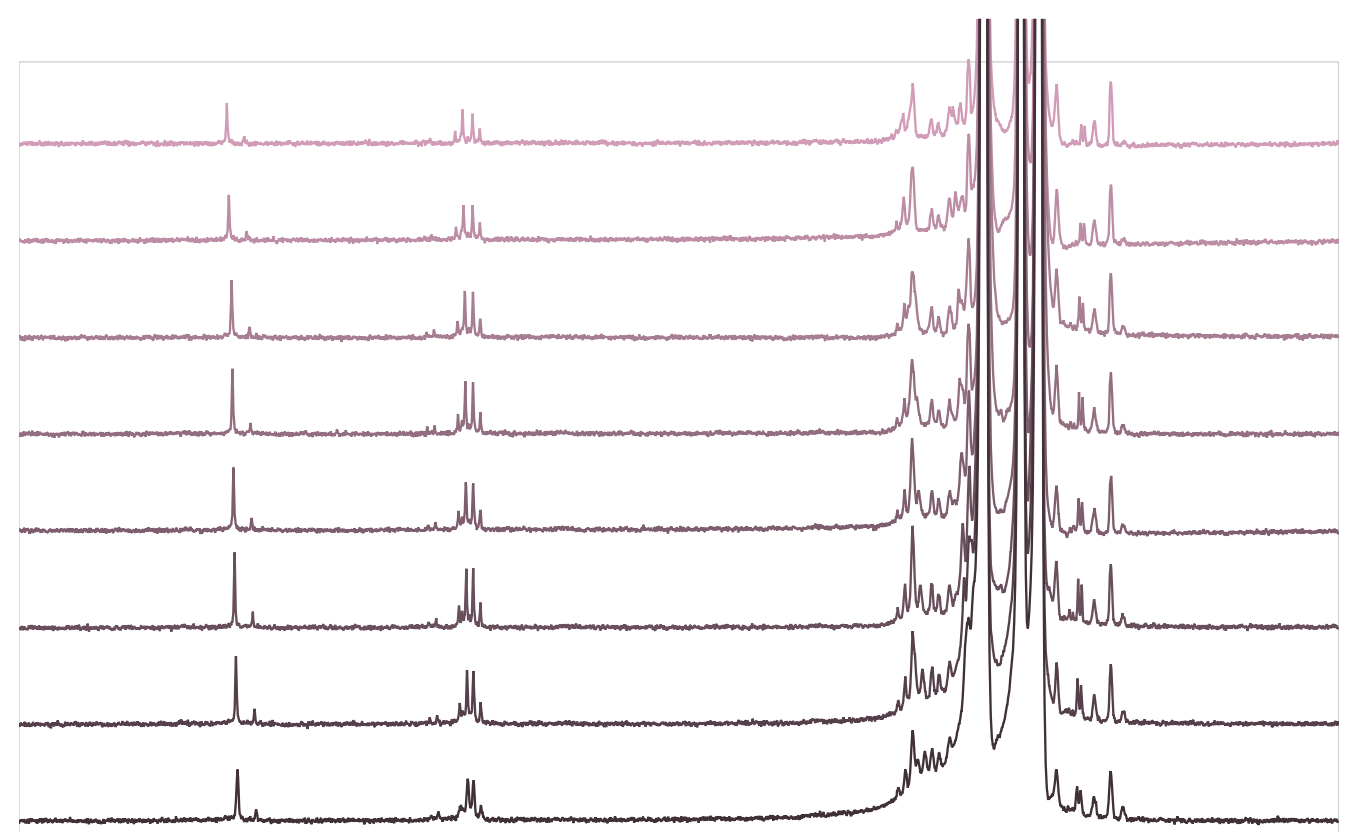

$\begin{array}{llllllllllllllllllllllllllllllllllllllll}9.1 & 9.0 & 8.9 & 8.8 & 8.7 & 8.6 & 8.5 & 8.4 & 8.3 & 8.2 & 8.1 & 8.0 & 7.9 & 7.8 & 7.7 & 7.6 & 7.5 & 7.4 & 7.3 & 7.2 & 7.1 & 7.0 & 6.9 & 6.8 & 6.7 & 6.6 & 6.5 & 6.4\end{array}$

Figure 9.14. The ${ }^{1} \mathrm{H}$ NMR of aryl-PBPB up to 3.4 equivalent of $\mathbf{C}_{70}$.
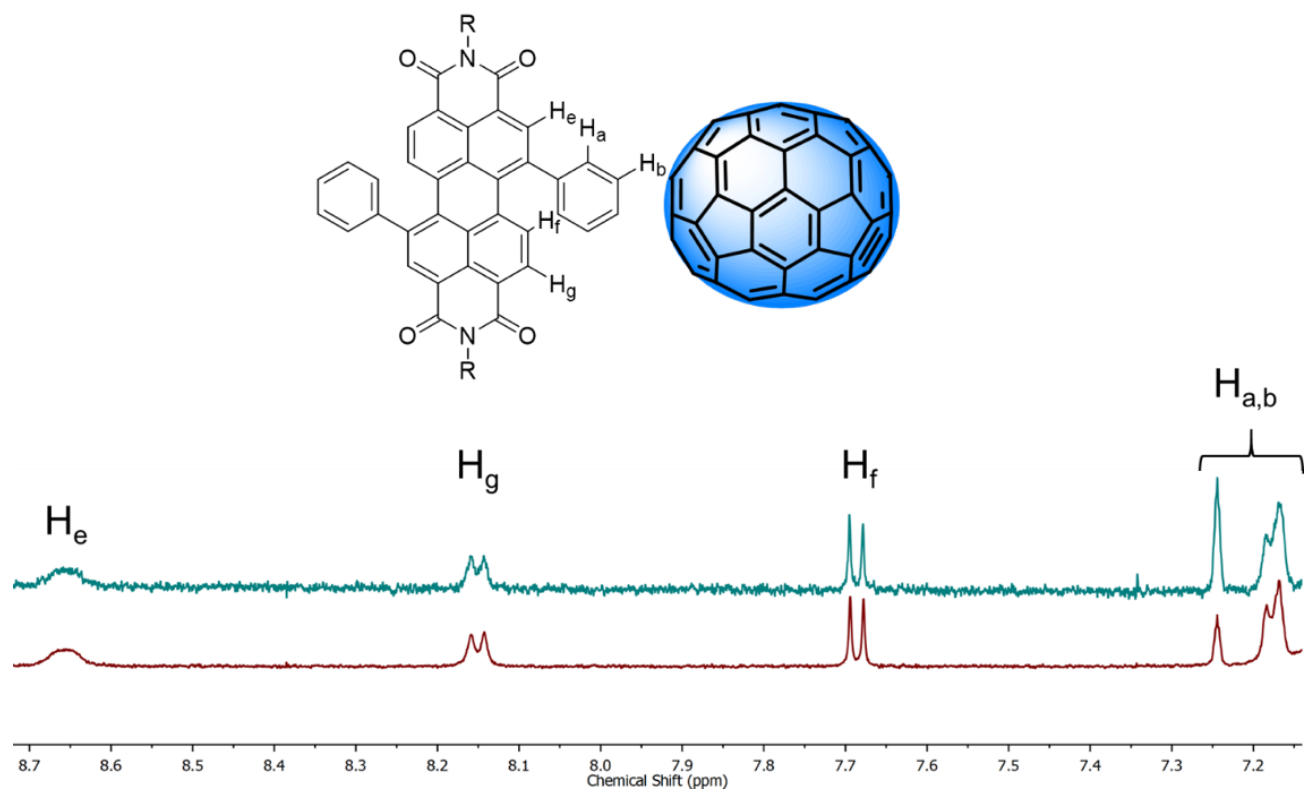

Figure 9.15. Truncated ${ }^{1} \mathrm{H}$ NMR spectra of Compound 3.4 (bottom) and after the addition of four equivalents of $\mathbf{C}_{70}$ fullerene (top) (d8-toluene, $298 \mathrm{~K}, 500 \mathrm{MHz}, \mathrm{R}=\mathrm{C}_{11} \mathrm{H}_{23}$ ). 


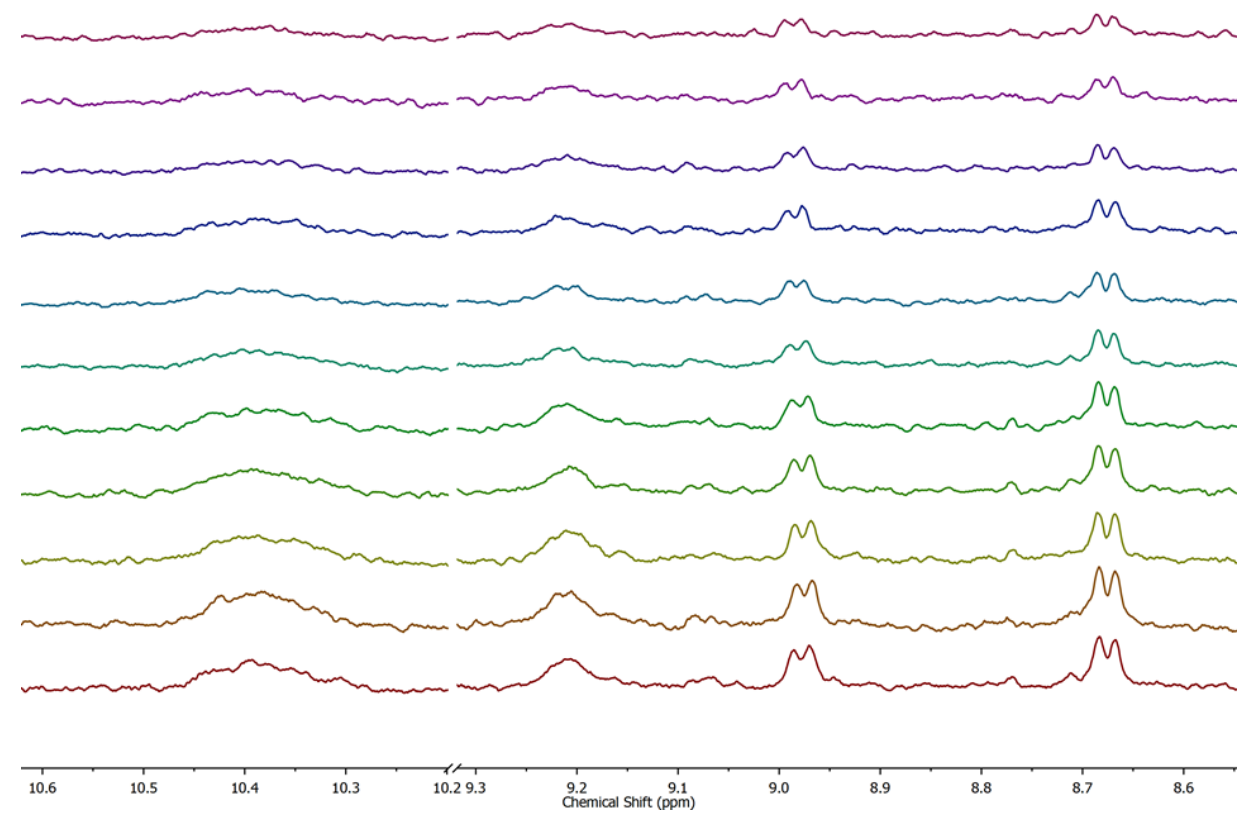

Figure 9.16. Truncated ${ }^{1} \mathrm{HNMR}$ spectra of trans-DBDB (Chapter 8) and after the addition of four equivalents of $\mathbf{C}_{70}$ fullerene ( $\mathrm{d}_{8}$-toluene, $298 \mathrm{~K}, 500 \mathrm{MHz}$ ). $\Delta \delta \mathrm{H}_{\mathrm{a}}=0.01 \mathrm{ppm}$ and so too small to be accurately fitted.

(a) $[\mathbf{P B P B}] \supset\left[\mathbf{C}_{70}\right]$

$K_{\mathrm{a}}=9,278(7 \%)$

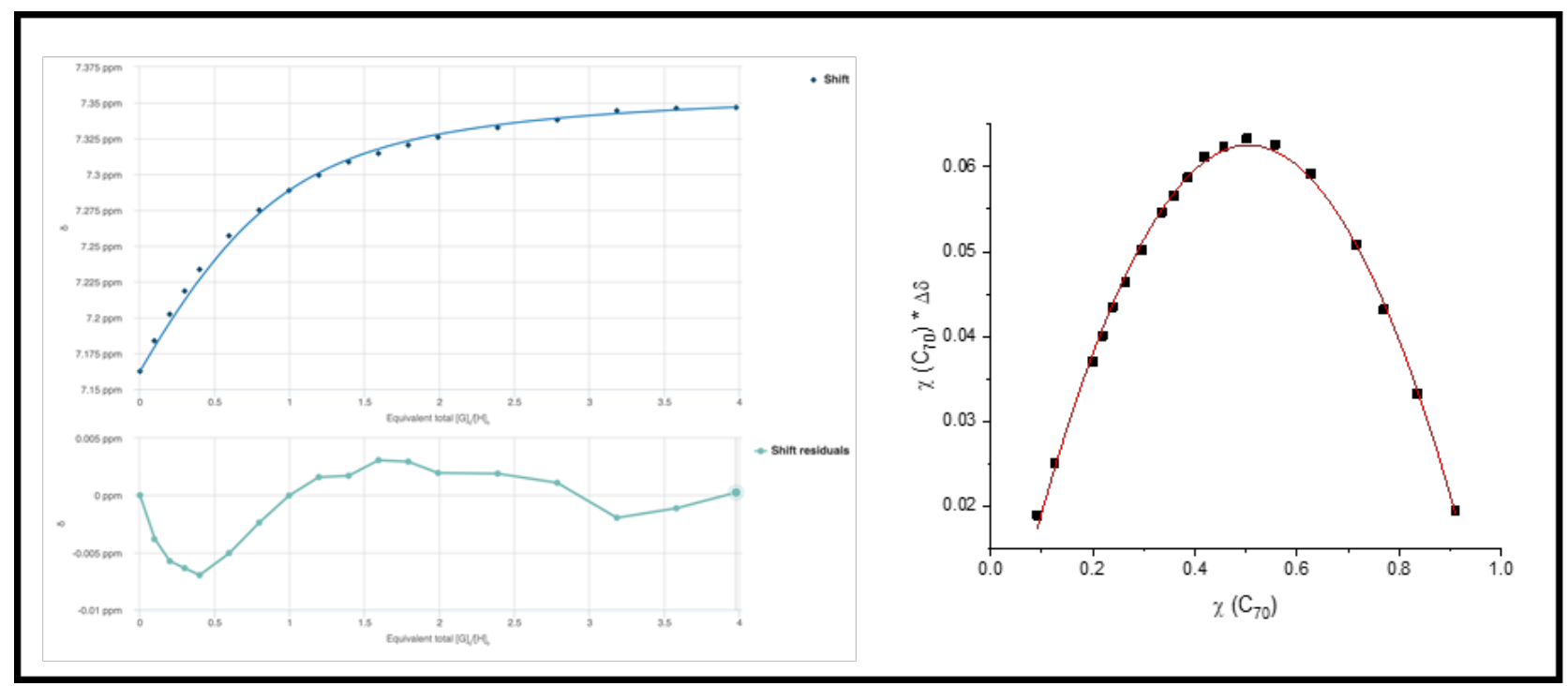

(b) $[\mathrm{PBPB}] \supset\left[\mathrm{C}_{60}\right]$

$K_{\mathrm{a}}=2,119(1 \%)$ 


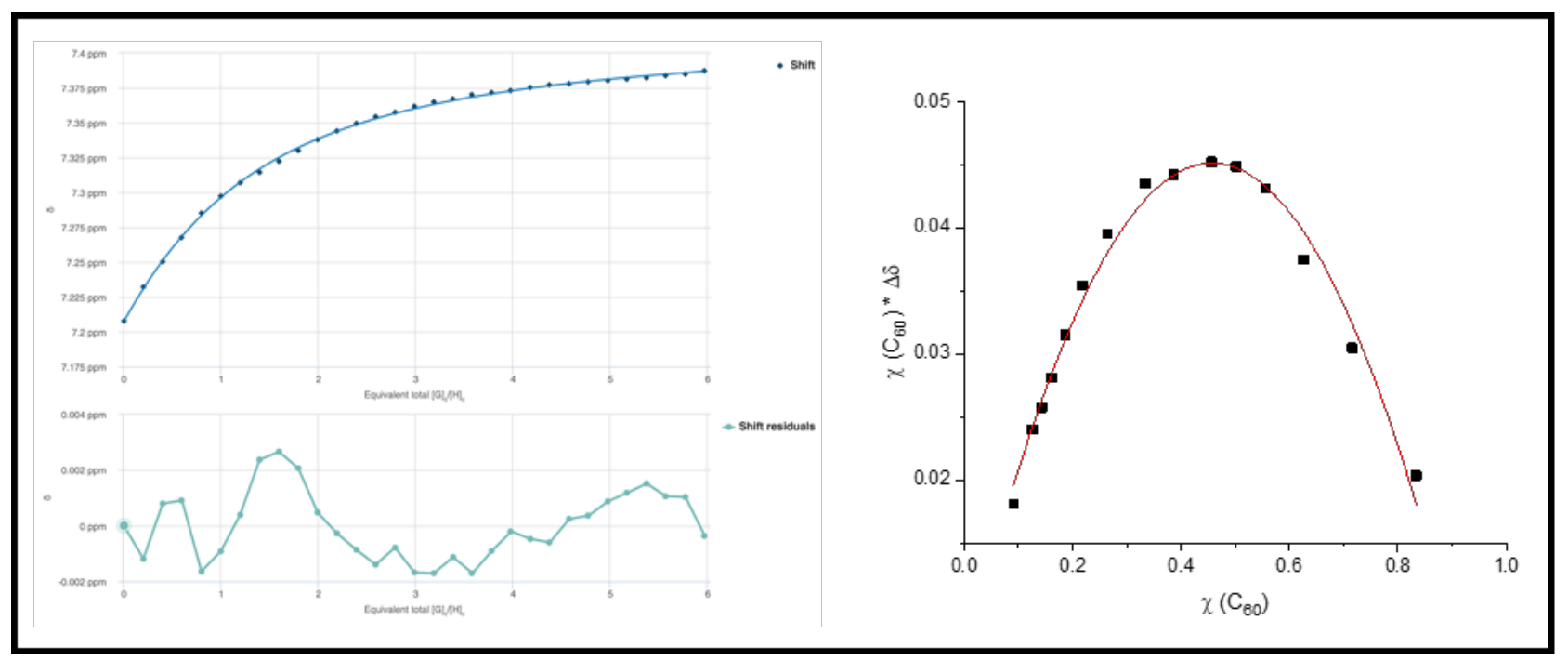

(c) $[$ PBPB $] \supset[$ PCBM $]$

$K_{\mathrm{a}}=1,336(2 \%)$

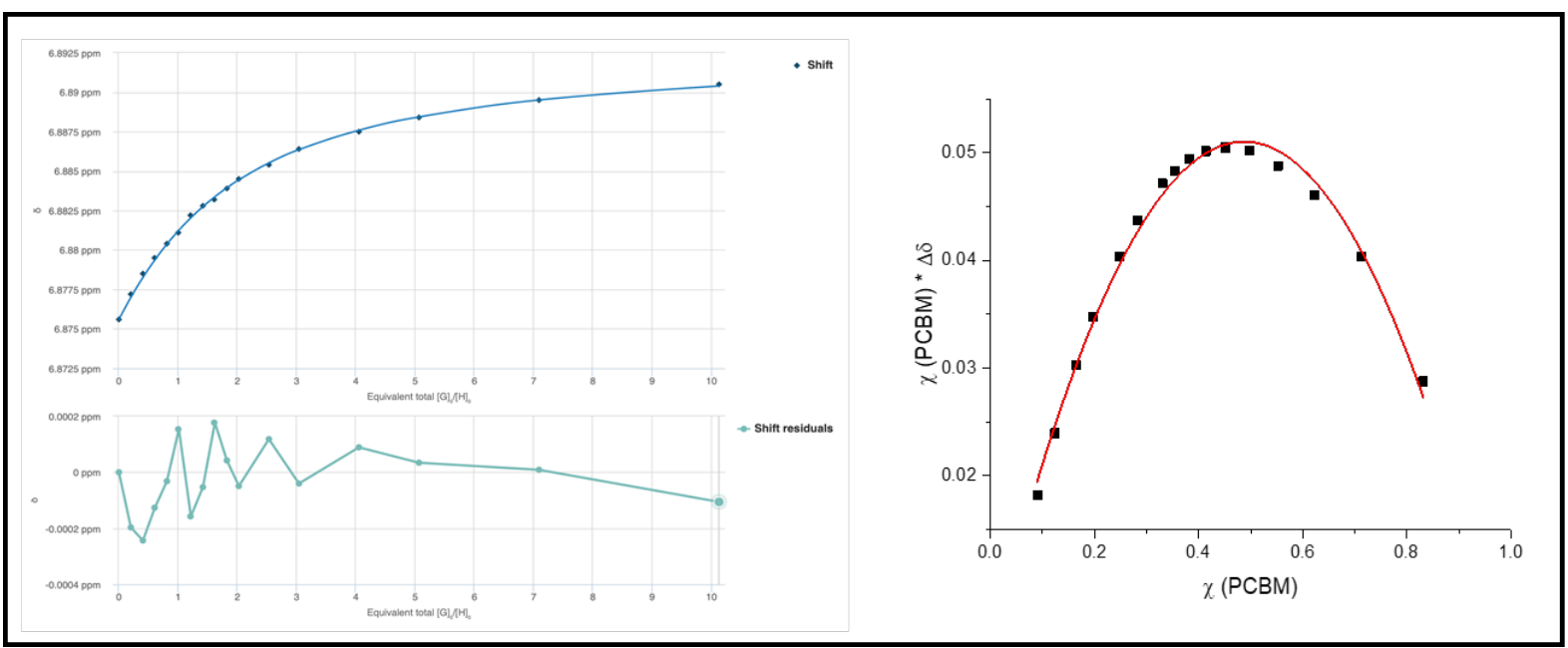

(d) $[$ aryl-PBPB $] \supset\left[\mathbf{C}_{70}\right]$

$K_{\mathrm{a}}=591(1 \%)$ 


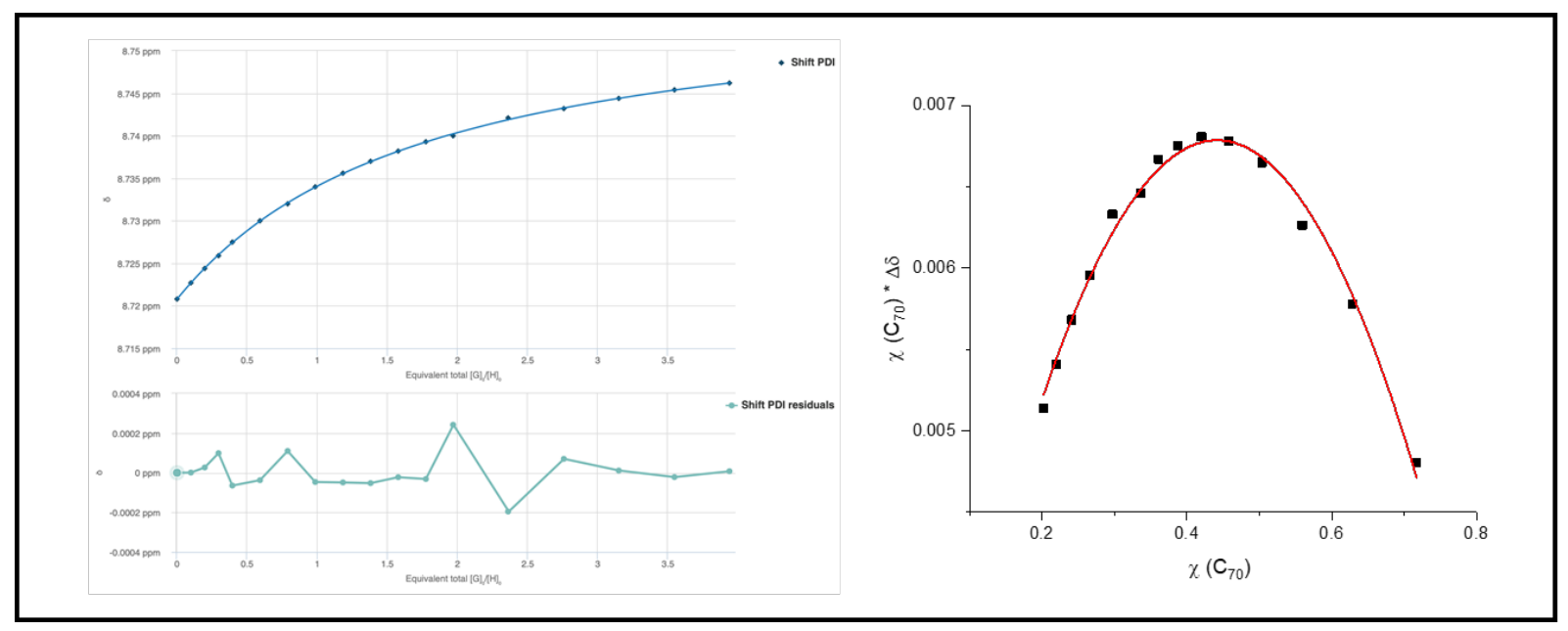

Figure 9.17. ${ }^{1} \mathrm{H}$ NMR spectroscopic titration curves and residuals for PBPB and aryl-PBPB upon titration with fullerene guests. Solid points represent experimental data, continuous line represents theoretical binding isotherm. Errors for association constants are given in brackets. Job's plots for complexes indicate a 1:1 binding stoichiometry; the maximum change in chemical shift occurs when the mole fraction of fullerene guest $\chi(F)=0.5$.
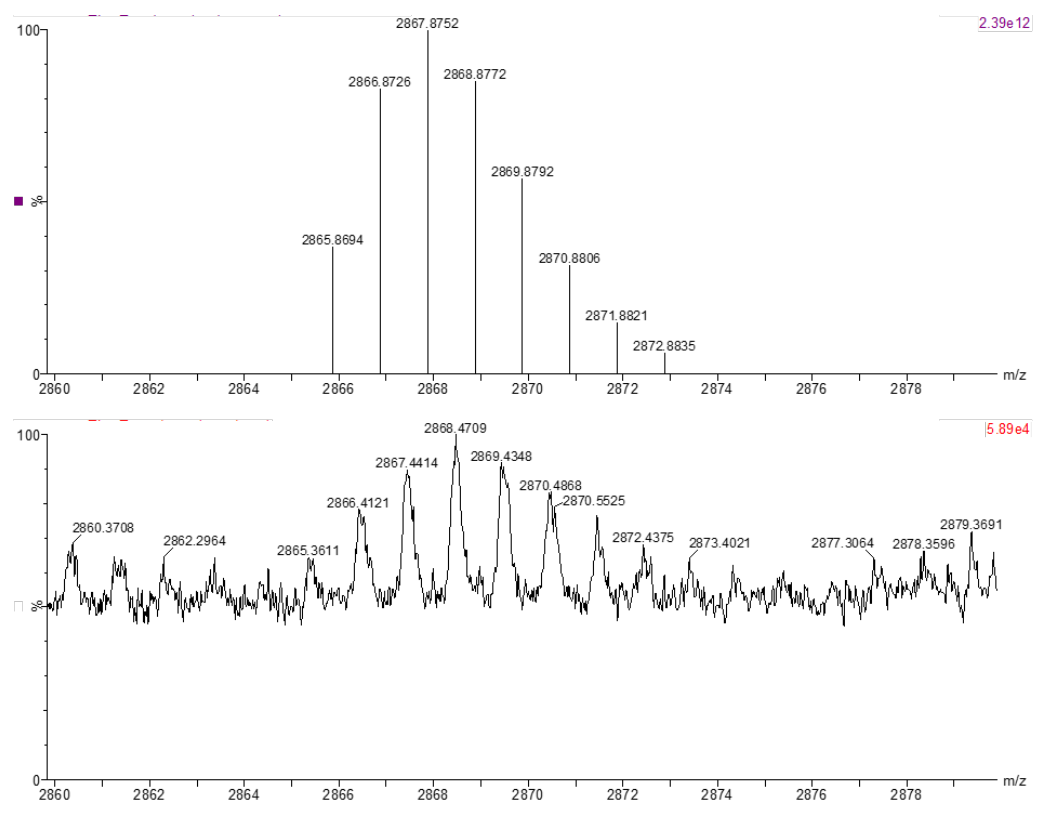

Figure 9.18. Top: The theoretical ESI mass spectrum of the 1:1 stoichiometric host guest complex $[\mathrm{PBPB}] \supset\left[\mathbf{C}_{70}\right]$. Bottom: Experimental ESI mass spectrum of the 1:1 stoichiometric host guest complex [PBPB $] \supset\left[\mathbf{C}_{70}\right]$. 


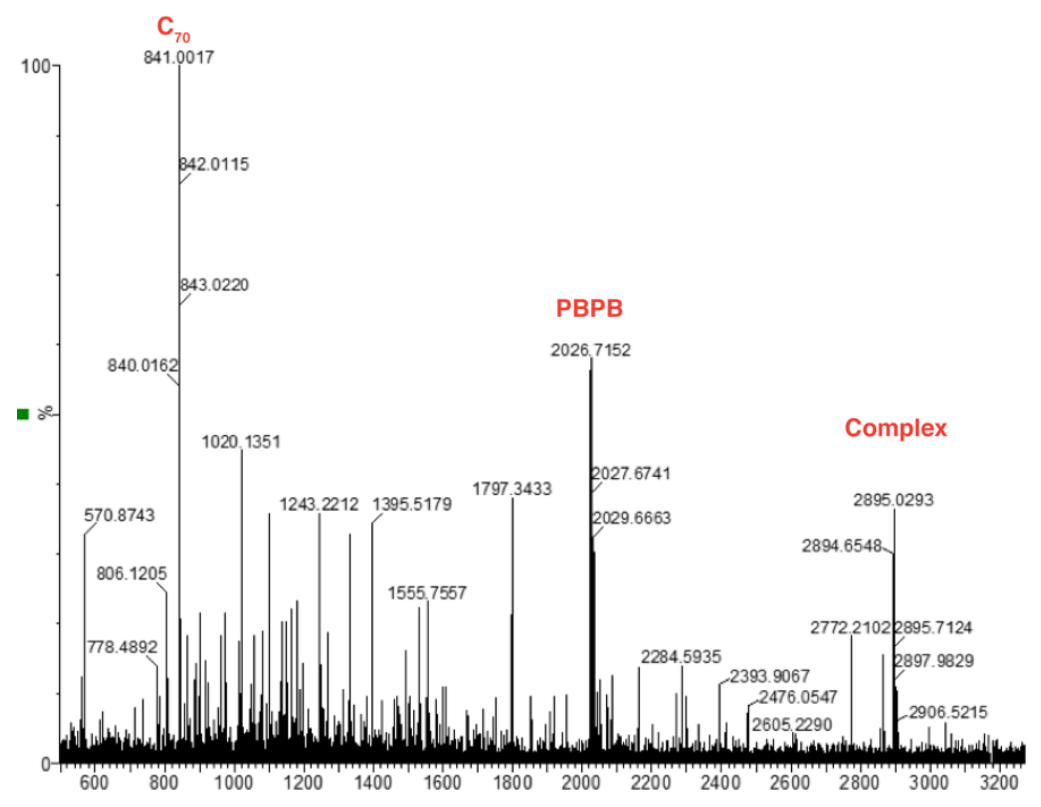

Figure 9.19. The ESI MS/MS spectrum of $[\mathbf{P B P B}] \supset\left[\mathbf{C}_{70}\right]$ as the complex is broken into its constituent parts (collision energy $=80-100 \mathrm{eV}$ ).

(a)

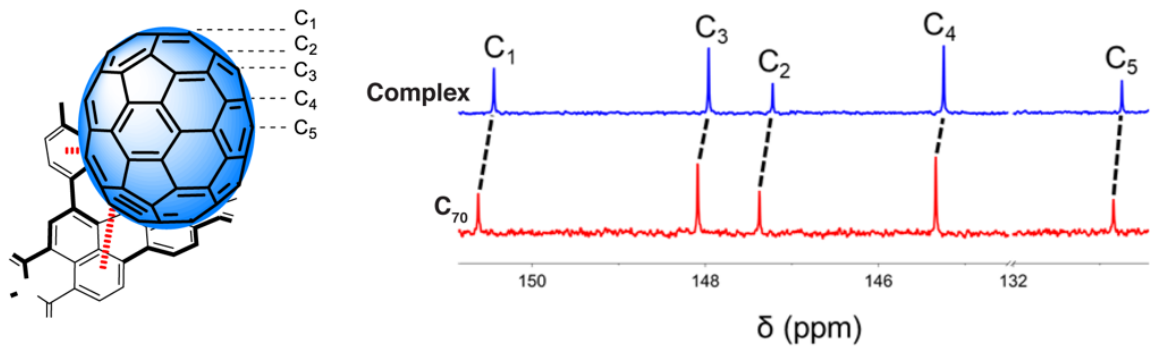

(b)

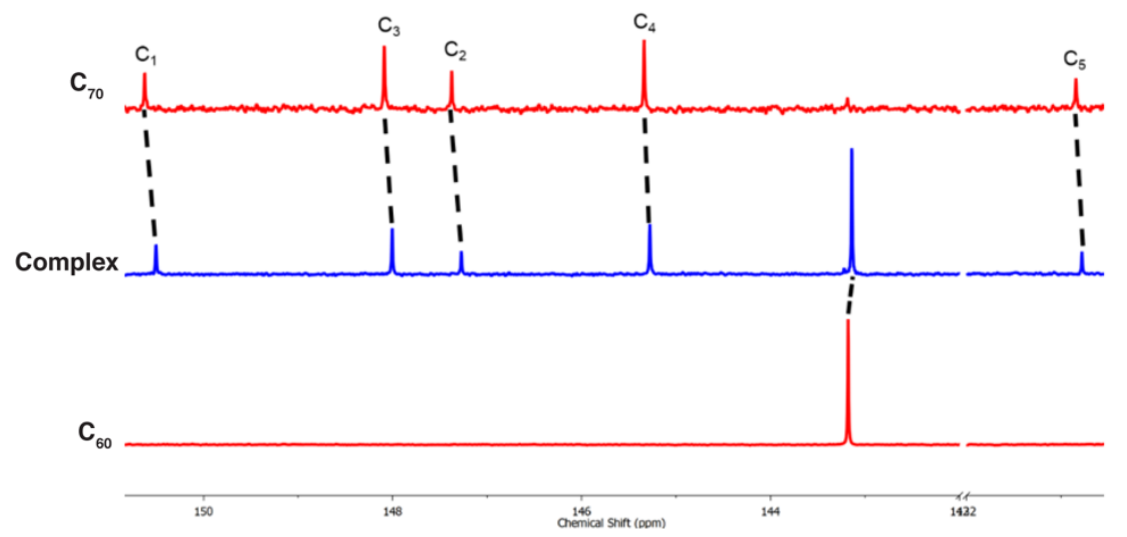

Figure 9.20. (a) Truncated ${ }^{13} \mathrm{C}$ NMR spectra showing $\mathbf{C}_{70}$ fullerene and a 1:1.5 PBPB: $\mathbf{C}_{70}$ stoichiometric mixture (d8-toluene, $298 \mathrm{~K}, 125 \mathrm{MHz}$ ). Carbon labels for $\mathbf{C}_{70}$ are also given. (b) Truncated ${ }^{13} \mathrm{C}$ NMR spectra showing $\mathbf{C}_{60}$ fullerene, $\mathbf{C}_{70}$ fullerene and a 1:1.5:1.5 PBPB: $\mathbf{C}_{60}: \mathbf{C}_{70}$ stoichiometric mixture ( $\mathrm{d}_{8}$-toluene, $298 \mathrm{~K}, 125 \mathrm{MHz}$ ). 

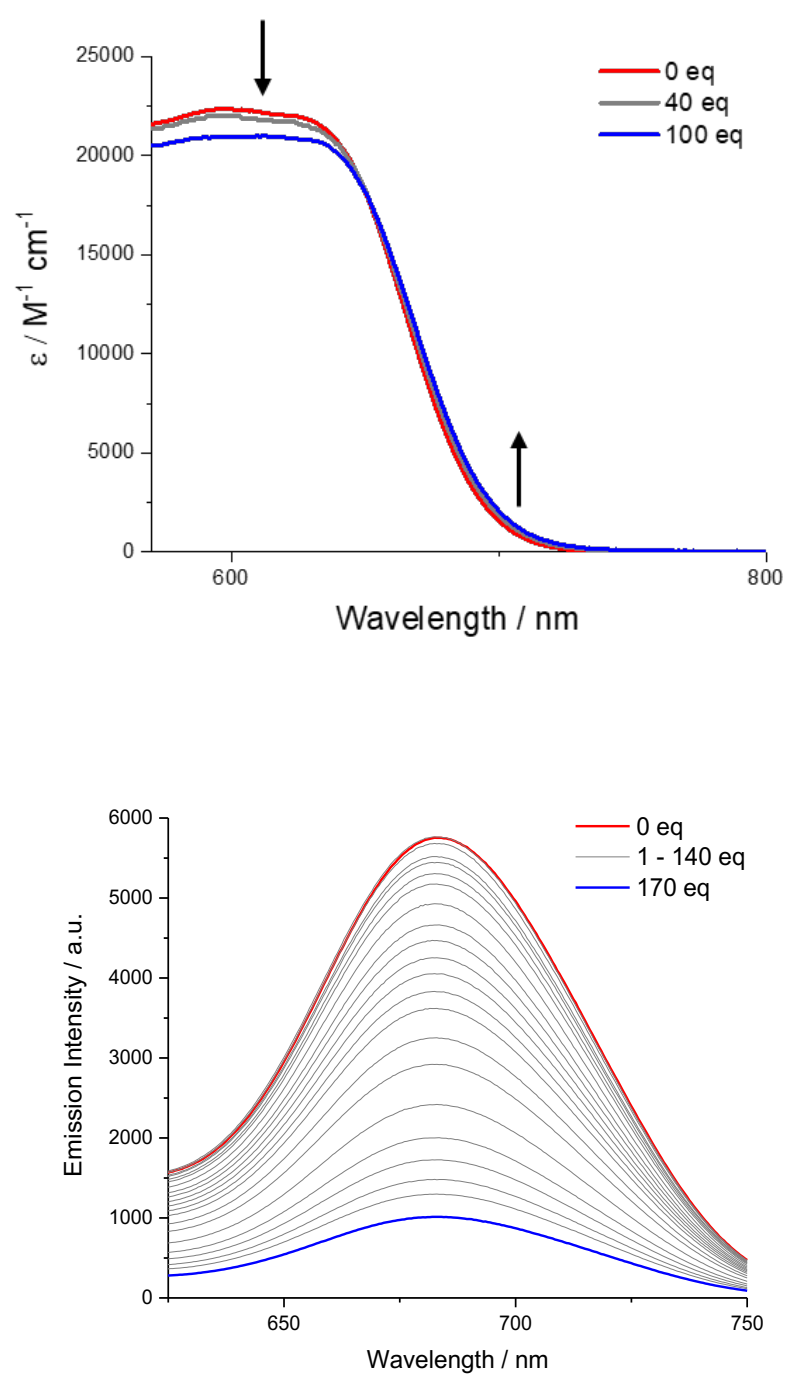

Figure 9.21. (top) Electronic absorption spectrum for PBPB upon addition of $\mathbf{C}_{60}$ correct for $\mathbf{C}_{60}$ absorptions; (bottom) Florescence emission spectrum of PBPB with titration of $\mathbf{C}_{60}$ (toluene, 4 $\left.\mu \mathrm{M}, \lambda_{\mathrm{ex}}=600 \mathrm{~nm}\right)$. 


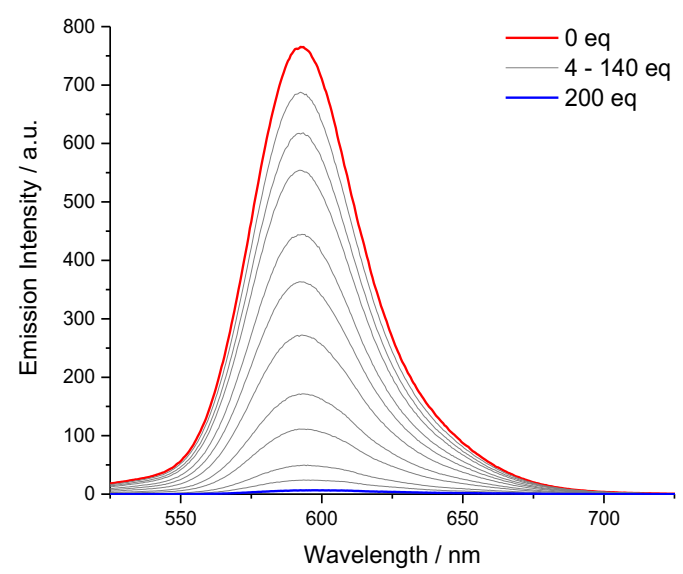

Figure 9.22. Florescence emission spectrum of $\mathbf{3 . 4}$ with titration of $\mathbf{C}_{60}$ (toluene, $4 \mu \mathrm{M}, \lambda_{\mathrm{ex}}=600$ $\mathrm{nm})$.

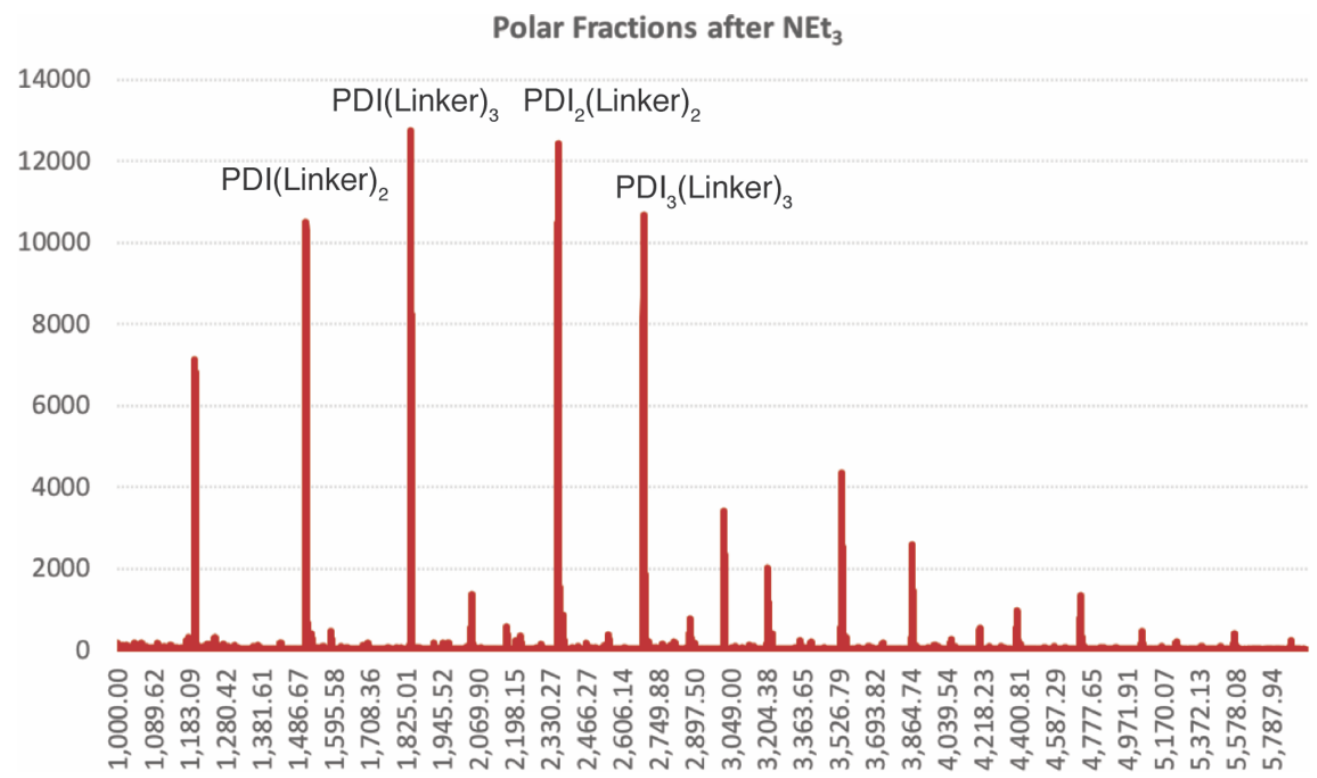

Figure 9.23. MALDI from first attempt to synthesize PDI-phenanthroline macrocycle showing many products formed due to the reversibility of the reaction.

\subsection{General Experimental Information}

Synthesis. All reactions were performed in oven-dried or flame-dried round bottom flasks, unless otherwise noted. The flasks were fitted with rubber septa and reactions were conducted under a positive pressure of nitrogen or argon, unless otherwise noted. Anhydrous and anaerobic solvents were obtained from a Glass Contour solvent system consisting of a Schlenk manifold with 
purification columns packed with activated alumina and supported copper catalyst. Reaction monitoring by thin layer chromatography (TLC) was performed on J.T. Baker Baker-flex Silica Gel IB2-F (25 mm x $75 \mathrm{~mm}$ ) TLC plates. TLC visualization was accomplished by visible observation and irradiation with a UV lamp. Commercial reagents were used without further purification. $\mathrm{Pt}(\mathrm{COD}) \mathrm{Cl}_{2}$ was purchased from Strem Chemicals, and all other reagents were purchased from Sigma-Aldrich. Compounds 3.4 (Chapter 3), trans-DBDB (Chapter 8) and PBPB (Chapter 2) have been characterized elsewhere in this thesis.

Purification. Automated flash chromatography was performed using a Teledyne Isco Combiflash Rf200 and Redisep Rf Silica columns. Preparative HPLC was performed on a Waters Prep150 instrument equipped with a UV-vis detector $(335 \mathrm{~nm})$, an automated fraction collector, and a Nacalai Tesque COSMOSIL Buckyprep column (20 mm I.D. x $250 \mathrm{~mm}, 5 \mu \mathrm{m})$.

Spectrometers. ${ }^{1} \mathrm{H}$ NMR spectra were recorded on a Bruker 400 or $500 \mathrm{MHz}$ spectrometer. ${ }^{13} \mathrm{C}$ NMR spectra were recorded on a Bruker 125 or $100 \mathrm{MHz}$ spectrometer with complete proton decoupling. NMR spectra were recorded at $300 \mathrm{~K}$ unless otherwise noted. Chemical shifts are reported in parts per million (ppm) Data are represented as follows: chemical shift, multiplicity $(\mathrm{s}=$ singlet, $\mathrm{d}=$ doublet, $\mathrm{dd}=$ doublet of doublets, $\mathrm{t}=$ triplet, $\mathrm{m}=$ multiplet $)$, coupling constants in $\mathrm{Hz}$, and integration. HRMS was performed on a Waters XEVO G2XS instrument equipped with a UPC SFC ESI and APCI ionization, and a QToF mass spectrometer. Absorption spectra were obtained on Shimadzu UV 1800 UV-Vis spectrophotometer and emission spectra were recorded in a Fluorolog-3 spectrophotometer. 


\subsection{NMR Spectra of aryl-PBPB}

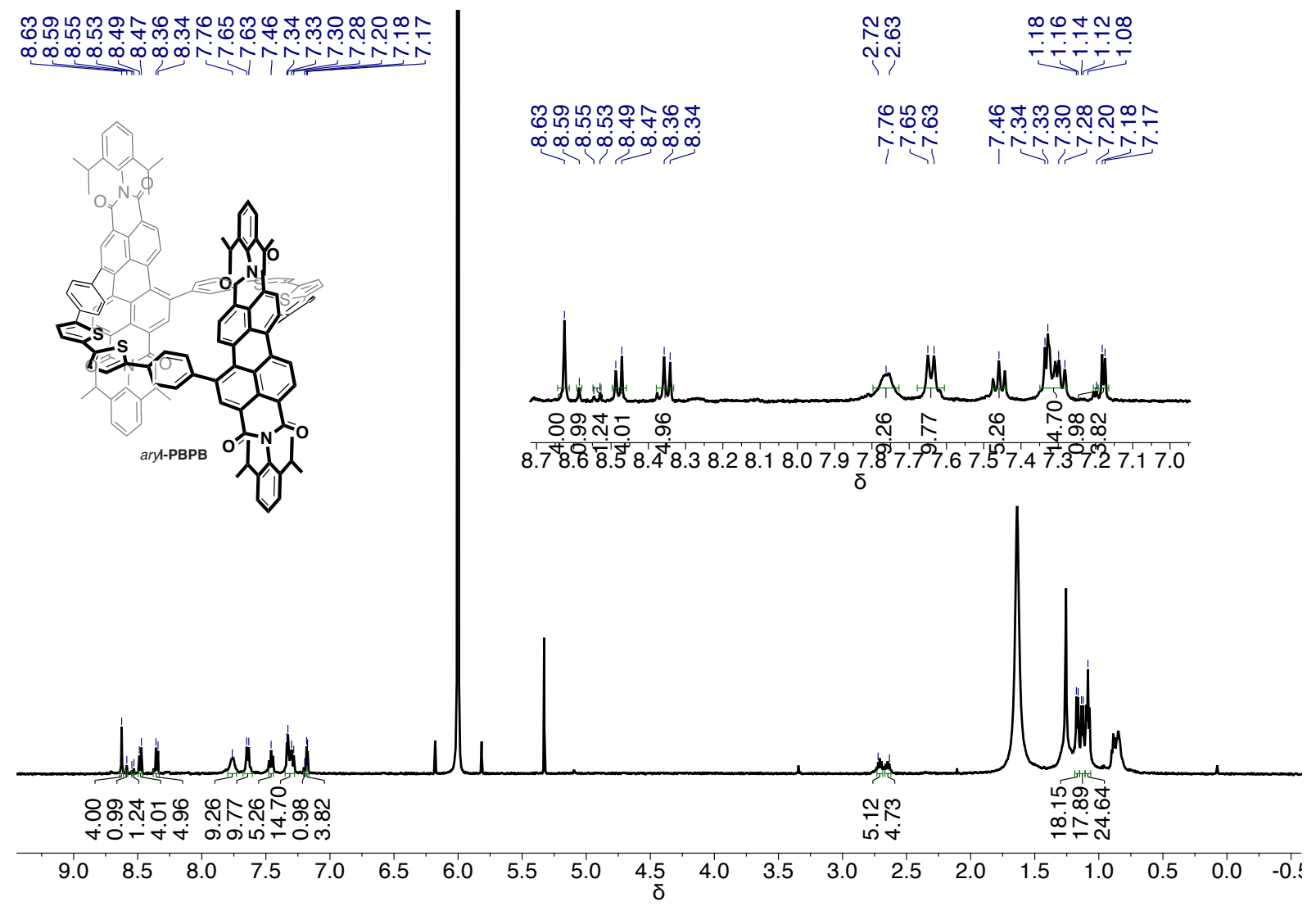

\subsection{DFT Output}

All quantum chemical calculations were performed using Jaguar, version 8.3, Schrodinger, Inc., New York, NY, 2013. (See A. D. Bochevarov, E. Harder, T. F. Hughes, J. R. Greenwood, D. A. Braden, D. M. Philipp, D. Rinaldo, M. D. Halls, J. Zhang, R. A. Friesner, "Jaguar: A High Performance Quantum Chemistry Software Program with Strengths in Life and Materials Sciences", Int. J. Quantum Chem., 2013, 113(18), 2110-2142). All geometries were optimized using the B3LYP functional and the $6-31 \mathrm{G}$ basis set. Below contains the optimized geometry for PBPB and $\mathbf{C}_{70}$, followed by the 30 lowest roots from TDDFT. The optimized geometry and excited state calculations for free PBPB can be found at the original reference and in Chapter 2. ${ }^{1}$ 


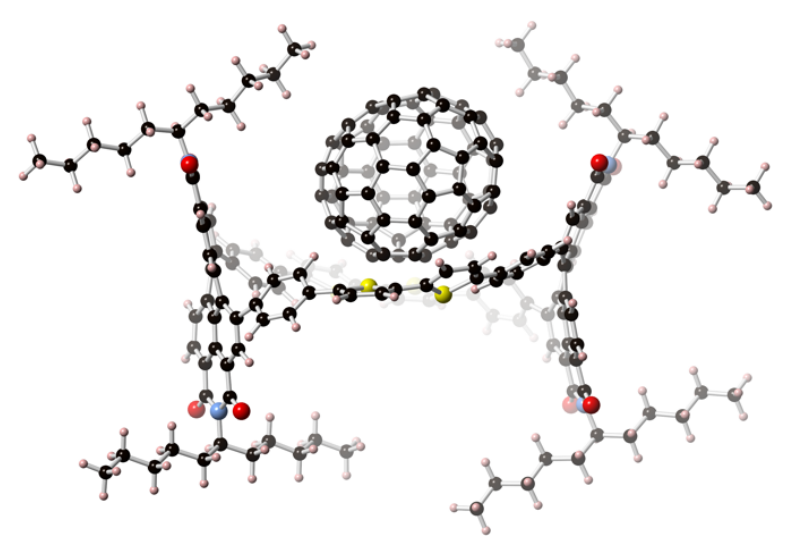

PBPB-C 70 Complex

Final Heat of Formation: -10188. 507539

$\begin{array}{rrrr}\text { C } & 15.124211 & 0.422396 & -2.251470 \\ \mathrm{C} & 14.577263 & 0.974609 & -1.073298 \\ \mathrm{C} & 14.370439 & 2.371112 & -1.091596 \\ \mathrm{C} & 14.443244 & 3.129872 & -2.301740 \\ \mathrm{C} & 14.728112 & 2.514977 & -3.543959 \\ \mathrm{C} & 15.200018 & 1.179271 & -3.467956 \\ \mathrm{C} & 15.020352 & 0.257013 & -4.566973 \\ \mathrm{C} & 14.852095 & -1.076525 & -4.032468 \\ \mathrm{C} & 14.900536 & -0.962568 & -2.595554 \\ \mathrm{C} & 14.080056 & -1.752628 & -1.807513 \\ \mathrm{C} & 13.439621 & -1.181934 & -0.643418 \\ \mathrm{C} & 13.709785 & 0.142683 & -0.235834 \\ \mathrm{C} & 12.697579 & 0.760516 & 0.533111 \\ \mathrm{C} & 12.568295 & 2.198035 & 0.554225 \\ \mathrm{C} & 13.384070 & 2.986760 & -0.238507 \\ \mathrm{C} & 12.854309 & 4.150958 & -0.909825 \\ \mathrm{C} & 13.496807 & 4.216744 & -2.203301 \\ \mathrm{C} & 12.781786 & 4.624411 & -3.317164 \\ \mathrm{C} & 12.979049 & 3.947878 & -4.579761 \\ \mathrm{C} & 13.963277 & 2.934309 & -4.723330 \\ \mathrm{C} & 13.719187 & 1.980063 & -5.739484 \\ \mathrm{C} & 14.282041 & 0.652466 & -5.668938 \\ \mathrm{C} & 13.364700 & -0.268979 & -6.290861 \\ \mathrm{C} & 13.206434 & -1.551512 & -5.786454 \\ \mathrm{C} & 13.970604 & -1.971726 & -4.632405 \\ \mathrm{C} & 13.107262 & -2.786044 & -3.808463 \\ \mathrm{C} & 13.170776 & -2.687923 & -2.421541 \\ \mathrm{C} & 11.961372 & -2.676991 & -1.631711 \\ \mathrm{C} & 12.142680 & -1.780163 & -0.502284 \\ \mathrm{C} & 11.045224 & -1.031888 & 0.001477 \\ \mathrm{C} & 11.383723 & 0.188815 & 0.648093 \\ \mathrm{C} & 10.444934 & 1.284086 & 0.727306\end{array}$




\begin{tabular}{|c|c|c|c|}
\hline 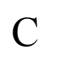 & & & \\
\hline $\mathrm{C}$ & & & \\
\hline & 506 & T & \\
\hline & 609 & & \\
\hline & 976 & 9 & \\
\hline & 832 & & \\
\hline $\mathrm{C}$ & 11.700377 & & \\
\hline & 11.3 & & \\
\hline $\mathrm{C}$ & & & \\
\hline & 12. & & \\
\hline & & & \\
\hline & & & \\
\hline & & & \\
\hline$U$ & & -2.5 & \\
\hline$c$ & & & \\
\hline $\mathrm{C}$ & & -2. & \\
\hline $\mathrm{C}$ & & -1 . & \\
\hline $\mathrm{C}$ & & & \\
\hline $\mathrm{C}$ & & -0.0 & \\
\hline $\mathrm{C}$ & & & \\
\hline $\mathrm{C}$ & & & \\
\hline $\mathrm{C}$ & & & \\
\hline $\mathrm{C}$ & & & \\
\hline $\mathrm{C}$ & & & \\
\hline $\mathrm{C}$ & & & \\
\hline $\mathrm{C}$ & & & \\
\hline $\mathrm{C}$ & & & \\
\hline $\mathrm{C}$ & & & \\
\hline $\mathrm{C}$ & & $-0 .($ & \\
\hline $\mathrm{C}$ & & & \\
\hline $\mathrm{C}$ & 2 & -2.1 & \\
\hline $\mathrm{C}$ & & -1.5 & \\
\hline $\mathrm{C}$ & & & \\
\hline $\mathrm{C}$ & & & \\
\hline $\mathrm{C}$ & & & \\
\hline $\mathrm{C}$ & & & -2.9 \\
\hline $\mathrm{C}$ & & & \\
\hline $\mathrm{C}$ & 1 & 0 & -4.2 \\
\hline $\mathrm{H}$ & 641 & -2.1 & \\
\hline $\mathrm{H}$ & & & \\
\hline $\mathrm{H}$ & & & \\
\hline $\mathrm{H}$ & & & \\
\hline $\mathrm{H}$ & 20. & 2.3 & \\
\hline - & & & \\
\hline $\mathrm{H}$ & 13.0 & -3.488072 & 44 \\
\hline & 16.264253 & -5.843290 & -0.43207 \\
\hline
\end{tabular}




$\begin{array}{ccrc}\mathrm{H} & 17.828697 & -4.054115 & 0.112839 \\ \mathrm{H} & 14.642332 & -1.828777 & 1.955501 \\ \mathrm{H} & 7.236548 & -2.235751 & 0.802217 \\ \mathrm{H} & 3.606567 & 3.208840 & 1.506785 \\ \mathrm{H} & 3.496373 & -1.381441 & 3.311009 \\ \mathrm{H} & 8.673431 & -4.163633 & 1.085977 \\ \mathrm{C} & 10.970599 & 9.821331 & 1.464467 \\ \mathrm{C} & 11.040724 & 9.999075 & 2.920062 \\ \mathrm{C} & 12.286203 & 9.779706 & 3.576018 \\ \mathrm{C} & 13.441283 & 9.960986 & 1.408350 \\ \mathrm{C} & 9.947651 & 10.421371 & 3.688240 \\ \mathrm{C} & 11.150621 & 10.046951 & 5.747994 \\ \mathrm{C} & 13.487644 & 9.570852 & 2.827264 \\ \mathrm{C} & 9.786151 & 9.564167 & 0.736234 \\ \mathrm{C} & 13.463831 & 9.260839 & 5.648366 \\ \mathrm{C} & 12.175762 & 10.062173 & 0.750945 \\ \mathrm{C} & 14.549615 & 8.876935 & 4.893074 \\ \mathrm{C} & 13.259655 & 11.016428 & -2.787466 \\ \mathrm{C} & 10.002294 & 10.462016 & 5.085722 \\ \mathrm{C} & 14.598238 & 9.001427 & 3.478561 \\ \mathrm{~N} & 11.991731 & 11.080248 & -3.391140 \\ \mathrm{C} & 14.529954 & 10.606165 & -0.687304 \\ \mathrm{C} & 14.589753 & 10.265083 & 0.669852 \\ \mathrm{C} & 13.311364 & 10.650029 & -1.348440 \\ \mathrm{C} & 10.780060 & 10.784539 & -2.722902 \\ \mathrm{C} & 12.292707 & 9.701512 & 4.996537 \\ \mathrm{C} & 11.170146 & 9.969262 & 7.230019 \\ \mathrm{C} & 11.344656 & -6.467341 & 4.533211 \\ \mathrm{C} & 12.580767 & -6.708037 & 5.167651 \\ \mathrm{C} & 13.462182 & -6.304758 & -1.266032 \\ \mathrm{C} & 16.647220 & -0.194814 & 2.110735 \\ \mathrm{C} & 17.204916 & 5.952363 & 2.120012 \\ \mathrm{C} & 15.667419 & 8.223846 & 2.791215 \\ \mathrm{C} & 16.079990 & 6.298327 & 1.357856 \\ \mathrm{C} & 17.237807 & -1.636837 & 1.286427 \\ \mathrm{C} & 14.143895 & -3.746284 & 1.195481 \\ \mathrm{C} & 12.128212 & 10.341019 & -0.643262 \\ \mathrm{C} & 10.872697 & 10.391842 & -1.295094 \\ \mathrm{C} & 9.937538 & -7.416545 & -1.589612 \\ \mathrm{C} & 12.340068 & 9.365961 & 9.331923 \\ \mathrm{O} & 14.483861 & 8.651522 & 7.698080 \\ \mathrm{C} & 9.757175 & 9.885236 & -0.642345 \\ \mathrm{C} & 11.179784 & -8.074853 & -3.627008 \\ \mathrm{C} & 10.824653 & -1.246354\end{array}$




\begin{tabular}{|c|c|c|c|}
\hline & & & \\
\hline $\mathrm{O}$ & 699498 & & \\
\hline & & & \\
\hline & 10.098007 & & \\
\hline & $14.2^{\prime}$ & & \\
\hline & 12.3 & & \\
\hline C & & & \\
\hline & 11.8 & 11.5 & \\
\hline & & & \\
\hline & 8.76 & -6.8 & \\
\hline & 6.6 & & \\
\hline & 13.6 & -6. & \\
\hline & 11. & & \\
\hline C & & & \\
\hline & & & \\
\hline $\mathrm{C}$ & 13.7 & & \\
\hline & & -7.3 & \\
\hline & 17.6 & & \\
\hline & 16.7 & & \\
\hline & & & \\
\hline$C$ & 16.9 & & \\
\hline $\mathrm{C}$ & & & \\
\hline $\mathrm{C}$ & & & \\
\hline & 13. & & \\
\hline $\mathrm{C}$ & & & \\
\hline & 18.4 & & \\
\hline $\mathrm{O}$ & & & -3. \\
\hline & 10.2 & & 700 \\
\hline $\mathrm{T}$ & 12.3 & & \\
\hline O & & & \\
\hline $\mathrm{C}$ & & & \\
\hline $\mathrm{C}$ & & & \\
\hline & & & \\
\hline $\mathrm{C}$ & & & \\
\hline 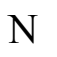 & & & \\
\hline $\mathrm{C}$ & & & \\
\hline $\mathrm{O}$ & 92 & & -3 \\
\hline$C$ & 14.5 & -4 . & \\
\hline $\mathrm{C}$ & 18.0 & & \\
\hline $\mathrm{C}$ & & -2.5 & \\
\hline $\mathrm{C}$ & 11.35 & -7.8 & \\
\hline $\mathrm{S}$ & & & \\
\hline 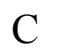 & & & 374 \\
\hline & & & \\
\hline 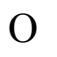 & 13.70 & 862 & 7.1 \\
\hline & 5.027939 & 4.895106 & 1.58919 \\
\hline
\end{tabular}




$\begin{array}{crrc}\mathrm{C} & 7.745393 & -4.871917 & 2.912382 \\ \mathrm{C} & 7.075004 & -2.890579 & 1.651881 \\ \mathrm{C} & 4.579520 & 3.574725 & 1.813329 \\ \mathrm{C} & 5.518774 & 2.759010 & 2.403918 \\ \mathrm{C} & 15.032328 & -2.738159 & 1.509223 \\ \mathrm{C} & 15.902787 & -4.982372 & 0.124749 \\ \mathrm{C} & 5.367242 & 1.331626 & 2.622296 \\ \mathrm{C} & 11.266732 & -6.226750 & 3.130481 \\ \mathrm{C} & 6.337015 & 5.117587 & 1.953304 \\ \mathrm{C} & 4.316961 & -0.725969 & 3.041222 \\ \mathrm{C} & 18.939797 & -0.072305 & 0.930120 \\ \mathrm{C} & 5.558828 & -1.195744 & 2.686969 \\ \mathrm{~S} & 7.022329 & 3.644156 & 2.653734 \\ \mathrm{C} & 4.210473 & 0.687469 & 3.009605 \\ \mathrm{~S} & 6.613704 & 0.154989 & 2.302302 \\ \mathrm{C} & 17.820043 & 4.631563 & 1.968522 \\ \mathrm{C} & 19.334283 & 2.862183 & 1.985001 \\ \mathrm{C} & 8.896705 & -5.713214 & 3.336855 \\ \mathrm{H} & 9.142308 & 10.762097 & 5.673752 \\ \mathrm{H} & 8.859798 & 9.709342 & -1.224386 \\ \mathrm{H} & 15.381389 & 8.437945 & 5.427061 \\ \mathrm{H} & 17.268959 & 8.665138 & 4.175392 \\ \mathrm{C} & 9.986343 & -6.075039 & 2.508787 \\ \mathrm{C} & 8.994834 & -5.990505 & 4.723858 \\ \mathrm{C} & 11.145514 & -6.457751 & 0.322663 \\ \mathrm{C} & 8.746121 & 5.756499 & 3.077531 \\ \mathrm{H} & 12.488378 & 10.622803 & -5.825480 \\ \mathrm{C} & 10.156946 & -6.460837 & 5.299858 \\ \mathrm{H} & 15.759751 & 5.649362 & 0.547991 \\ \mathrm{C} & 12.384328 & -6.076509 & 0.902725 \\ \mathrm{H} & 14.390530 & 7.573950 & 1.167179 \\ \mathrm{H} & 8.164690 & -5.778553 & 5.384291 \\ \mathrm{H} & 14.614586 & -6.297263 & 2.509843 \\ \mathrm{H} & 5.658247 & 7.383819 & 0.566024 \\ \mathrm{H} & 7.851236 & -7.749920 & -1.301575 \\ \mathrm{H} & 7.011949 & 9.354606 & 0.159535 \\ \mathrm{H} & 18.577880 & 6.663068 & 3.629135 \\ \mathrm{H} & 14.700640 & -6.792731 & 4.911830 \\ \mathrm{H} & 14.311205 & -6.150149 & -1.923357 \\ \mathrm{H} & 7.832114 & -6.860080 & 1.009778 \\ \mathrm{H} & 11.068665 & -5.337724 & 4.551070 \\ \mathrm{H} & -3.317543 & 4.345592 \\ \mathrm{H} & -8.788495 & -4.946681 \\ \mathrm{H} & 10.208205 & 9.996184\end{array}$




$\begin{array}{cccc}\mathrm{H} & 11.379225 & 9.801143 & 9.609485 \\ \mathrm{C} & 12.350932 & 7.891127 & 9.776844 \\ \mathrm{C} & 11.413596 & -7.814662 & -6.121265 \\ \mathrm{C} & 11.959034 & -9.301866 & 8.624489 \\ \mathrm{C} & 12.070693 & -6.957116 & 9.678239 \\ \mathrm{H} & 10.327405 & -7.919685 & 8.872197 \\ \mathrm{H} & 10.184484 & -9.191396 & -5.026440 \\ \mathrm{H} & 9.030424 & 10.700935 & 3.186888 \\ \mathrm{H} & 15.552232 & 10.263382 & 1.165073 \\ \mathrm{C} & 12.191507 & -9.964590 & -4.935898 \\ \mathrm{H} & 11.397202 & -8.421314 & -7.037648 \\ \mathrm{C} & 10.342874 & -6.718726 & -6.219260 \\ \mathrm{H} & 12.413742 & -7.374696 & -6.058995 \\ \mathrm{H} & 12.092963 & -10.464114 & -5.909679 \\ \mathrm{H} & 13.217000 & -9.586586 & -4.883734 \\ \mathrm{C} & 11.938281 & -10.980653 & -3.813441 \\ \mathrm{H} & 12.225581 & 13.361951 & -5.941976 \\ \mathrm{C} & 11.842619 & 14.031333 & -3.922595 \\ \mathrm{H} & 13.535994 & 13.018990 & -4.822309 \\ \mathrm{H} & 12.491189 & 11.154445 & -6.787587 \\ \mathrm{H} & 13.535338 & 10.413717 & -5.584622 \\ \mathrm{C} & 11.687493 & 9.322938 & -5.987839 \\ \mathrm{H} & 14.426509 & 9.755584 & 9.814753 \\ \mathrm{C} & 13.460918 & 11.678233 & 9.551418 \\ \mathrm{H} & 13.267400 & 10.160060 & 11.078765 \\ \mathrm{H} & 13.303168 & 7.428968 & 9.498369 \\ \mathrm{H} & 12.307063 & 7.890300 & 10.874480 \\ \mathrm{C} & 11.176991 & 7.072019 & 9.222266 \\ \mathrm{H} & 11.807928 & -9.704056 & 9.636055 \\ \mathrm{C} & 11.287322 & -10.229313 & 7.602386 \\ \mathrm{H} & 13.040098 & -9.273760 & 8.456711 \\ \mathrm{H} & 11.970619 & -7.458003 & 10.651799 \\ \mathrm{H} & 13.142535 & -6.893042 & 9.467823 \\ \mathrm{C} & 11.461229 & -5.550283 & 9.759557 \\ \mathrm{C} & 10.432706 & -5.881184 & -7.502371 \\ \mathrm{H} & 9.345562 & -7.175531 & -6.152088 \\ \mathrm{H} & 10.425023 & -6.047773 & -5.352964 \\ \mathrm{C} & 12.831688 & -12.226263 & -3.897415 \\ \mathrm{H} & 12.101522 & -10.499958 & -2.839242 \\ \mathrm{H} & 12.316589 & 15.474412 & -4.145303 \\ \mathrm{H} & 12.101188 & 13.733154 & -2.897037 \\ \mathrm{H} & -1563 & 8.723997 & -5.068366 \\ \mathrm{H} & 9.570726 & -6.117956\end{array}$




$\begin{array}{cccc}\mathrm{C} & 14.485827 & 12.541084 & 10.300465 \\ \mathrm{H} & 13.679744 & 11.731057 & 8.476505 \\ \mathrm{H} & 12.457621 & 12.109067 & 9.677273 \\ \mathrm{C} & 11.121889 & 5.632608 & 9.750476 \\ \mathrm{H} & 10.233115 & 7.583075 & 9.456940 \\ \mathrm{H} & 11.237714 & 7.042552 & 8.125779 \\ \mathrm{C} & 11.771351 & -11.683095 & 7.682376 \\ \mathrm{H} & 10.196615 & -10.198202 & 7.740063 \\ \mathrm{H} & 11.473150 & -9.851782 & 6.587432 \\ \mathrm{C} & 12.054382 & -4.684266 & 10.879672 \\ \mathrm{H} & 11.610756 & -5.034582 & 8.800680 \\ \mathrm{H} & 10.373736 & -5.628613 & 9.892435 \\ \mathrm{C} & 9.336214 & -4.810112 & -7.591022 \\ \mathrm{H} & 11.420469 & -5.400468 & -7.563075 \\ \mathrm{H} & 10.364018 & -6.543463 & -8.377783 \\ \mathrm{C} & 12.566346 & -13.228330 & -2.764684 \\ \mathrm{H} & 12.681707 & -12.721854 & -4.867743 \\ \mathrm{H} & 13.888649 & -11.921725 & -3.875302 \\ \mathrm{C} & 9.384231 & -3.992079 & -8.885189 \\ \mathrm{H} & 8.353004 & -5.292836 & -7.499321 \\ \mathrm{H} & 9.420353 & -4.133782 & -6.729612 \\ \mathrm{C} & 13.439299 & -14.484680 & -2.843039 \\ \mathrm{H} & 12.730812 & -12.731365 & -1.798375 \\ \mathrm{H} & 11.506360 & -13.518387 & -2.779558 \\ \mathrm{H} & 8.590152 & -3.238339 & -8.912404 \\ \mathrm{H} & 10.342140 & -3.468333 & -8.989620 \\ \mathrm{H} & 9.262009 & -4.633900 & -9.765710 \\ \mathrm{H} & 13.221738 & -15.175102 & -2.020750 \\ \mathrm{H} & 13.274241 & -15.025820 & -3.782074 \\ \mathrm{H} & 14.505314 & -14.232187 & -2.791244 \\ \mathrm{C} & 11.711041 & 16.465832 & -3.141169 \\ \mathrm{H} & 13.413146 & 15.513744 & -4.075020 \\ \mathrm{H} & 12.065907 & 15.791974 & -5.168198 \\ \mathrm{C} & 11.236753 & 7.266452 & -7.440925 \\ \mathrm{H} & 12.196697 & 9.093962 & -8.079961 \\ \mathrm{H} & 13.181156 & 8.117433 & -7.001820 \\ \mathrm{C} & 12.183227 & 17.908682 & -3.348413 \\ \mathrm{H} & 10.615337 & 16.424438 & -3.208626 \\ \mathrm{H} & 11.962326 & 16.143558 & -2.120636 \\ \mathrm{C} & 11.697851 & 6.393047 & -8.611673 \\ \mathrm{H} & 11.172194 & 6.655579 & -6.531532 \\ \mathrm{H} & 10.218198 & 7.629218 & -7.635613 \\ \mathrm{H} & 11.730132 & 18.587499 & -2.617696 \\ \mathrm{H} & 17.989335 & -3.245317 \\ \mathrm{H} & 18.273637 & -4.348408 \\ \mathrm{H} & 5.551640 & -8.775578\end{array}$




$\begin{array}{crcc}\mathrm{H} & 11.744951 & 6.968618 & -9.544015 \\ \mathrm{H} & 12.696284 & 5.978512 & -8.429630 \\ \mathrm{C} & 11.116938 & -12.587414 & 6.627698 \\ \mathrm{H} & 12.863622 & -11.711761 & 7.557332 \\ \mathrm{H} & 11.569154 & -12.086447 & 8.685700 \\ \mathrm{C} & 11.438679 & -3.278537 & 10.933179 \\ \mathrm{H} & 11.909214 & -5.183893 & 11.849224 \\ \mathrm{H} & 13.142637 & -4.599155 & 10.744128 \\ \mathrm{C} & 11.594270 & -14.041334 & 6.690749 \\ \mathrm{H} & 10.025301 & -12.554321 & 6.749405 \\ \mathrm{H} & 11.321284 & -12.178933 & 5.627682 \\ \mathrm{C} & 12.006908 & -2.402918 & 12.054948 \\ \mathrm{H} & 11.596267 & -2.779391 & 9.966502 \\ \mathrm{H} & 10.349869 & -3.366323 & 11.053020 \\ \mathrm{H} & 11.108750 & -14.655038 & 5.924062 \\ \mathrm{H} & 12.677402 & -14.110765 & 6.535022 \\ \mathrm{H} & 11.372021 & -14.491598 & 7.665629 \\ \mathrm{H} & 11.543845 & -1.410397 & 12.061556 \\ \mathrm{H} & 11.834555 & -2.855769 & 13.038886 \\ \mathrm{H} & 13.088758 & -2.263095 & 11.942098 \\ \mathrm{C} & 14.513273 & 13.995999 & 9.809535 \\ \mathrm{H} & 14.267685 & 12.523595 & 11.378073 \\ \mathrm{H} & 15.487268 & 12.101537 & 10.187335 \\ \mathrm{C} & 9.929186 & 4.843642 & 9.190678 \\ \mathrm{H} & 12.055605 & 5.110603 & 9.495660 \\ \mathrm{H} & 11.068923 & 5.642457 & 10.848740 \\ \mathrm{C} & 15.531463 & 14.872302 & 10.545747 \\ \mathrm{H} & 14.734224 & 14.008117 & 8.733174 \\ \mathrm{H} & 13.510667 & 14.432099 & 9.915934 \\ \mathrm{C} & 9.865371 & 3.396005 & 9.686595 \\ \mathrm{H} & 8.997720 & 5.362585 & 9.455372 \\ \mathrm{H} & 9.976453 & 4.850975 & 8.092769 \\ \mathrm{H} & 15.521196 & 15.900604 & 10.168705 \\ \mathrm{H} & 15.319492 & 14.911252 & 11.620547 \\ \mathrm{H} & 16.550001 & 14.485364 & 10.425310 \\ \mathrm{H} & 9.001544 & 2.868289 & 9.268344 \\ \mathrm{H} & 10.764617 & 2.837706 & 9.400986 \\ \mathrm{H} & 9.783987 & 3.352158 & 10.778974\end{array}$

\section{TDDFT Output for 30 Lowest Roots}

Restricted Singlet Excited State 1:

\footnotetext{
Excitation energy $=0.0575100274$ hartrees $\quad 1.56492747 \mathrm{eV} \quad 792.27 \mathrm{~nm}$ excitation $\mathrm{X}$ coeff.
} 


$$
\begin{aligned}
& 746=>749 \quad-0.10745 \\
& 748=>749 \quad 0.93795 \\
& 748=>750 \quad-0.28549
\end{aligned}
$$

Transition dipole moment (debye):

$$
\mathrm{X}=0.5066 \mathrm{Y}=-0.0922 \quad \mathrm{Z}=3.8091 \text { Tot }=3.8437
$$

Oscillator strength, $\mathrm{f}=\quad 0.0877$

Restricted Singlet Excited State 2:

Excitation energy $=0.0606414457$ hartrees $\quad 1.65013770 \mathrm{eV} \quad 751.36 \mathrm{~nm}$

excitation $\mathrm{X}$ coeff.

$748=>749 \quad 0.27441$

$748=>750 \quad 0.91951$

$748=>751 \quad-0.21721$

$748=>752 \quad-0.11629$

Transition dipole moment (debye):

$$
\mathrm{X}=3.5602 \mathrm{Y}=0.2888 \mathrm{Z}=-1.4775 \text { Tot }=3.8655
$$

Oscillator strength, $\mathrm{f}=\quad 0.0935$

\section{Restricted Singlet Excited State 3:}

Excitation energy $=0.0616435373$ hartrees $\quad 1.67740600 \mathrm{eV} \quad 739.14 \mathrm{~nm}$ excitation X coeff.

$$
\begin{aligned}
& 748=>750 \quad-0.23889 \\
& 748=>751 \quad-0.89632 \\
& 748=>752 \quad-0.17238 \\
& 748=>753 \quad-0.29460
\end{aligned}
$$

Transition dipole moment (debye):

$$
\mathrm{X}=-1.2780 \mathrm{Y}=-0.3623 \mathrm{Z}=0.4317 \mathrm{Tot}=1.3968
$$

Oscillator strength, $\mathrm{f}=\quad 0.0124$ 
Restricted Singlet Excited State 4:

Excitation energy $=0.0628412198$ hartrees $\quad 1.70999659 \mathrm{eV} \quad 725.06 \mathrm{~nm}$ excitation $\mathrm{X}$ coeff.

$748=>751 \quad-0.11876$

$748=>752 \quad 0.94676$

$748=>753 \quad-0.27216$

Transition dipole moment (debye):

$$
\mathrm{X}=0.4435 \mathrm{Y}=-0.0132 \mathrm{Z}=-0.2155 \mathrm{Tot}=0.4932
$$

Oscillator strength, $\mathrm{f}=0.0016$

Restricted Singlet Excited State 5:

Excitation energy $=0.0646824193$ hartrees $\quad 1.76009818 \mathrm{eV} \quad 704.42 \mathrm{~nm}$

excitation $\mathrm{X}$ coeff.

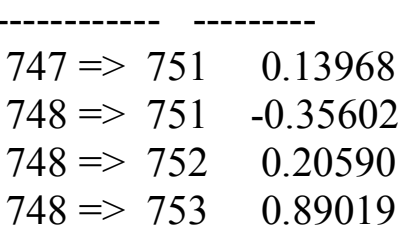

Transition dipole moment (debye):

$$
\mathrm{X}=0.1324 \mathrm{Y}=0.0296 \mathrm{Z}=0.1107 \mathrm{Tot}=0.1751
$$

Oscillator strength, $\mathrm{f}=\quad 0.0002$

Restricted Singlet Excited State 6:

Excitation energy $=0.0657336955$ hartrees $\quad 1.78870486 \mathrm{eV} \quad 693.15 \mathrm{~nm}$ excitation $\mathrm{X}$ coeff.

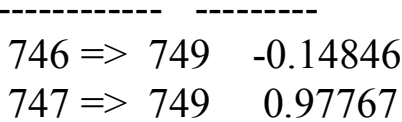


Transition dipole moment (debye):

$$
\mathrm{X}=-1.2422 \quad \mathrm{Y}=-5.0308 \quad \mathrm{Z}=-1.2131 \mathrm{Tot}=5.3220
$$

Oscillator strength, $\mathrm{f}=\quad 0.1921$

Restricted Singlet Excited State 7:

Excitation energy $=0.0679481166$ hartrees $\quad 1.84896233 \mathrm{eV} \quad 670.56 \mathrm{~nm}$

excitation X coeff

$746=>750 \quad 0.18304$

$747=>750 \quad-0.96613$

Transition dipole moment (debye):

$$
\mathrm{X}=0.0409 \quad \mathrm{Y}=-2.4380 \quad \mathrm{Z}=1.1339 \text { Tot }=2.6891
$$

Oscillator strength, $\mathrm{f}=\quad 0.0507$

Restricted Singlet Excited State 8:

Excitation energy $=0.0694372836$ hartrees $\quad 1.88948462 \mathrm{eV} \quad 656.18 \mathrm{~nm}$

excitation X coeff.

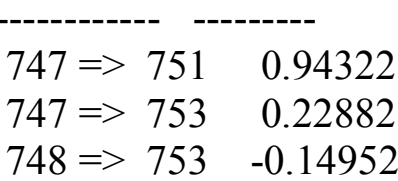

Transition dipole moment (debye):

$$
\mathrm{X}=0.0879 \quad \mathrm{Y}=0.3072 \quad \mathrm{Z}=-0.1368 \text { Tot }=0.3476
$$

Oscillator strength, $\mathrm{f}=\quad 0.0009$

Restricted Singlet Excited State 9:

Excitation energy $=0.0703871399$ hartrees $\quad 1.91533153 \mathrm{eV} \quad 647.32 \mathrm{~nm}$ excitation X coeff. 
$747=>752 \quad 0.95926$

$747=>753 \quad-0.23151$

Transition dipole moment (debye):

$\mathrm{X}=-0.0904 \quad \mathrm{Y}=0.4241 \quad \mathrm{Z}=-0.2290$ Tot $=0.4904$

Oscillator strength, $\mathrm{f}=\quad 0.0017$

Restricted Singlet Excited State 10:

Excitation energy $=0.0713236376$ hartrees $\quad 1.94081493 \mathrm{eV} \quad 638.83 \mathrm{~nm}$

excitation $\mathrm{X}$ coeff.

$746=>749 \quad-0.97084$

$747=>749 \quad-0.13613$

$748=>749 \quad-0.13229$

Transition dipole moment (debye):

$\mathrm{X}=2.0902 \mathrm{Y}=-1.6402 \mathrm{Z}=1.6768 \mathrm{Tot}=3.1418$

Oscillator strength, $\mathrm{f}=\quad 0.0727$

Restricted Singlet Excited State 11:

Excitation energy $=0.0723772617$ hartrees $\quad 1.96948550 \mathrm{eV} \quad 629.53 \mathrm{~nm}$

excitation $\mathrm{X}$ coeff.

$\begin{array}{lr}746=>750 & -0.12712 \\ 746=>751 & 0.13484 \\ 747=>751 & 0.25563 \\ 747=>752 & -0.20334 \\ 747=>753 & -0.90820\end{array}$

Transition dipole moment (debye):

$\mathrm{X}=-0.3088 \mathrm{Y}=0.3810 \mathrm{Z}=0.2518 \mathrm{Tot}=0.5513$

Oscillator strength, $\mathrm{f}=\quad 0.0023$

Restricted Singlet Excited State 12: 
Excitation energy $=0.0734500072$ hartrees $\quad 1.99867639 \mathrm{eV} \quad 620.33 \mathrm{~nm}$ excitation $\mathrm{X}$ coeff.

\begin{tabular}{|c|c|}
\hline $746=>750$ & -0.88972 \\
\hline $746=>751$ & 0.27220 \\
\hline $746=>752$ & 0.20410 \\
\hline $747=>750$ & -0.17378 \\
\hline $747 \Rightarrow 753$ & 0.18026 \\
\hline
\end{tabular}

Transition dipole moment (debye):
$\mathrm{X}=-0.5897 \mathrm{Y}=0.5790$
$\mathrm{Z}=$
4.3633 Tot $=4.4409$

Oscillator strength, $\mathrm{f}=0.1495$

Restricted Singlet Excited State 13:

Excitation energy $=0.0740039569$ hartrees $\quad 2.01375013 \mathrm{eV} \quad 615.69 \mathrm{~nm}$

excitation $\mathrm{X}$ coeff.

\begin{tabular}{|c|c|c|}
\hline $5=$ & 751 & 0.16039 \\
\hline 16 & 750 & -0.29822 \\
\hline $6=>$ & 751 & -0.88889 \\
\hline $6=>$ & 53 & -0.22828 \\
\hline $7=>$ & & -0.12065 \\
\hline
\end{tabular}

Transition dipole moment (debye):
$\mathrm{X}=-0.0768 \quad \mathrm{Y}=0.3021 \quad \mathrm{Z}=$
$\mathrm{Z}=1.8618$ Tot $=1.8877$

Oscillator strength, $\mathrm{f}=\quad 0.0272$

Restricted Singlet Excited State 14:

Excitation energy $=0.0750234086$ hartrees $\quad 2.04149082 \mathrm{eV} \quad 607.32 \mathrm{~nm}$ excitation $\mathrm{X}$ coeff.

$\begin{array}{ll}745=>752 & 0.21671 \\ 746=>750 & -0.20187\end{array}$




$$
746=>752-0.91516
$$

$746=>753 \quad 0.16932$

Transition dipole moment (debye):

$$
\mathrm{X}=-0.2348 \mathrm{Y}=0.3632 \mathrm{Z}=1.3373 \text { Tot }=1.4054
$$

Oscillator strength, $\mathrm{f}=0.0153$

Restricted Singlet Excited State 15:

Excitation energy $=0.0768266753$ hartrees $\quad 2.09056020 \mathrm{eV} \quad 593.07 \mathrm{~nm}$

\begin{tabular}{|c|c|}
\hline $742=>752$ & -0.16519 \\
\hline $3=>751$ & -0.14085 \\
\hline $4=>751$ & 0.14147 \\
\hline $4=>752$ & -0.15353 \\
\hline $5=>751$ & 0.29753 \\
\hline $5=>752$ & -0.14769 \\
\hline $5=>753$ & -0.24748 \\
\hline $6=>751$ & -0.15729 \\
\hline $6=>752$ & 0.11588 \\
\hline $6=>7$ & 0.81316 \\
\hline
\end{tabular}

excitation $\mathrm{X}$ coeff.

Transition dipole moment (debye):

$$
\mathrm{X}=-0.1549 \mathrm{Y}=0.3611 \mathrm{Z}=-0.1351 \text { Tot }=0.4155
$$

Oscillator strength, $\mathrm{f}=\quad 0.0014$

Restricted Singlet Excited State 16:

\begin{tabular}{|c|c|}
\hline & \\
\hline$>75$ & -0 . \\
\hline- & \\
\hline 74 & \\
\hline 743 & -0 \\
\hline 744 & \\
\hline $745=>$ & -0.1 \\
\hline
\end{tabular}

Excitation energy $=0.0772261905$ hartrees $\quad 2.10143156 \mathrm{eV} \quad 590.00 \mathrm{~nm}$

excitation $\mathrm{X}$ coeff. 


$$
745=>752 \quad 0.66353
$$

$746=>752 \quad 0.25150$

Transition dipole moment (debye):

$$
\mathrm{X}=-0.0023 \mathrm{Y}=0.0917 \mathrm{Z}=-0.1720 \text { Tot }=0.1949
$$

Oscillator strength, $\mathrm{f}=0.0003$

Restricted Singlet Excited State 17:

Excitation energy $=0.0772767679$ hartrees $\quad 2.10280784 \mathrm{eV} \quad 589.61 \mathrm{~nm}$

\begin{tabular}{|c|c|}
\hline $742=>751$ & 0.23048 \\
\hline $42=>752$ & -0.24916 \\
\hline $43=>751$ & 0.34079 \\
\hline $44=>751$ & 0.14057 \\
\hline $4=>752$ & 0.24876 \\
\hline $44=>753$ & -0.25801 \\
\hline $45=>751$ & -0.67466 \\
\hline $15=>753$ & -0.16484 \\
\hline $6=>751$ & -0.23128 \\
\hline $6=>75$ & 0.19406 \\
\hline
\end{tabular}

excitation X coeff.

Transition dipole moment (debye):

$$
\mathrm{X}=-0.2082 \mathrm{Y}=-0.1127 \mathrm{Z}=0.1785 \text { Tot }=0.2965
$$

Oscillator strength, $\mathrm{f}=0.0007$

Restricted Singlet Excited State 18:

\begin{tabular}{|c|c|}
\hline$>75$ & -0 . \\
\hline & \\
\hline 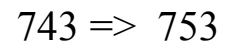 & \\
\hline$\Rightarrow$ & \\
\hline $44=>$ & -0.1 \\
\hline $745=>$ & 0.17 \\
\hline
\end{tabular}

Excitation energy $=0.0780985018$ hartrees $\quad 2.12516836 \mathrm{eV} \quad 583.41 \mathrm{~nm}$ excitation $\mathrm{X}$ coeff. 


$$
\begin{aligned}
& 745=>752 \quad-0.40047 \\
& 745=>753 \quad-0.42630 \\
& 746=>751 \quad 0.13783 \\
& 746=>752-0.11651 \\
& 746=>753 \quad-0.42926
\end{aligned}
$$

Transition dipole moment (debye):

$$
\mathrm{X}=0.2032 \mathrm{Y}=0.0128 \quad \mathrm{Z}=0.0713 \text { Tot }=0.2157
$$

\begin{tabular}{|c|c|}
\hline $742=>75$ & 0.60 \\
\hline 743 & \\
\hline $743=>7$ & 0. \\
\hline $744=>7$ & 0.6 \\
\hline $744=>$ & 0.28 \\
\hline $715-1$ & \\
\hline
\end{tabular}

Oscillator strength, $\mathrm{f}=\quad 0.0004$

Restricted Singlet Excited State 19:

Excitation energy $=0.0783937783$ hartrees $\quad 2.13320324 \mathrm{eV} \quad 581.21 \mathrm{~nm}$

excitation X coeff.

Transition dipole moment (debye):

$$
\mathrm{X}=0.0813 \mathrm{Y}=0.1870 \mathrm{Z}=-0.1903 \text { Tot }=0.2790
$$

Oscillator strength, $\mathrm{f}=0.0006$

Restricted Singlet Excited State 20:

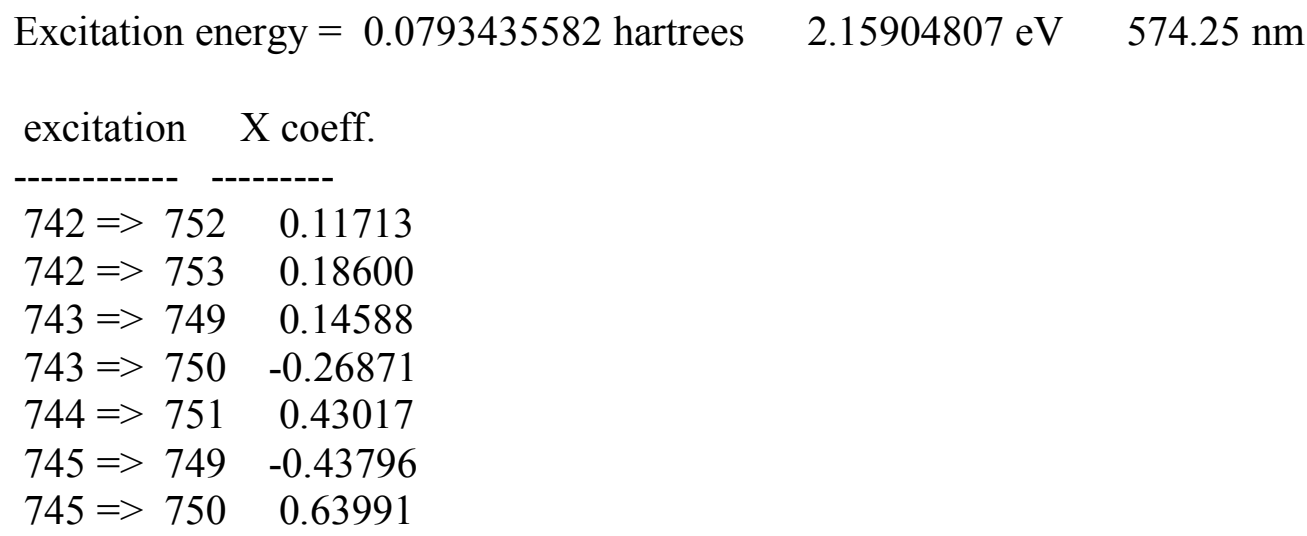


$745=>753 \quad 0.14400$

Transition dipole moment (debye):

$\mathrm{X}=0.4430 \quad \mathrm{Y}=-0.6923 \quad \mathrm{Z}=0.6589 \mathrm{Tot}=1.0534$

Oscillator strength, $\mathrm{f}=\quad 0.0091$

Restricted Singlet Excited State 21:

Excitation energy $=0.0799075748$ hartrees $\quad 2.17439574 \mathrm{eV} \quad 570.20 \mathrm{~nm}$

excitation X coeff.

\begin{tabular}{|c|c|}
\hline $742=>7$ & 0.20273 \\
\hline $742=>7$ & 0.47632 \\
\hline $743=>7$ & 0.11941 \\
\hline $743=>7$ & 0.14829 \\
\hline $743=>7$ & -0.14436 \\
\hline $744=>7$ & 0.49596 \\
\hline $744=>7$ & -0.10729 \\
\hline $745=>7$ & 0.33952 \\
\hline $745=>7$ & -0.31074 \\
\hline $745=>$ & -0.11686 \\
\hline $745=>$ & -0.23560 \\
\hline $745=$ & 0 \\
\hline
\end{tabular}

Transition dipole moment (debye):

$\mathrm{X}=-0.1971 \quad \mathrm{Y}=0.4948 \quad \mathrm{Z}=-0.5053 \quad \mathrm{Tot}=0.7342$

Oscillator strength, $f=0.0044$

Restricted Singlet Excited State 22:

Excitation energy $=0.0801574394$ hartrees $\quad 2.18119490 \mathrm{eV} \quad 568.42 \mathrm{~nm}$

excitation X coeff.

\begin{tabular}{|c|c|}
\hline $742=>75$ & 0.56279 \\
\hline $742=>75$ & 0.11351 \\
\hline $743=>7$ & -0.1617 \\
\hline $743=>$ & 0.13222 \\
\hline $744=$ & 0.2 \\
\hline
\end{tabular}




$$
\begin{aligned}
& 744=>752 \quad-0.56339 \\
& 744=>753 \quad 0.38607 \\
& 745=>749 \quad-0.12030 \\
& 745=>751 \quad-0.16399 \\
& 745=>752 \quad 0.11932 \\
& 745=>753 \quad-0.12428
\end{aligned}
$$

Transition dipole moment (debye):

$$
\mathrm{X}=0.0678 \mathrm{Y}=0.1004 \mathrm{Z}=0.1501 \text { Tot }=0.1929
$$

Oscillator strength, $\mathrm{f}=\quad 0.0003$

Restricted Singlet Excited State 23:

Excitation energy $=0.0808063308$ hartrees $\quad 2.19885214 \mathrm{eV} \quad 563.86 \mathrm{~nm}$

excitation $\mathrm{X}$ coeff.

$$
\begin{aligned}
& 742=>750 \quad-0.21610 \\
& 742=>752 \quad 0.48626 \\
& 742=>753 \quad-0.75290 \\
& 744=>751 \quad 0.26242 \\
& 744=>752 \quad-0.12271 \\
& 745=>752 \quad-0.14274
\end{aligned}
$$

Transition dipole moment (debye):

$$
\mathrm{X}=-0.1337 \mathrm{Y}=0.1607 \mathrm{Z}=-0.2572 \mathrm{Tot}=0.3315
$$

Oscillator strength, $\mathrm{f}=0.0009$

Restricted Singlet Excited State 24:

Excitation energy $=0.0814116035$ hartrees $\quad 2.21532244 \mathrm{eV} \quad 559.67 \mathrm{~nm}$ excitation X coeff.

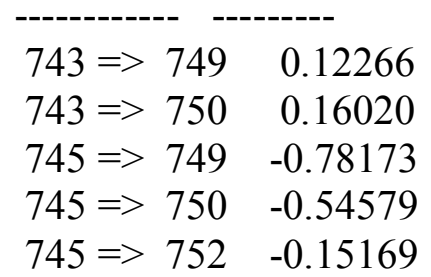


Transition dipole moment (debye):

$$
\mathrm{X}=0.0555 \mathrm{Y}=-0.2123 \quad \mathrm{Z}=1.5257 \mathrm{Tot}=1.5414
$$

Oscillator strength, $\mathrm{f}=\quad 0.0200$

Restricted Singlet Excited State 25:

Excitation energy $=0.0821100011$ hartrees $\quad 2.23432681 \mathrm{eV} \quad 554.91 \mathrm{~nm}$

excitation X coeff.

$742=>749 \quad 0.45164$

$743=>749 \quad-0.15026$

$744=>749 \quad-0.74898$

$744=>750 \quad 0.40004$

Transition dipole moment (debye):

$$
\mathrm{X}=0.0510 \mathrm{Y}=-0.1237 \quad \mathrm{Z}=0.1713 \text { Tot }=0.2174
$$

Oscillator strength, $\mathrm{f}=\quad 0.0004$

Restricted Singlet Excited State 26:

Excitation energy $=0.0826620185$ hartrees $\quad 2.24934797 \mathrm{eV} \quad 551.20 \mathrm{~nm}$

\begin{tabular}{|c|c|}
\hline 749 & -0.8 \\
\hline $\begin{array}{l}744=>749 \\
744=>750\end{array}$ & \\
\hline
\end{tabular}

excitation X coeff.

Transition dipole moment (debye):

$$
\mathrm{X}=-0.1895 \quad \mathrm{Y}=-0.1198 \quad \mathrm{Z}=-0.0019 \text { Tot }=0.2242
$$

Oscillator strength, $\mathrm{f}=\quad 0.0004$

Restricted Singlet Excited State 27:

Excitation energy $=0.0833227139$ hartrees $\quad 2.26732641 \mathrm{eV} \quad 546.83 \mathrm{~nm}$ 
excitation $\mathrm{X}$ coeff.

\begin{tabular}{|c|c|}
\hline $742=>75$ & 0.25769 \\
\hline $743=>74$ & -0.15693 \\
\hline $743=>750$ & -0.13708 \\
\hline $744=>74$ & -0.48738 \\
\hline $744=>$ & -0.765 \\
\hline $744=>752$ & -0.1864 \\
\hline
\end{tabular}

Transition dipole moment (debye):

$$
\mathrm{X}=0.0013 \quad \mathrm{Y}=-0.1128 \quad \mathrm{Z}=0.4322 \mathrm{Tot}=0.4467
$$

Oscillator strength, $\mathrm{f}=\quad 0.0017$

Restricted Singlet Excited State 28:

Excitation energy $=0.0838719224$ hartrees $\quad 2.28227113 \mathrm{eV} \quad 543.25 \mathrm{~nm}$

excitation X coeff.

$748=>754 \quad-0.98794$

Transition dipole moment (debye):

$$
\mathrm{X}=0.0298 \quad \mathrm{Y}=-0.0636 \quad \mathrm{Z}=-0.0815 \text { Tot }=0.1076
$$

Oscillator strength, $f=0.0001$

Restricted Singlet Excited State 29:

Excitation energy $=0.0841914008$ hartrees $\quad 2.29096458 \mathrm{eV} \quad 541.19 \mathrm{~nm}$

$$
\begin{aligned}
& \text { excitation } \mathrm{X} \text { coeff. } \\
& 742=>750 \quad 0.13863 \\
& 743=>749 \quad 0.92226 \\
& 744=>749 \quad-0.22666 \\
& 745=>749 \quad 0.18094
\end{aligned}
$$

Transition dipole moment (debye):

$$
\mathrm{X}=-0.2429 \quad \mathrm{Y}=0.6862 \quad \mathrm{Z}=-4.5972 \quad \mathrm{Tot}=4.6544
$$

Oscillator strength, $\mathrm{f}=0.1882$ 
Restricted Singlet Excited State 30:

Excitation energy $=0.0845547724$ hartrees $\quad 2.30085242 \mathrm{eV} \quad 538.86 \mathrm{~nm}$

excitation $\mathrm{X}$ coeff.

$742=>750 \quad-0.90033$

$742=>753 \quad 0.16493$

$743=>749 \quad 0.14839$

$744=>750 \quad-0.30324$

Transition dipole moment (debye):

$\mathrm{X}=-0.0231 \mathrm{Y}=0.2851 \mathrm{Z}=-1.1256 \mathrm{Tot}=1.1614$

Oscillator strength, $\mathrm{f}=\quad 0.0118$

\subsection{References}

1. Ball, M. et al. Chiral Conjugated Corrals. J. Am. Chem. Soc. 137, 9982-9987 (2015).

2. Haddon, R. C. et al. C60 thin film transistors. Appl. Phys. Lett. 67, 121-123 (1995).

3. Savoie, B. M. et al. Unequal Partnership: Asymmetric Roles of Polymeric Donor and Fullerene Acceptor in Generating Free Charge. J. Am. Chem. Soc. 136, 2876-2884 (2014).

4. Anthony, J. E. et al. n-Type Organic Semiconductors in Organic Electronics. Adv. Mater. 22, 3876-3892 (2010).

5. Huang, Q. et al. Photoconductive Curved-Nanographene/Fullerene Supramolecular Heterojunctions. Angew. Chem. Int. Ed. 58, 6244 (2019).

6. Li, H. et al. High-Mobility Field-Effect Transistors from Large-Area Solution-Grown Aligned C60 Single Crystals. J. Am. Chem. Soc. 134, 2760-2765 (2012).

7. Zhang, Y. et al. Development of fullerenes and their derivatives as semiconductors in fieldeffect transistors: exploring the molecular design. J. Mater. Chem. C 6, 3514-3537 (2018).

8. Zhao, Y., Guo, Y. \& Liu, Y. 25th Anniversary Article: Recent Advances in n-Type and Ambipolar Organic Field-Effect Transistors. Adv. Mater. 25, 5372-5391 (2013).

9. Tremblay, N. J. et al. Photovoltaic Universal Joints: Ball-and-Socket Interfaces in Molecular Photovoltaic Cells. ChemPhysChem 11, 799-803 (2010).

10. Kang, S. J. et al. A Supramolecular Complex in Small-Molecule Solar Cells based on Contorted Aromatic Molecules. Angew. Chem. Int. Ed. 51, 8594-8597 (2012).

11. Shimizu, H. et al. A Saturn-Like Complex Composed of Macrocyclic Oligothiophene and C60 Fullerene: Structure, Stability, and Photophysical Properties in Solution and the Solid 
State. Chem. Eur. J. 24, 3793-3801 (2018).

12. Toyota, S. et al. Exploration of Nano-Saturns: A Spectacular Sphere-Ring Supramolecular System. Chem. Eur. J. 25, 6878-6890 (2019).

13. Lu, X. et al. Bowl-Shaped Carbon Nanobelts Showing Size-Dependent Properties and Selective Encapsulation of C70. J. Am. Chem. Soc. 141, 5934-5941 (2019).

14. Ferrero, S. et al. Dual-Tweezer Behavior of an Octapodal Pyrene Porphyrin-Based System as a Host for Fullerenes. J. Org. Chem. 84, 6183-6190 (2019).

15. Selmani, S. \& Schipper, D. J. $\pi$-Concave Hosts for Curved Carbon Nanomaterials. Chem. Eur. J. 25, 6673-6692 (2019).

16. Xu, Y. et al. Concave-Convex $\pi-\pi$ Template Approach Enables the Synthesis of [10]Cycloparaphenylene-Fullerene [2]Rotaxanes. J. Am. Chem. Soc. 140, 13413-13420 (2018).

17. Würthner, F. et al. Perylene Bisimide Dye Assemblies as Archetype Functional Supramolecular Materials. Chem. Rev. 116, 962-1052 (2016).

18. Liu, Z. et al. Surveying macrocyclic chemistry: from flexible crown ethers to rigid cyclophanes. Chem. Soc. Rev. 46, 2459-2478 (2017).

19. Shi, Y. et al. Selective Extraction of C70 by a Tetragonal Prismatic Porphyrin Cage. J. Am. Chem. Soc. 140, 13835-13842 (2018).

20. Jiao, T. et al. Guest recognition enhanced by lateral interactions. Chem. Sci. 10, 5114-5123 (2019).

21. Samanta, J. et al. Cofacial Organic Click Cage to Intercalate Polycyclic Aromatic Hydrocarbons. Org. Lett. 18, 3394-3397 (2016).

22. Wu, Y. L. et al. Electron Delocalization in a Rigid Cofacial Naphthalene-1,8:4,5bis(dicarboximide) Dimer. Angew. Chem. Int. Ed. 53, 9476-9481 (2014).

23. Haino, T. et al. Fullerenes Enclosed in Bridged Calix[5]arenes. Angew. Chem. Int. Ed. 37, 997-998 (1998).

24. Spenst, P. et al. A Perylene Bisimide Cyclophane as a "Turn-On" and "Turn-Off" Fluorescence Probe. Angew. Chem. Int. Ed. 54, 10165-10168 (2015).

25. Spenst, P. et al. Perylene Bisimide Cyclophanes with High Binding Affinity for Large Planar Polycyclic Aromatic Hydrocarbons: Host-Guest Complexation versus SelfEncapsulation of Side Arms. Chem. Eur. J. 23, 1667-1675 (2017).

26. Song, J. et al. A Porphyrin Nanobarrel That Encapsulates C60. J. Am. Chem. Soc. 132, 16356-16357 (2010).

27. Xia, J. et al. Gram-scale synthesis and crystal structures of [8]- and [10]CPP, and the solidstate structure of C60@[10]CPP. Chem.Sci.3, 3018-3021 (2012).

28. Iwamoto, T. et al. Size-Selective Encapsulation of C60 by [10]Cycloparaphenylene: Formation of the Shortest Fullerene-Peapod. Angew. Chem. Int. Ed. 50, 8342-8344 (2011).

29. Ball, M. et al. Contorted Polycyclic Aromatics. Acc. Chem. Res. 48, 267-276 (2015). 
30. Shimizu, H. et al. Synthesis, Structures, and Photophysical Properties of $\pi$-Expanded Oligothiophene 8-mers and Their Saturn-Like C60 Complexes. J. Am. Chem. Soc. 137, 3877-3885 (2015).

31. Ball, M. et al. Macrocyclization in the Design of Organic n-Type Electronic Materials. $J$. Am. Chem. Soc. 138, 12861-12867 (2016).

32. Bindfit. Available at: http://supramolecular.org.

33. Diao, G. et al. The electrochemical reduction of fullerenes, C60 and C70. Talanta 43, 16331637 (1996).

34. Kishi, N. et al. Selective host-guest interactions of a transformable coordination capsule/tube with fullerenes. Angew. Chem. Int. Ed. 53, 3604-3607 (2014).

35. Tashiro, K. \& Aida, T. Metalloporphyrin hosts for supramolecular chemistry of fullerenes. Chem. Soc. Rev. 36, 189-197 (2007).

36. Ke, X.-S. et al. Flattened Calixarene-like Cyclic BODIPY Array: A New Photosynthetic Antenna Model. J. Am. Chem. Soc. 139, 13950-13956 (2017).

37. Moreira, L. et al. Unveiling the nature of supramolecular crown ether-C60 interactions. Chem. Sci. 6, 4426-4432 (2015).

38. Hua, J. et al. Novel soluble and thermally-stable fullerene dyad containing perylene. $J$. Mater. Chem. 14, 1849-1853 (2004).

39. Baffreau, J. et al. Fullerene C60-Perylene-3,4:9,10-bis(dicarboximide) Light-Harvesting Dyads: Spacer-Length and Bay-Substituent Effects on Intramolecular Singlet and Triplet Energy Transfer. Chem. Eur. J. 14, 4974-4992 (2008).

40. Hitosugi, S. et al. Asymmetric Autocatalysis Initiated by Finite Single-Wall Carbon Nanotube Molecules with Helical Chirality. Org. Lett. 16, 645-647 (2014).

41. Fan, Y.-Y. et al. An isolable catenane consisting of two Möbius conjugated nanohoops. Nat. Commun. 9, 3037 (2018).

42. Dietrich-Buchecker, C. et al. Quantitative Formation of [2]Catenanes Using Copper(I) and Palladium(II) as Templating and Assembling Centers: The Entwining Route and the Threading Approach. J. Am. Chem. Soc. 125, 5717-5725 (2003).

43. Darzi, E. R. et al. An Operationally Simple and Mild Oxidative Homocoupling of Aryl Boronic Esters To Access Conformationally Constrained Macrocycles. J. Am. Chem. Soc. 139, 3106-3114 (2017).

44. Kayahara, E. et al. Gram-Scale Syntheses and Conductivities of $\{[\} 10]$ Cycloparaphenylene and Its Tetraalkoxy Derivatives. J. Am. Chem. Soc. 139, 18480-18483 (2017).

45. Shekhar, S. et al. Distinct electronic effects on reductive eliminations of symmetrical and unsymmetrical bis-aryl platinum complexes. J. Am. Chem. Soc. 126, 13016-13027 (2004). 